\title{
An Intramolecular Wittig Approach toward Heteroarenes: Synthesis of Pyrazoles, Isoxazoles and Chromenone-oximes.
}

Pankaj V. Khairnar, Tsai-Hui Lung, Yi-Jung Lin, Chi-Yi Wu, Srinivasa Rao Koppolu, Athukuri Edukondalu, Praneeth Karanam and Wenwei Lin*

\section{Table of Contents:}

I. General information

II. Reaction conditions optimization

III. Experimental procedures

a. Typical procedure (TP-1) for the preparation of $\alpha$-halohydrazone derivatives $\mathbf{1}$

b. Typical procedure (TP-2) for the preparation of pyrazole derivatives $\mathbf{3}$

c. Typical procedure (TP-3) for the preparation of $\alpha$-haloketoxime derivatives $\mathbf{5}$

d. Typical procedure (TP-4) for the preparation of isoxazole derivatives 6

e. Typical procedure (TP-5) for the preparation of phosphonium salts 4 and 7

f. Typical procedure (TP-6) for the preparation of pyrazole or isoxazole $\mathbf{3}$ or $\mathbf{6}$ from phosphonium salt $\mathbf{4}$ or $\mathbf{7}$ 
g. Typical procedure (TP-7) for the preparation of $o$-hydroxy chloroacetophenone $\mathbf{S 1}$

h. Typical procedure (TP-8) for the preparation of compound

i. Typical procedure (TP-9) for the preparation of $\alpha$-haloketoxime S3

j. Typical procedure (TP-10) for the preparation of phosphonium salt 8a

k. Typical procedure (TP-11) for the preparation of compound S4

1. Typical procedure (TP-12) for the preparation of compounds S5-S8

m. Typical procedure (TP-13) for the preparation of bis-acyl compounds $\mathbf{1 3}$

n. Typical procedure (TP-14) for the preparation of isoxazole derivatives $\mathbf{1 2}$ from phosphonium salt $\mathbf{8 a}$

o. Typical procedure (TP-15) for the preparation of chromenone derivatives $\mathbf{1 1}$ from phosphonium salt $\mathbf{8 a}$

p. Typical procedure (TP-16) for the preparation of chromenone derivatives $\mathbf{1 1}$ from bis-acyl compounds $\mathbf{1 3}$

IV. Analytical data for all new compounds

V. References

VI. X-ray crystallographic data for selected compounds

VII. ${ }^{1} \mathrm{H}$ NMR and ${ }^{13} \mathrm{C}$ NMR spectra of all new compounds S82-S259 


\section{General Information:}

All reactions were carried out under argon atmosphere in an oven-dried glassware employing standard Schlenk techniques. Unless otherwise mentioned, all the starting materials that were purchased from commercial sources were used without further purification. THF was continuously refluxed and freshly distilled from sodium benzophenone ketyl under argon atmosphere. Yields refer to isolated yields of compounds estimated to be $>95 \%$ pure as determined by ${ }^{1} \mathrm{H}$ NMR. ${ }^{1} \mathrm{H},{ }^{31} \mathrm{P}$ NMR and

${ }^{13} \mathrm{C}$ spectra were recorded on an $\mathrm{AV}-400$ Bruker instrument using $\mathrm{CDCl}_{3}, \mathrm{MeOD}$ or DMSO- $\mathrm{d}_{6}$ as solvents at 400 and $100 \mathrm{MHz}$ respectively. Chemical shifts are reported in ppm relative to either TMS ( $\delta 0.00 \mathrm{ppm}$ for $\left.{ }^{1} \mathrm{H} \mathrm{NMR}\right), \mathrm{H}_{3} \mathrm{PO}_{4}\left(\delta 0.00 \mathrm{ppm}\right.$ for ${ }^{31} \mathrm{P}$ $\mathrm{NMR}), \mathrm{C}_{6} \mathrm{H}_{5} \mathrm{~F}$ ( $\delta-113.15 \mathrm{ppm}$ for $\left.{ }^{19} \mathrm{~F} \mathrm{NMR}\right)$ or residual solvent $\mathrm{CDCl}_{3}(\delta 77.0 \mathrm{ppm}$ for ${ }^{13} \mathrm{C} \mathrm{NMR}$ ), MeOD ( $\delta 3.31 \mathrm{ppm}$ for ${ }^{1} \mathrm{H}$ NMR and $49.0 \mathrm{ppm}$ for ${ }^{13} \mathrm{C} \mathrm{NMR}$ ) or DMSO ( $\delta 2.50 \mathrm{ppm}$ for ${ }^{1} \mathrm{H}$ NMR and $39.5 \mathrm{ppm}$ for $\left.{ }^{13} \mathrm{C} \mathrm{NMR}\right)$. Analytical thin layer chromatography (TLC) was performed using Merck 60 F254 pre-coated silica gel plate (0.2 mm thickness). Flash chromatography was performed using Merck silica gel 60 . 


\section{Reaction conditions optimization:}

Table S1. Optimization of the reaction conditions for the synthesis of pyrazole ${ }^{a}$<smiles>O=[N+]([O-])c1ccc(NN=C(CCl)c2ccccc2)cc1</smiles>

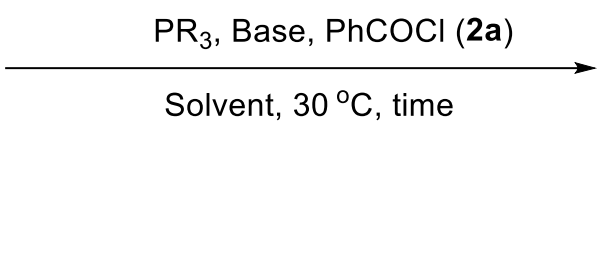

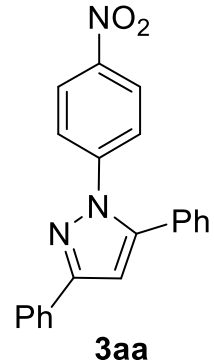

3aa

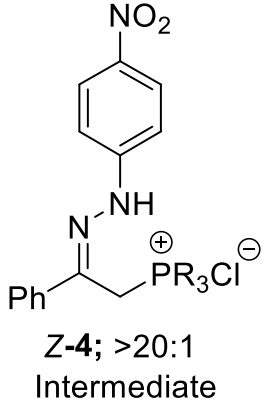

$3 \mathbf{a a}^{b}(4)^{b}(\%)$

$61(29)$

$39(32)$

$34(32)$

$80(0)$

$71(0)$

$26(63)$

$34(35)$

trace (50)

$20(53)$

$60(13)$

$81(0)$

$50(17)$

$67(0)$

$26(68)$

$17^{8}$

$\mathrm{PPh}_{3}$

$\mathrm{Et}_{3} \mathrm{~N}$

$\mathrm{CH}_{2} \mathrm{Cl}_{2}$

5

${ }^{a}$ To a solution of $\mathbf{1 a}(0.3 \mathrm{mmol}), \mathrm{PR}_{3}(1.1$ equiv) in dry solvent $(3 \mathrm{~mL})$, base (3.0 equiv) and $\mathrm{PhCOCl}(\mathbf{2 a})(1.2$
equiv) were added sequentially under argon atmosphere at $30{ }^{\circ} \mathrm{C}$. ${ }^{b}$ Yield of $\mathbf{3 a a}$ and $Z-\mathbf{4}$ was determined by NMR analysis of the crude reaction mixture using $\mathrm{Ph}_{3} \mathrm{CH}$ as an internal standard. ${ }^{c} 2$ equiv of $\mathrm{Et}_{3} \mathrm{~N}$. ${ }^{d} 2.5$ equiv of $\mathrm{Et}_{3} \mathrm{~N}$. ${ }^{e} 1.5 \mathrm{~mL} \mathrm{CH}_{2} \mathrm{Cl}_{2} .{ }^{f} 4.5 \mathrm{~mL} \mathrm{CH}_{2} \mathrm{Cl}_{2} .{ }^{g} \mathrm{PhCOCl}, \mathrm{Et}_{3} \mathrm{~N}$ were sequentially added. 
Table S2. Optimization of the reaction conditions for the synthesis of isoxazole ${ }^{a}$

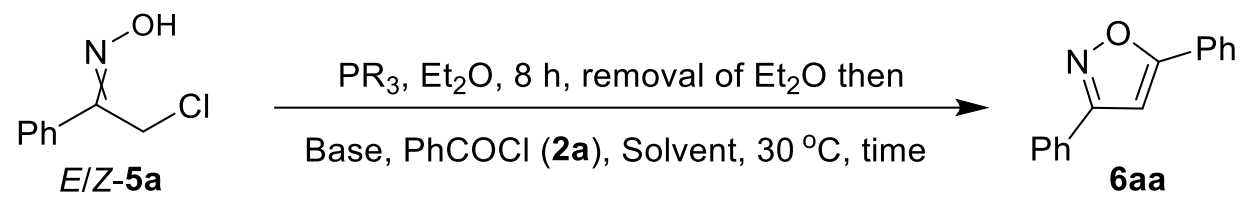

\begin{tabular}{|c|c|c|c|c|c|}
\hline entry & $\mathbf{P R}_{3}$ & base & solvent & $t(\mathbf{h})$ & $6 a^{b}(\%)$ \\
\hline 1 & $\mathrm{PBu}_{3}$ & DBU & THF & 2 & 67 \\
\hline 2 & $\mathrm{PEt}_{2} \mathrm{Ph}$ & DBU & THF & 2 & 46 \\
\hline 3 & $\mathrm{PEtPh}_{2}$ & DBU & THF & 2 & 10 \\
\hline 4 & $\mathrm{PPh}_{3}$ & DBU & THF & 2 & 07 \\
\hline 5 & $\mathrm{PBu}_{3}$ & $\mathrm{Et}_{3} \mathrm{~N}$ & THF & 1 & 05 \\
\hline 6 & $\mathrm{PBu}_{3}$ & DIPEA & THF & 1 & 0 \\
\hline 7 & $\mathrm{PBu}_{3}$ & Pyridine & THF & 1 & $05(80)^{c}$ \\
\hline 8 & $\mathrm{PBu}_{3}$ & TBD & THF & 3 & 53 \\
\hline 9 & $\mathrm{PBu}_{3}$ & MTBD & THF & 3 & 86 \\
\hline 10 & $\mathrm{PBu}_{3}$ & TMG & THF & 3 & $37(15)^{c}$ \\
\hline 11 & $\mathrm{PBu}_{3}$ & MTBD & $\mathrm{Et}_{2} \mathrm{O}$ & 3 & 76 \\
\hline 12 & $\mathrm{PBu}_{3}$ & MTBD & toluene & 3 & 66 \\
\hline 13 & $\mathrm{PBu}_{3}$ & MTBD & $\mathrm{CH}_{2} \mathrm{Cl}_{2}$ & 3 & 50 \\
\hline 14 & $\mathrm{PBu}_{3}$ & MTBD & $\mathrm{CH}_{3} \mathrm{CN}$ & 3 & 54 \\
\hline 15 & $\mathrm{PBu}_{3}$ & MTBD & EtOAC & 3 & 74 \\
\hline $16^{d}$ & $\mathrm{PBu}_{3}$ & MTBD & THF & 1 & 62 \\
\hline $17^{e}$ & $\mathrm{PBu}_{3}$ & MTBD & THF & 3 & 65 \\
\hline $18^{f}$ & $\mathrm{PBu}_{3}$ & MTBD & THF & 1 & 77 \\
\hline $19^{g}$ & $\mathrm{PBu}_{3}$ & MTBD & THF & 1 & 76 \\
\hline $20^{h}$ & $\mathrm{PBu}_{3}$ & MTBD & THF & 3 & $10(68)^{c}$ \\
\hline
\end{tabular}

${ }^{a}$ To a solution of $\mathbf{5 a}(0.2 \mathrm{mmol}), \mathrm{PR}_{3}$ (1.1 equiv) in dry solvent $(2 \mathrm{~mL})$, base (4 equiv) and $\mathrm{PhCOCl}$ (1.2 equiv) were added sequentially under argon atmosphere at $30{ }^{\circ} \mathrm{C} .{ }^{b}$ Yield of $6 \mathbf{a a}$ was determined by ${ }^{1} \mathrm{H}$ NMR analysis of the crude reaction mixture using $\mathrm{Ph}_{3} \mathrm{CH}$ as an internal standard. ${ }^{c}$ The numbers in parentheses refer to the yields of corresponding unreacted phosphonium salt Z-7a. ${ }^{d} 2$ equiv of MTBD. ${ }^{e} 3$ equiv of MTBD. ${ }^{f} 1 \mathrm{~mL}$ THF. g $3 \mathrm{~mL}$ THF was used. ${ }^{h} \mathrm{PhCOCl}$, MTBD were sequentially added. 
Scheme S1. Optimization studies on isoxazole 6aa synthesis in one step<smiles>O/N=C(/CCl)c1ccccc1</smiles>

THF: $48 \%, 3 \mathrm{~h}$

$\mathrm{Et}_{2} \mathrm{O}: 51 \%, 3 \mathrm{~h}$

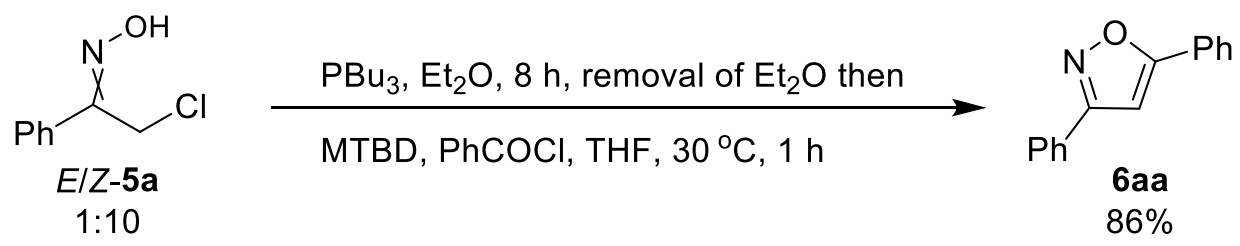

Scheme S2. Synthesis of pyrazole 3aa from the phosphonium salt Z-4a

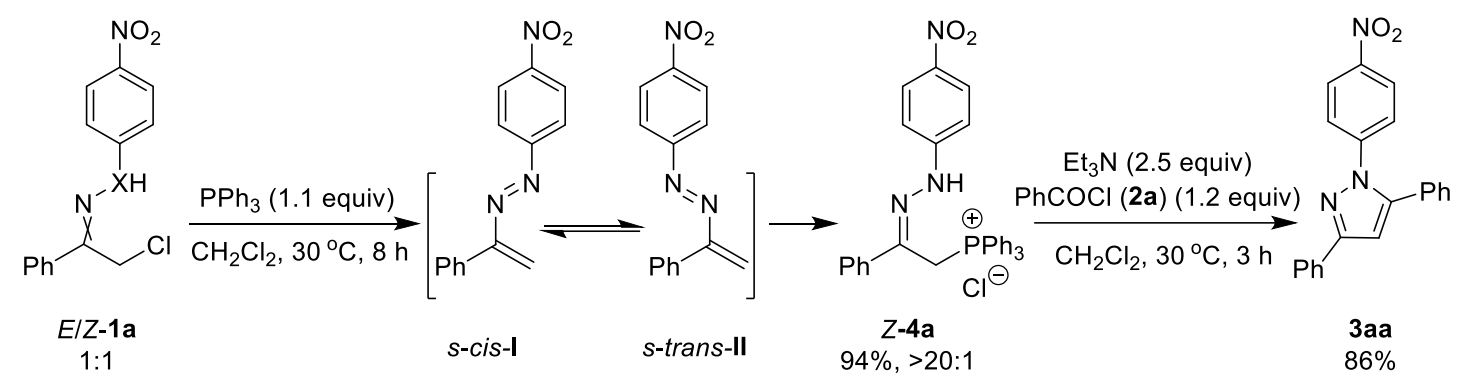

Scheme S3. Synthesis of isoxazole 6aa from the phosphonium salt Z-7a

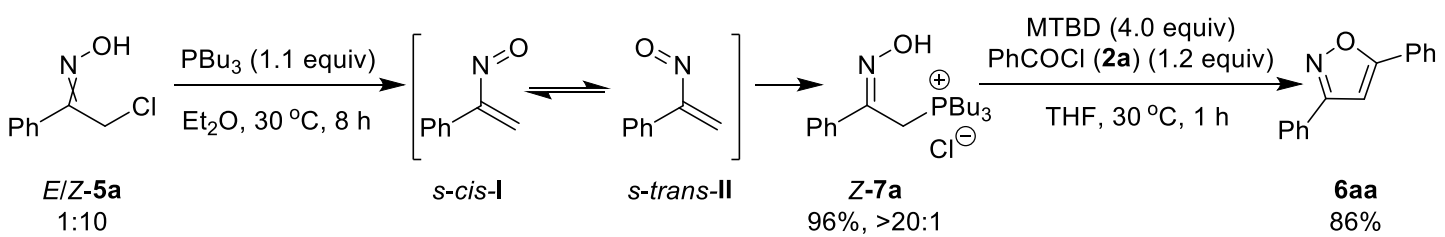

Scheme S4. Formation of rearranged isoxazole 12a without acyl chloride $\left(\mathrm{R}^{3} \mathrm{COCl}\right)$<smiles>O[13CH]C/C(=N\Oc1ccccc1Oc1ccccc1)c1ccccc1</smiles>

$8 \mathbf{a}$

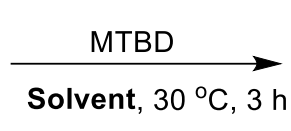

THF: $\quad 55 \%$

$\mathrm{CH}_{2} \mathrm{Cl}_{2}: \quad 55 \%$ 
Table S3. Optimization of the reaction conditions for the synthesis of isoxazole and chromenone-oxime derivatives $^{a}$<smiles>O=C(C/C(=N\O)c1ccccc1OC(=O)c1ccccc1)c1ccccc1</smiles>

$8 \mathbf{a}$<smiles>C=C(/C=C/c1ccccc1)c1ccccc1OC(=O)c1ccccc1</smiles>

12aa<smiles>O=C(O/N=c1\cc(-c2ccccc2)oc2ccccc12)c1ccccc1</smiles>

11 aa

\begin{tabular}{|c|c|c|c|c|c|}
\hline entry & base & solvent & $t(\mathbf{h})$ & $12 \mathbf{a a}^{b}(\%)$ & $11 \mathbf{a a}^{b}(\%)$ \\
\hline 1 & MTBD & THF & 5 & 57 & 19 \\
\hline 2 & MTBD & $\mathrm{CH}_{2} \mathrm{Cl}_{2}$ & 3 & 9 & 61 \\
\hline 3 & MTBD & toluene & 3 & 31 & 46 \\
\hline 4 & MTBD & $\mathrm{CH}_{3} \mathrm{CN}$ & 3 & 38 & 10 \\
\hline 5 & MTBD & $\mathrm{Et}_{2} \mathrm{O}$ & 5 & 49 & 24 \\
\hline 6 & MTBD & EtOAC & 3 & 48 & 18 \\
\hline 7 & MTBD & DMF & 3 & 44 & 7 \\
\hline 8 & MTBD & acetone & 3 & 29 & 38 \\
\hline 9 & DBU & THF & 5 & 39 & 30 \\
\hline 10 & TMG & THF & 5 & 11 & 15 \\
\hline 11 & $\mathrm{Et}_{3} \mathrm{~N}$ & $\mathrm{THF}$ & 5 & 0 & 23 \\
\hline $12^{c}$ & MTBD & $\mathrm{THF}$ & 5 & 51 & 29 \\
\hline $13^{d}$ & MTBD & THF & 5 & 54 & 21 \\
\hline $14^{d}$ & MTBD & $\mathrm{CH}_{2} \mathrm{Cl}_{2}$ & 3 & 11 & 61 \\
\hline
\end{tabular}

${ }^{a}$ To a solution of compound $\mathbf{8 a}(0.2 \mathrm{mmol})$ in dry solvent $(2 \mathrm{~mL})$, base (4 equiv) and $\mathrm{PhCOCl}$ (1.2 equiv) were added sequentially under argon atmosphere at $30{ }^{\circ} \mathrm{C}$. ${ }^{b}$ Yield was determined by ${ }^{1} \mathrm{H}$-NMR analysis of crude reaction mixture. ${ }^{c} 3.0$ equiv of MTBD was used. ${ }^{d} 2.5$ equiv of MTBD was used. 


\section{Experimental procedures:}

a. Typical procedure (TP-1) for the preparation of $\alpha$-halohydrazone derivatives $1:^{1}$

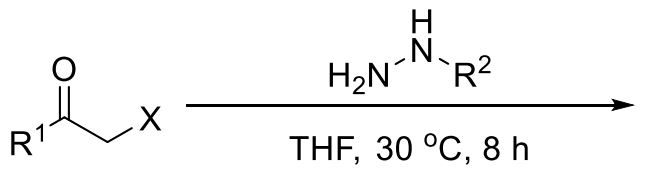<smiles>[X]CC([R])NN[R]</smiles>

1

To a solution of substituted $\alpha$-haloacetophenone $(20 \mathrm{mmol})$ in THF $(1.6 \mathrm{~mL} / \mathrm{mmol}$ ketone) was added hydrazine (1.1 equiv). The mixture was stirred at $30{ }^{\circ} \mathrm{C}$ for $8 \mathrm{~h}$. Thereafter, solvent was removed by evaporation in vacuo and the product $\mathbf{1}$ was obtained after washing with cold $\mathrm{Et}_{2} \mathrm{O}$ and hexane.

b. Typical procedure (TP-2) for the preparation of pyrazole derivatives 3:<smiles>[X]CC([R])NN[R]</smiles>

1

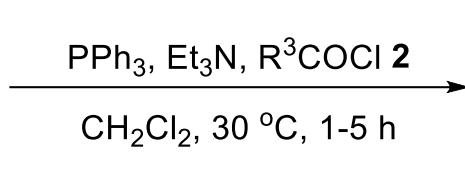

$\mathrm{CH}_{2} \mathrm{Cl}_{2}, 30{ }^{\circ} \mathrm{C}, 1-5 \mathrm{~h}$<smiles>[R]c1cc([R])n([R])n1</smiles>

3

A dry and argon-flushed $10 \mathrm{~mL}$ Schlenk flask equipped with a magnetic stir bar and septum was sequentially charged with $1(0.3 \mathrm{mmol})$, anhydrous $\mathrm{CH}_{2} \mathrm{Cl}_{2}(3 \mathrm{~mL}), \mathrm{PPh}_{3}$ (1.1 equiv), $\mathrm{Et}_{3} \mathrm{~N}$ (2.5 equiv) and acyl chloride 2 (1.2 equiv). The reaction mixture was stirred for $1-5 \mathrm{~h}$ at $30{ }^{\circ} \mathrm{C}$. After completion of the reaction, solvent was removed in vacuo and the crude residue was subjected to flash column chromatography on silica gel to obtain the product 3 .

c. Typical procedure (TP-3) for the preparation of $\alpha$-haloketoxime derivatives $5:^{2}$

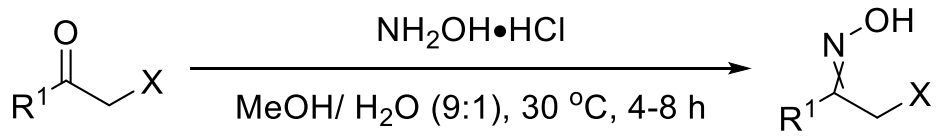


A solution of substituted $\alpha$-haloacetophenone $(0.3 \mathrm{mmol})$ in methanol $(1.6 \mathrm{~mL} / \mathrm{mmol}$ ketone) was added with a solution of hydroxylamine hydrochloride (3.0 equiv) in water $(0.2 \mathrm{~mL} / \mathrm{mmol}$ ketone $)$. The mixture was stirred at $30{ }^{\circ} \mathrm{C}$ for $8 \mathrm{~h}$. After completion of the reaction, solvent was removed in vacuo. The resulting solids were dissolved in water $(3 \mathrm{~mL})$ and extracted with $\mathrm{CH}_{2} \mathrm{Cl}_{2}(2 \times 10 \mathrm{~mL})$, the organic layer was dried over anhydrous sodium sulfate and concentrated under reduced pressure. The resulting solids were washed with hexanes $(3 \times 3 \mathrm{~mL})$ and dried in vacuo to yield the product 5 .

\section{d. Typical procedure (TP-4) for the preparation of isoxazole derivatives 6:}

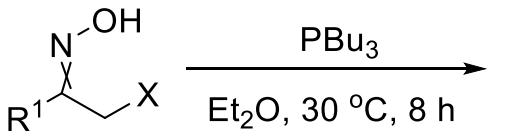

5

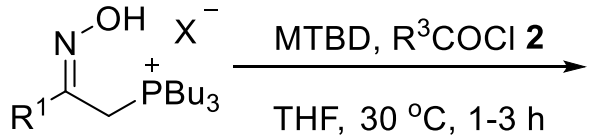

7<smiles>[R]c1cc([R])on1</smiles>

A dry and argon-flushed $10 \mathrm{~mL}$ Schlenk flask equipped with a magnetic stir bar and septum was sequentially charged with $5(0.2 \mathrm{mmol})$, anhydrous $\mathrm{Et}_{2} \mathrm{O}(2.0 \mathrm{~mL})$, and $\mathrm{PBu}_{3}$ (1.1 equiv). The reaction mixture was stirred for $8 \mathrm{~h}$ at $30{ }^{\circ} \mathrm{C}$. Then the solvent was removed in vacuo and dissolved in anhydrous THF (2.0 mL), MTBD (4.0 equiv) and acyl chloride (1.2 equiv) were added sequentially. The reaction mixture was stirred for $1-4 \mathrm{~h}$ at $30{ }^{\circ} \mathrm{C}$. After the completion of reaction, solvent was removed in vacuo and the crude residue was subjected to flash column chromatography on silica gel to obtain the product 6 .

\section{e. Typical procedure (TP-5) for the preparation of phosphonium salts 4 and 7:}<smiles>[X]CC([R])N[X]</smiles>

1 or 5

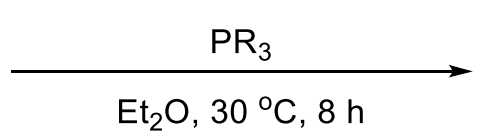

$\mathrm{X}=-\mathrm{NR}^{2}$ or $-\mathrm{O}-$<smiles>[Y]N=C([R])C[R16]([H])=O</smiles>

4 or 7

A dry and argon-flushed $10 \mathrm{~mL}$ round-bottomed flask equipped with a magnetic stirring bar and a septum was charged with a solution of hydrazone $\mathbf{1}$ or 
$\alpha$-haloketoxime $5(0.3 \mathrm{mmol})$ in $\mathrm{CH}_{2} \mathrm{Cl}_{2}$ or $\mathrm{Et}_{2} \mathrm{O}(3 \mathrm{~mL})$ was added $\mathrm{PPh}_{3}$ or $\mathrm{PBu}_{3}(1.1$ equiv). The mixture was stirred at $30{ }^{\circ} \mathrm{C}$ for $8 \mathrm{~h}$. Thereafter, the solvent was removed by evaporation in vacuo and the corresponding phosphonium salt $\mathbf{4}$ or $\mathbf{7}$ was obtained in quantitative yield after washing with hexane.

\section{f. Typical procedure (TP-6) for the preparation of pyrazole or isoxazole 3 or 6} from phosphonium salt 4 or 7 :

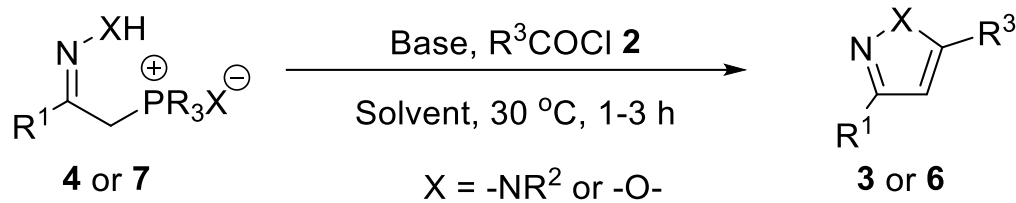

A dry and argon-flushed $10 \mathrm{~mL}$ Schlenk flask equipped with a magnetic stir bar and septum was sequentially charged with phosphonium salt 4 or $7(0.3 \mathrm{mmol})$, anhydrous $\mathrm{CH}_{2} \mathrm{Cl}_{2}$ or THF (3 mL), Et $3 \mathrm{~N}$ (2.5 equiv) or MTBD (4 equiv) and acyl chloride (1.2 equiv). The reaction mixture was stirred for the $1-3 \mathrm{~h}$ at $30^{\circ} \mathrm{C}$. After completion of the reaction, solvent was removed in vacuo and the crude residue was subjected to flash column chromatography on silica gel to obtain the product $\mathbf{3}$ or $\mathbf{6}$.

g. Typical procedure (TP-7) for the preparation of o-hydroxy chloroacetophenone $\mathbf{S 1}:^{3,4}$
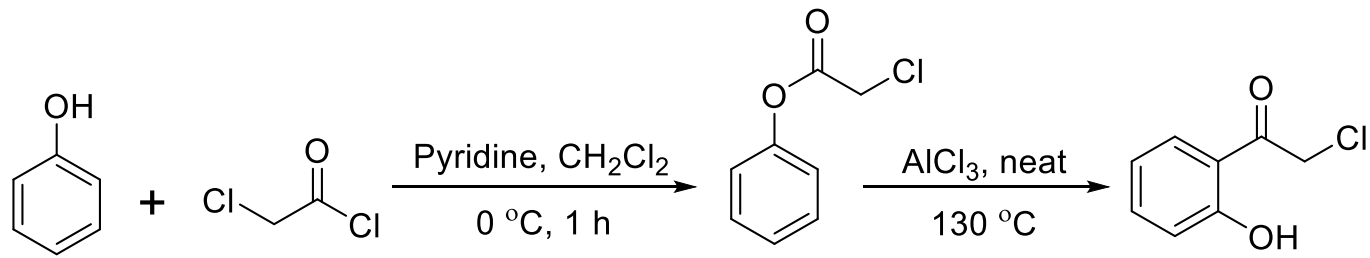

S1

To a solution of phenol $(10 \mathrm{mmol})$ in $\mathrm{CH}_{2} \mathrm{Cl}_{2}(60 \mathrm{~mL})$ chloroacetyl chloride $(1.2 \mathrm{~mL}$, $15 \mathrm{mmol})$ and pyridine $(1.21 \mathrm{~mL}, 15 \mathrm{mmol})$ was added at $0{ }^{\circ} \mathrm{C}$. The mixture was brought to room temperature and stirred for $1 \mathrm{~h}$. Then the reaction was quenched by addition of water $(60 \mathrm{~mL})$. The organic layer was separated, washed with water (50 
$\mathrm{mL}$ ) and dried over anhydrous sodium sulfate. The solvent was evaporated in vacuo to afford the products which as such used for the next step.

A mixture of powdered anhydrous aluminum chloride $(60 \mathrm{mmol})$ and phenyl chloroacetate $(30 \mathrm{mmol})$ was heated at $135-140{ }^{\circ} \mathrm{C}$ for $7 \mathrm{~h}$. The reaction mixture was quenched with cooled water at $0{ }^{\circ} \mathrm{C}$, then the reaction mixture was extracted with EtOAc (15 mL x 3) and organic layer was dried over anhydrous sodium sulfate. The solvent was evaporated in vacuo and the crude residue was subjected to flash column chromatography on silica gel to obtain the product $\mathbf{S 1}$.

h. Typical procedure (TP-8) for the preparation of compound S2:<smiles>O=C(CCl)c1ccccc1O</smiles>

S1

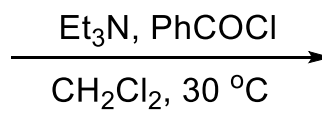<smiles>O=C(Oc1ccccc1C(=O)CCl)c1ccccc1</smiles>

S2

A dry and argon-flushed $10 \mathrm{~mL}$ Schlenk flask equipped with a magnetic stir bar and septum was sequentially charged with $\mathbf{S 1}(3 \mathrm{mmol})$, anhydrous $\mathrm{CH}_{2} \mathrm{Cl}_{2}(7 \mathrm{~mL}), \mathrm{Et}_{3} \mathrm{~N}$ (1.5 equiv) and benzoyl chloride (1.2 equiv). The reaction mixture was stirred for $8 \mathrm{~h}$ at $30{ }^{\circ} \mathrm{C}$. After completion of the reaction, solvent was removed in vacuo and the crude residue was subjected to flash column chromatography on silica gel to obtain the product $\mathbf{S 2}$.

\section{i. Typical procedure (TP-9) for the preparation of $\alpha$-haloketoxime S3: ${ }^{2}$}<smiles>O=C(Oc1ccccc1C(=O)CCl)c1ccccc1</smiles>

S2

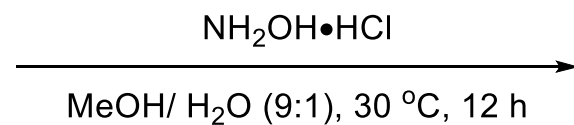

$\mathrm{MeOH} / \mathrm{H}_{2} \mathrm{O}(9: 1), 30{ }^{\circ} \mathrm{C}, 12 \mathrm{~h}$<smiles>O=C(Oc1ccccc1/C(CCl)=N/O)c1ccccc1</smiles>

S3 
To a solution of $\mathbf{S 2}(5 \mathrm{mmol})$ in $\mathrm{MeOH}(18 \mathrm{~mL})$, a solution of hydroxylamine hydrochloride (3.0 equiv) in water $(2 \mathrm{~mL})$ was added. The mixture was stirred at 30 ${ }^{\circ} \mathrm{C}$ for $12 \mathrm{~h}$. After completion of the reaction, solvent was removed in vacuo. The resulting solids were dissolved in water $(30 \mathrm{~mL})$ and extracted with $\mathrm{CH}_{2} \mathrm{Cl}_{2}(3 \times 15$ $\mathrm{mL}$ ), the organic layer was dried over anhydrous sodium sulfate and concentrated under reduced pressure. The product $\mathbf{S 3}$ was obtained after washing with hexanes $(3 \mathrm{x}$ $5 \mathrm{~mL}$ ) and dried in vacuo.

\section{j. Typical procedure (TP-10) for the preparation of phosphonium salt 8a:}<smiles>O=C(Oc1ccccc1/C(CCl)=N/O)c1ccccc1</smiles>

S3

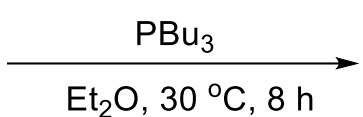

$\mathrm{Et}_{2} \mathrm{O}, 30^{\circ} \mathrm{C}, 8 \mathrm{~h}$<smiles>CC(C)(Cl)[Pb]C/C(=N\O)c1ccccc1OC(=O)c1ccccc1</smiles>

A dry and argon-flushed $10 \mathrm{~mL}$ Schlenk flask equipped with a magnetic stir bar and septum was sequentially charged with $\alpha$-haloketoxime $\mathbf{S 3}(2 \mathrm{mmol}), \mathrm{Et}_{2} \mathrm{O}(10 \mathrm{~mL})$ and $\mathrm{PBu}_{3}\left(1.1\right.$ equiv). The mixture was stirred for $8 \mathrm{~h}$ at $30{ }^{\circ} \mathrm{C}$. Thereafter, the solvent was removed by evaporation in vacuo and the corresponding phosphonium salt $\mathbf{8 a}$ was obtained in quantitative yield after washing with hexane.

\section{k. Typical procedure (TP-11) for the preparation of compound S4: ${ }^{2}$}<smiles>O=C(CCl)c1ccccc1O</smiles>

S1

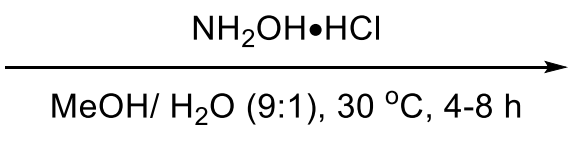<smiles>O/N=C(\CCl)c1ccccc1O</smiles>

S4

A solution of $\mathbf{S 1}(5 \mathrm{mmol})$ in $\mathrm{MeOH}(18 \mathrm{~mL})$ was added with a solution of hydroxylamine hydrochloride (3.0 equiv) in water $(2 \mathrm{~mL})$. The mixture was stirred at 
$30{ }^{\circ} \mathrm{C}$ for $8 \mathrm{~h}$. After completion of the reaction, solvent was removed in vacuo. The resulting solids were dissolved in water $(30 \mathrm{~mL})$ and extracted with $\mathrm{CH}_{2} \mathrm{Cl}_{2}(3 \times 15$ $\mathrm{mL}$ ), the organic layer was dried over anhydrous sodium sulfate and concentrated under reduced pressure. The resulting solids were washed with hexanes ( $3 \times 5 \mathrm{~mL})$ and dried in vacuo to yield the product $\mathbf{S 4}$.

\section{Typical procedure (TP-12) for the preparation of compounds S5-S8:}<smiles>O/N=C(\CCl)c1ccccc1O</smiles>

S4

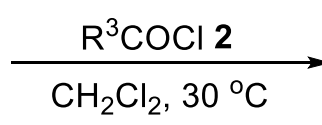$$
\mathrm{CH}_{2} \mathrm{Cl}_{2}, 30^{\circ} \mathrm{C}
$$

A dry and argon-flushed $10 \mathrm{~mL}$ Schlenk flask equipped with a magnetic stir bar and septum was sequentially charged with $\mathbf{S 4}(3 \mathrm{mmol})$, anhydrous $\mathrm{CH}_{2} \mathrm{Cl}_{2}(7 \mathrm{~mL})$, acyl chloride (1.2 equiv). The reaction mixture was stirred for $3-5 \mathrm{~h}$ at $30{ }^{\circ} \mathrm{C}$. After completion of the reaction, solvent was removed in vacuo and the crude residue was subjected to flash column chromatography on silica gel to obtain the products S5-S8.

m. Typical procedure (TP-13) for the preparation of compounds 13:<smiles>[R]C(=O)O/N=C(\CCl)c1ccccc1O</smiles>

S5

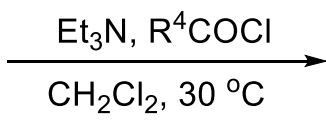

A dry and argon-flushed $10 \mathrm{~mL}$ Schlenk flask equipped with a magnetic stir bar and septum was sequentially charged with $\mathbf{S 5}(3 \mathrm{mmol})$, anhydrous $\mathrm{CH}_{2} \mathrm{Cl}_{2}(7 \mathrm{~mL}), \mathrm{Et}_{3} \mathrm{~N}$ 
(1.5 equiv) and acyl chloride (1.2 equiv). The reaction mixture was stirred for 6-12 h at $30{ }^{\circ} \mathrm{C}$. After completion of the reaction, solvent was removed in vacuo and the crude residue subjected to flash column chromatography on silica gel to obtain the product 13 .

n. Typical procedure (TP-14) for the preparation of isoxazole derivatives 12 from phosphonium salt 8a:

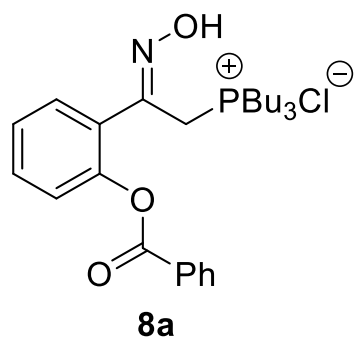

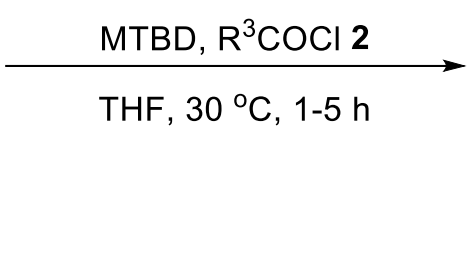<smiles>[R]C(=O)Oc1ccccc1-c1cc(-c2ccccc2)on1</smiles>

A dry and argon-flushed $10 \mathrm{~mL}$ Schlenk flask equipped with a magnetic stir bar and septum was sequentially charged with phosphonium salt $8 \mathbf{a}(0.2 \mathrm{mmol})$, anhydrous THF (2 mL), MTBD (2.5 equiv) and acyl chloride (1.2 equiv). The reaction mixture was stirred for $1-5 \mathrm{~h}$ at $30{ }^{\circ} \mathrm{C}$. After completion of the reaction, solvent was removed in vacuo and the crude residue subjected to flash column chromatography on silica gel to obtain the product 12 .

o. Typical procedure (TP-15) for the preparation of chromenone derivatives 11 from phosphonium salt 8a:

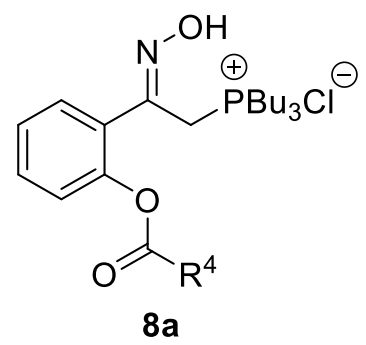

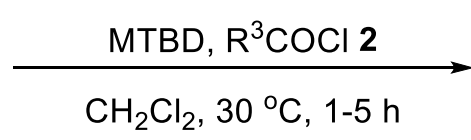<smiles>[R]C(=O)O/N=c1\cc([Z10])oc2ccccc12</smiles>

11

A dry and argon-flushed $10 \mathrm{~mL}$ Schlenk flask equipped with a magnetic stir bar and septum was sequentially charged with phosphonium salt 8a $(0.2 \mathrm{mmol})$, anhydrous 
$\mathrm{CH}_{2} \mathrm{Cl}_{2}$ (2 mL), MTBD (2.5 equiv) and acyl chloride (1.2 equiv). The reaction mixture was stirred for $1-5 \mathrm{~h}$ at $30{ }^{\circ} \mathrm{C}$. After completion of the reaction, solvent was removed in vacuo and the crude residue subjected to flash column chromatography on silica gel to obtain the product $\mathbf{1 1}$.

p. Typical procedure (TP-16) for the preparation of chromenone derivatives 11 from bis-acyl compounds 13:<smiles>[R]C(=O)O/N=C(\CCl)c1ccccc1OC([R])=O</smiles>

13

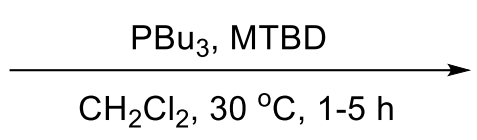

A dry and argon-flushed $10 \mathrm{~mL}$ Schlenk flask equipped with a magnetic stir bar and septum was sequentially charged with bis-acyl compound 13 (0.2 mmol), anhydrous $\mathrm{CH}_{2} \mathrm{Cl}_{2}(2 \mathrm{~mL}), \mathrm{PBu}_{3}$ (1.1 equiv), MTBD (1.5 equiv). The reaction mixture was stirred for $1-5 \mathrm{~h}$ at $30{ }^{\circ} \mathrm{C}$. After completion of the reaction, solvent was removed in vacuo and the crude residue subjected to flash column chromatography on silica gel to obtain the product 11 .

\section{Analytical data for all new compounds:}

(E/Z)-1-(2-Chloro-1-phenylethylidene)-2-(4-nitrophenyl)hydrazine (1a)<smiles>O=[N+]([O-])c1ccc(NN=C(CCl)c2ccccc2)cc1</smiles>

Following the TP-1, 1a was obtained from 2-chloroacetophenone (3.1 g, $20 \mathrm{mmol})$ 
and 4-nitrophenyl hydrazine ( $3.3 \mathrm{~g}, 1.1$ equiv) as a brown solid (5.5 g, 98\% yield); $\mathrm{R}_{f}$ $=0.40($ EA:Hex $=1: 9) ; \mathrm{mp}=143.3-144.1{ }^{\circ} \mathrm{C}$.

${ }^{1} \mathbf{H}$ NMR (400 MHz, $\left.\mathrm{CDCl}_{3}, 25{ }^{\circ} \mathrm{C}\right) \delta / \mathrm{ppm}: 8.38(\mathrm{~s}, 1 \mathrm{H}$, major), $8.22(\mathrm{~d}, J=9.2 \mathrm{~Hz}$, 2H, major), $8.13(\mathrm{~d}, J=9.0 \mathrm{~Hz}, 2 \mathrm{H}$, minor), $7.92(\mathrm{~s}, 1 \mathrm{H}$, minor), $7.78(\mathrm{~d}, J=7.0 \mathrm{~Hz}$, $2 \mathrm{H}$, major), 7.60-7.51 (m, 3H, minor), 7.47-7.39 (m, 3H, major and $2 \mathrm{H}$, minor), 7.27 (d, $J=9.2 \mathrm{~Hz}, 2 \mathrm{H}$, major), $7.03(\mathrm{~d}, J=9.0 \mathrm{~Hz}, 2 \mathrm{H}$, minor), $4.58(\mathrm{~s}, 2 \mathrm{H}), 4.51(\mathrm{~s}, 2 \mathrm{H})$. ${ }^{13} \mathrm{C}$ NMR $\left(100 \mathrm{MHz}, \mathrm{CDCl}_{3}, 25{ }^{\circ} \mathrm{C}\right) \delta$ (ppm, major + minor) 149.2, 148.8, 145.3, $142.3,141.4,140.9,135.8,130.8,130.3,129.9,129.4,128.9,127.9,125.98,125.97$, $125.7,113.0,112.2,48.1,34.1$

IR $(\mathrm{KBr}) \tilde{v}\left(\mathrm{~cm}^{-1}\right): 3315,2360,1601,1480,1316,1273,1153,1110,837,764,681$.

HRMS (ESI) for $\mathrm{C}_{14} \mathrm{H}_{12}{ }^{35} \mathrm{ClN}_{3} \mathrm{O}_{2},[\mathrm{M}]^{+}$(289.0618) found: 289.0616 .

HRMS (ESI) for $\mathrm{C}_{14} \mathrm{H}_{12}{ }^{37} \mathrm{ClN}_{3} \mathrm{O}_{2},[\mathrm{M}]^{+}$(291.0589) found: 291.0592.

(E/Z)-1-(2-Chloro-1-(4-methoxyphenyl)ethylidene)-2-(4-nitrophenyl)hydrazine (1b)

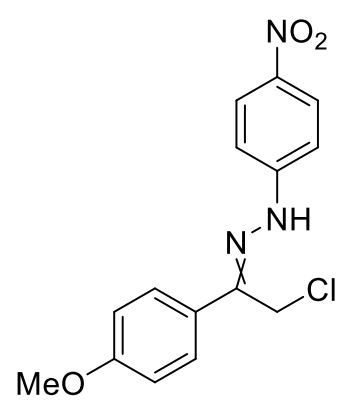

Following the TP-1, 1b was obtained from 2-chloro-1-(4-methoxyphenyl)ethan-1-one (200 mg, $1.0 \mathrm{mmol}$ ) and 4-nitrophenyl hydrazine (261 mg, 1.1 equiv) as a white solid (298 mg, 86\% yield); $\mathrm{R}_{f}=0.30(\mathrm{EA}: \mathrm{Hex}=1: 9) ; \mathrm{mp}=142.0-143.3{ }^{\circ} \mathrm{C}$.

${ }^{1} \mathbf{H}$ NMR $\left(400 \mathrm{MHz}, \mathrm{CDCl}_{3}, 25{ }^{\circ} \mathrm{C}\right) \delta / \mathrm{ppm}: 8.30$ (s, $1 \mathrm{H}$, minor), $8.24(\mathrm{~d}, J=9.2 \mathrm{~Hz}$, 2H, minor), 8.16 (d, $J=9.2 \mathrm{~Hz}, 2 \mathrm{H}$, major), 7.99 (s, 1H, major), 7.76 (d, $J=8.8 \mathrm{~Hz}$, 2H, minor), 7.37 (d, $J=8.8 \mathrm{~Hz}, 2 \mathrm{H}$, major), $7.27(\mathrm{~d}, J=8.8 \mathrm{~Hz}, 2 \mathrm{H}$, minor), 7.10 (d, $J=8.8 \mathrm{~Hz}, 2 \mathrm{H}$, major), $7.05(\mathrm{~d}, J=9.2 \mathrm{~Hz}, 2 \mathrm{H}$, major), $6.99(\mathrm{~d}, J=8.8 \mathrm{~Hz}, 2 \mathrm{H}$, minor), 4.58 (s, 2H, minor), 4.52 (s, $2 \mathrm{H}$, major), 3.91 (s, 3H, major), 3.89 (s, 3H, 
minor).

${ }^{13} \mathrm{C}$ NMR $\left(100 \mathrm{MHz}, \mathrm{CDCl}_{3}, 25{ }^{\circ} \mathrm{C}\right) \delta$ (ppm, major + minor): 160.8, 160.7, 149.4, $148.9,145.3,142.5,141.2,140.8,129.3,128.2,127.2,126.0,122.5,115.2,114.3$, $112.8,112.1,55.4,48.2,34.0$.

IR $(\mathrm{KBr}) \tilde{v}\left(\mathrm{~cm}^{-1}\right): 3309,2359,1600,1482,1317,1273,1152,1110,834,744,493$.

HRMS (ESI) for $\mathrm{C}_{15} \mathrm{H}_{14}{ }^{35} \mathrm{ClN}_{3} \mathrm{O}_{3},[\mathrm{M}]^{+}$(319.0724) found: 319.0722 .

HRMS (ESI) for $\mathrm{C}_{15} \mathrm{H}_{14}{ }^{37} \mathrm{ClN}_{3} \mathrm{O}_{3},[\mathrm{M}]^{+}$(321.0694) found: 321.0697.

(E/Z)-1-(2-Chloro-1-(4-fluorophenyl)ethylidene)-2-(4-nitrophenyl)hydrazine (1c)<smiles>O=[N+]([O-])c1ccc(NN=C(CCl)c2ccc(F)cc2)cc1</smiles>

Following the TP-1, 1c was obtained from 2-Chloro-4'-fluoroacetophenone (500 mg, $2.8 \mathrm{mmol}$ ) and 4-nitrophenyl hydrazine (487 mg, 1.1 equiv) as a brown solid (786 mg, $88 \%$ yield $) ; \mathrm{R}_{f}=0.40(\mathrm{EA}: \mathrm{Hex}=1: 9) ; \mathrm{mp}=151.6-152.1{ }^{\circ} \mathrm{C}$.

${ }^{1} \mathbf{H}$ NMR $\left(400 \mathrm{MHz}, \mathrm{CDCl}_{3}, 25{ }^{\circ} \mathrm{C}\right) \delta / \mathrm{ppm}: 8.34$ (s, 1H, major), $8.22(\mathrm{~d}, J=9.1 \mathrm{~Hz}$, 2H, major), 8.15 (d, $J=9.1 \mathrm{~Hz}, 2 \mathrm{H}$, minor), 7.83 (s, 1H, minor), 7.79-7.75 (m, 2H, major), 7.43-7.39 (m, 2H, minor), 7.30-7.25 (m, 2H, major; 2H, minor), 7.4 (t, $J=8.3$ $\mathrm{Hz}, 2 \mathrm{H}$, major), 7.04 (d, $J=9.1 \mathrm{~Hz}, 2 \mathrm{H}$, minor), 4.56 (s, 2H, major), 4.49 (s, 2H, minor).

${ }^{13} \mathrm{C}$ NMR $\left(100 \mathrm{MHz}, \mathrm{CDCl}_{3}, 25{ }^{\circ} \mathrm{C}\right) \delta$ (ppm, major + minor): 164.7, 162.2, 149.1, 148.7, 144.2, 141.5, 141.4, 141.1, 131.9 (d, $J=3.1 \mathrm{~Hz}), 130.1(\mathrm{~d}, J=8.5 \mathrm{~Hz}), 127.6$ $(\mathrm{d}, J=8.4 \mathrm{~Hz}), 126.6(\mathrm{~d}, J=3.7 \mathrm{~Hz}), 125.9(\mathrm{~d}, J=2.5 \mathrm{~Hz}), 117.1(\mathrm{~d}, J=21.7 \mathrm{~Hz})$, $115.9(\mathrm{~d}, J=21.9 \mathrm{~Hz}), 112.9,112.3,48.0,34.3$.

IR $(\mathrm{KBr}) \tilde{v}\left(\mathrm{~cm}^{-1}\right): 3309,2359,1600,1482,1317,1273,1152,1110,834,744,493$. 
HRMS (ESI) for $\mathrm{C}_{14} \mathrm{H}_{11}{ }^{35} \mathrm{ClFN}_{3} \mathrm{O}_{2},[\mathrm{M}]^{+}$(307.0524) found: 307.0526.

HRMS (ESI) for $\mathrm{C}_{14} \mathrm{H}_{11}{ }^{37} \mathrm{ClFN}_{3} \mathrm{O}_{2},[\mathrm{M}]^{+}$(309.0503) found: 309.0506.

(Z)-1-(2-Bromo-1-(4-nitrophenyl)ethylidene)-2-(4-nitrophenyl)hydrazine (1d)<smiles>O=[N+]([O-])c1ccc(N/N=C(\CBr)c2ccc([N+](=O)[O-])cc2)cc1</smiles>

Following the TP-1, 1d was obtained from 2-chloro-1-(4-methoxyphenyl)ethan-1-one (200 mg, $1.0 \mathrm{mmol}$ ) and 4-nitrophenyl hydrazine (261 mg, 1.1 equiv) as a white solid $(312 \mathrm{mg}, 82 \%$ yield $) ; \mathrm{R}_{f}=0.20(\mathrm{EA}: \mathrm{Hex}=2: 8) ; \mathrm{mp}=128.9-129.5^{\circ} \mathrm{C}$.

${ }^{1} \mathbf{H}$ NMR $\left(400 \mathrm{MHz},\left(\mathrm{CD}_{3}\right)_{2} \mathrm{CO}, 25{ }^{\circ} \mathrm{C}\right) \delta / \mathrm{ppm}: 8.30(\mathrm{~d}, J=8.9 \mathrm{~Hz}, 2 \mathrm{H}), 8.22(\mathrm{~d}, J=$ $8.9 \mathrm{~Hz}, 2 \mathrm{H}), 8.20(\mathrm{~d}, J=9.2 \mathrm{~Hz}, 2 \mathrm{H}), 7.57(\mathrm{~d}, J=9.2 \mathrm{~Hz}, 2 \mathrm{H}), 4.84(\mathrm{~s}, 2 \mathrm{H})$.

IR (KBr) $\tilde{v}\left(\mathrm{~cm}^{-1}\right):$ 3417, 2354, 1643, 1305, 1248, 1099, 744, 493.

HRMS (ESI) for $\mathrm{C}_{14} \mathrm{H}_{11}{ }^{79} \mathrm{BrN}_{4} \mathrm{O}_{4},[\mathrm{M}]^{+}$(377.9763) found: 377.9761 .

HRMS (ESI) for $\mathrm{C}_{14} \mathrm{H}_{11}{ }^{81} \mathrm{BrN}_{4} \mathrm{O}_{4},[\mathrm{M}]^{+}$(379.8955) found: 379.8952.

(Z)-1-(1-Chloropropan-2-ylidene)-2-(4-nitrophenyl)hydrazine (1e)<smiles>C/C(CCl)=N/Nc1ccc([N+](=O)[O-])cc1</smiles>

Following the TP-1, 1e was obtained from 1-chloropropan-2-one (1.0 g, $10.8 \mathrm{mmol})$ and 4-nitrophenyl hydrazine ( $2.5 \mathrm{~g}, 1.1$ equiv) as a brown solid ( $2.2 \mathrm{~g}, 93 \%$ yield); $\mathrm{R}_{f}$ $=0.70($ EA:Hex $=1: 9) ; \mathrm{mp}=145.8-146.3{ }^{\circ} \mathrm{C}$.

${ }^{1} \mathbf{H}$ NMR (400 MHz, $\left.\mathrm{CDCl}_{3}, 25{ }^{\circ} \mathrm{C}\right) \delta / \mathrm{ppm}: 8.17(\mathrm{~d}, J=9.0 \mathrm{~Hz}, 2 \mathrm{H}), 7.59(\mathrm{bs}, 1 \mathrm{H})$, $7.10(\mathrm{~d}, J=9.0 \mathrm{~Hz}, 2 \mathrm{H}), 4.26(\mathrm{~s}, 2 \mathrm{H}), 2.05(\mathrm{~s}, 3 \mathrm{H})$. 
${ }^{13} \mathrm{C}$ NMR (100 MHz, $\left.\mathrm{CDCl}_{3}, 25{ }^{\circ} \mathrm{C}\right) \delta / \mathrm{ppm}: 149.3,143.8,140.9,126.0,112.3,49.1$, 12.6 .

IR $(\mathrm{KBr}) \tilde{v}\left(\mathrm{~cm}^{-1}\right):$ 3328, 2359, 1602, 1502, 1324, 1261, 1166, 840, 751, 693.

HRMS (ESI) for $\mathrm{C}_{9} \mathrm{H}_{10}{ }^{35} \mathrm{ClN}_{3} \mathrm{O}_{2}$, [M] $]^{+}$(227.0459) found: 227.0461 .

HRMS (ESI) for $\mathrm{C}_{9} \mathrm{H}_{10}{ }^{37} \mathrm{ClN}_{3} \mathrm{O}_{2},[\mathrm{M}]^{+}$(229.0437) found: 229.0440 .

(Z)-1-(2-Chloro-1-phenylethylidene)-2-(2,4-dinitrophenyl)hydrazine (1f) ${ }^{5}$<smiles>O=[N+]([O-])c1ccc(N/N=C(\CCl)c2ccccc2)c([N+](=O)[O-])c1</smiles>

Following the TP-1, 1f was obtained from 2-chloroacetophenone (800 mg, $5 \mathrm{mmol}$ ) and 2,4-dinitrophenyl hydrazine (1.1. g, 1.1 equiv) as orange solid (1.6 g, 97\% yield); $\mathrm{R}_{f}=0.20(\mathrm{EA}: \operatorname{Hex}=1: 9) ; \mathrm{mp}=210.6-211.8^{\circ} \mathrm{C}$.

${ }^{1} \mathbf{H}$ NMR (400 MHz, $\left.\mathrm{CDCl}_{3}, 25{ }^{\circ} \mathrm{C}\right) \delta / \mathrm{ppm}: 11.70(\mathrm{~s}, 1 \mathrm{H}), 9.20(\mathrm{~s} 1 \mathrm{H}), 8.41(\mathrm{~d}, J=$ $9.6 \mathrm{~Hz}, 1 \mathrm{H}), 8.14(\mathrm{~d}, J=9.5 \mathrm{~Hz} 1 \mathrm{H}), 7.88-7.82(\mathrm{~m}, 2 \mathrm{H}), 7.53-7.49(\mathrm{~m}, 3 \mathrm{H}), 4.6(\mathrm{~s}$, $2 \mathrm{H})$.

IR $(\mathrm{KBr}) \tilde{v}\left(\mathrm{~cm}^{-1}\right): 1615,1505,1117,838,742,559$.

HRMS (ESI) for $\mathrm{C}_{14} \mathrm{H}_{11}{ }^{35} \mathrm{ClN}_{4} \mathrm{O}_{4},[\mathrm{M}]^{+}$(334.0469) found: 334.0470 .

HRMS (ESI) for $\mathrm{C}_{14} \mathrm{H}_{11}{ }^{37} \mathrm{ClN}_{4} \mathrm{O}_{4},[\mathrm{M}]^{+}$(336.0444) found: 336.0446 .

(E/Z)- $N^{\prime}$-(2-Chloro-1-phenylethylidene)-4-methylbenzenesulfonohydrazide (1g) ${ }^{1}$<smiles>Cc1ccc(S(=O)(=O)NNC(CCl)c2ccccc2)cc1</smiles> 
Following the TP-1, $1 \mathrm{~g}$ was obtained from 2-chloroacetophenone (462 mg, $3 \mathrm{mmol}$ ) and tosyl hydrazide (614 $\mathrm{mg}, 1.1$ equiv) as a white solid ( $879 \mathrm{mg}, 88 \%$ yield); $\mathrm{R}_{f}=$ $0.20(\mathrm{EA}: \mathrm{Hex}=1: 9) ; \mathrm{mp}=132.0-133.3^{\circ} \mathrm{C}$.

${ }^{1} \mathbf{H}$ NMR $\left(400 \mathrm{MHz}, \mathrm{CDCl}_{3}, 25{ }^{\circ} \mathrm{C}\right)(\delta / \mathrm{ppm}$, major + minor $):$ 8.10-7.28 (m, 16H), 7.24-7.15 (m, 2H), $4.38(\mathrm{~s}, 2 \mathrm{H}), 4.32(\mathrm{~s}, 2 \mathrm{H}), 2.45(\mathrm{~s}, 3 \mathrm{H}), 2.41(\mathrm{~s}, 3 \mathrm{H})$.

IR $(\mathrm{KBr}) \tilde{v}\left(\mathrm{~cm}^{-1}\right):$ 3171, 2359, 1403, 1337, 1172, 1053, 879, 698, 590.

HRMS (ESI) for $\mathrm{C}_{15} \mathrm{H}_{15}{ }^{35} \mathrm{ClN}_{2} \mathrm{O}_{2} \mathrm{~S},[\mathrm{M}]^{+}$(322.0541) found: 322.0543 .

HRMS (ESI) for $\mathrm{C}_{14} \mathrm{H}_{12}{ }^{37} \mathrm{ClN}_{2} \mathrm{O}_{2},[\mathrm{M}]^{+}$(324.0501) found: 324.0503 .

(E/Z)- $N^{\prime}$-(2-Chloro-1-(4-methoxyphenyl)ethylidene)-4-methylbenzenesulfonohyd razide $(\mathbf{1 h})^{6}$

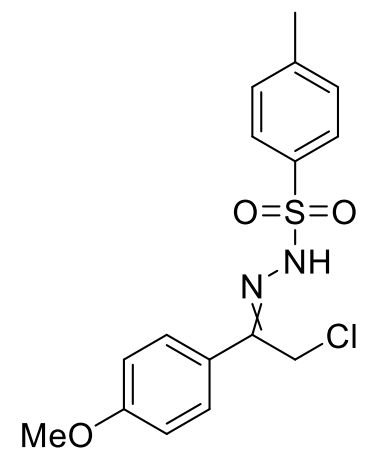

Following the TP-1, $\mathbf{1 h}$ was obtained from 2-chloro-1-(4-methoxyphenyl)ethan-1-one (200 mg, $1.0 \mathrm{mmol}$ ) and tosyl hydrazide (221 mg, 1.1 equiv) as a white solid (312 $\mathrm{mg}$, $82 \%$ yield $) ; \mathrm{R}_{f}=0.20(\mathrm{EA}: \mathrm{Hex}=2: 8) ; \mathrm{mp}=128.9-129.4^{\circ} \mathrm{C}$.

${ }^{1} \mathbf{H}$ NMR $\left(400 \mathrm{MHz}, \mathrm{CDCl}_{3}, 25{ }^{\circ} \mathrm{C}\right) \delta / \mathrm{ppm}: 7.91$ (s, $1 \mathrm{H}$, minor), $7.88(\mathrm{~d}, J=8.3 \mathrm{~Hz}$, 2H, minor), 7.79 (d, $J=8.3 \mathrm{~Hz}, 2 \mathrm{H}$, major), 7.68 (s, 1H, major), 7.60 (d, $J=8.8 \mathrm{~Hz}$, 2H, minor), 7.33-7.30 (m, 2H, major; $2 \mathrm{H}$, minor), 7.18 (d, $J=8.8 \mathrm{~Hz}, 2 \mathrm{H}$, major), $6.98(\mathrm{~d}, J=8.8 \mathrm{~Hz}, 2 \mathrm{H}$, major), $6.88(\mathrm{~d}, J=8.8 \mathrm{~Hz}, 2 \mathrm{H}$, minor), 4.36 (s, 2H, minor), 4.31 (s, 2H, major), 3.85 (s, 3H, major), 3.83 (s, 3H, minor), 2.45 (s, 3H, major), 2.41 (s, 3H, minor).

IR (KBr) $\tilde{v}\left(\mathrm{~cm}^{-1}\right):$ 3434, 2367, 2341, 1611, 1318, 1251, 1162, 1084, 666. 
HRMS (ESI) for $\mathrm{C}_{16} \mathrm{H}_{17}{ }^{35} \mathrm{ClN}_{2} \mathrm{O}_{3} \mathrm{~S},[\mathrm{M}]^{+}$(352.0648) found: 352.0650 .

HRMS (ESI) for $\mathrm{C}_{16} \mathrm{H}_{17}{ }^{37} \mathrm{ClN}_{2} \mathrm{O}_{3} \mathrm{~S},[\mathrm{M}]^{+}$(354.0619) found: 354.0616 .

(Z)-(2-(2-(4-Nitrophenyl)hydrazono)-2-phenylethyl)triphenylphosphonium

chloride (4a)

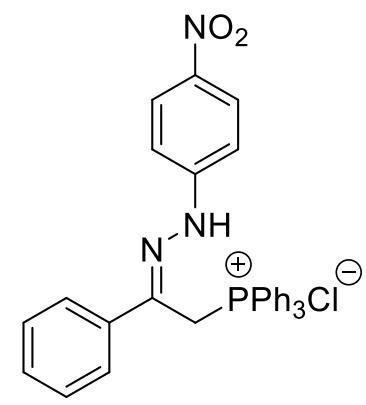

Following the TP-5, 4a was obtained from $1 \mathbf{a}(200 \mathrm{mg}, 0.7 \mathrm{mmol})$ and $\mathrm{PPh}_{3}(200 \mathrm{mg}$, 1.1 equiv) as a yellow solid (342 mg, 90\% yield); $\mathrm{R}_{f}=0.50\left(\mathrm{MeOH}: \mathrm{CH}_{2} \mathrm{Cl}_{2}=1: 9\right)$; $\mathrm{mp}=234.3-235.0^{\circ} \mathrm{C}$.

${ }^{1} \mathbf{H}$ NMR (400 MHz, $\left.\mathrm{CDCl}_{3}, 25{ }^{\circ} \mathrm{C}\right) \delta / \mathrm{ppm}: 11.68(\mathrm{~s}, 1 \mathrm{H}), 7.9(\mathrm{~d}, J=9.0 \mathrm{~Hz}, 2 \mathrm{H})$, $7.86(\mathrm{dd}, J=12.7,7.8 \mathrm{~Hz}, 6 \mathrm{H}), 7.61-7.56(\mathrm{~m}, 2 \mathrm{H}), 7.56-7.49(\mathrm{~m}, 3 \mathrm{H}), 7.43(\mathrm{td}, J=$ 7.68, 3.20 Hz, 6H), $7.31(\mathrm{~d}, J=9.0 \mathrm{~Hz}, 2 \mathrm{H}), 7.22-7.17(\mathrm{~m}, 3 \mathrm{H}), 6.15(\mathrm{~d}, J=17.0 \mathrm{~Hz}$, $2 \mathrm{H})$.

${ }^{13} \mathrm{C}$ NMR $\left(100 \mathrm{MHz}, \mathrm{CDCl}_{3}, 25{ }^{\circ} \mathrm{C}\right) \delta / \mathrm{ppm}: 150.7,140.2,138.5(\mathrm{~d}, J=1.81 \mathrm{~Hz})$, $134.8(\mathrm{~d}, J=2.95 \mathrm{~Hz}), 134.4(\mathrm{~d}, J=10.97 \mathrm{~Hz}), 129.6(\mathrm{~d}, J=12.54 \mathrm{~Hz}), 128.6,128.5$, $127.1,125.3,118.0,117.2,112.8,26.7(\mathrm{~d}, J=46.81 \mathrm{~Hz})$.

${ }^{31} \mathbf{P}\left(100 \mathrm{MHz}, \mathrm{CDCl}_{3}, 25^{\circ} \mathrm{C}\right) \delta / \mathrm{ppm}: 18.0$.

IR $(\mathrm{KBr}) \tilde{v}\left(\mathrm{~cm}^{-1}\right): 3309,2362,1706,1594,1320,1267,1107,834,762$.

HRMS (ESI) for $\mathrm{C}_{32} \mathrm{H}_{27} \mathrm{~N}_{3} \mathrm{O}_{2} \mathrm{P},[\mathrm{M}]^{+}$(516.1841) found: 516.1838 .

(Z)-Ethyl(2-(2-(4-nitrophenyl)hydrazono)-2-phenylethyl)diphenylphosphonium chloride (4b) 


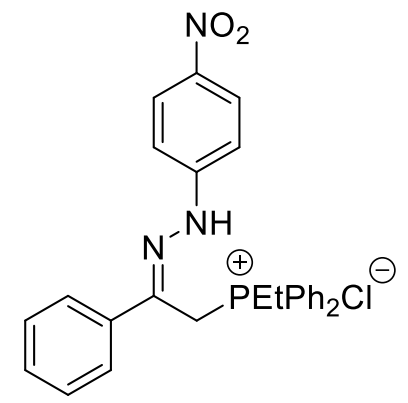

Following the TP-5, 4b was obtained from 1a (200 mg, $0.7 \mathrm{mmol})$ and $\mathrm{PEt}_{2} \mathrm{Ph}(160$ $\mu \mathrm{L}, 1.1$ equiv) as a brown solid (303 mg, 88\% yield); $\mathrm{R}_{f}=0.50\left(\mathrm{MeOH}: \mathrm{CH}_{2} \mathrm{Cl}_{2}=1: 9\right)$; $\mathrm{mp}=252.1-253.6^{\circ} \mathrm{C}$.

${ }^{1} \mathbf{H}$ NMR $\left(400 \mathrm{MHz}, \mathrm{CDCl}_{3}, 25^{\circ} \mathrm{C}\right) \delta / \mathrm{ppm}: 11.46(\mathrm{~s}, 1 \mathrm{H}), 8.00(\mathrm{~d}, J=9.0 \mathrm{~Hz}, 2 \mathrm{H})$, $7.87(\mathrm{dd}, J=12.1,7.7 \mathrm{~Hz}, 4 \mathrm{H}), 7.61-7.40(\mathrm{~m}, 10 \mathrm{H}), 7.21(\mathrm{~d}, J=4.01 \mathrm{~Hz}, 3 \mathrm{H}), 5.78(\mathrm{~d}$, $J=17.20 \mathrm{~Hz}, 2 \mathrm{H}), 3.20(\mathrm{~m}, 2 \mathrm{H}), 1.07(\mathrm{dt}, J=20.1,7.2 \mathrm{~Hz}, 3 \mathrm{H})$.

${ }^{13} \mathrm{C}$ NMR $\left(100 \mathrm{MHz}, \mathrm{CDCl}_{3}, 25{ }^{\circ} \mathrm{C}\right) \delta / \mathrm{ppm}: 150.5,140.3,137.8(\mathrm{~d}, J=1.5 \mathrm{~Hz})$, $134.8(\mathrm{~d}, J=2.9 \mathrm{~Hz}), 133.7(\mathrm{~d}, J=9.6 \mathrm{~Hz}), 129.7(\mathrm{~d}, J=12.3 \mathrm{~Hz}), 128.7,128.6$, 126.7, 125.4, 116.4, 115.6, 112.9, $25.0(\mathrm{~d}, J=45.2 \mathrm{~Hz}), 16.2(\mathrm{~d}, J=48.4 \mathrm{~Hz}), 6.2(\mathrm{~d}, J$ $=5.3 \mathrm{~Hz})$.

${ }^{31} \mathbf{P}\left(100 \mathrm{MHz}, \mathrm{CDCl}_{3}, 25^{\circ} \mathrm{C}\right) \delta / \mathrm{ppm}: 27.2$.

IR $(\mathrm{KBr}) \tilde{v}\left(\mathrm{~cm}^{-1}\right): 3413,2358,1552,1324,1265,1109,751$.

HRMS (ESI) for $\mathrm{C}_{28} \mathrm{H}_{27} \mathrm{~N}_{3} \mathrm{O}_{2} \mathrm{P},[\mathrm{M}]^{+}$(468.1841) found: 468.1849 .

(Z)-Diethyl(2-(2-(4-nitrophenyl)hydrazono)-2-phenylethyl)(phenyl)phosphonium chloride (4c)

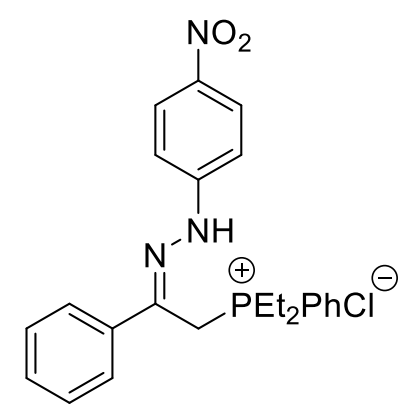

Following the TP-5, 4c was obtained from 1a (200 mg, $0.7 \mathrm{mmol})$ and $\mathrm{PEt}_{2} \mathrm{Ph}(140$ 
$\mu \mathrm{L}, 1.1$ equiv) as a yellow solid (299 mg, 96\% yield); $\mathrm{R}_{f}=0.50\left(\mathrm{MeOH}: \mathrm{CH}_{2} \mathrm{Cl}_{2}=\right.$ $1: 9) ; \mathrm{mp}=221.8-222.4^{\circ} \mathrm{C}$.

${ }^{1} \mathbf{H}$ NMR (400 MHz, $\left.\mathrm{CDCl}_{3}, 25^{\circ} \mathrm{C}\right) \delta / \mathrm{ppm}: 11.25(\mathrm{~s}, 1 \mathrm{H}), 8.00(\mathrm{~d}, J=9.2 \mathrm{~Hz}, 2 \mathrm{H})$, $7.80(\mathrm{dd}, J=11.8,7.5 \mathrm{~Hz}, 2 \mathrm{H}), 7.70(\mathrm{t}, J=3.9 \mathrm{~Hz}, 2 \mathrm{H}), 7.50(\mathrm{~d}, J=8.9 \mathrm{~Hz}, 2 \mathrm{H})$, 7.43-7.34 (m, 3H), 7.34-7.27 (m, 3H), $5.3(\mathrm{~d}, J=16.9 \mathrm{~Hz}, 2 \mathrm{H}), 3.10-2.87(\mathrm{~m}, 2 \mathrm{H})$, 2.86-2.63 (m, 2H), $1.25(\mathrm{dt}, J=19.4,7.8 \mathrm{~Hz}, 6 \mathrm{H})$.

${ }^{13} \mathrm{C}$ NMR $\left(100 \mathrm{MHz}, \mathrm{CDCl}_{3}, 25{ }^{\circ} \mathrm{C}\right) \delta / \mathrm{ppm}: 150.4,140.4,137.3(\mathrm{~d}, J=1.7 \mathrm{~Hz})$, $134.6(\mathrm{~d}, J=2.9 \mathrm{~Hz}), 133.2(\mathrm{~d}, J=9.5 \mathrm{~Hz}), 132.3(\mathrm{~d}, J=8.9 \mathrm{~Hz}), 129.5(\mathrm{~d}, J=11.9$ $\mathrm{Hz}), 129.1,128.9,126.3,125.5,116.1,115.3,112.8,23.6$ (d, $J=44.2 \mathrm{~Hz}), 12.7(\mathrm{~d}, J=$ $48.3 \mathrm{~Hz}), 6.0(\mathrm{~d}, J=5.2 \mathrm{~Hz})$.

${ }^{31} \mathbf{P}\left(100 \mathrm{MHz}, \mathrm{CDCl}_{3}, 25^{\circ} \mathrm{C}\right) \delta / \mathrm{ppm}: 31.3$.

IR $(\mathrm{KBr}) \tilde{v}\left(\mathrm{~cm}^{-1}\right): 3413,3198,2959,2362,1597,1322,1111,753$.

HRMS (ESI) for $\mathrm{C}_{24} \mathrm{H}_{27} \mathrm{~N}_{3} \mathrm{O}_{2} \mathrm{P},[\mathrm{M}]^{+}$(420.1841) found: 420.1843.

\section{(Z)-Tributyl(2-(2-(4-nitrophenyl)hydrazono)-2-phenylethyl)phosphonium} chloride (4d)

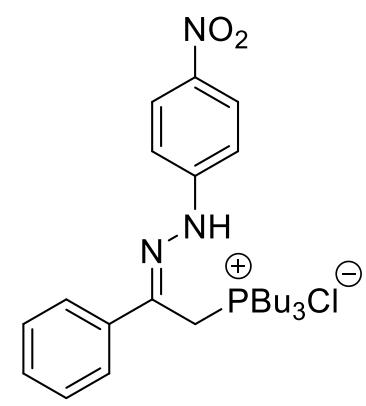

Following the TP-5, 4d was obtained from $1 \mathbf{a}(200 \mathrm{mg}, 0.7 \mathrm{mmol})$ and $\mathrm{PBu}_{3}(220 \mu \mathrm{L}$, 1.1 equiv) as a yellow solid (312 mg, 92\% yield); $\mathrm{R}_{f}=0.40\left(\mathrm{MeOH}: \mathrm{CH}_{2} \mathrm{Cl}_{2}=1: 9\right)$; $\mathrm{mp}=215.2-216.5^{\circ} \mathrm{C}$.

${ }^{1} \mathbf{H}$ NMR $\left(400 \mathrm{MHz}, \mathrm{CDCl}_{3}, 25^{\circ} \mathrm{C}\right) \delta / \mathrm{ppm}: 11.84(\mathrm{~s}, 1 \mathrm{H}), 8.14(\mathrm{~d}, J=9.2 \mathrm{~Hz}, 2 \mathrm{H})$, $7.79(\mathrm{~d}, J=7.4 \mathrm{~Hz}, 2 \mathrm{H}), 7.79(\mathrm{~d}, J=9.2 \mathrm{~Hz}, 2 \mathrm{H}), 7.50-7.33(\mathrm{~m}, 3 \mathrm{H}), 5.00(\mathrm{~d}, J=17.0$ $\mathrm{Hz}, 2 \mathrm{H}), 2.4-2.1(\mathrm{~m}, 6 \mathrm{H}), 1.60-1.20(\mathrm{~m}, 12 \mathrm{H}), 0.8(\mathrm{t}, J=7.3 \mathrm{~Hz}, 9 \mathrm{H})$. 
${ }^{13} \mathrm{C}$ NMR $\left(100 \mathrm{MHz}, \mathrm{CDCl}_{3}, 25{ }^{\circ} \mathrm{C}\right) \delta / \mathrm{ppm}: 150.8,140.6,137.8,133.9(\mathrm{~d}, J=9.6$ $\mathrm{Hz}), 129.4,129.1,126.4,125.7,113.1,23.9(\mathrm{~d}, J=15.9 \mathrm{~Hz}), 23.4(\mathrm{~d}, J=4.7 \mathrm{~Hz})$, $21.4(\mathrm{~d}, J=43.9 \mathrm{~Hz}), 19.6(\mathrm{~d}, J=45.1 \mathrm{~Hz}), 13.1$.

${ }^{31} \mathbf{P}\left(100 \mathrm{MHz}, \mathrm{CDCl}_{3}, 25{ }^{\circ} \mathrm{C}\right) \delta / \mathrm{ppm}: 33.4$.

IR $(\mathrm{KBr}) \tilde{v}\left(\mathrm{~cm}^{-1}\right):$ 3434, 2358, 1597, 1497, 1324, 1263, 1152, 1111, 744.

HRMS (ESI) for $\mathrm{C}_{26} \mathrm{H}_{39} \mathrm{~N}_{3} \mathrm{O}_{2} \mathrm{P},[\mathrm{M}]^{+}$(456.2780) found: 456.2778 .

1-(4-Nitrophenyl)-3,5-diphenyl-1H-pyrazole (3aa)

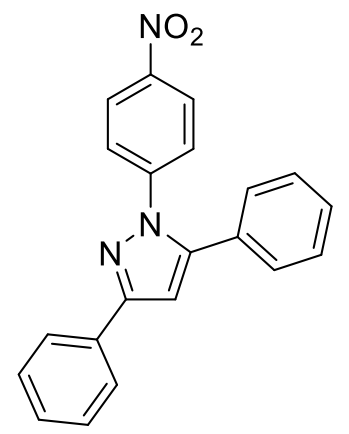

Following the TP-2, 3aa was obtained from $1 \mathbf{a}(86.7 \mathrm{mg}, 0.3 \mathrm{mmol}), \mathrm{PPh}_{3}(87.4 \mathrm{mg}$, 1.1 equiv), $\mathrm{Et}_{3} \mathrm{~N}(105 \mu \mathrm{L}, 2.5$ equiv) and $\mathrm{PhCOCl} \mathbf{2 a}(41.79 \mu \mathrm{L}, 1.2$ equiv) as a yellow solid in (83.1 mg, 81\% yield; $\mathrm{R}_{f}=0.60(\mathrm{EA}: \mathrm{Hex}=1: 9) ; \mathrm{mp}=106.5-107.1{ }^{\circ} \mathrm{C}$. ${ }^{1} \mathbf{H}$ NMR $\left(400 \mathrm{MHz}, \mathrm{CDCl}_{3}, 25{ }^{\circ} \mathrm{C}\right) \delta / \mathrm{ppm}: 8.19(\mathrm{~d}, J=9.0 \mathrm{~Hz}, 2 \mathrm{H}), 7.92(\mathrm{~d}, J=7.5$ $\mathrm{Hz}, 2 \mathrm{H}), 7.55(\mathrm{~d}, J=9.0 \mathrm{~Hz}, 2 \mathrm{H}), 7.46(\mathrm{t}, J=7.5 \mathrm{~Hz}, 2 \mathrm{H}), 7.43-7.35(\mathrm{~m}, 4 \mathrm{H})$, 7.34-7.28 (m, 2H), $6.86(\mathrm{~s}, 1 \mathrm{H})$.

${ }^{13} \mathrm{C}$ NMR (100 MHz, $\left.\mathrm{CDCl}_{3}, 25{ }^{\circ} \mathrm{C}\right) \delta / \mathrm{ppm}: 153.3,145.8,144.95,144.92,132.3$, $130.1,129.1,128.9,128.8,128.7,128.6,125.9,124.5,124.4,107.2$.

IR $(\mathrm{KBr}) \tilde{v}\left(\mathrm{~cm}^{-1}\right): 3063,2927,1595,1519,1500,1340,1172,1081,968,854,762$.

HRMS (ESI) for $\mathrm{C}_{21} \mathrm{H}_{16} \mathrm{~N}_{3} \mathrm{O}_{2},[\mathrm{M}+\mathrm{H}]^{+}$(342.1237) found: 342.1247.

3-(4-Methoxyphenyl)-1-(4-nitrophenyl)-5-phenyl-1H-pyrazole (3ba) 


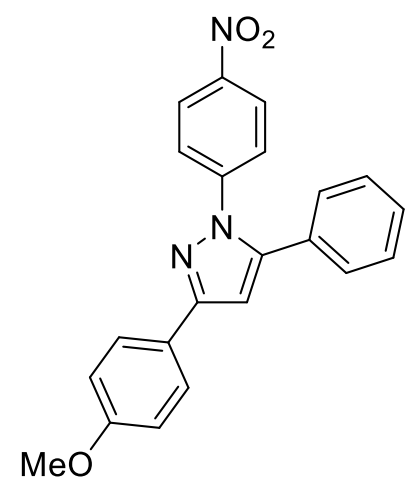

Following the TP-2, 3ba was obtained from $\mathbf{1 b}(95.7 \mathrm{mg}, 0.3 \mathrm{mmol}), \mathrm{PPh}_{3}(87.4 \mathrm{mg}$, 1.1 equiv), $\mathrm{Et}_{3} \mathrm{~N}$ (105 $\mu \mathrm{L}, 2.5$ equiv) and $\mathrm{PhCOCl}$ 2a (41.79 $\mu \mathrm{L}, 1.2$ equiv) as a yellow solid in $\left(88.1 \mathrm{mg}, 79 \%\right.$ yield; $\mathrm{R}_{f}=0.40(\mathrm{EA}: \mathrm{Hex}=1: 9) ; \mathrm{mp}=132.8-133.5^{\circ} \mathrm{C}$. ${ }^{1} \mathbf{H}$ NMR $\left(400 \mathrm{MHz}, \mathrm{CDCl}_{3}, 25{ }^{\circ} \mathrm{C}\right) \delta / \mathrm{ppm}: 8.18(\mathrm{~d}, J=9.0 \mathrm{~Hz}, 2 \mathrm{H}), 7.85(\mathrm{~d}, J=8.8$ $\mathrm{Hz}, 2 \mathrm{H}), 7.53(\mathrm{~d}, J=9.0 \mathrm{~Hz}, 2 \mathrm{H}), 7.42-7.37(\mathrm{~m}, 3 \mathrm{H}), 7.31-7.29(\mathrm{~m}, 2 \mathrm{H}), 6.98(\mathrm{~d}, J=$ $8.8 \mathrm{~Hz}, 2 \mathrm{H}), 6.79(\mathrm{~s}, 1 \mathrm{H}), 3.86(\mathrm{~s}, 3 \mathrm{H})$.

${ }^{13} \mathrm{C}$ NMR $\left(100 \mathrm{MHz}, \mathrm{CDCl}_{3}, 25{ }^{\circ} \mathrm{C}\right) \delta / \mathrm{ppm}: 160.0,153.1,145.7,144.9,144.8,130.2$, $129.0,128.9,128.8,127.2,124.9,124.4,124.3,114.2,106.9,55.3$.

IR (KBr) $\tilde{v}\left(\mathrm{~cm}^{-1}\right):$ 2934, 1995, 1434, 1111, 1030, 967, 854, 699.

HRMS (ESI) for $\mathrm{C}_{22} \mathrm{H}_{18} \mathrm{~N}_{3} \mathrm{O}_{3},[\mathrm{M}+\mathrm{H}]^{+}$(372.1343) found: 372.1351 .

\section{3-(4-Fluorophenyl)-1-(4-nitrophenyl)-5-phenyl-1H-pyrazole (3ca)}

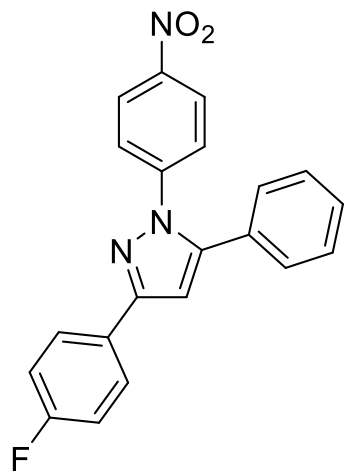

Following the TP-2, 3ca was obtained from 1 c $(92.3 \mathrm{mg}, 0.3 \mathrm{mmol}), \mathrm{PPh}_{3}(87.4 \mathrm{mg}$, 1.1 equiv), $\mathrm{Et}_{3} \mathrm{~N}(105 \mu \mathrm{L}, 2.5$ equiv) and $\mathrm{PhCOCl} \mathbf{2 a}(41.79 \mu \mathrm{L}, 1.2$ equiv) as a yellow solid in $\left(78.8 \mathrm{mg}, 73 \%\right.$ yield; $\mathrm{R}_{f}=0.60(\mathrm{EA}: \mathrm{Hex}=1: 9) ; \mathrm{mp}=132.6-133.1^{\circ} \mathrm{C}$. ${ }^{1} \mathbf{H}$ NMR $\left(400 \mathrm{MHz}, \mathrm{CDCl}_{3}, 2{ }^{\circ} \mathrm{C}\right) \delta / \mathrm{ppm}: 8.18(\mathrm{~d}, J=8.8 \mathrm{~Hz}, 2 \mathrm{H}), 7.89(\mathrm{dd}, J=8.0$, 
$5.5 \mathrm{~Hz}, 2 \mathrm{H}), 7.53(\mathrm{~d}, J=8.5 \mathrm{~Hz}, 2 \mathrm{H}), 7.46-7.36(\mathrm{~m}, 3 \mathrm{H}), 7.34-7.26(\mathrm{~m}, 2 \mathrm{H}), 7.14(\mathrm{t}, J$ $=8.5 \mathrm{~Hz}, 2 \mathrm{H}), 6.80(\mathrm{~s}, 1 \mathrm{H})$.

${ }^{13} \mathrm{C}$ NMR $\left(100 \mathrm{MHz}, \mathrm{CDCl}_{3}, 25{ }^{\circ} \mathrm{C}\right) \delta / \mathrm{ppm}: 164.3,161.8,152.3,145.8,145.0,144.8$, 129.9, 129.1, $128.9(\mathrm{~d}, J=16.1 \mathrm{~Hz}), 128.5(\mathrm{~d}, J=3.7 \mathrm{~Hz}), 127.6(\mathrm{~d}, J=8.0 \mathrm{~Hz})$, $124.4(\mathrm{~d}, J=2.9 \mathrm{~Hz}), 115.8,115.6,106.9$.

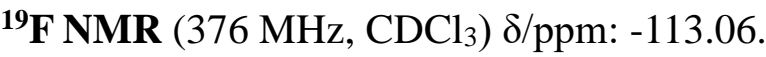

IR $(\mathrm{KBr}) \tilde{v}\left(\mathrm{~cm}^{-1}\right): 3083,1597,1435,1111,968,854,764,699,613$.

HRMS (ESI) for $\left(\mathrm{C}_{21} \mathrm{H}_{15} \mathrm{FN}_{3} \mathrm{O}_{2}\right)[\mathrm{M}+\mathrm{H}]^{+}(360.1143)$ found: 360.1148 .

\section{1,3-Bis(4-nitrophenyl)-5-phenyl-1H-pyrazole (3da)}

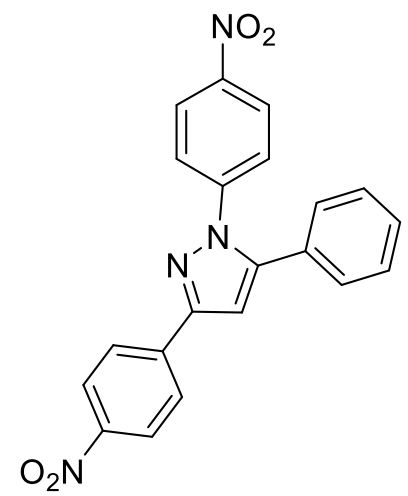

Following the TP-2, 3da was obtained from $1 d(113.75 \mathrm{mg}, 0.3 \mathrm{mmol}), \mathrm{PPh}_{3}(87.4$ mg, 1.1 equiv), $\mathrm{Et}_{3} \mathrm{~N}(105 \mu \mathrm{L}, 2.5$ equiv) and $\mathrm{PhCOCl} \mathbf{2 a}(41.79 \mu \mathrm{L}, 1.2$ equiv) as a yellow solid in $\left(70.7 \mathrm{mg}, 61 \%\right.$ yield; $\mathrm{R}_{f}=055(\mathrm{EA}: \mathrm{Hex}=1: 9) ; \mathrm{mp}=186.3-187.1^{\circ} \mathrm{C}$. ${ }^{1} \mathbf{H}$ NMR $\left(400 \mathrm{MHz}, \mathrm{CDCl}_{3}, 25{ }^{\circ} \mathrm{C}\right) \delta / \mathrm{ppm}: 8.32(\mathrm{~d}, J=8.8 \mathrm{~Hz}, 2 \mathrm{H}), 8.26(\mathrm{~d}, J=9.0$ $\mathrm{Hz}, 2 \mathrm{H}), 8.08(\mathrm{~d}, J=8.8 \mathrm{~Hz}, 2 \mathrm{H}), 7.56(\mathrm{~d}, J=9.0 \mathrm{~Hz}, 2 \mathrm{H}), 7.46-7.37(\mathrm{~m}, 2 \mathrm{H}), 7.35(\mathrm{t}$, $J=8.0 \mathrm{~Hz}, 1 \mathrm{H}), 7.12(\mathrm{dt}, J=7.5,1.4, \mathrm{~Hz}, 1 \mathrm{H}), 6.97(\mathrm{~s}, 1 \mathrm{H})$.

${ }^{13} \mathrm{C}$ NMR (100 MHz, $\left.\mathrm{CDCl}_{3}, 25{ }^{\circ} \mathrm{C}\right) \delta / \mathrm{ppm}: 151.0,147.8,146.5,144.1,140.0,138.3$, $135.1,131.2,130.3,129.6,128.8,126.9,126.4,124.8,124.7,124.2,107.9$.

IR $(\mathrm{KBr}) \tilde{v}\left(\mathrm{~cm}^{-1}\right): 3434,2354,1641,1516,1339,1107,856,753$.

HRMS (ESI) for $\left(\mathrm{C}_{21} \mathrm{H}_{15} \mathrm{~N}_{4} \mathrm{O}_{4}\right)[\mathrm{M}+\mathrm{H}]^{+}$(387.1018) found: 387.1016.

3-Methyl-1-(4-nitrophenyl)-5-phenyl-1H-pyrazole (3ea) 


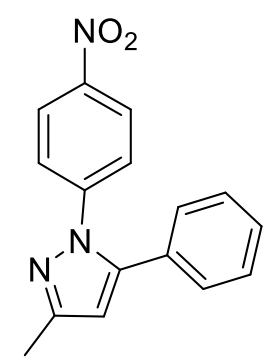

Following the TP-2, 3ea was obtained from 1 e $(68.3 \mathrm{mg}, 0.3 \mathrm{mmol}), \mathrm{PPh}_{3}(87.4 \mathrm{mg}$, 1.1 equiv), $\mathrm{Et}_{3} \mathrm{~N}$ (105 $\mu \mathrm{L}, 2.5$ equiv) and $\mathrm{PhCOCl} \mathbf{2 a}(41.79 \mu \mathrm{L}, 1.2$ equiv) as a white solid in (44.6 mg, 54\% yield; $\mathrm{R}_{f}=0.65(\mathrm{EA}: \mathrm{Hex}=1: 9) ; \mathrm{mp}=126.6-127.1{ }^{\circ} \mathrm{C}$.

${ }^{\mathbf{1}} \mathbf{H}$ NMR $\left(400 \mathrm{MHz}, \mathrm{CDCl}_{3}, 25{ }^{\circ} \mathrm{C}\right) \delta / \mathrm{ppm}: 8.15(\mathrm{~d}, J=9.2 \mathrm{~Hz}, 2 \mathrm{H}), 7.43(\mathrm{~d}, J=9.0$ $\mathrm{Hz}, 2 \mathrm{H}), 7.40-7.31(\mathrm{~m}, 3 \mathrm{H}), 7.25-7.21(\mathrm{~m}, 2 \mathrm{H}), 6.35$ (s, 1H).

${ }^{13} \mathrm{C}$ NMR (100 MHz, $\left.\mathrm{CDCl}_{3}, 25{ }^{\circ} \mathrm{C}\right) \delta / \mathrm{ppm}: 151.1,145.5,144.9,144.2,130.2,128.83$, $128.80,128.7,124.3,124.2,109.8,13.5$.

IR $(\mathrm{KBr}) \tilde{v}\left(\mathrm{~cm}^{-1}\right): 2928,2405,1599,1525,1343,856,786,689$.

HRMS (ESI) for $\left(\mathrm{C}_{16} \mathrm{H}_{14} \mathrm{~N}_{3} \mathrm{O}_{2}\right)[\mathrm{M}+\mathrm{H}]^{+}$(280.1086) found: 280.1089.

\section{1-(2,4-Dinitrophenyl)-3,5-diphenyl-1H-pyrazole (3fa)}

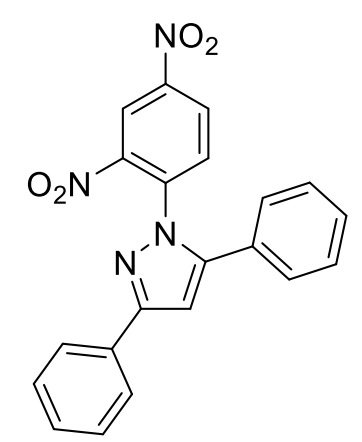

Following the TP-2, 3fa was obtained from 1 f (100.4 mg, $0.3 \mathrm{mmol}), \mathrm{PPh}_{3}(87.4 \mathrm{mg}$, 1.1 equiv), $\mathrm{Et}_{3} \mathrm{~N}$ (105 $\mu \mathrm{L}, 2.5$ equiv) and $\mathrm{PhCOCl} \mathbf{2 a}(41.79 \mu \mathrm{L}, 1.2$ equiv) as a yellow solid in (98.6 mg, 85\% yield; $\mathrm{R}_{f}=0.30(\mathrm{EA}: \mathrm{Hex}=1: 9) ; \mathrm{mp}=149.7-150.3{ }^{\circ} \mathrm{C}$.

${ }^{\mathbf{1}} \mathbf{H}$ NMR $\left(400 \mathrm{MHz}, \mathrm{CDCl}_{3}, 25^{\circ} \mathrm{C}\right) \delta / \mathrm{ppm}: 8.73(\mathrm{~d}, J=2.4 \mathrm{~Hz}, 1 \mathrm{H}), 8.35(\mathrm{dd}, J=8.8$, $2.4 \mathrm{~Hz}, 1 \mathrm{H}), 7.84(\mathrm{~d}, J=7.3 \mathrm{~Hz}, 2 \mathrm{H}), 7.48(\mathrm{~d}, J=8.8 \mathrm{~Hz}, 1 \mathrm{H}), 7.46-7.34(\mathrm{~m}, 6 \mathrm{H})$, 7.31-7.26 (m, 2H), $6.90(\mathrm{~s}, 1 \mathrm{H})$.

${ }^{13} \mathrm{C}$ NMR $\left(100 \mathrm{MHz}, \mathrm{CDCl}_{3}, 25{ }^{\circ} \mathrm{C}\right) \delta / \mathrm{ppm}: 154.7,146.0,145.9,145.3,138.1,131.8$, 
$129.6,129.4,129.1,128.9,128.8,128.7,128.6,127.0,126.0,120.9,106.6$.

IR $(\mathrm{KBr}) \tilde{v}\left(\mathrm{~cm}^{-1}\right):$ 3084, 2923, 1607, 1537, 1346, 1178, 1076, 833, 764, 696.

HRMS (ESI) for $\mathrm{C}_{21} \mathrm{H}_{15} \mathrm{~N}_{4} \mathrm{O}_{4},[\mathrm{M}+\mathrm{H}]^{+}$(387.1088) found: 387.1096 .

3,5-Diphenyl-1-tosyl-1H-pyrazole (3ga)

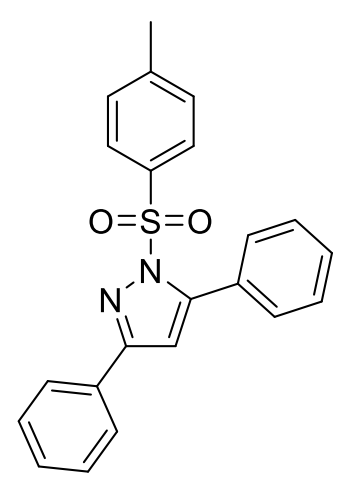

Following the TP-2, 3ga was obtained from $1 \mathrm{~g}(96.9 \mathrm{mg}, 0.3 \mathrm{mmol}), \mathrm{PPh}_{3}(87.4 \mathrm{mg}$, 1.1 equiv), $\mathrm{Et}_{3} \mathrm{~N}$ (105 $\mu \mathrm{L}, 2.5$ equiv) and $\mathrm{PhCOCl} \mathbf{2 a}(41.79 \mu \mathrm{L}, 1.2$ equiv) as a white solid in $\left(92.1 \mathrm{mg}, 82 \%\right.$ yield; $\mathrm{R}_{f}=0.40(\mathrm{EA}: \mathrm{Hex}=1: 9) ; \mathrm{mp}=119.2-120.6{ }^{\circ} \mathrm{C}$.

${ }^{1} \mathbf{H}$ NMR $\left(400 \mathrm{MHz}, \mathrm{CDCl}_{3}, 25{ }^{\circ} \mathrm{C}\right) \delta / \mathrm{ppm}: 7.84(\mathrm{~d}, J=7.5 \mathrm{~Hz}, 2 \mathrm{H}), 7.62(\mathrm{~d}, J=8.2$ $\mathrm{Hz}, 2 \mathrm{H}), 7.52-7.33$ (m, 8H), $7.20(\mathrm{~d}, J=8.2 \mathrm{~Hz}, 2 \mathrm{H}), 6.60(\mathrm{~s}, 1 \mathrm{H}), 2.36$ (s, 3H).

${ }^{13} \mathrm{C}$ NMR (100 MHz, $\left.\mathrm{CDCl}_{3}, 25{ }^{\circ} \mathrm{C}\right) \delta / \mathrm{ppm}: 155.1,149.4,145.2,134.9,131.4,129.9$, $129.6,129.4,129.2,128.7,128.0,127.8,126.4,109.4,21.6$.

IR $(\mathrm{KBr}) \tilde{v}\left(\mathrm{~cm}^{-1}\right):$ 3063, 2360, 1596, 1460, 1271, 1125, 1027, 944, 813, 660.

HRMS (ESI) for $\mathrm{C}_{22} \mathrm{H}_{19} \mathrm{~N}_{2} \mathrm{O}_{2} \mathrm{~S},[\mathrm{M}+\mathrm{H}]^{+}$(375.1162) found: 375.1169 .

3-(4-Methoxyphenyl)-5-phenyl-1-tosyl-1H-pyrazole (3ha)

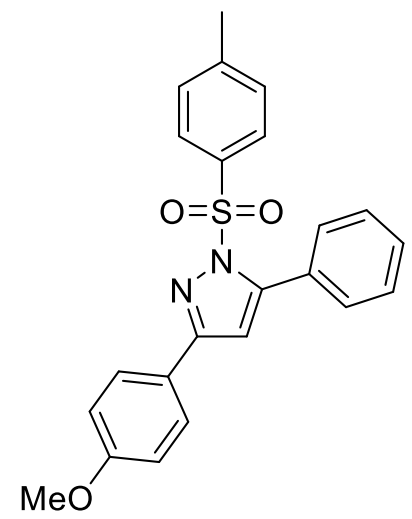


Following the TP-2, 3ha was obtained from $\mathbf{1 h}(105.8 \mathrm{mg}, 0.3 \mathrm{mmol}), \mathrm{PPh}_{3}(87.4 \mathrm{mg}$, 1.1 equiv), $\mathrm{Et}_{3} \mathrm{~N}(105 \mu \mathrm{L}, 2.5$ equiv) and $\mathrm{PhCOCl} \mathbf{2 a}(41.79 \mu \mathrm{L}, 1.2$ equiv) as a white solid in (102.1 mg, 84\% yield; $\mathrm{R}_{f}=0.20(\mathrm{EA}: \mathrm{Hex}=1: 9) ; \mathrm{mp}=126.3-127.1^{\circ} \mathrm{C}$.

${ }^{1} \mathbf{H}$ NMR $\left(400 \mathrm{MHz}, \mathrm{CDCl}_{3}, 25{ }^{\circ} \mathrm{C}\right) \delta / \mathrm{ppm}: 7.78(\mathrm{~d}, J=8.8 \mathrm{~Hz}, 2 \mathrm{H}), 7.61(\mathrm{~d}, J=8.3$ $\mathrm{Hz}, 2 \mathrm{H}), 7.49-7.41(\mathrm{~m}, 5 \mathrm{H}), 7.19(\mathrm{~d}, J=8.3 \mathrm{~Hz}, 2 \mathrm{H}), 6.93(\mathrm{~d}, J=8.8 \mathrm{~Hz}, 2 \mathrm{H}), 6.54(\mathrm{~s}$, $1 \mathrm{H}), 3.84(\mathrm{~s}, 3 \mathrm{H}), 2.36(\mathrm{~s}, 3 \mathrm{H})$.

${ }^{13} \mathrm{C}$ NMR $\left(100 \mathrm{MHz}, \mathrm{CDCl}_{3}, 25{ }^{\circ} \mathrm{C}\right) \delta / \mathrm{ppm}: 160.5,155.1,149.5,145.1,134.9,129.9$, $129.7,129.5,129.3,127.9,127.8,127.7,124.0,114.0,109.3,55.3,21.6$.

IR $(\mathrm{KBr}) \tilde{v}\left(\mathrm{~cm}^{-1}\right):$ 3060, 2933, 2554, 1733, 1612, 1296, 1125, 1029, 943, 763, 696.

HRMS (ESI) for $\mathrm{C}_{23} \mathrm{H}_{21} \mathrm{~N}_{2} \mathrm{O}_{3} \mathrm{~S},[\mathrm{M}+\mathrm{H}]^{+}$(405.1267) found: 405.1275 .

1-(4-Nitrophenyl)-3-phenyl-5-( $p$-tolyl)-1H-pyrazole (3ab)

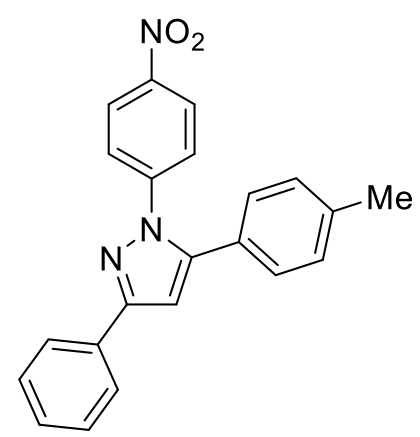

Following the TP-2, 3ab was obtained from $\mathbf{1 a}(86.7 \mathrm{mg}, 0.3 \mathrm{mmol}), \mathrm{PPh}_{3}(87.4 \mathrm{mg}$, 1.1 equiv), $\mathrm{Et}_{3} \mathrm{~N}$ (105 $\mu \mathrm{L}, 2.5$ equiv) and 4-methyl benzoyl chloride $2 \mathbf{b}$ (47.8 $\mu \mathrm{L}, 1.2$ equiv) as a yellow solid in $\left(87.4 \mathrm{mg}, 82 \%\right.$ yield; $\mathrm{R}_{f}=0.70(\mathrm{EA}: \mathrm{Hex}=1: 9) ; \mathrm{mp}=$ $170.9-171.6^{\circ} \mathrm{C}$

${ }^{1} \mathbf{H}$ NMR $\left(400 \mathrm{MHz}, \mathrm{CDCl}_{3}, 25{ }^{\circ} \mathrm{C}\right) \delta / \mathrm{ppm}: 8.19(\mathrm{~d}, J=9.0 \mathrm{~Hz}, 2 \mathrm{H}), 7.91(\mathrm{~d}, J=7.2$ $\mathrm{Hz}, 2 \mathrm{H}), 7.55(\mathrm{~d}, J=9.0 \mathrm{~Hz}, 2 \mathrm{H}), 7.45(\mathrm{t}, J=7.2 \mathrm{~Hz}, 2 \mathrm{H}), 7.37(\mathrm{t}, J=7.2 \mathrm{~Hz}, 1 \mathrm{H})$, 7.14-7.24 (m, 4H), $6.82(\mathrm{~s}, 1 \mathrm{H}), 2.40(\mathrm{~s}, 3 \mathrm{H})$.

${ }^{13} \mathrm{C}$ NMR $\left(100 \mathrm{MHz}, \mathrm{CDCl}_{3}, 25{ }^{\circ} \mathrm{C}\right) \delta / \mathrm{ppm}: 153.3,145.7,145.06,145.03,139.2$, $132.4,129.6,128.8,128.7,128.5,127.1,125.9,124.5,124.4,107.0,21.3$.

IR $(\mathrm{KBr}) \tilde{v}\left(\mathrm{~cm}^{-1}\right):$ 2918, 1594, 1497, 1338, 1108, 967, 855, 690. 
HRMS (ESI) for $\mathrm{C}_{22} \mathrm{H}_{18} \mathrm{~N}_{3} \mathrm{O}_{2},[\mathrm{M}+\mathrm{H}]^{+}$(356.1394) found: 356.1401 .

\section{5-(4-Methoxyphenyl)-1-(4-nitrophenyl)-3-phenyl-1H-pyrazole (3ac)}

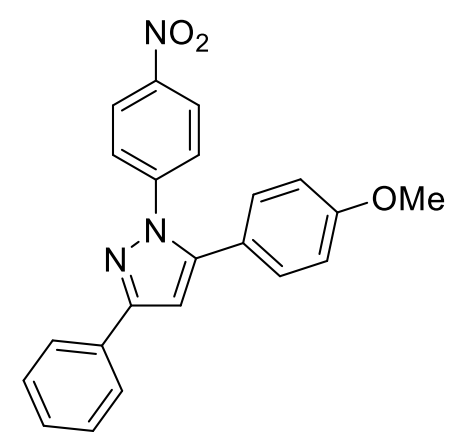

Following the TP-2, 3ac was obtained from $1 \mathbf{a}(86.7 \mathrm{mg}, 0.3 \mathrm{mmol}), \mathrm{PPh}_{3}(87.4 \mathrm{mg}$, 1.1 equiv), $\mathrm{Et}_{3} \mathrm{~N}$ (105 $\mu \mathrm{L}, 2.5$ equiv) and 4-methoxy benzoyl chloride $2 \mathrm{c}$ (49.2 $\mu \mathrm{L}, 1.2$ equiv) as a yellow solid in $\left(78.0 \mathrm{mg}, 70 \%\right.$ yield; $\mathrm{R}_{f}=0.50(\mathrm{EA}: \mathrm{Hex}=1: 9) ; \mathrm{mp}=$ $138.4-139.1^{\circ} \mathrm{C}$.

${ }^{1} \mathbf{H}$ NMR $\left(400 \mathrm{MHz}, \mathrm{CDCl}_{3}, 25{ }^{\circ} \mathrm{C}\right) \delta / \mathrm{ppm}: 8.20(\mathrm{~d}, J=8.9 \mathrm{~Hz}, 2 \mathrm{H}), 7.91(\mathrm{~d}, J=7.5$ $\mathrm{Hz}, 2 \mathrm{H}), 7.55(\mathrm{~d}, J=8.9 \mathrm{~Hz}, 2 \mathrm{H}), 7.45(\mathrm{t}, J=7.5 \mathrm{~Hz}, 2 \mathrm{H}), 7.37(\mathrm{t}, J=7.2 \mathrm{~Hz}, 1 \mathrm{H})$, $7.22(\mathrm{~d}, J=8.5 \mathrm{~Hz}, 2 \mathrm{H}), 6.91(\mathrm{~d}, J=8.5 \mathrm{~Hz}, 2 \mathrm{H}), 6.80(\mathrm{~s}, 1 \mathrm{H}), 3.85(\mathrm{~s}, 3 \mathrm{H})$.

${ }^{13} \mathrm{C}$ NMR $\left(100 \mathrm{MHz}, \mathrm{CDCl}_{3}, 25^{\circ} \mathrm{C}\right) \delta / \mathrm{ppm}: 160.2,153.2,145.7,145.0,144.8,132.4$, $130.1,128.8,128.5,125.9,124.42,124.40,122.3,114.4,106.8,55.3$.

IR $(\mathrm{KBr}) \tilde{v}\left(\mathrm{~cm}^{-1}\right):$ 3060, 2935, 1613, 1496, 1253, 1111, 1029, 968, 854, 693.

HRMS (ESI) for $\mathrm{C}_{22} \mathrm{H}_{18} \mathrm{~N}_{3} \mathrm{O}_{3},[\mathrm{M}+\mathrm{H}]^{+}$(372.1343) found: 372.1346 .

\section{1,5-Bis(4-nitrophenyl)-3-phenyl-1H-pyrazole (3ad)}

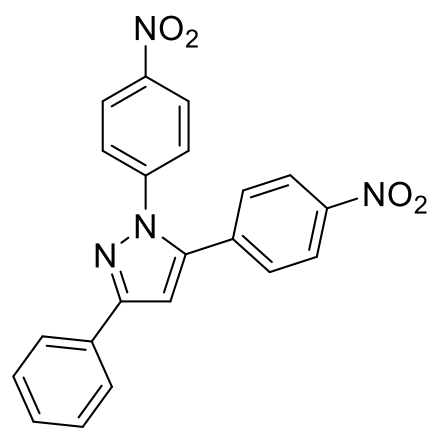

Following the TP-2, 3ad was obtained from $1 \mathbf{1 a}(86.7 \mathrm{mg}, 0.3 \mathrm{mmol}), \mathrm{PPh}_{3}(87.4 \mathrm{mg}$,

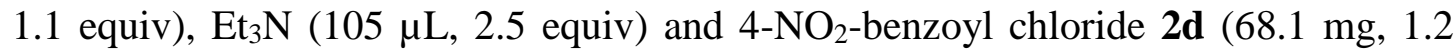


equiv) as a yellow solid in $\left(93.9 \mathrm{mg}, 81 \%\right.$ yield; $\mathrm{R}_{f}=0.40(\mathrm{EA}: \mathrm{Hex}=1: 9) ; \mathrm{mp}=$ $252.2-253.4{ }^{\circ} \mathrm{C}$.

${ }^{1} \mathbf{H}$ NMR $\left(400 \mathrm{MHz}, \mathrm{CDCl}_{3}, 25^{\circ} \mathrm{C}\right) \delta / \mathrm{ppm}: 8.28-8.24(\mathrm{~m}, 4 \mathrm{H}), 7.92(\mathrm{~d}, J=7.24 \mathrm{~Hz}$, 2H), 7.55-7.39 (m, 7H), $6.98(\mathrm{~s}, 1 \mathrm{H})$.

${ }^{13} \mathrm{C}$ NMR (100 MHz, $\left.\mathrm{CDCl}_{3}, 25{ }^{\circ} \mathrm{C}\right) \delta / \mathrm{ppm}: 153.8,147.9,146.4,144.3,142.4,136.2$ $131.7,129.5,128.97,128.91,125.9,124.9,124.8,124.2,108.2$.

IR $(\mathrm{KBr}) \tilde{v}\left(\mathrm{~cm}^{-1}\right): 2361,1594,1515,1337,1111,851,695$.

HRMS (ESI) for $\mathrm{C}_{21} \mathrm{H}_{15} \mathrm{~N}_{4} \mathrm{O}_{4},[\mathrm{M}+\mathrm{H}]^{+}$(387.1088) found: 387.1094 .

5-(4-Chlorophenyl)-1-(4-nitrophenyl)-3-phenyl-1H-pyrazole (3ae)

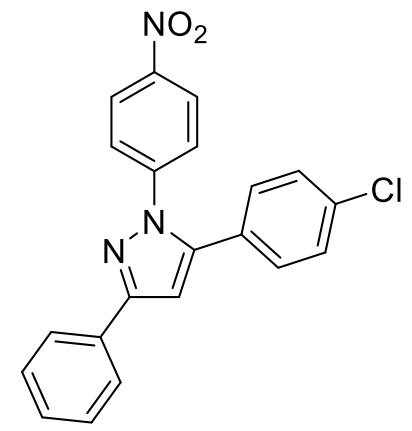

Following the TP-2, 3ae was obtained from $1 \mathbf{a}(86.7 \mathrm{mg}, 0.3 \mathrm{mmol}), \mathrm{PPh}_{3}(87.4 \mathrm{mg}$, 1.1 equiv), $\mathrm{Et}_{3} \mathrm{~N}$ (105 $\mu \mathrm{L}, 2.5$ equiv) and 4-Cl-benzoyl chloride $2 \mathrm{e}(46.8 \mu \mathrm{L}, 1.2$ equiv) as a yellow solid in $\left(87.9 \mathrm{mg}, 78 \%\right.$ yield; $\mathrm{R}_{f}=0.70(\mathrm{EA}: \mathrm{Hex}=1: 9) ; \mathrm{mp}=188.2-189.4$ ${ }^{\circ} \mathrm{C}$.

${ }^{1} \mathbf{H}$ NMR $\left(400 \mathrm{MHz}, \mathrm{CDCl}_{3}, 25{ }^{\circ} \mathrm{C}\right) \delta / \mathrm{ppm}: 8.20(\mathrm{~d}, J=9.0 \mathrm{~Hz}, 2 \mathrm{H}), 7.90(\mathrm{~d}, J=7.4$ $\mathrm{Hz}, 2 \mathrm{H}), 7.54(\mathrm{~d}, J=9.0 \mathrm{~Hz}, 2 \mathrm{H}), 7.46(\mathrm{t}, J=7.4 \mathrm{~Hz}, 2 \mathrm{H}), 7.40-7.37(\mathrm{~m}, 3 \mathrm{H}), 7.24(\mathrm{~d}$, $J=8.8 \mathrm{~Hz}, 2 \mathrm{H}), 6.85(\mathrm{~s}, 1 \mathrm{H})$.

${ }^{13} \mathrm{C}$ NMR (100 MHz, $\left.\mathrm{CDCl}_{3}, 25{ }^{\circ} \mathrm{C}\right) \delta / \mathrm{ppm:} \mathrm{153.4,} \mathrm{146.0,} \mathrm{144.7,} \mathrm{143.7,} \mathrm{135.4,} \mathrm{132.1,}$ $130.1,129.3,128.8,128.7,128.5,125.9,124.6,124.59,107.3$.

IR $(\mathrm{KBr}) \tilde{v}\left(\mathrm{~cm}^{-1}\right):$ 3135, 2924, 1594, 1337, 1108, 967, 767, 688.

HRMS (ESI) for $\mathrm{C}_{21} \mathrm{H}_{15}{ }^{35} \mathrm{ClN}_{3} \mathrm{O}_{2},[\mathrm{M}+\mathrm{H}]^{+}$(376.0847) found: 376.0858 .

HRMS (ESI) for $\mathrm{C}_{21} \mathrm{H}_{15}{ }^{37} \mathrm{ClN}_{3} \mathrm{O}_{2},[\mathrm{M}+\mathrm{H}]^{+}$(378.0818) found: 378.0837 . 


\section{5-(4-Bromophenyl)-1-(4-nitrophenyl)-3-phenyl-1H-pyrazole (3af)}

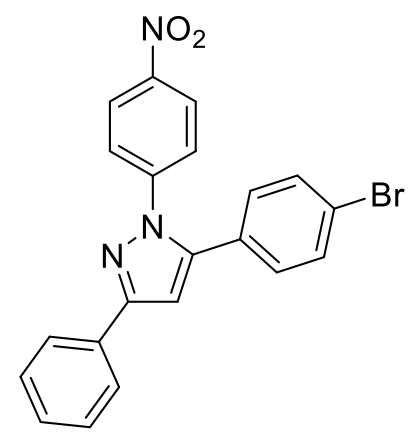

Following the TP-2, 3af was obtained from $1 \mathbf{a}(86.7 \mathrm{mg}, 0.3 \mathrm{mmol}), \mathrm{PPh}_{3}(87.4 \mathrm{mg}$, 1.1 equiv), $\mathrm{Et}_{3} \mathrm{~N}$ (105 $\mu \mathrm{L}, 2.5$ equiv) and 4-Br-benzoyl chloride $2 \mathbf{f}(80.6 \mu \mathrm{L}, 1.2$ equiv) as a yellow solid in (98.3 mg, 78\% yield; $\mathrm{R}_{f}=0.70(\mathrm{EA}: \mathrm{Hex}=1: 9) ; \mathrm{mp}=201.6-202.1$ ${ }^{\circ} \mathrm{C}$.

${ }^{1} \mathbf{H}$ NMR $\left(400 \mathrm{MHz}, \mathrm{CDCl}_{3}, 25{ }^{\circ} \mathrm{C}\right) \delta / \mathrm{ppm}: 8.21(\mathrm{~d}, J=9.0 \mathrm{~Hz}, 2 \mathrm{H}), 7.90(\mathrm{~d}, J=7.6$ $\mathrm{Hz}, 2 \mathrm{H}), 7.53(\mathrm{~d}, J=8.5 \mathrm{~Hz}, 4 \mathrm{H}), 7.45(\mathrm{t}, J=7.6 \mathrm{~Hz}, 2 \mathrm{H}), 7.38(\mathrm{t}, J=7.3 \mathrm{~Hz}, 1 \mathrm{H})$, $7.17(\mathrm{~d}, J=8.5 \mathrm{~Hz}, 2 \mathrm{H}), 6.85(\mathrm{~s}, 1 \mathrm{H})$.

${ }^{13} \mathrm{C}$ NMR $\left(100 \mathrm{MHz}, \mathrm{CDCl}_{3}, 25{ }^{\circ} \mathrm{C}\right) \delta / \mathrm{ppm}: 153.4,146.0,144.6,143.7,132.2,132.1$, $130.3,128.9,128.8,128.7,125.9,124.6,123.5,107.3$

IR $(\mathrm{KBr}) \tilde{v}\left(\mathrm{~cm}^{-1}\right):$ 2360, 1593, 1338, 1170, 966, 767, 690.

HRMS (ESI) for $\left(\mathrm{C}_{21} \mathrm{H}_{15}{ }^{79} \mathrm{BrN}_{3} \mathrm{O}_{2}\right)[\mathrm{M}+\mathrm{H}]^{+}$(420.0348) found: 420.0346.

HRMS (ESI) for $\left(\mathrm{C}_{21} \mathrm{H}_{15}{ }^{81} \mathrm{BrN}_{3} \mathrm{O}_{2}\right)[\mathrm{M}+\mathrm{H}]^{+}$(422.0329) found: 422.0328.

\section{5-(3-Chlorophenyl)-1-(4-nitrophenyl)-3-phenyl-1H-pyrazole (3ag)}

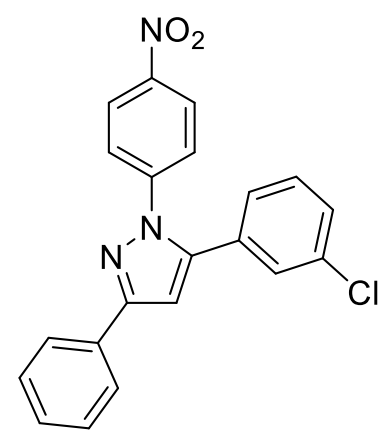

Following the TP-2, 3ag was obtained from $1 \mathbf{a}(86.7 \mathrm{mg}, 0.3 \mathrm{mmol}), \mathrm{PPh}_{3}(87.4 \mathrm{mg}$, 1.1 equiv), Et ${ }_{3} \mathrm{~N}$ (105 $\mu \mathrm{L}, 2.5$ equiv) and 3-Cl-benzoyl chloride $2 \mathrm{~g}$ ( $47.5 \mu \mathrm{L}, 1.2$ equiv) 
as a yellow solid in $\left(94.5 \mathrm{mg}, 84 \%\right.$ yield; $\mathrm{R}_{f}=0.60(\mathrm{EA}: \mathrm{Hex}=1: 9) ; \mathrm{mp}=147.1-148.6$ ${ }^{\circ} \mathrm{C}$.

${ }^{1} \mathbf{H}$ NMR $\left(400 \mathrm{MHz}, \mathrm{CDCl}_{3}, 25{ }^{\circ} \mathrm{C}\right) \delta / \mathrm{ppm}: 8.22(\mathrm{~d}, J=8.5 \mathrm{~Hz}, 2 \mathrm{H}), 7.91(\mathrm{~d}, J=7.9$ $\mathrm{Hz}, 2 \mathrm{H}), 7.54(\mathrm{~d}, J=8.5 \mathrm{~Hz}, 2 \mathrm{H}), 7.46(\mathrm{t}, J=7.5 \mathrm{~Hz}, 2 \mathrm{H}), 7.42-7.35(\mathrm{~m}, 3 \mathrm{H}), 7.31(\mathrm{t}$, $J=7.7 \mathrm{~Hz}, 1 \mathrm{H}), 7.12(\mathrm{~d}, J=7.7 \mathrm{~Hz}, 1 \mathrm{H}), 6.87(\mathrm{~s}, 1 \mathrm{H})$.

${ }^{13} \mathrm{C}$ NMR $\left(100 \mathrm{MHz}, \mathrm{CDCl}_{3}, 25^{\circ} \mathrm{C}\right) \delta / \mathrm{ppm}: 153.4,146.0,144.5,143.3,134.9,132.0$, $131.8,130.2,129.2,128.8,128.75,128.72,126.9,125.9,124.54,124.51,107.6$.

IR $(\mathrm{KBr}) \tilde{v}\left(\mathrm{~cm}^{-1}\right): 3064,2926,2360,1596,1359,1111,985,854,693$.

HRMS (ESI) for $\mathrm{C}_{21} \mathrm{H}_{15}{ }^{35} \mathrm{ClN}_{3} \mathrm{O}_{2},[\mathrm{M}+\mathrm{H}]^{+}$(376.0853) found: 376.0849 .

HRMS (ESI) for $\mathrm{C}_{21} \mathrm{H}_{15}{ }^{37} \mathrm{ClN}_{3} \mathrm{O}_{2},[\mathrm{M}+\mathrm{H}]^{+}$(378.0827) found: 378.0824 .

5-(2-Chlorophenyl)-1-(4-nitrophenyl)-3-phenyl-1H-pyrazole (3ah)

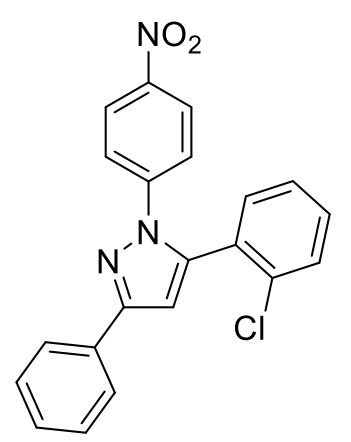

Following the TP-2, 3ah was obtained from $\mathbf{1 a}(86.7 \mathrm{mg}, 0.3 \mathrm{mmol}), \mathrm{PPh}_{3}(87.4 \mathrm{mg}$, 1.1 equiv), $\mathrm{Et}_{3} \mathrm{~N}$ (105 $\mu \mathrm{L}, 2.5$ equiv) and 2-Cl-benzoyl chloride $2 \mathbf{h}$ (45.0 $\mu \mathrm{L}, 1.2$ equiv) as a yellow solid in $\left(53.0 \mathrm{mg}, 47 \%\right.$ yield; $\mathrm{R}_{f}=0.50(\mathrm{EA}: \mathrm{Hex}=1: 9) ; \mathrm{mp}=$ $147.9-148.3^{\circ} \mathrm{C}$

${ }^{1} \mathbf{H}$ NMR $\left(400 \mathrm{MHz}, \mathrm{CDCl}_{3}, 25{ }^{\circ} \mathrm{C}\right) \delta / \mathrm{ppm}: 8.14(\mathrm{~d}, J=8.7 \mathrm{~Hz}, 2 \mathrm{H}), 7.93(\mathrm{~d}, J=7.4$ $\mathrm{Hz}, 2 \mathrm{H}), 7.50-7.33(\mathrm{~m}, 9 \mathrm{H}), 6.86(\mathrm{~s}, 1 \mathrm{H})$.

${ }^{13} \mathrm{C}$ NMR $\left(100 \mathrm{MHz}, \mathrm{CDCl}_{3}, 25^{\circ} \mathrm{C}\right) \delta / \mathrm{ppm}: 153.0,145.6,145.0,141.6,133.8,132.1$, $131.7,130.9,130.3,129.7,128.7,128.6,127.3,125.9,124.5,122.9,108.4$.

IR $(\mathrm{KBr}) \tilde{v}\left(\mathrm{~cm}^{-1}\right):$ 3064, 2923, 2850, 1595, 1458, 1111, 970, 854, 692.

HRMS (ESI) for $\mathrm{C}_{21} \mathrm{H}_{15}{ }^{35} \mathrm{ClN}_{3} \mathrm{O}_{2},[\mathrm{M}+\mathrm{H}]^{+}$(376.0853) found: 376.0858 . 
HRMS (ESI) for $\mathrm{C}_{21} \mathrm{H}_{15}{ }^{37} \mathrm{ClN}_{3} \mathrm{O}_{2},[\mathrm{M}+\mathrm{H}]^{+}$(378.0835) found: 378.0838 .

5-(2-Bromophenyl)-1-(4-nitrophenyl)-3-phenyl-1H-pyrazole (3ai)

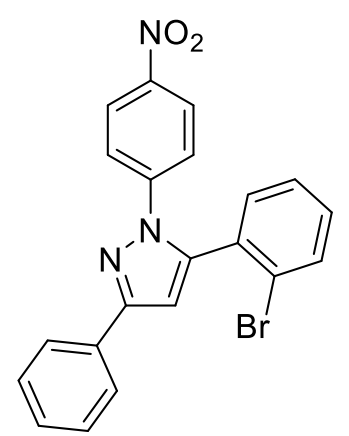

Following the TP-2, 3ai was obtained from $1 \mathbf{a}(86.7 \mathrm{mg}, 0.3 \mathrm{mmol}), \mathrm{PPh}_{3}(87.4 \mathrm{mg}$, 1.1 equiv), $\mathrm{Et}_{3} \mathrm{~N}$ (105 $\mu \mathrm{L}, 2.5$ equiv) and 2-bromo benzoyl chloride $2 \mathbf{i}$ (48.0 $\mu \mathrm{L}, 1.2$ equiv) as a yellow solid in $\left(50.3 \mathrm{mg}, 40 \%\right.$ yield; $\mathrm{R}_{f}=0.50(\mathrm{EA}: \mathrm{Hex}=1: 9) ; \mathrm{mp}=$ $162.5-163.2^{\circ} \mathrm{C}$

${ }^{1} \mathbf{H}$ NMR $\left(400 \mathrm{MHz}, \mathrm{CDCl}_{3}, 25{ }^{\circ} \mathrm{C}\right) \delta / \mathrm{ppm}: 8.15(\mathrm{~d}, J=9.0 \mathrm{~Hz}, 2 \mathrm{H}), 7.90-8.03(\mathrm{~m}$, 2H), $7.65(\mathrm{~d}, J=8.1 \mathrm{~Hz}, 1 \mathrm{H}), 7.56-7.30(\mathrm{~m}, 8 \mathrm{H}), 6.87(\mathrm{~s}, 1 \mathrm{H})$.

${ }^{13} \mathrm{C}$ NMR (100 MHz, $\left.\mathrm{CDCl}_{3}, 25{ }^{\circ} \mathrm{C}\right) \delta / \mathrm{ppm}: 152.9,145.7,145.0,143.1,133.5,132.1$, $131.9,131.1,128.8,128.7,127.8,125.9,124.5,123.9,122.9,108.4$.

IR $(\mathrm{KBr}) \tilde{v}\left(\mathrm{~cm}^{-1}\right): 3060,2925,1595,1457,1340,1112,1027,969,854,691,644$.

HRMS (ESI) for $\left(\mathrm{C}_{21} \mathrm{H}_{15}{ }^{79} \mathrm{BrN}_{3} \mathrm{O}_{2}\right)[\mathrm{M}+\mathrm{H}]^{+}$(420.0348) found: 420.0348 .

HRMS (ESI) for $\left(\mathrm{C}_{21} \mathrm{H}_{15}{ }^{81} \mathrm{BrN}_{3} \mathrm{O}_{2}\right)[\mathrm{M}+\mathrm{H}]^{+}$(422.0331) found: 422.0331

\section{1-(4-Nitrophenyl)-5-(perfluorophenyl)-3-phenyl-1H-pyrazole (3aj)}

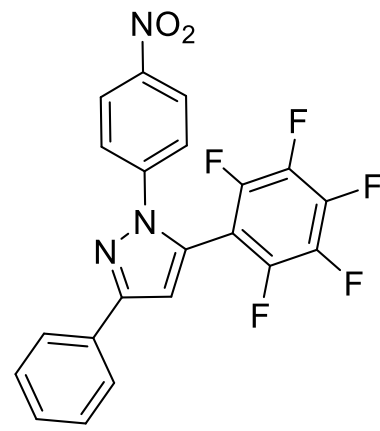

Following the TP-2, 3aj was obtained from $1 \mathbf{a}(86.7 \mathrm{mg}, 0.3 \mathrm{mmol}), \mathrm{PPh}_{3}(87.4 \mathrm{mg}$, 1.1 equiv), $\mathrm{Et}_{3} \mathrm{~N}$ (105 $\mu \mathrm{L}, 2.5$ equiv) and pentafluro benzoyl chloride $2 \mathbf{j}$ (54.8 $\mu \mathrm{L}, 1.2$ 
equiv) as a yellow solid in $\left(104.7 \mathrm{mg}, 81 \%\right.$ yield; $\mathrm{R}_{f}=0.40(\mathrm{EA}: \mathrm{Hex}=1: 9) ; \mathrm{mp}=$ $189.6-190.1^{\circ} \mathrm{C}$

${ }^{1} \mathbf{H}$ NMR $\left(400 \mathrm{MHz}, \mathrm{CDCl}_{3}, 25{ }^{\circ} \mathrm{C}\right) \delta / \mathrm{ppm}: 8.28(\mathrm{~d}, J=8.9 \mathrm{~Hz}, 2 \mathrm{H}), 7.91(\mathrm{~d}, J=7.6$ $\mathrm{Hz}, 2 \mathrm{H}), 7.55(\mathrm{~d}, J=8.9 \mathrm{~Hz}, 2 \mathrm{H}), 7.50(\mathrm{t}, J=7.6 \mathrm{~Hz}, 2 \mathrm{H}), 7.40(\mathrm{t}, J=7.6 \mathrm{~Hz}, 1 \mathrm{H})$, $7.0(\mathrm{~s}, 1 \mathrm{H})$.

${ }^{13} \mathrm{C}$ NMR $\left(100 \mathrm{MHz}, \mathrm{CDCl}_{3}, 25^{\circ} \mathrm{C}\right) \delta / \mathrm{ppm}: 153.8,146.5,144.4,131.5,129.0,128.9$, 128.5, 126.0, 125.0, 123.4, 110.1, 105.7 (td, $J=17.7,4.0 \mathrm{~Hz}$ ), 135-150 (broadened peaks due to the multiple ${ }^{13} \mathrm{C}-{ }^{19} \mathrm{~F}$ couplings of the perfluorophenyl rings).

${ }^{19}$ F NMR $\left(376 \mathrm{MHz}, \mathrm{CDCl}_{3}\right) \delta / \mathrm{ppm}:-136.35--138.98(\mathrm{~m}, 2 \mathrm{~F}),-149.89(\mathrm{t}, J=20.3$, 1F), $-158.10--161.36(\mathrm{~m}, 2 \mathrm{~F})$.

IR $(\mathrm{KBr}) \tilde{v}\left(\mathrm{~cm}^{-1}\right): 3438,2362,1643,1506,1343,989,699$.

HRMS (ESI) for $\mathrm{C}_{21} \mathrm{H}_{11} \mathrm{~F}_{5} \mathrm{~N}_{3} \mathrm{O}_{2}[\mathrm{M}+\mathrm{H}]^{+}$(432.0769) found: 432.0769.

\section{5-Methyl-1-(4-nitrophenyl)-3-phenyl-1H-pyrazole (3ak)}

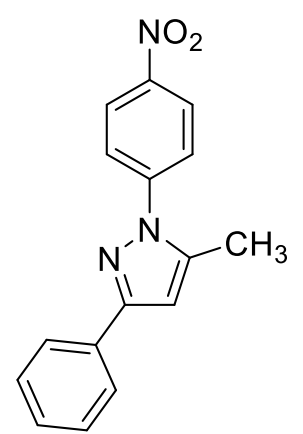

Following the TP-2, 3ak was obtained from $\mathbf{1 a}(86.7 \mathrm{mg}, 0.3 \mathrm{mmol}), \mathrm{PPh}_{3}(87.4 \mathrm{mg}$, 1.1 equiv), $\mathrm{Et}_{3} \mathrm{~N}(105 \mu \mathrm{L}, 2.5$ equiv) and acetic anhydride $2 \mathbf{k}(34.3 \mu \mathrm{L}, 1.2$ equiv) as a white solid in (27.6 mg, 51\% yield; $\mathrm{R}_{f}=0.50(\mathrm{EA}: \mathrm{Hex}=1: 9) ; \mathrm{mp}=121.3-122.1{ }^{\circ} \mathrm{C}$. ${ }^{1} \mathbf{H}$ NMR $\left(400 \mathrm{MHz}, \mathrm{CDCl}_{3}, 25{ }^{\circ} \mathrm{C}\right) \delta / \mathrm{ppm}: 8.35(\mathrm{~d}, J=9.1 \mathrm{~Hz}, 2 \mathrm{H}), 7.85(\mathrm{~d}, J=8.2$ $\mathrm{Hz}, 2 \mathrm{H}), 7.78(\mathrm{~d}, J=9.1 \mathrm{~Hz}, 2 \mathrm{H}), 7.42(\mathrm{t}, J=8.2 \mathrm{~Hz}, 2 \mathrm{H}), 7.38-7.31(\mathrm{~m}, 1 \mathrm{H}), 6.60(\mathrm{~s}$, $1 \mathrm{H}), 2.51(\mathrm{~s}, 3 \mathrm{H})$.

${ }^{13} \mathrm{C}$ NMR $\left(100 \mathrm{MHz}, \mathrm{CDCl}_{3}, 25{ }^{\circ} \mathrm{C}\right) \delta / \mathrm{ppm}: 152.9,145.9,145.0,140.5,132.5,128.7$, $128.4,125.8,124.7,123.8,106.6,13.3$. 
IR $(\mathrm{KBr}) \tilde{v}\left(\mathrm{~cm}^{-1}\right): 3084,2814,1464,1346,1111,884,769,691$.

HRMS (ESI) for $\mathrm{C}_{16} \mathrm{H}_{14} \mathrm{~N}_{3} \mathrm{O}_{2},[\mathrm{M}+\mathrm{H}]^{+}$(280.1086) found: 280.1090.

1-(4-Nitrophenyl)-3-phenyl-5-(trifluoromethyl)-1H-pyrazole (3al)

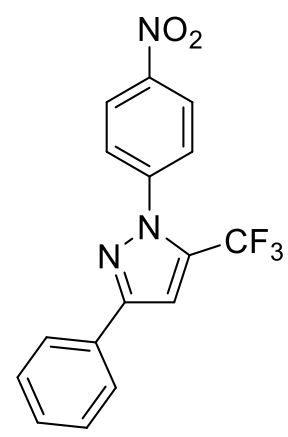

Following the TP-2, 3al was obtained from $1 \mathbf{a}(86.7 \mathrm{mg}, 0.3 \mathrm{mmol}), \mathrm{PPh}_{3}(87.4 \mathrm{mg}$, 1.1 equiv), $\mathrm{Et}_{3} \mathrm{~N}$ (105 $\mu \mathrm{L}, 2.5$ equiv) and TFAA $2 \mathbf{l}(51.3 \mu \mathrm{L}, 1.2$ equiv) as a white solid in $\left(69.0 \mathrm{mg}, 69 \%\right.$ yield; $\mathrm{R}_{f}=0.70(\mathrm{EA}: \mathrm{Hex}=1: 9) ; \mathrm{mp}=102.5-103.2{ }^{\circ} \mathrm{C}$.

${ }^{1} \mathbf{H}$ NMR $\left(400 \mathrm{MHz}, \mathrm{CDCl}_{3}, 25{ }^{\circ} \mathrm{C}\right) \delta / \mathrm{ppm}: 8.36(\mathrm{~d}, J=8.9 \mathrm{~Hz}, 2 \mathrm{H}), 7.85(\mathrm{~d}, J=7.2$ $\mathrm{Hz}, 2 \mathrm{H}), 7.79(\mathrm{~d}, J=8.9 \mathrm{~Hz}, 2 \mathrm{H}), 7.51-7.36(\mathrm{~m}, 3 \mathrm{H}), 7.19(\mathrm{~s}, 1 \mathrm{H})$.

${ }^{13} \mathrm{C}$ NMR $\left(100 \mathrm{MHz}, \mathrm{CDCl}_{3}, 25{ }^{\circ} \mathrm{C}\right) \delta / \mathrm{ppm}: 152.8,147.4,143.9,133.9$ (q, $J=39.6$ $\mathrm{Hz}), 130.9,129.2,128.9,125.9,125.5,124.7,119.6(\mathrm{q}, J=269.4 \mathrm{~Hz}), 107.9(\mathrm{~d}, J=$ $2.4 \mathrm{~Hz})$.

${ }^{19}$ F NMR $\left(376 \mathrm{MHz}, \mathrm{CDCl}_{3}\right) \delta / \mathrm{ppm}:-57.16$.

IR $(\mathrm{KBr}) \tilde{v}\left(\mathrm{~cm}^{-1}\right): 3093,2924,1598,1446,1208,988,824,769,691$.

HRMS (ESI) for $\mathrm{C}_{16} \mathrm{H}_{11} \mathrm{~F}_{3} \mathrm{~N}_{3} \mathrm{O}_{2},[\mathrm{M}+\mathrm{H}]^{+}$(334.0803) found: 334.0806 .

1-(4-Nitrophenyl)-3,5-diphenyl-1H-pyrazole (3aa)

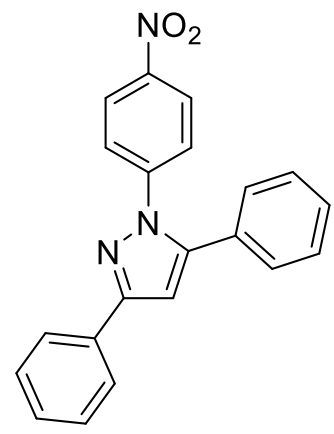

Following the TP-6, 3aa was obtained from $4 \mathbf{a}(165.3 \mathrm{mg}, 0.3 \mathrm{mmol}), \mathrm{Et}_{3} \mathrm{~N}$ (105 $\mu \mathrm{L}$, 
2.5 equiv) and $\mathrm{PhCOCl} \mathbf{2 a}(41.79 \mu \mathrm{L}, 1.2$ equiv) as a yellow solid in (140.2 mg, 86\% yield).

(E/Z)-2-Chloro-1-phenylethan-1-one oxime (5a) $)^{2}$<smiles>ON=C(CCl)c1ccccc1</smiles>

Following the TP-3, 5a was obtained from 2-chloroacetophenone (3.1 g, $20 \mathrm{mmol})$ and hydroxylamine hydrochloride (4.0 g, 3.0 equiv) as a white solid ( $2.3 \mathrm{~g}, 70 \%$ yield); $\mathrm{R}_{f}=0.725(\mathrm{DCM}: \mathrm{Hex}=3: 1) ; \mathrm{mp}=86.6-87.7^{\circ} \mathrm{C}$.

${ }^{1} \mathbf{H}$ NMR $\left(400 \mathrm{MHz}, \mathrm{CDCl}_{3}, 25{ }^{\circ} \mathrm{C}\right) \delta / \mathrm{ppm}: 9.00(\mathrm{~s}, 1 \mathrm{H}), 7.78-7.64(\mathrm{~m}, 2 \mathrm{H})$, 7.54-7.38 (m, 3H), $4.62(\mathrm{~s}, 2 \mathrm{H})$. (Minor peaks also observed in ${ }^{1} \mathrm{H}$ NMR spectra $E / Z=1: 10)$

IR $(\mathrm{KBr}) \tilde{v}\left(\mathrm{~cm}^{-1}\right): 3241,3063,2923,1636,1499,1266,742$.

HRMS (ESI) for $\mathrm{C}_{8} \mathrm{H}_{9}{ }^{35} \mathrm{ClNO},[\mathrm{M}+\mathrm{H}]^{+}$(170.0373) found: 170.0374

HRMS (ESI) for $\mathrm{C}_{8} \mathrm{H}_{9}{ }^{37} \mathrm{ClNO},[\mathrm{M}+\mathrm{H}]^{+}$(172.0343) found: 172.0345 .

(Z)-2-Chloro-1-(4-methoxyphenyl)ethan-1-one oxime (5b)<smiles>COc1ccc(/C(CCl)=N/O)cc1</smiles>

Following the TP-3, 5b was obtained from 4'-methoxy-2-chloroacetophenone (200.0 $\mathrm{mg}, 1.1 \mathrm{mmol})$ and hydroxylamine hydrochloride $(228.0 \mathrm{mg}, 3.0$ equiv) as a white solid (0.14 g, 66\% yield); $\mathrm{R}_{f}=0.175(\mathrm{DCM}: \mathrm{Hex}=3: 1) ; \mathrm{mp}=87.7-88.4{ }^{\circ} \mathrm{C}$.

${ }^{1} \mathbf{H}$ NMR $\left(400 \mathrm{MHz}, \mathrm{CDCl}_{3}, 25{ }^{\circ} \mathrm{C}\right) \delta / \mathrm{ppm}: 7.94(\mathrm{~s}, 1 \mathrm{H}), 7.64(\mathrm{~d}, J=8.8 \mathrm{~Hz}, 2 \mathrm{H})$, $6.94(\mathrm{~d}, J=8.8 \mathrm{~Hz}, 2 \mathrm{H}), 4.59(\mathrm{~s}, 2 \mathrm{H}), 3.84(\mathrm{~s}, 3 \mathrm{H})$.

IR $(\mathrm{KBr}) \tilde{v}\left(\mathrm{~cm}^{-1}\right):$ 3224, 3064, 2934, 1608, 1516, 1259, 959, 911, 830.

HRMS (ESI) for $\mathrm{C}_{9} \mathrm{H}_{11}{ }^{35} \mathrm{ClNO}_{2},[\mathrm{M}+\mathrm{H}]^{+}$(200.0478) found: 200.0480 . 
HRMS (ESI) for $\mathrm{C}_{9} \mathrm{H}_{11}{ }^{37} \mathrm{ClNO}_{2},[\mathrm{M}+\mathrm{H}]^{+}$(202.0449) found: 202.0451 .

\section{(Z)-2-Chloro-1-(4-fluorophenyl)ethan-1-one oxime $(5 \mathrm{c})^{8}$}<smiles>ON=C(CCl)c1ccc(F)cc1</smiles>

Following the TP-3, 5c was obtained from 2-chloro-4'-fluoroacetophenone (1.0 g, 5.8 mmol) and hydroxylamine hydrochloride (1.2 g, 3.0 equiv) as a white solid (1.0 g, $90 \%$ yield $) ; \mathrm{R}_{f}=0.700(\mathrm{DCM}: \mathrm{Hex}=3: 1) ; \mathrm{mp}=68.6-69.0^{\circ} \mathrm{C}$.

${ }^{1} \mathbf{H}$ NMR $\left(400 \mathrm{MHz}, \mathrm{CDCl}_{3}, 25{ }^{\circ} \mathrm{C}\right) \delta / \mathrm{ppm}: 8.33(\mathrm{~s}, 1 \mathrm{H}), 7.69(\mathrm{dd}, J=8.4,5.5 \mathrm{~Hz}$, $2 \mathrm{H}), 7.11(\mathrm{t}, J=8.6 \mathrm{~Hz}, 2 \mathrm{H}), 4.59(\mathrm{~s}, 2 \mathrm{H})$.

IR $(\mathrm{KBr}) \tilde{v}\left(\mathrm{~cm}^{-1}\right): 3314,3080,2943,1682,1594,1509,1285,1211,1160,1102,866$. HRMS (ESI) for $\mathrm{C}_{8} \mathrm{H}_{8}{ }^{35} \mathrm{ClFNO},[\mathrm{M}+\mathrm{H}]^{+}$(188.0278) found: 188.0279.

HRMS (ESI) for $\mathrm{C}_{8} \mathrm{H}_{8}{ }^{37} \mathrm{ClFNO},[\mathrm{M}+\mathrm{H}]^{+}$(190.0249) found: 190.0247 .

(E/Z)-2-Bromo-1-(4-nitrophenyl)ethan-1-one oxime $(5 \mathrm{~d})^{7}$<smiles>O=[N+]([O-])c1ccc(/C(CBr)=N/O)cc1</smiles>

Following the TP-3, 5d was obtained from 2-bromo-4'-nitroacetophenone (500.0 mg, $2.1 \mathrm{mmol}$ ) and hydroxylamine hydrochloride (425.0 $\mathrm{mg}, 3.0$ equiv) as a white solid (376.8 mg, 71\% yield); $\mathrm{R}_{f}=0.175$ (DCM:Hex $\left.=3: 1\right) ; \mathrm{mp}=130.0-131.0{ }^{\circ} \mathrm{C}$.

${ }^{1} \mathbf{H}$ NMR $\left(400 \mathrm{MHz}, \mathrm{CDCl}_{3}, 25^{\circ} \mathrm{C}\right) \delta / \mathrm{ppm}: 8.28(\mathrm{~d}, J=8.8 \mathrm{~Hz}, 2 \mathrm{H}$, major), 8.14 (s, 1H, minor), 8.07 (s, 1H, major), 7.89 (d, $J=8.8 \mathrm{~Hz}, 2 \mathrm{H}$, major), 4.64 (s, 2H, major), 4.44 (s, 2H, minor).

IR (KBr) $\tilde{v}\left(\mathrm{~cm}^{-1}\right): 3220,3068,2914,1596,1516,1339,857,691$.

HRMS (EI) for $\mathrm{C}_{8} \mathrm{H}_{7}{ }^{79} \mathrm{BrN}_{2} \mathrm{O}_{3},[\mathrm{M}]^{+}$(257.9640) found: 257.9640 .

HRMS (EI) for $\mathrm{C}_{8} \mathrm{H}_{7}{ }^{81} \mathrm{BrN}_{2} \mathrm{O}_{3},[\mathrm{M}]^{+}$(259.9620) found: 259.9620. 
(Z)-Tributyl(2-(hydroxyimino)-2-phenylethyl)phosphonium chloride (7a)<smiles>O/N=C(/C[18OH])c1ccccc1</smiles>

Following the TP-5, 7a was obtained from $5 \mathbf{a}(500 \mathrm{mg}, 3.0 \mathrm{mmol})$ and $\mathrm{PBu}_{3}(1.0 \mathrm{~mL}$, 1.1 equiv), as a white solid (1.0 g, 97\% yield); $\mathrm{Rf}=0.462(\mathrm{DCM}: \mathrm{MeOH}=9: 1) ; \mathrm{mp}=$ $194.5-195.5^{\circ} \mathrm{C}$.

${ }^{1} \mathbf{H}$ NMR (400 MHz, $\left.\mathrm{CDCl}_{3}, 25{ }^{\circ} \mathrm{C}\right) \delta / \mathrm{ppm}: 12.48(\mathrm{~s}, 1 \mathrm{H})$, 7.95-7.69 (m, 2H), 7.43-7.29 (m, 3H), $4.20(\mathrm{~d}, J=16.7 \mathrm{~Hz}, 2 \mathrm{H}), 2.22-2.08(\mathrm{~m}, 6 \mathrm{H}), 1.43-1.22(\mathrm{~m}, 12 \mathrm{H})$, $0.81(\mathrm{t}, J=6.9 \mathrm{~Hz}, 9 \mathrm{H})$.

${ }^{13} \mathrm{C}$ NMR $\left(100 \mathrm{MHz}, \mathrm{CDCl}_{3}, 25{ }^{\circ} \mathrm{C}\right) \delta / \mathrm{ppm}: 147.0(\mathrm{~d}, J=9.7 \mathrm{~Hz}), 134.6(\mathrm{~d}, J=2 \mathrm{~Hz})$, 129.7, 128.7, 126.7, $23.7(\mathrm{~d}, J=15.6 \mathrm{~Hz}), 23.4(\mathrm{~d}, J=4.8 \mathrm{~Hz}), 19.8(\mathrm{~d}, J=46.3 \mathrm{~Hz})$, $18.6(\mathrm{~d}, J=46.8 \mathrm{~Hz}), 13.2$.

${ }^{31} \mathbf{P}$ NMR $\left(162 \mathrm{MHz}, \mathrm{CDCl}_{3}, 25^{\circ} \mathrm{C}\right) \delta / \mathrm{ppm}: 33.4$.

IR $(\mathrm{KBr}) \tilde{v}\left(\mathrm{~cm}^{-1}\right): 3086,2962,1608,1461,1403,1228,955,700$.

HRMS (ESI) for $\mathrm{C}_{20} \mathrm{H}_{35} \mathrm{NOP},[\mathrm{M}]^{+}$(336.2456) found: 336.2455 .

(Z)-Diethyl(2-(hydroxyimino)-2-phenylethyl)(phenyl)phosphonium chloride (7b)<smiles>O/N=C(\C[Pb]=[Po])c1ccccc1</smiles>

Following the TP-5, 7b was obtained from 5a $(500 \mathrm{mg}, 3.0 \mathrm{mmol})$ and $\mathrm{PEt}_{2} \mathrm{Ph}(540 \mu \mathrm{l}$, 1.1 equiv) as white solid (921 mg, 94\% yield); $\mathrm{R}_{f}=0.325(\mathrm{DCM}: \mathrm{MeOH}=9: 1) ; \mathrm{mp}=$ $178.0-180.1^{\circ} \mathrm{C}$.

${ }^{1} \mathbf{H}$ NMR $\left(400 \mathrm{MHz}, \mathrm{CDCl}_{3}, 25{ }^{\circ} \mathrm{C}\right) \delta / \mathrm{ppm}: 12.44(\mathrm{brs}, 1 \mathrm{H}), 7.72(\mathrm{dd}, J=11.6,7.9 \mathrm{~Hz}$, 2H), 7.59-7.45 (m, 3H), 7.45-7.35 (m, 2H), 7.25-7.02 (m, 3H), $4.41(\mathrm{~d}, J=16.6 \mathrm{~Hz}$, 2H), 2.88-2.50 (m, 4H), 1.09 (dt, $J=19.4,7.4 \mathrm{~Hz}, 6 \mathrm{H})$. 
${ }^{13} \mathrm{C}$ NMR $\left(100 \mathrm{MHz}, \mathrm{CDCl}_{3}, 25{ }^{\circ} \mathrm{C}\right) \delta / \mathrm{ppm}: 147.1(\mathrm{~d}, J=9.8 \mathrm{~Hz}), 134.2,132.0(\mathrm{~d}, J$ $=8.9 \mathrm{~Hz}), 129.8(\mathrm{~d}, J=12.0 \mathrm{~Hz}), 129.3,128.4,126.7,117.4,116.6,20.6(\mathrm{~d}, J=47.5$ $\mathrm{Hz}), 13.6(\mathrm{~d}, J=49.2 \mathrm{~Hz}), 5.8(\mathrm{~d}, J=5.0 \mathrm{~Hz})$.

${ }^{31} \mathbf{P}$ NMR $\left(162 \mathrm{MHz}, \mathrm{CDCl}_{3}, 25^{\circ} \mathrm{C}\right) \delta / \mathrm{ppm}: 32.3$.

IR $(\mathrm{KBr}) \tilde{v}\left(\mathrm{~cm}^{-1}\right):$ 3398, 3090, 2822, 1624, 1438, 1303, 956, 869, 757, 740, 693.

HRMS (ESI) for $\mathrm{C}_{18} \mathrm{H}_{23} \mathrm{NOP},[\mathrm{M}]^{+}$(300.1517) found: 300.1516 .

(Z)-Ethyl(2-(hydroxyimino)-2-phenylethyl)diphenylphosphonium chloride (7c)<smiles>O/N=C(\C[PbH])c1ccccc1</smiles>

Following the TP-5, 7c was obtained from 5a (500 mg, $3.0 \mathrm{mmol})$ and $\mathrm{PEtPh}_{2}(698 \mu \mathrm{l}$, 1.1 equiv) as a white solid (1.10 gm, $98 \%$ yield); $\mathrm{R}_{f}=0.286(\mathrm{DCM}: \mathrm{MeOH}=9: 1) ; \mathrm{mp}$ $=179.5-181.2^{\circ} \mathrm{C}$.

${ }^{1} \mathbf{H}$ NMR (400 MHz, $\left.\mathrm{CDCl}_{3}, 25^{\circ} \mathrm{C}\right) \delta / \mathrm{ppm}:$ 7.75-7.56 (m, 6H), 7.53-7.43 (m, 4H), $7.37(\mathrm{~d}, J=7.7 \mathrm{~Hz}, 2 \mathrm{H}), 7.15-7.01(\mathrm{~m}, 3 \mathrm{H}), 4.78(\mathrm{~d}, J=16.4 \mathrm{~Hz}, 2 \mathrm{H}), 2.92(\mathrm{dq}, J=$ $13.8,6.8 \mathrm{~Hz}, 2 \mathrm{H}), 1.03(\mathrm{dt}, J=20.6,7.4 \mathrm{~Hz}, 3 \mathrm{H})$.

${ }^{13} \mathrm{C}$ NMR $\left(100 \mathrm{MHz}, \mathrm{CDCl}_{3}, 25{ }^{\circ} \mathrm{C}\right) \delta / \mathrm{ppm}: 146.9(\mathrm{~d}, J=9.4 \mathrm{~Hz}), 134.5,133.2(\mathrm{~d}, J$ $=9.6 \mathrm{~Hz}), 129.9(\mathrm{~d}, J=12.4 \mathrm{~Hz}), 129.1,128.2,126.9,118.2,117.3,22.2(\mathrm{~d}, J=50.1$ $\mathrm{Hz}), 17.3(\mathrm{~d}, J=50.6 \mathrm{~Hz}), 6.3(\mathrm{~d}, J=5.2 \mathrm{~Hz})$.

${ }^{31}$ P NMR (162 MHz, CD $\left.30 D, 25^{\circ} \mathrm{C}\right) \delta / p p m: 27.7$.

IR $(\mathrm{KBr}) \tilde{v}\left(\mathrm{~cm}^{-1}\right): 3129,1592,1438,956,740,691$.

HRMS (ESI) for $\mathrm{C}_{22} \mathrm{H}_{23} \mathrm{NOP},[\mathrm{M}]^{+}$(348.1517) found: 348.1518 .

(Z)-(2-Hydroxyimino)-2-phenylethyl)triphenylphosphonium chloride (7d)<smiles>O/N=C(/C[Pb])c1ccccc1</smiles> 
Following the TP-5, 7d was obtained from 5a (500 mg, $3.0 \mathrm{mmol})$ and $\mathrm{PPh}_{3}(866 \mathrm{mg}$, 1.1 equiv) as a white solid (1.19 gm, 94\% yield); $\mathrm{R}_{f}=0.286(\mathrm{DCM}: \mathrm{MeOH}=9: 1) ; \mathrm{mp}$ $=226.6-228.4{ }^{\circ} \mathrm{C}$.

${ }^{1} \mathbf{H}$ NMR $\left(400 \mathrm{MHz}, \mathrm{CD}_{3} \mathrm{OD}, 25{ }^{\circ} \mathrm{C}\right) \delta / \mathrm{ppm}:$ 7.88-7.80 (m, 3H), 7.77-7.71 (m, 4H), 7.71-7.64 (m, 9H), $7.45(\mathrm{~d}, J=7.2 \mathrm{~Hz}, 2 \mathrm{H}), 7.31(\mathrm{t}, J=7.2 \mathrm{~Hz}, 1 \mathrm{H}), 7.25(\mathrm{t}, J=7.4 \mathrm{~Hz}$, 2H), $4.98(\mathrm{~d}, J=16.2 \mathrm{~Hz}, 2 \mathrm{H})$.

${ }^{13} \mathrm{C}$ NMR $\left(100 \mathrm{MHz}, \mathrm{CD}_{3} \mathrm{OD}, 25{ }^{\circ} \mathrm{C}\right) \delta / \mathrm{ppm}: 148.3(\mathrm{~d}, J=9.9 \mathrm{~Hz}), 136.5(\mathrm{~d}, J=3.0$ $\mathrm{Hz}), 135.8(\mathrm{~d}, J=2.4 \mathrm{~Hz}), 135.2(\mathrm{~d}, J=10.3 \mathrm{~Hz}), 131.3(\mathrm{~d}, J=13.0 \mathrm{~Hz}), 131.0,129.9$, $127.8,120.4,119.5$.

${ }^{31} \mathbf{P}$ NMR $\left(162 \mathrm{MHz}, \mathrm{CD}_{3} \mathrm{OD}, 25^{\circ} \mathrm{C}\right) \delta / \mathrm{ppm}: 21.0$.

IR $(\mathrm{KBr}) \tilde{v}\left(\mathrm{~cm}^{-1}\right):$ 3435, 2974, 2813, 1605, 1586, 1432, 954, 744, 722, 694.

HRMS (ESI) for $\mathrm{C}_{26} \mathrm{H}_{23} \mathrm{NOP},[\mathrm{M}]^{+}$(396.1517) found: 396.1519.

\section{3,5-Diphenylisoxazole (6aa)}

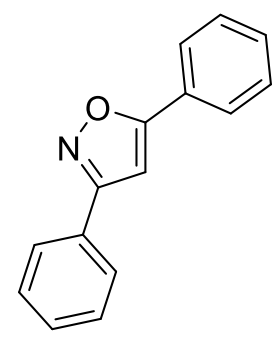

Following the TP-4, 6aa was obtained from $5 \mathbf{a}(33.7 \mathrm{mg}, 0.2 \mathrm{mmol}), \mathrm{PBu}_{3}(61.0 \mu \mathrm{L}$, 1.1 equiv), 7-methyl-1,5,7-triazabicyclo[4.4.0]dec-5-ene (121.0 $\mu \mathrm{L}, 40.0$ equiv) and benzoyl chloride $2 \mathbf{a}\left(28.0 \mu \mathrm{L}, 1.2\right.$ equiv) as a white solid $\left(38.17 \mathrm{mg}, 86 \%\right.$ yield); $\mathrm{R}_{f}=$ 0.300 (DCM:Hex = 1:2); $\mathrm{mp}=141.5-142.5^{\circ} \mathrm{C}$.

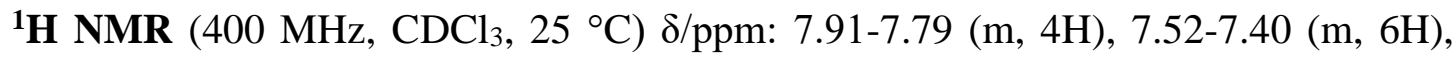
$6.82(\mathrm{~s}, 1 \mathrm{H})$.

${ }^{13} \mathrm{C}$ NMR (100 MHz, $\left.\mathrm{CDCl}_{3}, 25{ }^{\circ} \mathrm{C}\right) \delta / \mathrm{ppm}: 170.4,162.9,130.2,130.0,129.1,129.0$ $128.9,127.4,126.8,125.8,97.4$.

IR $(\mathrm{KBr}) \tilde{v}\left(\mathrm{~cm}^{-1}\right): 3115,1451,914,763$. 
HRMS (ESI) for $\mathrm{C}_{15} \mathrm{H}_{12} \mathrm{NO},[\mathrm{M}+\mathrm{H}]^{+}$(222.0913) found: 222.0919.

\section{3-(4-Methoxyphenyl)-5-phenylisoxazole (6ba)}

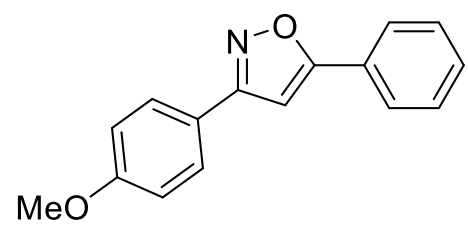

Following the TP-4, $\mathbf{6 b a}$ was obtained from $\mathbf{5 b}(39.8 \mathrm{mg}, 0.2 \mathrm{mmol}), \mathrm{PBu}_{3}(61.0 \mu \mathrm{L}$, 1.1 equiv), 7-methyl-1,5,7-triazabicyclo[4.4.0]dec-5-ene (121.0 $\mu \mathrm{L}, 4.0$ equiv) and benzoyl chloride $2 \mathbf{a}\left(28.0 \mu \mathrm{L}, 1.2\right.$ equiv) as a white solid $\left(40.80 \mathrm{mg}, 81 \%\right.$ yield); $\mathrm{R}_{f}=$ 0.075 (DCM:Hex = 1:3); $\mathrm{mp}=121.1-122.3{ }^{\circ} \mathrm{C}$.

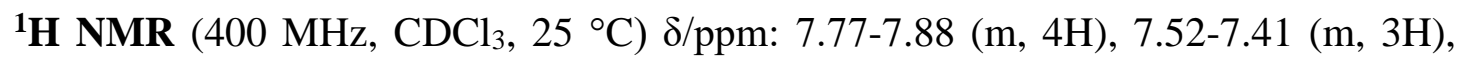
$6.99(\mathrm{~d}, J=8.8 \mathrm{~Hz}, 2 \mathrm{H}), 6.78(\mathrm{~s}, 1 \mathrm{H}), 3.87(\mathrm{~s}, 3 \mathrm{H})$.

${ }^{13} \mathrm{C}$ NMR $\left(100 \mathrm{MHz}, \mathrm{CDCl}_{3}, 25^{\circ} \mathrm{C}\right) \delta / \mathrm{ppm}: 170.1,162.6,161.0,130.1,129.0,128.2$ $127.5,125.8,121.6,114.3,97.2,55.3$.

IR $(\mathrm{KBr}) \tilde{v}\left(\mathrm{~cm}^{-1}\right): 3112,2932,1614,1447,1252,836,763$.

HRMS (ESI) for $\mathrm{C}_{16} \mathrm{H}_{14} \mathrm{NO}_{2},[\mathrm{M}+\mathrm{H}]^{+}$(252.1019) found: 252.1025 .

3-(4-Fluorophenyl)-5-phenylisoxazole (6ca)

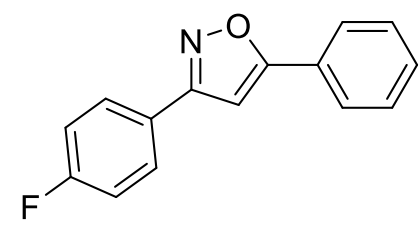

Following the TP-4, 6ca was obtained from $\mathbf{5 c}(37.51 \mathrm{mg}, 0.2 \mathrm{mmol}), \mathrm{PBu}_{3}(61.0 \mu \mathrm{L}$, 1.1 equiv), 7-methyl-1,5,7-triazabicyclo[4.4.0]dec-5-ene (121.0 $\mu \mathrm{L}, 4.0$ equiv) and benzoyl chloride $2 \mathbf{a}\left(28.0 \mu \mathrm{L}, 1.2\right.$ equiv) as a white solid (30.49 mg, $64 \%$ yield); $\mathrm{R}_{f}=$ 0.150 (DCM:Hex = 1:5); $\mathrm{mp}=169.3-170.0{ }^{\circ} \mathrm{C}$.

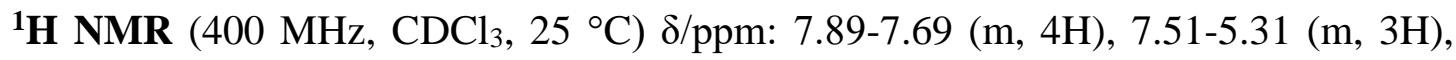
$7.09(\mathrm{t}, J=8.5 \mathrm{~Hz}, 2 \mathrm{H}), 6.71(\mathrm{~s}, 1 \mathrm{H})$.

${ }^{13} \mathrm{C}$ NMR (100 MHz, $\left.\mathrm{CDCl}_{3}, 25{ }^{\circ} \mathrm{C}\right) \delta / \mathrm{ppm}: 170.6,163.8(\mathrm{~d}, J=249.9 \mathrm{~Hz}), 162.0$, 
130.3, 129.0, 128.7 (d, $J=8.6 \mathrm{~Hz}), 127.3,125.8,125.4(\mathrm{~d}, J=3.4 \mathrm{~Hz}), 116.0(\mathrm{~d}, J=$ $21.9 \mathrm{~Hz}), 97.3$.

${ }^{19}$ F NMR $\left(376 \mathrm{MHz}, \mathrm{CDCl}_{3}\right) \delta / \mathrm{ppm}:-110.62$.

IR $(\mathrm{KBr}) \tilde{v}\left(\mathrm{~cm}^{-1}\right): 3112,2360,1448,845,817,766$.

HRMS (ESI) for $\mathrm{C}_{15} \mathrm{H}_{11} \mathrm{FNO},[\mathrm{M}+\mathrm{H}]^{+}$(240.0819) found: 240.0828.

3-(4-Nitrophenyl)-5-phenylisoxazole (6da)

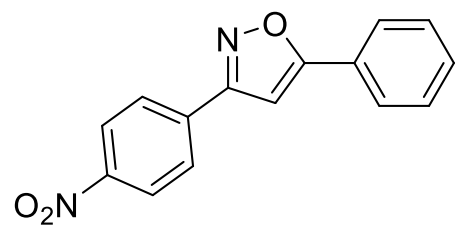

Following the TP-4, $\mathbf{6 d a}$ was obtained from $\mathbf{5 d}(51.81 \mathrm{mg}, 0.2 \mathrm{mmol}), \mathrm{PBu}_{3}(61.0 \mu \mathrm{L}$, 1.1 equiv), 7-methyl-1,5,7-triazabicyclo[4.4.0]dec-5-ene (121.0 $\mu \mathrm{L}, 4.0$ equiv) and benzoyl chloride $2 \mathbf{a}\left(28.0 \mu \mathrm{L}, 1.2\right.$ equiv) as a white solid $(33.10 \mathrm{mg}, 62 \%$ yield $) ; \mathrm{R}_{f}=$ $0.225(\mathrm{DCM}: \mathrm{Hex}=3: 5) ; \mathrm{mp}=226.9-227.3^{\circ} \mathrm{C}$.

${ }^{1} \mathbf{H}$ NMR $\left(400 \mathrm{MHz}, \mathrm{CDCl}_{3}, 25{ }^{\circ} \mathrm{C}\right) \delta / \mathrm{ppm}: 8.35(\mathrm{~d}, J=8.7 \mathrm{~Hz}, 2 \mathrm{H}), 8.06(\mathrm{~d}, J=8.7$ $\mathrm{Hz}, 2 \mathrm{H}), 7.82-7.92(\mathrm{~m}, 2 \mathrm{H}), 7.59-7.48(\mathrm{~m}, 3 \mathrm{H}), 6.90(\mathrm{~s}, 1 \mathrm{H})$.

${ }^{13} \mathrm{C}$ NMR $\left(100 \mathrm{MHz}, \mathrm{CDCl}_{3}, 25^{\circ} \mathrm{C}\right) \delta / \mathrm{ppm}: 171.5,161.2,148.7,135.2,130.7,129.2$ $127.7,127.0,125.9,124.2,97.4$

IR $(\mathrm{KBr}) \tilde{v}\left(\mathrm{~cm}^{-1}\right): 3085,1568,1520,1347,861$.

HRMS (ESI) for $\mathrm{C}_{15} \mathrm{H}_{11} \mathrm{~N}_{2} \mathrm{O}_{3},[\mathrm{M}+\mathrm{H}]^{+}$(267.0764) found: 267.0772 .

\section{3-Phenyl-5-(p-tolyl)isoxazole (6ab)}

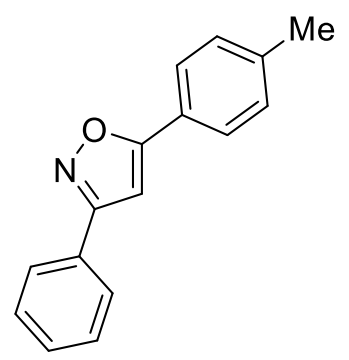

Following the TP-4, 6ab was obtained from $5 \mathbf{a}(33.7 \mathrm{mg}, 0.2 \mathrm{mmol}), \mathrm{PBu}_{3}(61.0 \mu \mathrm{L}$, 1.1 equiv), 7-methyl-1,5,7-triazabicyclo[4.4.0]dec-5-ene (121.0 $\mu \mathrm{L}, 4.0$ equiv) and 
p-toluoyl chloride $\mathbf{2 b}\left(31.9 \mu \mathrm{L}, 1.2\right.$ equiv) as a white solid $\left(33.81 \mathrm{mg}, 72 \%\right.$ yield); $\mathrm{R}_{f}$ $=0.100(\mathrm{DCM}: \mathrm{He} \mathrm{x}=1: 5) ; \mathrm{mp}=133.8-134.5^{\circ} \mathrm{C}$.

${ }^{1} \mathbf{H}$ NMR $\left(400 \mathrm{MHz}, \mathrm{CDCl}_{3}, 25{ }^{\circ} \mathrm{C}\right) \delta / \mathrm{ppm}: 7.84-7.81(\mathrm{~m}, 2 \mathrm{H}), 7.72(\mathrm{~d}, J=8.1 \mathrm{~Hz}$, 2H), 7.54-7.41 (m, 3H), $7.27(\mathrm{~d}, J=8.0 \mathrm{~Hz}, 2 \mathrm{H}), 6.77(\mathrm{~s}, 1 \mathrm{H}), 2.41(\mathrm{~s}, 3 \mathrm{H})$.

${ }^{13} \mathrm{C}$ NMR $\left(100 \mathrm{MHz}, \mathrm{CDCl}_{3}, 25{ }^{\circ} \mathrm{C}\right) \delta / \mathrm{ppm:} \mathrm{170.6,} \mathrm{162.9,} \mathrm{140.5,} \mathrm{129.9,} \mathrm{129.7,} \mathrm{129.2,}$ $128.9,126.8,125.7,124.7,96.9,21.5$.

IR $(\mathrm{KBr}) \tilde{v}\left(\mathrm{~cm}^{-1}\right): 3112,2914,1597,1463,816,766$.

HRMS (ESI) for $\mathrm{C}_{16} \mathrm{H}_{14} \mathrm{NO},[\mathrm{M}+\mathrm{H}]^{+}{ }^{(236.1070)}$ found: 236.1079 .

\section{5-(4-Methoxyphenyl)-3-phenylisoxazole (6ac)}

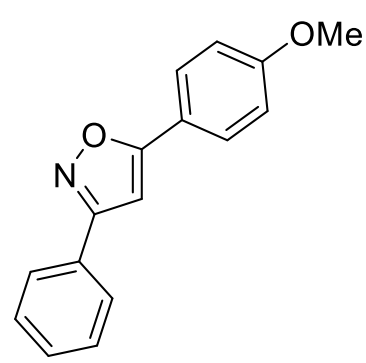

Following the TP-4, 6ac was obtained from $5 \mathbf{a}(33.7 \mathrm{mg}, 0.2 \mathrm{mmol}), \mathrm{PBu}_{3}(61.0 \mu \mathrm{L}$, 1.1 equiv), 7-methyl-1,5,7-triazabicyclo[4.4.0]dec-5-ene (121.0 $\mu \mathrm{L}, 4.0$ equiv) and 4-methoxybenzoyl chloride $2 \mathbf{c}(32.80 \mu \mathrm{L}, 1.2$ equiv) as a white solid (41.71 $\mathrm{mg}, 83 \%$ yield); $\mathrm{R}_{f}=0.100(\mathrm{DCM}: \mathrm{Hex}=1: 3) ; \mathrm{mp}=126.7-127.3^{\circ} \mathrm{C}$.

${ }^{1} \mathbf{H}$ NMR $\left(400 \mathrm{MHz}, \mathrm{CDCl}_{3}, 25^{\circ} \mathrm{C}\right) \delta / \mathrm{ppm}: 7.94-7.82(\mathrm{~m}, 2 \mathrm{H}), 7.77(\mathrm{~d}, J=8.6 \mathrm{~Hz}$, 2H), 7.57-7.39 (m, 3H), $6.99(\mathrm{~d}, J=8.6 \mathrm{~Hz}, 2 \mathrm{H}), 6.71(\mathrm{~s}, 1 \mathrm{H}), 3.87(\mathrm{~s}, 3 \mathrm{H})$.

${ }^{13} \mathrm{C}$ NMR $\left(100 \mathrm{MHz}, \mathrm{CDCl}_{3}, 25{ }^{\circ} \mathrm{C}\right) \delta / \mathrm{ppm}: 170.4,162.9,161.1,129.9,129.3,128.9$, $127.4,126.8,120.3,114.4,96.1,55.4$

IR $(\mathrm{KBr}) \tilde{v}\left(\mathrm{~cm}^{-1}\right): 3107,2917,1615,1466,1179,1034,802,768$.

HRMS (ESI) for $\mathrm{C}_{16} \mathrm{H}_{14} \mathrm{NO}_{2},[\mathrm{M}+\mathrm{H}]^{+}$(252.1019) found: 252.1027.

5-(4-Nitrophenyl)-3-phenylisoxazole (6ad) 


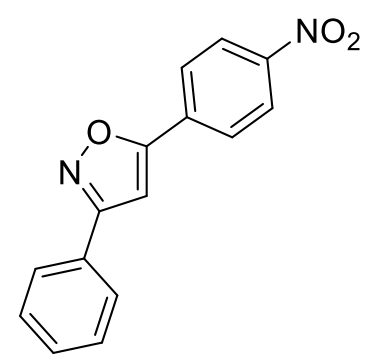

Following the TP-4, 6ad was obtained from $5 \mathbf{a}(33.7 \mathrm{mg}, 0.2 \mathrm{mmol}), \mathrm{PBu}_{3}(61.0 \mu \mathrm{L}$, 1.1 equiv) 7-methyl-1,5,7-triazabicyclo[4.4.0]dec-5-ene (121.0 $\mu \mathrm{L}, 4.0$ equiv) and 4-nitrobenzoyl chloride $2 \mathbf{d}$ (45.4 mg, 1.2 equiv) as a white solid (38.3 mg, 72\% yield); $\mathrm{R}_{f}=0.075(\mathrm{DCM}: \mathrm{Hex}=1: 3) ; \mathrm{mp}=223.9-224.4{ }^{\circ} \mathrm{C}$.

${ }^{1} \mathbf{H}$ NMR $\left(400 \mathrm{MHz}, \mathrm{CDCl}_{3}, 25{ }^{\circ} \mathrm{C}\right) \delta / \mathrm{ppm}: 8.37(\mathrm{~d}, J=8.6 \mathrm{~Hz}, 2 \mathrm{H}), 8.02(\mathrm{~d}, J=8.6$ $\mathrm{Hz}, 2 \mathrm{H})$, 7.92-7.86 (m, 2H), 7.61-7.45 (m, 3H), $7.02(\mathrm{~s}, 1 \mathrm{H})$.

${ }^{13} \mathrm{C}$ NMR (100 MHz, $\left.\mathrm{CDCl}_{3}, 25^{\circ} \mathrm{C}\right) \delta / \mathrm{ppm}: 167.8,163.4,148.5,132.8,130.4,129.1$, $128.5,126.8,126.6,124.5,100.1$.

IR $(\mathrm{KBr}) \tilde{v}\left(\mathrm{~cm}^{-1}\right): 3106,2362,1575,1519,1358,824$.

HRMS (ESI) for $\mathrm{C}_{15} \mathrm{H}_{11} \mathrm{~N}_{2} \mathrm{O}_{3},[\mathrm{M}+\mathrm{H}]^{+}$(267.0764) found: 267.0772 .

\section{5-(4-Chlorophenyl)-3-phenylisoxazole (6ae)}

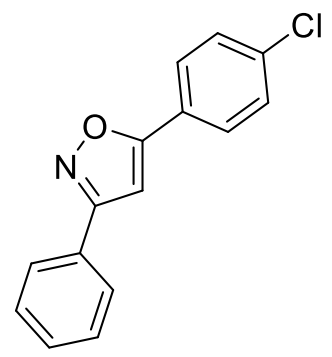

Following the TP-4, 6ae was obtained from $5 \mathbf{a}(33.7 \mathrm{mg}, 0.2 \mathrm{mmol}), \mathrm{PBu}_{3}(61.0 \mu \mathrm{L}$, 1.1 equiv), 7-methyl-1,5,7-triazabicyclo[4.4.0]dec-5-ene (121.0 $\mu \mathrm{L}, 4.0$ equiv) and 4-chlorobenzoyl chloride $2 \mathrm{e}(31.1 \mu \mathrm{L}, 1.2$ equiv) as a white solid (41.2 $\mathrm{mg}, 80 \%$ yield); $\mathrm{R}_{f}=0.225(\mathrm{DCM}: \mathrm{Hex}=1: 3) ; \mathrm{mp}=176.9-177.7^{\circ} \mathrm{C}$.

${ }^{1} \mathbf{H}$ NMR $\left(400 \mathrm{MHz}, \mathrm{CDCl}_{3}, 25^{\circ} \mathrm{C}\right) \delta / \mathrm{ppm}: 7.91-7.82(\mathrm{~m}, 2 \mathrm{H}), 7.77(\mathrm{~d}, J=8.4 \mathrm{~Hz}$, 2H), 7.54-7.42 (m, 5H), $6.82(\mathrm{~s}, 1 \mathrm{H})$.

${ }^{13} \mathrm{C}$ NMR $\left(100 \mathrm{MHz}, \mathrm{CDCl}_{3}, 25^{\circ} \mathrm{C}\right) \delta / \mathrm{ppm}: 169.3,163.1,136.3,130.1,129.3,129.0$, 
$128.9,127.1,126.8,125.9,97.8$.

IR $(\mathrm{KBr}) \tilde{v}\left(\mathrm{~cm}^{-1}\right): 3106,1411,1094,820$.

HRMS (ESI) for $\mathrm{C}_{15} \mathrm{H}_{11}{ }^{35} \mathrm{CINO},[\mathrm{M}+\mathrm{H}]^{+}$(256.0524) found: 256.0528 .

HRMS (ESI) for $\mathrm{C}_{15} \mathrm{H}_{11}{ }^{37} \mathrm{ClNO},[\mathrm{M}+\mathrm{H}]^{+}$(258.0500) found: 258.0501.

\section{5-(4-Bromophenyl)-3-phenylisoxazole (6af)}

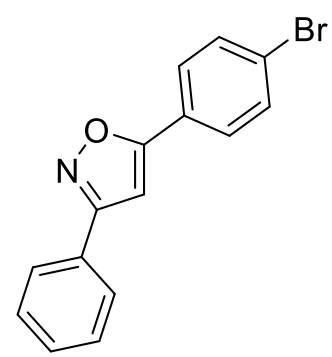

Following the TP-4, 6af was obtained from $5 \mathbf{a}(33.7 \mathrm{mg}, 0.2 \mathrm{mmol}), \mathrm{PBu}_{3}(61.0 \mu \mathrm{L}$, 1.1 equiv), 7-methyl-1,5,7-triazabicyclo[4.4.0]dec-5-ene (121.0 $\mu \mathrm{L}, 4.0$ equiv) and 4-bromobenzoyl chloride $2 \mathrm{f}$ (54.0 $\mathrm{mg}, 1.2$ equiv) as a white solid (46.3 $\mathrm{mg}, 78 \%$ yield $) ; \mathrm{R}_{f}=0.159(\mathrm{DCM}: \mathrm{Hex}=1: 5) ; \mathrm{mp}=187.6-188.4{ }^{\circ} \mathrm{C}$.

${ }^{1} \mathbf{H}$ NMR $\left(400 \mathrm{MHz}, \mathrm{CDCl}_{3}, 25^{\circ} \mathrm{C}\right) \delta / \mathrm{ppm}: 7.91-7.79(\mathrm{~m}, 2 \mathrm{H}), 7.70(\mathrm{~d}, J=8.7 \mathrm{~Hz}$, 2H), $7.62(\mathrm{~d}, J=8.7 \mathrm{~Hz}, 2 \mathrm{H}), 7.53-7.44(\mathrm{~m}, 3 \mathrm{H}), 6.83(\mathrm{~s}, 1 \mathrm{H})$.

${ }^{13} \mathrm{C}$ NMR $\left(100 \mathrm{MHz}, \mathrm{CDCl}_{3}, 25^{\circ} \mathrm{C}\right) \delta / \mathrm{ppm}: 169.3,163.1,132.3,130.1,129.0,128.9$, $127.3,126.8,126.3,124.6,97.9$.

IR $(\mathrm{KBr}) \tilde{v}\left(\mathrm{~cm}^{-1}\right): 3189,2841,2597,1545,1412,1052,768,440$.

HRMS (EI) for $\mathrm{C}_{15} \mathrm{H}_{11}{ }^{79} \mathrm{BrNO},[\mathrm{M}+\mathrm{H}]^{+}$(300.0027) found: 300.0024

HRMS (EI) for $\mathrm{C}_{15} \mathrm{H}_{11}{ }^{81} \mathrm{BrNO},[\mathrm{M}+\mathrm{H}]^{+}$(302.0011) found: 302.0009 .

5-(3-Chlorophenyl)-3-phenylisoxazole (6ag)

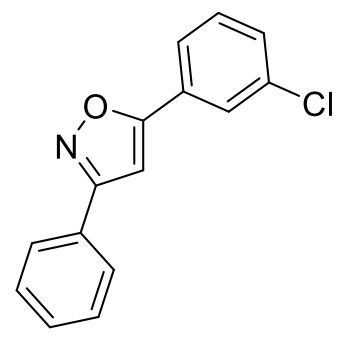

Following the TP-4, $\mathbf{6 a g}$ was obtained from $5 \mathbf{a}(33.7 \mathrm{mg}, 0.2 \mathrm{mmol}), \mathrm{PBu}_{3}(61.0 \mu \mathrm{L}$, 
1.1 equiv), 7-methyl-1,5,7-triazabicyclo[4.4.0]dec-5-ene (121.0 $\mu \mathrm{L}, 4.0$ equiv) and 3-chlorobenzoyl chloride $2 \mathrm{~g}$ (31.7 $\mu \mathrm{L}, 1.2$ equiv) as a white solid (43.2 $\mathrm{mg}, 84 \%$ yield); $\mathrm{R}_{f}=0.150(\mathrm{DCM}: \mathrm{Hex}=1: 5) ; \mathrm{mp}=135.5-136.5^{\circ} \mathrm{C}$.

${ }^{1} \mathbf{H}$ NMR $\left(400 \mathrm{MHz}, \mathrm{CDCl}_{3}, 25{ }^{\circ} \mathrm{C}\right) \delta / \mathrm{ppm}: 7.93-7.83(\mathrm{~m}, 2 \mathrm{H}), 7.82(\mathrm{~s}, 1 \mathrm{H}), 7.72(\mathrm{t}, J$ $=4.3 \mathrm{~Hz}, 1 \mathrm{H}), 7.53-7.45(\mathrm{~m}, 3 \mathrm{H}), 7.45-7.40(\mathrm{~m}, 2 \mathrm{H}), 6.85(\mathrm{~s}, 1 \mathrm{H})$.

${ }^{13} \mathrm{C}$ NMR $\left(100 \mathrm{MHz}, \mathrm{CDCl}_{3}, 25{ }^{\circ} \mathrm{C}\right) \delta / \mathrm{ppm}: 168.9,163.0,135.1,130.3,130.2,130.1$, $129.01,128.96,128.8,126.8,125.9,123.9,98.3$.

IR $(\mathrm{KBr}) \tilde{v}\left(\mathrm{~cm}^{-1}\right): 3102,1564,1412,1080,798,695$.

HRMS (ESI) for $\mathrm{C}_{15} \mathrm{H}_{11}{ }^{35} \mathrm{CINO},[\mathrm{M}+\mathrm{H}]^{+}$(256.0529) found: 256.0530 .

HRMS (ESI) for $\mathrm{C}_{15} \mathrm{H}_{11}{ }^{37} \mathrm{CINO},[\mathrm{M}+\mathrm{H}]^{+}$(258.0500) found: 258.0502 .

\section{5-(3-Bromophenyl)-3-phenylisoxazole (6am)}

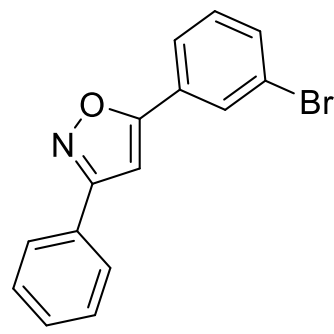

Following the TP-4, 6am was obtained from $\mathbf{5 a}(33.7 \mathrm{mg}, 0.2 \mathrm{mmol}), \mathrm{PBu}_{3}(61.0 \mu \mathrm{L}$, 1.1 equiv), 7-methyl-1,5,7-triazabicyclo[4.4.0]dec-5-ene (121.0 $\mu \mathrm{L}, 4.0$ equiv) and 3-bromobenzoyl chloride $2 \mathrm{~m}(32.0 \mu \mathrm{L}, 1.2$ equiv) as a white solid (48.0 $\mathrm{mg}, 81 \%$ yield); $\mathrm{R}_{f}=0.162(\mathrm{DCM}: \mathrm{Hex}=1: 5) ; \mathrm{mp}=136.8-137.3^{\circ} \mathrm{C}$.

${ }^{1} \mathbf{H}$ NMR $\left(400 \mathrm{MHz}, \mathrm{CDCl}_{3}, 25{ }^{\circ} \mathrm{C}\right) \delta / \mathrm{ppm}: 7.96(\mathrm{t}, J=1.8 \mathrm{~Hz}, 1 \mathrm{H}), 7.89-7.81(\mathrm{~m}$, 2H), $7.75(\mathrm{dt}, J=7.9,1.0 \mathrm{~Hz}, 1 \mathrm{H}), 7.60-7.53(\mathrm{~m}, 1 \mathrm{H}), 7.52-7.43(\mathrm{~m}, 3 \mathrm{H}), 7.34(\mathrm{t}, J=$ $7.9 \mathrm{~Hz}, 1 \mathrm{H}), 6.83(\mathrm{~s}, 1 \mathrm{H})$.

${ }^{13} \mathrm{C}$ NMR $\left(100 \mathrm{MHz}, \mathrm{CDCl}_{3}, 25^{\circ} \mathrm{C}\right) \delta / \mathrm{ppm}: 168.7,163.0,133.1,130.5,130.1,129.2$ $128.9,128.8,128.7,126.8,124.3,123.0,98.2$

IR $(\mathrm{KBr}) \tilde{v}\left(\mathrm{~cm}^{-1}\right):$ 3201, 2922, 2618, 1545, 1410, 1218, 1052, 899, 432.

HRMS (EI) for $\mathrm{C}_{15} \mathrm{H}_{11}{ }^{79} \mathrm{BrNO},[\mathrm{M}+\mathrm{H}]^{+}$(300.0027) found: 300.0024 . 
HRMS (EI) for $\mathrm{C}_{15} \mathrm{H}_{11}{ }^{81} \mathrm{BrNO},[\mathrm{M}+\mathrm{H}]^{+}$(302.0009) found: 302.0007 .

\section{5-(2-Chlorophenyl)-3-phenylisoxazole (6ah)}

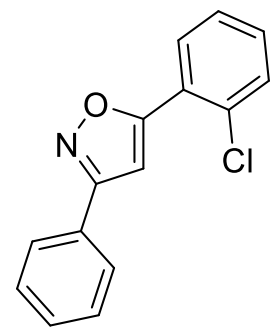

Following the TP-4, 6ah was obtained from $5 \mathbf{a}(33.7 \mathrm{mg}, 0.2 \mathrm{mmol}), \mathrm{PBu}_{3}(61.0 \mu \mathrm{L}$, 1.1 equiv), 7-methyl-1,5,7-triazabicyclo[4.4.0]dec-5-ene (121.0 $\mu \mathrm{L}, 4.0$ equiv) and 2-chlorobenzoyl chloride $\mathbf{2 h}(31.1 \mu \mathrm{L}, 1.2$ equiv) as a white solid (36.8 $\mathrm{mg}, 70 \%$ yield $) ; \mathrm{R}_{f}=0.175(\mathrm{DCM}: \mathrm{Hex}=1: 5) ; \mathrm{mp}=59.6-60.0^{\circ} \mathrm{C}$.

${ }^{1} \mathbf{H}$ NMR $\left(400 \mathrm{MHz}, \mathrm{CDCl}_{3}, 25^{\circ} \mathrm{C}\right) \delta / \mathrm{ppm}: 8.01(\mathrm{dd}, J=7.3,1.8 \mathrm{~Hz}, 1 \mathrm{H}), 7.96-7.84$ (m, 2H), 7.59-7.46 (m, 4H), 7.46-7.35 (m, 2H), $7.27(\mathrm{~s}, 1 \mathrm{H})$.

${ }^{13} \mathrm{C}$ NMR $\left(100 \mathrm{MHz}, \mathrm{CDCl}_{3}, 25^{\circ} \mathrm{C}\right) \delta / \mathrm{ppm}: 166.6,162.9,131.7,130.8,130.1,129.4$, $129.0,128.9,127.2,126.9,126.3,102.5$

IR $(\mathrm{KBr}) \tilde{v}\left(\mathrm{~cm}^{-1}\right): 3069,1605,1577,1482,1034,759$.

HRMS (ESI) for $\mathrm{C}_{15} \mathrm{H}_{11}{ }^{35} \mathrm{CINO},[\mathrm{M}+\mathrm{H}]^{+}$(256.0529) found: 256.0531 .

HRMS (ESI) for $\mathrm{C}_{15} \mathrm{H}_{11}{ }^{37} \mathrm{CINO},[\mathrm{M}+\mathrm{H}]^{+}$(258.0500) found: 258.0503 .

\section{5-(2-Bromophenyl)-3-phenylisoxazole (6ai)}

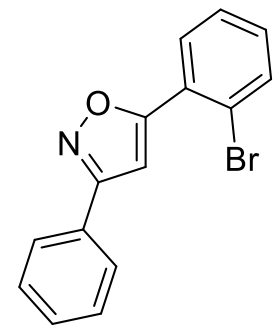

Following the TP-4, 6ai was obtained from $\mathbf{5 a}(33.7 \mathrm{mg}, 0.2 \mathrm{mmol}), \mathrm{PBu}_{3}(61.0 \mu \mathrm{L}$, 1.1 equiv), 7-methyl-1,5,7-triazabicyclo[4.4.0]dec-5-ene (121.0 $\mu \mathrm{L}, 4.0$ equiv) and 2-bromobenzoyl chloride $2 \mathbf{i}(32.0 \mu \mathrm{L}, 1.2$ equiv) as a white solid (49.1 $\mathrm{mg}, 82 \%$ yield $) ; \mathrm{R}_{f}=0.150(\mathrm{DCM}: \mathrm{Hex}=1: 5) ; \mathrm{mp}=53.2-54.2{ }^{\circ} \mathrm{C}$. 
${ }^{1} \mathbf{H}$ NMR $\left(400 \mathrm{MHz}, \mathrm{CDCl}_{3}, 25{ }^{\circ} \mathrm{C}\right) \delta / \mathrm{ppm}: 8.01-7.82(\mathrm{~m}, 3 \mathrm{H}), 7.73(\mathrm{~d}, J=8.0 \mathrm{~Hz}$, 1H), 7.58-7.42 (m, 4H), 7.38-7.28 (m, 2H).

${ }^{13} \mathrm{C}$ NMR $\left(100 \mathrm{MHz}, \mathrm{CDCl}_{3}, 25^{\circ} \mathrm{C}\right) \delta / \mathrm{ppm}: 168.0,162.7,134.2,131.1,130.1,130.0$ $129.1,128.9,128.4,127.7,126.9,121.1,102.3$.

IR $(\mathrm{KBr}) \tilde{v}\left(\mathrm{~cm}^{-1}\right): 3064,1602,1479,1076,1059,758$.

HRMS (EI) for $\mathrm{C}_{15} \mathrm{H}_{10}{ }^{79} \mathrm{BrNO},[\mathrm{M}]^{+}$(298.9946) found: 298.9945.

HRMS (EI) for $\mathrm{C}_{15} \mathrm{H}_{10}{ }^{81} \mathrm{BrNO},[\mathrm{M}]^{+}$(300.9923) found: 300.9921.

\section{5-Methyl-3-phenylisoxazole (6an)}

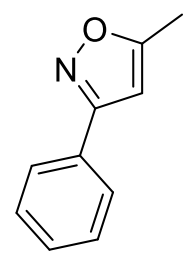

Following the TP-4, 6an was obtained from $\mathbf{5 a}(33.7 \mathrm{mg}, 0.2 \mathrm{mmol}), \mathrm{PBu}_{3}(61.0 \mu \mathrm{L}$, 1.1 equiv), 7-methyl-1,5,7-triazabicyclo[4.4.0]dec-5-ene (121.0 $\mu \mathrm{L}, 4.0$ equiv) and acetyl chloride $2 \mathrm{n}\left(17.5 \mu \mathrm{L}, 1.2\right.$ equiv) as a white solid (18.5 mg, $58 \%$ yield); $\mathrm{R}_{f}=$ $0.275(\mathrm{DCM}: \mathrm{Hex}=1: 2) ; \mathrm{mp}=36.9-38.7^{\circ} \mathrm{C}$.

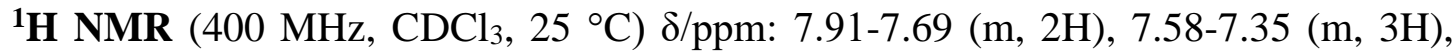
$6.28(\mathrm{~s}, 1 \mathrm{H}), 2.47(\mathrm{~s}, 3 \mathrm{H})$.

${ }^{13} \mathrm{C}$ NMR $\left(100 \mathrm{MHz}, \mathrm{CDCl}_{3}, 25{ }^{\circ} \mathrm{C}\right) \delta / \mathrm{ppm}: 169.9,162.6,129.8,129.4,128.8,126.7$, $99.7,12.4$.

IR $(\mathrm{KBr}) \tilde{v}\left(\mathrm{~cm}^{-1}\right): 3131,3070,2928,1613,1471,820,771$.

HRMS (ESI) for $\mathrm{C}_{10} \mathrm{H}_{10} \mathrm{NO},[\mathrm{M}+\mathrm{H}]^{+}$(160.0757) found: 160.0764 .

\section{3-Phenyl-5-(trifluoromethyl)isoxazole (6al)}

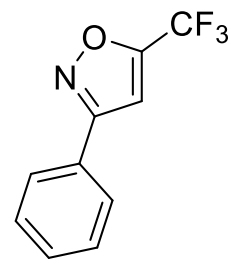


Following the TP-4, 6al was obtained from $\mathbf{5 a}(33.7 \mathrm{mg}, 0.2 \mathrm{mmol}), \mathrm{PBu}_{3}(61.0 \mu \mathrm{L}$, 1.1 equiv), 7-methyl-1,5,7-triazabicyclo[4.4.0]dec-5-ene (121.0 $\mu \mathrm{L}, 4.0$ equiv) and trifluoroacetic anhydride (TFAA) $2 \mathbf{l}(41.7 \mu \mathrm{L}, 1.5$ equiv) as a white solid (31.0 mg, $73 \%$ yield $) ; \mathrm{R}_{f}=0.200(100 \% \mathrm{Hex}) ; \mathrm{mp}=73.3-74.0^{\circ} \mathrm{C}$.

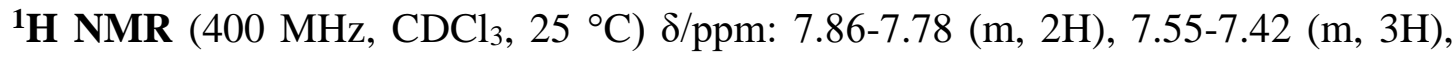
$7.00(\mathrm{~s}, 1 \mathrm{H})$.

${ }^{13} \mathrm{C}$ NMR $\left(100 \mathrm{MHz}, \mathrm{CDCl}_{3}, 25{ }^{\circ} \mathrm{C}\right) \delta / \mathrm{ppm}: 162.6,159.2(\mathrm{q}, J=42.5 \mathrm{~Hz}), 130.9$, $129.2,127.3,126.9,117.9(\mathrm{q}, J=270.3 \mathrm{~Hz}), 103.4(\mathrm{~d}, J=1.5 \mathrm{~Hz})$.

${ }^{19}$ F NMR $\left(376 \mathrm{MHz}, \mathrm{CDCl}_{3}\right) \delta / \mathrm{ppm}:-64.25$.

IR $(\mathrm{KBr}) \tilde{v}\left(\mathrm{~cm}^{-1}\right): 3119,3060,2926,1623,1470,1445,770,741$.

HRMS (ESI) for $\mathrm{C}_{10} \mathrm{H}_{7} \mathrm{~F}_{3} \mathrm{NO},[\mathrm{M}]^{+}$(213.0399) found: 213.0399 .

\section{(E)-3-Phenyl-5-styrylisoxazole (6ao)}

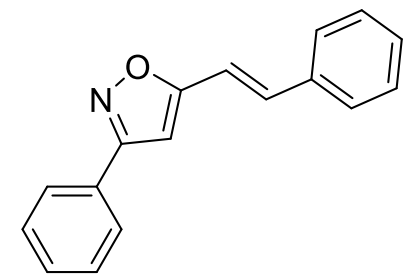

Following the TP-4, $6 \mathbf{a o}$ was obtained from $5 \mathbf{a}(33.7 \mathrm{mg}, 0.2 \mathrm{mmol}), \mathrm{PBu}_{3}(61.0 \mu \mathrm{L}$, 1.1 equiv), 7-methyl-1,5,7-triazabicyclo[4.4.0]dec-5-ene (121.0 $\mu \mathrm{L}, 4.0$ equiv) and cinnamoyl chloride $2 \mathrm{o}\left(40.0 \mu \mathrm{L}, 1.2\right.$ equiv) as a white solid $(22.3 \mathrm{mg}, 45 \%$ yield $)$; $\mathrm{R}_{f}$ $=0.60(\mathrm{DCM}: \mathrm{Hex}=2: 4) ; \mathrm{mp}=140.3-141.1^{\circ} \mathrm{C}$.

${ }^{1} \mathbf{H}$ NMR $\left(400 \mathrm{MHz}, \mathrm{CDCl}_{3}, 25^{\circ} \mathrm{C}\right) \delta / \mathrm{ppm}: 7.91-7.81(\mathrm{~m}, 2 \mathrm{H}), 7.57(\mathrm{~d}, J=7.8 \mathrm{~Hz}$, 2H), 7.53-7.50 (m, 3H), 7.46-7.34 (m, 4H), $7.03(\mathrm{~d}, J=16.4 \mathrm{~Hz}, 1 \mathrm{H}), 6.60(\mathrm{~s}, 1 \mathrm{H})$.

${ }^{13} \mathrm{C}$ NMR $\left(100 \mathrm{MHz}, \mathrm{CDCl}_{3}, 25^{\circ} \mathrm{C}\right) \delta / \mathrm{ppm}: 168.9,162.7,135.5,134.9,129.9,129.2$ $129.1,128.9,127.1,126.8,113.1,99.4$.

IR $(\mathrm{KBr}) \tilde{v}\left(\mathrm{~cm}^{-1}\right): 3438,2358,1647,1462,959,761$.

HRMS (ESI) for $\mathrm{C}_{17} \mathrm{H}_{14} \mathrm{NO},[\mathrm{M}+\mathrm{H}]^{+}{ }^{(248.1075)}$ found: 248.1077. 


\section{3,5-Diphenylisoxazole (6aa)}

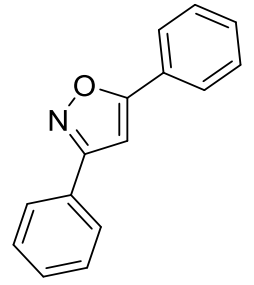

Following the TP-6, 6aa was obtained from $7 \mathbf{a}(74.4 \mathrm{mg}, 0.2 \mathrm{mmol})$, 7-methyl-1,5,7-triazabicyclo[4.4.0]dec-5-ene (121.0 $\mu \mathrm{L}, 4.0$ equiv) and benzoyl chloride $\mathbf{2 a}\left(28.0 \mu \mathrm{L}, 1.2\right.$ equiv) as a white solid ( $39.1 \mathrm{mg}, 88 \%$ yield); $\mathrm{R}_{f}=0.300$ (DCM:Hex = 1:2); $\mathrm{mp}=141.5-142.5{ }^{\circ} \mathrm{C}$.

\section{2-Chloro-1-(2-hydroxyphenyl)ethan-1-one (S1)}<smiles>O=C(CCl)c1ccccc1O</smiles>

Following the TP-7, S1 was obtained from phenyl chloroacetate $(5.0 \mathrm{~g}, 30 \mathrm{mmol})$ and $\mathrm{AlCl}_{3}\left(7.8 \mathrm{~g}, 2.5\right.$ equiv) as a white solid (4.4 g, 87\% yield); $\mathrm{R}_{f}=0.512(\mathrm{EA}: \mathrm{Hex}=2: 8)$; $\mathrm{mp}=80.6-81.2^{\circ} \mathrm{C}$.

${ }^{1} \mathbf{H}$ NMR $\left(400 \mathrm{MHz}, \mathrm{CDCl}_{3}, 25^{\circ} \mathrm{C}\right) \delta / \mathrm{ppm}: 11.67(\mathrm{~s}, 1 \mathrm{H}), 7.70(\mathrm{dd}, J=8.0,1.4 \mathrm{~Hz}$, 1H), 7.53 (ddd, $J=8.8,7.0,1.4 \mathrm{~Hz}, 1 \mathrm{H}), 7.04(\mathrm{dd}, J=8.4,1.0 \mathrm{~Hz}, 1 \mathrm{H}), 6.94(\mathrm{ddd}, J=$ 8.4, 7.0, $1.0 \mathrm{~Hz}, 1 \mathrm{H}), 4.72(\mathrm{~s}, 2 \mathrm{H})$.

${ }^{13} \mathrm{C}$ NMR (100 MHz, $\left.\mathrm{CDCl}_{3}, 25{ }^{\circ} \mathrm{C}\right) \delta / \mathrm{ppm}: 196.5,162.9,137.4,129.6,119.3,118.9$, $117.2,45.2$

IR $(\mathrm{KBr}) \tilde{v}\left(\mathrm{~cm}^{-1}\right):$ 1653, 1616, 1484, 1401, 1267, 1159, 752, 695

HRMS (ESI) for $\mathrm{C}_{8} \mathrm{H}_{7}{ }^{35} \mathrm{ClO}_{2},[\mathrm{M}]^{+}$(170.0136) found: 170.0136 .

HRMS (ESI) for $\mathrm{C}_{8} \mathrm{H}_{7}{ }^{37} \mathrm{ClO}_{2},[\mathrm{M}]^{+}$(172.0117) found: 172.0117.

\section{2-(2-Chloroacetyl)phenyl benzoate (S2)}




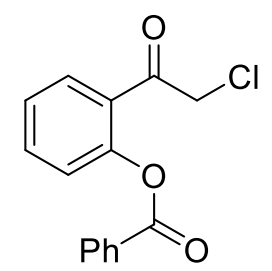

Following the TP-8, S2 was obtained from $o$-hydroxy chloroacetophenone $\mathbf{S 1}$ (1.0 g,

$5.8 \mathrm{mmol}), \mathrm{Et}_{3} \mathrm{~N}$ (1.5 equiv) and $\mathrm{PhCOCl}$ (1.2 equiv) as a pale yellow liquid (1.6 $\mathrm{g}$, $91 \%$ yield $) ; \mathrm{R}_{f}=0.725(\mathrm{EA}: \mathrm{Hex}=2: 8)$.

${ }^{1} \mathbf{H}$ NMR $\left(400 \mathrm{MHz}, \mathrm{CDCl}_{3}, 25^{\circ} \mathrm{C}\right) \delta / \mathrm{ppm}: 8.19(\mathrm{~d}, J=8.0 \mathrm{~Hz}, 2 \mathrm{H}), 7.84(\mathrm{~d}, J=8.0$ $\mathrm{Hz}, 1 \mathrm{H}), 7.70-7.58(\mathrm{~m}, 2 \mathrm{H}), 7.52(\mathrm{t}, J=7.5 \mathrm{~Hz}, 2 \mathrm{H}), 7.37(\mathrm{t}, J=7.5 \mathrm{~Hz}, 1 \mathrm{H}), 7.28(\mathrm{~d}$, $J=8.4 \mathrm{~Hz}, 1 \mathrm{H}), 4.60(\mathrm{~s}, 2 \mathrm{H})$.

${ }^{13} \mathrm{C}$ NMR (100 MHz, $\left.\mathrm{CDCl}_{3}, 25{ }^{\circ} \mathrm{C}\right) \delta / \mathrm{ppm}: 190.9,164.8,149.5,134.14,134.11$, $130.3,130.2,128.8,128.7,126.3,124.0,48.3$.

IR $(\mathrm{KBr}) \tilde{v}\left(\mathrm{~cm}^{-1}\right):$ 3068, 2941, 2372, 1735, 1482, 1264, 1023, 756.

HRMS (ESI) for $\mathrm{C}_{15} \mathrm{H}_{11}{ }^{35} \mathrm{ClO}_{3} \mathrm{Na},[\mathrm{M}+\mathrm{Na}]^{+}$(297.0294) found: 297.0296.

HRMS (ESI) for $\mathrm{C}_{15} \mathrm{H}_{11}{ }^{37} \mathrm{ClO}_{3} \mathrm{Na},[\mathrm{M}+\mathrm{Na}]^{+}$(299.0273) found: 299.0277 .

(Z)-2-(2-Chloro-1-(hydroxyimino)ethyl)phenyl benzoate (S3)<smiles>O=C(Oc1ccccc1/C(CCl)=N/O)c1ccccc1</smiles>

Following the TP-9, S3 was obtained from $o$-benzoyl- $\alpha$-chloroacetonphenone S2 (5.4 g, $20 \mathrm{mmol}$ ) and hydroxylamine hydrochloride (4.2 g, 3.0 equiv) as a white solid (5.4 $\mathrm{g}, 88 \%$ yield $) ; \mathrm{R}_{f}=0.335(\mathrm{EA}: \mathrm{Hex}=2: 8) ; \mathrm{mp}=78.9-79.5^{\circ} \mathrm{C}$.

${ }^{1} \mathbf{H}$ NMR (400 MHz, $\left.\mathrm{CDCl}_{3}, 25{ }^{\circ} \mathrm{C}\right) \delta / \mathrm{ppm}: 8.25(\mathrm{bs}, 1 \mathrm{H}), 8.17(\mathrm{dd}, J=8.0,1.0 \mathrm{~Hz}$, 2H), $7.64(\mathrm{tt}, J=7.5,1.4 \mathrm{~Hz}, 1 \mathrm{H}), 7.56(\mathrm{dd}, J=7.8,1.4 \mathrm{~Hz}, 1 \mathrm{H}), 7.54-7.46(\mathrm{~m}, 3 \mathrm{H})$, $7.34(\mathrm{td}, J=7.5,1.4 \mathrm{~Hz}, 1 \mathrm{H}), 7.28(\mathrm{dd}, J=8.0,1.0 \mathrm{~Hz}, 1 \mathrm{H}), 4.52(\mathrm{~s}, 2 \mathrm{H})$.

${ }^{13} \mathrm{C}$ NMR $\left(100 \mathrm{MHz}, \mathrm{CDCl}_{3}, 25{ }^{\circ} \mathrm{C}\right) \delta / \mathrm{ppm}: 164.9,153.0,148.5,133.8,130.6,130.2$, 
$130.0,129.0,128.7,127.3,126.2,123.2,35.0$.

IR $(\mathrm{KBr}) \tilde{v}\left(\mathrm{~cm}^{-1}\right): 3367,1739,1259,1202,1057,706,432$.

HRMS (ESI) for $\mathrm{C}_{15} \mathrm{H}_{12}{ }^{35} \mathrm{ClNO}_{3} \mathrm{Na}$, [M+Na] ${ }^{+}$(312.0403) found: 312.0403 .

HRMS (ESI) for $\mathrm{C}_{15} \mathrm{H}_{12}{ }^{37} \mathrm{ClNO}_{3} \mathrm{Na},[\mathrm{M}+\mathrm{Na}]^{+}$(314.0375) found: 314.0375 .

\section{(Z)-(2-(2-(Benzoyloxy)phenyl)-2-(hydroxyimino)ethyl)tributylphosphonium}

chloride (8a)<smiles>O=C(Oc1ccccc1/C(C[10B]Cl)=N\O)c1ccccc1</smiles>

Following the TP-10, 8a was obtained from $\mathbf{S 3}(853 \mathrm{mg}, 5.0 \mathrm{mmol})$ and tri- $n$-butylphosphine (1.1 equiv) in $\mathrm{Et}_{2} \mathrm{O}$, as a white solid $\left(2.2 \mathrm{~g}, 97 \%\right.$ yield); $\mathrm{R}_{f}=$ $0.445(\mathrm{MeOH}: \mathrm{DCM}=1: 9) ; \mathrm{mp}=167.3-168.0^{\circ} \mathrm{C}$.

${ }^{1} \mathbf{H}$ NMR (400 MHz, $\left.\mathrm{CDCl}_{3}, 25^{\circ} \mathrm{C}\right) \delta / \mathrm{ppm}: 12.41$ (bs, 1H), 8.19 (d, $\left.J=8.0 \mathrm{~Hz}, 2 \mathrm{H}\right)$, $7.94(\mathrm{~d}, J=8.0 \mathrm{~Hz}, 1 \mathrm{H}), 7.71-7.50(\mathrm{~m}, 3 \mathrm{H}), 7.41-7.29(\mathrm{~m}, 2 \mathrm{H}), 7.15(\mathrm{dd}, J=8.0,1.3$ $\mathrm{Hz}, 1 \mathrm{H}), 4.21(\mathrm{~d}, J=16.7 \mathrm{~Hz}, 2 \mathrm{H}), 2.13-1.93(\mathrm{~m}, 6 \mathrm{H}), 1.39-1.18(\mathrm{~m}, 12 \mathrm{H}), 0.76(\mathrm{t}, J$ $=7.5 \mathrm{~Hz}, 3 \mathrm{H})$.

${ }^{13} \mathrm{C}$ NMR $\left(100 \mathrm{MHz}, \mathrm{CDCl}_{3}, 25{ }^{\circ} \mathrm{C}\right) \delta / \mathrm{ppm}: 166.3,148.2,143.8(\mathrm{~d}, J=9.7 \mathrm{~Hz})$, $134.0,130.5,130.4,130.3,129.0,128.9,128.0,126.6,123.5,23.6(\mathrm{~d}, J=16.0 \mathrm{~Hz})$, $23.3(\mathrm{~d}, J=4.5 \mathrm{~Hz}), 19.9(\mathrm{~d}, J=47.0 \mathrm{~Hz}), 19.3(\mathrm{~d}, J=46.3 \mathrm{~Hz}), 13.1$.

${ }^{31} \mathbf{P}$ NMR $\left(162 \mathrm{MHz}, \mathrm{CDCl}_{3}, 25^{\circ} \mathrm{C}\right) \delta / \mathrm{ppm}: 34.1$.

IR $(\mathrm{KBr}) \tilde{v}\left(\mathrm{~cm}^{-1}\right): 2961,2370,1420,1251,958,776$.

HRMS (ESI) for $\mathrm{C}_{27} \mathrm{H}_{39} \mathrm{NO}_{3} \mathrm{P},[\mathrm{M}]^{+}$(456.2668) found: 456.2668 .

(Z)-2-Chloro-1-(2-hydroxyphenyl)ethan-1-one oxime (S4)<smiles>O/N=C(\CCl)c1ccccc1O</smiles> 
Following the TP-11, S4 was obtained from $o$-hydroxy- $\alpha$-chloroacetophenone $\mathbf{S 1}$ (500 $\mathrm{mg}, 3.0 \mathrm{mmol}$ ) and hydroxylamine hydrochloride (3.0 equiv) as a white solid (447 mg, $82 \%$ yield $) ; \mathrm{R}_{f}=0.412(\mathrm{EA}: \mathrm{Hex}=2: 8) ; \mathrm{mp}=148.6-149.0^{\circ} \mathrm{C}$.

${ }^{1} \mathbf{H}$ NMR $\left(400 \mathrm{MHz}, \mathrm{CDCl}_{3}, 25^{\circ} \mathrm{C}\right) \delta / \mathrm{ppm}: 10.67(\mathrm{~s}, 1 \mathrm{H}), 7.95(\mathrm{~s}, 1 \mathrm{H}), 7.51(\mathrm{~d}, J=$ $8.0 \mathrm{~Hz}, 1 \mathrm{H}), 7.32(\mathrm{t}, J=7.5 \mathrm{~Hz}, 1 \mathrm{H}), 7.00(\mathrm{~d}, J=8.3 \mathrm{~Hz}, 1 \mathrm{H}) .6 .97(\mathrm{t}, J=7.5 \mathrm{~Hz}, 1 \mathrm{H})$, $4.67(\mathrm{~s}, 2 \mathrm{H})$.

${ }^{13} \mathrm{C}$ NMR $\left(100 \mathrm{MHz}, \mathrm{CDCl}_{3}, 25{ }^{\circ} \mathrm{C}\right) \delta / \mathrm{ppm}: 157.8,157.2,131.6,127.5,119.7,117.7$, 115.6, 30.3.

IR $(\mathrm{KBr}) \tilde{v}\left(\mathrm{~cm}^{-1}\right): 3415,1642,1211,978,750,430$.

HRMS (ESI) for $\mathrm{C}_{8} \mathrm{H}_{9}{ }^{35} \mathrm{ClNO}_{2},[\mathrm{M}+\mathrm{H}]^{+}$(186.0322); found: 186.0324 .

HRMS (ESI) for $\mathrm{C}_{8} \mathrm{H}_{9}{ }^{37} \mathrm{ClNO}_{2},[\mathrm{M}+\mathrm{H}]^{+}$(188.0293); found: 188.0296 .

(Z)-2-Chloro-1-(2-hydroxyphenyl)ethan-1-one $O$-benzoyl oxime (S5)<smiles>O=C(O/N=C(\CCl)c1ccccc1O)c1ccccc1</smiles>

Following the TP-12, S5 was obtained from $o$-hydroxy- $\alpha$-chloroketoxime S4 (556 mg, $3.0 \mathrm{mmol})$, and benzoyl chloride ( 1.1 equiv) as a white solid ( $850 \mathrm{mg}, 91 \%$ yield); $\mathrm{R}_{f}$ $=0.683(\mathrm{EA}: \mathrm{Hex}=2: 8) ; \mathrm{mp}=149.3-150.1^{\circ} \mathrm{C}$.

${ }^{1} \mathbf{H}$ NMR $\left(400 \mathrm{MHz}, \mathrm{CDCl}_{3}, 2{ }^{\circ} \mathrm{C}\right) \delta / \mathrm{ppm}: 10.87(\mathrm{~s}, 1 \mathrm{H}), 8.15(\mathrm{~d}, J=7.0 \mathrm{~Hz}, 2 \mathrm{H})$, $7.67(\mathrm{t}, J=7.5 \mathrm{~Hz}, 1 \mathrm{H}), 7.61-7.51(\mathrm{~m}, 3 \mathrm{H}), 7.41(\mathrm{t}, J=8.0 \mathrm{~Hz}, 1 \mathrm{H}), 7.11(\mathrm{~d}, J=8.4$ $\mathrm{Hz}, 1 \mathrm{H}), 7.00(\mathrm{t}, J=7.5 \mathrm{~Hz}, 1 \mathrm{H}), 4.75(\mathrm{~s}, 2 \mathrm{H})$.

${ }^{13} \mathrm{C}$ NMR $\left(100 \mathrm{MHz}, \mathrm{CDCl}_{3}, 25{ }^{\circ} \mathrm{C}\right) \delta / \mathrm{ppm}: 162.2,161.6,159.2,134.1,133.2,129.9$, $128.9,128.1,127.6,119.6,118.6,114.5,31.9$.

IR $(\mathrm{KBr}) \tilde{v}\left(\mathrm{~cm}^{-1}\right): 3411,1752,1601,1246,1044,741,421$.

HRMS (ESI) for $\mathrm{C}_{15} \mathrm{H}_{13}{ }^{35} \mathrm{ClNO}_{3},[\mathrm{M}+\mathrm{Na}]^{+}$(312.0403) found: 312.0405 . 
HRMS (ESI) for $\mathrm{C}_{15} \mathrm{H}_{13}{ }^{37} \mathrm{ClNO}_{3},[\mathrm{M}+\mathrm{Na}]^{+}$(314.0374) found: 314.0378 .

(Z)-2-Chloro-1-(2-hydroxyphenyl)ethan-1-one $O$-(4-methoxybenzoyl) oxime (S6)<smiles>COc1ccc(C(=O)O/N=C(\CCl)c2ccccc2O)cc1</smiles>

Following the TP-12, S6 was obtained from $o$-hydroxy- $\alpha$-chloroketoxime S4 (186 mg, $1.0 \mathrm{mmol}$ ), and 4-methoxy benzoyl chloride (1.1 equiv) as a white solid (299 $\mathrm{mg}$, $93 \%$ yield $) ; \mathrm{R}_{f}=0.693(\mathrm{EA}: \mathrm{Hex}=2: 8) ; \mathrm{mp}=164.6-165.3{ }^{\circ} \mathrm{C}$.

${ }^{1} \mathbf{H}$ NMR $\left(400 \mathrm{MHz}, \mathrm{CDCl}_{3}, 2{ }^{\circ} \mathrm{C}\right) \delta / \mathrm{ppm}: 10.93(\mathrm{~s}, 1 \mathrm{H}), 8.10(\mathrm{~d}, J=8.6 \mathrm{~Hz}, 2 \mathrm{H})$, $7.56(\mathrm{~d}, J=8.0 \mathrm{~Hz}, 1 \mathrm{H}), 7.39(\mathrm{t}, J=7.7 \mathrm{~Hz}, 1 \mathrm{H}), 7.09(\mathrm{~d}, J=8.2 \mathrm{~Hz}, 1 \mathrm{H}), 7.04-6.65$ (m, 3H), $4.73(\mathrm{~s}, 2 \mathrm{H}), 3.90(\mathrm{~s}, 3 \mathrm{H})$.

${ }^{13} \mathrm{C}$ NMR $\left(100 \mathrm{MHz}, \mathrm{CDCl}_{3}, 25^{\circ} \mathrm{C}\right) \delta / \mathrm{ppm}: 164.2,161.9,161.0,159.1,133.0,132.0$, $128.0,119.6,119.5,118.5,114.6,114.2,55.5,31.9$.

IR $(\mathrm{KBr}) \tilde{v}\left(\mathrm{~cm}^{-1}\right): 3326,2369,1758,1523,1263,1037,886,669$.

HRMS (ESI) for $\mathrm{C}_{16} \mathrm{H}_{14}{ }^{35} \mathrm{ClNO}_{4},[\mathrm{M}+\mathrm{Na}]^{+}$(342.0508) found: 342.0505 .

HRMS (ESI) for $\mathrm{C}_{16} \mathrm{H}_{14}{ }^{37} \mathrm{ClNO}_{4},[\mathrm{M}+\mathrm{Na}]^{+}$(344.0481) found: 344.0483 .

(Z)-2-Chloro-1-(2-hydroxyphenyl)ethan-1-one $O$-pivaloyl oxime (S7)<smiles>CC(C)(C)C(=O)O/N=C(/CCl)c1ccccc1O</smiles>

Following the TP-12, S7 was obtained from $o$-hydroxy- $\alpha$-chloroketoxime S4 (186 mg, $1.0 \mathrm{mmol}$ ), and pivaloyl chloride (1.1 equiv) as a white solid (233 $\mathrm{mg}, 86 \%$ yield); $\mathrm{R}_{f}$ $=0.622(\mathrm{EA}: \mathrm{Hex}=2: 8) ; \mathrm{mp}=93.5-94.2{ }^{\circ} \mathrm{C}$.

${ }^{1} \mathbf{H}$ NMR $\left(400 \mathrm{MHz}, \mathrm{CDCl}_{3}, 25{ }^{\circ} \mathrm{C}\right) \delta / \mathrm{ppm}: 10.81(\mathrm{~s}, 1 \mathrm{H}), 7.51(\mathrm{~d}, J=8.4 \mathrm{~Hz}, 1 \mathrm{H})$, 
$7.38(\mathrm{t}, J=7.6 \mathrm{~Hz}, 1 \mathrm{H}), 7.07(\mathrm{~d}, J=8.4 \mathrm{~Hz}, 1 \mathrm{H}), 6.96(\mathrm{t}, J=7.6 \mathrm{~Hz}, 1 \mathrm{H}), 4.61(\mathrm{~s}, 2 \mathrm{H})$, $1.37(\mathrm{~s}, 9 \mathrm{H})$.

${ }^{13} \mathrm{C}$ NMR $\left(100 \mathrm{MHz}, \mathrm{CDCl}_{3}, 25{ }^{\circ} \mathrm{C}\right) \delta / \mathrm{ppm}: 173.3,161.1,159.1,133.0,128.0,119.5$, $118.5,114.5,38.7,31.6,27.1$.

IR $(\mathrm{KBr}) \tilde{v}\left(\mathrm{~cm}^{-1}\right):$ 2978, 2371, 1773, 1605, 1464, 1087, 939, 751.

HRMS (ESI) for $\mathrm{C}_{13} \mathrm{H}_{16}{ }^{35} \mathrm{ClNO}_{3}$, [M+Na $]^{+}$(292.0716) found: 292.0717.

HRMS (ESI) for $\mathrm{C}_{13} \mathrm{H}_{16}{ }^{37} \mathrm{ClNO}_{3}$, [M+Na $]^{+}$(294.0684) found: 292.0686.

(Z)-2-Chloro-1-(2-hydroxyphenyl)ethan-1-one $O$-acetyl oxime (S8)<smiles>CC(=O)O/N=C(\CCl)c1ccccc1O</smiles>

Following the TP-12, S8 was obtained from $o$-hydroxy- $\alpha$-chloroketoxime S4 (186 mg, $1.0 \mathrm{mmol})$, and acetyl chloride (1.1 equiv) as a white solid (203 $\mathrm{mg}, 89 \%$ yield); $\mathrm{R}_{f}=$ $0.701(\mathrm{EA}: \mathrm{Hex}=2: 8) ; \mathrm{mp}=125.1-125.9^{\circ} \mathrm{C}$.

${ }^{1} \mathbf{H}$ NMR $\left(400 \mathrm{MHz}, \mathrm{CDCl}_{3}, 25{ }^{\circ} \mathrm{C}\right) \delta / \mathrm{ppm}: 10.80(\mathrm{~s}, 1 \mathrm{H}), 7.53(\mathrm{dd}, J=8.0,1.4 \mathrm{~Hz}$, 1H), $7.38(\mathrm{ddd}, J=8.4,7.0,1.4 \mathrm{~Hz}, 1 \mathrm{H}), 7.07(\mathrm{dd}, J=8.4,1.0 \mathrm{~Hz}, 1 \mathrm{H}), 6.97$ (ddd, $J=$ 8.0, 7.0, 1.0 Hz, 1H), $4.64(\mathrm{~s}, 2 \mathrm{H}), 2.30(\mathrm{~s}, 3 \mathrm{H})$.

${ }^{13} \mathrm{C}$ NMR $\left(100 \mathrm{MHz}, \mathrm{CDCl}_{3}, 25{ }^{\circ} \mathrm{C}\right) \delta / \mathrm{ppm}: 166.0,160.8,159.1,133.1,128.2,119.5$, $118.5,114.4,31.5,19.1$

IR $(\mathrm{KBr}) \tilde{v}\left(\mathrm{~cm}^{-1}\right):$ 2927, 2370, 1793, 1606, 1250, 1175, 967, 744.

HRMS (ESI) for $\mathrm{C}_{10} \mathrm{H}_{10}{ }^{35} \mathrm{ClNO}_{3},[\mathrm{M}+\mathrm{Na}]^{+}$(250.0213) found: 250.0214 .

HRMS (ESI) for $\mathrm{C}_{10} \mathrm{H}_{10}{ }^{37} \mathrm{ClNO}_{3},[\mathrm{M}+\mathrm{Na}]^{+}$(252.0171) found: 252.0169.

(Z)-2-(1-((Benzoyloxy)imino)-2-chloroethyl)phenyl benzoate (13aa) 
<smiles>O=C(O/N=C(\CCl)c1ccccc1OC(=O)c1ccccc1)c1ccccc1</smiles>

Following the TP-13, 13aa was obtained from S5 (300 mg, $1.0 \mathrm{mmol}), \mathrm{Et}_{3} \mathrm{~N}(1.2$ equiv) and benzoyl chloride (1.1 equiv) in $\mathrm{CH}_{2} \mathrm{Cl}_{2}$ as a white solid (298 $\mathrm{mg}, 73 \%$ yield); $\mathrm{R}_{f}=0.445(\mathrm{EA}: \mathrm{Hex}=2: 8) ; \mathrm{mp}=67.3-68.1^{\circ} \mathrm{C}$.

${ }^{1} \mathbf{H}$ NMR $\left(400 \mathrm{MHz}, \mathrm{CDCl}_{3}, 25{ }^{\circ} \mathrm{C}\right) \delta / \mathrm{ppm}: 8.20(\mathrm{~d}, J=7.5 \mathrm{~Hz}, 2 \mathrm{H}), 8.10(\mathrm{~d}, J=7.5$ $\mathrm{Hz}, 2 \mathrm{H}), 7.73(\mathrm{dd}, J=7.8,1.6 \mathrm{~Hz}, 1 \mathrm{H}), 7.66-7.54(\mathrm{~m}, 3 \mathrm{H}), 7.54-7.44(\mathrm{~m}, 4 \mathrm{H})$, 7.42-7.33 (m, 2H), $4.60(\mathrm{~s}, 2 \mathrm{H})$.

${ }^{13} \mathrm{C}$ NMR $\left(100 \mathrm{MHz}, \mathrm{CDCl}_{3}, 2{ }^{\circ} \mathrm{C}\right) \delta / \mathrm{ppm}: 164.6,162.6,160.4,148.8,133.9,133.7$, $131.7,130.7,130.3,129.8,128.9,128.68,128.65,128.3,126.2,126.2,123.2,36.5$.

IR $(\mathrm{KBr}) \tilde{v}\left(\mathrm{~cm}^{-1}\right): 1752,1243,1202,1059,1024,702,443$.

HRMS (ESI) for $\mathrm{C}_{22} \mathrm{H}_{16}{ }^{35} \mathrm{ClNO}_{4} \mathrm{Na}$, [M+Na] ${ }^{+}$(416.0666) found: 416.0664 .

HRMS (ESI) for $\mathrm{C}_{22} \mathrm{H}_{16}{ }^{37} \mathrm{ClNO}_{4} \mathrm{Na},[\mathrm{M}+\mathrm{Na}]^{+}$(418.0645) found: 418.0643.

(Z)-2-(2-Chloro-1-(((4-methoxybenzoyl)oxy)imino)ethyl)phenyl pivalate (13bc)<smiles>COc1ccc(C(=O)O/N=C(\CCl)c2ccccc2OC(=O)C(C)(C)C)cc1</smiles>

Following the TP-13, 13bc was obtained from S6 $(320 \mathrm{mg}, 1.0 \mathrm{mmol}), \mathrm{Et}_{3} \mathrm{~N}(1.2$ equiv) and pivaloyl chloride (1.1 equiv) in $\mathrm{CH}_{2} \mathrm{Cl}_{2}$ as a colorless liquid (352 $\mathrm{mg}, 87 \%$ yield $) ; \mathrm{R}_{f}=0.432(\mathrm{EA}: \mathrm{Hex}=2: 8)$.

${ }^{1} \mathbf{H}$ NMR $\left(400 \mathrm{MHz}, \mathrm{CDCl}_{3}, 25{ }^{\circ} \mathrm{C}\right) \delta / \mathrm{ppm}: 7.71(\mathrm{~d}, J=8.9 \mathrm{~Hz}, 2 \mathrm{H}), 7.58-7.49(\mathrm{~m}$, 
1H), 7.43-7.24 (m, 3H), $6.82(\mathrm{~d}, J=8.9 \mathrm{~Hz}, 2 \mathrm{H}), 4.56(\mathrm{~s}, 2 \mathrm{H}), 3.81(\mathrm{~s}, 3 \mathrm{H}), 1.23(\mathrm{~s}$, 9H).

${ }^{13} \mathrm{C}$ NMR $\left(100 \mathrm{MHz}, \mathrm{CDCl}_{3}, 25{ }^{\circ} \mathrm{C}\right) \delta / \mathrm{ppm}: 176.1,163.8,162.9,158.9,147.3,131.9$, $130.9,127.9,125.6,124.6,122.6,120.0,113.8,55.4,44.1,39.0,26.9$.

IR $(\mathrm{KBr}) \tilde{v}\left(\mathrm{~cm}^{-1}\right): 3316,2963,2822,1715,1640,1501,1111,889,443$.

HRMS (ESI) for $\mathrm{C}_{21} \mathrm{H}_{22}{ }^{35} \mathrm{ClNO}_{5} \mathrm{Na},[\mathrm{M}+\mathrm{Na}]^{+}$(426.1084) found: 426.1083 .

HRMS (ESI) for $\mathrm{C}_{21} \mathrm{H}_{22}{ }^{37} \mathrm{ClNO}_{5} \mathrm{Na},[\mathrm{M}+\mathrm{Na}]^{+}$(428.1062) found: 428.1062 .

(Z)-2-(2-Chloro-1-((pivaloyloxy)imino)ethyl)phenyl 4-bromobenzoate (13cp)<smiles>CC(C)(C)C(=O)O/N=C(\CCl)c1ccccc1OC(=O)c1ccc(Br)cc1</smiles>

Following the TP-13, 13cp was obtained from S7 $(270 \mathrm{mg}, 1.0 \mathrm{mmol}), \mathrm{Et}_{3} \mathrm{~N}$ (1.2 equiv) and 4-bromo benzoyl chloride (1.1 equiv) in $\mathrm{CH}_{2} \mathrm{Cl}_{2}$ as a yellow liquid (436 mg, 92\% yield); $\mathrm{R}_{f}=0.691(\mathrm{EA}: \mathrm{Hex}=2: 8)$.

${ }^{1} \mathbf{H}$ NMR $\left(400 \mathrm{MHz}, \mathrm{CDCl}_{3}, 25{ }^{\circ} \mathrm{C}\right) \delta / \mathrm{ppm}: 8.03(\mathrm{~d}, \mathrm{~J}=8.1 \mathrm{~Hz}, 2 \mathrm{H})$, 7.71-7.60 (m, $3 \mathrm{H}), 7.53(\mathrm{dt}, \mathrm{J}=7.5,1.5 \mathrm{~Hz}, 1 \mathrm{H}), 7.36(\mathrm{dt}, \mathrm{J}=7.5,1.5 \mathrm{~Hz}, 1 \mathrm{H}), 7.31(\mathrm{~d}, \mathrm{~J}=8.0 \mathrm{~Hz}$, $1 \mathrm{H}), 4.42(\mathrm{~s}, 2 \mathrm{H}), 1.26(\mathrm{~s}, 9 \mathrm{H})$.

${ }^{13} \mathrm{C}$ NMR (100 MHz, $\left.\mathrm{CDCl}_{3}, 25^{\circ} \mathrm{C}\right) \delta / \mathrm{ppm:} \mathrm{173.7,} \mathrm{164.2,} \mathrm{159.3,} \mathrm{148.5,} \mathrm{131.9,} \mathrm{131.8,}$ 131.6, 130.3, 129.1, 127.9, 126.3, 126.1, 123.2, 38.7, 36.2, 27.0.

IR $(\mathrm{KBr}) \tilde{v}\left(\mathrm{~cm}^{-1}\right): 3402,1631,1402,1118,759,456$.

HRMS (ESI) for $\mathrm{C}_{20} \mathrm{H}_{19}{ }^{79} \mathrm{Br}^{35} \mathrm{ClNO}_{4} \mathrm{Na},[\mathrm{M}+\mathrm{Na}]^{+}$(474.0084) found: 474.0085 .

HRMS (ESI) for $\mathrm{C}_{20} \mathrm{H}_{19}{ }^{79} \mathrm{Br}^{37} \mathrm{ClNO}_{4} \mathrm{Na} / \mathrm{C}_{20} \mathrm{H}_{19}{ }^{81} \mathrm{Br}^{35} \mathrm{ClNO}_{4} \mathrm{Na},[\mathrm{M}+\mathrm{Na}]^{+}$(476.0065) found: 476.0065 . 
HRMS (ESI) for $\mathrm{C}_{20} \mathrm{H}_{19}{ }^{81} \mathrm{Br}^{37} \mathrm{ClNO}_{4} \mathrm{Na},[\mathrm{M}+\mathrm{Na}]^{+}$(478.0045) found: 478.0046 .

(Z)-2-(2-Chloro-1-((pivaloyloxy)imino)ethyl)phenyl 4-methoxybenzoate (13dp)<smiles>COc1ccc(C(=O)Oc2ccccc2/C(CCl)=N/OC(=O)C(C)(C)C)cc1</smiles>

Following the TP-13, 13dp was obtained from S7 (270 mg, $1.0 \mathrm{mmol}), \mathrm{Et}_{3} \mathrm{~N}$ (1.2 equiv) and 4-methoxy benzoyl chloride (1.1 equiv) in $\mathrm{CH}_{2} \mathrm{Cl}_{2}$ as a light yello liquid (337 mg, 79\% yield); $\mathrm{R}_{f}=0.482(\mathrm{EA}: \mathrm{Hex}=2: 8)$.

${ }^{1} \mathbf{H}$ NMR $\left(400 \mathrm{MHz}, \mathrm{CDCl}_{3}, 25^{\circ} \mathrm{C}\right) \delta / \mathrm{ppm}: 8.12(\mathrm{~d}, J=8.8 \mathrm{~Hz}, 2 \mathrm{H}), 7.65(\mathrm{~d}, J=7.8$ $\mathrm{Hz}, 1 \mathrm{H}), 7.52$ (t, $J=7.8 \mathrm{~Hz}, 1 \mathrm{H}), 7.37-7.27(\mathrm{~m}, 2 \mathrm{H}), 6.97$ (d, $J=8.8 \mathrm{~Hz}, 2 \mathrm{H}), 4.44$ (s, 2H), $3.88(\mathrm{~s}, 3 \mathrm{H}), 1.28(\mathrm{~s}, 9 \mathrm{H})$.

${ }^{13} \mathrm{C}$ NMR $\left(100 \mathrm{MHz}, \mathrm{CDCl}_{3}, 25{ }^{\circ} \mathrm{C}\right) \delta / \mathrm{ppm}: 173.8,164.4,164.1,159.9,148.9,132.5$, $131.5,130.4,126.2,125.9,123.2,121.2,113.9,55.5,38.7,36.3,27.0$.

IR $(\mathrm{KBr}) \tilde{v}\left(\mathrm{~cm}^{-1}\right): 1744,1536,1220,1111,988,724$.

HRMS (ESI) for $\mathrm{C}_{21} \mathrm{H}_{22}{ }^{35} \mathrm{ClNO}_{5} \mathrm{Na},[\mathrm{M}+\mathrm{Na}]^{+}$(426.1084) found: 426.1087.

HRMS (ESI) for $\mathrm{C}_{21} \mathrm{H}_{22}{ }^{37} \mathrm{ClNO}_{5} \mathrm{Na},[\mathrm{M}+\mathrm{Na}]^{+}$(428.1064) found: 428.1066.

(Z)-2-(1-(Acetoxyimino)-2-chloroethyl)phenyl 4-chlorobenzoate (13en)<smiles>CC(=O)O/N=C(\CCl)c1ccccc1OC(=O)c1ccc(Cl)cc1</smiles>

Following the TP-13, 13en was obtained from S8 $(228 \mathrm{mg}, 1.0 \mathrm{mmol}), \mathrm{Et}_{3} \mathrm{~N}(1.2$ 
equiv) and 4-chloro benzoyl chloride (1.1 equiv) in $\mathrm{CH}_{2} \mathrm{Cl}_{2}$ as a pale yellow liquid (322 mg, 88\% yield); $\mathrm{R}_{f}=0.561(\mathrm{EA}: \mathrm{Hex}=2: 8)$.

${ }^{1} \mathbf{H}$ NMR $\left(400 \mathrm{MHz}, \mathrm{CDCl}_{3}, 25^{\circ} \mathrm{C}\right) \delta / \mathrm{ppm}: 8.10(\mathrm{~d}, J=8.8 \mathrm{~Hz}, 2 \mathrm{H}), 7.64(\mathrm{dd}, J=8.1$, $1.7 \mathrm{~Hz}, 1 \mathrm{H}), 7.54(\mathrm{td}, J=8.1,1.7 \mathrm{~Hz}, 1 \mathrm{H}), 7.49(\mathrm{~d}, J=8.8 \mathrm{~Hz}, 2 \mathrm{H}), 7.37(\mathrm{td}, J=8.1$, $1.0 \mathrm{~Hz}, 1 \mathrm{H}), 7.31(\mathrm{dd}, J=8.1,1.0 \mathrm{~Hz}, 1 \mathrm{H}), 4.49(\mathrm{~s}, 2 \mathrm{H}), 2.19(\mathrm{~s}, 3 \mathrm{H})$.

${ }^{13} \mathrm{C}$ NMR $\left(100 \mathrm{MHz}, \mathrm{CDCl}_{3}, 25^{\circ} \mathrm{C}\right) \delta / \mathrm{ppm}: 167.6,163.8,159.0,148.4,140.5,131.6$, $130.3,129.0,127.4,126.4,125.9,123.3,36.1,19.4$.

IR $(\mathrm{KBr}) \tilde{v}\left(\mathrm{~cm}^{-1}\right): 3389,1712,1620,1411,1285,1040,889,664$.

HRMS (ESI) for $\mathrm{C}_{17} \mathrm{H}_{13}{ }^{35} \mathrm{Cl}^{35} \mathrm{ClNO}_{4} \mathrm{Na},[\mathrm{M}+\mathrm{Na}]^{+}$(388.0119) found: 388.0120 .

HRMS (ESI) for $\mathrm{C}_{17} \mathrm{H}_{13}{ }^{35} \mathrm{Cl}^{37} \mathrm{ClNO}_{4} \mathrm{Na} / \mathrm{C}_{17} \mathrm{H}_{13}{ }^{37} \mathrm{Cl}^{35} \mathrm{ClNO}_{4} \mathrm{Na}$, $[\mathrm{M}+\mathrm{Na}]^{+}$(390.0092) found: 390.0092 .

HRMS (ESI) for $\mathrm{C}_{17} \mathrm{H}_{13}{ }^{37} \mathrm{Cl}^{37} \mathrm{ClNO}_{4} \mathrm{Na},[\mathrm{M}+\mathrm{Na}]^{+}$(392.0069) found: 392.0071 .

\section{2-(5-Phenylisoxazol-3-yl)phenyl benzoate (12aa)}

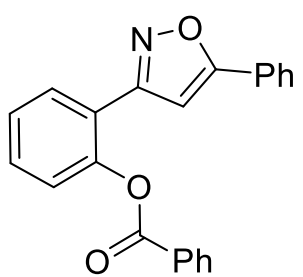

Following the TP-14, 12aa was obtained from 8a (98.4 mg, $0.2 \mathrm{mmol}$ ), 7-methyl-1,5,7-triazabicyclo[4.4.0]dec-5-ene (2.5 equiv) and benzoyl chloride 2a (1.2 equiv) as a white solid (36.9 $\mathrm{mg}, 54 \%$ yield); $\mathrm{R}_{f}=0.500$ (EA:Hex $\left.=2: 8\right) ; \mathrm{mp}=$ $122.9-123.5^{\circ} \mathrm{C}$.

${ }^{1} \mathbf{H}$ NMR $\left(400 \mathrm{MHz}, \mathrm{CDCl}_{3}, 25^{\circ} \mathrm{C}\right) \delta / \mathrm{ppm}: 8.23(\mathrm{~d}, J=7.5 \mathrm{~Hz}, 2 \mathrm{H}), 7.92(\mathrm{dd}, J=7.8$, $1.7 \mathrm{~Hz}, 1 \mathrm{H}), 7.69-7.62(\mathrm{~m}, 3 \mathrm{H}), 7.59-7.49(\mathrm{~m}, 3 \mathrm{H}), 7.44-7.38(\mathrm{~m}, 4 \mathrm{H}), 7.36(\mathrm{~d}, J=8.0$ $\mathrm{Hz}, 1 \mathrm{H}), 6.75$ (s, 1H).

${ }^{13} \mathrm{C}$ NMR $\left(100 \mathrm{MHz}, \mathrm{CDCl}_{3}, 25^{\circ} \mathrm{C}\right) \delta / \mathrm{ppm}: 170.0,165.0,159.8,148.6,133.8,131.0$, $130.3,130.1,129.9,129.3,128.9,128.7,127.3,126.5,125.7,123.6,122.7,99.5$. 
IR $(\mathrm{KBr}) \tilde{v}\left(\mathrm{~cm}^{-1}\right): 3059,1736,1590,1460,1236,1061,759,441$.

HRMS (ESI) for $\mathrm{C}_{22} \mathrm{H}_{16} \mathrm{NO}_{3},[\mathrm{M}+\mathrm{H}]^{+}$(342.1130) found: 342.1132 .

2-(5-Phenylisoxazol-3-yl)phenyl 4-methoxybenzoate (12ac)<smiles>COc1ccc(C(=O)Oc2ccccc2-c2cc(-c3ccccc3)on2)cc1</smiles>

Following the TP-14, 12ac was obtained from 8a (98.4 mg, $0.2 \mathrm{mmol}$ ), 7-methyl-1,5,7-triazabicyclo[4.4.0]dec-5-ene (2.5 equiv) and 4-OMe-benzoyl chloride

2c (1.2 equiv) as a pale yellow liquid (31.52 mg, $40 \%$ yield); $\mathrm{R}_{f}=0.416$ (EA:Hex $=2: 8$.

${ }^{1} \mathbf{H}$ NMR $\left(400 \mathrm{MHz}, \mathrm{CDCl}_{3}, 25^{\circ} \mathrm{C}\right) \delta / \mathrm{ppm}: 8.18(\mathrm{~d}, J=8.8 \mathrm{~Hz}, 2 \mathrm{H}), 7.92(\mathrm{dd}, J=7.8$, $1.7 \mathrm{~Hz}, 1 \mathrm{H}), 7.71-7.64(\mathrm{~m}, 2 \mathrm{H}), 7.53(\mathrm{dt}, J=7.8,1.7 \mathrm{~Hz}, 1 \mathrm{H}), 7.44-7.38(\mathrm{~m}, 4 \mathrm{H}), 7.35$ $(\mathrm{dt}, J=7.8,1.3 \mathrm{~Hz}, 1 \mathrm{H}), 6.99(\mathrm{~d}, J=8.8 \mathrm{~Hz}, 2 \mathrm{H}), 6.74(\mathrm{~s}, 1 \mathrm{H}), 3.89(\mathrm{~s}, 3 \mathrm{H})$.

${ }^{13} \mathrm{C}$ NMR $\left(100 \mathrm{MHz}, \mathrm{CDCl}_{3}, 25^{\circ} \mathrm{C}\right) \delta / \mathrm{ppm}: 169.9,164.7,164.1,160.0,148.7,132.5$, $130.9,130.1,129.8,129.0,127.4,126.3,125.7,123.7,122.7,121.6,114.0,99.6,55.5$. IR $(\mathrm{KBr}) \tilde{v}\left(\mathrm{~cm}^{-1}\right): 3432,1730,1601,1254,1167,761,439$.

HRMS (ESI) for $\mathrm{C}_{23} \mathrm{H}_{17} \mathrm{NO}_{4} \mathrm{Na},[\mathrm{M}+\mathrm{Na}]^{+}$(394.1055) found: 394.1056.

\section{2-(5-Phenylisoxazol-3-yl)phenyl 4-chlorobenzoate (12ae)}<smiles>O=C(Oc1ccccc1-c1cc(-c2ccccc2)on1)c1ccc(Cl)cc1</smiles>

Following the TP-14, 12ae was obtained from 8a (98.4 mg, $0.2 \mathrm{mmol}$ ), 7-methyl-1,5,7-triazabicyclo[4.4.0]dec-5-ene (2.5 equiv) and 4-Cl-benzoyl chloride 2e (1.2 equiv) as a white solid (39.9 $\mathrm{mg}, 53 \%$ yield); $\mathrm{R}_{f}=0.551(\mathrm{EA}: \mathrm{Hex}=2: 8) ; \mathrm{mp}=$ 
$119.9-120.3^{\circ} \mathrm{C}$.

${ }^{1} \mathbf{H}$ NMR $\left(400 \mathrm{MHz}, \mathrm{CDCl}_{3}, 25^{\circ} \mathrm{C}\right) \delta / \mathrm{ppm}: 8.16(\mathrm{~d}, J=8.8 \mathrm{~Hz}, 2 \mathrm{H}), 7.88(\mathrm{dd}, J=8.0$, $1.7 \mathrm{~Hz}, 1 \mathrm{H}), 7.70-7.66(\mathrm{~m}, 2 \mathrm{H}), 7.54(\mathrm{td}, J=8.0,1.7 \mathrm{~Hz}, 1 \mathrm{H}), 7.49(\mathrm{~d}, J=8.8 \mathrm{~Hz}$, 2H), 7.45-7.39 (m, 4H), $7.34(\mathrm{dd}, J=8.0,1.3 \mathrm{~Hz}, 1 \mathrm{H}), 6.71(\mathrm{~s}, 1 \mathrm{H})$.

${ }^{13} \mathrm{C}$ NMR $\left(100 \mathrm{MHz}, \mathrm{CDCl}_{3}, 25{ }^{\circ} \mathrm{C}\right) \delta / \mathrm{ppm}: 170.0,164.3,159.9,148.3,140.3,131.7$, $131.0,130.2,129.9,129.0,128.9,127.8,127.2,126.6,125.7,123.6,122.7,99.2$.

IR $(\mathrm{KBr}) \tilde{v}\left(\mathrm{~cm}^{-1}\right): 3438,1741,1596,1401,1261,1068,849,761$.

HRMS (ESI) for $\mathrm{C}_{22} \mathrm{H}_{15}{ }^{35} \mathrm{ClNO}_{3},[\mathrm{M}+\mathrm{H}]^{+}$(376.0740) found: 376.0740 .

HRMS (ESI) for $\mathrm{C}_{22} \mathrm{H}_{15}{ }^{37} \mathrm{ClNO}_{3},[\mathrm{M}+\mathrm{H}]^{+}$(378.0718) found: 378.0718 .

\section{2-(5-Phenylisoxazol-3-yl)phenyl 4-bromobenzoate (12af)}<smiles>O=C(Oc1ccccc1-c1cc(-c2ccccc2)on1)c1ccc(Br)cc1</smiles>

Following the TP-14, 12af was obtained from 8a (98.4 mg, $0.2 \mathrm{mmol}$ ), 7-methyl-1,5,7-triazabicyclo[4.4.0]dec-5-ene (2.5 equiv) and 4-Br-benzoyl chloride $2 \mathbf{f}$ (1.2 equiv) as a white solid (45.4 mg, 54\% yield); $\mathrm{R}_{f}=0.558(\mathrm{EA}: \mathrm{Hex}=2: 8) ; \mathrm{mp}=$ $125.9-126.5^{\circ} \mathrm{C}$.

${ }^{1} \mathbf{H}$ NMR $\left(400 \mathrm{MHz}, \mathrm{CDCl}_{3}, 25^{\circ} \mathrm{C}\right) \delta / \mathrm{ppm}: 8.07(\mathrm{~d}, J=8.8 \mathrm{~Hz}, 2 \mathrm{H}), 7.87(\mathrm{dd}, J=7.8$, $1.8 \mathrm{~Hz}, 1 \mathrm{H}), 7.70-7.63(\mathrm{~m}, 4 \mathrm{H}), 7.53(\mathrm{td}, J=7.8,1.8 \mathrm{~Hz}, 1 \mathrm{H}), 7.44-7.38(\mathrm{~m}, 4 \mathrm{H}), 7.34$ (dd, $J=7.8,1.0 \mathrm{~Hz}, 1 \mathrm{H}), 6.71(\mathrm{~s}, 1 \mathrm{H})$.

${ }^{13} \mathrm{C}$ NMR $\left(100 \mathrm{MHz}, \mathrm{CDCl}_{3}, 25{ }^{\circ} \mathrm{C}\right) \delta / \mathrm{ppm}: 170.0,164.4,159.9,148.4,132.1,131.8$ $131.0,130.3,130.0,129.0,128.3,127.2,126.7,125.8,123.6,122.7,99.2$.

IR $(\mathrm{KBr}) \tilde{v}\left(\mathrm{~cm}^{-1}\right): 3436,1741,1592,1261,1011,759,430$.

HRMS (ESI) for $\mathrm{C}_{22} \mathrm{H}_{15}{ }^{79} \mathrm{BrNO}_{3},[\mathrm{M}+\mathrm{H}]^{+}$(420.0235) found: 420.0237 .

HRMS (ESI) for $\mathrm{C}_{22} \mathrm{H}_{15}{ }^{81} \mathrm{BrNO}_{3},[\mathrm{M}+\mathrm{H}]^{+}$(422.0220) found: 422.0222 . 


\section{2-(5-Phenylisoxazol-3-yl)phenyl 3-chlorobenzoate (12ag)}<smiles>O=C(Oc1ccccc1-c1cc(-c2ccccc2)on1)c1cccc(Cl)c1</smiles>

Following the TP-14, 12ag was obtained from 8a (98.4 mg, $0.2 \mathrm{mmol}$ ), 7-methyl-1,5,7-triazabicyclo[4.4.0]dec-5-ene (2.5 equiv) and 3-Cl-benzoyl chloride $2 \mathrm{~g}$ (1.2 equiv) as a white solid (36.1 $\mathrm{mg}, 48 \%$ yield); $\mathrm{R}_{f}=0.521(\mathrm{EA}: \mathrm{Hex}=2: 8) ; \mathrm{mp}=$ 89.7-90.4 ${ }^{\circ} \mathrm{C}$.

${ }^{1} \mathbf{H}$ NMR $\left(400 \mathrm{MHz}, \mathrm{CDCl}_{3}, 25^{\circ} \mathrm{C}\right) \delta / \mathrm{ppm}: 8.20(\mathrm{t}, J=1.7 \mathrm{~Hz}, 1 \mathrm{H}), 8.10(\mathrm{~d}, J=7.7$ $\mathrm{Hz}, 1 \mathrm{H}), 7.88(\mathrm{dd}, J=7.8,1.7 \mathrm{~Hz}, 1 \mathrm{H}), 7.73-7.66(\mathrm{~m}, 2 \mathrm{H}), 7.61(\mathrm{~d}, J=8.0 \mathrm{~Hz}, 1 \mathrm{H})$, $7.54(\mathrm{td}, J=7.8,1.7 \mathrm{~Hz}, 1 \mathrm{H}), 7.49-7.39(\mathrm{~m}, 5 \mathrm{H}), 7.35(\mathrm{~d}, J=7.8 \mathrm{~Hz}, 1 \mathrm{H}), 6.74(\mathrm{~s}$, $1 \mathrm{H})$.

${ }^{13} \mathrm{C}$ NMR $\left(100 \mathrm{MHz}, \mathrm{CDCl}_{3}, 25^{\circ} \mathrm{C}\right) \delta / \mathrm{ppm}: 170.1,163.9,159.9,148.3,134.8,133.7$, $131.1,131.0,130.3,130.2,130.0,129.9129 .0,128.4,127.2,126.7,125.8,123.5$, 122.7, 99.3.

IR $(\mathrm{KBr}) \tilde{v}\left(\mathrm{~cm}^{-1}\right): 3432,1741,1248,1195,761,443$.

HRMS (ESI) for $\mathrm{C}_{22} \mathrm{H}_{15}{ }^{35} \mathrm{ClNO}_{3},[\mathrm{M}+\mathrm{H}]^{+}$(376.0740) found: 376.0740 .

HRMS (ESI) for $\mathrm{C}_{22} \mathrm{H}_{15}{ }^{37} \mathrm{ClNO}_{3},[\mathrm{M}+\mathrm{H}]^{+}$(378.0717) found: 378.0717.

\section{2-(5-Phenylisoxazol-3-yl)phenyl acetate (12an)}

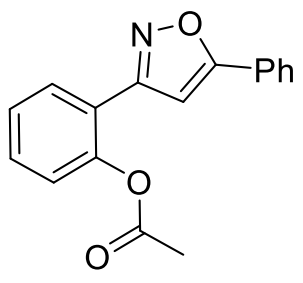

Following the TP-14, 12an was obtained from 8a (98.4 mg, $0.2 \mathrm{mmol}$ ), 7-methyl-1,5,7-triazabicyclo[4.4.0]dec-5-ene (2.5 equiv) and acetyl chloride 2n (1.2 
equiv) as a pale yellow liquid (30.3 mg, 54\% yield); $\mathrm{R}_{f}=0.522(\mathrm{EA}: \mathrm{Hex}=2: 8)$.

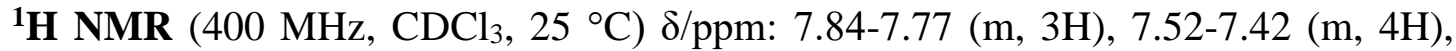
$7.36(\mathrm{td}, J=7.5,1.4 \mathrm{~Hz}, 1 \mathrm{H}), 7.21(\mathrm{dd}, J=8.0,1.4 \mathrm{~Hz}, 1 \mathrm{H}), 6.78(\mathrm{~s}, 1 \mathrm{H}), 2.35(\mathrm{~s}$, $3 \mathrm{H})$.

${ }^{13} \mathrm{C}$ NMR (100 MHz, $\left.\mathrm{CDCl}_{3}, 25{ }^{\circ} \mathrm{C}\right) \delta / \mathrm{ppm:} \mathrm{169.9,} \mathrm{169.4,} \mathrm{160.1,} \mathrm{148.3,} \mathrm{130.9,} \mathrm{130.3,}$ $129.8,129.0,127.3,126.4,125.8,123.6,122.6,99.1,21.2$.

IR $(\mathrm{KBr}) \tilde{v}\left(\mathrm{~cm}^{-1}\right): 3753,2364,1747,1189,766,430$.

HRMS (ESI) for $\mathrm{C}_{17} \mathrm{H}_{14} \mathrm{NO}_{3},[\mathrm{M}+\mathrm{H}]^{+}$(280.0974) found: 280.0975 .

\section{2-(5-Phenylisoxazol-3-yl)phenyl pivalate (12ap)}

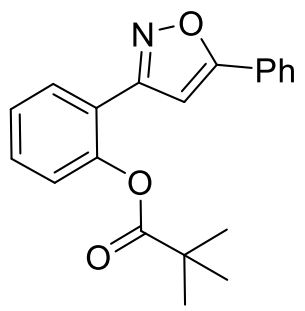

Following the TP-14, 12ap was obtained from 8a $(98.4 \mathrm{mg}, 0.2 \mathrm{mmol}$ ), 7-methyl-1,5,7-triazabicyclo[4.4.0]dec-5-ene (2.5 equiv) and pivaloyl chloride $\mathbf{2 p}$ (1.2 equiv) as a pale yellow liquid (30.3 mg, 53\% yield); $\mathrm{R}_{f}=0.536(\mathrm{EA}: \mathrm{Hex}=2: 8)$.

${ }^{1} \mathbf{H}$ NMR $\left(400 \mathrm{MHz}, \mathrm{CDCl}_{3}, 25{ }^{\circ} \mathrm{C}\right) \delta / \mathrm{ppm}: 7.84-7.73(\mathrm{~m}, 3 \mathrm{H}), 7.52-7.42(\mathrm{~m}, 4 \mathrm{H})$, $7.34(\mathrm{t}, J=7.7 \mathrm{~Hz}, 1 \mathrm{H}), 7.14(\mathrm{~d}, J=8.1 \mathrm{~Hz}, 1 \mathrm{H}), 6.73(\mathrm{~s}, 1 \mathrm{H}), 1.35(\mathrm{~s}, 9 \mathrm{H})$.

${ }^{13} \mathrm{C}$ NMR $\left(100 \mathrm{MHz}, \mathrm{CDCl}_{3}, 25{ }^{\circ} \mathrm{C}\right) \delta / \mathrm{ppm}: 176.8,169.7,160.0,148.9,130.8,130.2$ 130.0, 129.0, 127.4, 126.1, 125.7, 123.3, 122.9, 99.9, 39.1, 27.2.

IR $(\mathrm{KBr}) \tilde{v}\left(\mathrm{~cm}^{-1}\right):$ 2975, 2376, 1752, 1270, 1114, 950, 687.

HRMS (ESI) for $\mathrm{C}_{20} \mathrm{H}_{20} \mathrm{NO}_{3},[\mathrm{M}+\mathrm{H}]^{+}$(322.1443) found: 322.1447.

\section{2-(5-Phenylisoxazol-3-yl)phenol (12a)}<smiles>Oc1ccccc1-c1cc(-c2ccccc2)on1</smiles>

Following the TP-14, 12a was obtained from 8a $(98.4 \mathrm{mg}, 0.2 \mathrm{mmol}$ ), 
7-methyl-1,5,7-triazabicyclo[4.4.0]dec-5-ene (2.5 equiv) and TFAA $2 \mathrm{l}$ (1.2 equiv) as a white solid (28.5 mg, 59\% yield); $\mathrm{R}_{f}=0.381(\mathrm{EA}: \mathrm{Hex}=2: 8) ; \mathrm{mp}=125.6-126.3{ }^{\circ} \mathrm{C}$.

${ }^{1} \mathbf{H}$ NMR $\left(400 \mathrm{MHz}, \mathrm{CDCl}_{3}, 25{ }^{\circ} \mathrm{C}\right) \delta / \mathrm{ppm}: 9.57$ (s, 1H), 7.89-7.82 (m, 2H), $7.58(\mathrm{dd}$, $J=7.9,1.7 \mathrm{~Hz}, 1 \mathrm{H}), 7.55-7.46(\mathrm{~m}, 3 \mathrm{H}), 7.40-7.32(\mathrm{~m}, 1 \mathrm{H}), 7.11(\mathrm{~d}, J=8.4 \mathrm{~Hz}, 1 \mathrm{H})$, $7.00(\mathrm{t}, J=7.4 \mathrm{~Hz}, 1 \mathrm{H}), 6.92(\mathrm{~s}, 1 \mathrm{H})$.

${ }^{13} \mathrm{C}$ NMR $\left(100 \mathrm{MHz}, \mathrm{CDCl}_{3}, 25^{\circ} \mathrm{C}\right) \delta / \mathrm{ppm}: 169.4,163.0,156.6,131.7,130.7,129.1$, $127.9,126.8,126.0,119.8,117.6,113.3,97.0$.

IR $(\mathrm{KBr}) \tilde{v}\left(\mathrm{~cm}^{-1}\right): 3317,2862,2318,1520,1261,1008,688,441$.

HRMS (ESI) for $\mathrm{C}_{15} \mathrm{H}_{12} \mathrm{NO}_{2},[\mathrm{M}+\mathrm{H}]^{+}$(238.0868) found: 238.0870 .

\section{2-(5-Phenylisoxazol-3-yl)phenol (12a)}<smiles>Oc1ccccc1-c1cc(-c2ccccc2)on1</smiles>

Following the TP-14, 12a was obtained from 8a (98.4 mg, $0.2 \mathrm{mmol})$, 7-methyl-1,5,7-triazabicyclo[4.4.0]dec-5-ene (2.5 equiv) in dry THF, as a white solid (26.1 mg, 55\% yield); $\mathrm{R}_{f}=0.381(\mathrm{EA}: \mathrm{Hex}=2: 8) ; \mathrm{mp}=125.6-126.3{ }^{\circ} \mathrm{C}$.

\section{2-(5-Phenylisoxazol-3-yl)phenol (12a)}<smiles>Oc1ccccc1-c1cc(-c2ccccc2)on1</smiles>

Following the TP-14, 12a was obtained from 8a (98.4 mg, $0.2 \mathrm{mmol}$ ), 7-methyl-1,5,7-triazabicyclo[4.4.0]dec-5-ene (2.5 equiv) in dry $\mathrm{CH}_{2} \mathrm{Cl}_{2}$, as a white solid $(26.1 \mathrm{mg}, 55 \%$ yield $) ; \mathrm{R}_{f}=0.381(\mathrm{EA}: \mathrm{Hex}=2: 8) ; \mathrm{mp}=125.6-126.3{ }^{\circ} \mathrm{C}$ (E)-2-Phenyl-4H-chromen-4-one $O$-benzoyl oxime (11aa) 


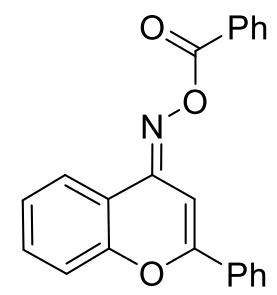

Following the TP-15, 11aa was obtained from 8a (98.4 mg, $0.2 \mathrm{mmol}$ ), 7-methyl-1,5,7-triazabicyclo[4.4.0]dec-5-ene (2.5 equiv) and benzoyl chloride 2a (1.2 equiv) as a colorless liquid (41.7 mg, 61\% yield); $\mathrm{R}_{f}=0.351$ (EA:Hex $=2: 8$ ).

${ }^{1} \mathbf{H}$ NMR $\left(400 \mathrm{MHz}, \mathrm{CDCl}_{3}, 25^{\circ} \mathrm{C}\right) \delta / \mathrm{ppm}: 8.38(\mathrm{dd}, \mathrm{J}=8.0,1.8 \mathrm{~Hz}, 1 \mathrm{H}), 8.19(\mathrm{~d}, \mathrm{~J}=$ $7.2 \mathrm{~Hz}, 2 \mathrm{H}), 7.96-7.88(\mathrm{~m}, 2 \mathrm{H}), 7.63(\mathrm{t}, \mathrm{J}=7.4 \mathrm{~Hz}, 1 \mathrm{H}), 7.58-7.49(\mathrm{~m}, 6 \mathrm{H}), 7.41(\mathrm{~d}, \mathrm{~J}$ $=8.0 \mathrm{~Hz}, 1 \mathrm{H}), 7.35(\mathrm{t}, \mathrm{J}=7.4 \mathrm{~Hz}, 1 \mathrm{H}), 7.16(\mathrm{~s}, 1 \mathrm{H})$.

${ }^{13} \mathrm{C}$ NMR $\left(100 \mathrm{MHz}, \mathrm{CDCl}_{3}, 25^{\circ} \mathrm{C}\right) \delta / \mathrm{ppm}: 164.3,157.7,152.5,150.7,133.0,132.3$, 132.0, 131.1, 129.7, 129.6, 128.9, 128.6, 126.1, 125.2, 124.5, 117.6, 116.7, 93.7.

IR $(\mathrm{KBr}) \tilde{v}\left(\mathrm{~cm}^{-1}\right): 3368,1736,1631,1239,1044,768,421$.

HRMS (ESI) for $\mathrm{C}_{22} \mathrm{H}_{16} \mathrm{NO}_{3},[\mathrm{M}+\mathrm{H}]^{+}$(342.1130) found: 342.1130 .

(E)-2-Phenyl-4H-chromen-4-one $O$-(4-methoxybenzoyl) oxime (11ac)<smiles>COc1ccc(C(=O)O/N=c2\cc(-c3ccccc3)oc3ccccc23)cc1</smiles>

Following the TP-15, 11ac was obtained from 8a (98.4 mg, $0.2 \mathrm{mmol}$ ), 7-methyl-1,5,7-triazabicyclo[4.4.0]dec-5-ene (2.5 equiv) and 4-OMe-benzoyl chloride 2c (1.2 equiv) as a yellow liquid (26.0 mg, 35\% yield); $\mathrm{R}_{f}=0.354$ (EA:Hex $\left.=2: 8\right)$.

${ }^{1} \mathbf{H}$ NMR $\left(400 \mathrm{MHz}, \mathrm{CDCl}_{3}, 25^{\circ} \mathrm{C}\right) \delta / \mathrm{ppm}: 8.37(\mathrm{dd}, J=8.0,1.4 \mathrm{~Hz}, 1 \mathrm{H}), 8.14(\mathrm{~d}, J$ $=8.8 \mathrm{~Hz}, 2 \mathrm{H}), 7.94-7.88(\mathrm{~m}, 2 \mathrm{H}),(\mathrm{ddd}, J=8.6,7.0,1.5 \mathrm{~Hz}, 1 \mathrm{H}), 7.54-7.48(\mathrm{~m}, 3 \mathrm{H})$, $7.40(\mathrm{dd}, J=8.0,1.0 \mathrm{~Hz}, 1 \mathrm{H}), 7.34(\mathrm{ddd}, J=8.3,7.0,1.0 \mathrm{~Hz}, 1 \mathrm{H}), 7.15(\mathrm{~s}, 1 \mathrm{H}), 7.01$ $(\mathrm{d}, J=8.8 \mathrm{~Hz}, 2 \mathrm{H}), 3.90(\mathrm{~s}, 3 \mathrm{H})$. 
${ }^{13} \mathrm{C}$ NMR $\left(100 \mathrm{MHz}, \mathrm{CDCl}_{3}, 25^{\circ} \mathrm{C}\right) \delta / \mathrm{ppm}: 164.0,163.5,157.5,152.5,150.5,132.4$, 131.9, 131.6, 131.0, 128.9, 126.1, 125.2, 124.5, 121.9, 117.6, 116.8, 113.9, 93.8, 55.5. IR $(\mathrm{KBr}) \tilde{v}\left(\mathrm{~cm}^{-1}\right): 3432,1629,1254,1077,761,437$.

HRMS (ESI) for $\mathrm{C}_{23} \mathrm{H}_{18} \mathrm{NO}_{4},[\mathrm{M}+\mathrm{H}]^{+}$(372.1236) found: 372.1235 .

(E)-2-Phenyl-4H-chromen-4-one $O$-(4-chlorobenzoyl) oxime (11ae)<smiles>O=C(O/N=c1\cc(-c2ccccc2)oc2ccccc12)c1ccc(Cl)cc1</smiles>

Following the TP-15, 11ae was obtained from 8a (98.4 mg, $0.2 \mathrm{mmol}$ ), 7-methyl-1,5,7-triazabicyclo[4.4.0]dec-5-ene (2.5 equiv) and 4-Cl-benzoyl chloride 2e (1.2 equiv) as a pale yellow solid (40.6 mg, 54\% yield); $\mathrm{R}_{f}=0.451$ (EA:Hex $=2: 8$ ); $\mathrm{mp}=165.8-166.2^{\circ} \mathrm{C}$.

${ }^{1} \mathbf{H}$ NMR $\left(400 \mathrm{MHz}, \mathrm{CDCl}_{3}, 25^{\circ} \mathrm{C}\right) \delta / \mathrm{ppm}: 8.35(\mathrm{dd}, J=8.4,1.8 \mathrm{~Hz}, 1 \mathrm{H}), 8.11(\mathrm{~d}, \mathrm{~J}=$ 8.7 Hz, 2H), 7.95-7.87 (m, 2H), 7.57 (ddd, $J=8.7,7.0,1.4 \mathrm{~Hz}, 1 \mathrm{H}), 7.54-7.47$ (m, 5H), $7.41(\mathrm{dd}, J=8.3,1.0 \mathrm{~Hz}, 1 \mathrm{H}), 7.34(\mathrm{t}, J=7.7 \mathrm{~Hz}, 1 \mathrm{H}), 7.11(\mathrm{~s}, 1 \mathrm{H})$.

${ }^{13} \mathrm{C}$ NMR $\left(100 \mathrm{MHz}, \mathrm{CDCl}_{3}, 25{ }^{\circ} \mathrm{C}\right) \delta / \mathrm{ppm}: 163.4,157.8,152.5,150.9,139.5,132.2$, $132.1,131.2,130.9,129.0,128.9,128.1,126.1,125.3,124.4,117.6,116.6,93.5$.

IR $(\mathrm{KBr}) \tilde{v}\left(\mathrm{~cm}^{-1}\right): 3428,1633,1259,768,752,439$.

HRMS (ESI) for $\mathrm{C}_{22} \mathrm{H}_{15}{ }^{35} \mathrm{ClNO}_{3},[\mathrm{M}+\mathrm{H}]^{+}$(376.0740) found: 376.0741 .

HRMS (ESI) for $\mathrm{C}_{22} \mathrm{H}_{15}{ }^{37} \mathrm{ClNO}_{3},[\mathrm{M}+\mathrm{H}]^{+}$(378.0702) found: 378.0704 .

(E)-2-Phenyl-4H-chromen-4-one $O$-acetyl oxime (11an)<smiles>CC(=O)O/N=c1\cc(-c2ccccc2)oc2ccccc12</smiles> 
Following the TP-15, 11an was obtained from 8a (98.4 mg, $0.2 \mathrm{mmol}$ ), 7-methyl-1,5,7-triazabicyclo[4.4.0]dec-5-ene (2.5 equiv) and acetyl chloride 2n (1.2 equiv) as a pale yellow solid (28.0 mg, 50\% yield); $\mathrm{R}_{f}=0.416(\mathrm{EA}: \mathrm{Hex}=2: 8) ; \mathrm{mp}=$ $111.4-112.8^{\circ} \mathrm{C}$.

${ }^{1} \mathbf{H}$ NMR $\left(400 \mathrm{MHz}, \mathrm{CDCl}_{3}, 25{ }^{\circ} \mathrm{C}\right) \delta / \mathrm{ppm}: 8.24(\mathrm{dd}, J=8.0,1.5 \mathrm{~Hz}, 1 \mathrm{H}), 7.94-7.88$ (m, 2H), 7.57-7.48 (m, 4H), $7.38(\mathrm{dd}, J=8.3,1.0 \mathrm{~Hz}, 1 \mathrm{H}), 7.30(\mathrm{ddd}, J=8.3,7.0,1.3$ $\mathrm{Hz}, 1 \mathrm{H}), 7.08(\mathrm{~s}, 1 \mathrm{H}), 2.33(\mathrm{~s}, 3 \mathrm{H})$.

${ }^{13} \mathrm{C}$ NMR $\left(100 \mathrm{MHz}, \mathrm{CDCl}_{3}, 25{ }^{\circ} \mathrm{C}\right) \delta / \mathrm{ppm}: 169.4,157.4,152.5,149.5,132.2,131.9$, $131.0,128.8,126.1,125.1,124.2,117.6,116.6,93.6,20.0$

IR $(\mathrm{KBr}) \tilde{v}\left(\mathrm{~cm}^{-1}\right): 3753,3498,3063,2370,1762,1632,1210,765,433$.

HRMS (ESI) for $\mathrm{C}_{17} \mathrm{H}_{14} \mathrm{NO}_{3},[\mathrm{M}+\mathrm{H}]^{+}$(280.0974) found: 280.0975 .

(E)-2-Phenyl-4H-chromen-4-one $O$-pivaloyl oxime (11ap)

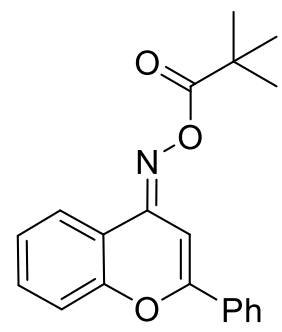

Following the TP-15, 11ap was obtained from 8a (98.4 mg, $0.2 \mathrm{mmol}$ ), 7-methyl-1,5,7-triazabicyclo[4.4.0]dec-5-ene (2.5 equiv) and pivaloyl chloride $\mathbf{2 p}$ (1.2 equiv) as a white solid (39.2 $\mathrm{mg}, 61 \%$ yield); $\mathrm{R}_{f}=0.450$ (EA:Hex $\left.=2: 8\right) ; \mathrm{mp}=$ $113.3-114.8^{\circ} \mathrm{C}$

${ }^{1} \mathbf{H}$ NMR $\left(400 \mathrm{MHz}, \mathrm{CDCl}_{3}, 25{ }^{\circ} \mathrm{C}\right) \delta / \mathrm{ppm}: 8.29(\mathrm{dd}, J=8.0,1.7 \mathrm{~Hz}, 1 \mathrm{H}), 7.92-7.83$ $(\mathrm{m}, 2 \mathrm{H}), 7.59-7.47(\mathrm{~m}, 4 \mathrm{H}), 7.38(\mathrm{~d}, J=8.3 \mathrm{~Hz}, 1 \mathrm{H}), 7.31(\mathrm{ddd}, J=8.3,7.1,1.0 \mathrm{~Hz}$ 1H), $7.02(\mathrm{~s}, 1 \mathrm{H}), 1.40(\mathrm{~s}, 9 \mathrm{H})$.

${ }^{13} \mathrm{C}$ NMR $\left(100 \mathrm{MHz}, \mathrm{CDCl}_{3}, 25{ }^{\circ} \mathrm{C}\right) \delta / \mathrm{ppm}: 175.3,157.4,152.4,150.4,132.3,131.9$, 131.0, 128.9, 126.0, 125.1, 124.4, 117.6, 116.7, 93.6, 39.0, 27.5.

IR $(\mathrm{KBr}) \tilde{v}\left(\mathrm{~cm}^{-1}\right): 3753,2973,2369,1751,1379,1116,889.438$. 
HRMS (ESI) for $\mathrm{C}_{20} \mathrm{H}_{20} \mathrm{NO}_{3},[\mathrm{M}+\mathrm{H}]^{+}$(322.1443) found: 322.1443.

(E)-2-Phenyl-4H-chromen-4-one $O$-benzoyl oxime (11aa)<smiles>O=C(O/N=c1\cc(-c2ccccc2)oc2ccccc12)c1ccccc1</smiles>

Following the TP-16, 11aa was obtained from 13aa $(78.8 \mathrm{mg}, 0.2 \mathrm{mmol}), \mathrm{PBu}_{3}(1.1$ equiv) and 7-methyl-1,5,7-triazabicyclo[4.4.0]dec-5-ene (1.5 equiv) as a colorless liquid (45.67 mg, 67\% yield); $\mathrm{R}_{f}=0.351(\mathrm{EA}: \mathrm{Hex}=2: 8)$.

HRMS (ESI) for $\mathrm{C}_{22} \mathrm{H}_{16} \mathrm{NO}_{3},[\mathrm{M}+\mathrm{H}]^{+}$(342.1126) found: 342.1128 .

(E)-2-(tert-Butyl)-4H-chromen-4-one $O$-(4-methoxybenzoyl) oxime (11bc)<smiles>COc1ccc(C(=O)O/N=c2\cc(C(C)(C)C)oc3ccccc23)cc1</smiles>

Following the TP-16, 11bc was obtained from $13 \mathbf{b c}(80.8 \mathrm{mg}, 0.2 \mathrm{mmol}), \mathrm{PBu}_{3}(1.1$ equiv) and 7-methyl-1,5,7-triazabicyclo[4.4.0]dec-5-ene (1.5 equiv) as a colorless liquid (36.5 mg, 52\% yield); $\mathrm{R}_{f}=0.401(\mathrm{EA}: \mathrm{Hex}=2: 8)$.

${ }^{1} \mathbf{H}$ NMR $\left(400 \mathrm{MHz}, \mathrm{CDCl}_{3}, 25^{\circ} \mathrm{C}\right) \delta / \mathrm{ppm}: 8.30(\mathrm{dd}, J=8.8,1.7 \mathrm{~Hz}, 1 \mathrm{H}), 8.10(\mathrm{~d}, J$ $=8.8 \mathrm{~Hz}, 2 \mathrm{H}), 7.50(\mathrm{ddd}, J=8.3,7.0,1.3 \mathrm{~Hz}, 1 \mathrm{H}), 7.31-7.26(\mathrm{~m}, 2 \mathrm{H}), 6.98(\mathrm{~d}, J=8.8$ $\mathrm{Hz}, 2 \mathrm{H}), 6.58(\mathrm{~s}, 1 \mathrm{H}), 3.89$ (s, 3H), 1.35 (s, 9H).

${ }^{13} \mathrm{C}$ NMR $\left(100 \mathrm{MHz}, \mathrm{CDCl}_{3}, 25^{\circ} \mathrm{C}\right) \delta / \mathrm{ppm}: 169.6,164.0,163.4,152.7,150.8,131.6$, $131.5,124.7,124.3,122.0,117.3,116.5,113.8,91.9,55.5,36.3,27.8$.

IR $(\mathrm{KBr}) \tilde{v}\left(\mathrm{~cm}^{-1}\right):$ 2968, 2368, 1735, 1607, 1250, 1160, 1028, 882, 691. HRMS (ESI) for $\mathrm{C}_{21} \mathrm{H}_{22} \mathrm{NO}_{4},[\mathrm{M}+\mathrm{H}]^{+}$(352.1549) found: 352.1550 . 


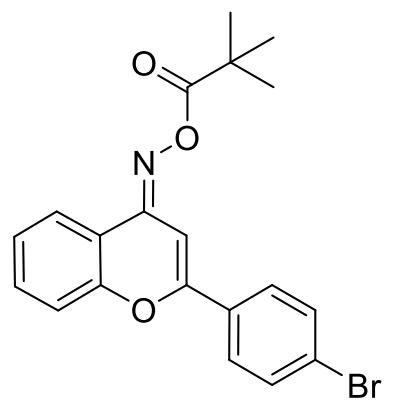

Following the TP-16, 11cp was obtained from $13 \mathbf{c p}(90.5 \mathrm{mg}, 0.2 \mathrm{mmol}), \mathrm{PBu}_{3}(1.1$ equiv) and 7-methyl-1,5,7-triazabicyclo[4.4.0]dec-5-ene (1.5 equiv) as a yellow solid $(66.3 \mathrm{mg}, 83 \%$ yield $) ; \mathrm{R}_{f}=0.453(\mathrm{EA}: \mathrm{Hex}=2: 8) ; \mathrm{mp}=164.0-164.4{ }^{\circ} \mathrm{C}$.

${ }^{1} \mathbf{H}$ NMR $\left(400 \mathrm{MHz}, \mathrm{CDCl}_{3}, 25^{\circ} \mathrm{C}\right) \delta / \mathrm{ppm}: 8.28(\mathrm{~d}, J=8.5 \mathrm{~Hz}, 1 \mathrm{H}), 7.73(\mathrm{~d}, \mathrm{~J}=8.8$ $\mathrm{Hz}, 2 \mathrm{H}), 7.65(\mathrm{~d}, J=8.8 \mathrm{~Hz}, 2 \mathrm{H}), 7.54(\mathrm{t}, J=8.5 \mathrm{~Hz}, 1 \mathrm{H}), 7.36(\mathrm{~d}, J=8.5 \mathrm{~Hz}, 1 \mathrm{H})$, $7.31(\mathrm{t}, J=8.5 \mathrm{~Hz}, 1 \mathrm{H}), 6.99(\mathrm{~s}, 1 \mathrm{H}), 1.40(\mathrm{~s}, 9 \mathrm{H})$.

${ }^{13} \mathrm{C}$ NMR $\left(100 \mathrm{MHz}, \mathrm{CDCl}_{3}, 25{ }^{\circ} \mathrm{C}\right) \delta / \mathrm{ppm}: 175.2,156.3,152.3,150.1,132.2,132.0$ $131.3,127.4,125.6,125.3,124.4,117.6,116.7,93.8,39.0,27.5$.

IR $(\mathrm{KBr}) \tilde{v}\left(\mathrm{~cm}^{-1}\right): 3402,2736,1631,1118,759,456$.

HRMS (ESI) for $\mathrm{C}_{20} \mathrm{H}_{19}{ }^{79} \mathrm{BrNO}_{3},[\mathrm{M}+\mathrm{H}]^{+}$(400.0548) found: 400.0551

HRMS (ESI) for $\mathrm{C}_{20} \mathrm{H}_{19}{ }^{81} \mathrm{BrNO}_{3},[\mathrm{M}+\mathrm{H}]^{+}$(402.0530) found: 402.0533

(E)-2-(4-Methoxyphenyl)-4H-chromen-4-one $O$-pivaloyl oxime (11dp)<smiles>COc1ccc(-c2c/c(=N\OC(=O)C(C)(C)C)c3ccccc3o2)cc1</smiles>

Following the TP-16, 11dp was obtained from 13dp (80.8 mg, $0.2 \mathrm{mmol}), \mathrm{PBu}_{3}(1.1$ equiv) and 7-methyl-1,5,7-triazabicyclo[4.4.0]dec-5-ene (1.5 equiv) as a white solid $(46.4 \mathrm{mg}, 66 \%$ yield $) ; \mathrm{R}_{f}=0.416(\mathrm{EA}: \mathrm{Hex}=2: 8) ; \mathrm{mp}=115.9-116.6{ }^{\circ} \mathrm{C}$.

${ }^{1} \mathbf{H}$ NMR $\left(400 \mathrm{MHz}, \mathrm{CDCl}_{3}, 25{ }^{\circ} \mathrm{C}\right) \delta / \mathrm{ppm}: 8.27(\mathrm{dd}, J=8.0,1.7 \mathrm{~Hz}, 1 \mathrm{H}), 7.82(\mathrm{~d}, J$ 
$=8.9 \mathrm{~Hz}, 2 \mathrm{H}), 7.52(\mathrm{ddd}, J=8.4,7.0,1.4 \mathrm{~Hz}, 1 \mathrm{H}), 7.36(\mathrm{~d}, J=8.4 \mathrm{~Hz}, 1 \mathrm{H}), 7.29(\mathrm{t}, J$ $=7.6 \mathrm{~Hz}, 1 \mathrm{H}), 7.01(\mathrm{~d}, J=8.9 \mathrm{~Hz}, 2 \mathrm{H}), 6.92(\mathrm{~s}, 1 \mathrm{H}), 3.88(\mathrm{~s}, 3 \mathrm{H}), 1.40(\mathrm{~s}, 9 \mathrm{H})$.

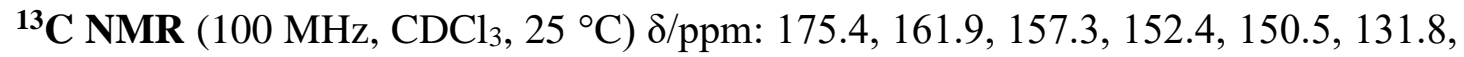
$127.6,125.0,124.6,124.3,117.5,116.8,114.3,92.0,55.4,39.0,27.5$.

IR $(\mathrm{KBr}) \tilde{v}\left(\mathrm{~cm}^{-1}\right): 3238,2971,1754,1631,1377,1259,1116,761,441$.

HRMS (ESI) for $\mathrm{C}_{21} \mathrm{H}_{22} \mathrm{NO}_{4},[\mathrm{M}+\mathrm{H}]^{+}$(352.1549) found: 352.1552.

(E)-2-(4-Chlorophenyl)-4H-chromen-4-one $O$-acetyl oxime (11en)<smiles>CC(=O)O/N=c1\cc(-c2ccc(Cl)cc2)oc2ccccc12</smiles>

Following the TP-16, 11en was obtained from 13en (73.24 mg, $0.2 \mathrm{mmol}), \mathrm{PBu}_{3}(1.1$ equiv) and 7-methyl-1,5,7-triazabicyclo[4.4.0]dec-5-ene (1.5 equiv) as an orange solid (42.4 mg, 68\% yield); $\mathrm{R}_{f}=0.491(\mathrm{EA}: \mathrm{Hex}=2: 8) ; \mathrm{mp}=144.2-145.8{ }^{\circ} \mathrm{C}$.

${ }^{1} \mathbf{H}$ NMR $\left(400 \mathrm{MHz}, \mathrm{CDCl}_{3}, 25^{\circ} \mathrm{C}\right) \delta / \mathrm{ppm}: 8.23(\mathrm{~d}, J=7.5 \mathrm{~Hz}, 1 \mathrm{H}), 7.84(\mathrm{~d}, J=8.4$ $\mathrm{Hz}, 2 \mathrm{H}), 7.54(\mathrm{t}, J=7.5 \mathrm{~Hz}, 1 \mathrm{H}), 7.47(\mathrm{~d}, J=8.4 \mathrm{~Hz}, 2 \mathrm{H}), 7.36(\mathrm{~d}, J=7.5 \mathrm{~Hz}, 1 \mathrm{H})$, $7.30(\mathrm{t}, J=7.5 \mathrm{~Hz}, 1 \mathrm{H}), 7.05(\mathrm{~s}, 1 \mathrm{H}), 2.34(\mathrm{~s}, 3 \mathrm{H})$.

${ }^{13} \mathrm{C}$ NMR $\left(100 \mathrm{MHz}, \mathrm{CDCl}_{3}, 25^{\circ} \mathrm{C}\right) \delta / \mathrm{ppm:} \mathrm{169.4,} \mathrm{156.2,} \mathrm{152.3,} \mathrm{149.2,} \mathrm{137.2,} \mathrm{132.0,}$ $130.6,129.1,127.3,125.2,124.1,117.6,116.5,93.7,20.0$.

IR $(\mathrm{KBr}) \tilde{v}\left(\mathrm{~cm}^{-1}\right): 3337,2851,1742,1655,1290,1259,1110,756,441$.

HRMS (ESI) for $\mathrm{C}_{17} \mathrm{H}_{13} \mathrm{ClNO}_{3},[\mathrm{M}+\mathrm{H}]^{+}$(314.0584) found: 314.0583 .

\section{References:}

1. Hatcher, J. M.; Coltart, D. M. Copper(I)-Catalyzed Addition of Grignard Reagents to in Situ-Derived $\mathrm{N}$-Sulfonyl Azoalkenes: An Umpolung Alkylation Procedure Applicable to the Formation of Up to Three Contiguous Quaternary Centers. J. Am. Chem. Soc. 2010, 132 (13), 4546-4547. 
2. Zhang, Y.; Stephens, D.; Hernandez, G.; Mendoza, R.; Larionov, O. V. Catalytic Diastereo and Enantioselective Annulations between Transient Nitrosoalkenes and Indoles. Chem. Eur. J. 2012, 18 (52), 16612-16615.

3. Vargha, L.; Ramonczai, J.; Bathory, J. Studies on Furan Compounds. II. Conversion of 2-Aceto-benzofuran to 2-Methyl-3-hydroxychromone. J. Am. Chem. Soc. 1949, 71 (8), 2652-2655.

4. Hu, Z.; Zhang, S.; Zhou, W.; Ma, X.; Xiang, G. Synthesis and Antibacterial Activity of 3-Benzylamide Derivatives as FtsZ Inhibitors. Bioorg. Med. Chem. Lett. 2017, 27 (8), 1854-1858.

5. Allen, C. F. H.; Richmond, J. H. Some Limitations of 2,4-Dinitrophenylhydrazine as a Reagent for Carbonyl Groups. J. Org. Chem. 1937, 2 (3), 222-226.

6. Ojha, D. P.; Prabhu, K. R. Regioselective Synthesis of Vinyl Halides, Vinyl Sulfones, and Alkynes: A Tandem Intermolecular Nucleophilic and Electrophilic Vinylation of Tosylhydrazones. Org. Lett. 2015, 17 (1), 18-21.

7. a) Liu, Y.-Y.; Yang, X.-H.; Yang, J.; Songa, R.-J.; Li, J.-H. Silver-mediated radical cyclization: construction of $\Delta^{2}$-isoxazolines from $\alpha$-halo ketoximes and 1,3-dicarbonyl compounds. Chem. Commun. 2014, 50 (52), 6906-6908. b) Wabnitz, T. C.; Saaby, S.; Jørgensen, K. A. The first catalytic inverse-electron demand hetero-Diels-Alder reaction of nitroso alkenes using pyrrolidine as an organocatalyst. Org. Biomol. Chem. 2004, 2 (6), 828-834.

8. Perrone, S.; Capua, M.; Messa, F.; Salomone, A.; Troisi, L. Green synthesis of 2-pyrazinones in deep eutectic solvents: From $\alpha$-chloro oximes to peptidomimetic scaffolds. Tetrahedron 2017, 73 (43), 6193-6198. 


\section{X-ray crystallographic data for selected compounds:}

a) 4a (CCDC no. 1588092): The thermal ellipsoid drawn at 30\% probability level.

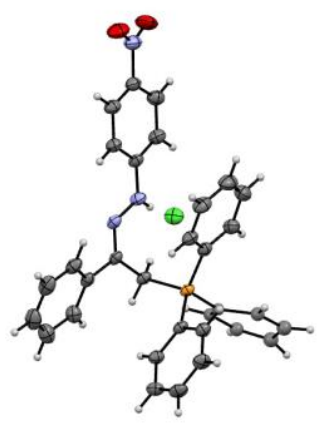

Empirical formula

Formula weight

Temperature

Wavelength

Crystal system

Space group

Unit cell dimensions

Volume

$\mathrm{Z}$

Density (calculated)

Absorption coefficient

$\mathrm{F}(000)$

Crystal size

Theta range for data collection

Index ranges

Reflections collected

Independent reflections

Completeness to theta $=25.07^{\circ}$

Absorption correction

Max. and min. transmission

Refinement method

Data / restraints / parameters

Goodness-of-fit on $\mathrm{F}^{2}$

Final R indices [I $>2 \operatorname{sigma}(\mathrm{I})]$

$\mathrm{R}$ indices (all data)

Largest diff. peak and hole

\section{$\mathrm{C}_{32} \mathrm{H}_{27} \mathrm{ClN}_{3} \mathrm{O}_{2} \mathrm{P}$}

551.99

200(2) K

$0.71073 \AA$

Triclinic

P -1

$\mathrm{a}=9.4968(12) \AA$ $\alpha=71.773(4)^{\circ}$.

$\mathrm{b}=12.0703(16) \AA$ $\beta=89.375(4)^{\circ}$.

$\mathrm{c}=14.5611(19) \AA$ $\gamma=82.036(4)^{\circ}$.

1569.2(4) $\AA^{3}$

2

$1.168 \mathrm{Mg} / \mathrm{m}^{3}$

$0.204 \mathrm{~mm}^{-1}$

576

$0.12 \times 0.11 \times 0.03 \mathrm{~mm}^{3}$

2.58 to $25.07^{\circ}$.

$-11<=\mathrm{h}<=11,-14<=\mathrm{k}<=14,-17<=\mathrm{l}<=17$

40586

$5563[\mathrm{R}($ int $)=0.0669]$

$99.7 \%$

multi-scan

0.9939 and 0.9760

Full-matrix least-squares on $\mathrm{F}^{2}$

5563 / 6 / 346

1.068

$\mathrm{R} 1=0.0697, \mathrm{wR} 2=0.1878$

$\mathrm{R} 1=0.1064, \mathrm{wR} 2=0.2135$

0.315 and -0.462 e. $\AA^{-3}$ 
b) 3fa: The thermal ellipsoid drawn at 30\% probability level. (The crystal structure was reported by Albayati et al. in 2015, for reference see: Mohamed, S. K.; Mague, J. T.; Akkurt, M.; Albayati, M. R.; Mohamed, A. F. Acta Crystallogr E. 2015, 71, o931.)

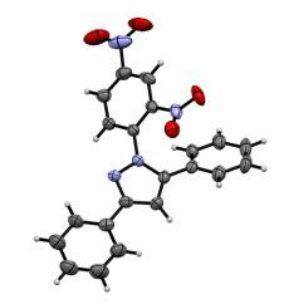

Empirical formula

Formula weight

Temperature

Wavelength

Crystal system

Space group

Unit cell dimensions

Volume

Z

Density (calculated)

Absorption coefficient

$\mathrm{F}(000)$

Crystal size

Theta range for data collection

Index ranges

Reflections collected

Independent reflections

Completeness to theta $=25.14^{\circ}$

Absorption correction

Max. and min. transmission

Refinement method

Data / restraints / parameters

Goodness-of-fit on $\mathrm{F}^{2}$

Final $\mathrm{R}$ indices [I $>2 \operatorname{sigma}(\mathrm{I})]$

$\mathrm{R}$ indices (all data)

Absolute structure parameter

Largest diff. peak and hole
$\mathrm{C}_{21} \mathrm{H}_{14} \mathrm{~N}_{4} \mathrm{O}_{4}$

386.36

298(2) K

$0.71073 \AA$

Orthorhombic

P 212121

$\mathrm{a}=7.409(3) \AA$ $\alpha=90^{\circ}$.

$\mathrm{b}=12.951(5) \AA$ $\beta=90^{\circ}$.

$\mathrm{c}=19.273(7) \AA$ $\gamma=90^{\circ}$.

1849.4(11) $\AA^{3}$

4

$1.388 \mathrm{Mg} / \mathrm{m}^{3}$

$0.099 \mathrm{~mm}^{-1}$

800

$0.24 \times 0.08 \times 0.05 \mathrm{~mm}^{3}$

2.63 to $25.14^{\circ}$.

$-5<=\mathrm{h}<=8,-15<=\mathrm{k}<=15,-22<=1<=22$

20590

$3299[\mathrm{R}(\mathrm{int})=0.1619]$

$99.8 \%$

multi-scan

0.9951 and 0.9766

Full-matrix least-squares on $\mathrm{F}^{2}$

3299 / 0 / 262

0.899

$\mathrm{R} 1=0.0678, \mathrm{wR} 2=0.1504$

$\mathrm{R} 1=0.2001, \mathrm{wR} 2=0.2184$

$0(4)$

0.169 and -0.215 e. $\AA^{-3}$ 
c) 7a (CCDC no. 1845897): The thermal ellipsoid drawn at $60 \%$ probability level.

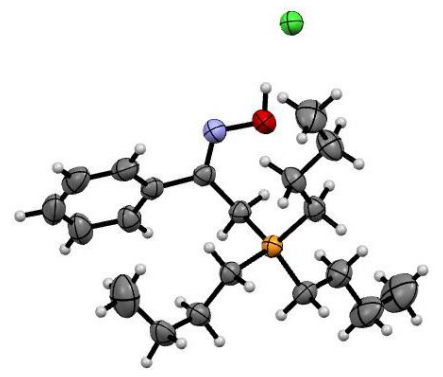

Empirical formula

Formula weight

Temperature

Wavelength

Crystal system

Space group

Unit cell dimensions

Volume

$\mathrm{Z}$

Density (calculated)

Absorption coefficient

$\mathrm{F}(000)$

Crystal size

Theta range for data collection

Index ranges

Reflections collected

Independent reflections

Completeness to theta $=25.03^{\circ}$

Absorption correction

Max. and min. transmission

Refinement method

Data / restraints / parameters

Goodness-of-fit on $\mathrm{F}^{2}$

Final R indices [I $>2 \operatorname{sigma(I)]~}$

$\mathrm{R}$ indices (all data)

Largest diff. peak and hole
$\mathrm{C}_{21} \mathrm{H}_{37} \mathrm{Cl}_{3} \mathrm{NOP}$

456.84

200(2) K

$0.71073 \AA$

Monoclinic

P 21/c

$\mathrm{a}=8.3324(6) \AA$

$\alpha=90^{\circ}$.

$\mathrm{b}=12.7398(9) \AA$

$\beta=98.883(2)^{\circ}$.

$\mathrm{c}=24.4859(18) \AA$

$\gamma=90^{\circ}$.
4

$1.182 \mathrm{Mg} / \mathrm{m}^{3}$

$0.430 \mathrm{~mm}^{-1}$

976

$0.79 \times 0.04 \times 0.02 \mathrm{~mm}^{3}$

2.32 to $25.03^{\circ}$.

$-9<=\mathrm{h}<=9,-15<=\mathrm{k}<=15,-28<=1<=29$

33790

$4515[\mathrm{R}(\mathrm{int})=0.0933]$

$99.7 \%$

multi-scan

0.9915 and 0.7275

Full-matrix least-squares on $\mathrm{F}^{2}$

4515 / 6 / 241

0.996

$\mathrm{R} 1=0.0551, \mathrm{wR} 2=0.1218$

$\mathrm{R} 1=0.0897, \mathrm{wR} 2=0.1417$

0.731 and -0.536 e. $\AA^{-3}$ 
d) 6da (CCDC no. 1839883): The thermal ellipsoid drawn at 60\% probability level.

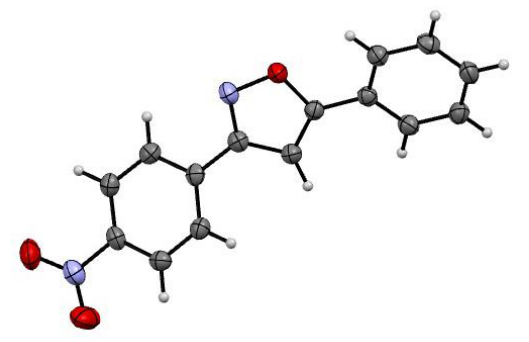

Empirical formula

Formula weight

Temperature

Wavelength

Crystal system

Space group

Unit cell dimensions

Volume

$\mathrm{Z}$

Density (calculated)

Absorption coefficient

$\mathrm{F}(000)$

Crystal size

Theta range for data collection

Index ranges

Reflections collected

Independent reflections

Completeness to theta $=25.03^{\circ}$

Absorption correction

Max. and min. transmission

Refinement method

Data / restraints / parameters

Goodness-of-fit on $\mathrm{F}^{2}$

Final $\mathrm{R}$ indices [I>2sigma(I)]

$\mathrm{R}$ indices (all data)

Extinction coefficient

Largest diff. peak and hole

$$
\mathrm{C}_{15} \mathrm{H}_{10} \mathrm{~N}_{2} \mathrm{O}_{3}
$$

266.25

200(2) K

$0.71073 \AA$

Triclinic

$\mathrm{P}-1$

$\mathrm{a}=5.7835(4) \AA \quad \alpha=100.099(2)^{\circ}$.

$\mathrm{b}=7.1214(5) \AA \quad \beta=100.309(2)^{\circ}$.

$\mathrm{c}=14.9191(10) \AA \quad \gamma=90.338(2)^{\circ}$.

594.74(7) $\AA^{3}$

2

$1.487 \mathrm{Mg} / \mathrm{m}^{3}$

$0.106 \mathrm{~mm}^{-1}$

276

$0.11 \times 0.07 \times 0.01 \mathrm{~mm}^{3}$

2.82 to $25.03^{\circ}$.

$-6<=\mathrm{h}<=6,-8<=\mathrm{k}<=8,-17<=\mathrm{l}<=17$

13673

$2089[\mathrm{R}(\mathrm{int})=0.0560]$

$99.7 \%$

multi-scan

0.9989 and 0.9884

Full-matrix least-squares on $\mathrm{F}^{2}$

2089 / 0 / 182

1.071

$\mathrm{R} 1=0.0412, \mathrm{wR} 2=0.0927$

$\mathrm{R} 1=0.0647, \mathrm{wR} 2=0.1062$

$0.058(6)$

0.223 and -0.168 e. $\AA^{-3}$ 
e) 6ad (CCDC no. 1588093): The thermal ellipsoid drawn at $60 \%$ probability level.

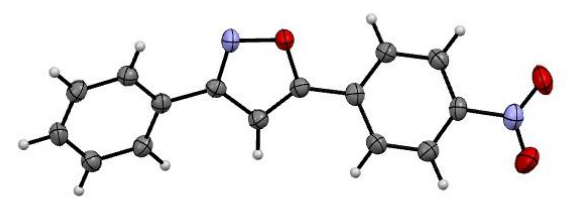

Empirical formula

Formula weight

Temperature

Wavelength

Crystal system

Space group

Unit cell dimensions

Volume

$\mathrm{Z}$

Density (calculated)

Absorption coefficient

$\mathrm{F}(000)$

Crystal size

Theta range for data collection

Index ranges

Reflections collected

Independent reflections

Completeness to theta $=25.05^{\circ}$

Absorption correction

Max. and min. transmission

Refinement method

Data / restraints / parameters

Goodness-of-fit on $\mathrm{F}^{2}$

Final $\mathrm{R}$ indices [I>2sigma(I)]

$\mathrm{R}$ indices (all data)

Extinction coefficient

Largest diff. peak and hole
$\mathrm{C}_{15} \mathrm{H}_{10} \mathrm{~N}_{2} \mathrm{O}_{3}$

266.25

200(2) K

$0.71073 \AA$

Triclinic

P -1

$\mathrm{a}=5.7891(4) \AA \quad \alpha=100.152(3)^{\circ}$.

$\mathrm{b}=7.1100(6) \AA \quad \beta=98.481(2)^{\circ}$.

$\mathrm{c}=14.8092(12) \AA \quad \gamma=90.527(3)^{\circ}$.

593.06(8) $\AA^{3}$

2

$1.491 \mathrm{Mg} / \mathrm{m}^{3}$

$0.106 \mathrm{~mm}^{-1}$

276

$0.40 \times 0.13 \times 0.01 \mathrm{~mm}^{3}$

2.83 to $25.05^{\circ}$.

$-6<=\mathrm{h}<=6,-8<=\mathrm{k}<=8,-17<=1<=17$

11027

$2088[\mathrm{R}(\mathrm{int})=0.0641]$

$99.3 \%$

multi-scan

0.9989 and 0.9587

Full-matrix least-squares on $\mathrm{F}^{2}$

2088 / 0 / 182

1.038

$\mathrm{R} 1=0.0442, \mathrm{wR} 2=0.1069$

$\mathrm{R} 1=0.0658, \mathrm{wR} 2=0.1209$

0.072(11)

0.214 and -0.196 e. $\AA^{-3}$ 
f) 12aa (CCDC no. 1893512): The thermal ellipsoid drawn at $60 \%$ probability level.

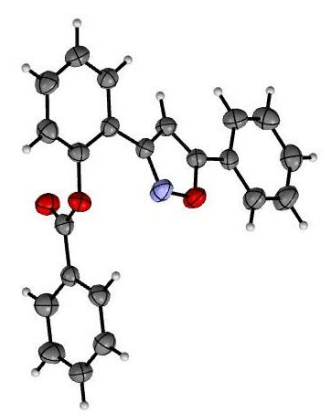

Empirical formula

Formula weight

Temperature

Wavelength

Crystal system

Space group

Unit cell dimensions

Volume

Z

Density (calculated)

Absorption coefficient

$\mathrm{F}(000)$

Crystal size

Theta range for data collection

Index ranges

Reflections collected

Independent reflections

Completeness to theta $=25.04^{\circ}$

Absorption correction

Max. and min. transmission

Refinement method

Data / restraints / parameters

Goodness-of-fit on $\mathrm{F}^{2}$

Final $\mathrm{R}$ indices [I $>2 \operatorname{sigma}(\mathrm{I})]$

$\mathrm{R}$ indices (all data)

Largest diff. peak and hole
$\mathrm{C}_{22} \mathrm{H}_{15} \mathrm{NO}_{3}$

341.35

200(2) K

$0.71073 \AA$

Orthorhombic

$\mathrm{Pbc} \mathrm{a}$

$\mathrm{a}=7.7573(10) \AA \quad \alpha=90^{\circ}$.

$\mathrm{b}=20.131(4) \AA \quad \beta=90^{\circ}$.

$\mathrm{c}=22.010(2) \AA \quad \gamma=90^{\circ}$.

3437.2(8) $\AA^{3}$

8

$1.319 \mathrm{Mg} / \mathrm{m}^{3}$

$0.088 \mathrm{~mm}^{-1}$

1424

$0.69 \times 0.05 \times 0.02 \mathrm{~mm}^{3}$

2.22 to $25.04^{\circ}$.

$-9<=\mathrm{h}<=8,-23<=\mathrm{k}<=23,-25<=1<=26$

20563

$3027[\mathrm{R}($ int $)=0.0777]$

$99.7 \%$

multi-scan

0.9982 and 0.9416

Full-matrix least-squares on $\mathrm{F}^{2}$

3027 / 0 / 235

1.107

$\mathrm{R} 1=0.0458, \mathrm{wR} 2=0.1018$

$\mathrm{R} 1=0.0934, \mathrm{wR} 2=0.1289$

0.189 and -0.211 e. $\AA^{-3}$ 
g) 11aa (CCDC no. 1882452): The thermal ellipsoid drawn at $60 \%$ probability level.

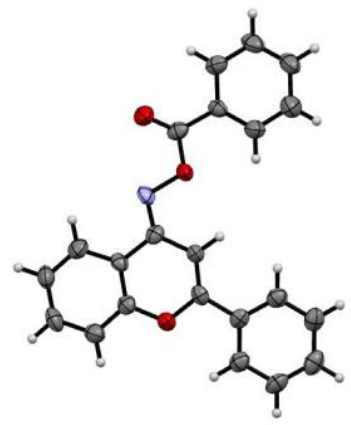

Empirical formula

Formula weight

Temperature

Wavelength

Crystal system

Space group

Unit cell dimensions

Volume

Z

Density (calculated)

Absorption coefficient

$\mathrm{F}(000)$

Crystal size

Theta range for data collection

Index ranges

Reflections collected

Independent reflections

Completeness to theta $=25.01^{\circ}$

Max. and min. transmission

Refinement method

Data / restraints / parameters

Goodness-of-fit on $\mathrm{F}^{2}$

Final $\mathrm{R}$ indices [I $>2 \operatorname{sigma}(\mathrm{I})]$

$\mathrm{R}$ indices (all data)

Absolute structure parameter

Largest diff. peak and hole
$\mathrm{C}_{22} \mathrm{H}_{15} \mathrm{NO}_{3}$

341.35

200(2) K

$0.71073 \AA$

Orthorhombic

P 212121

$\mathrm{a}=5.4097(13) \AA \quad \alpha=90^{\circ}$.

$\mathrm{b}=14.635(6) \AA \quad \beta=90^{\circ}$.

$\mathrm{c}=21.092(6) \AA \quad \gamma=90^{\circ}$.

1669.8(9) $\AA^{3}$

4

$1.358 \mathrm{Mg} / \mathrm{m}^{3}$

$0.091 \mathrm{~mm}^{-1}$

712

$0.08 \times 0.05 \times 0.01 \mathrm{~mm}^{3}$

2.38 to $25.01^{\circ}$.

$-6<=\mathrm{h}<=6,-17<=\mathrm{k}<=15,-23<=\mathrm{l}<=25$

9859

$2930[\mathrm{R}(\mathrm{int})=0.0892]$

99.4 \% Absorption correction multi-scan

0.9991 and 0.9928

Full-matrix least-squares on $\mathrm{F}^{2}$

$2930 / 0 / 235$

1.031

$\mathrm{R} 1=0.0571, \mathrm{wR} 2=0.0956$

$\mathrm{R} 1=0.1105, \mathrm{wR} 2=0.1128$

$-1(2)$

0.176 and -0.189 e. $\AA^{-3}$ 
h) 12ac (CCDC no. 1893511): The thermal ellipsoid drawn at $60 \%$ probability level.

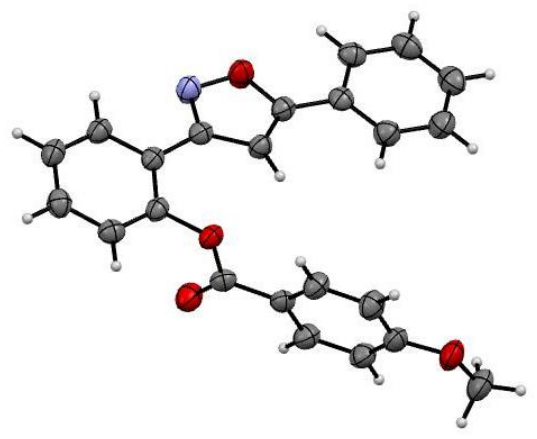

Empirical formula

Formula weight

Temperature

Wavelength

Crystal system

Space group

Unit cell dimensions

Volume

Z

Density (calculated)

Absorption coefficient

$\mathrm{F}(000)$

Crystal size

Theta range for data collection

Index ranges

Reflections collected

Independent reflections

Completeness to theta $=25.07^{\circ}$

Absorption correction

Max. and min. transmission

Refinement method

Data / restraints / parameters

Goodness-of-fit on $\mathrm{F}^{2}$

Final $\mathrm{R}$ indices [I $>2 \operatorname{sigma}(\mathrm{I})]$

$\mathrm{R}$ indices (all data)

Largest diff. peak and hole
$\mathrm{C}_{23} \mathrm{H}_{17} \mathrm{NO}_{4}$

371.38

200(2) K

$0.71073 \AA$

Monoclinic

P 21/c

$\mathrm{a}=17.7987(9) \AA$

$\alpha=90^{\circ}$.

$\mathrm{b}=5.2502(2) \AA$

$\beta=109.8060(10)^{\circ}$.

$\mathrm{c}=20.3996(11) \AA$

$\gamma=90^{\circ}$.

1793.51(15) $\AA^{3}$

4

$1.375 \mathrm{Mg} / \mathrm{m}^{3}$

$0.095 \mathrm{~mm}^{-1}$

776

$0.69 \times 0.25 \times 0.14 \mathrm{~mm}^{3}$

2.43 to $25.07^{\circ}$.

$-21<=\mathrm{h}<=21,-6<=\mathrm{k}<=6,-24<=1<=24$

24664

$3163[\mathrm{R}(\mathrm{int})=0.0619]$

$99.3 \%$

multi-scan

0.9869 and 0.9375

Full-matrix least-squares on $\mathrm{F}^{2}$

3163 / 0 / 254

1.048

$\mathrm{R} 1=0.0388, \mathrm{wR} 2=0.0844$

$\mathrm{R} 1=0.0549, \mathrm{wR} 2=0.0941$

0.145 and -0.204 e. $\AA^{-3}$ 
i) 11ac (CCDC no. 1904919): The thermal ellipsoid drawn at 60\% probability level.

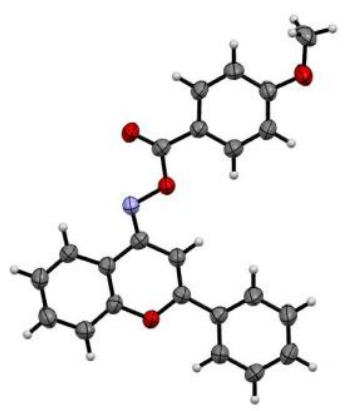

Empirical formula

Formula weight

Temperature

Wavelength

Crystal system

Space group

Unit cell dimensions

Volume

Z

Density (calculated)

Absorption coefficient

$\mathrm{F}(000)$

Crystal size

Theta range for data collection

Index ranges

Reflections collected

Independent reflections

Completeness to theta $=25.13^{\circ}$

Absorption correction

Max. and min. transmission

Refinement method

Data / restraints / parameters

Goodness-of-fit on $\mathrm{F}^{2}$

Final $\mathrm{R}$ indices [I $>2 \operatorname{sigma}(\mathrm{I})]$

$\mathrm{R}$ indices (all data)

Largest diff. peak and hole
$\mathrm{C}_{23} \mathrm{H}_{17} \mathrm{NO}_{4}$

371.38

210(2) K

$0.71073 \AA$

Triclinic

$\mathrm{P}-1$

$\mathrm{a}=5.8475(4) \AA$ $\alpha=87.674(3)^{\circ}$.

$\mathrm{b}=9.2693(7) \AA$ $\beta=80.955(2)^{\circ}$.

$c=16.6177(15) \AA$ $\gamma=85.745(2)^{\circ}$.
2

$1.391 \mathrm{Mg} / \mathrm{m}^{3}$

$0.096 \mathrm{~mm}^{-1}$

388

$0.70 \times 0.08 \times 0.02 \mathrm{~mm}^{3}$

2.48 to $25.13^{\circ}$.

$-6<=\mathrm{h}<=6,-11<=\mathrm{k}<=11,-19<=\mathrm{l}<=19$

22615

$3144[\mathrm{R}($ int $)=0.0799]$

$99.6 \%$

multi-scan

0.9981 and 0.9360

Full-matrix least-squares on $\mathrm{F}^{2}$

3144 / 0 / 254

1.004

$\mathrm{R} 1=0.0478, \mathrm{wR} 2=0.1195$

$\mathrm{R} 1=0.0817, \mathrm{wR} 2=0.1485$

0.244 and -0.192 e. $\AA^{-3}$ 


\section{VII. ${ }^{1} \mathrm{H}$ NMR and ${ }^{13} \mathrm{C}$ NMR spectra of new compounds:}
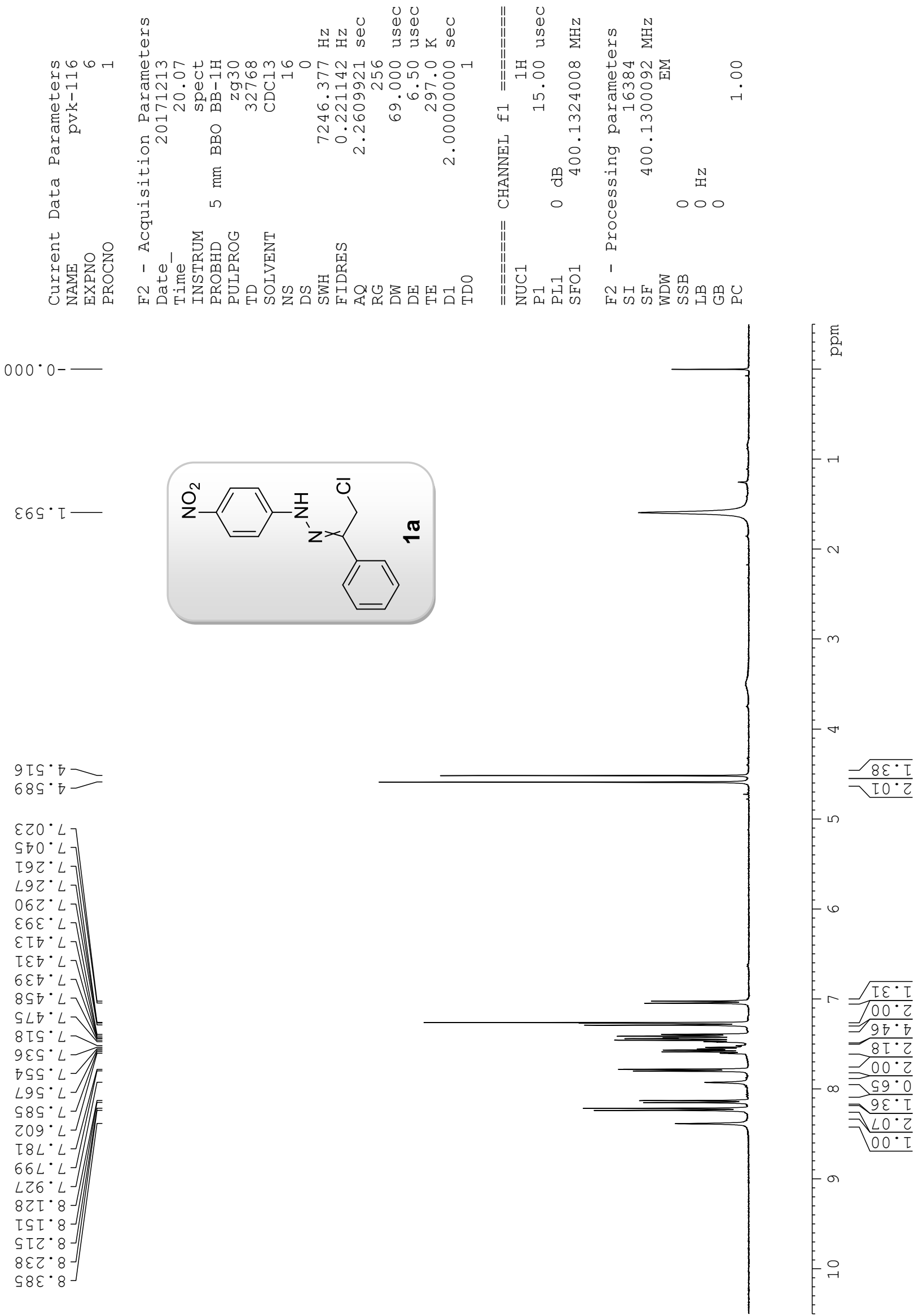

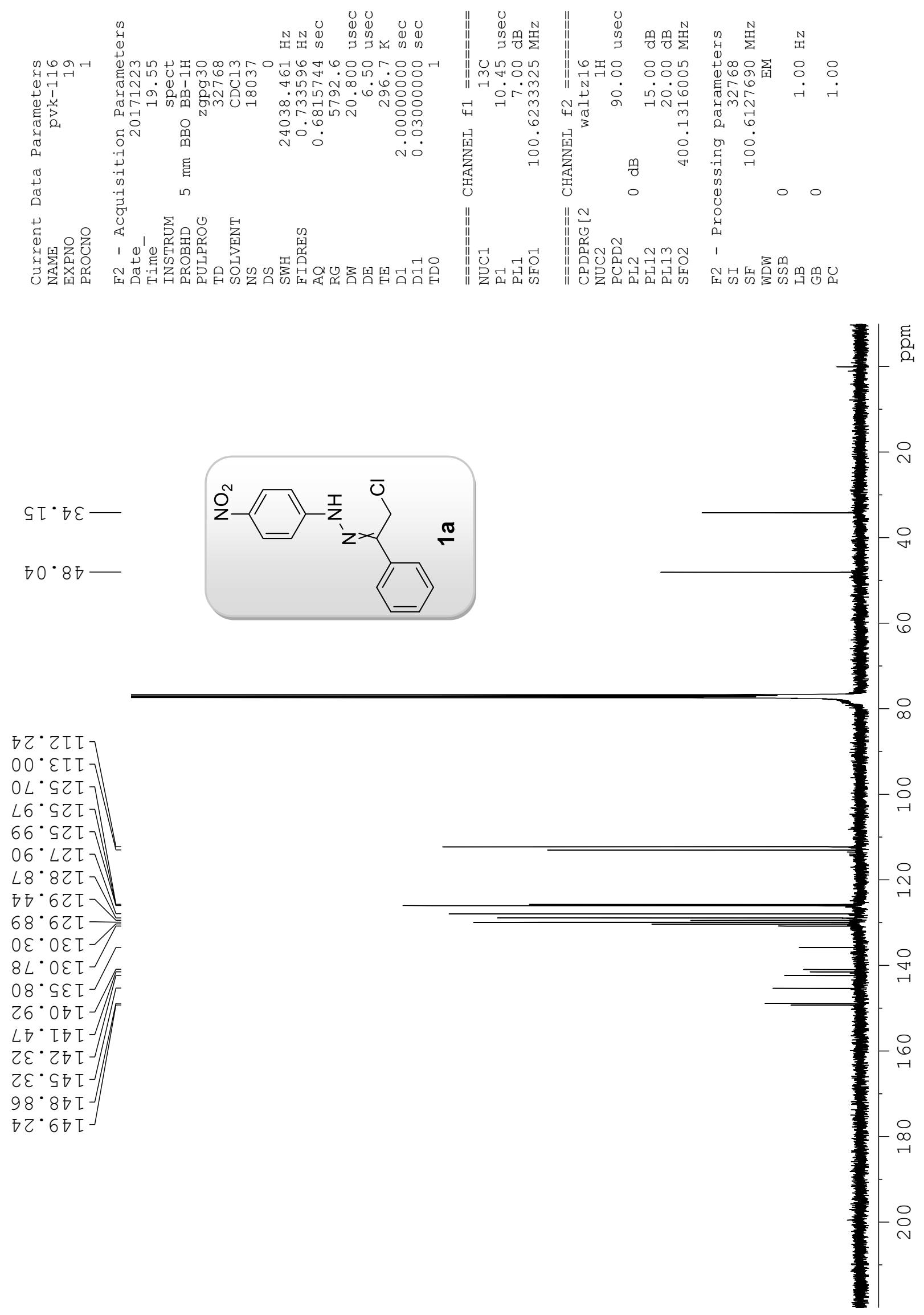


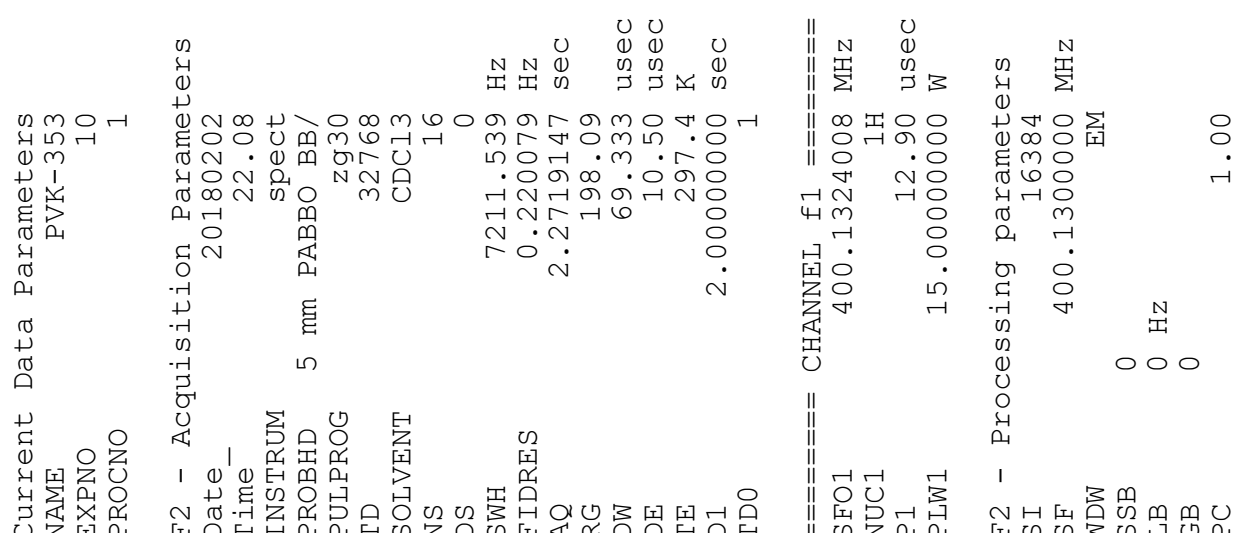

$20 \cdot 0$
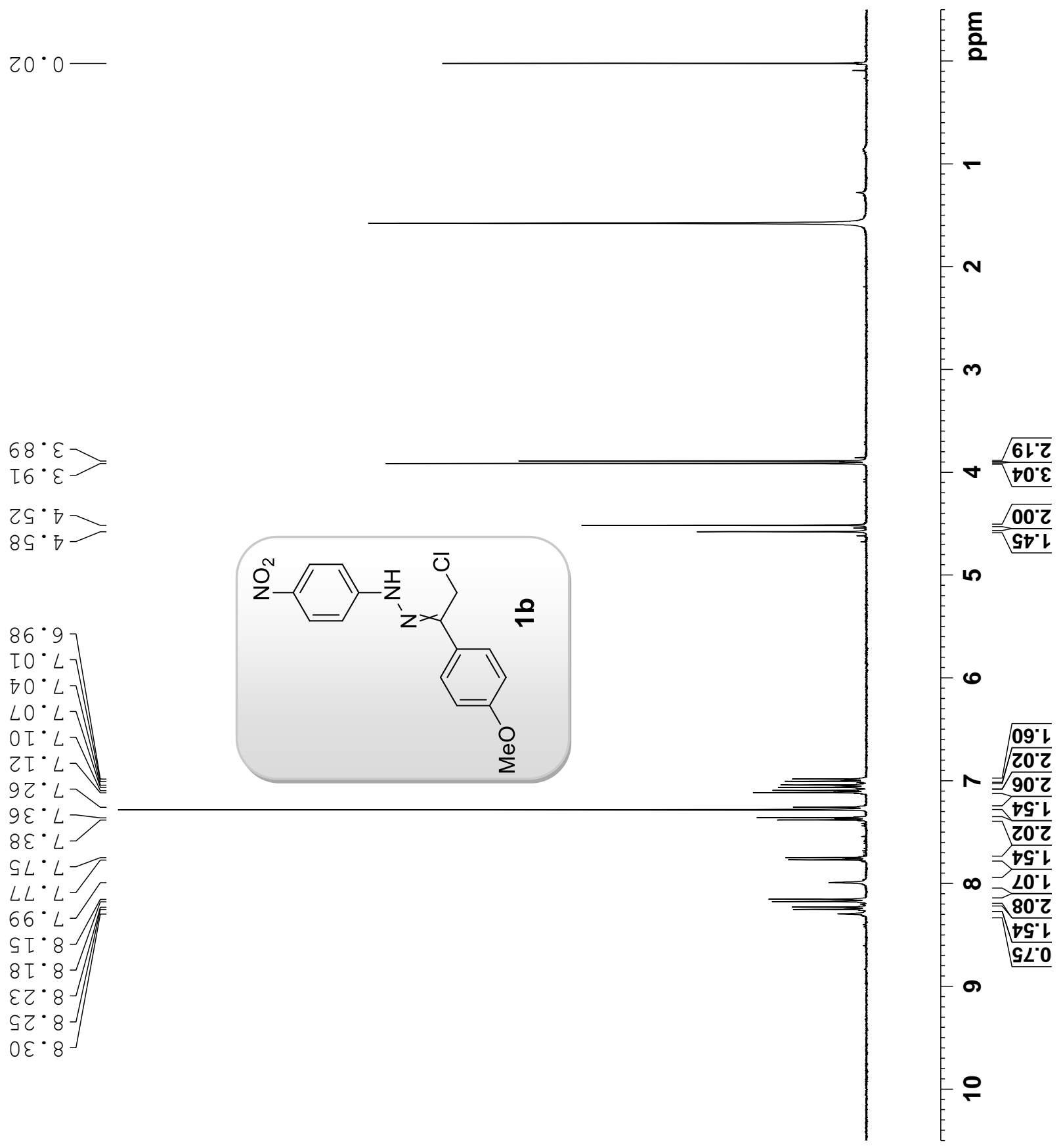

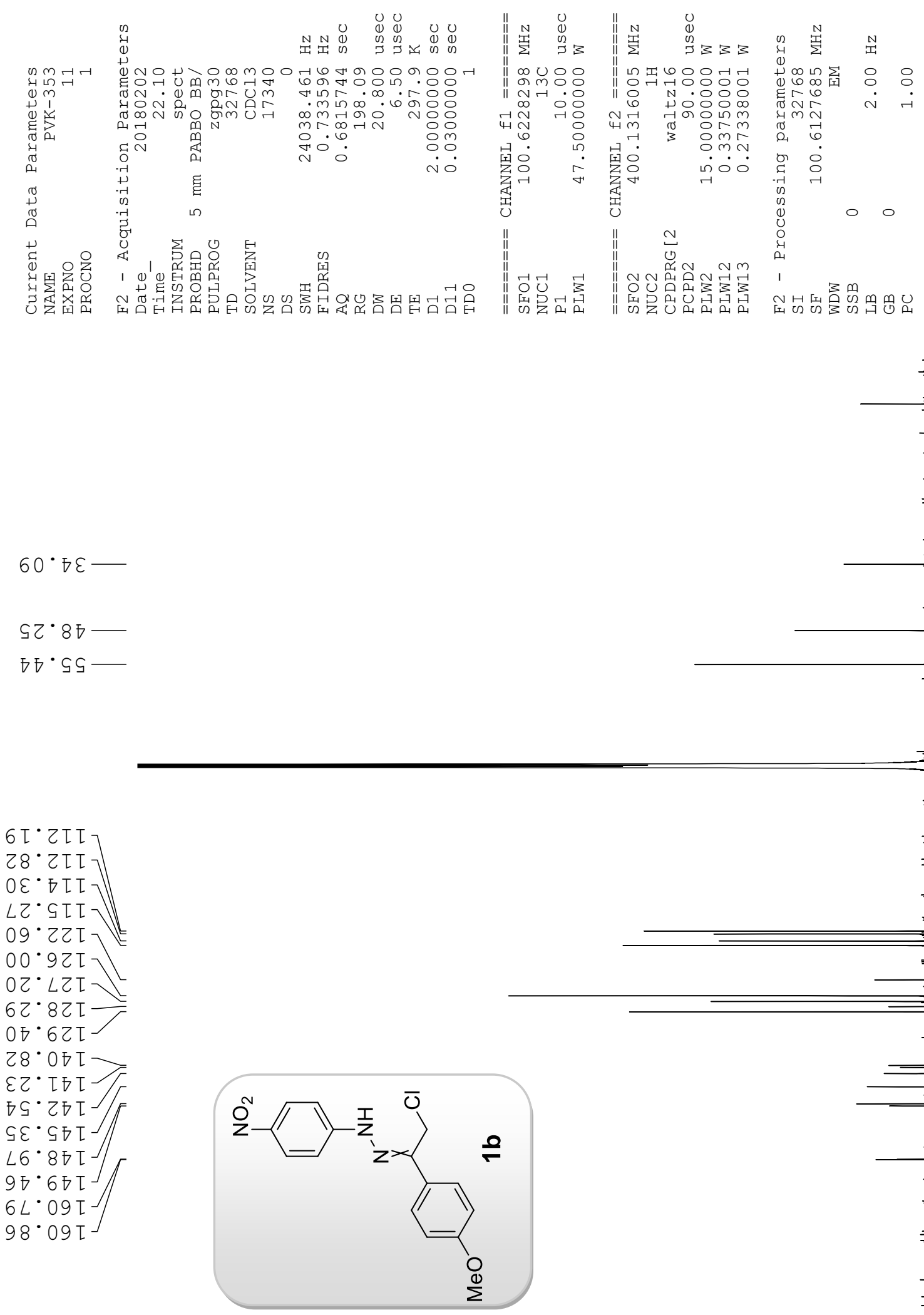


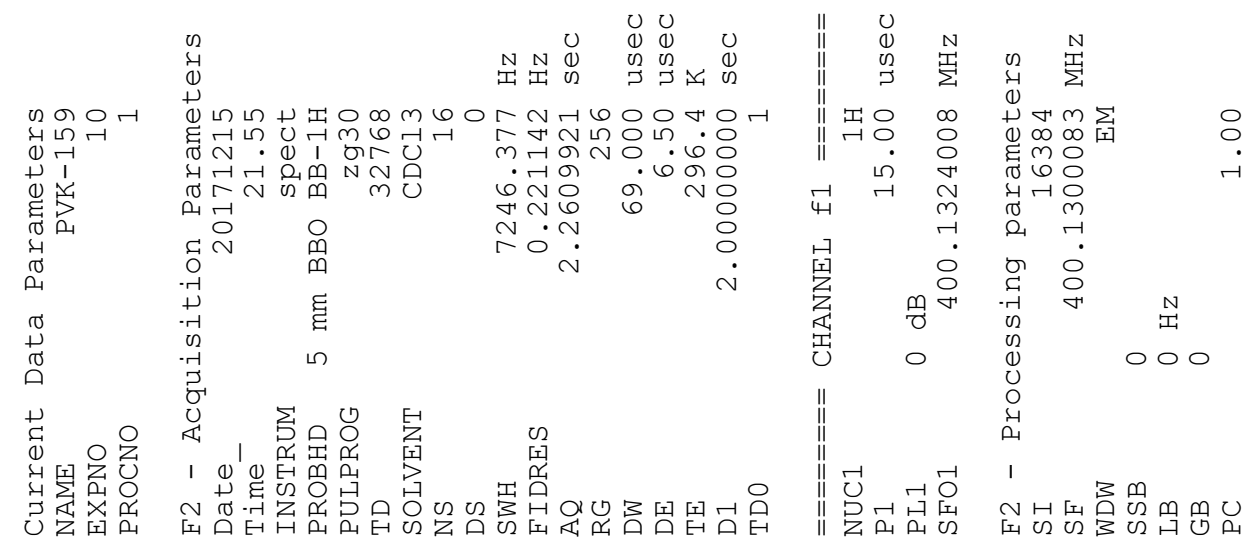

$000 \cdot 0$

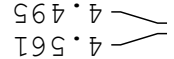
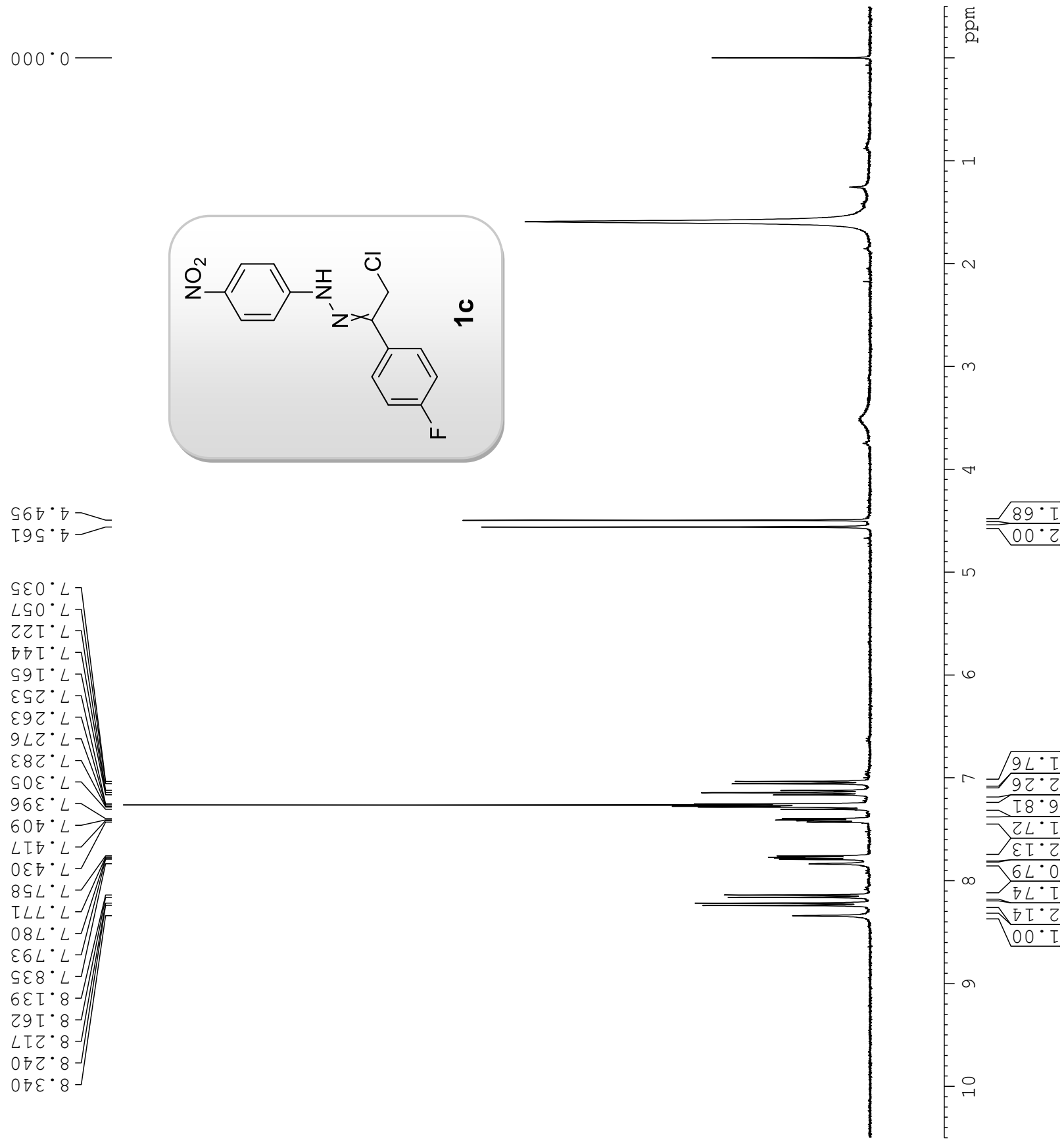

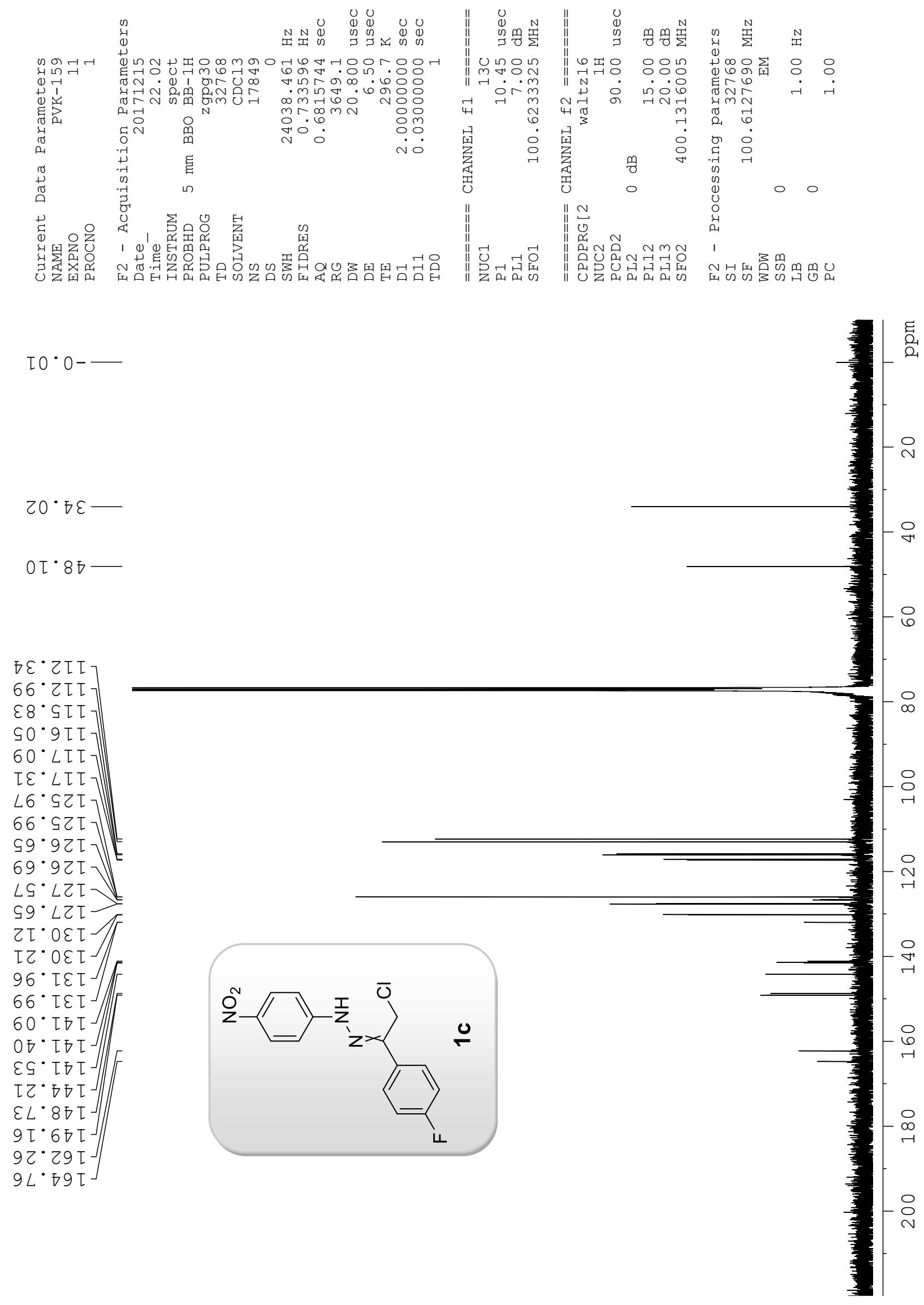

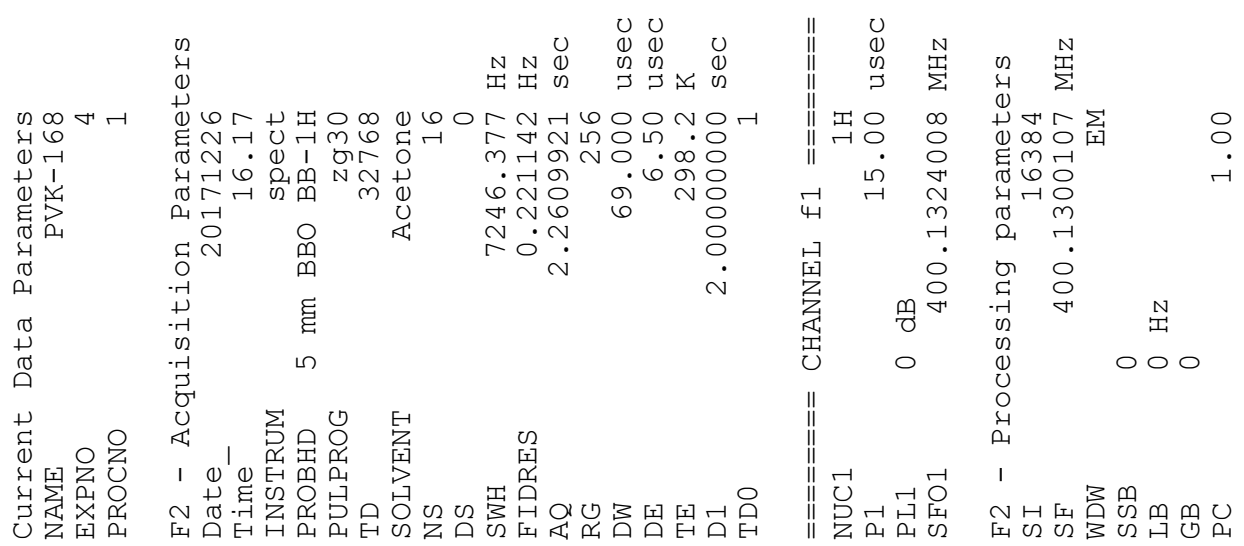

$\left.\begin{array}{l}s \varepsilon 0^{\circ} z \\ 070 \cdot r \\ 970 \cdot z\end{array}\right\rangle$

8ย $8^{\circ}$ 万—
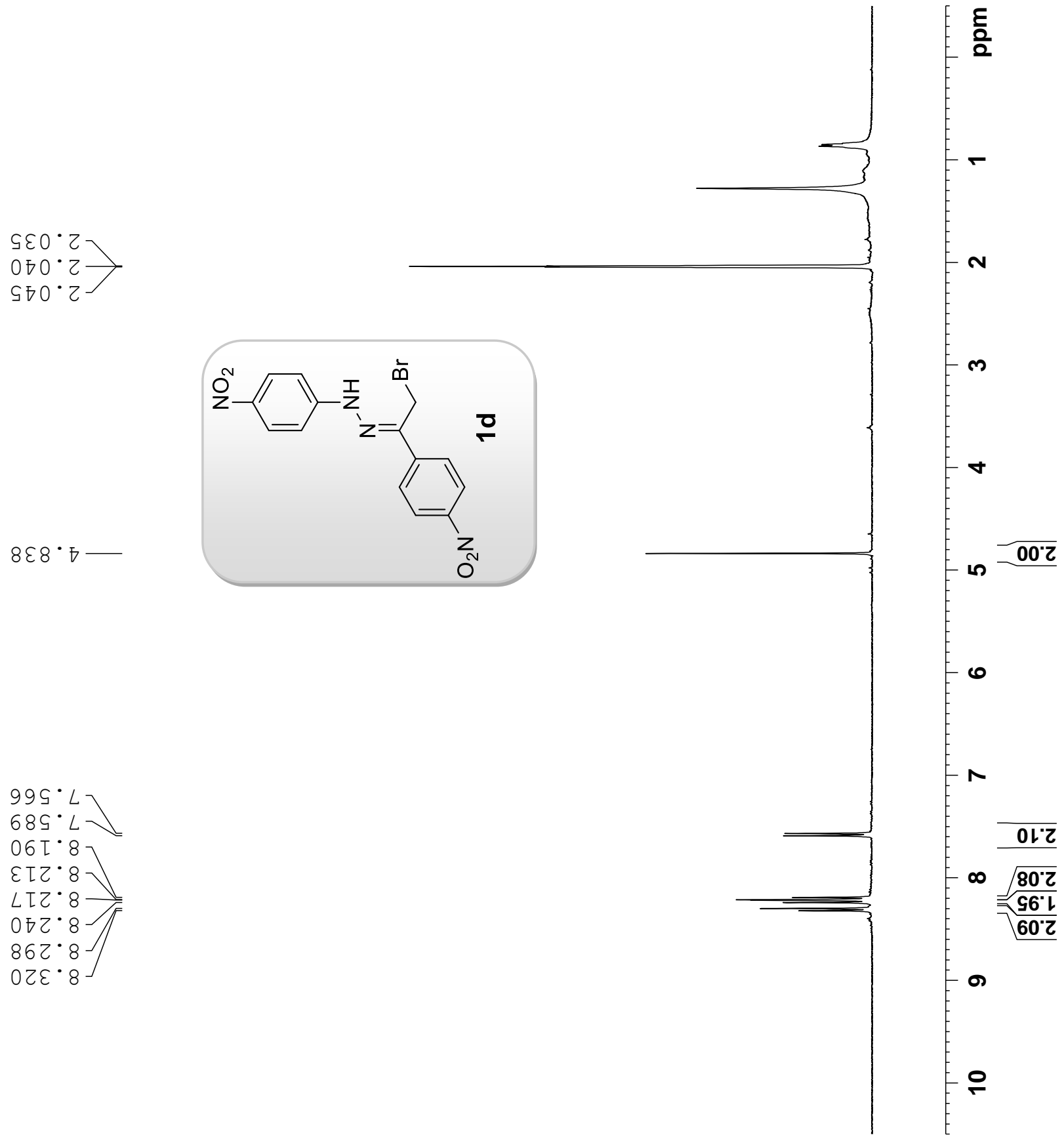


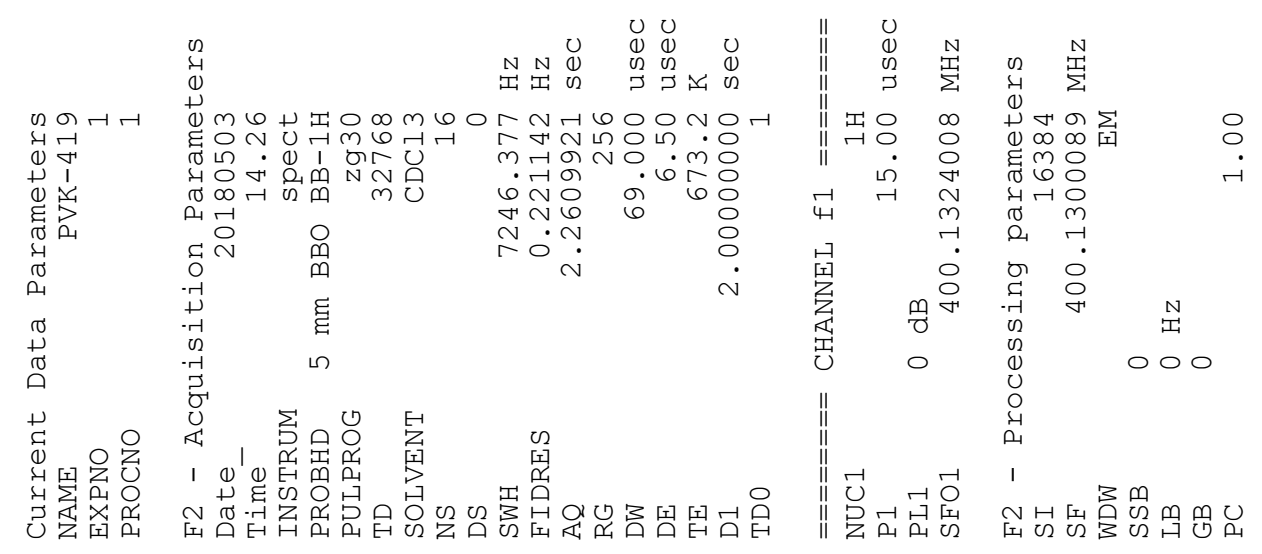

$00 \cdot 0$

so $2-$

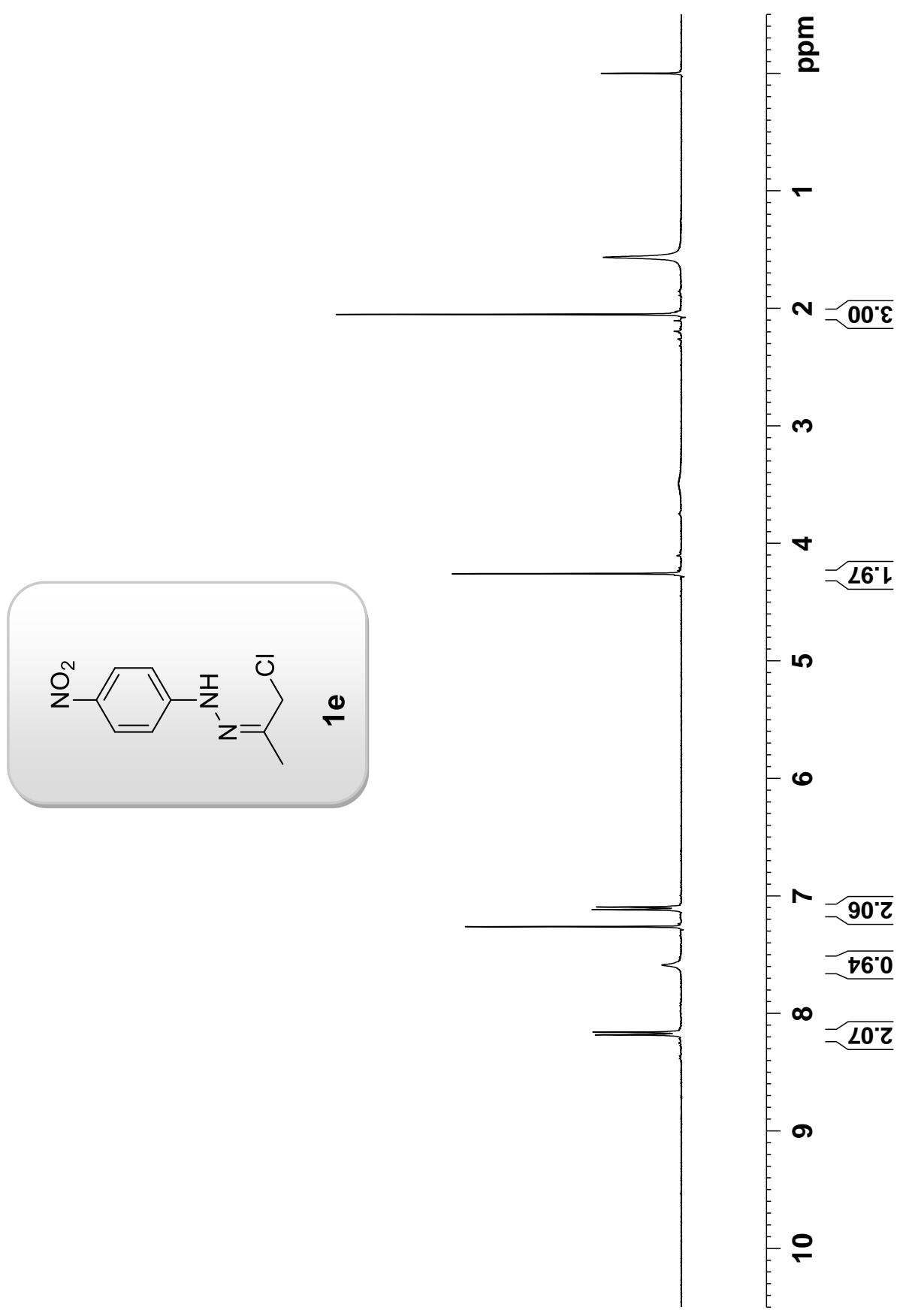

$92 \cdot \nabla \div$

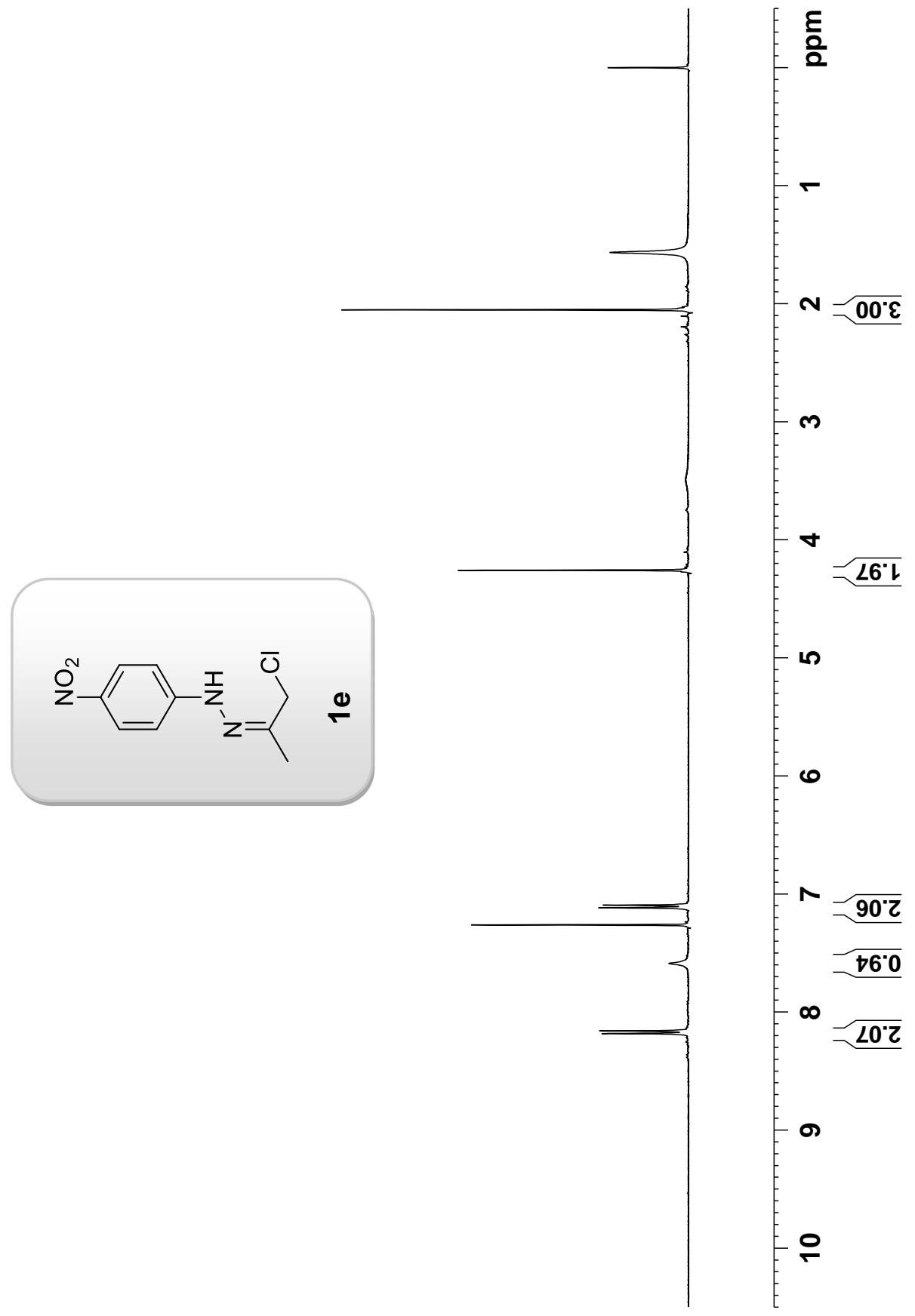

$60^{\circ} L$

$Z I \cdot L$

$92^{\circ} L$

$6 S^{\circ} L$

$9 \tau \cdot 8$

$8 I \cdot 8>$ 

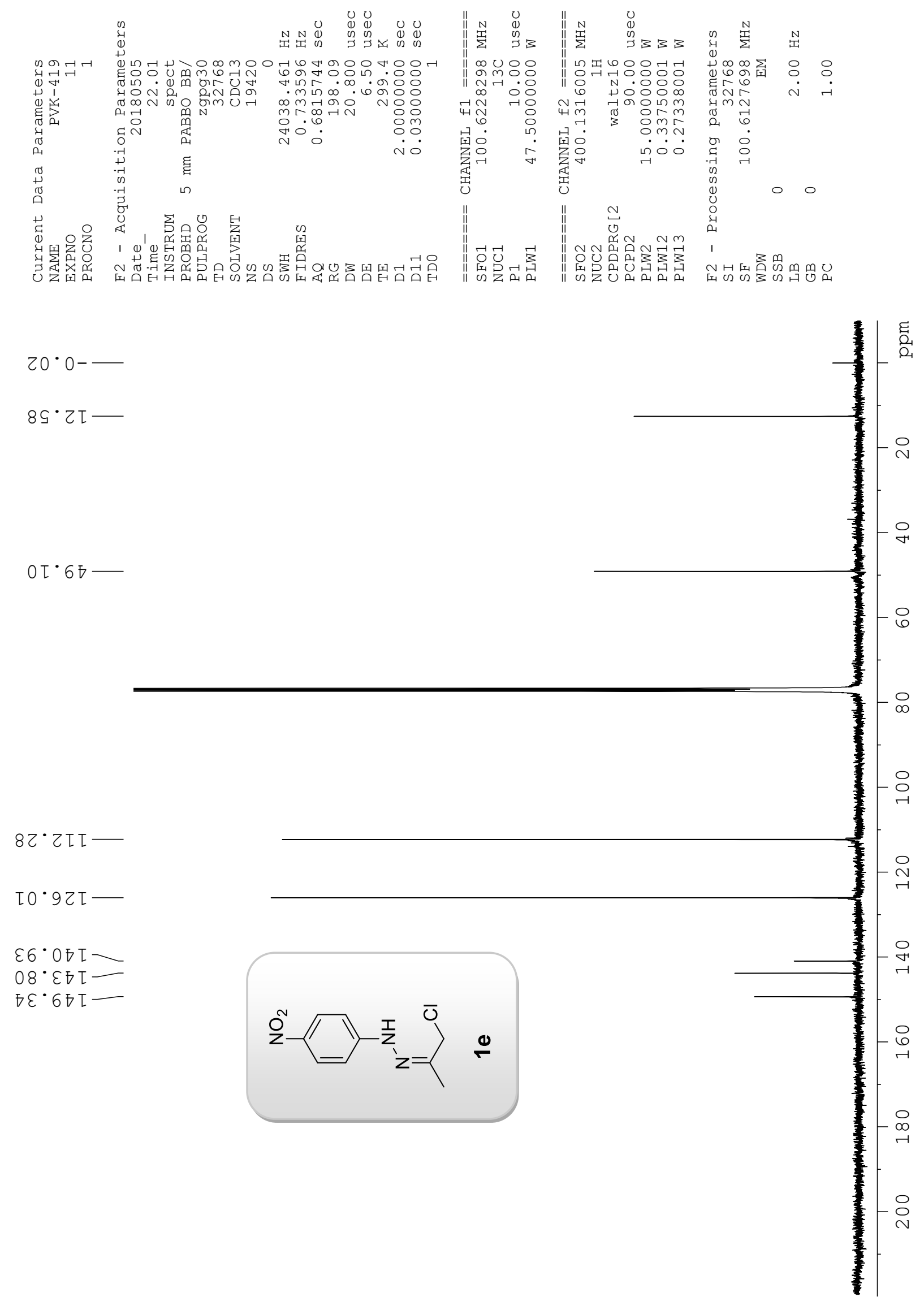


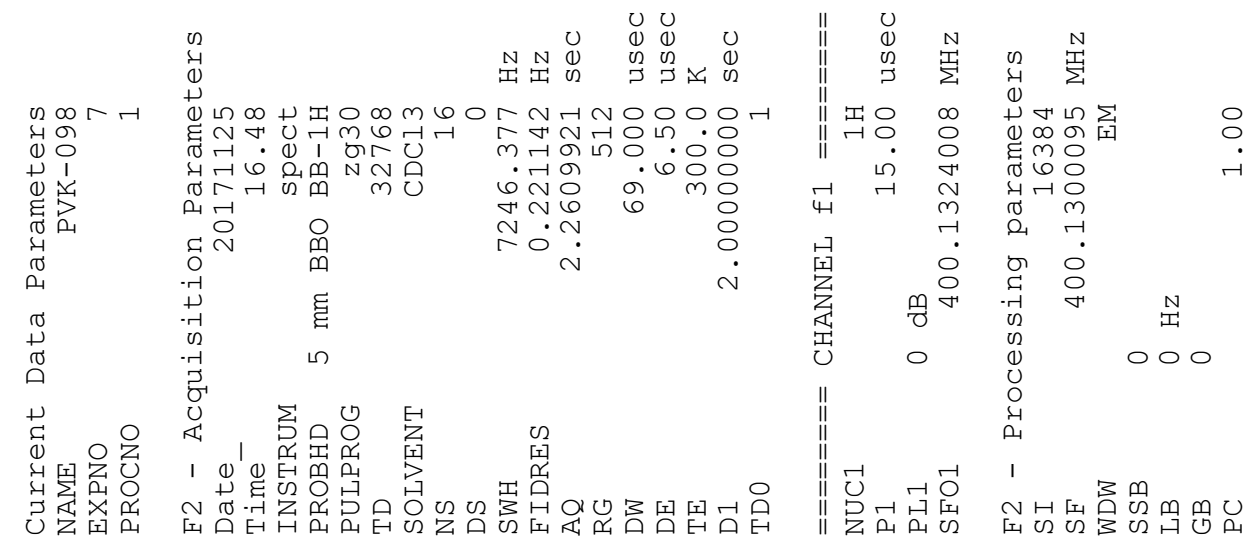

$000 \cdot 0$

$8 \varepsilon 9^{\circ}$ 万—

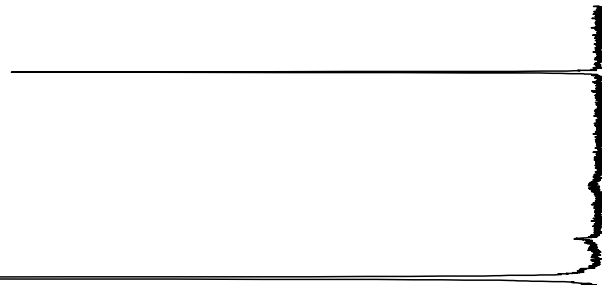

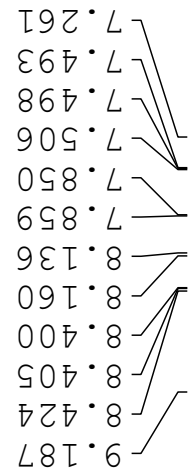

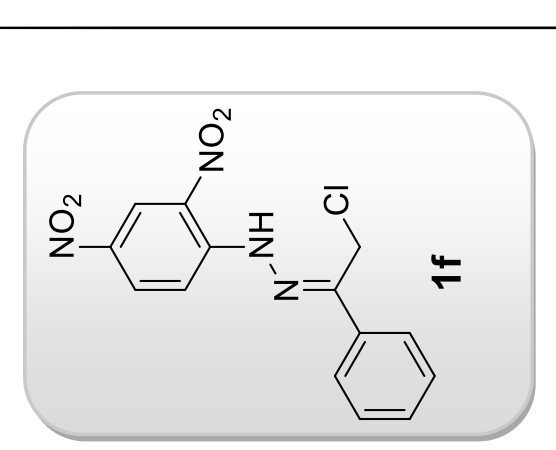

$\varepsilon 69^{\circ} \mathrm{II}$

$N$

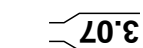

$\infty \longdiv { 0 0 ^ { \circ } z }$

$\frac{96^{\circ} 0}{\angle 6^{\circ} 0}$

$\sigma$

$-16^{\circ} 0$

우

$F$

$\simeq 00 \%$ 

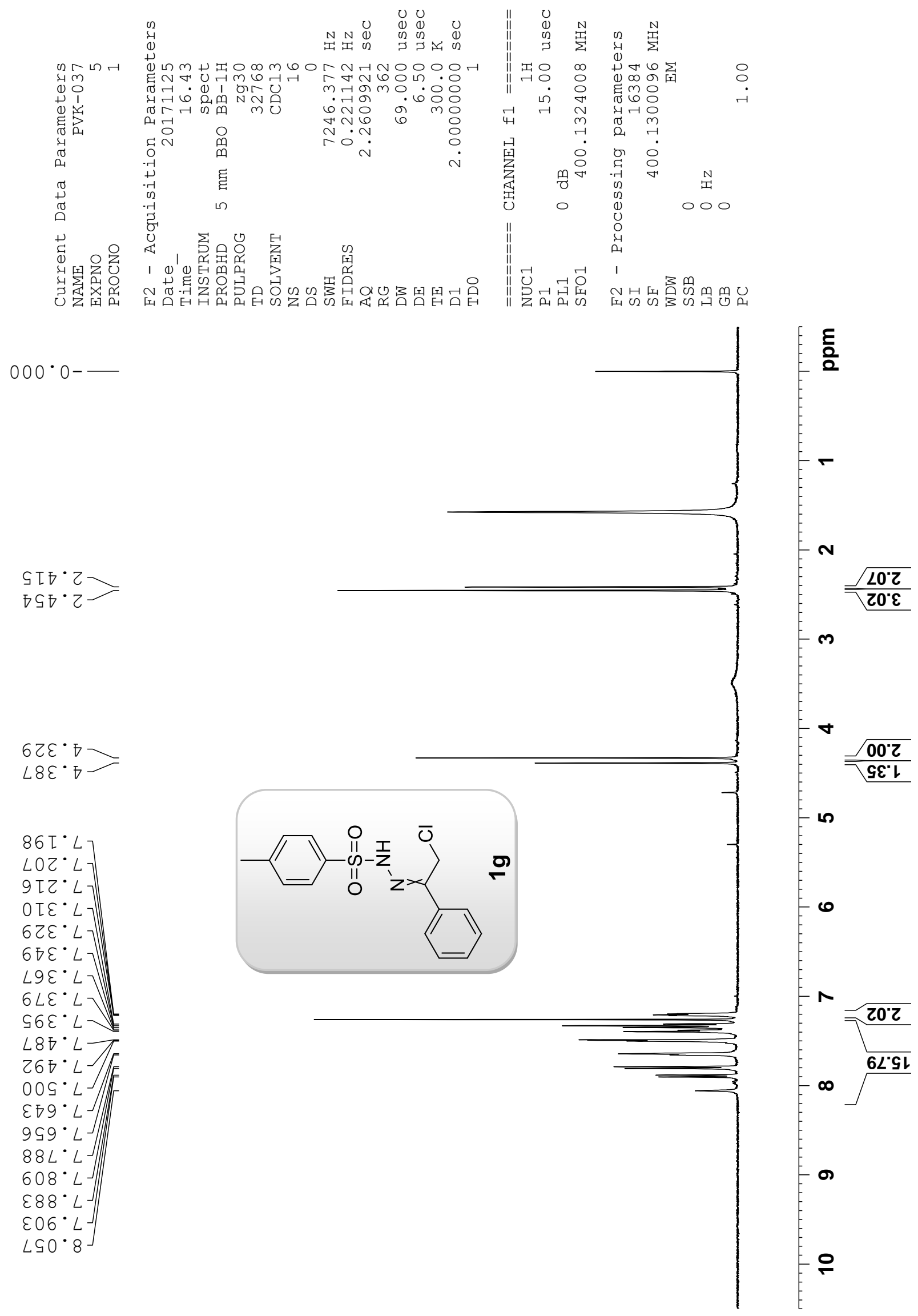


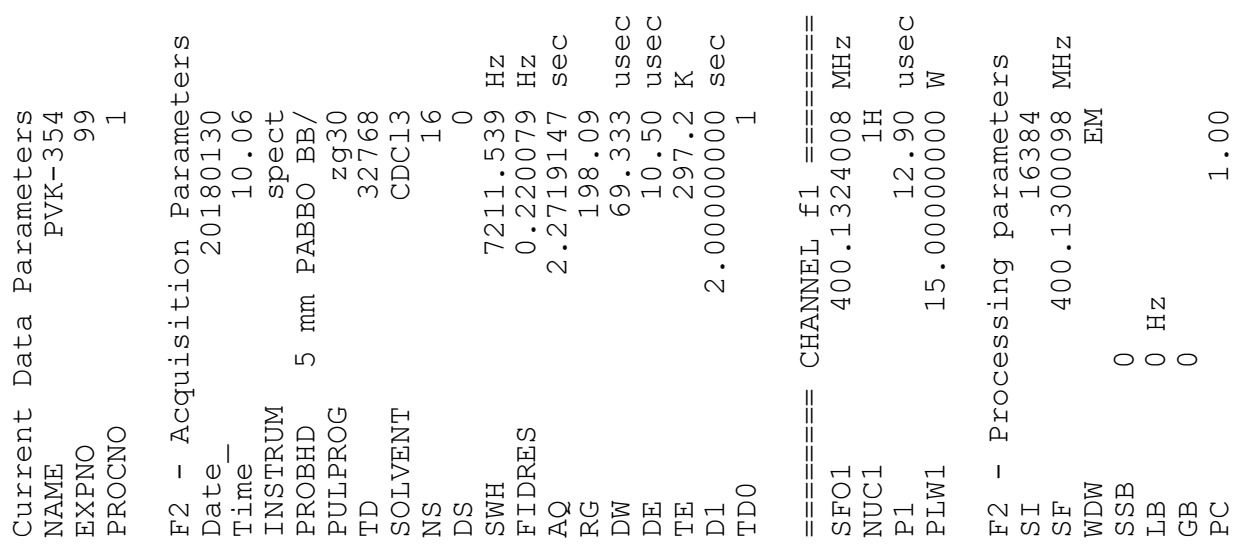

$00 \cdot 0-$

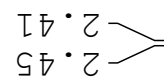

$\varepsilon 8^{\circ} \varepsilon>$

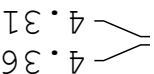
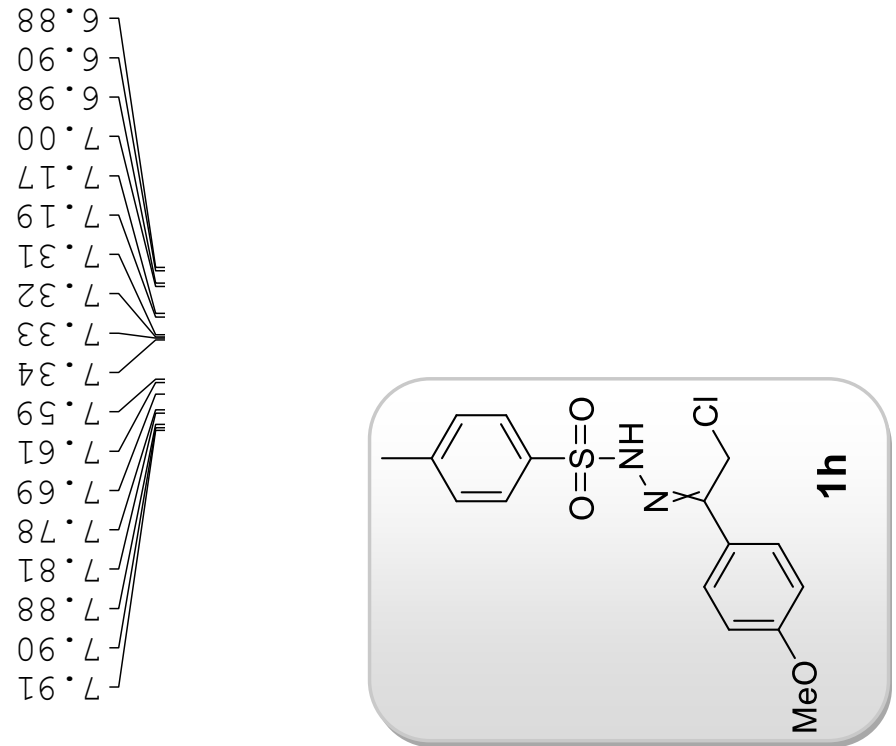

틍

(n)

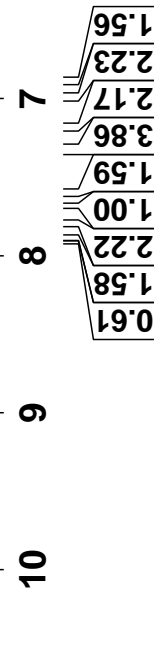



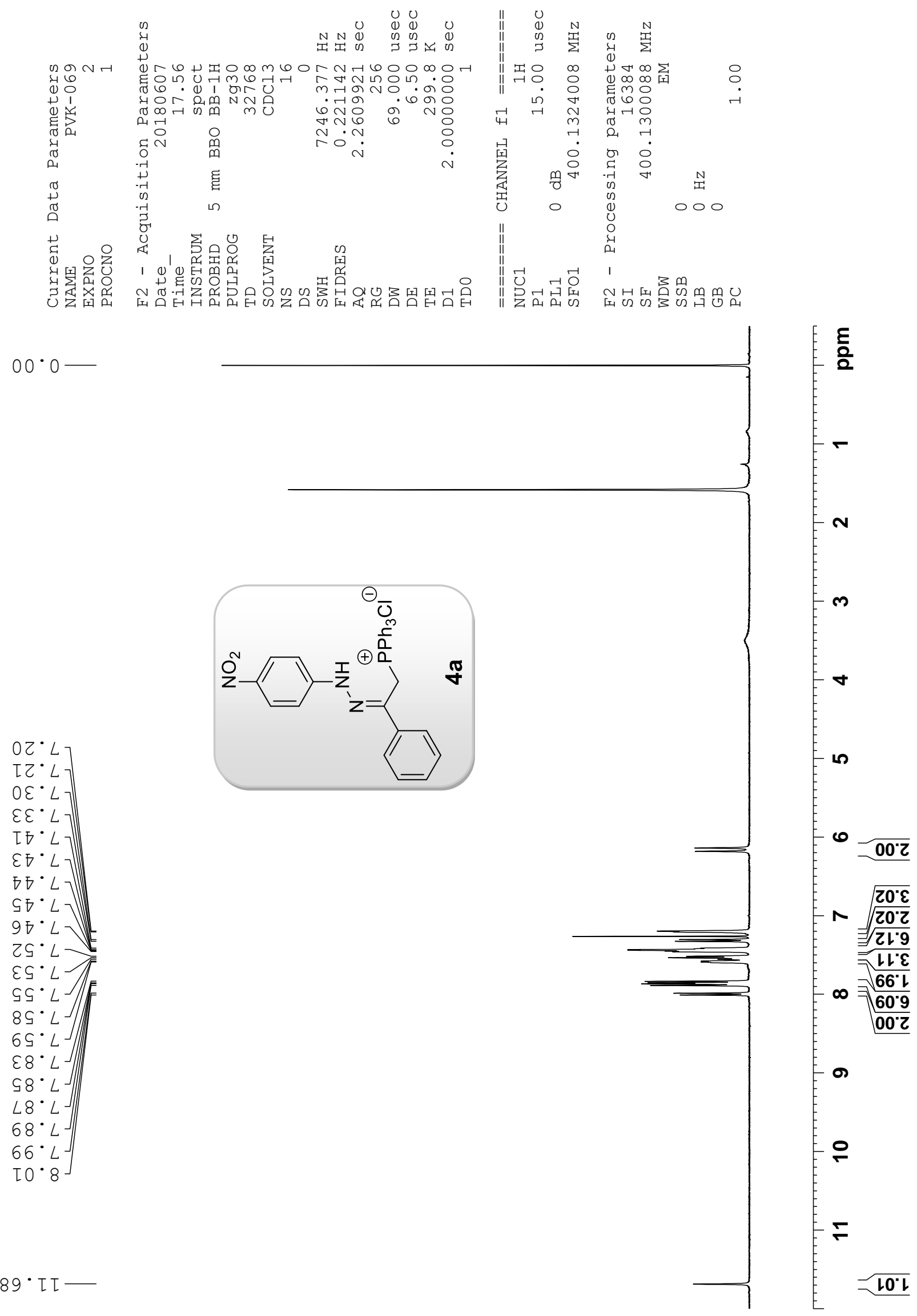

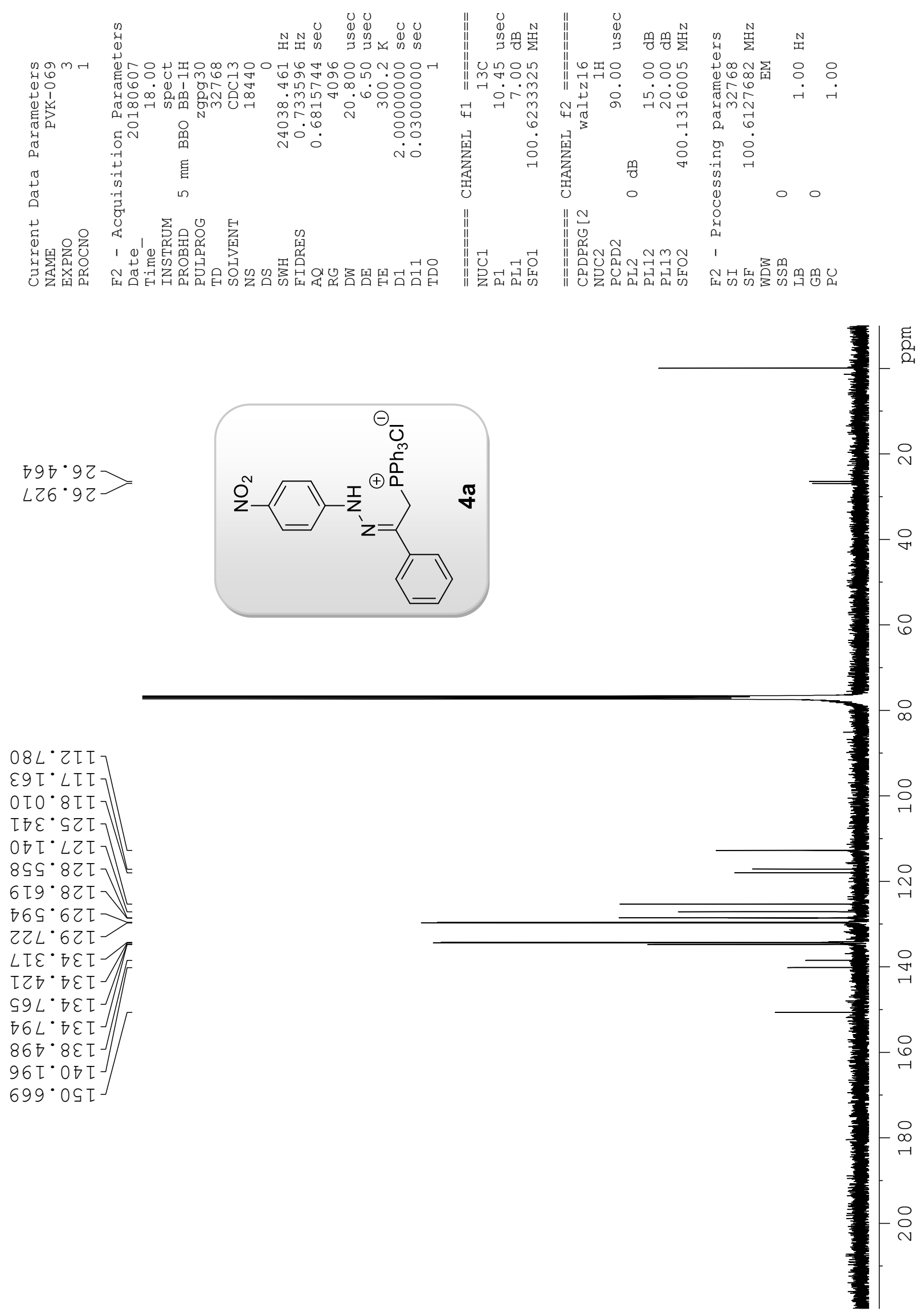


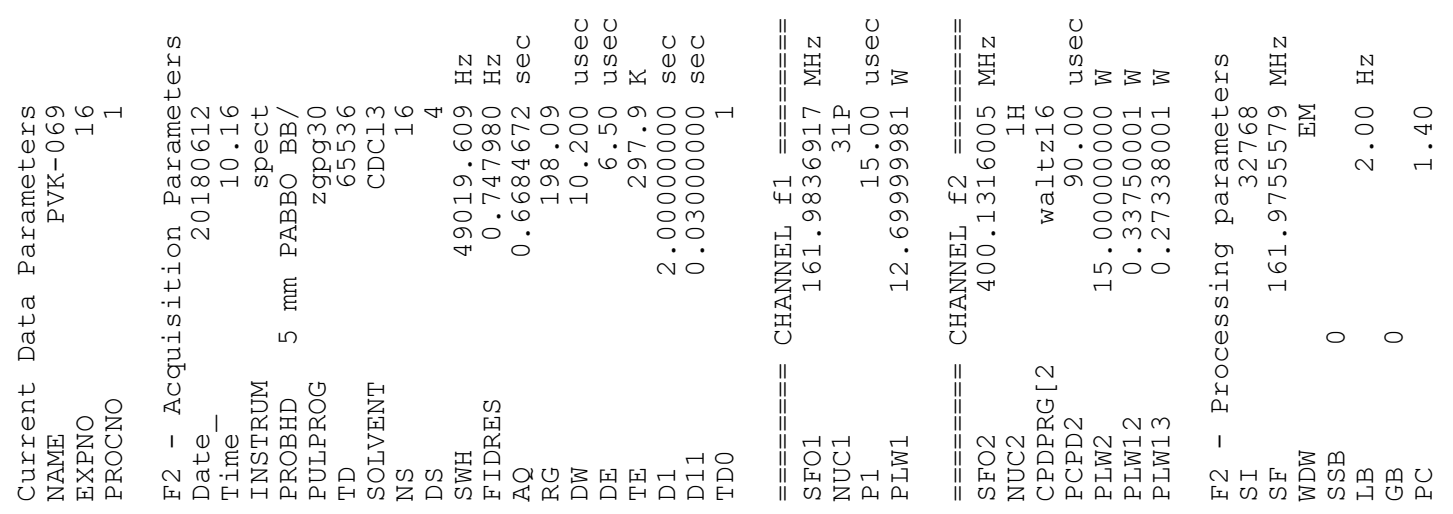

$500 \cdot 0-$
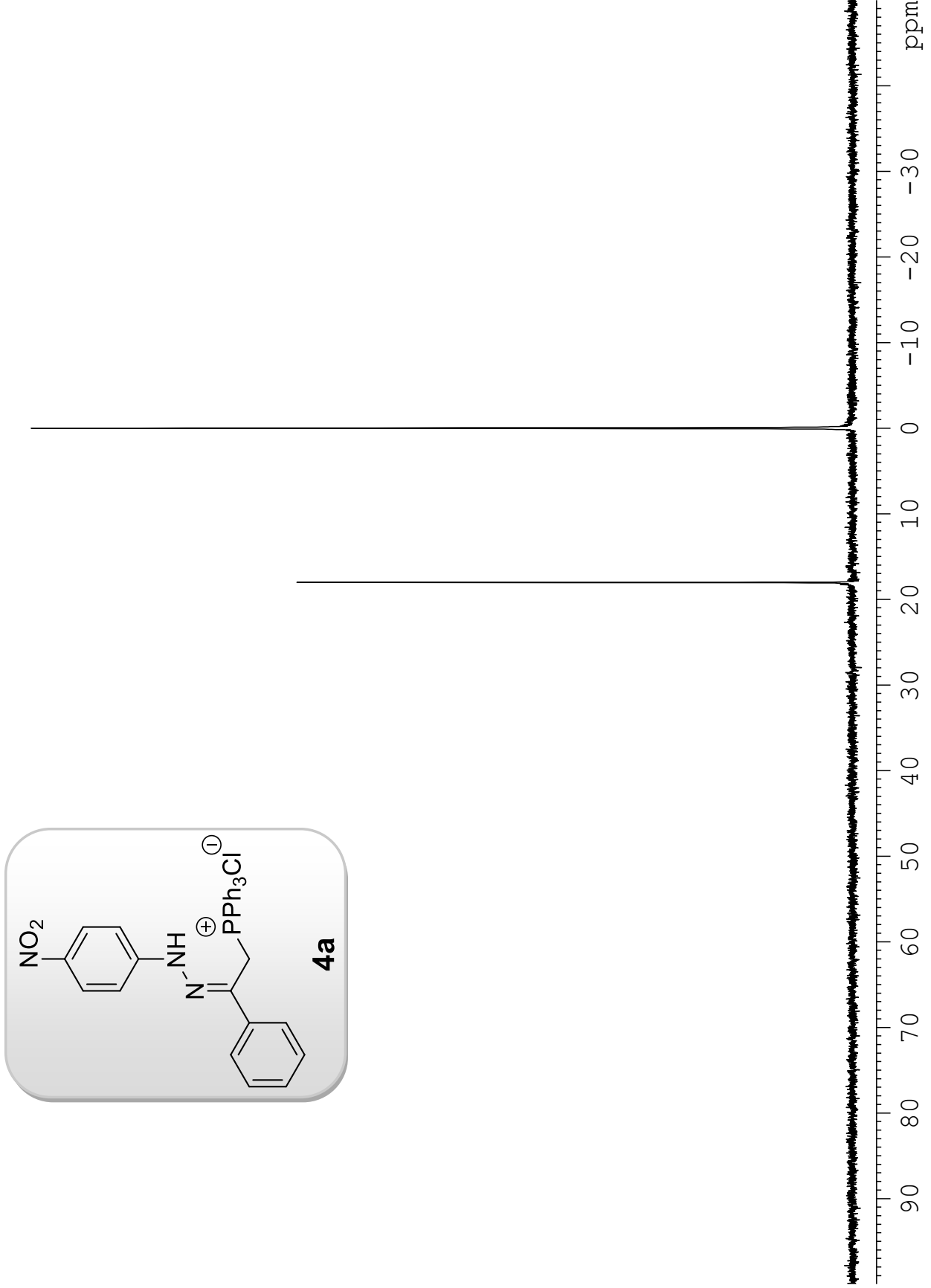


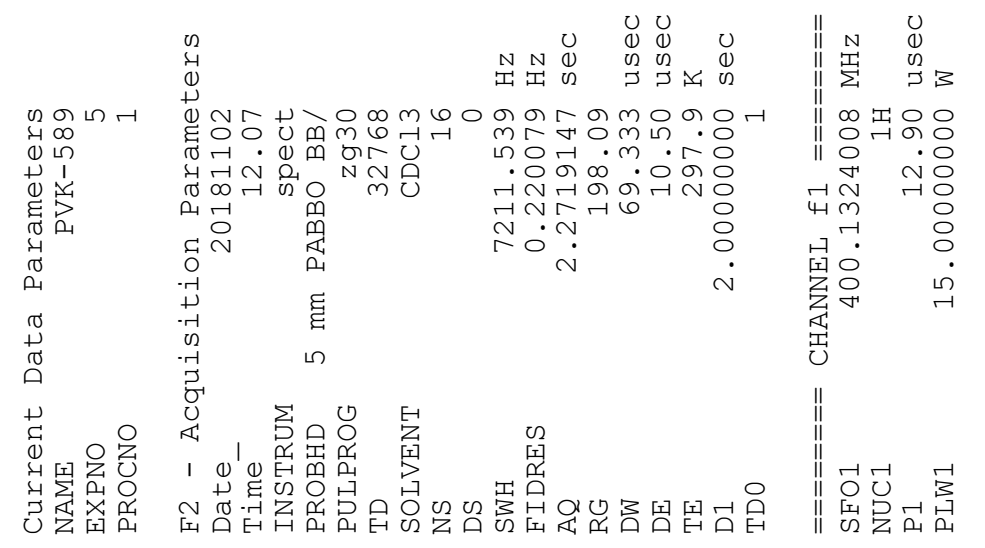

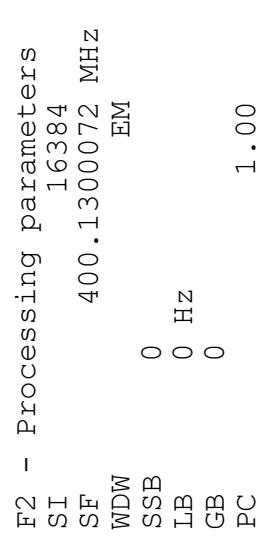

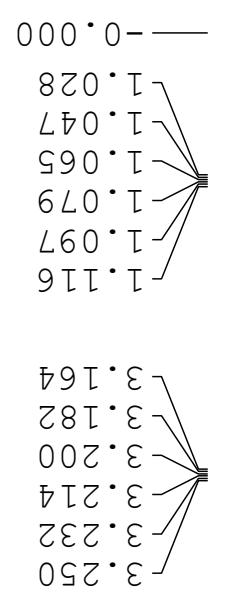

$\left.\begin{array}{l}09 L \cdot G \\ 208 \cdot G \\ 802 \cdot L]\end{array}\right]$

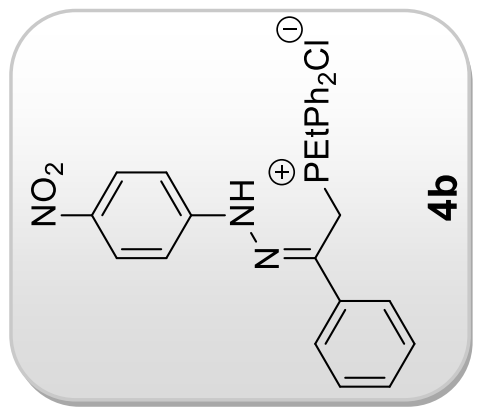

틍

[Ē $\left.{ }^{\circ} L\right]$

万S๐ ${ }^{\circ} L$

$897^{\circ} L$

$S \angle \nabla^{\circ} L$

$\angle O S^{\circ} L$

SZS $L \bigvee$

$\varepsilon \nabla S^{\circ} L$

$24 S^{\circ}$

28 $L$

$098^{\circ} L$

$698^{\circ} L J$

$088^{\circ} L$

$668^{\circ} \mathrm{L}$

LEO 8

$690 \cdot 8$
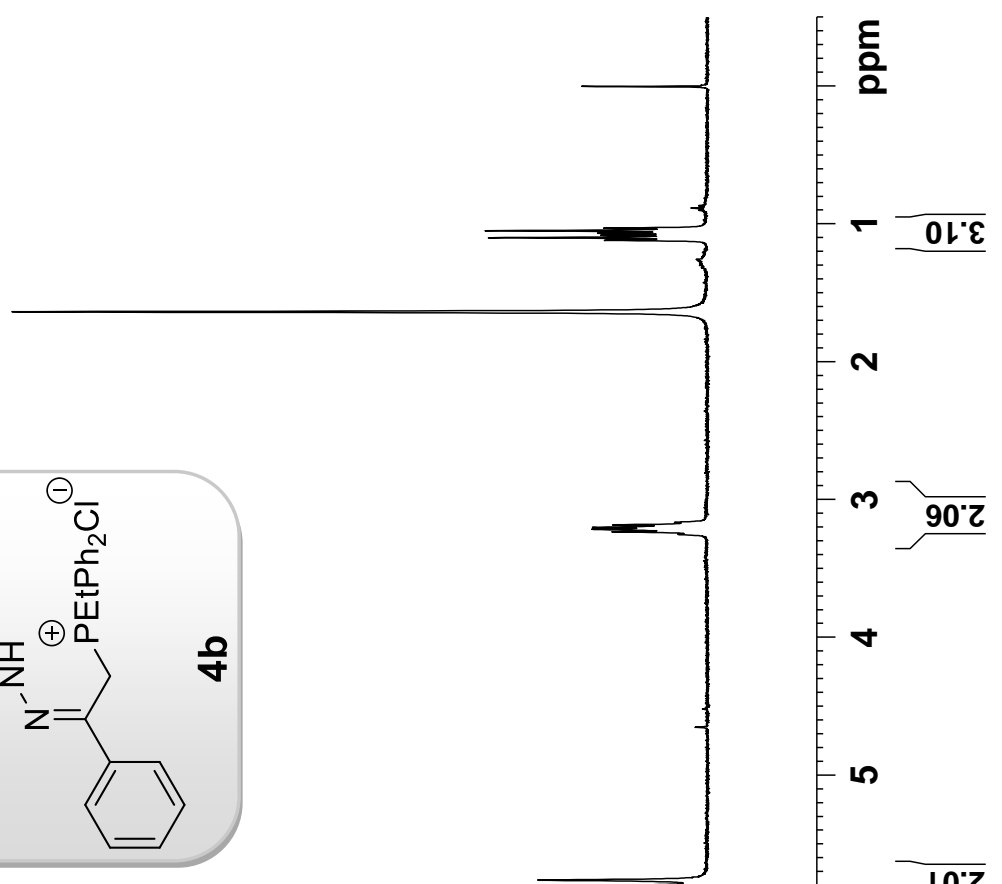

N
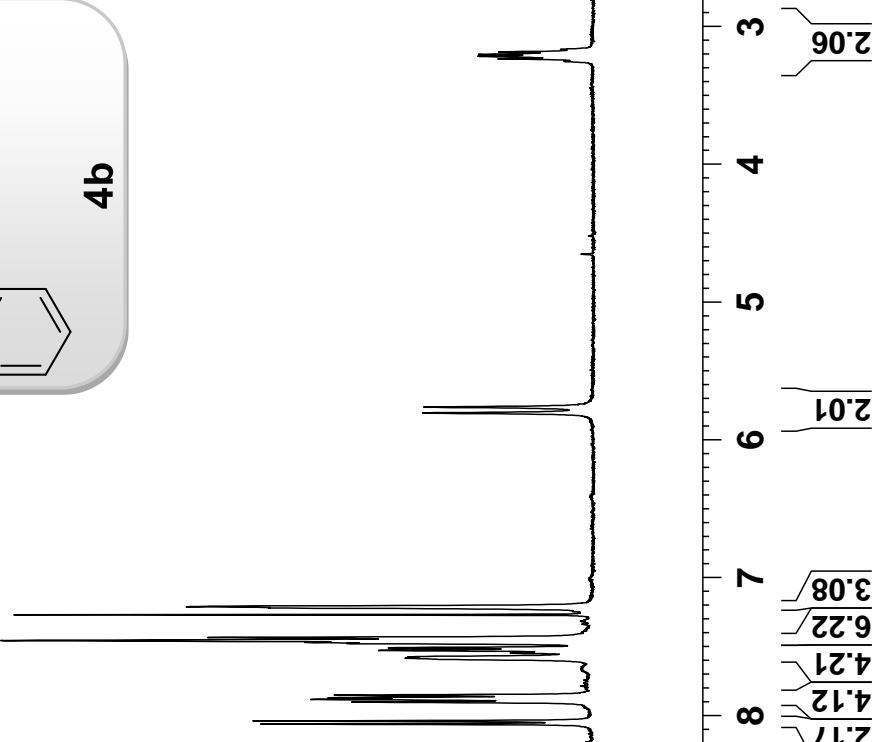

$\frac{\sqrt{80^{\circ} \varepsilon}}{\frac{2 Z^{\prime} 9}{L Z^{\prime} t}}$
$\frac{2 L^{\prime} t}{2 L^{\prime} \tau}$

$\infty$

$\angle L ' 2$

a

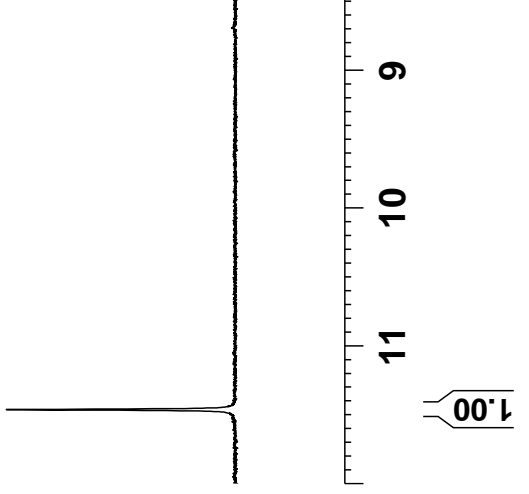

697 

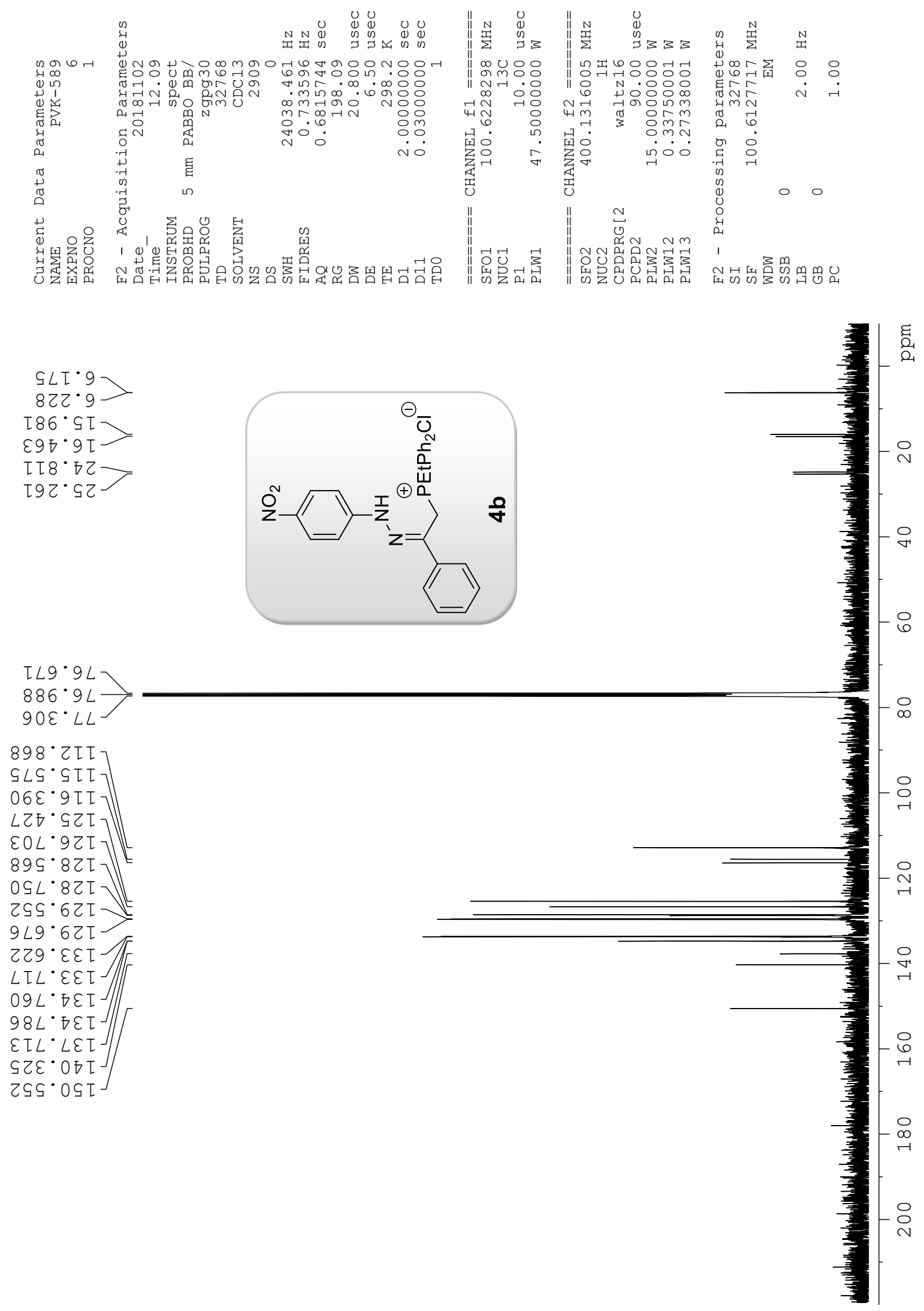

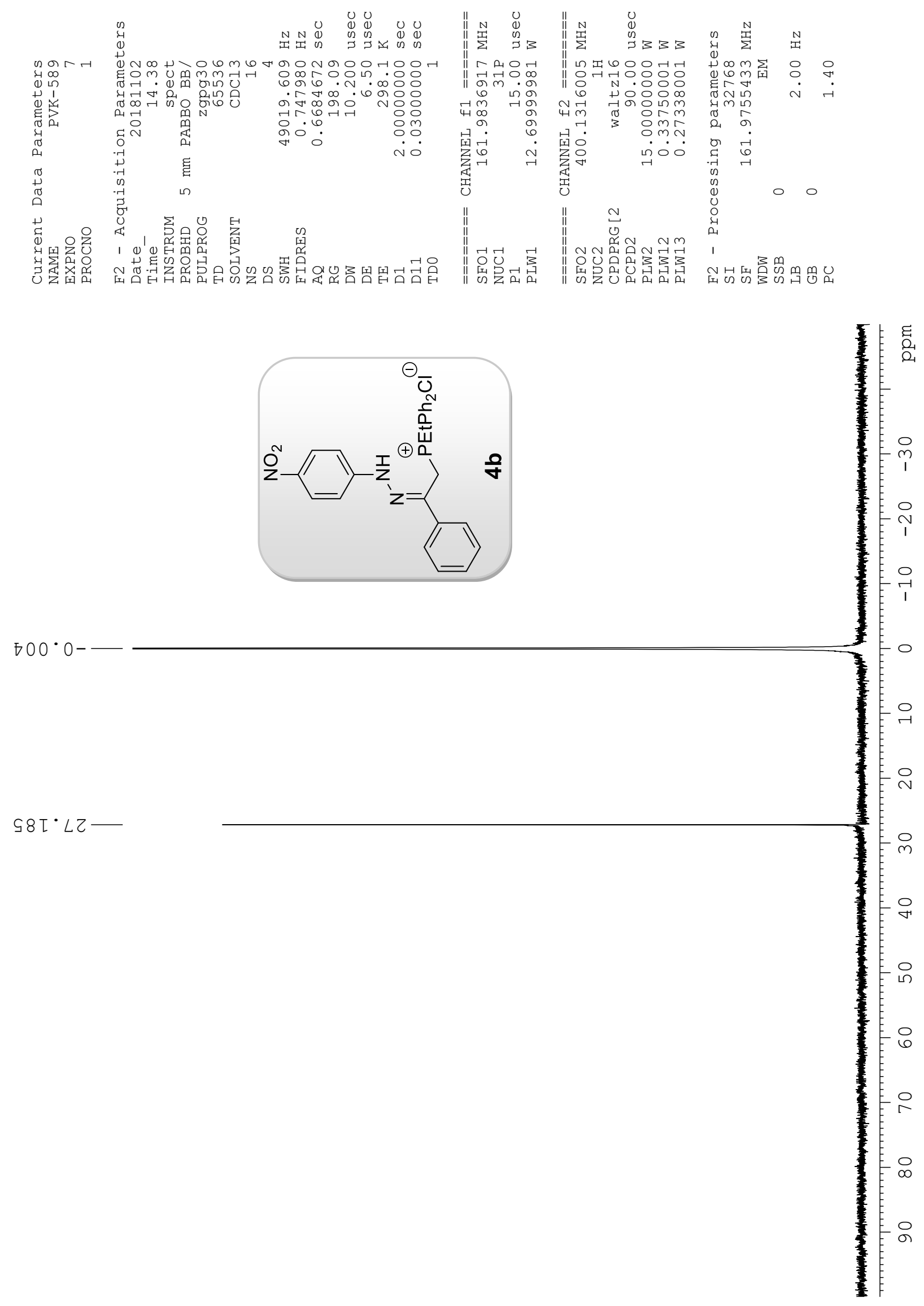

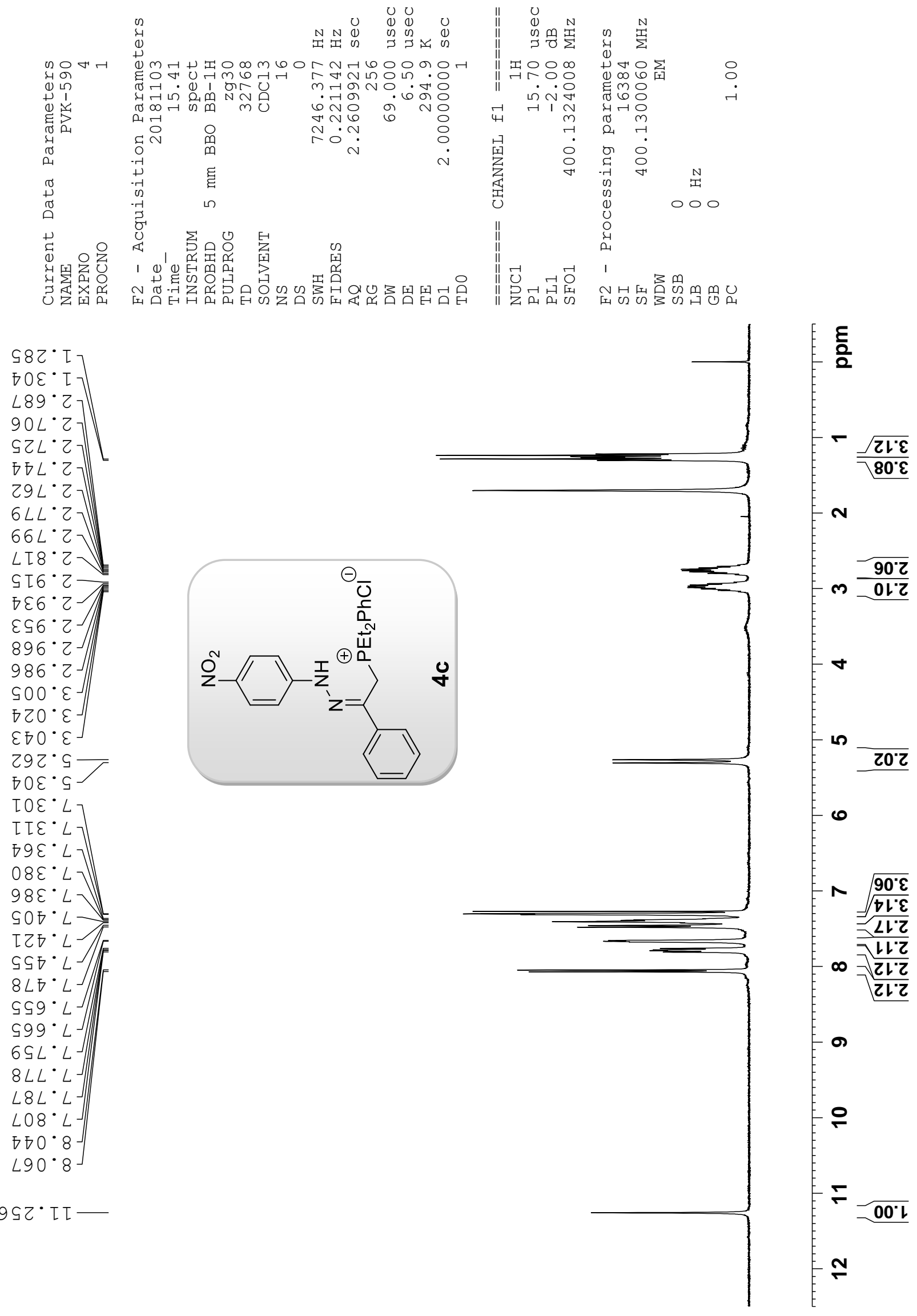

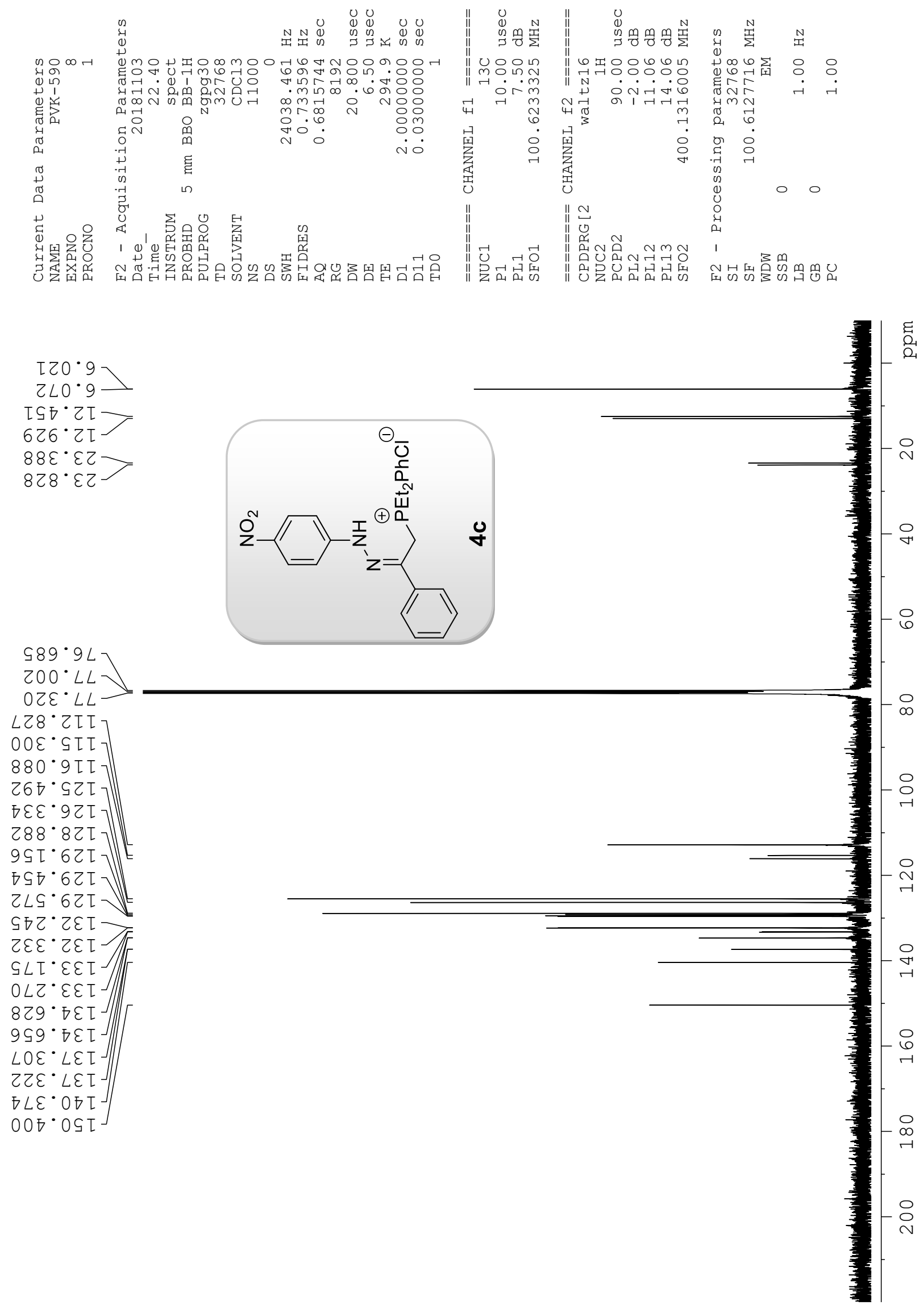

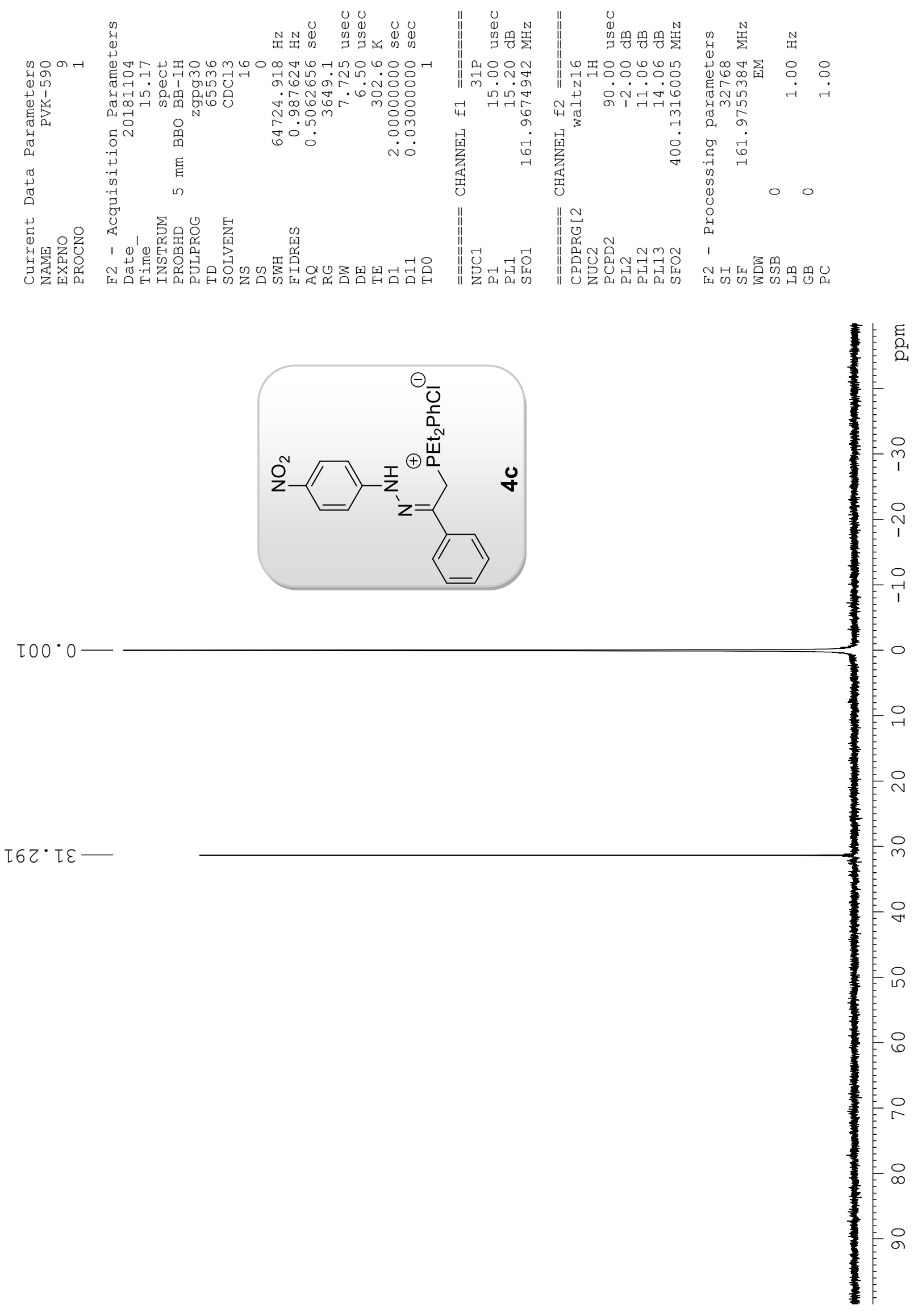

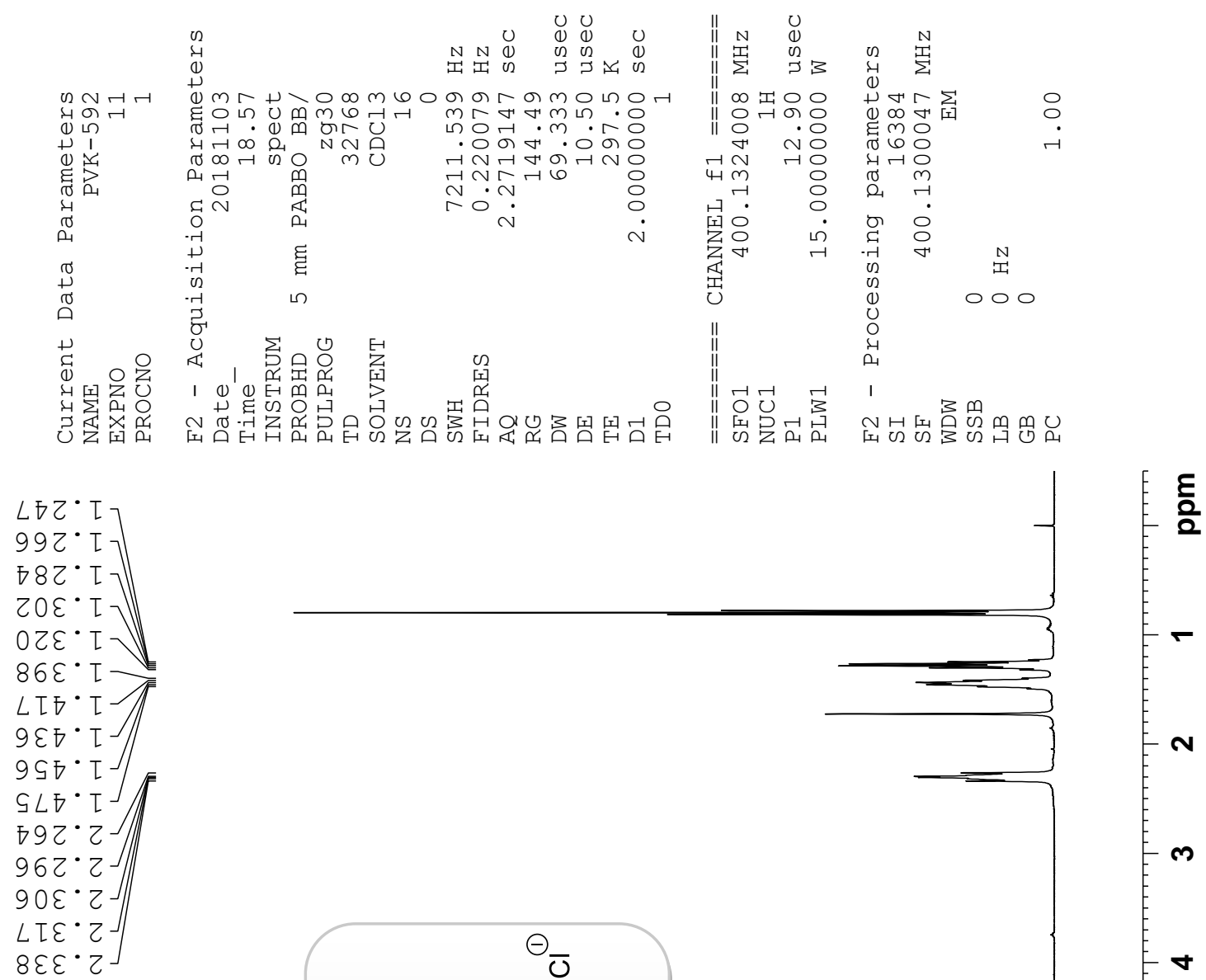

$900^{\circ}$
$870^{\circ}$

$\varepsilon 8 \varepsilon^{\circ}$

$00 \nabla^{\circ} L$

$\angle\left[\sigma^{\circ} L\right]$

ஏてゅ・L

E

$\varepsilon I L \cdot L$

$S \varepsilon L \cdot L \perp$

$68 L \cdot L$

$\angle 08^{\circ}$

$0 \varepsilon[\cdot 8$

ZSI: 8
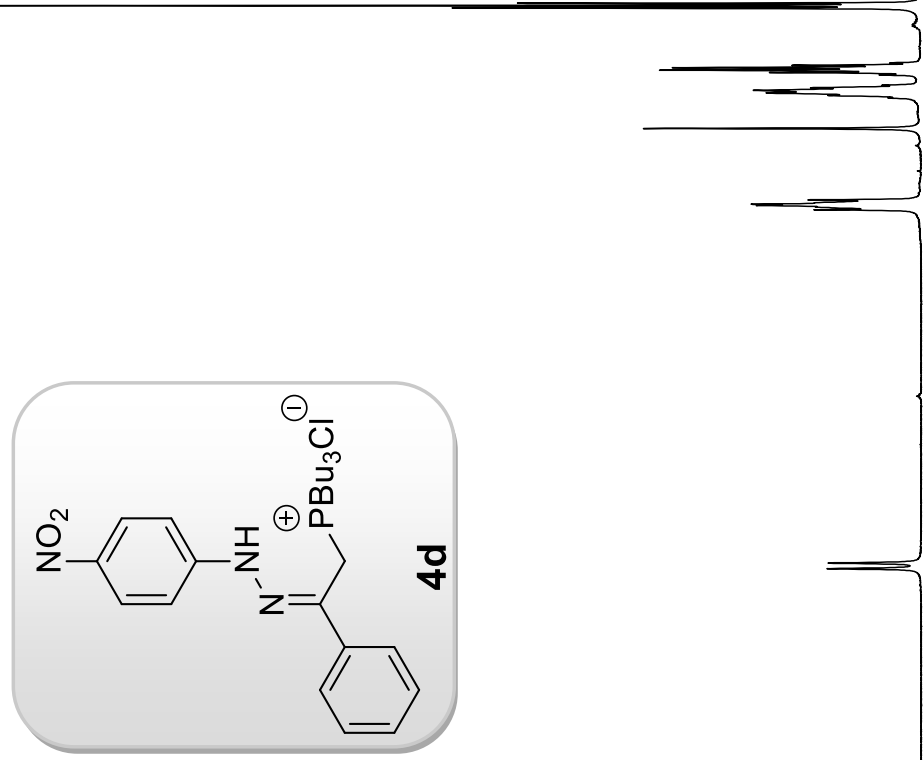

$-0{ }^{\circ} 6$

N

tเ.9

$m$

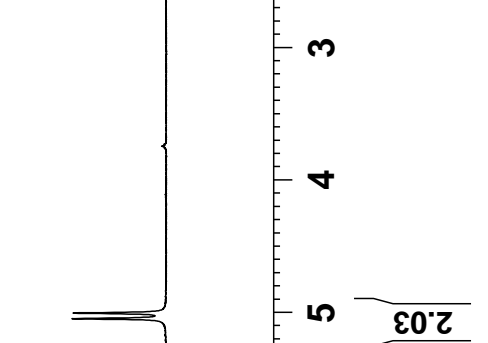

E† $8^{\circ}$ IT

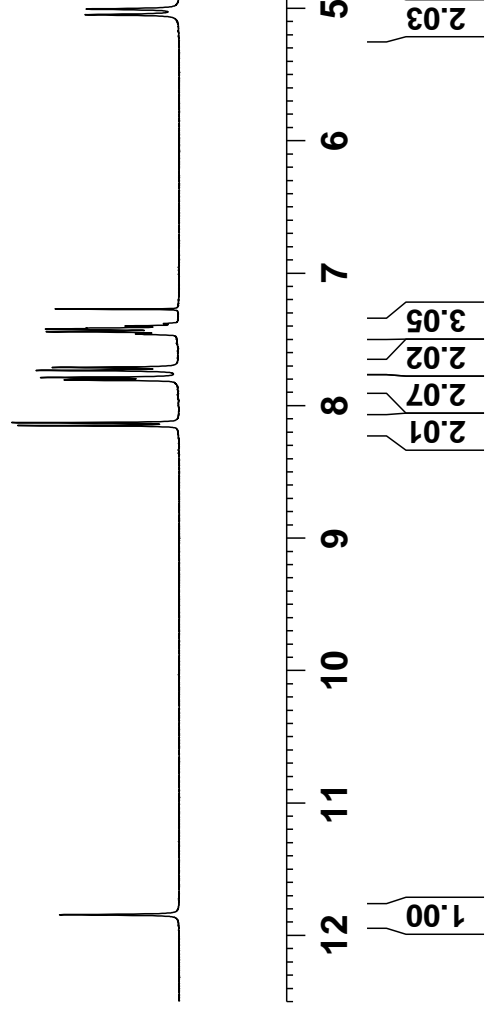



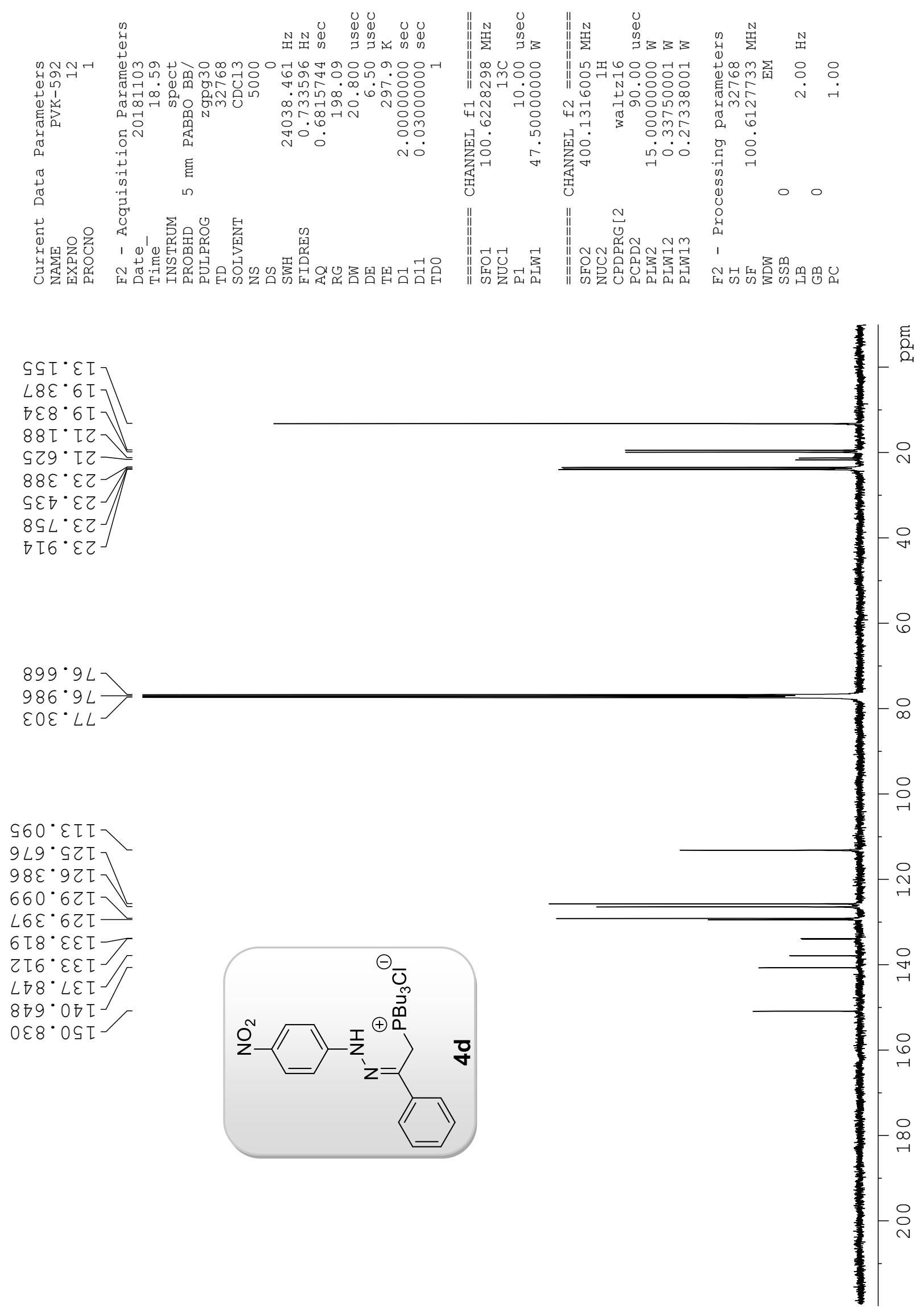

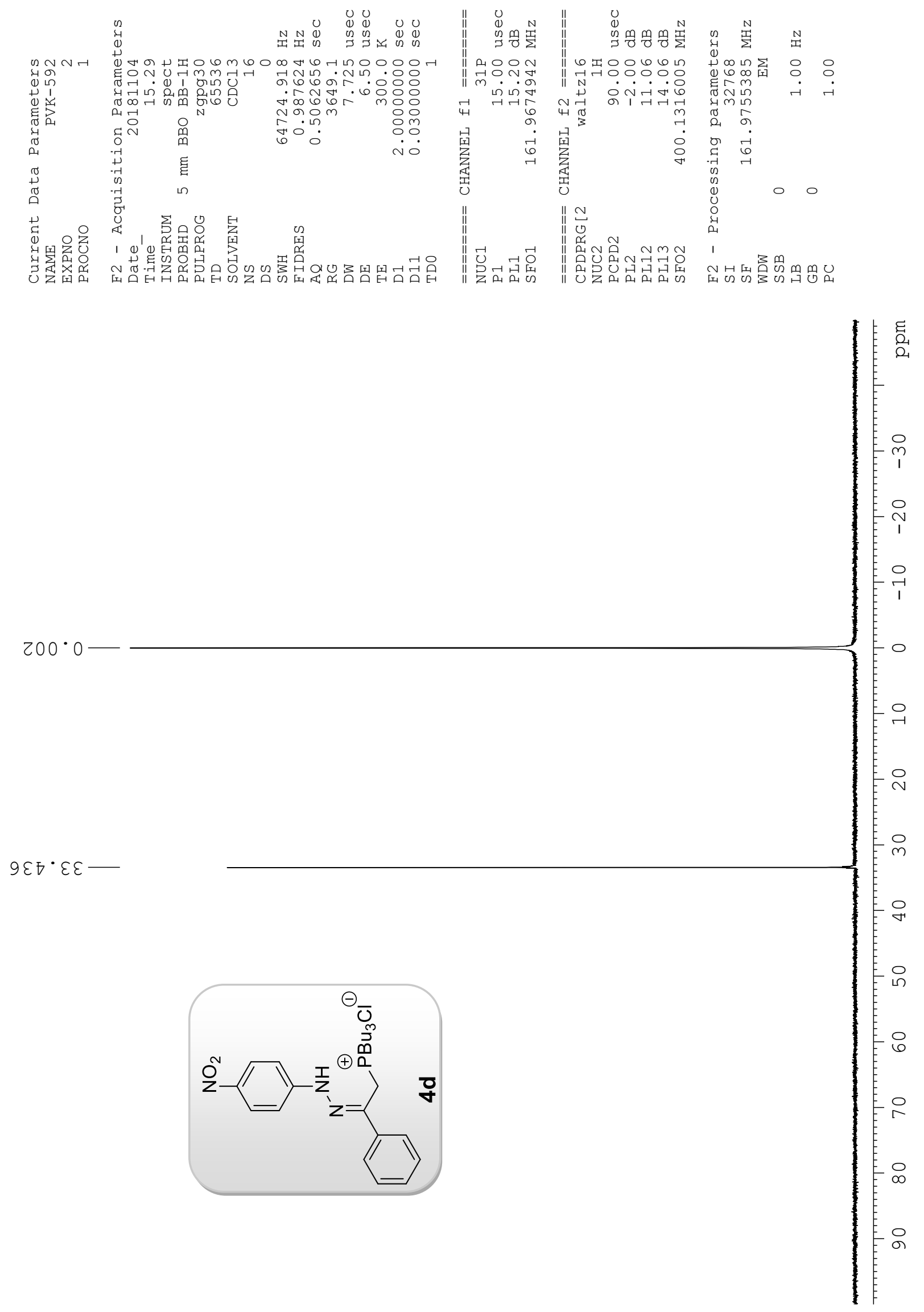


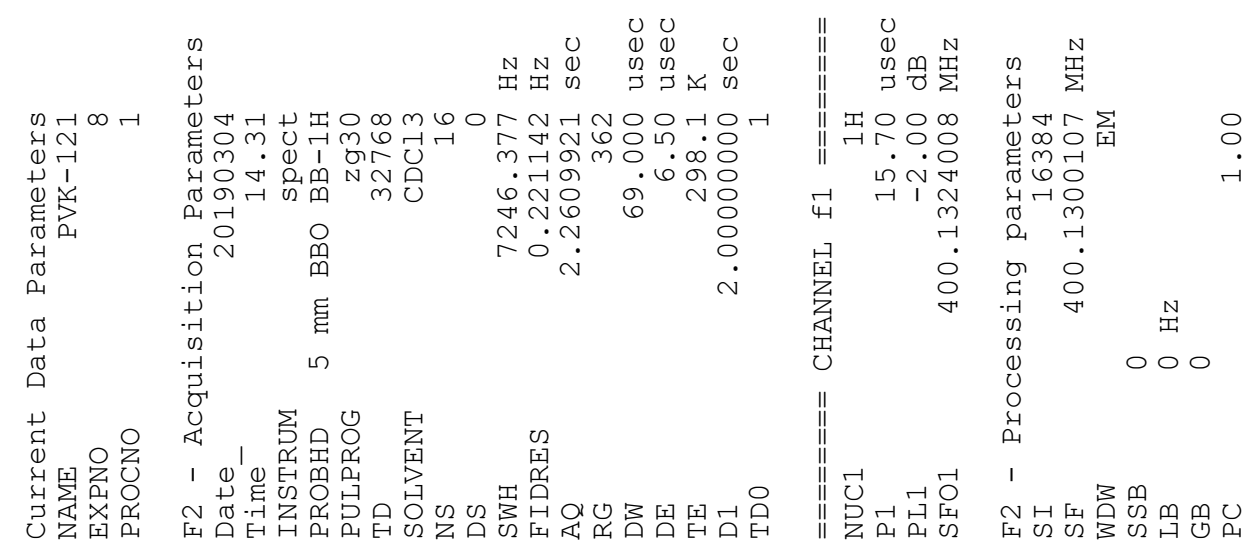

$000 \cdot 0-$

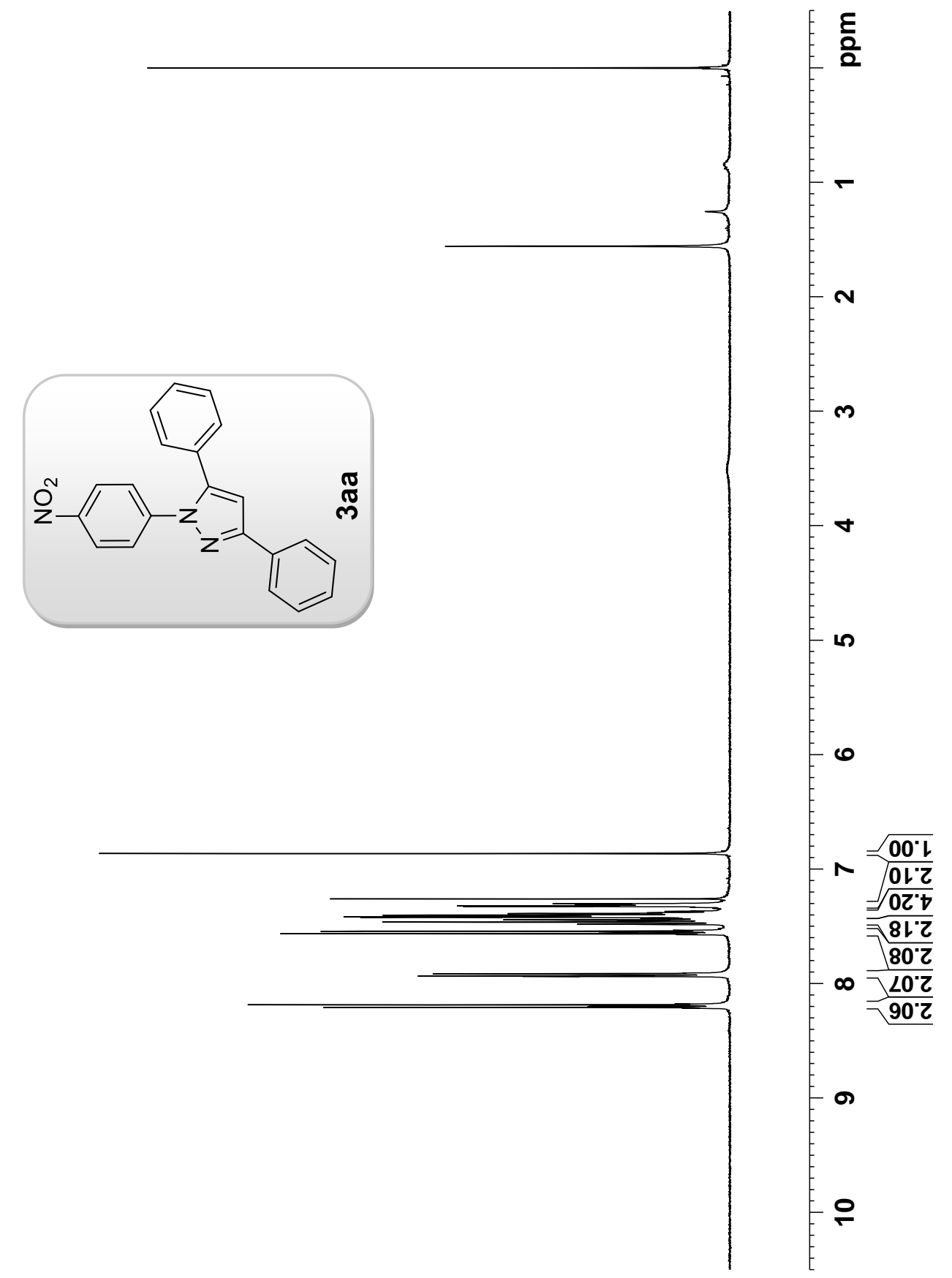




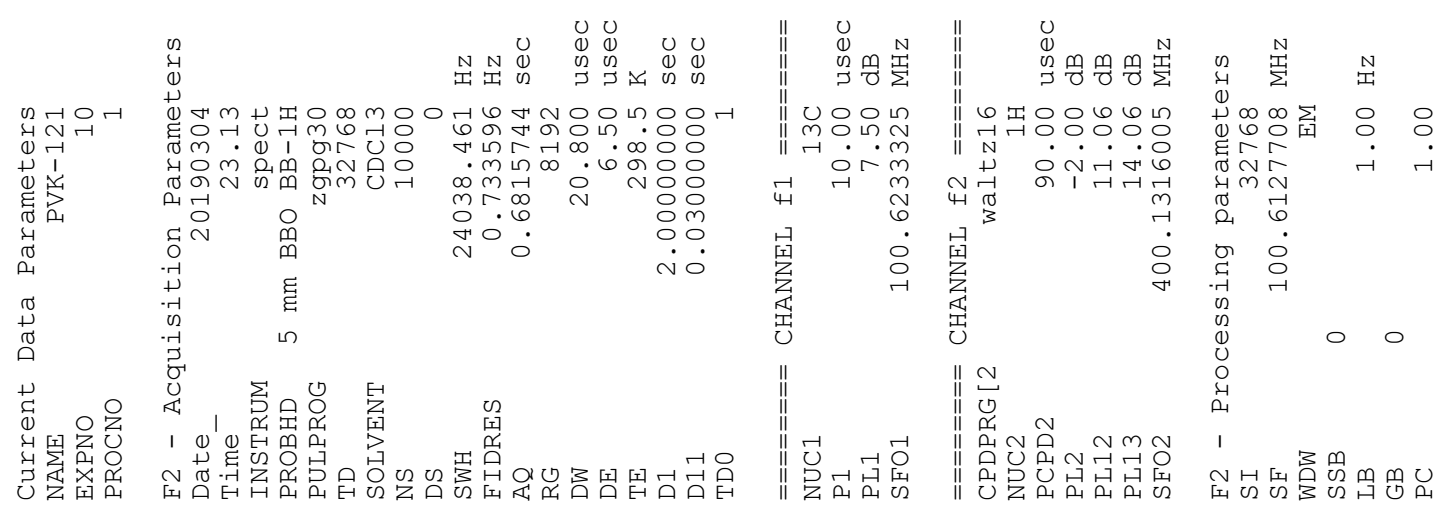

$6[0 \cdot 0-$
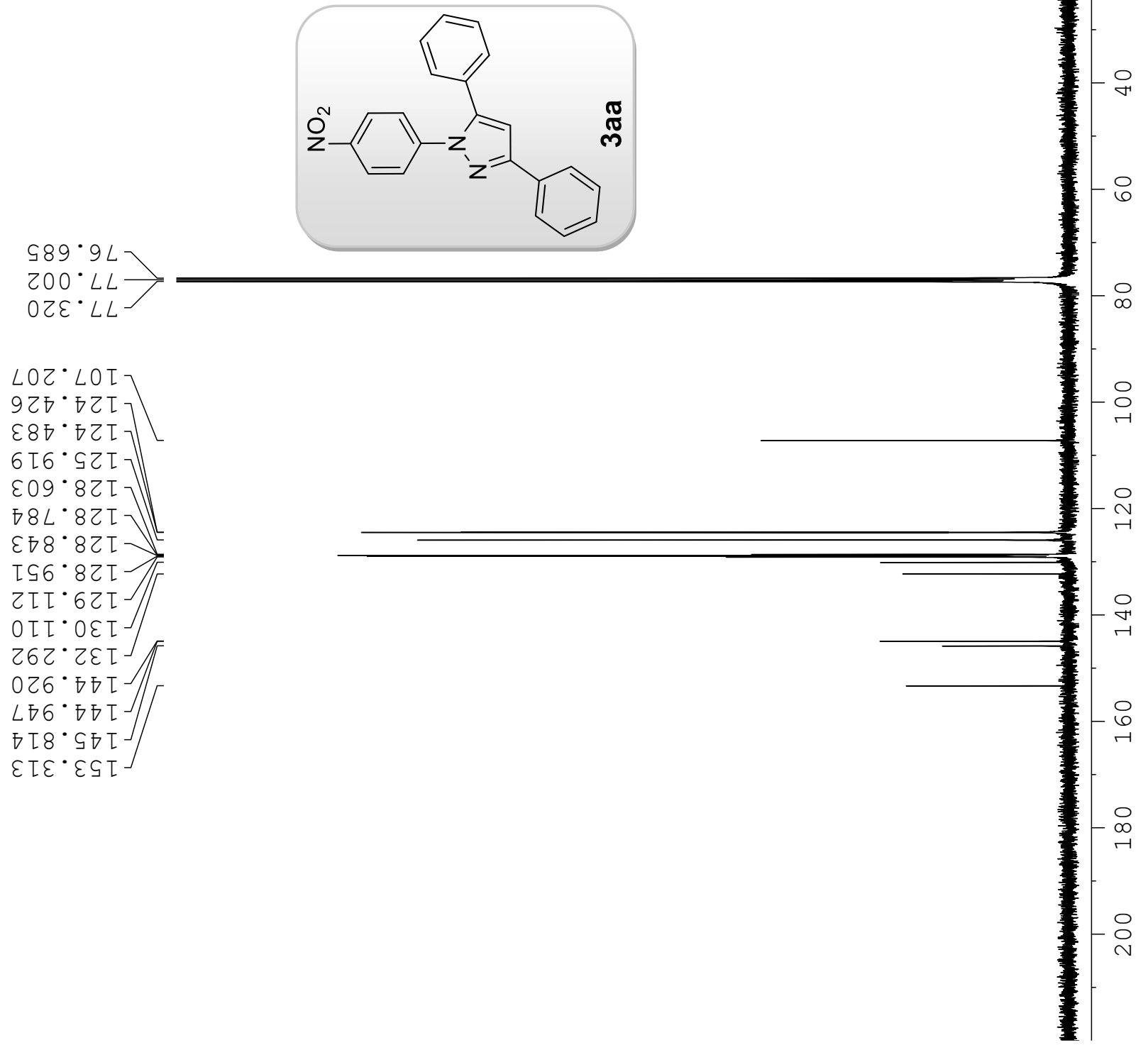


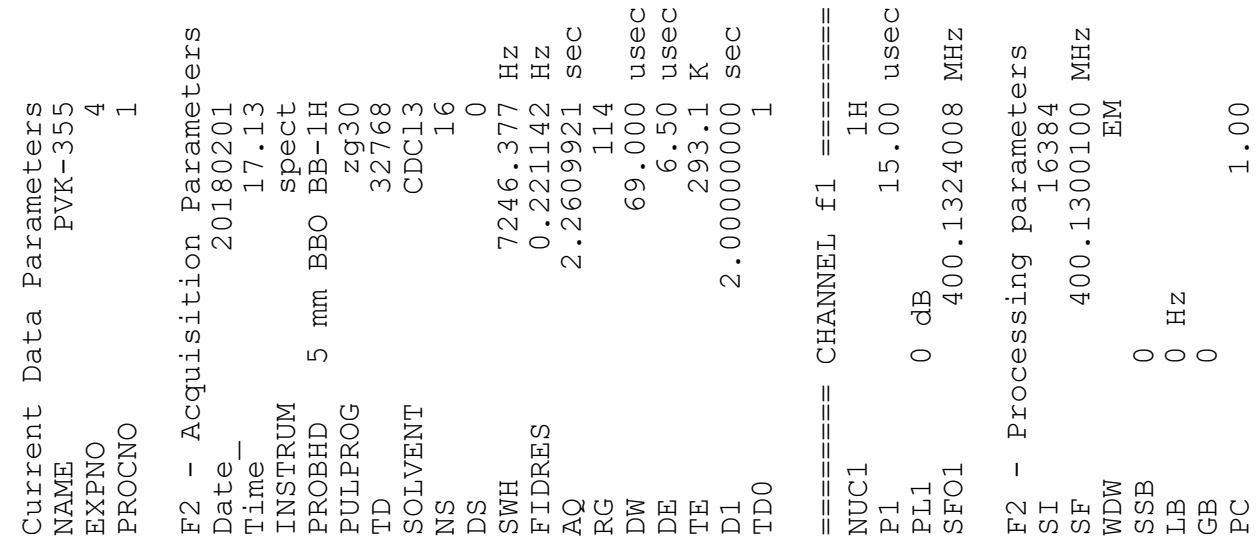

$00 \cdot 0$

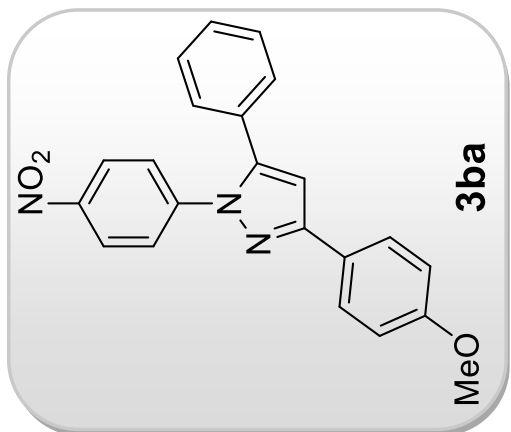

등

$80^{\circ} \varepsilon$
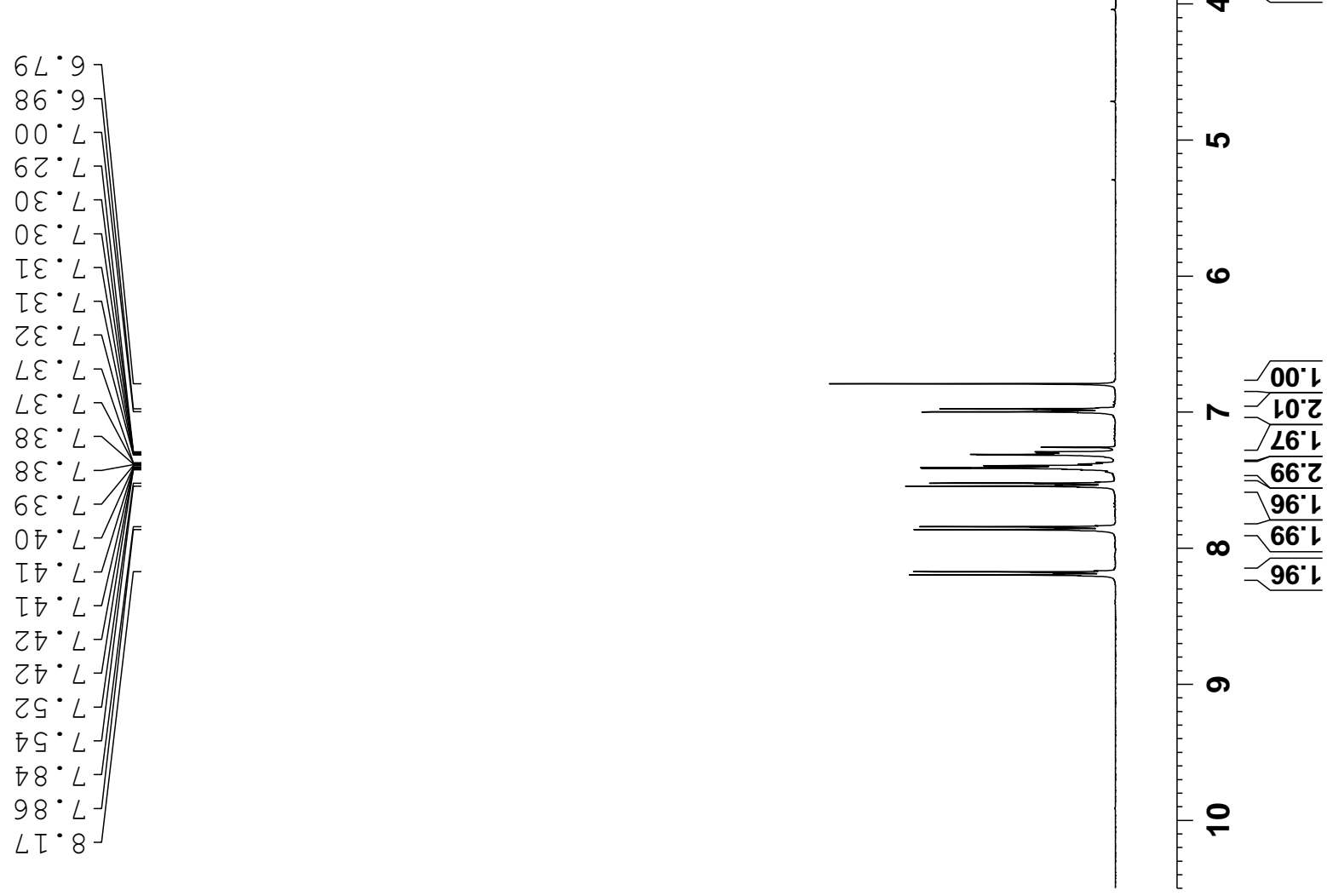

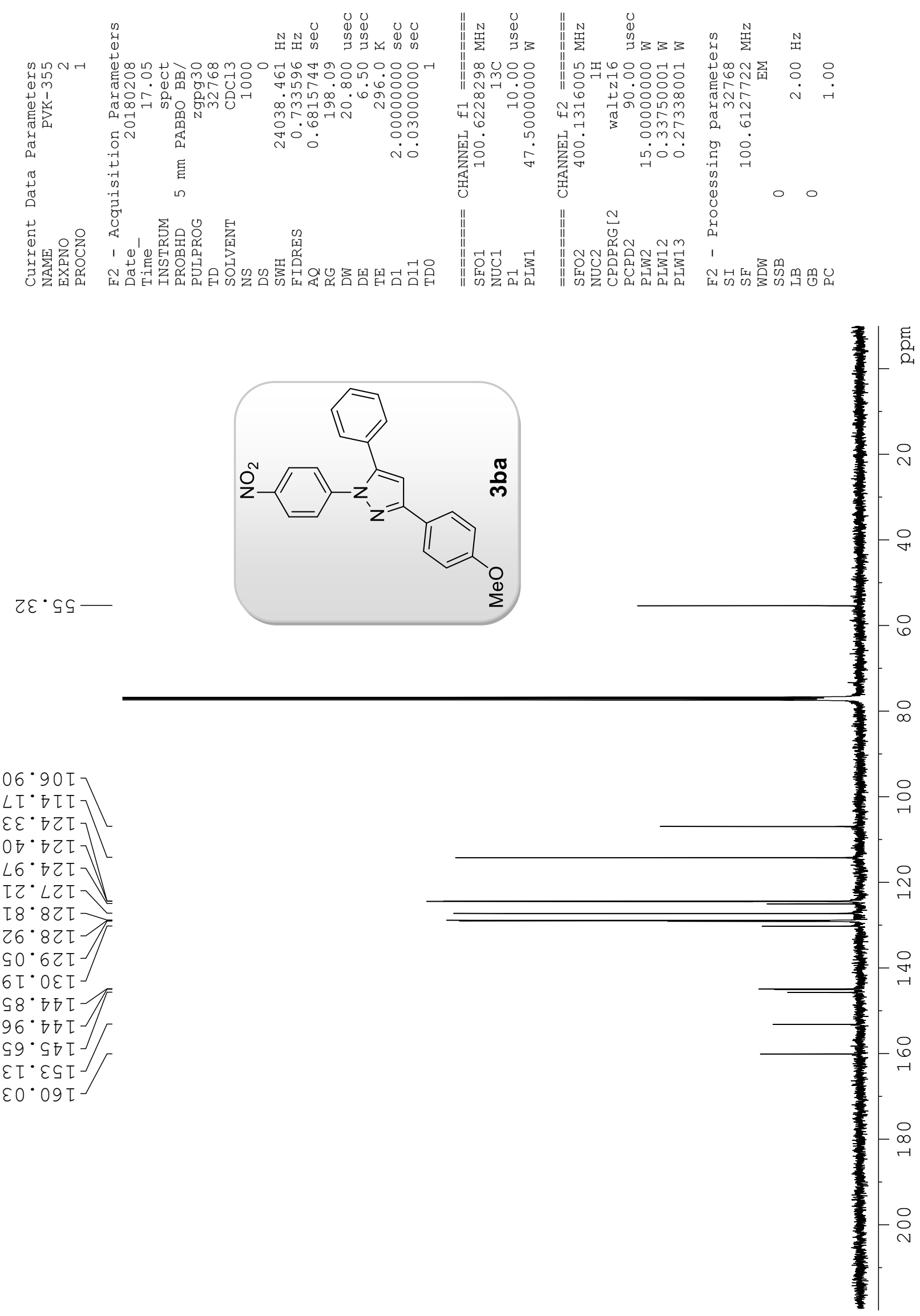


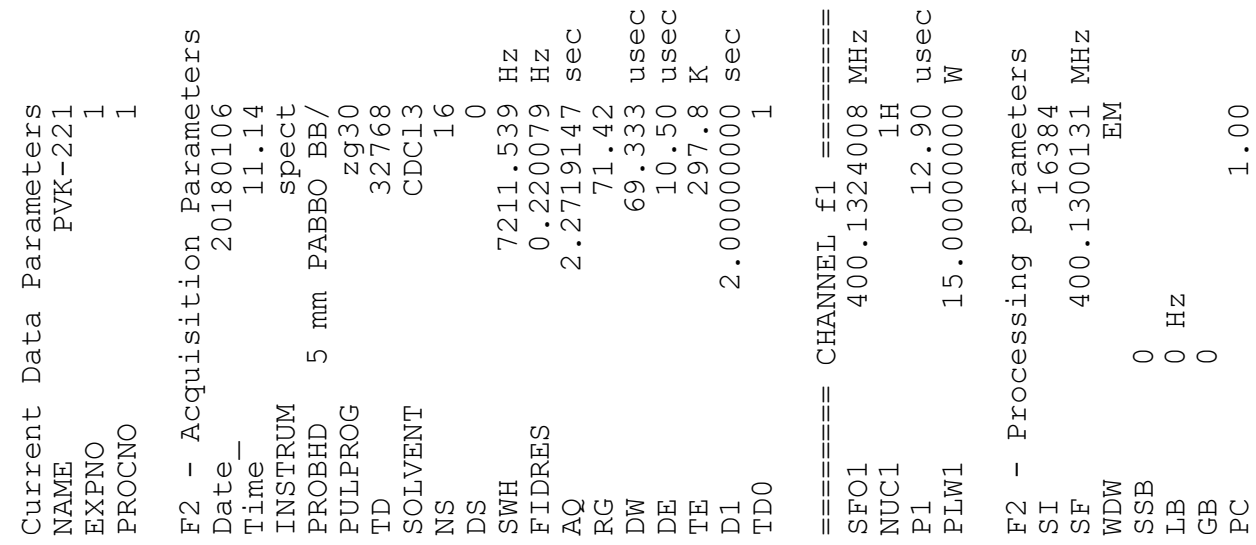

$00 \cdot 0-$

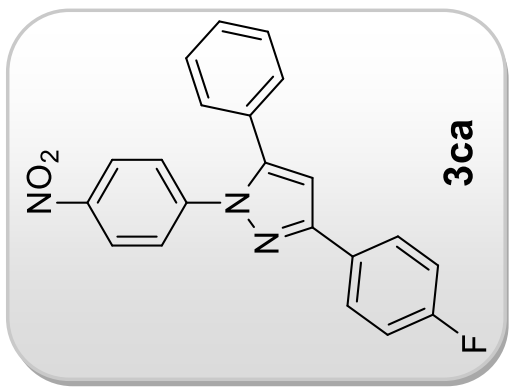

틍

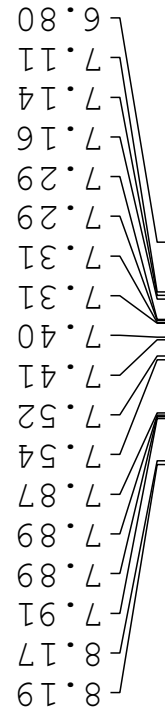

00

$\wedge \sqrt{\varepsilon 0^{\circ} \tau}$

$\varepsilon 0^{\circ} \mathrm{Z}$

$\varepsilon 0^{\circ} \varepsilon$

$00^{\circ} \mathrm{z}$

$\infty \frac{10.2}{66^{\circ} t}$

66.1

$\sigma$

으 


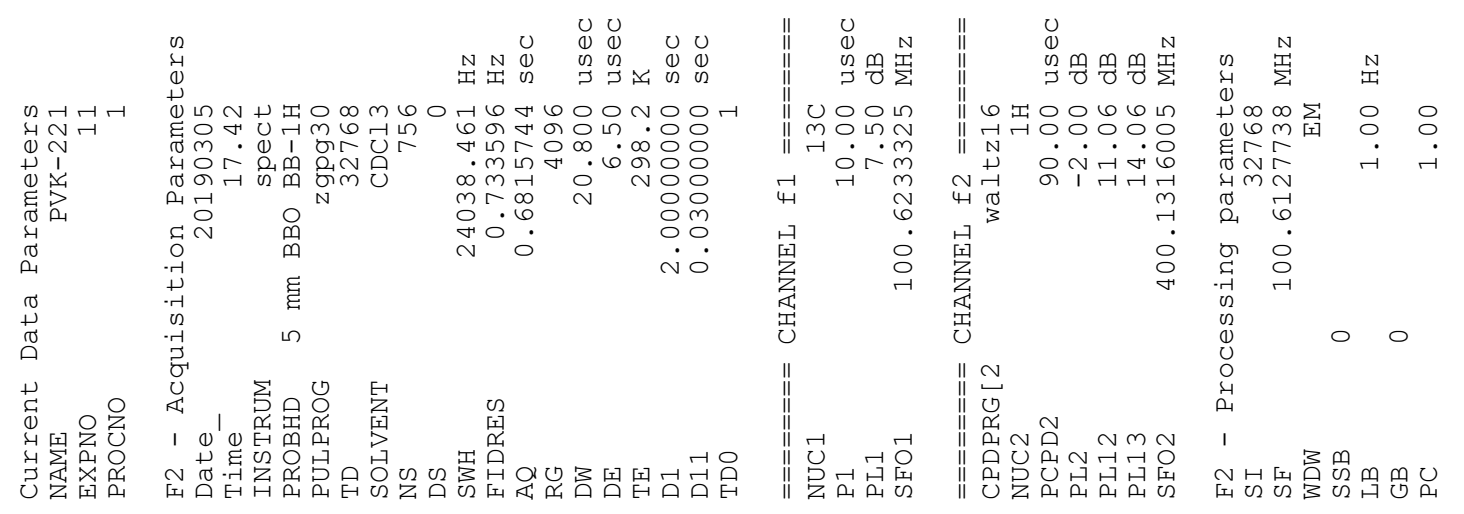

$070 \cdot 0-$
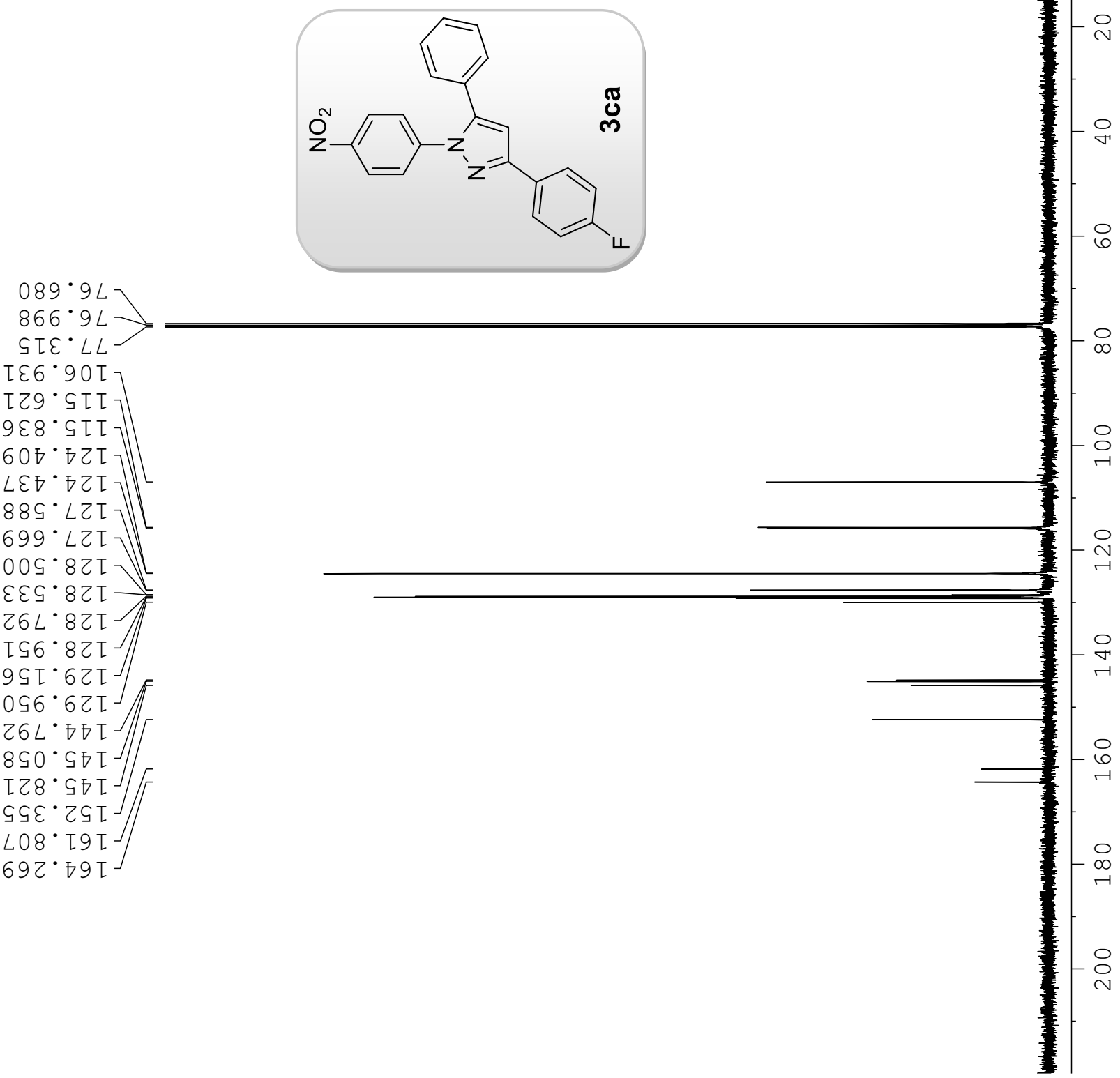

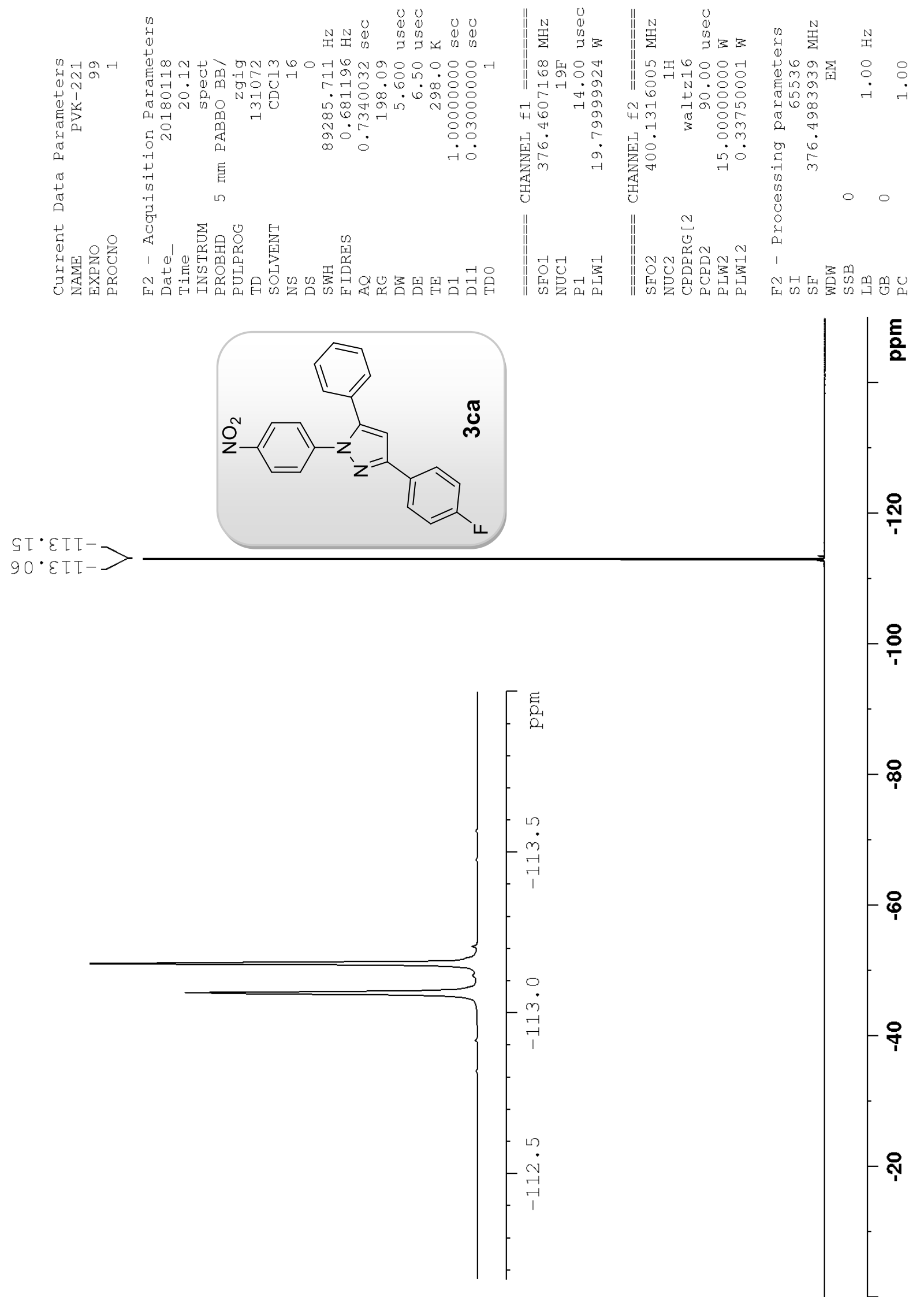


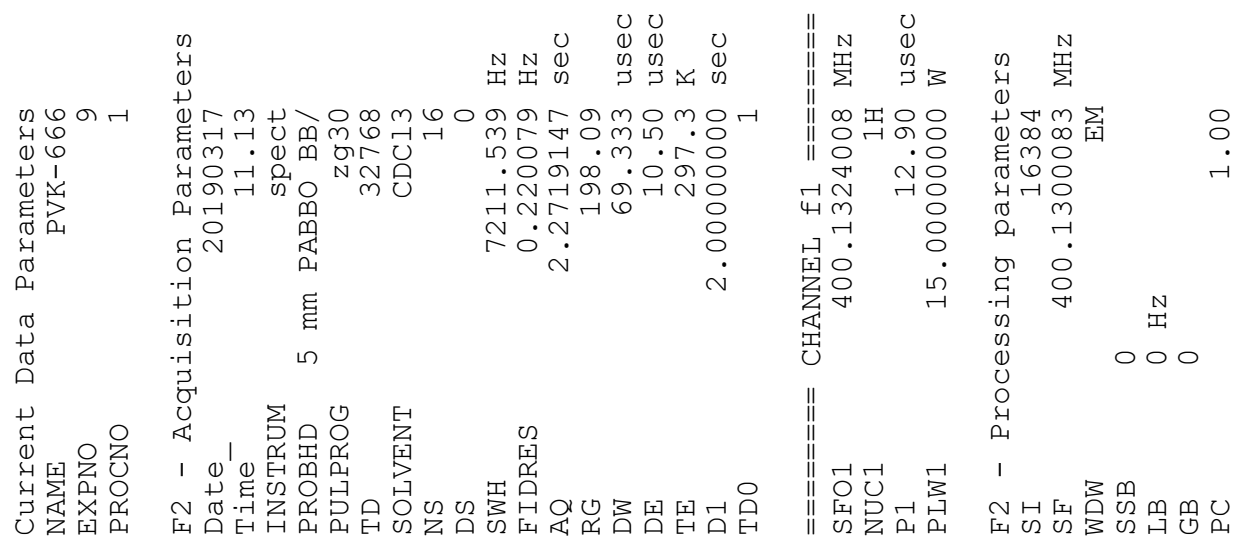

$000 \cdot 0$
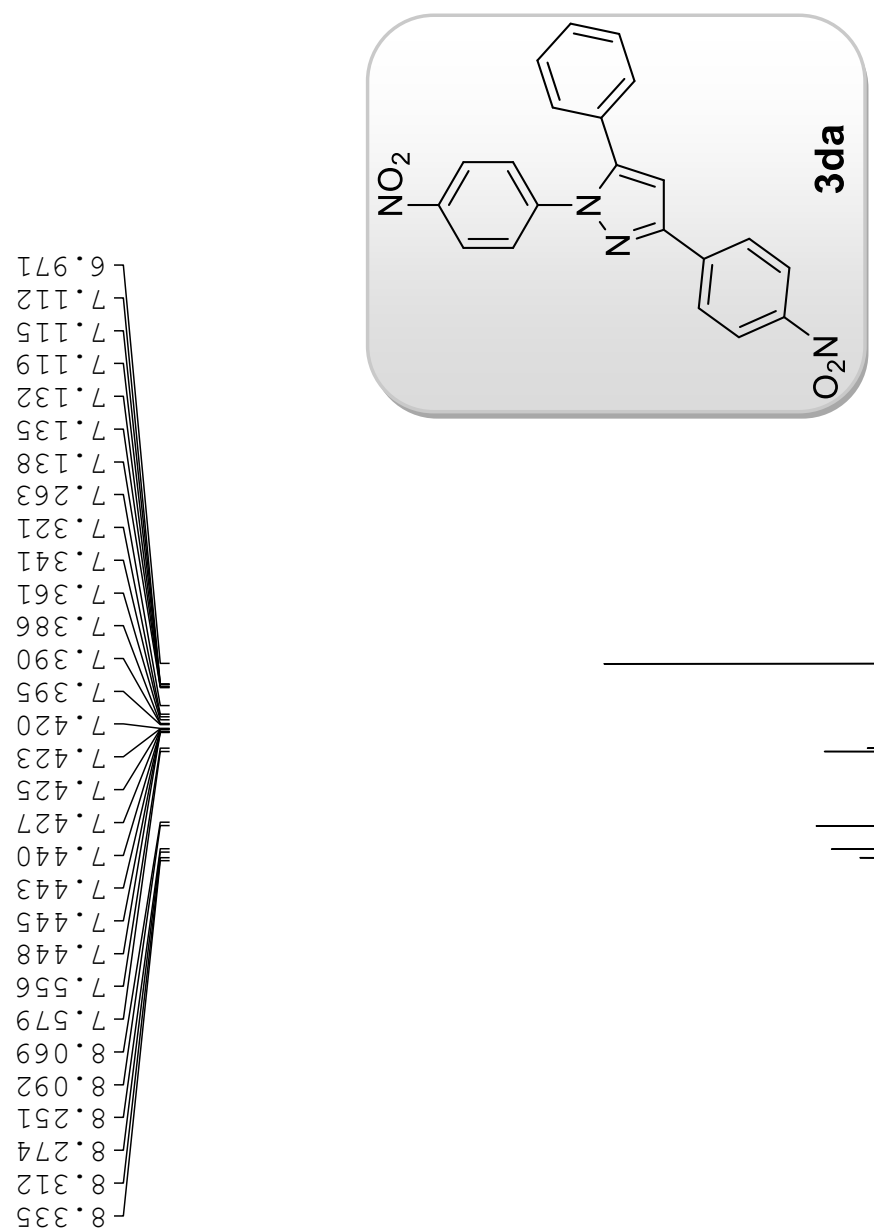


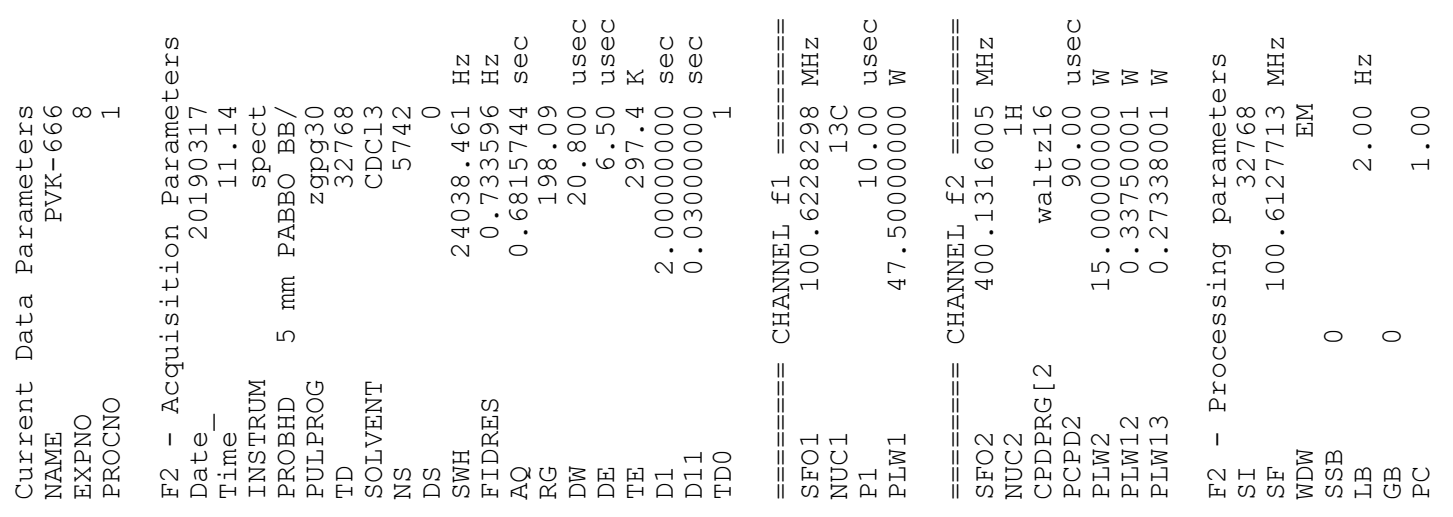

E $0^{\circ} 0--$
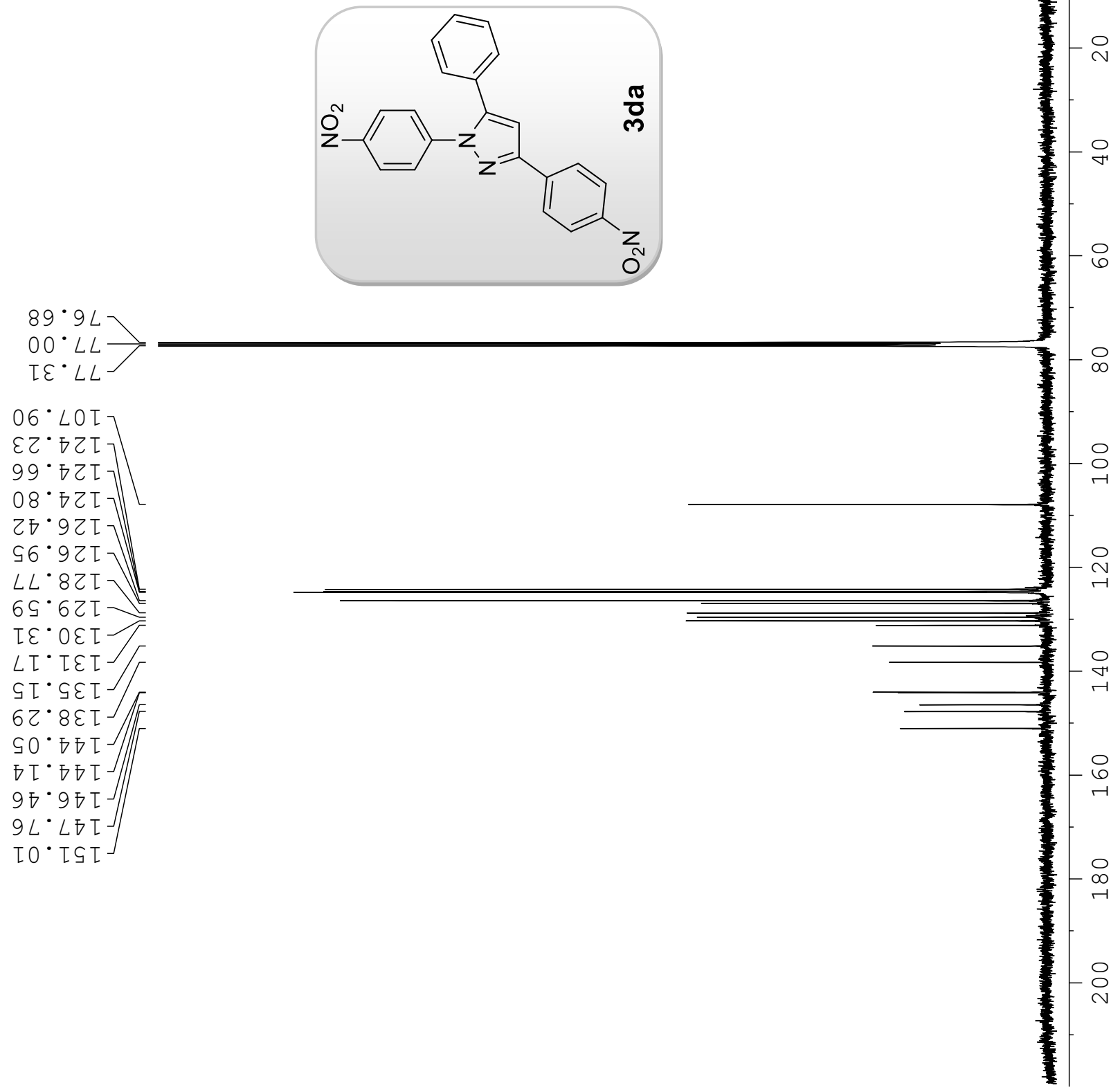

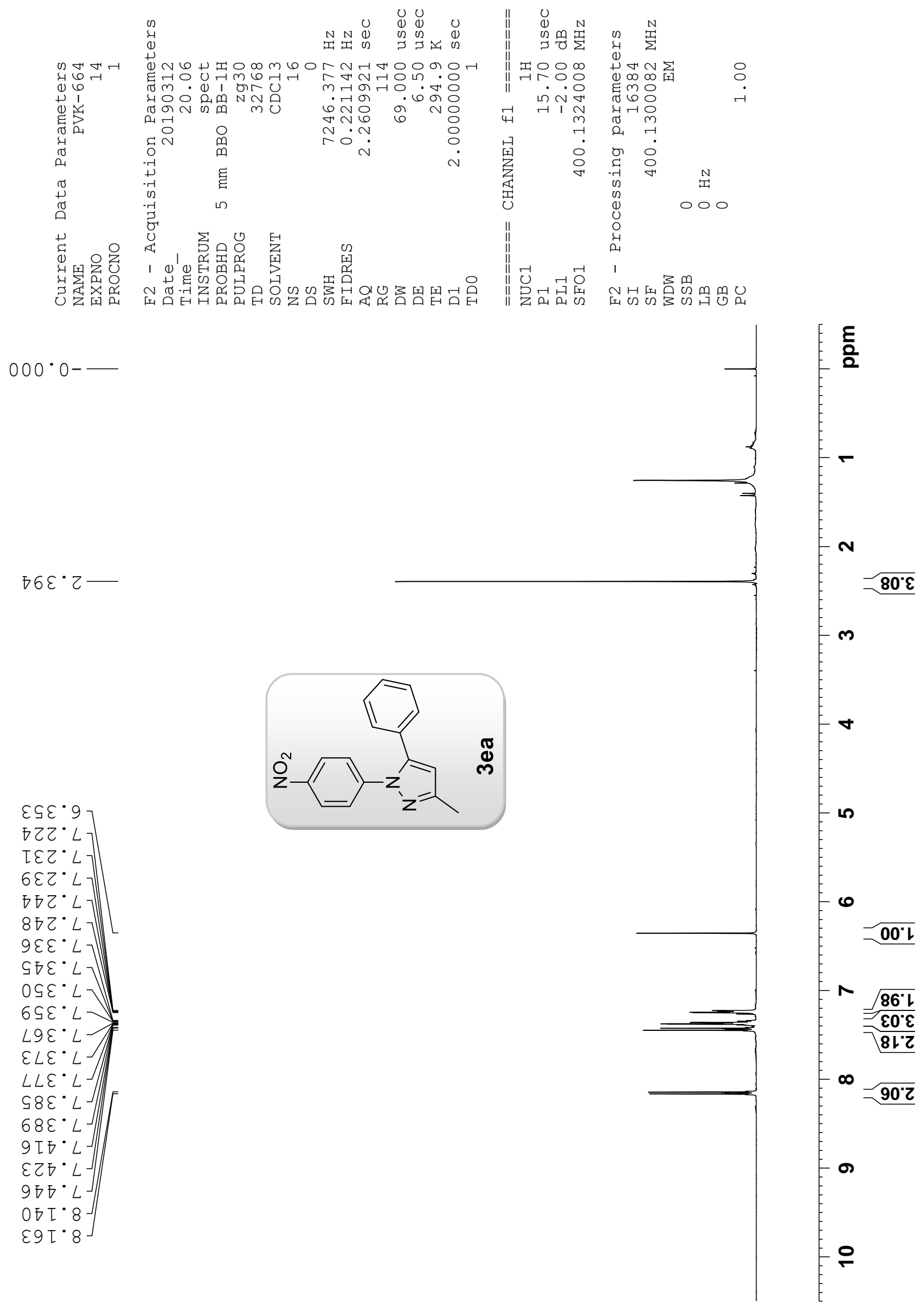


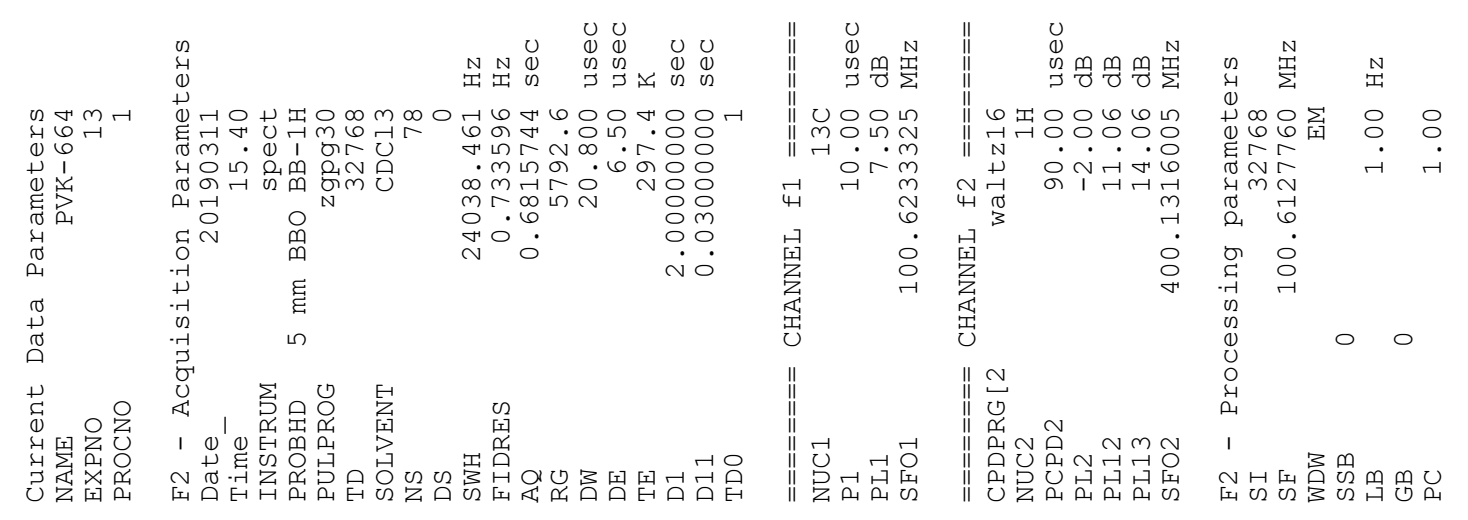

$\tau \varepsilon \mathcal{G}^{\bullet} \varepsilon \tau-$
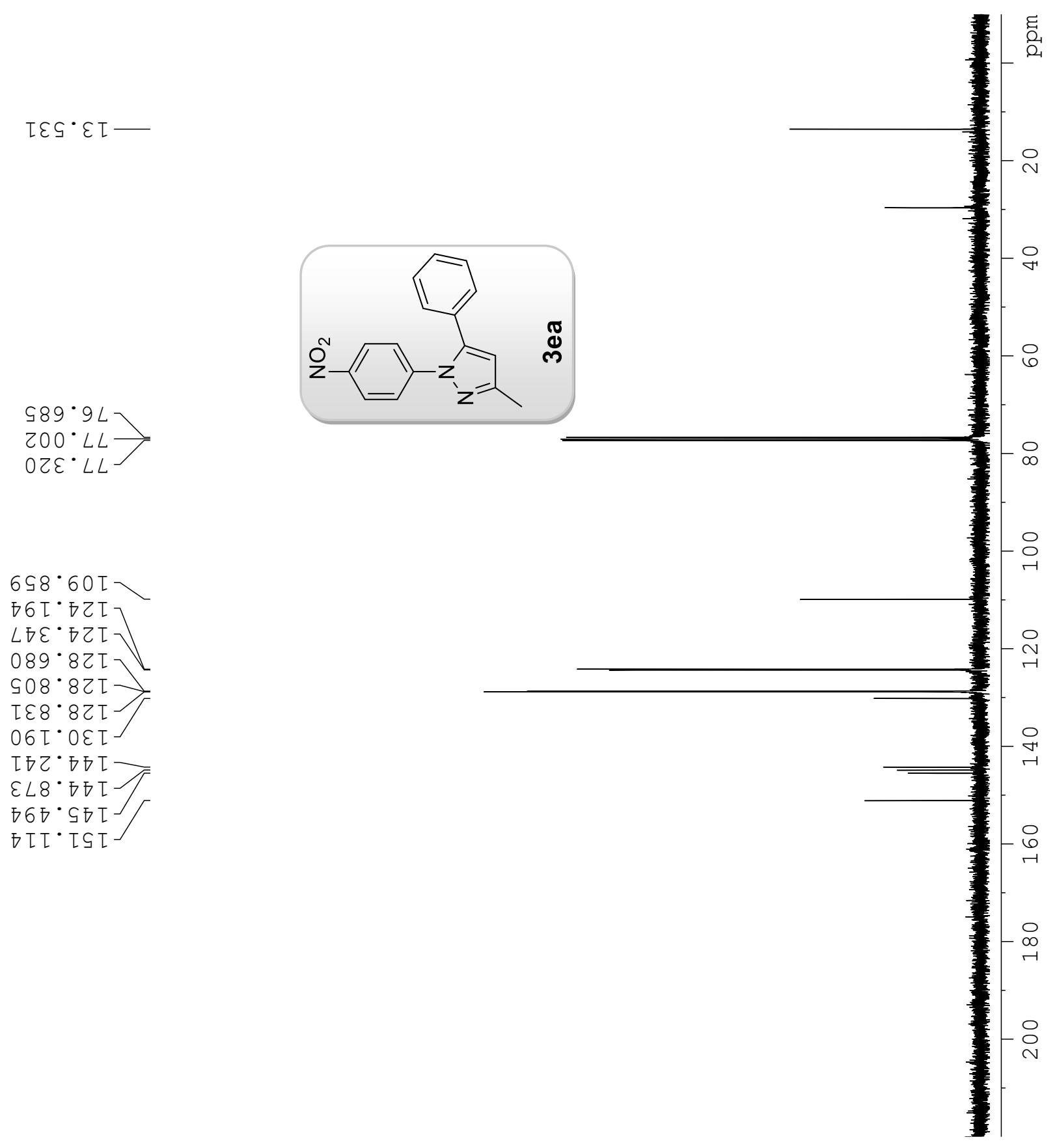

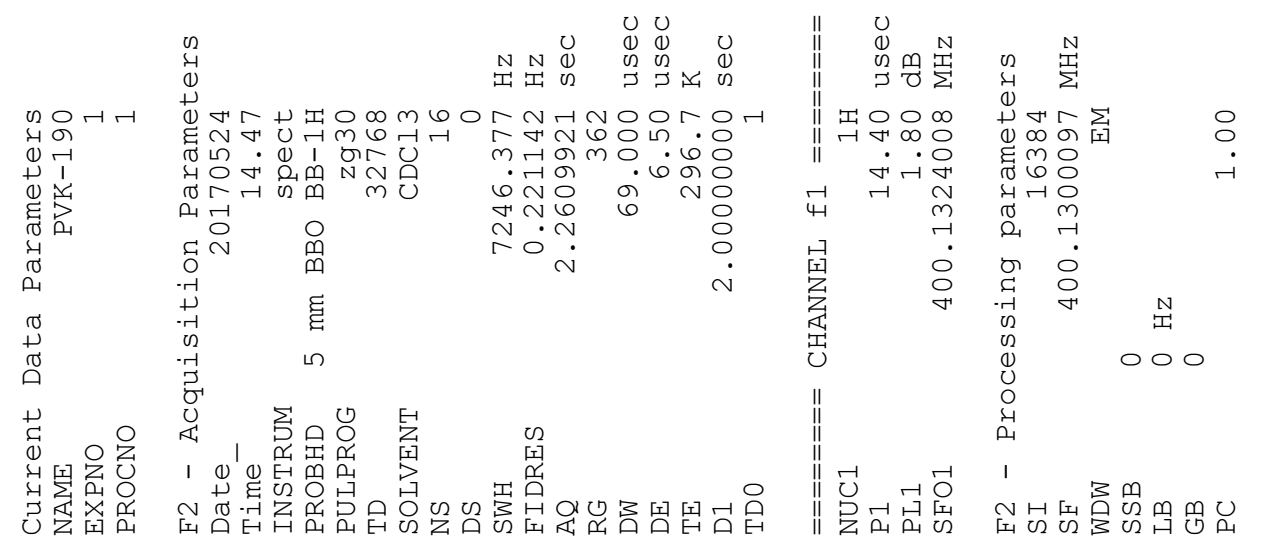

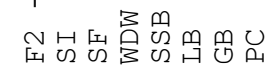

$000 \cdot 0$
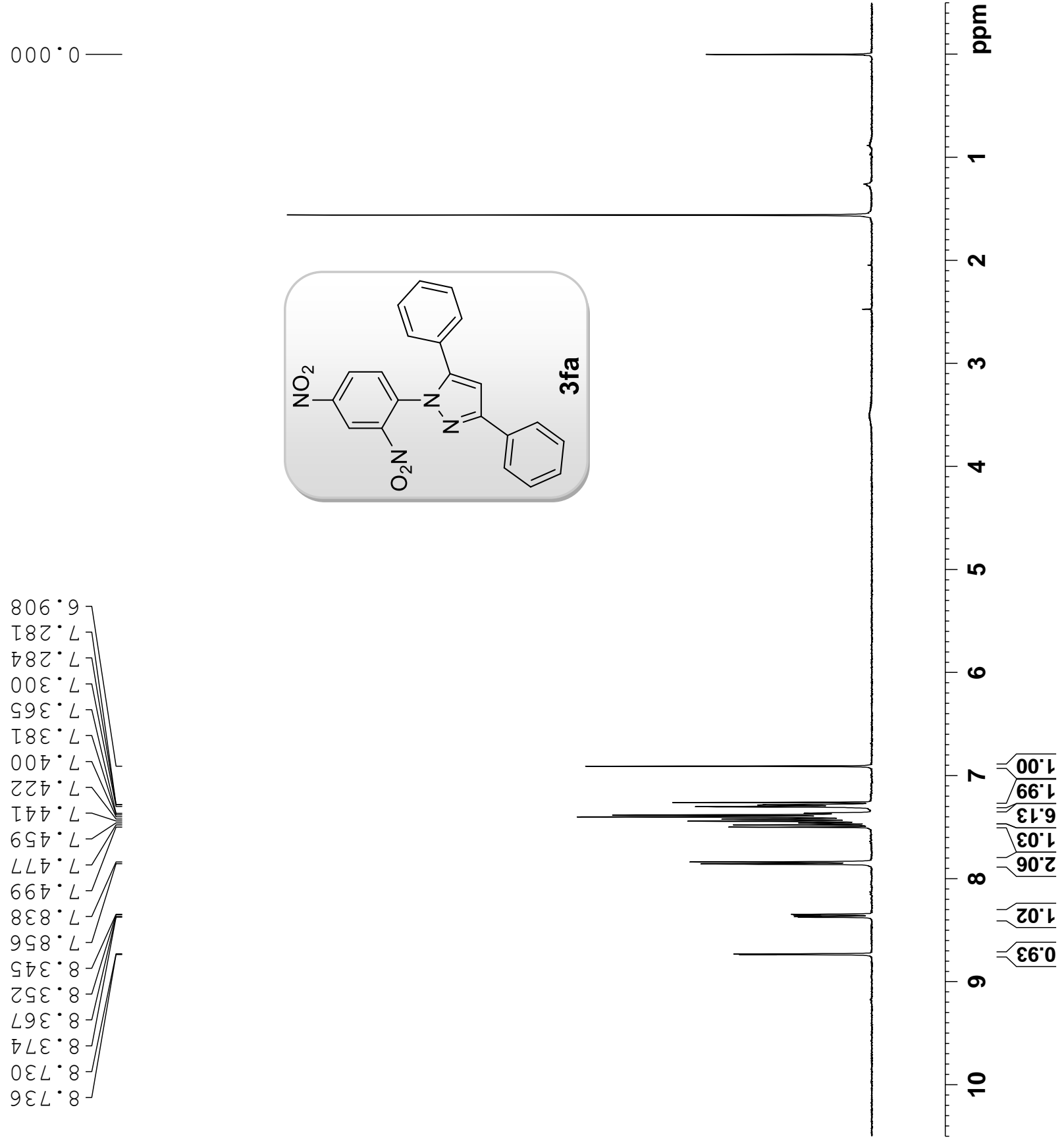


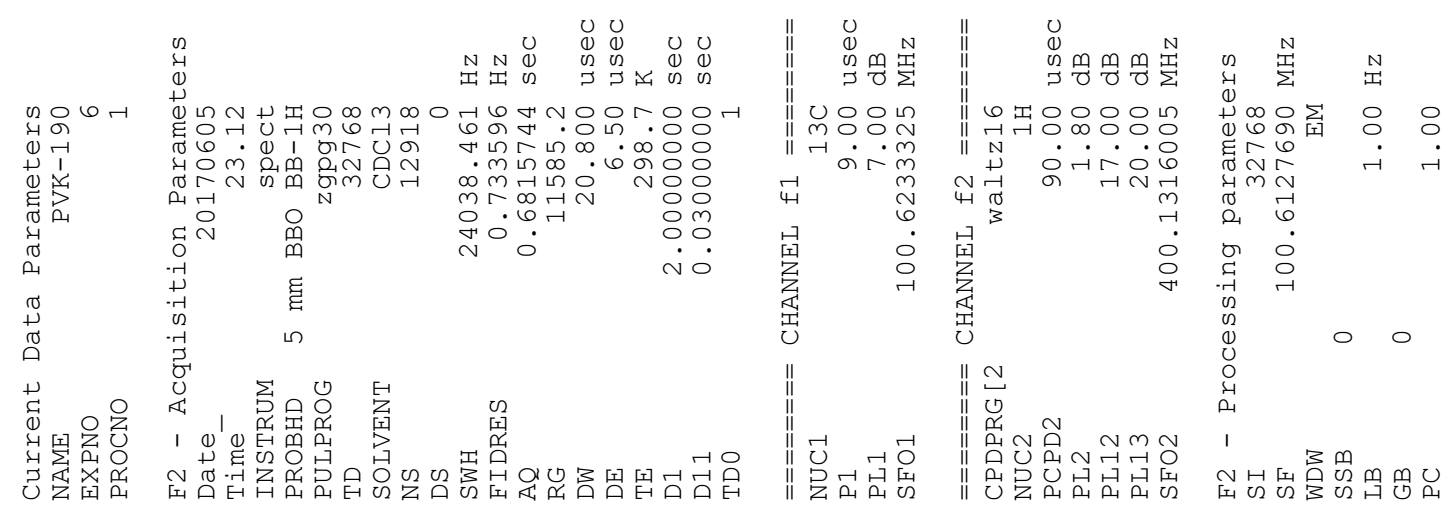

$\varepsilon 0 \cdot 0-$
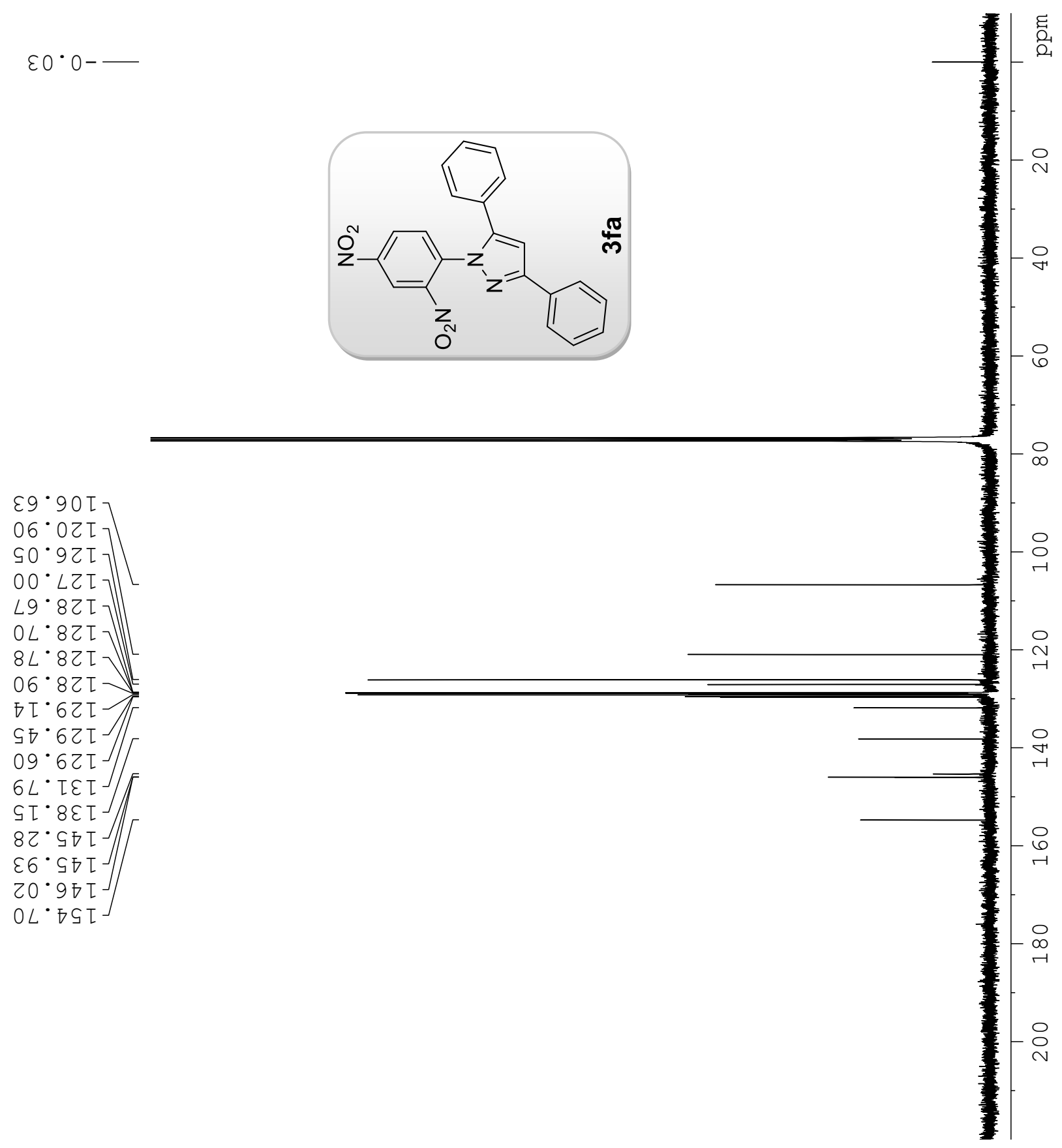


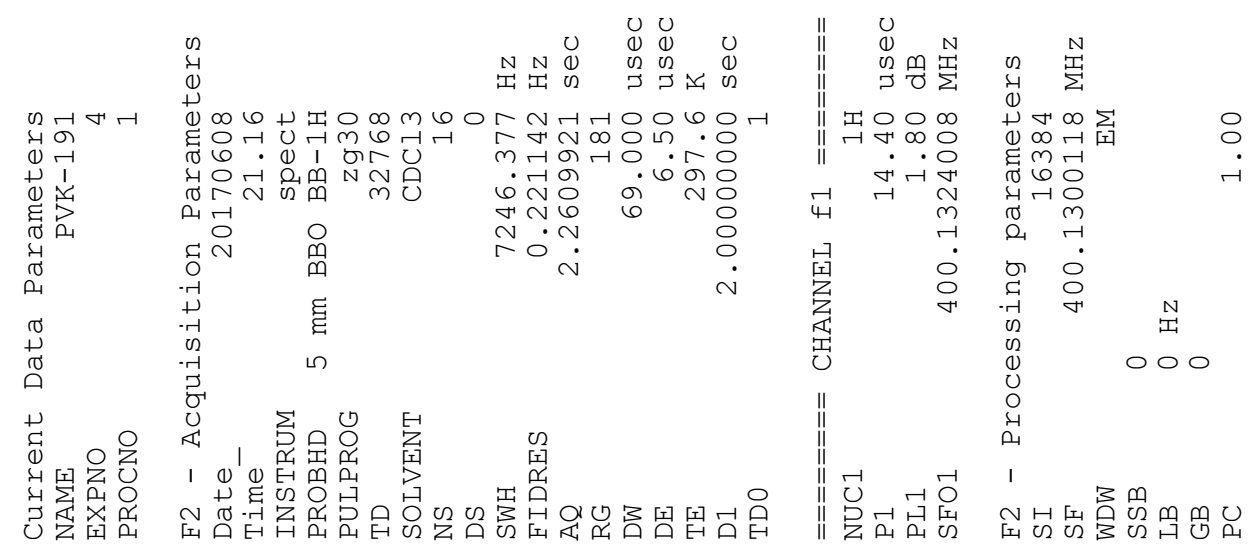

$500 \cdot 0-$
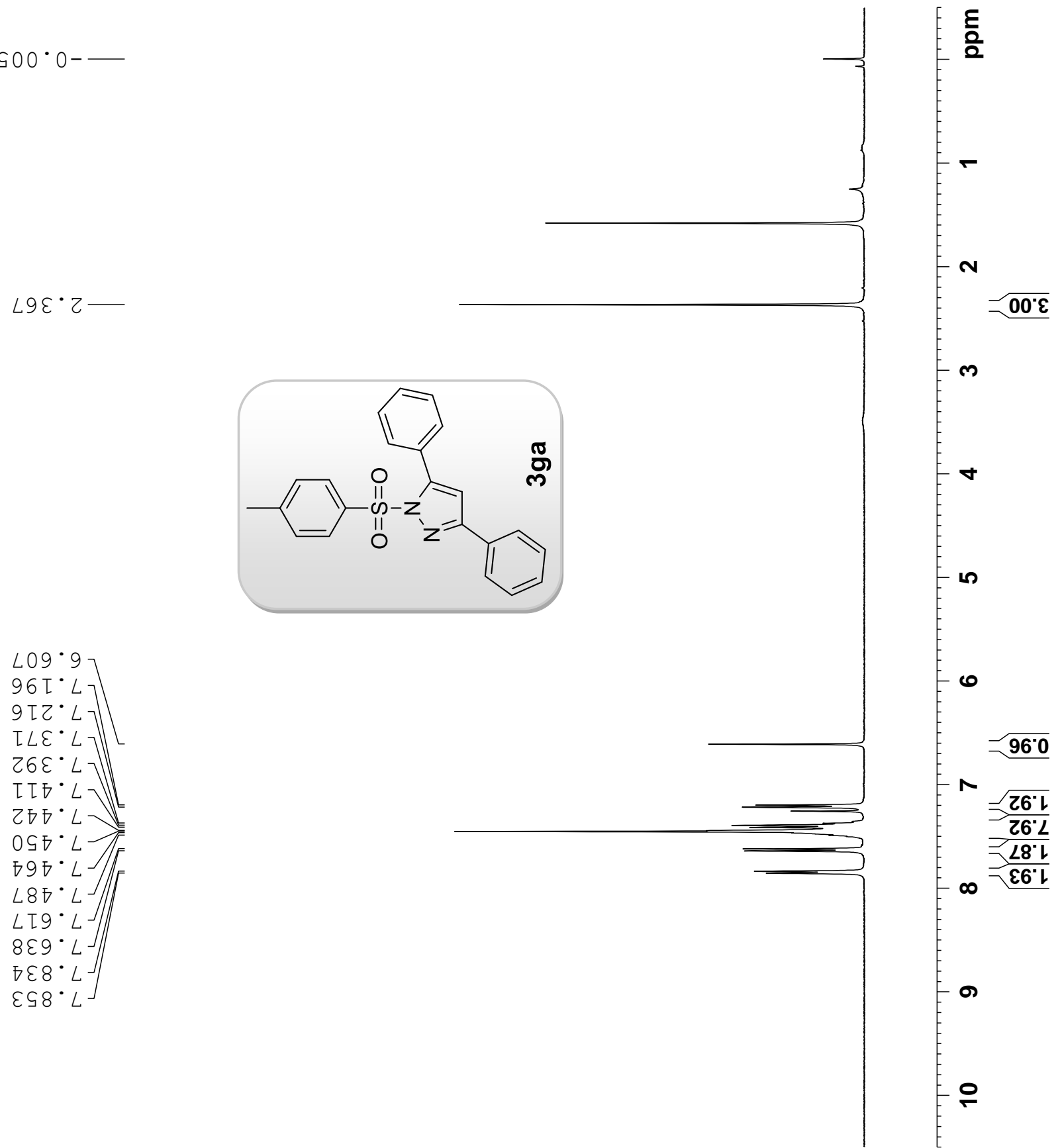

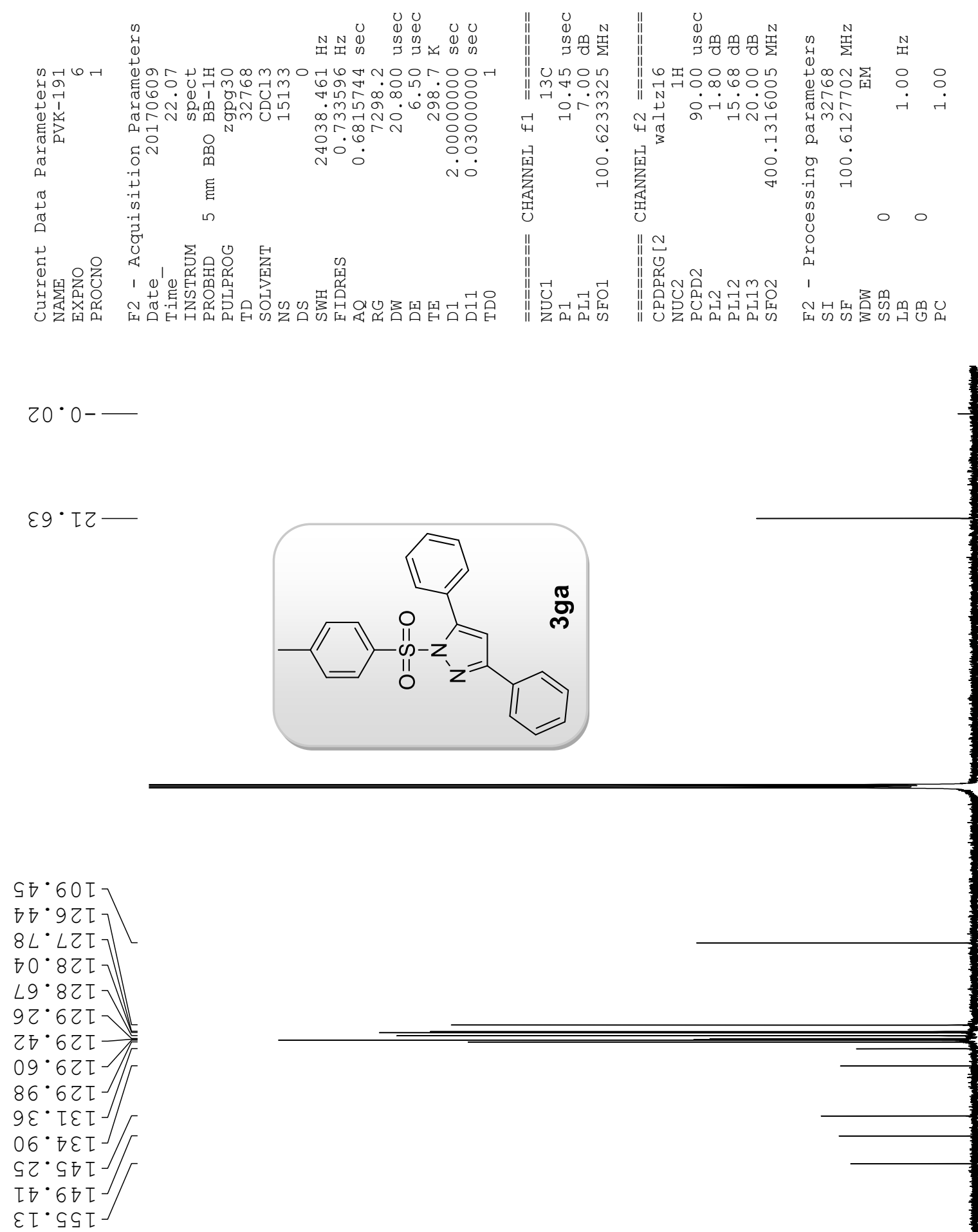

$-\infty$

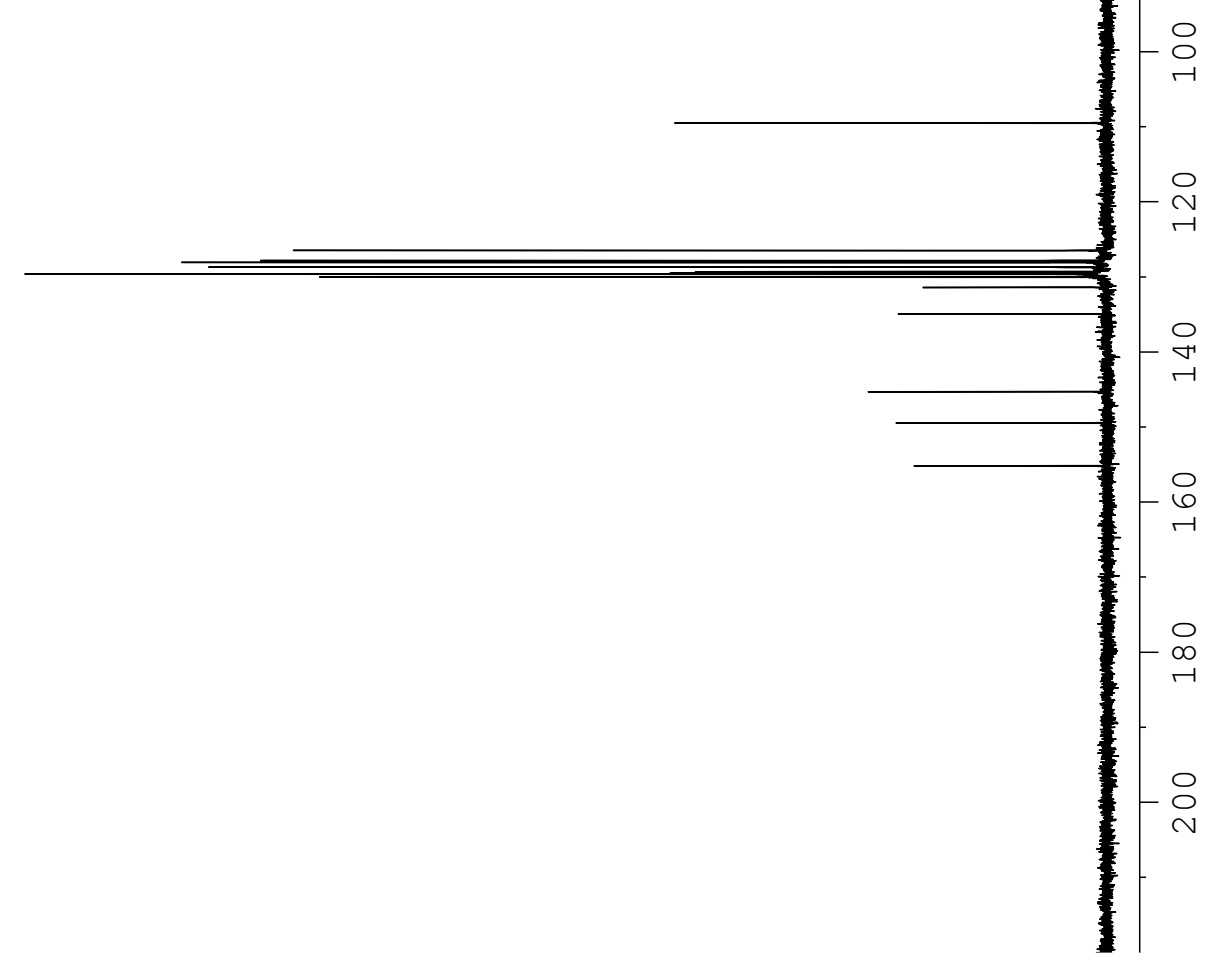




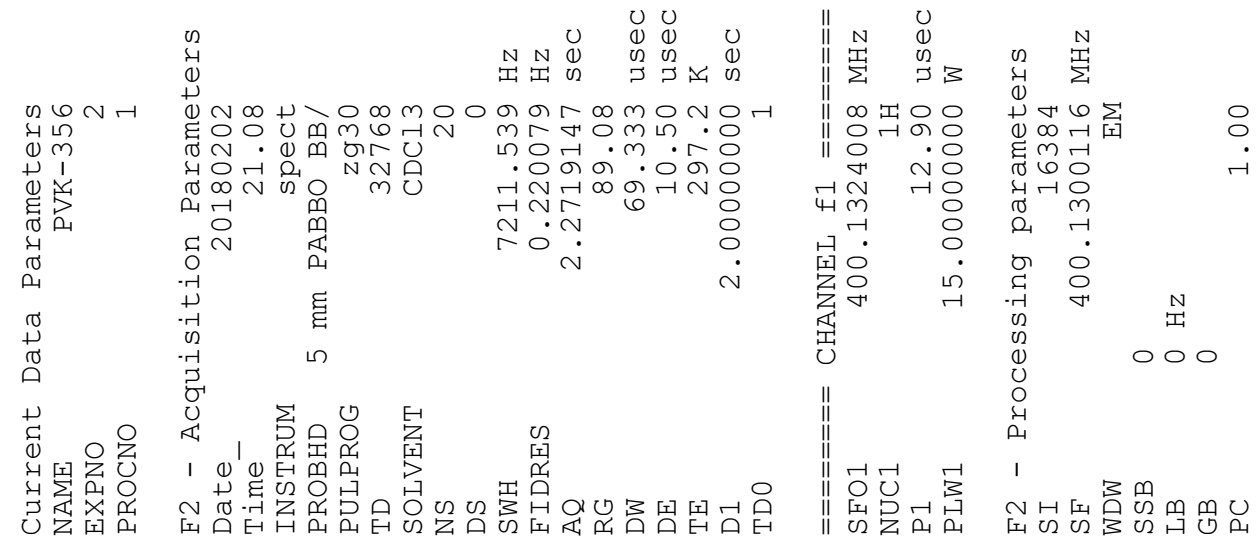

$00 \cdot 0-$

$9 \varepsilon \cdot 乙-$

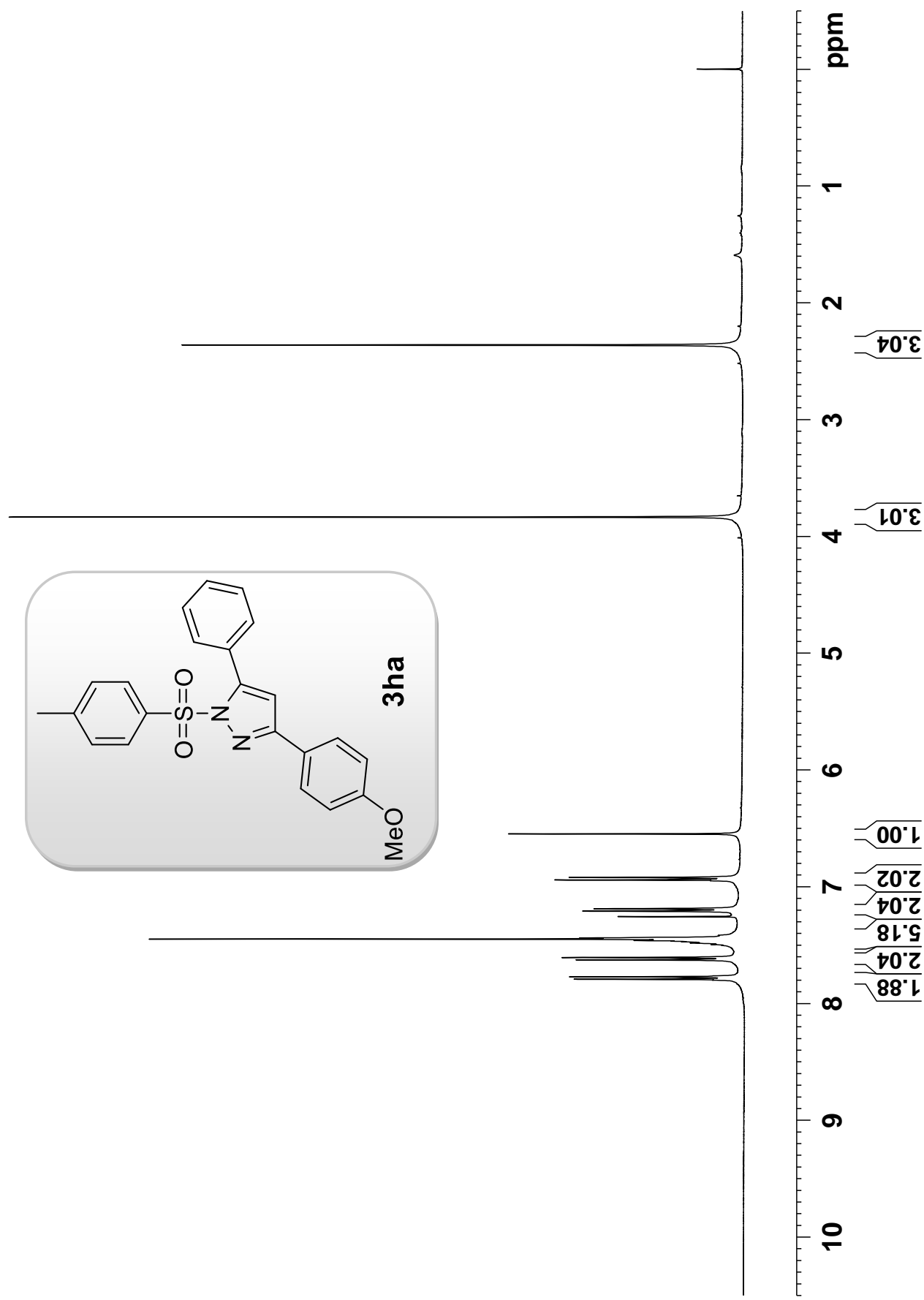

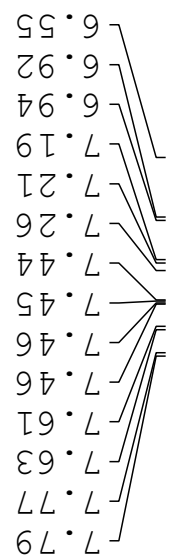



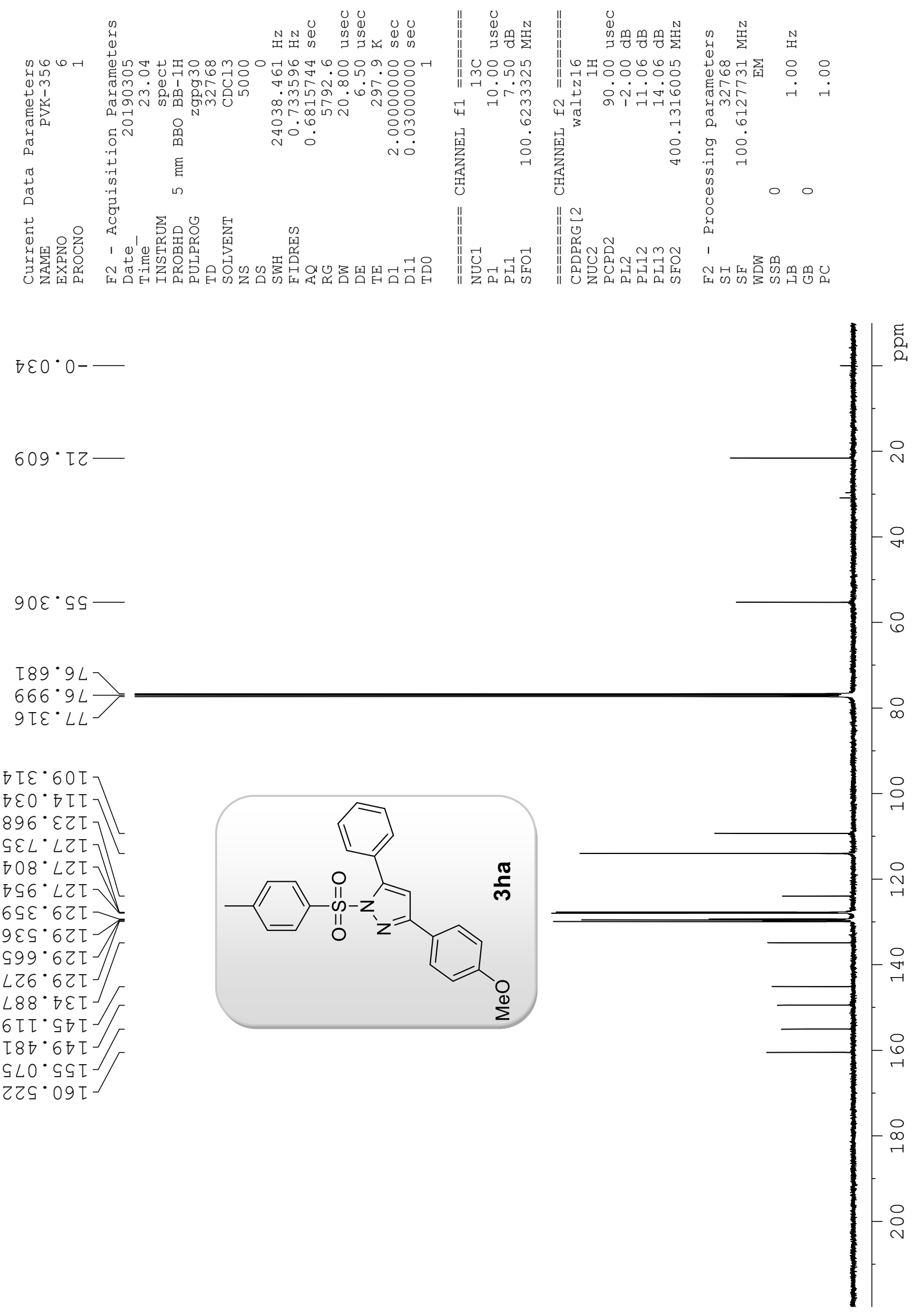


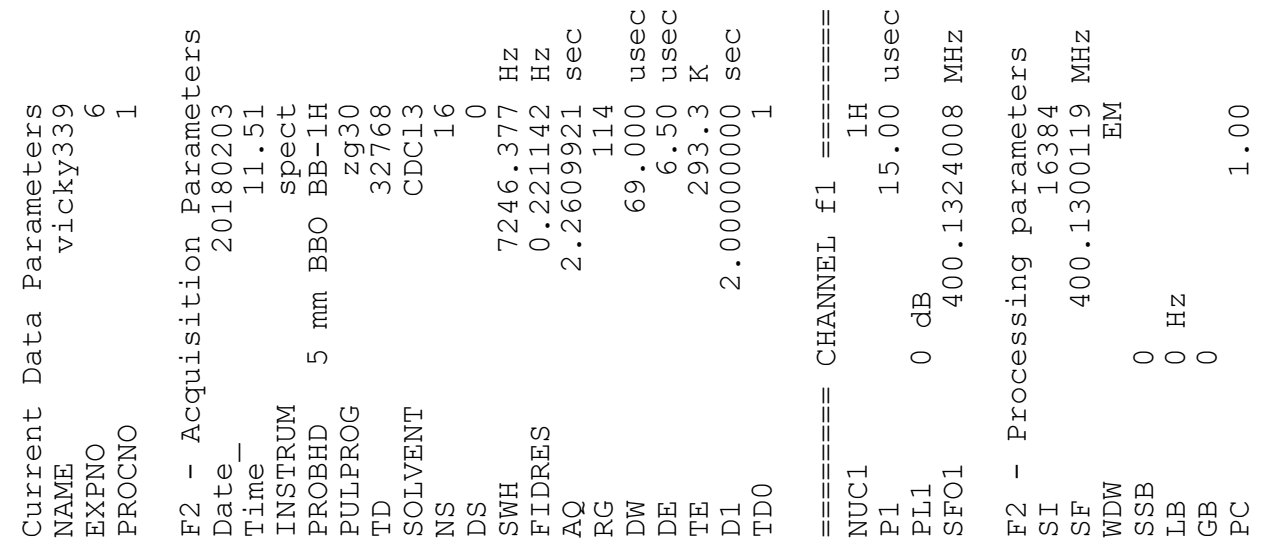

$00 \cdot 0$

$0 \nabla^{*} 2$
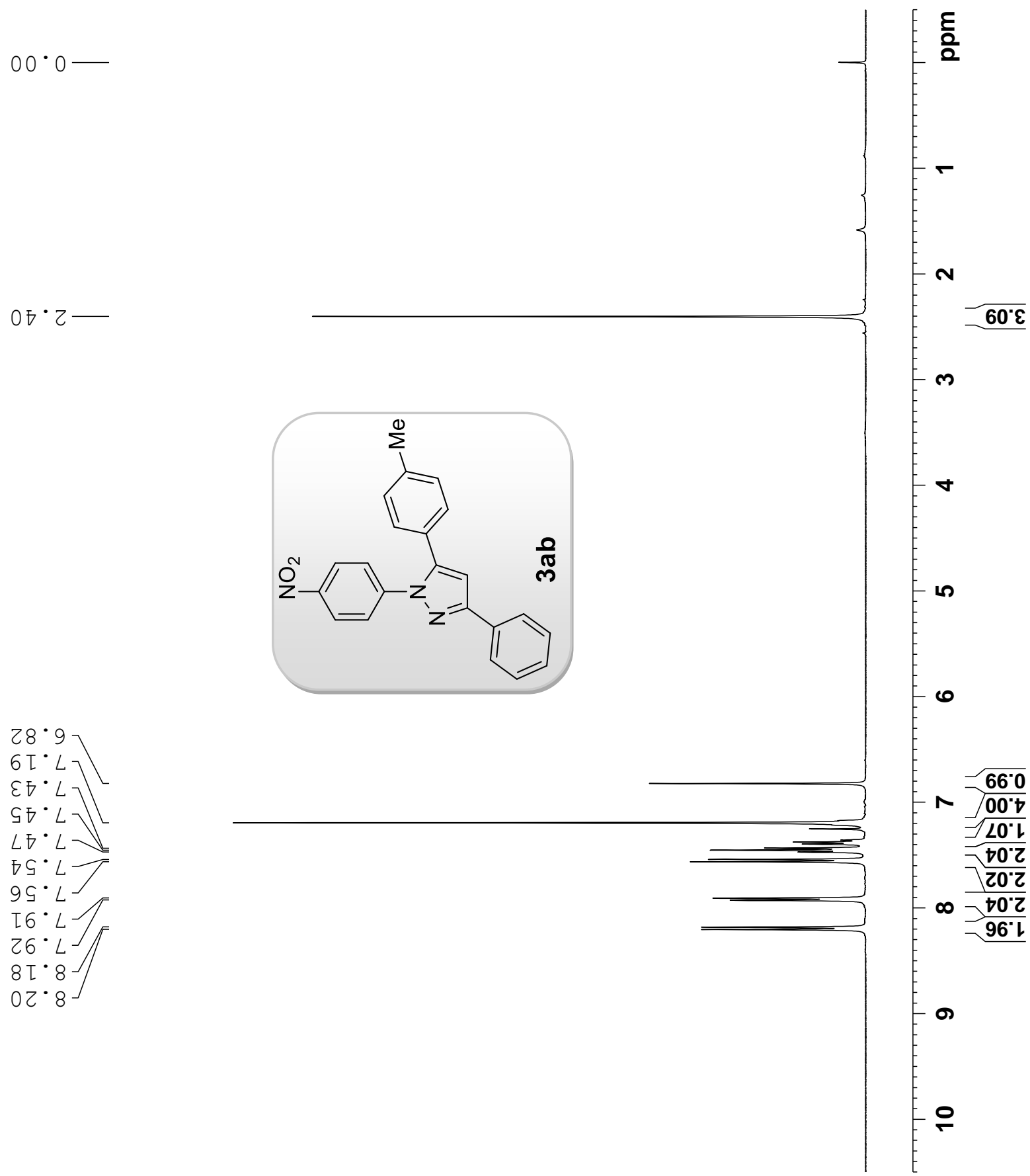


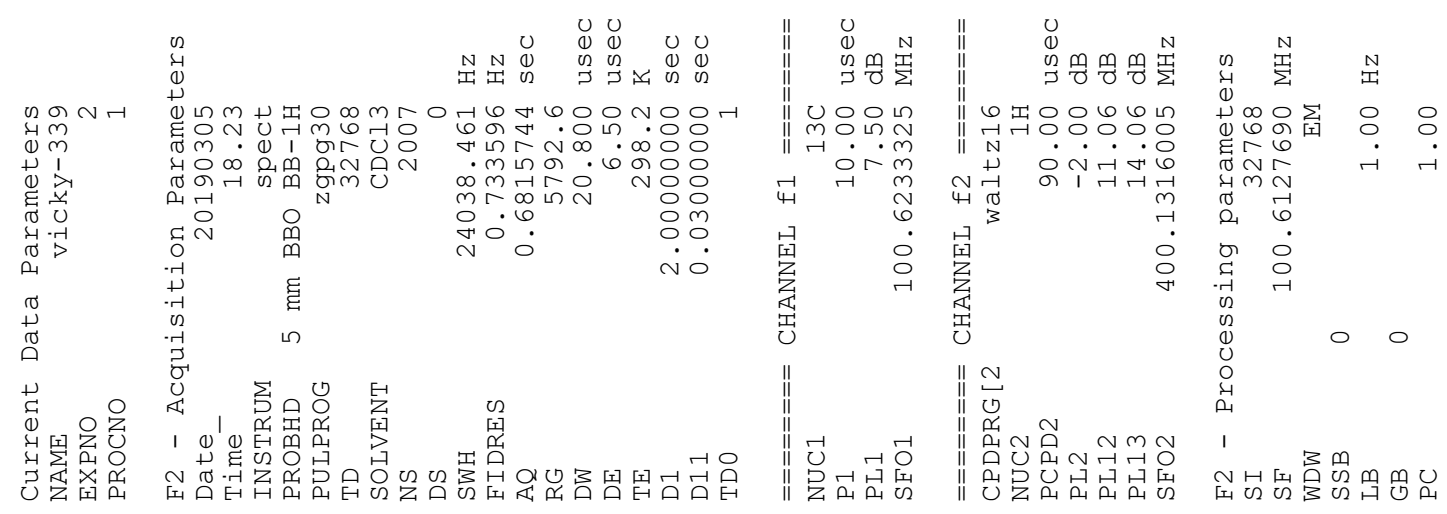

$220 \cdot 0-$

$6 乙 \varepsilon^{\bullet} \mathrm{\tau}$
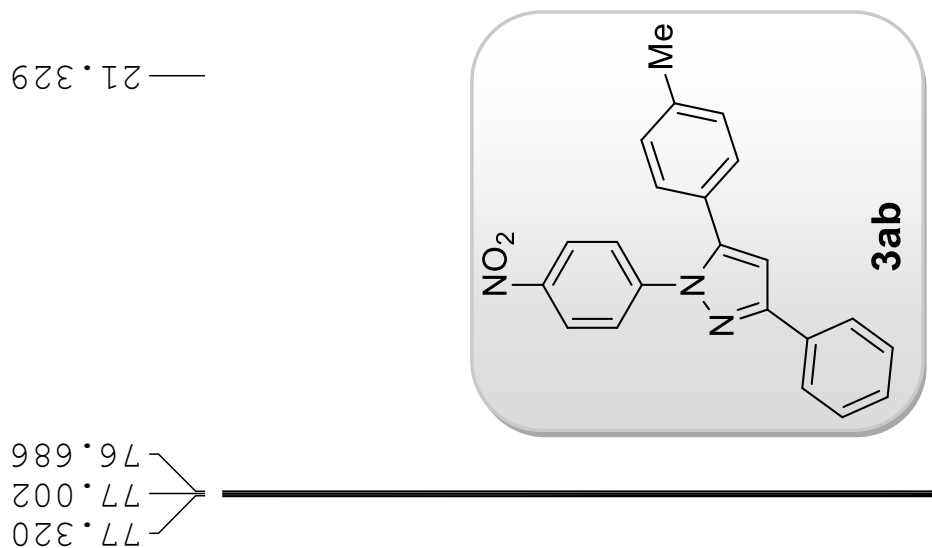

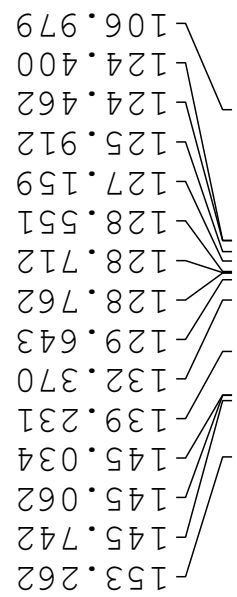

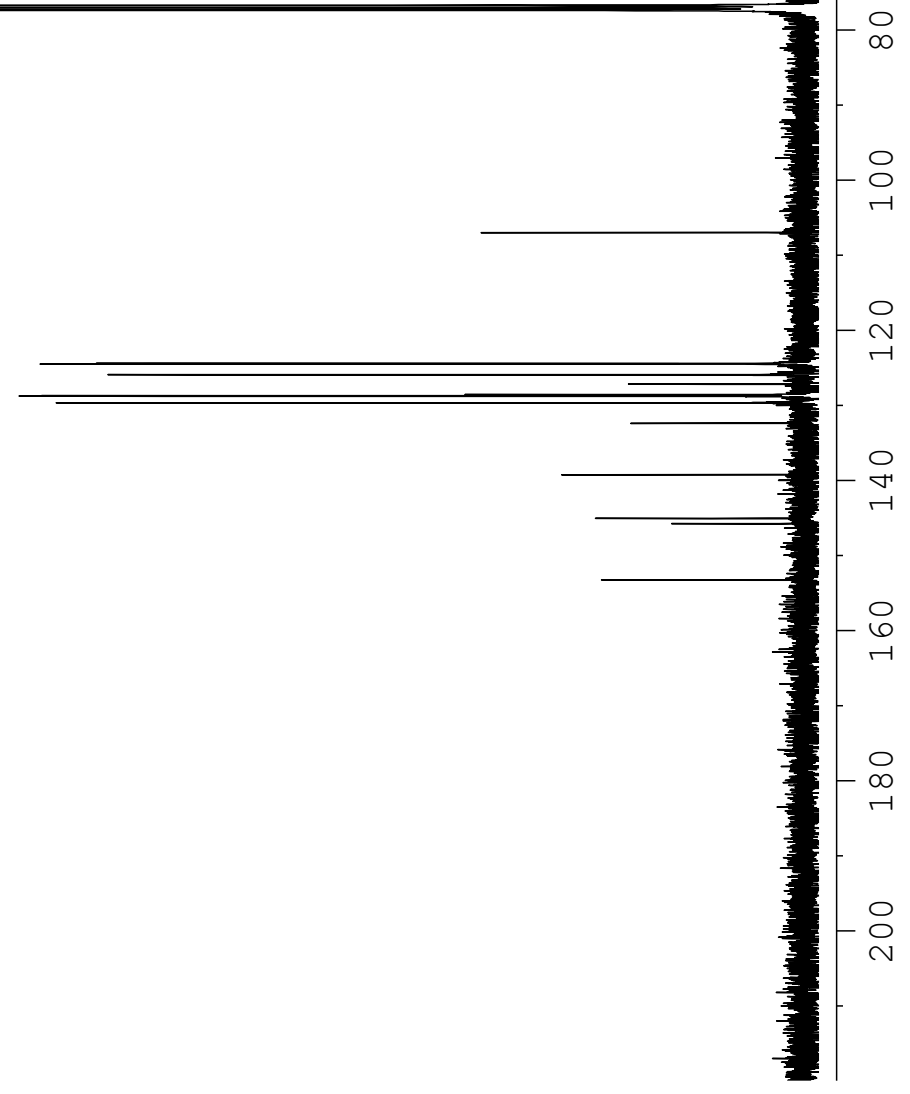




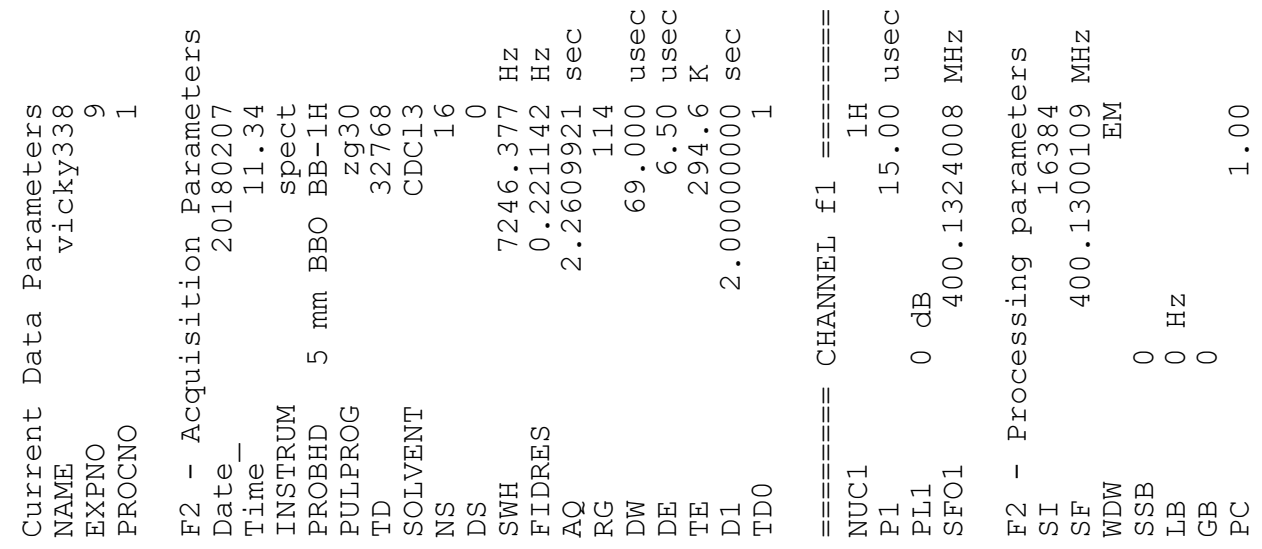

$00 \cdot 0$

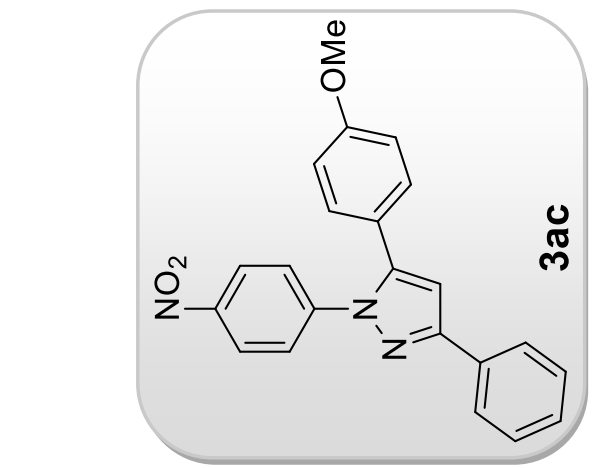

$98^{\circ} \varepsilon$
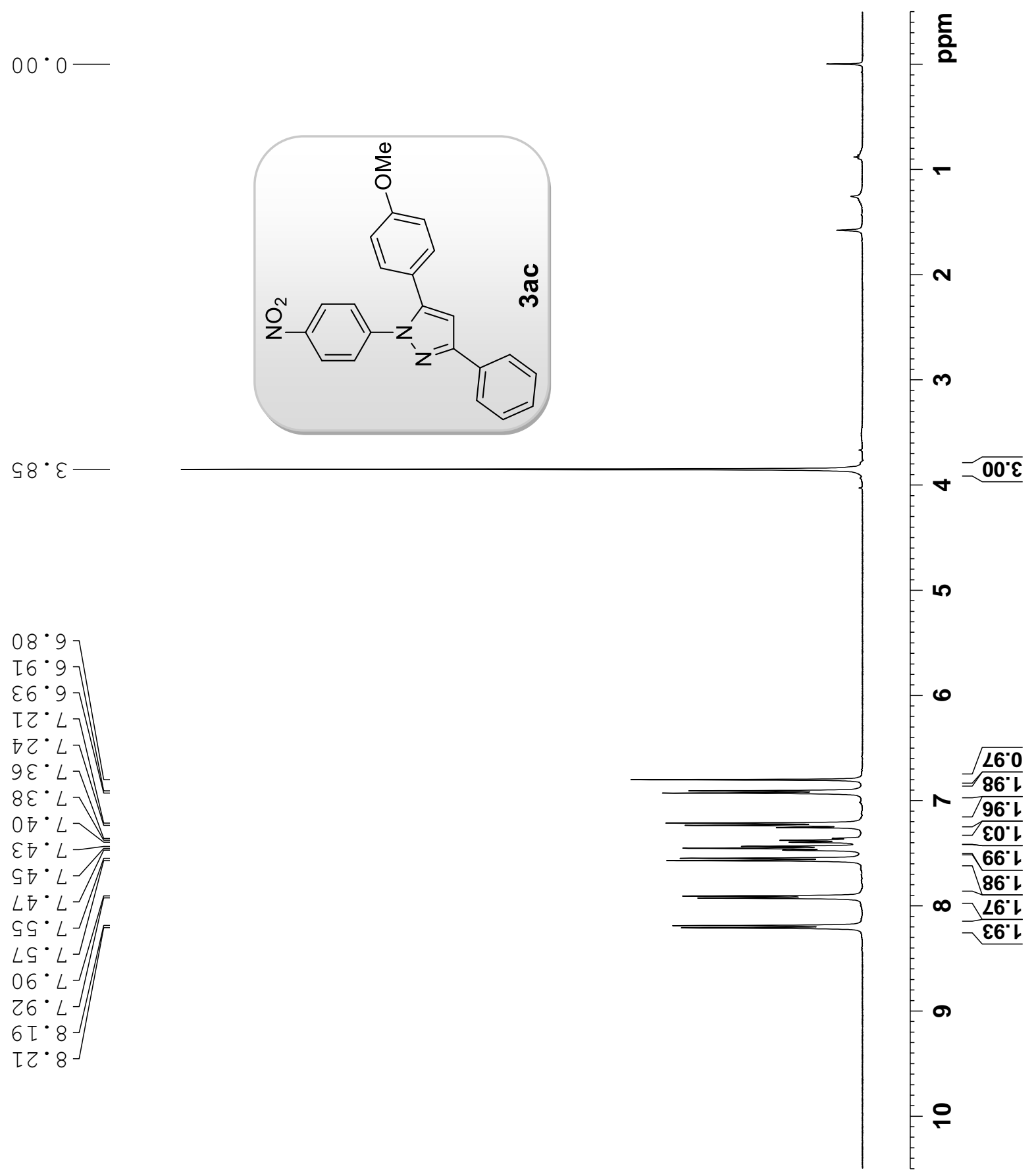

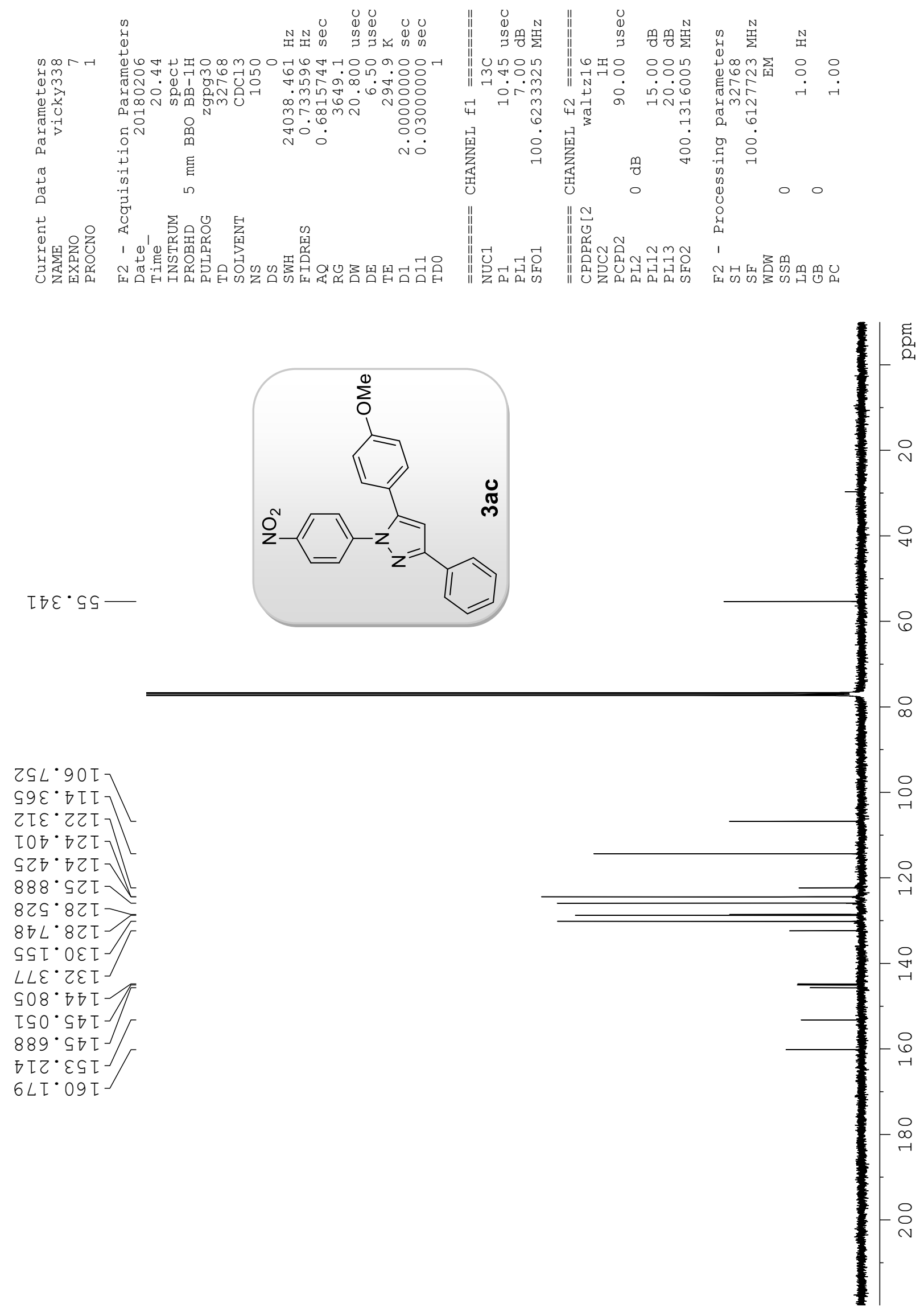

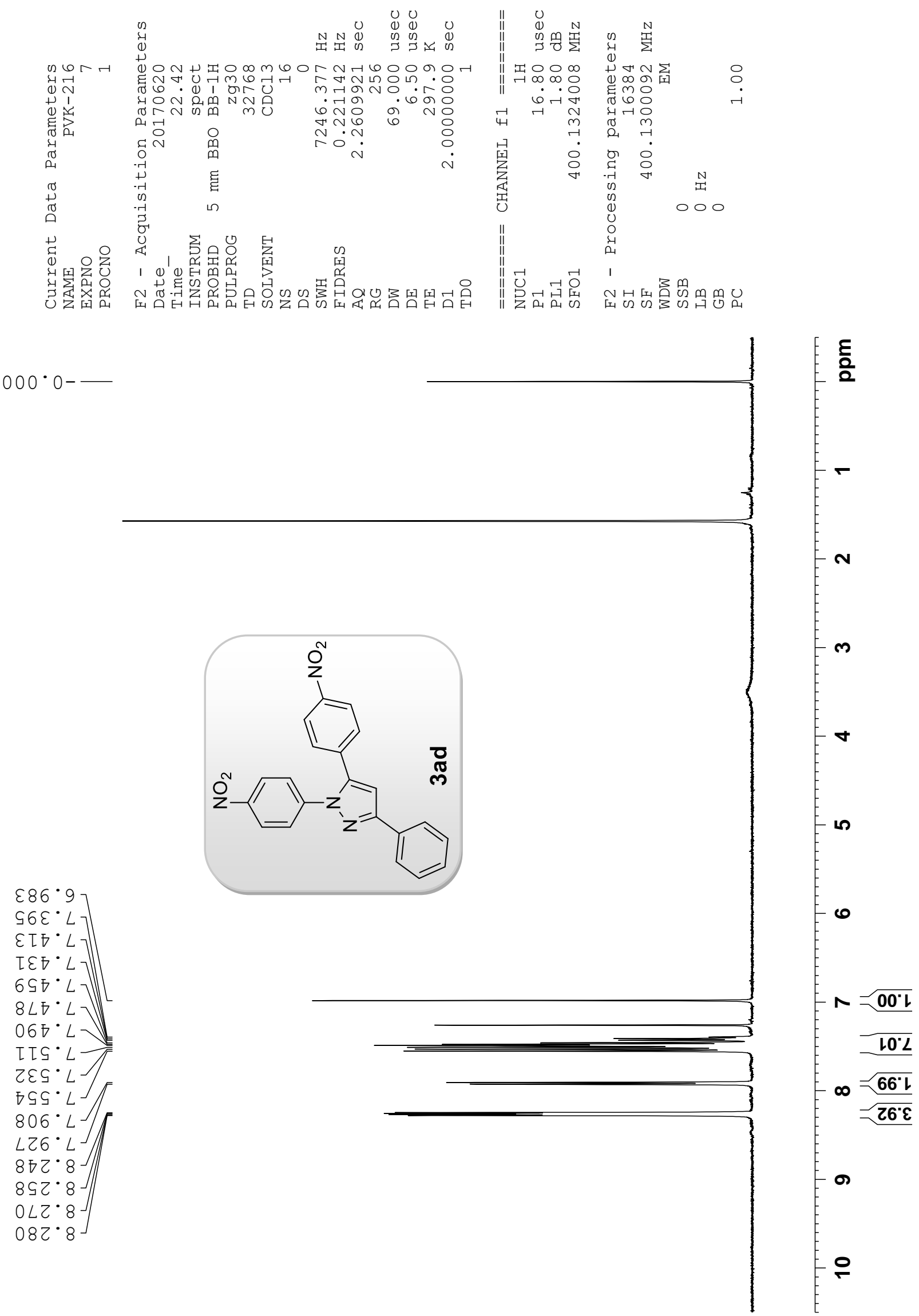


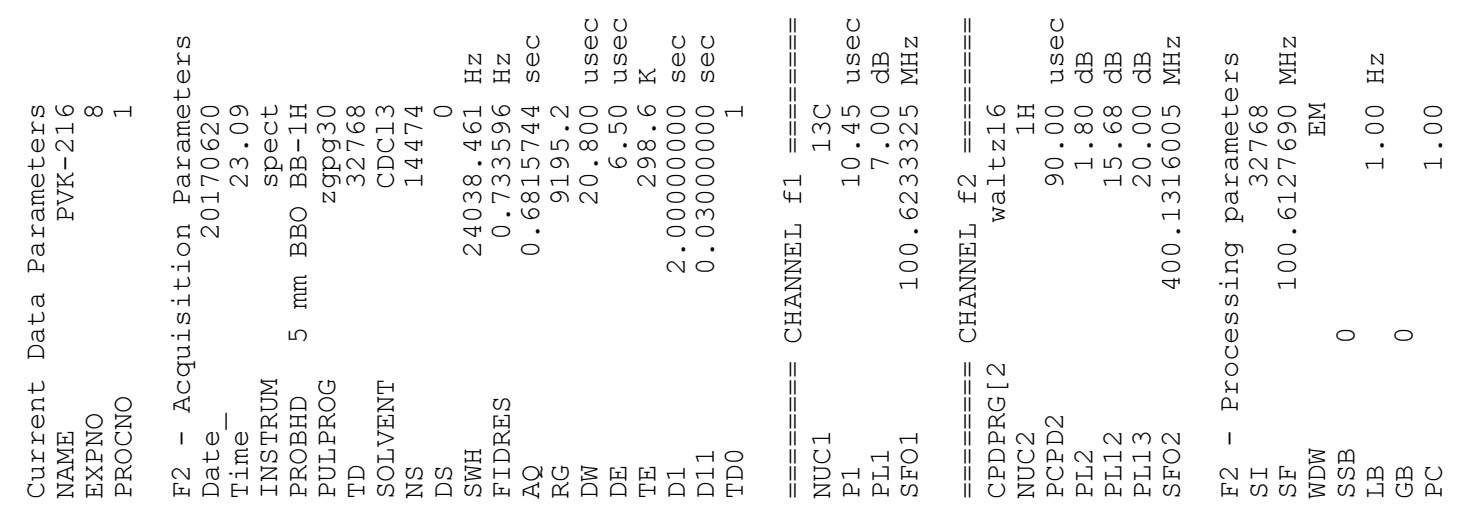

LO $0-$
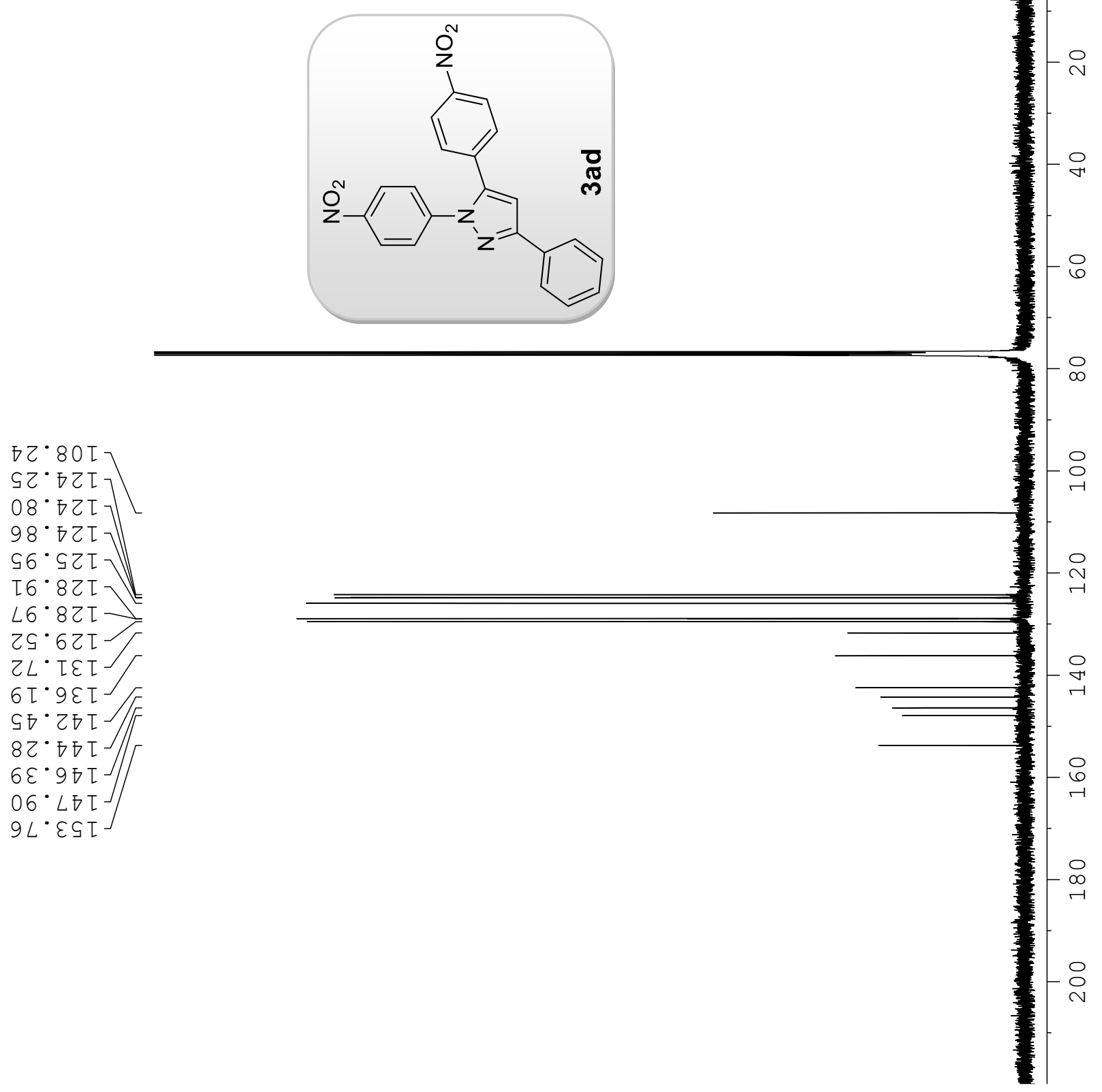


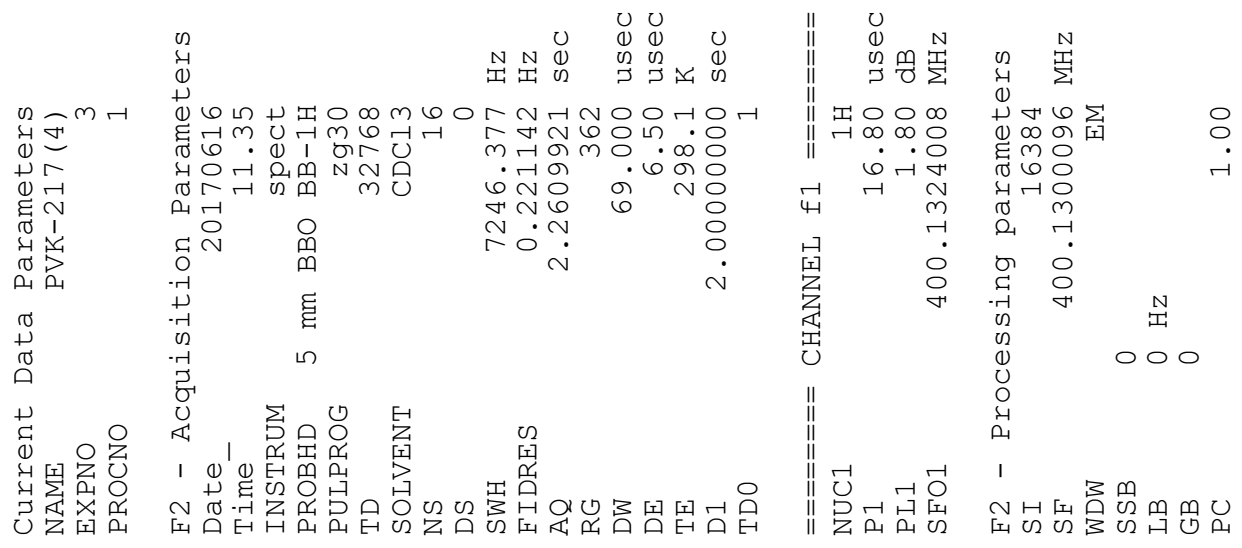

$000 \cdot 0-$
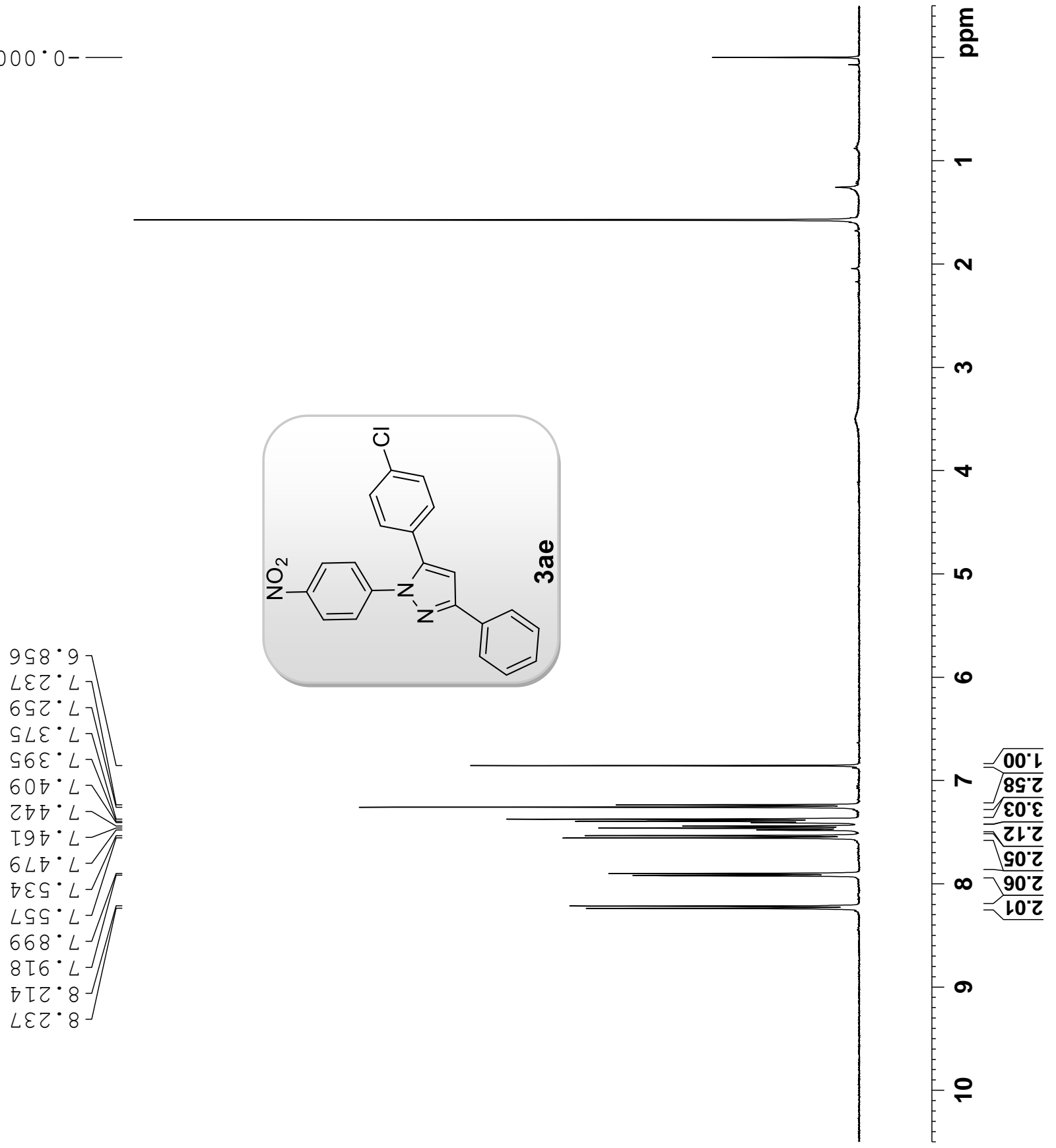

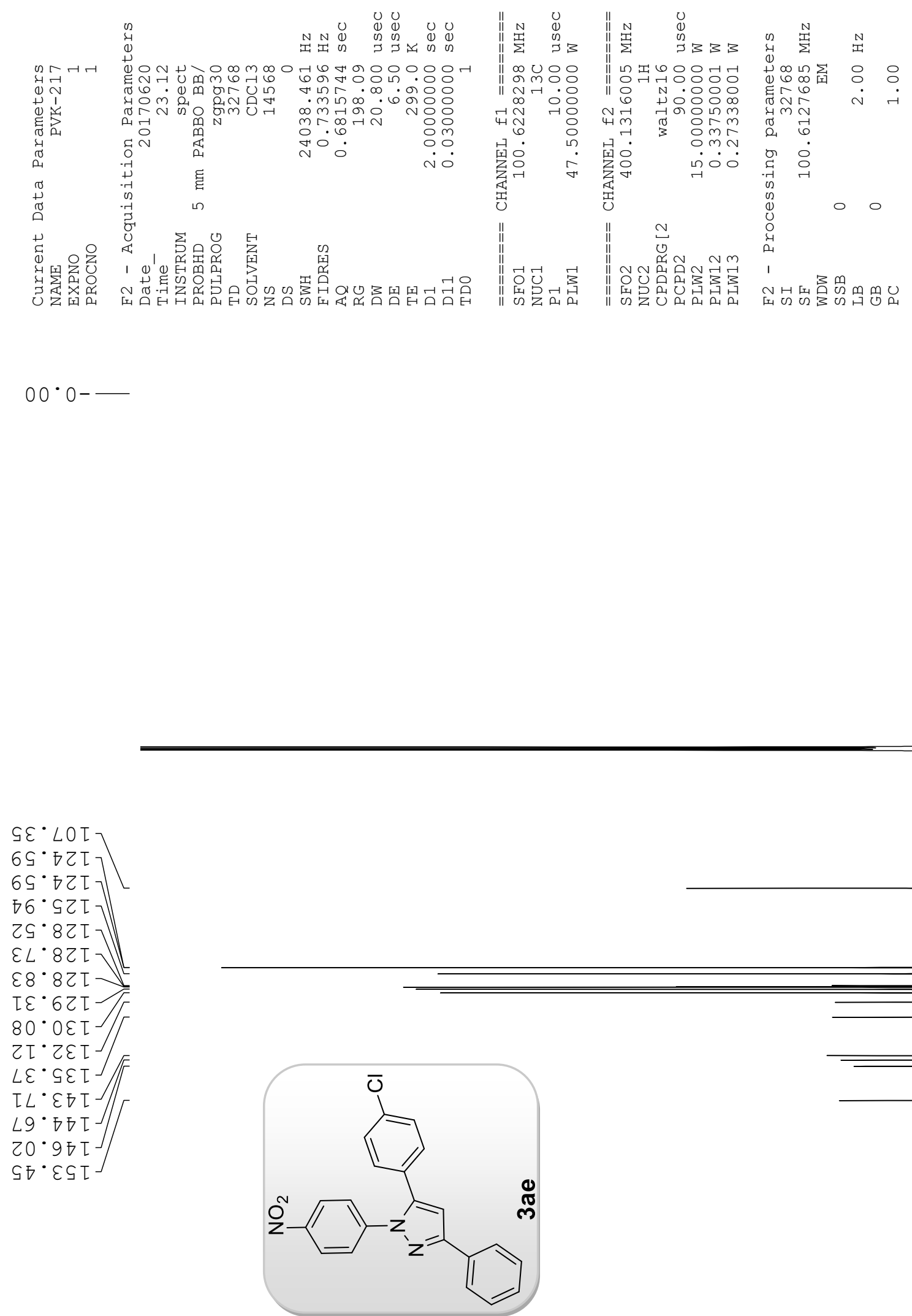

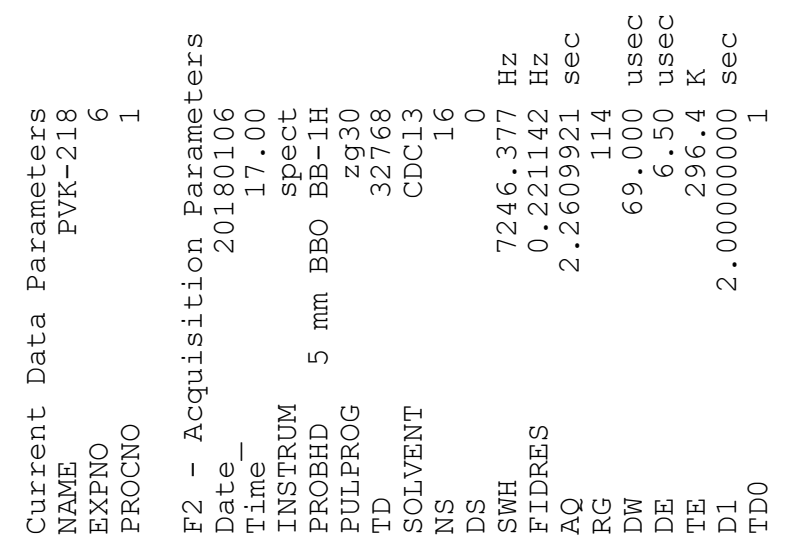

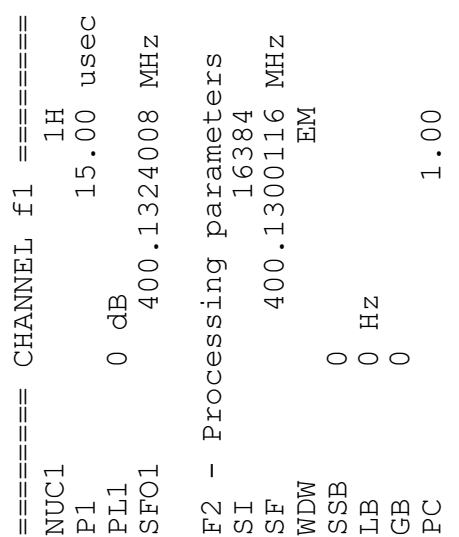
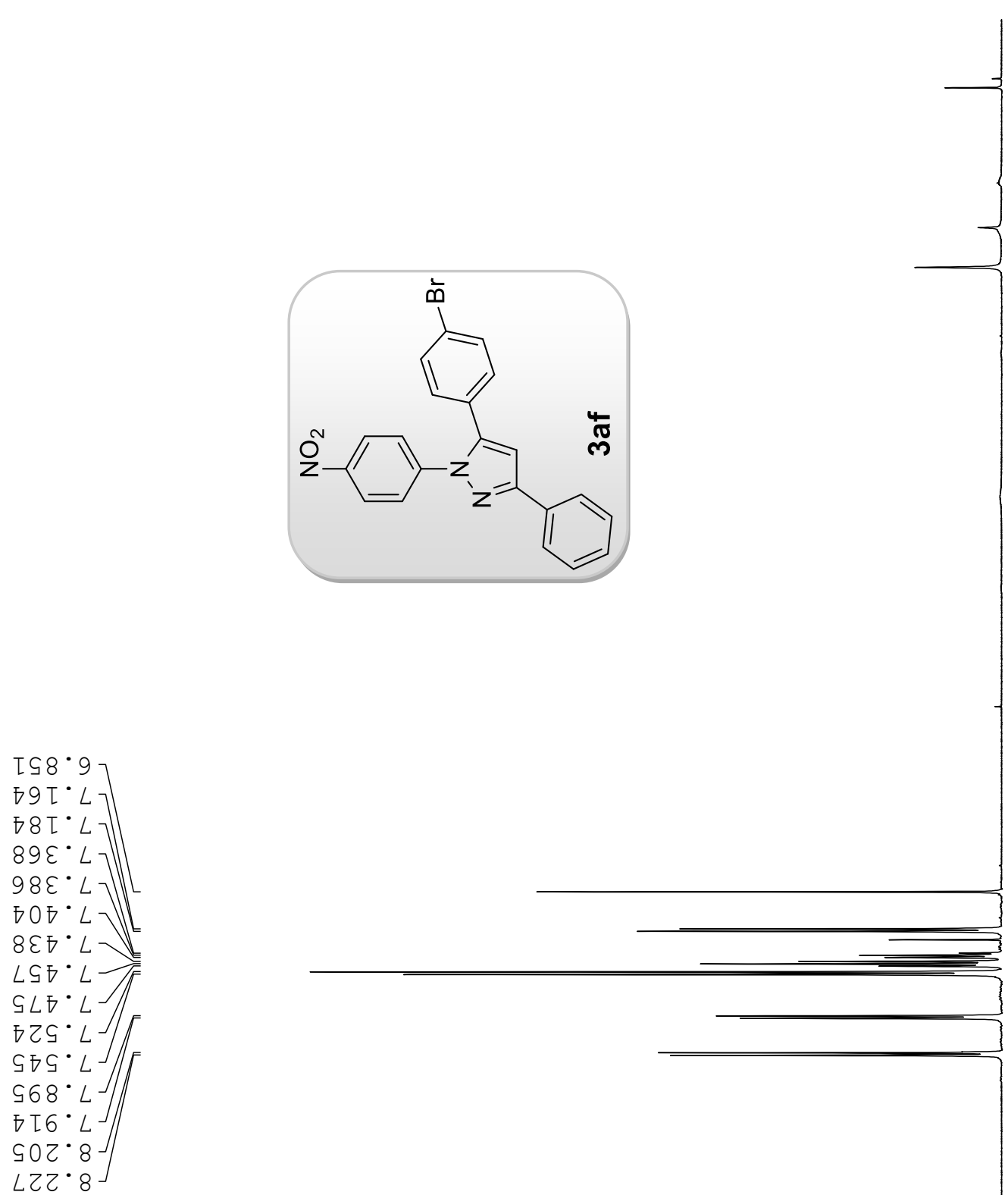

$N$

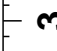

0

$\wedge \frac{\sqrt{00.1}}{10^{\circ} \mathrm{Z}}$ 10.1 $\sqrt{20 \cdot z}$

$\infty$

$20^{\circ} z$ $66^{\circ}$

0

으 

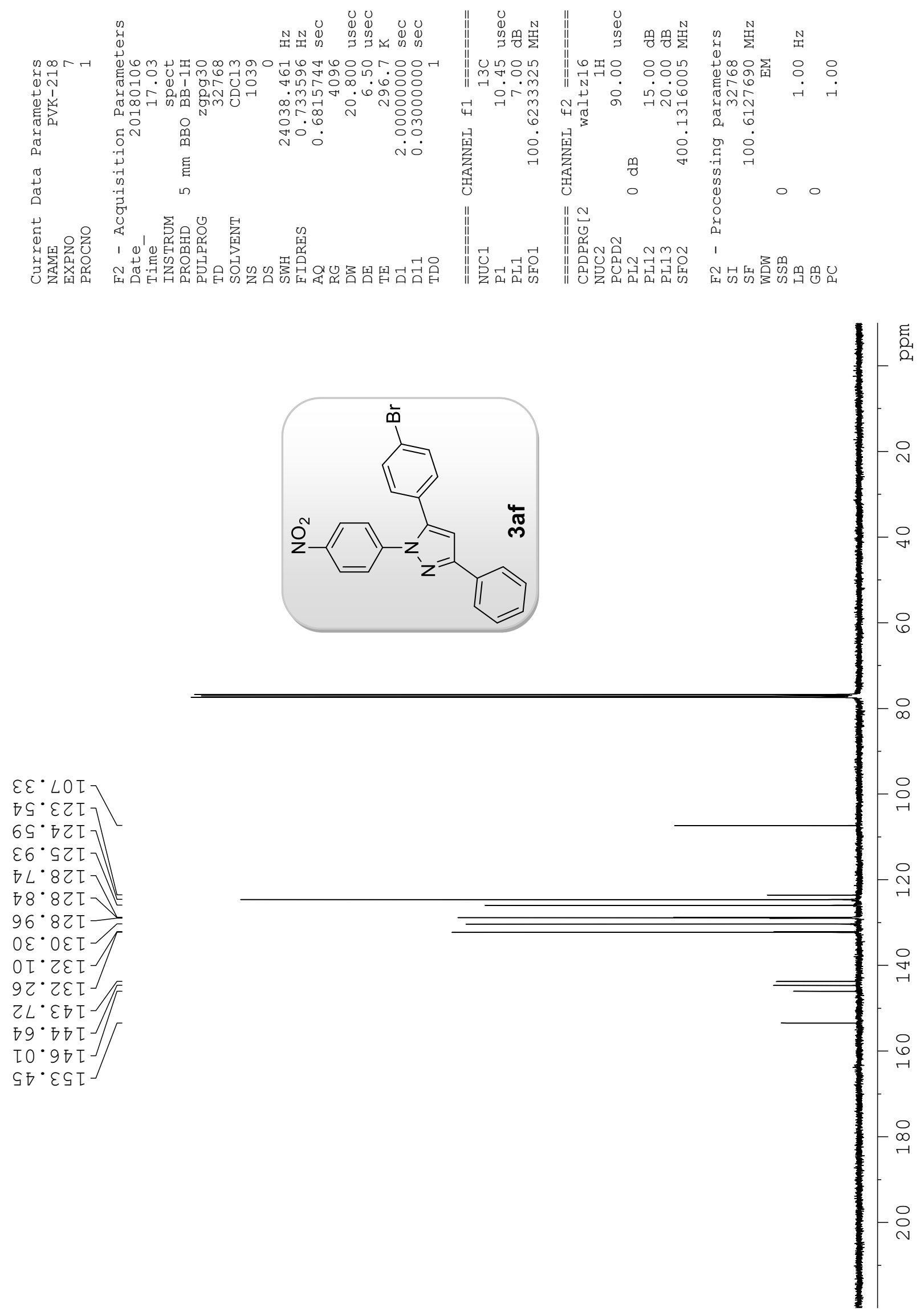


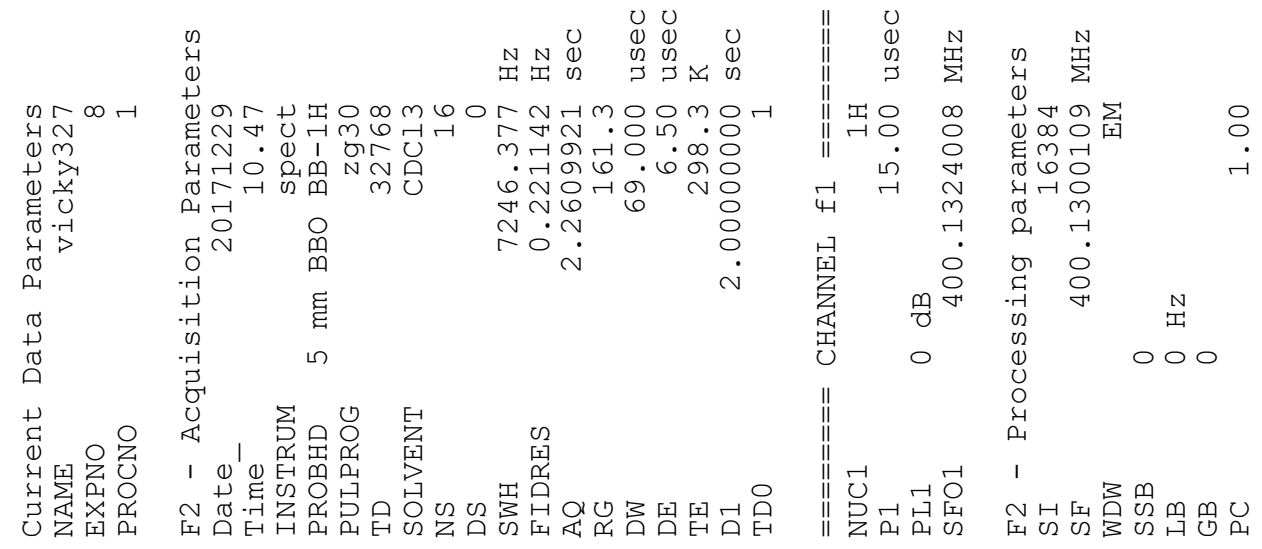

$00 \cdot 0$
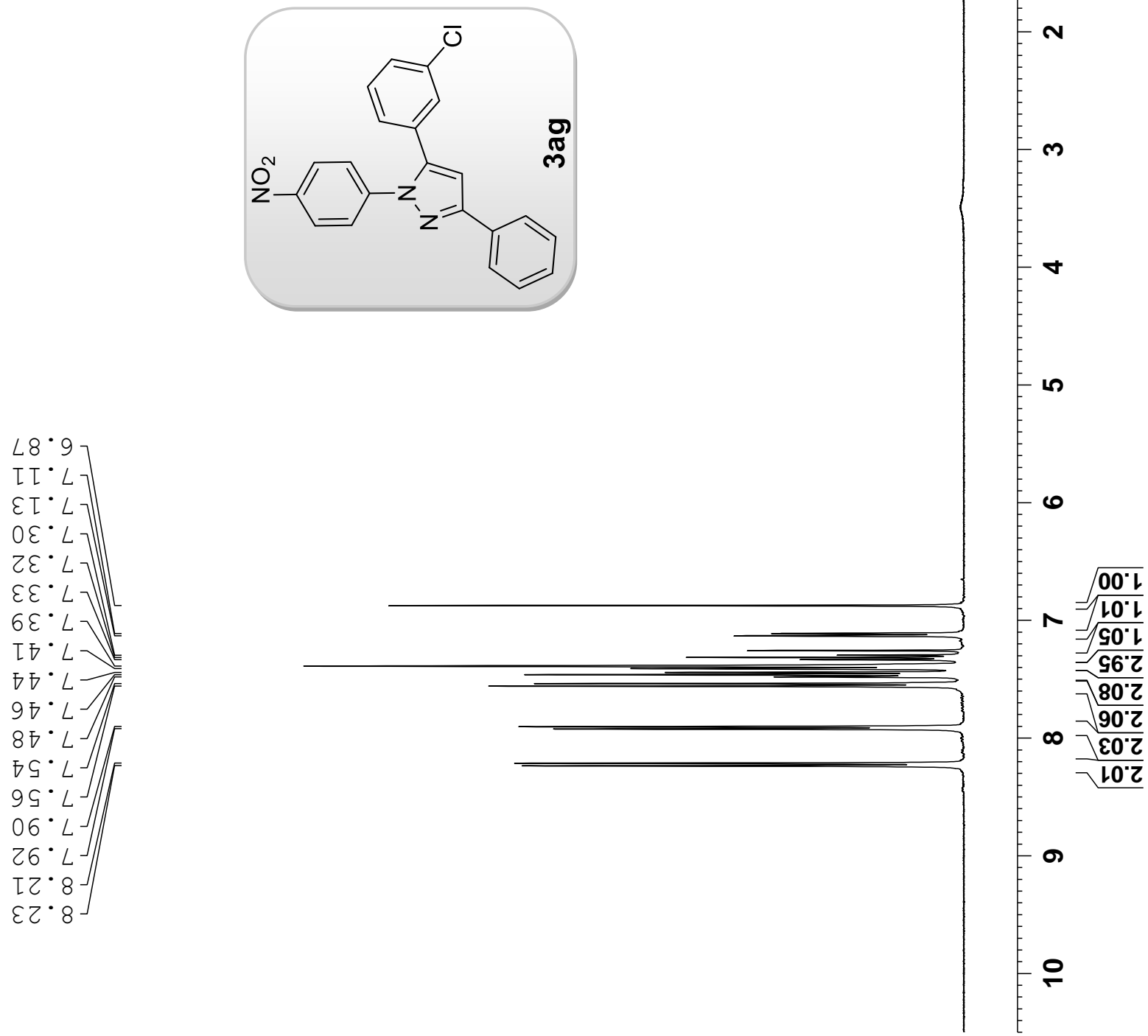

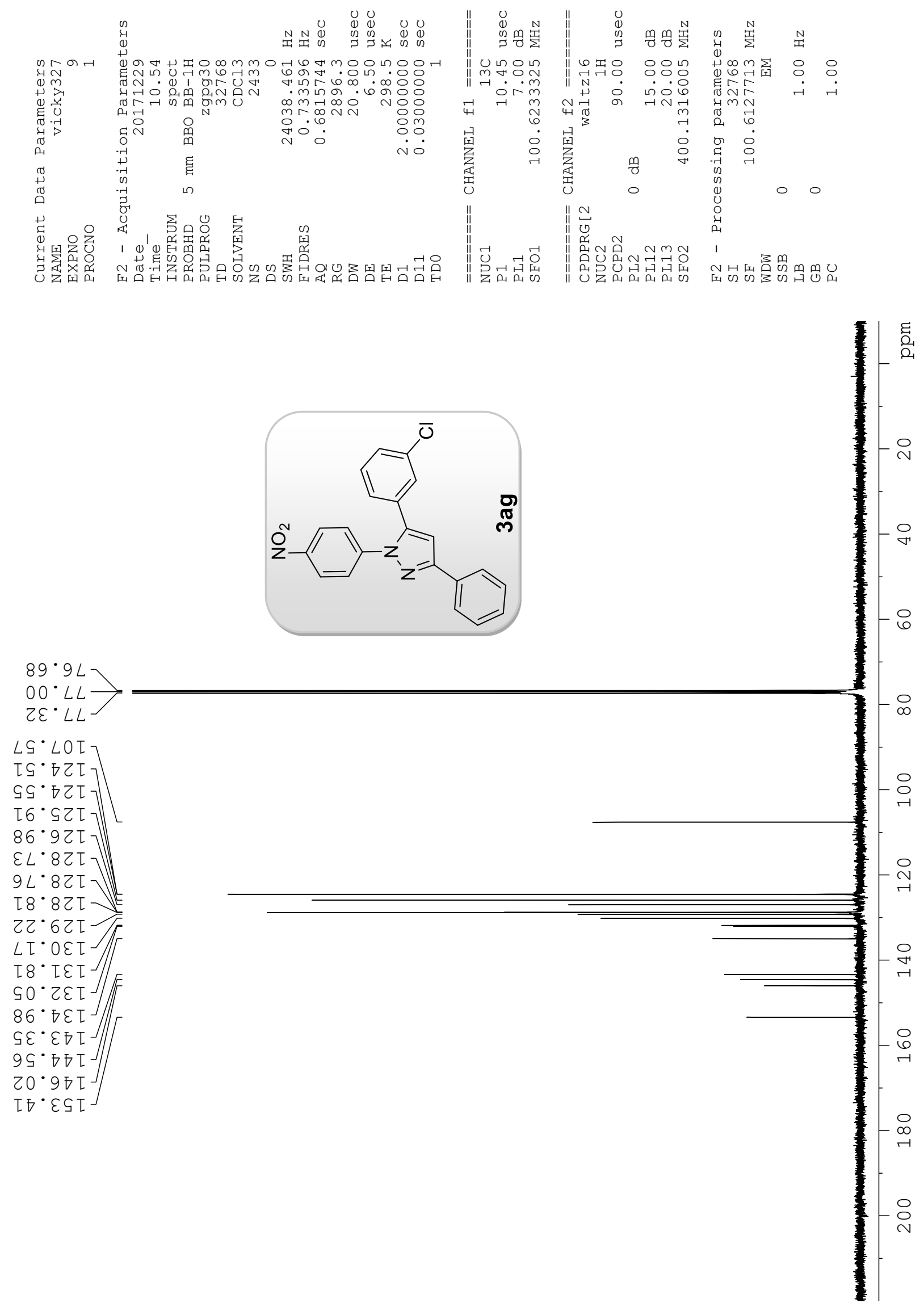


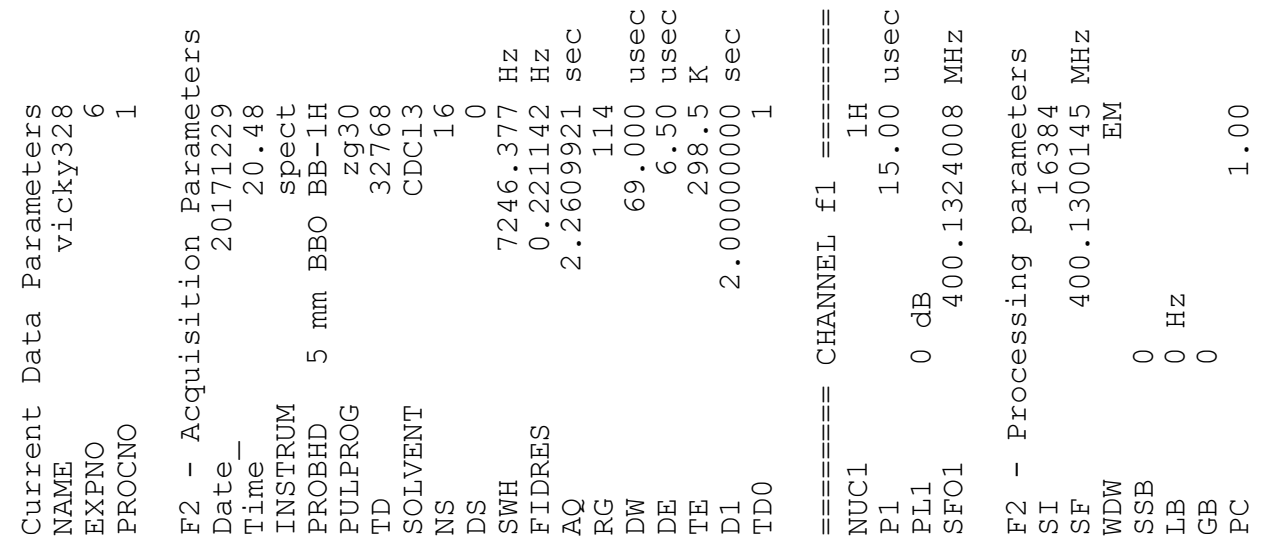

$00 \cdot 0-$

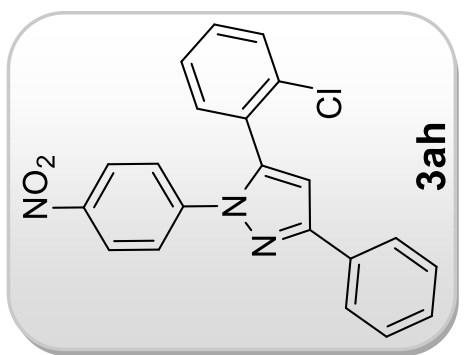

흥
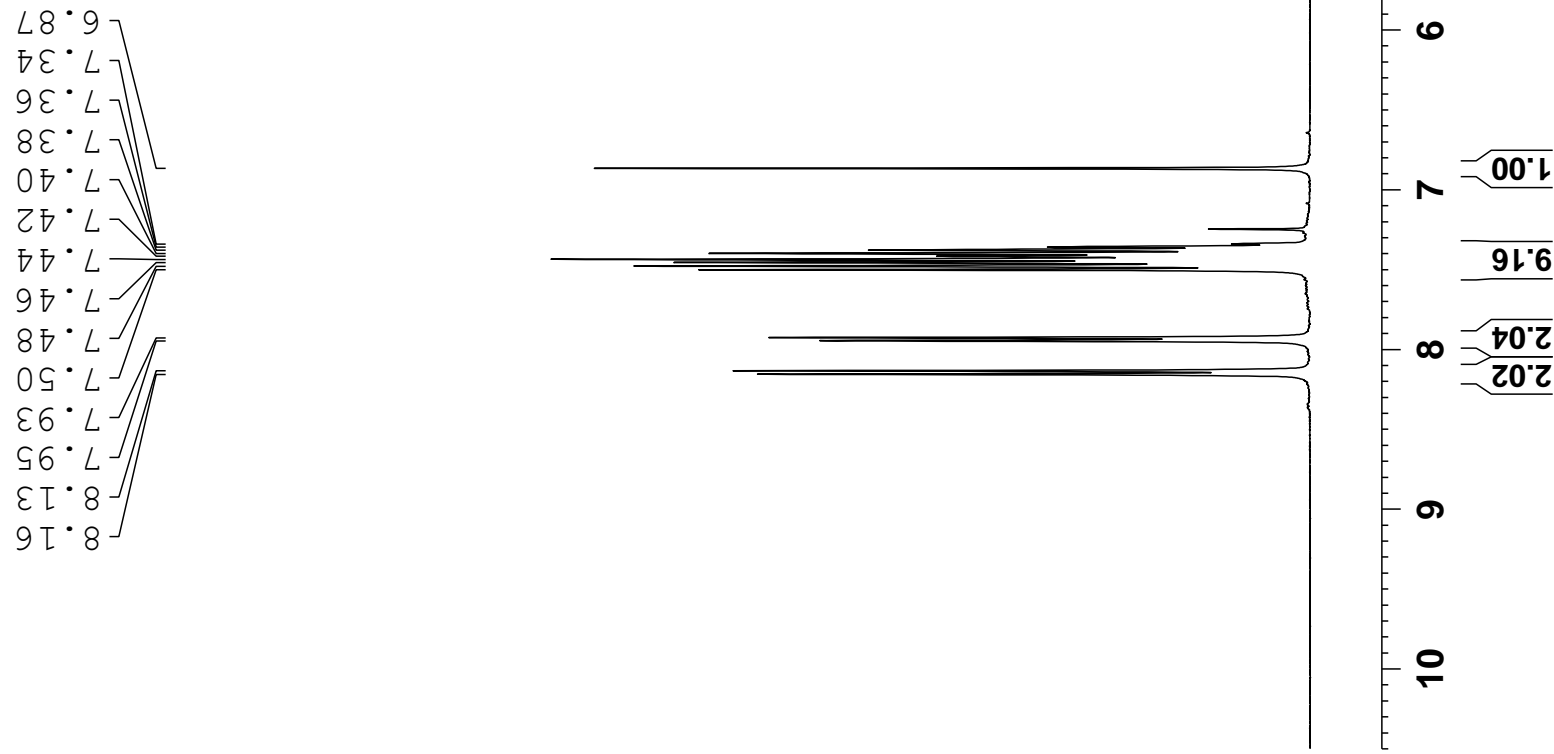

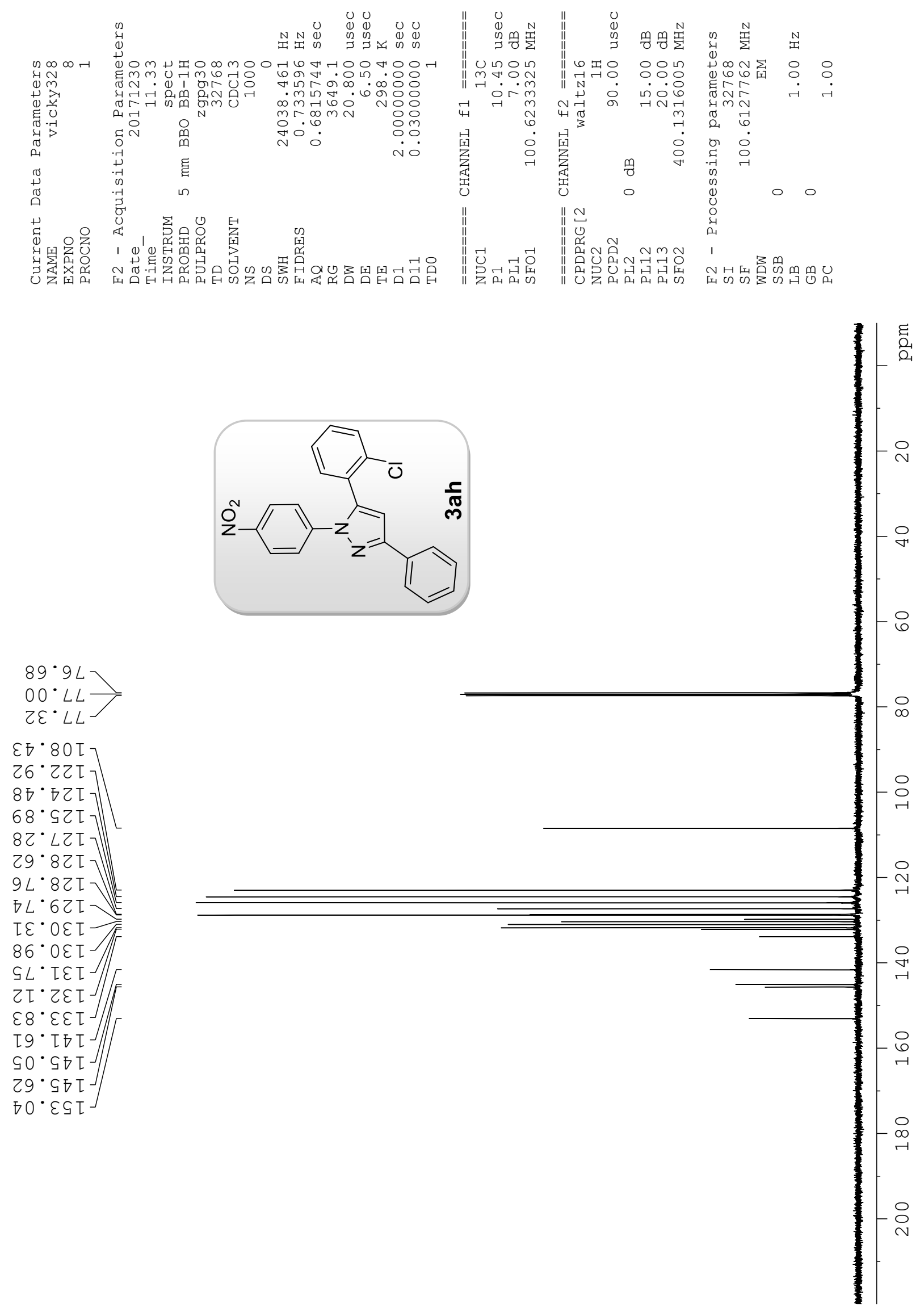


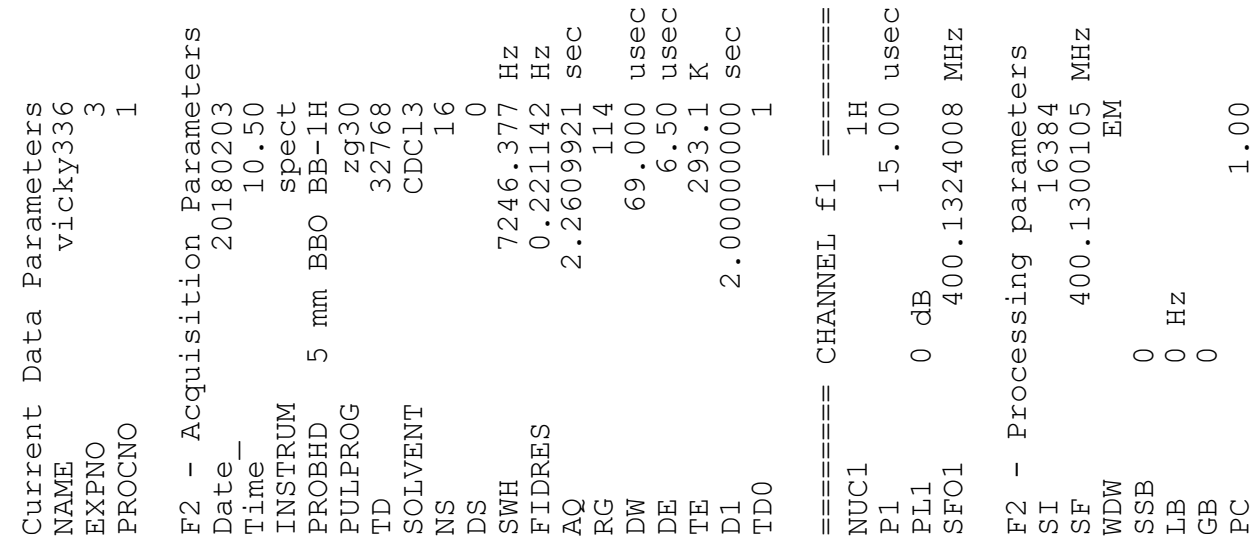

$00 \cdot 0$
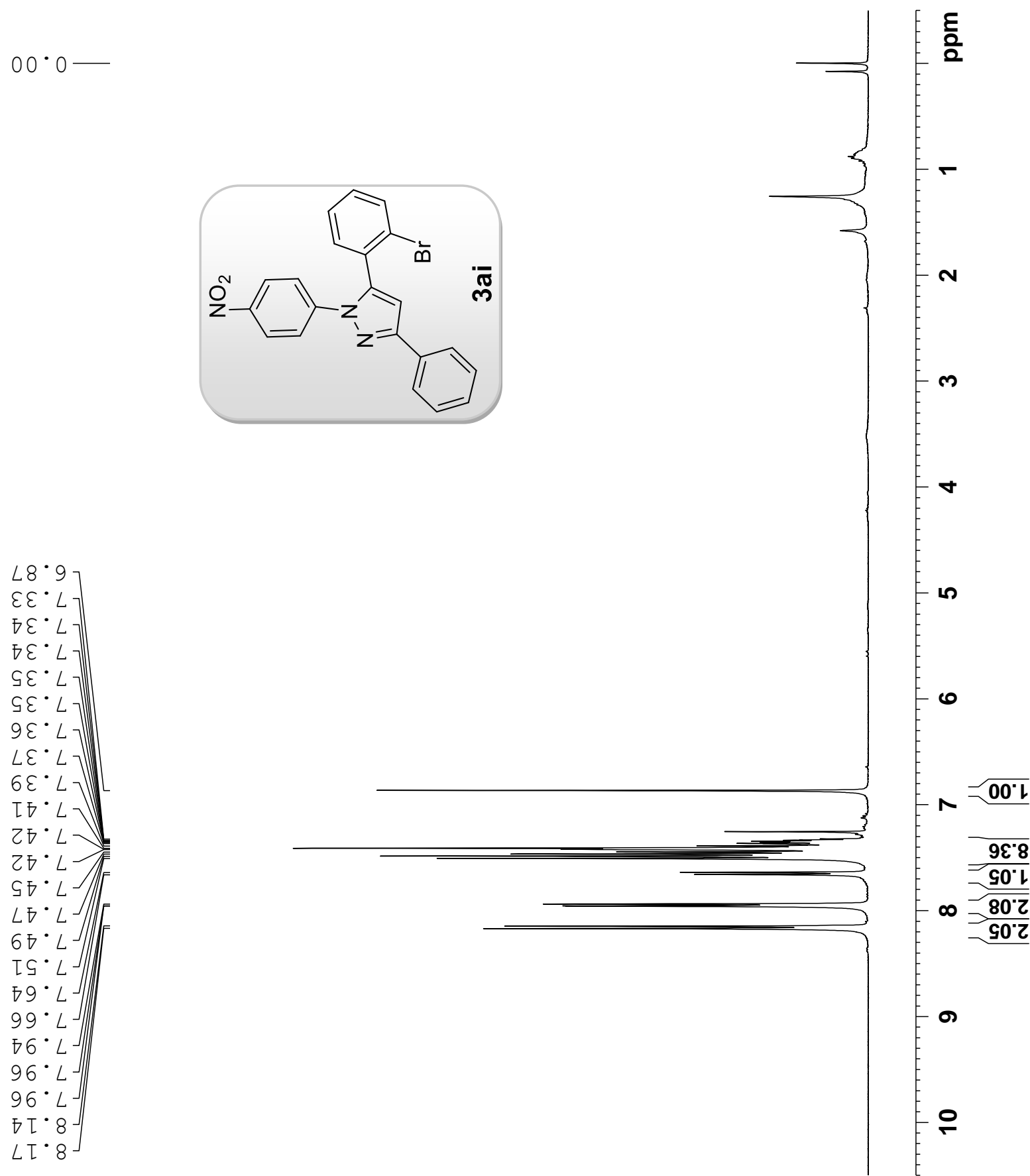

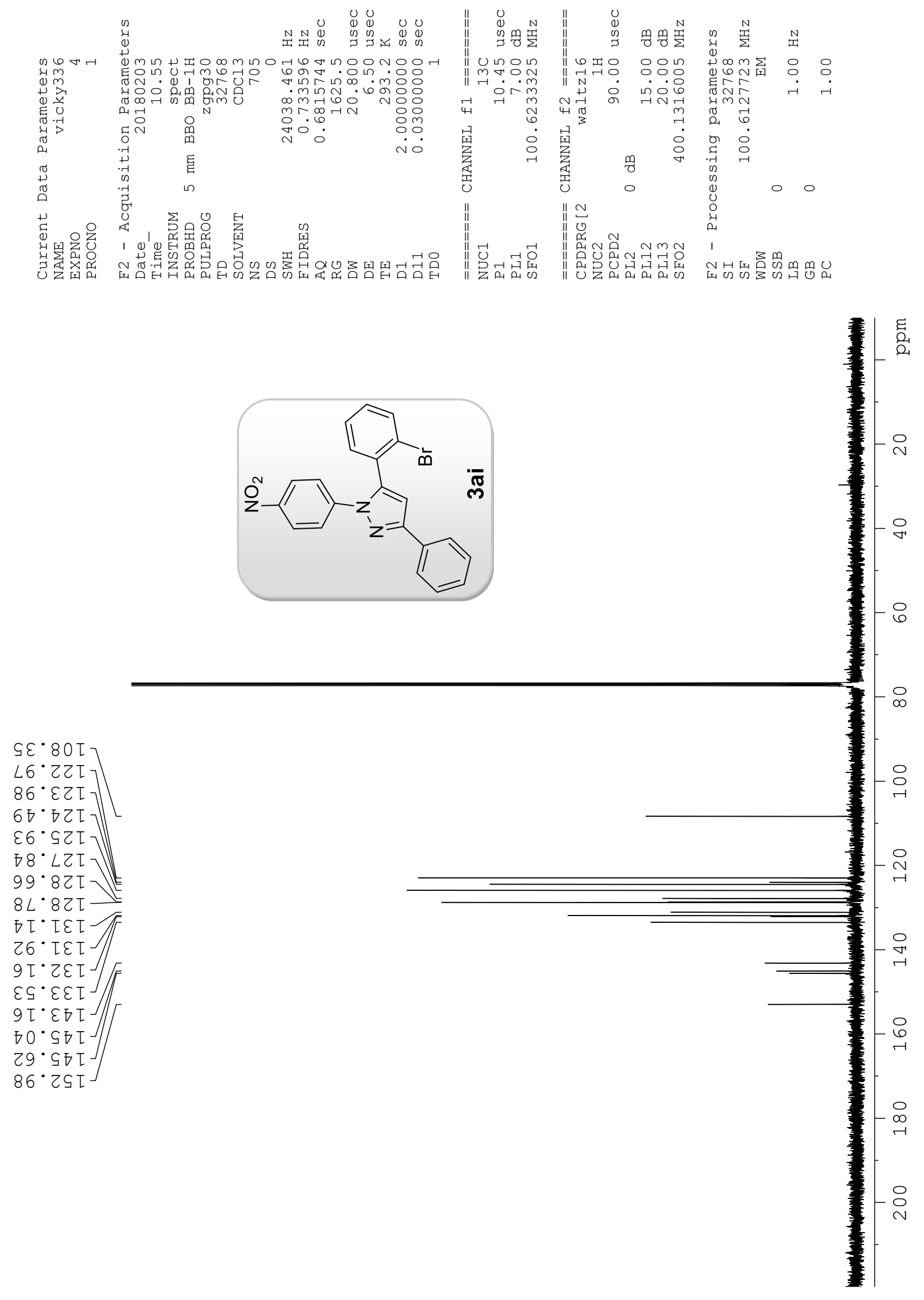


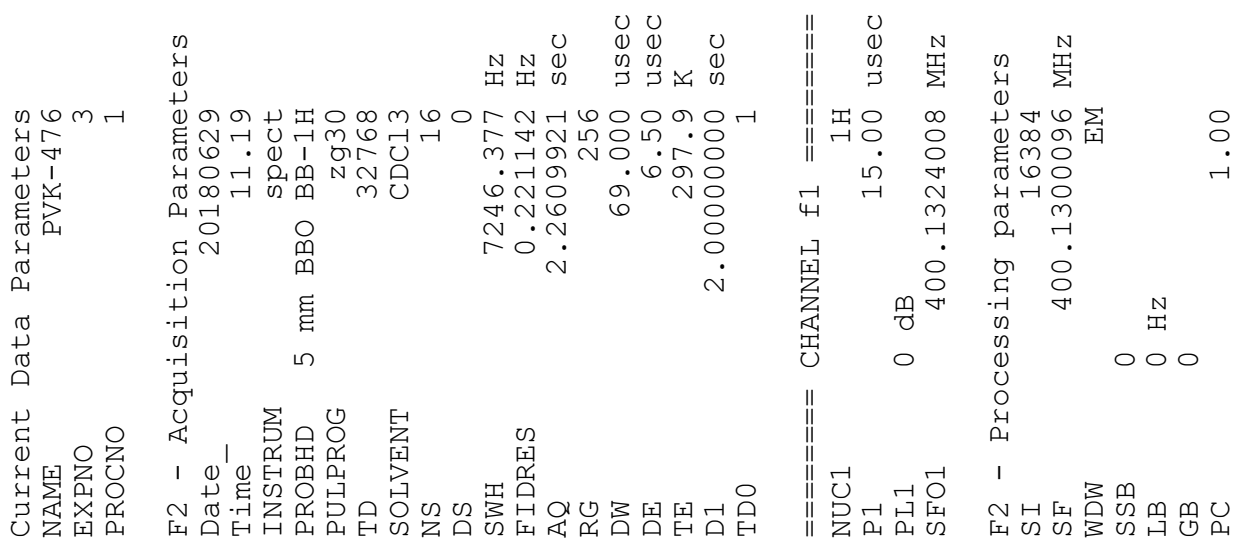

$000 \cdot 0-$

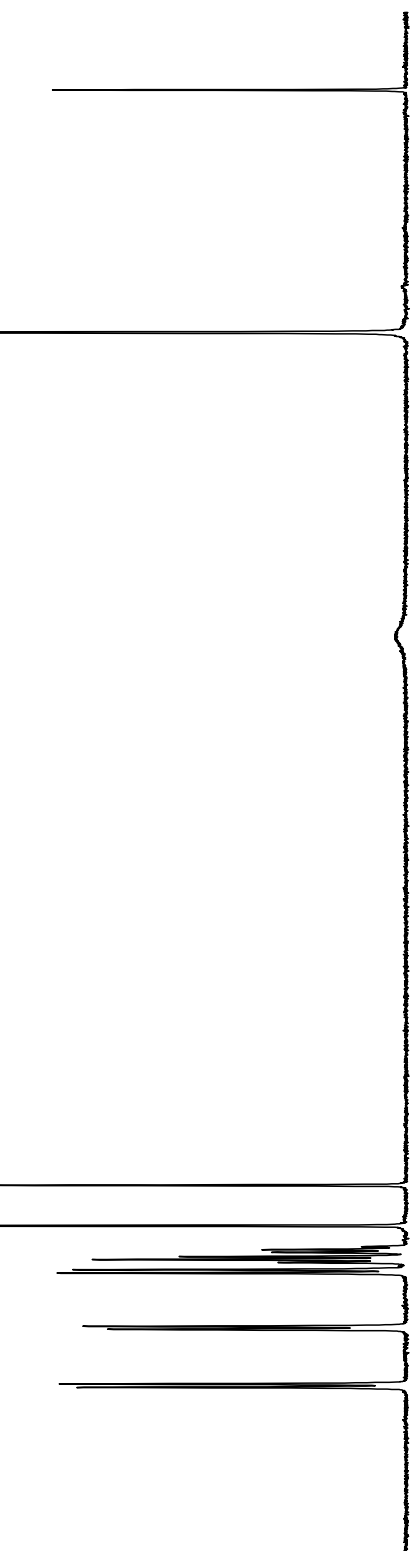

틍

$\tau 00^{\circ} L$

$\left.\varepsilon 6 \varepsilon^{\circ} L_{]}\right]$

โโ品 $L]$

OEF ${ }^{\circ}$

$9 \angle \sigma \cdot L]$

76 ${ }^{\circ} L$

$0 \nabla S^{\circ} L$

$299^{\circ} \mathrm{L}$

$206^{\circ} \mathrm{L} J$

โZ6 ${ }^{\circ}$

[LZ・8

$\varepsilon 6 Z \cdot 8$

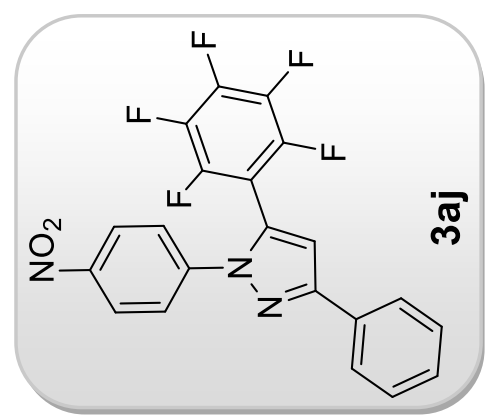

$m$

$-N$

ก

0

$\infty \quad 86^{\prime \prime}$

$\sqrt{96.1}$

$\sigma$

으 

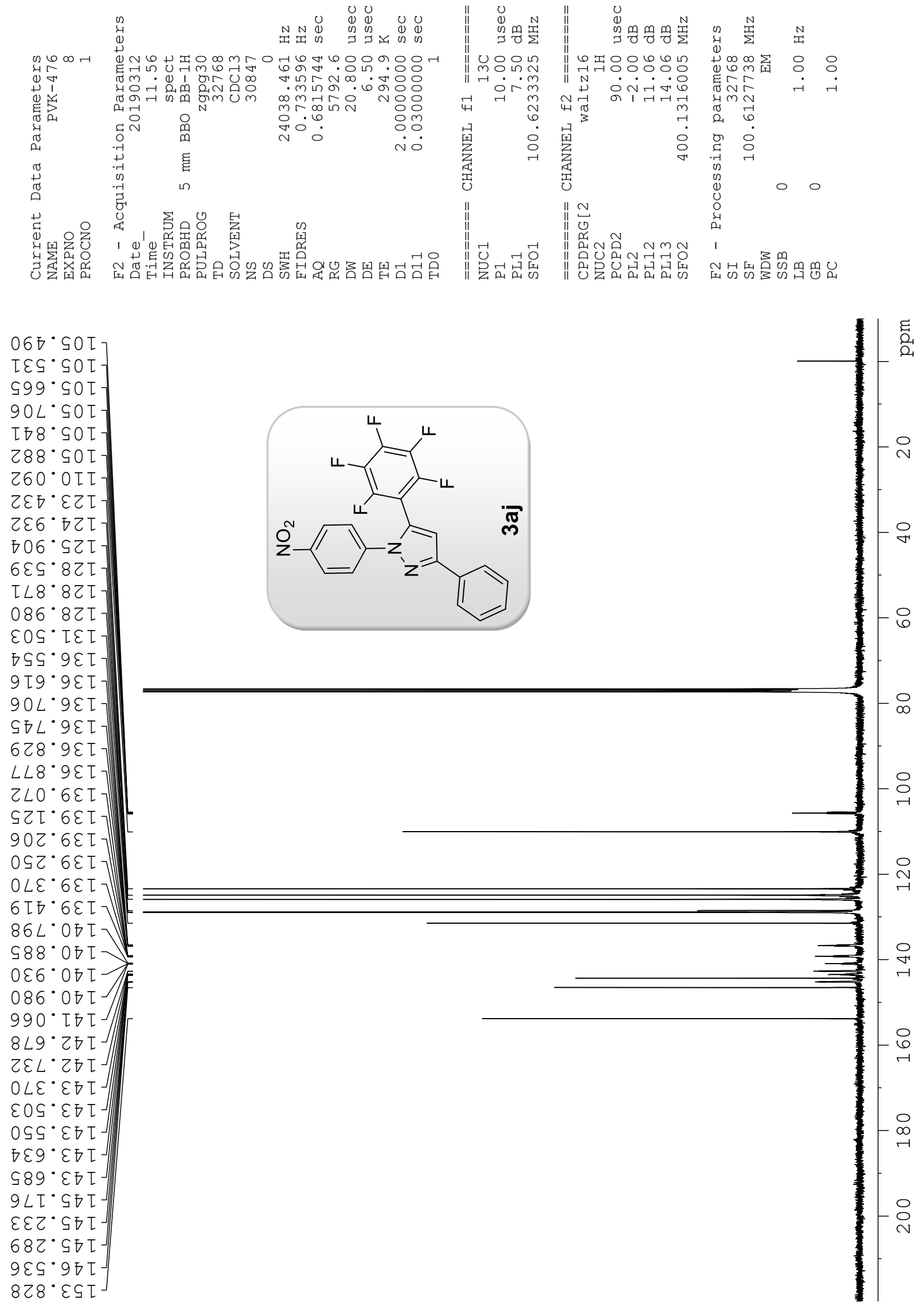

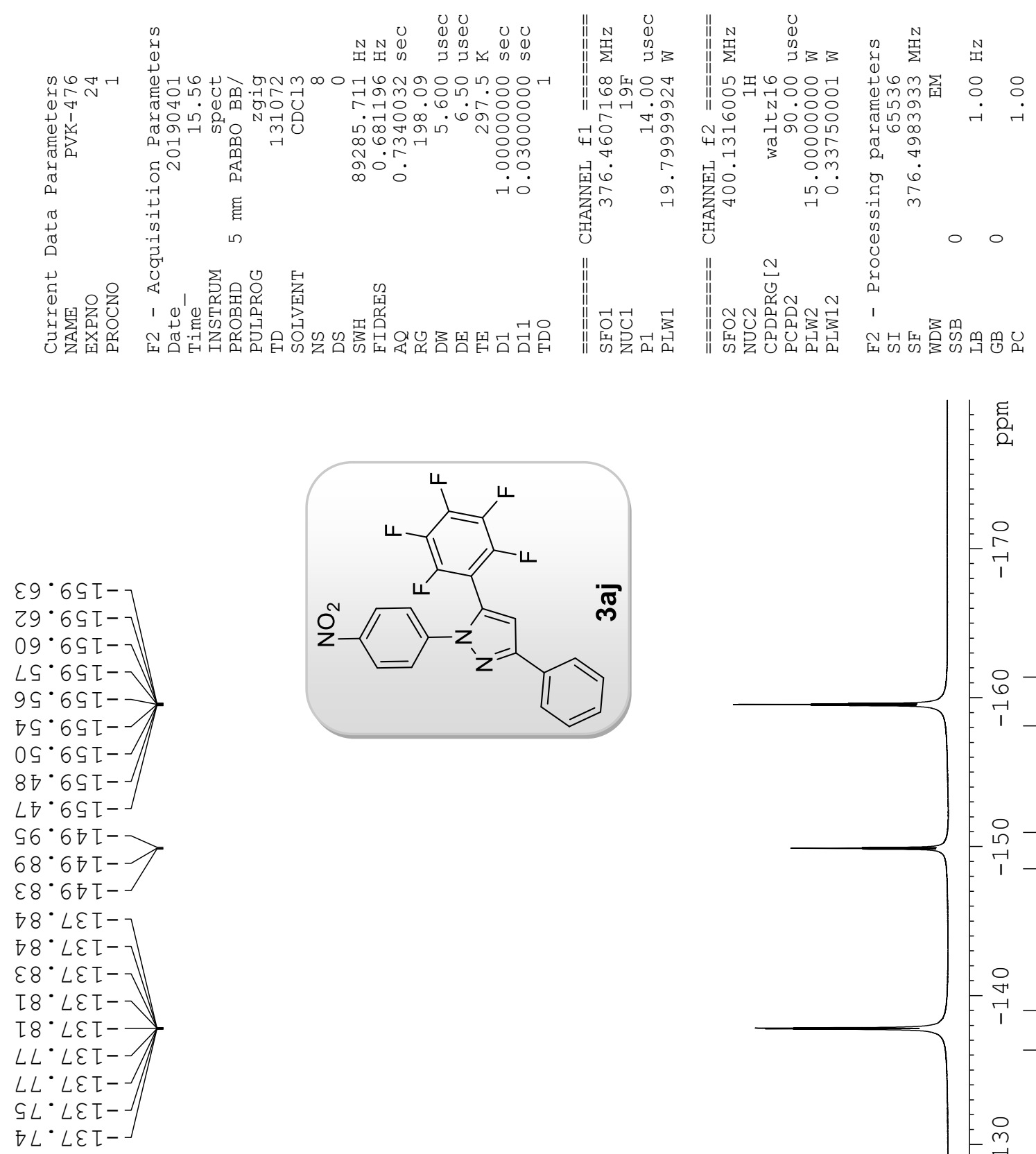

ڤ્a

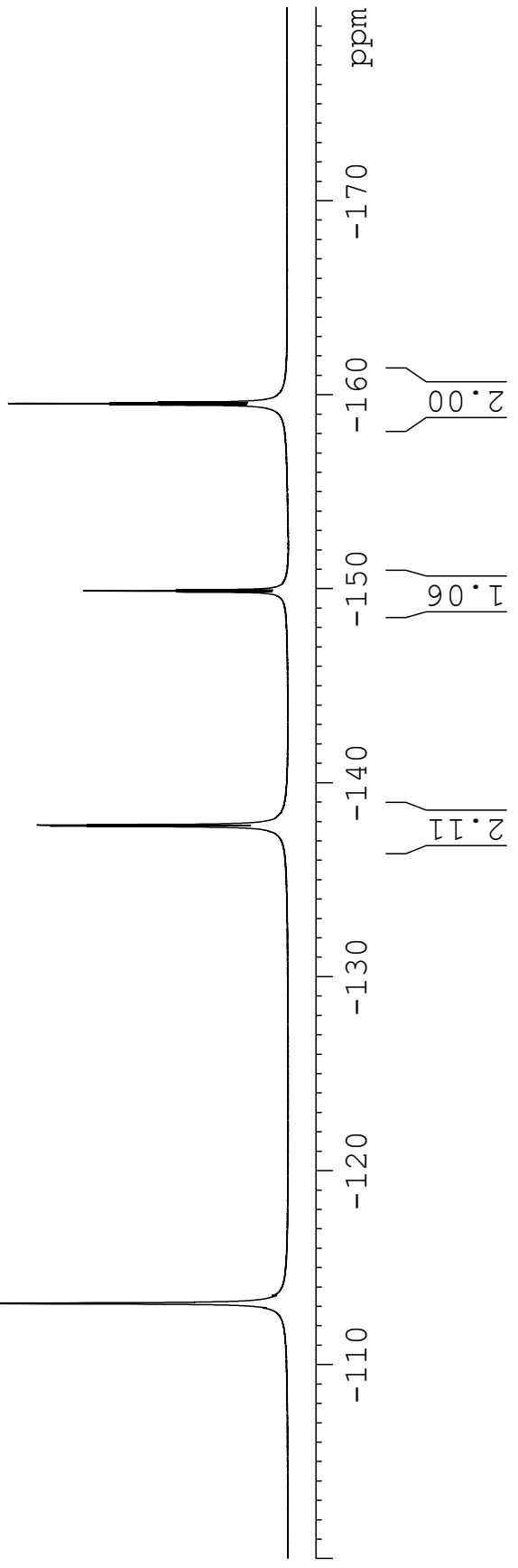




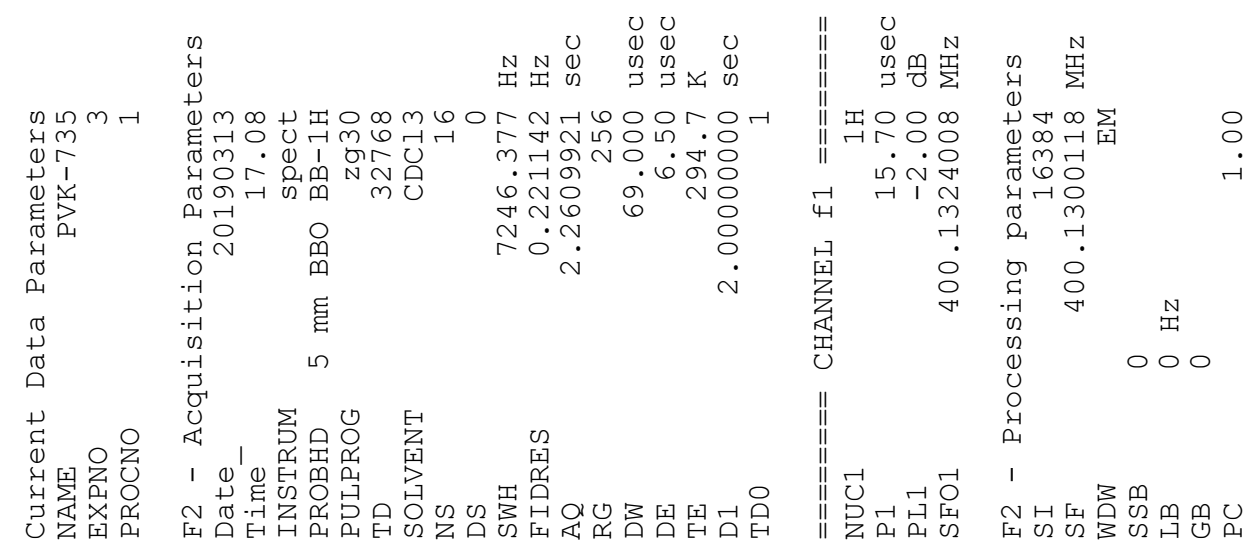

$500 \cdot 0-$

ITS'Z—

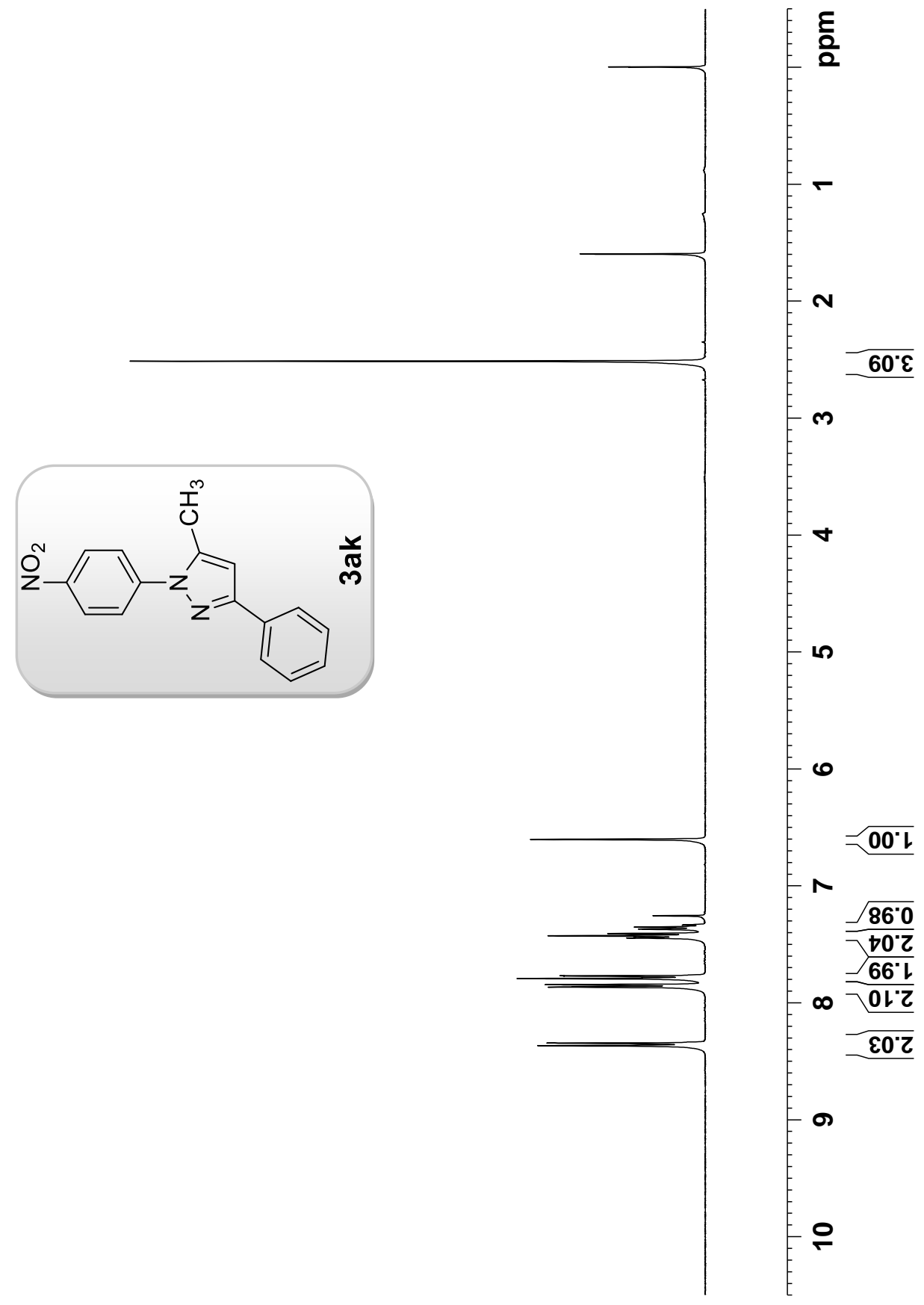




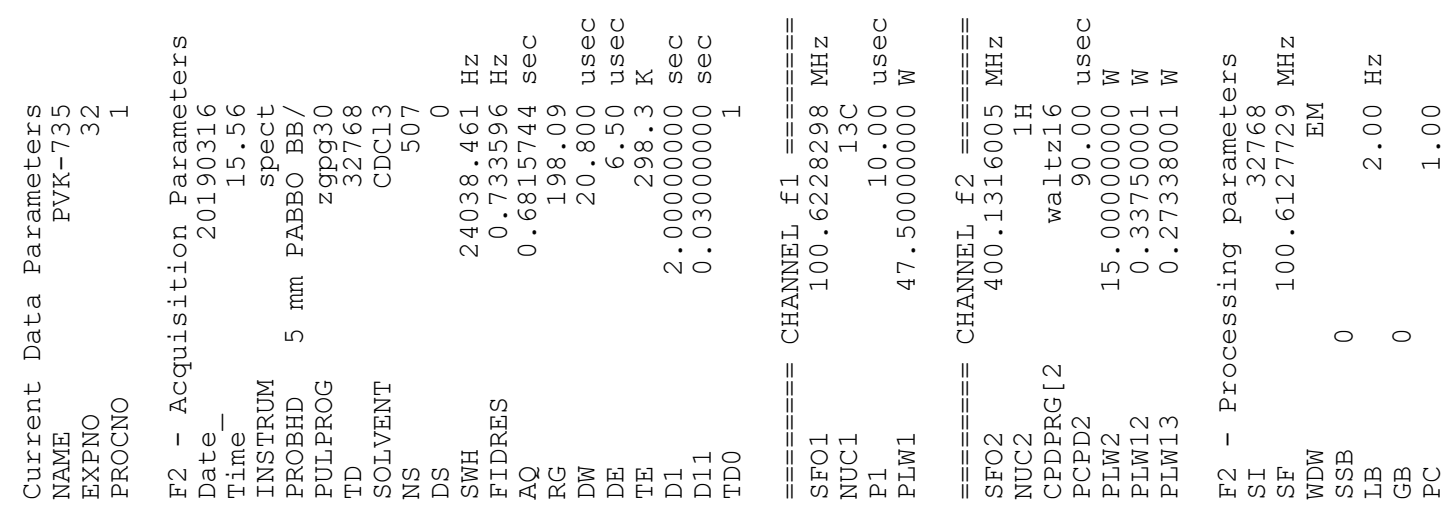

$\tau \tau \varepsilon \cdot \varepsilon \tau$

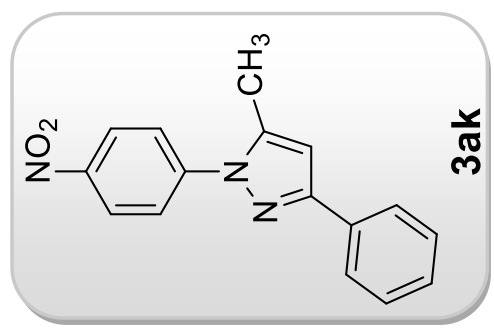

$089^{\circ} 9 L$
$866^{\circ} 9 L$
$9 \tau \varepsilon^{\circ} L L$

$289^{\circ} 90 \mathrm{~L}$

$8\left[8^{\circ} \varepsilon 乙[]\right.$

ง०8・

$6 L \varepsilon^{\circ} 8 Z \mathrm{Z}$

$\varepsilon 89 \cdot 8 Z \mathrm{~T}$

ZOS ZET

SSG.0DI

$666^{\circ}$ D田

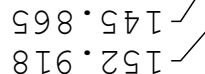

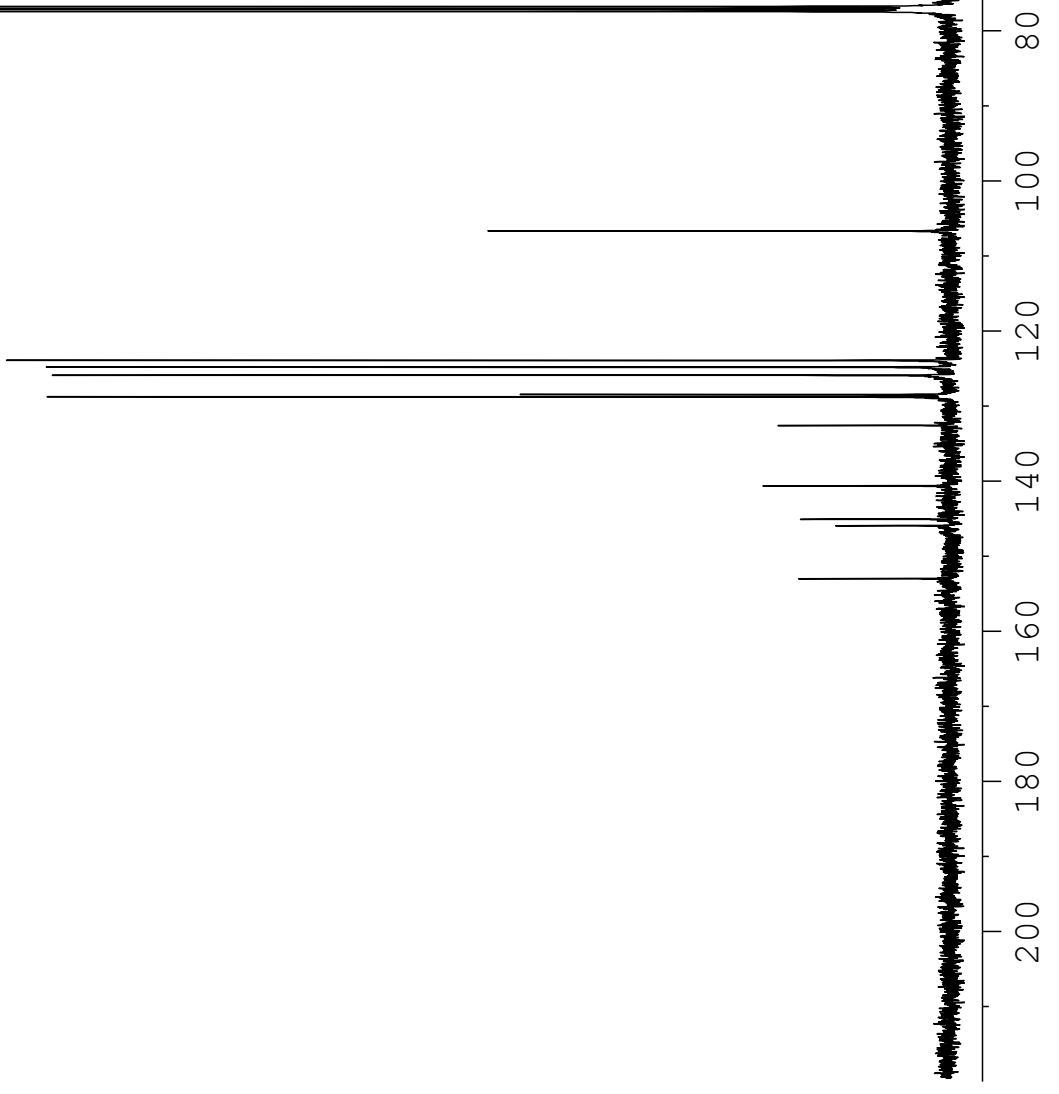




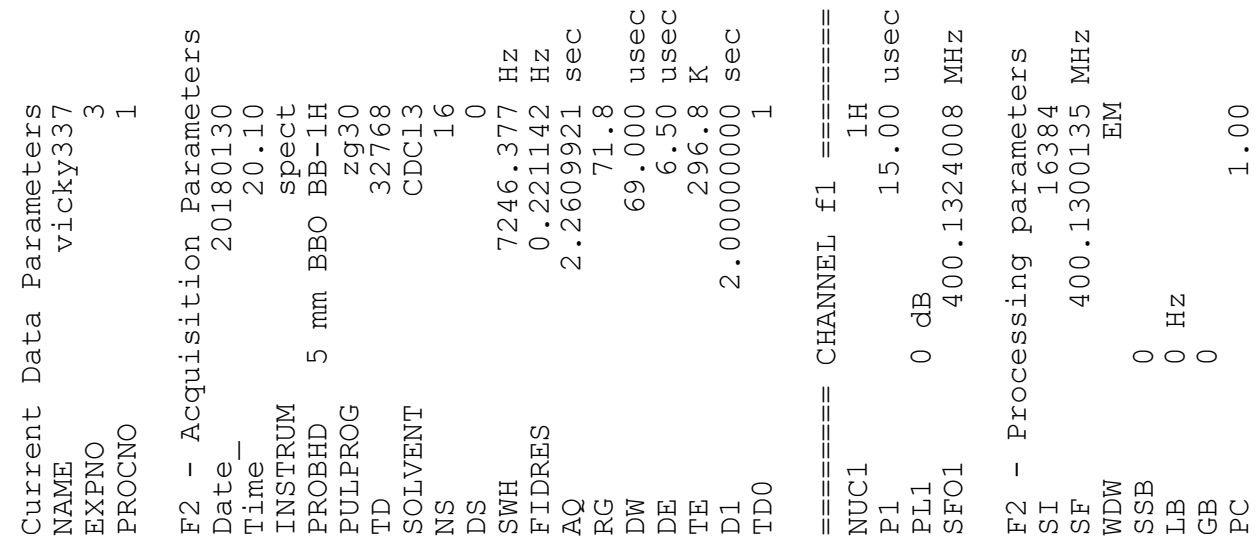

$00 \cdot 0-$
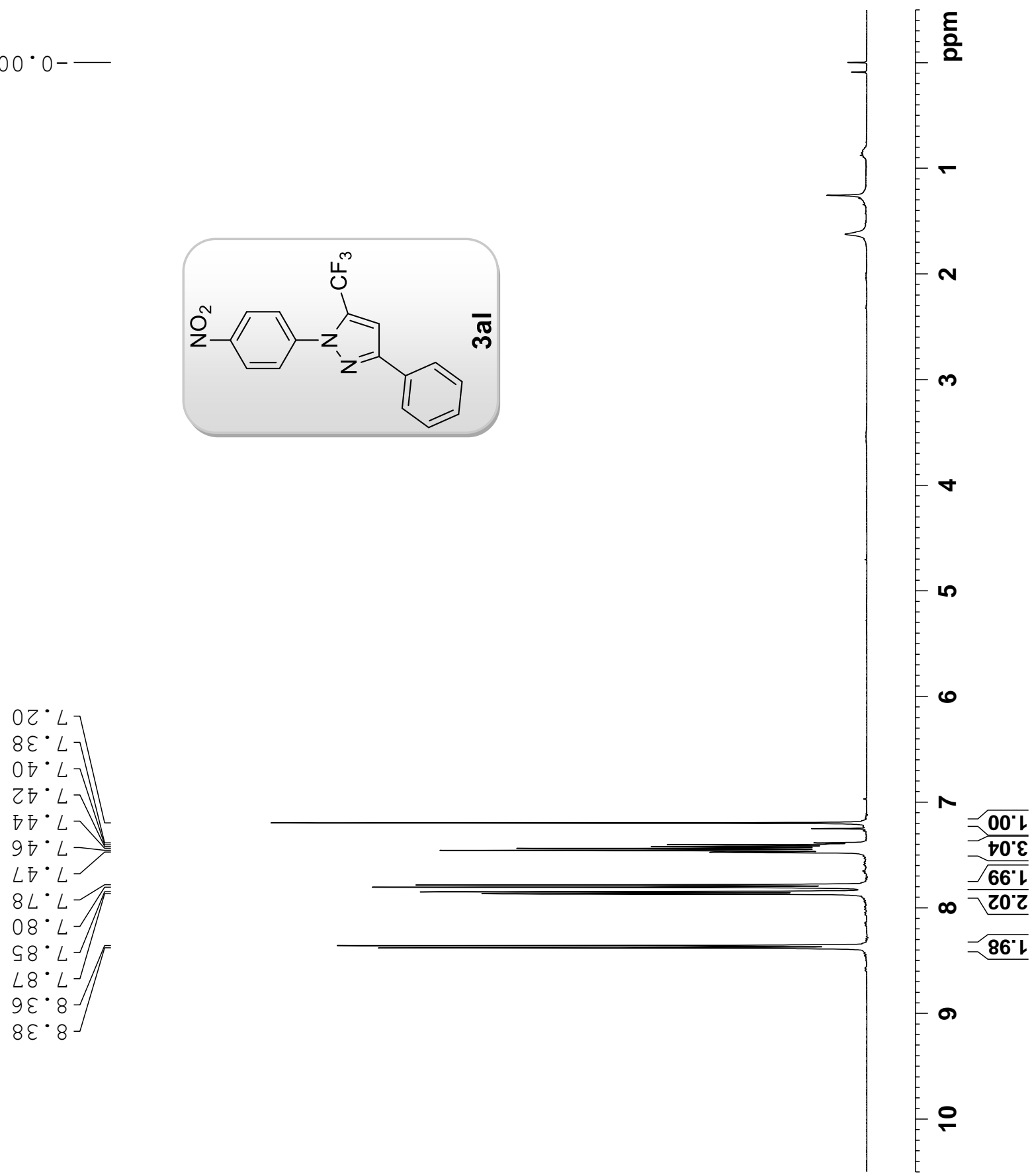

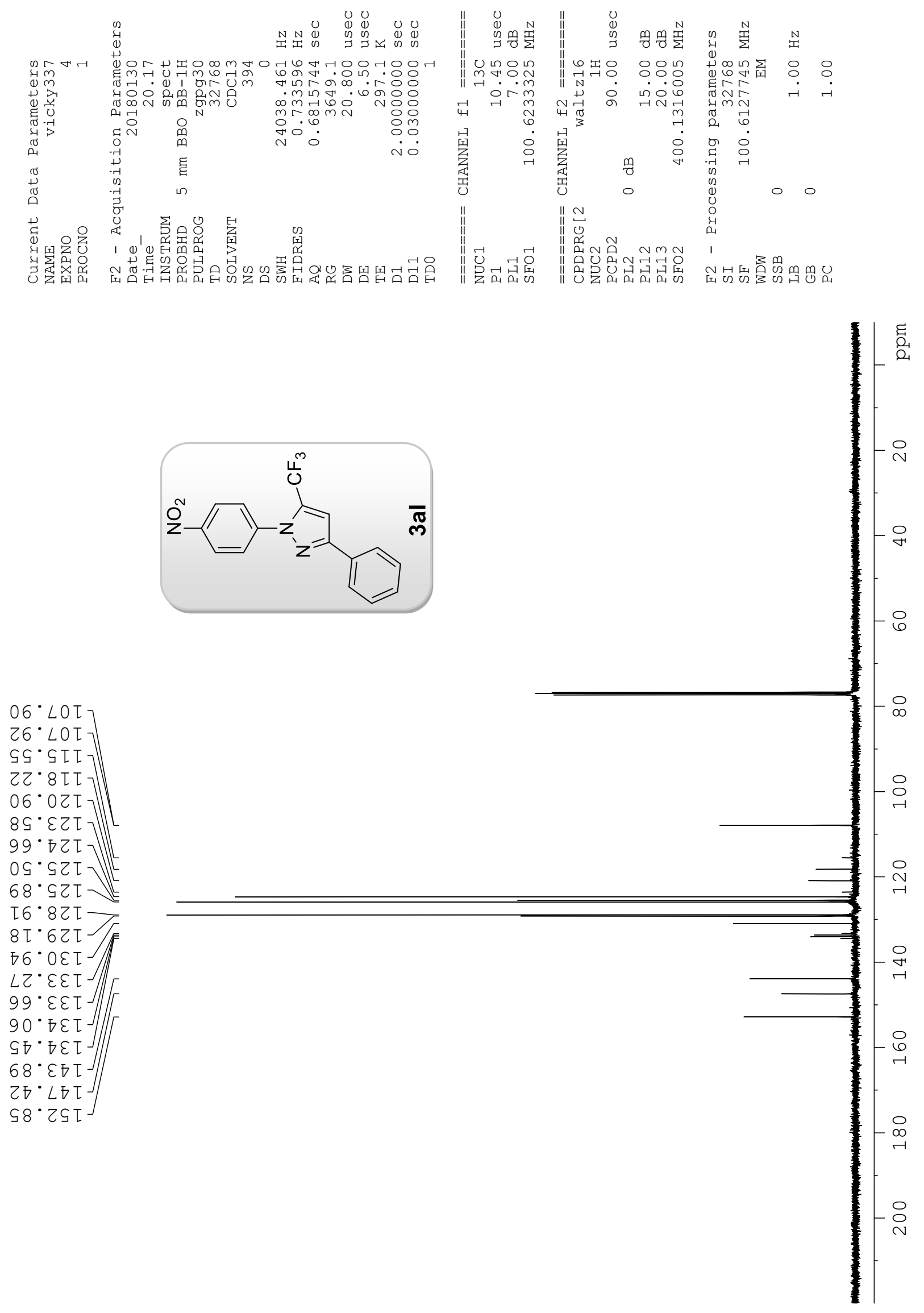

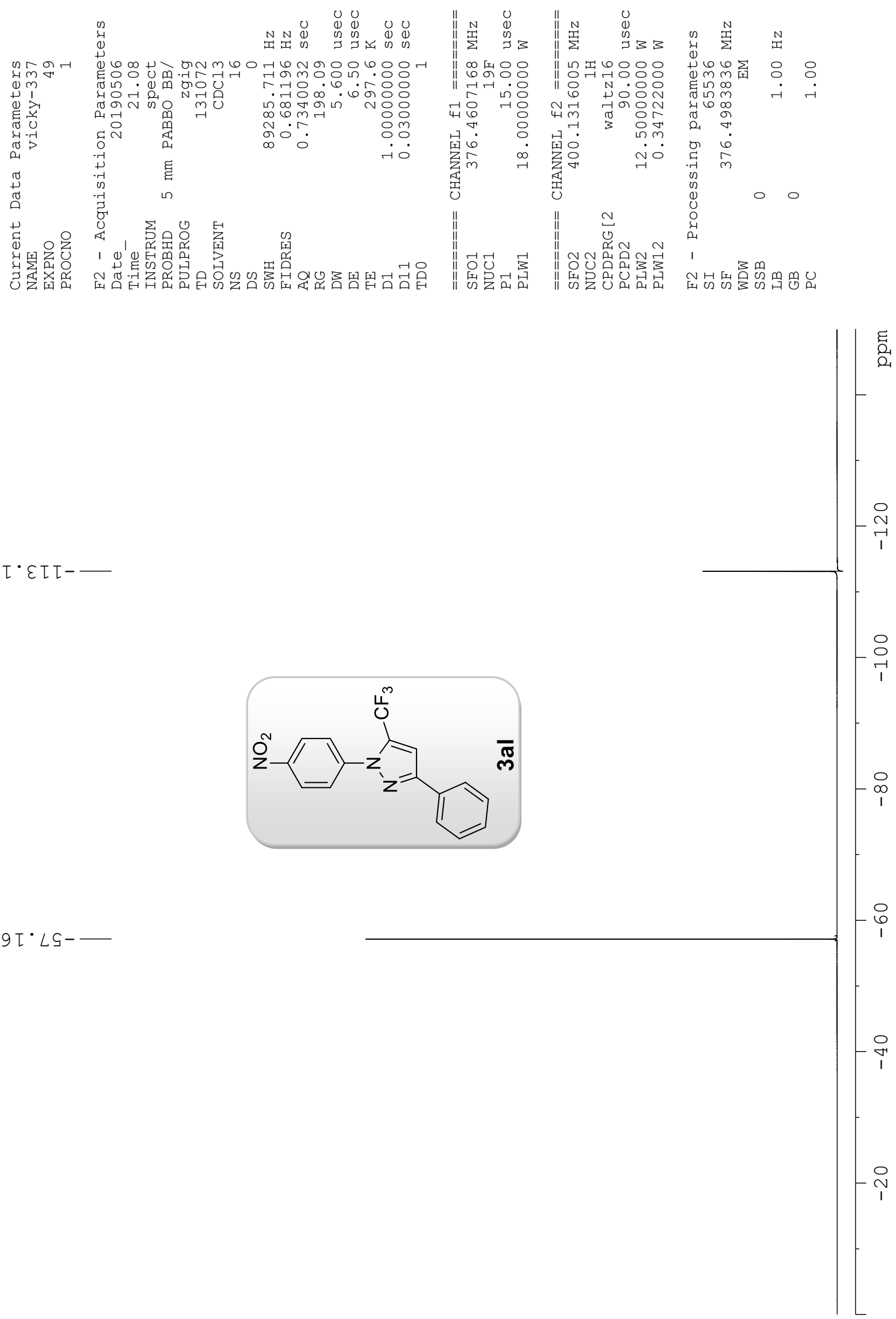


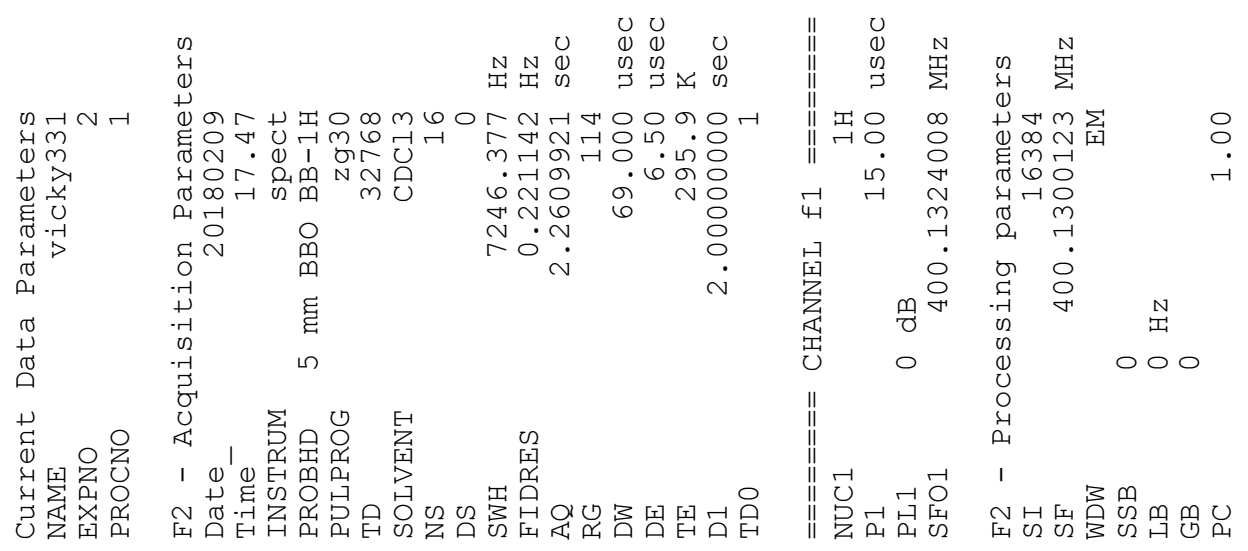

$000^{\circ} 0$

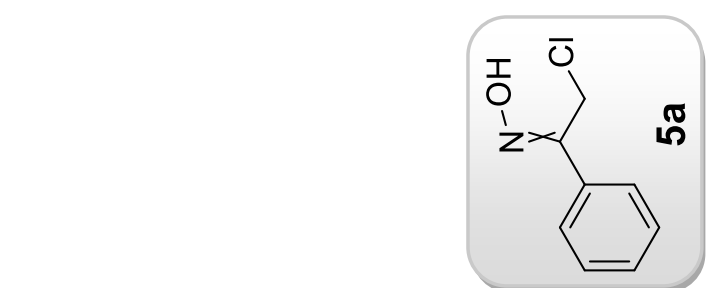

흥
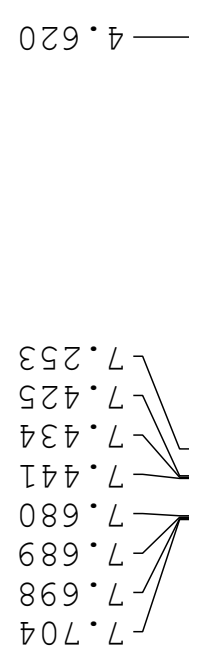

$200 \cdot 6$

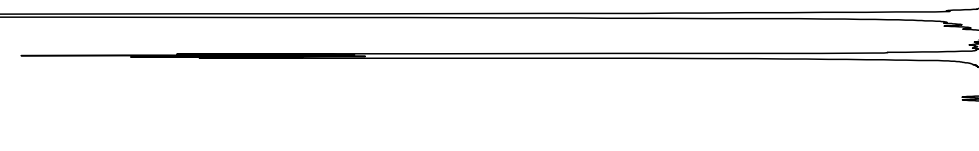




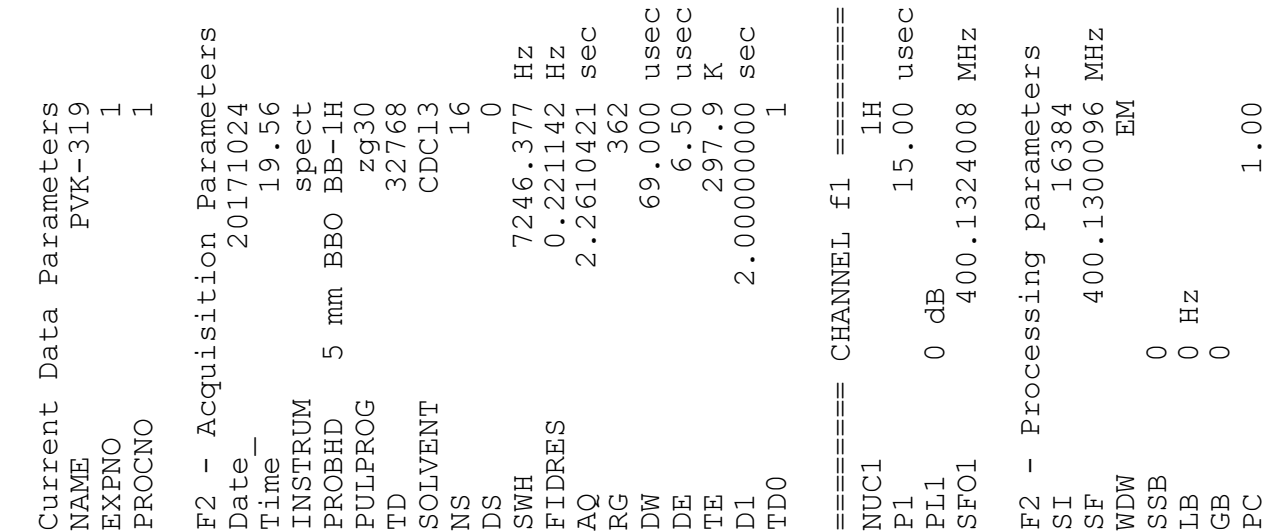

$000 \cdot 0$

$9 \nabla 8^{\circ} \varepsilon$

I6G'

$426 \cdot 9$

$676 \cdot 9$

$092^{\circ} L$

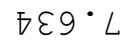

$999^{\circ} L$

$8 \varepsilon 6^{\circ} \mathrm{L}$
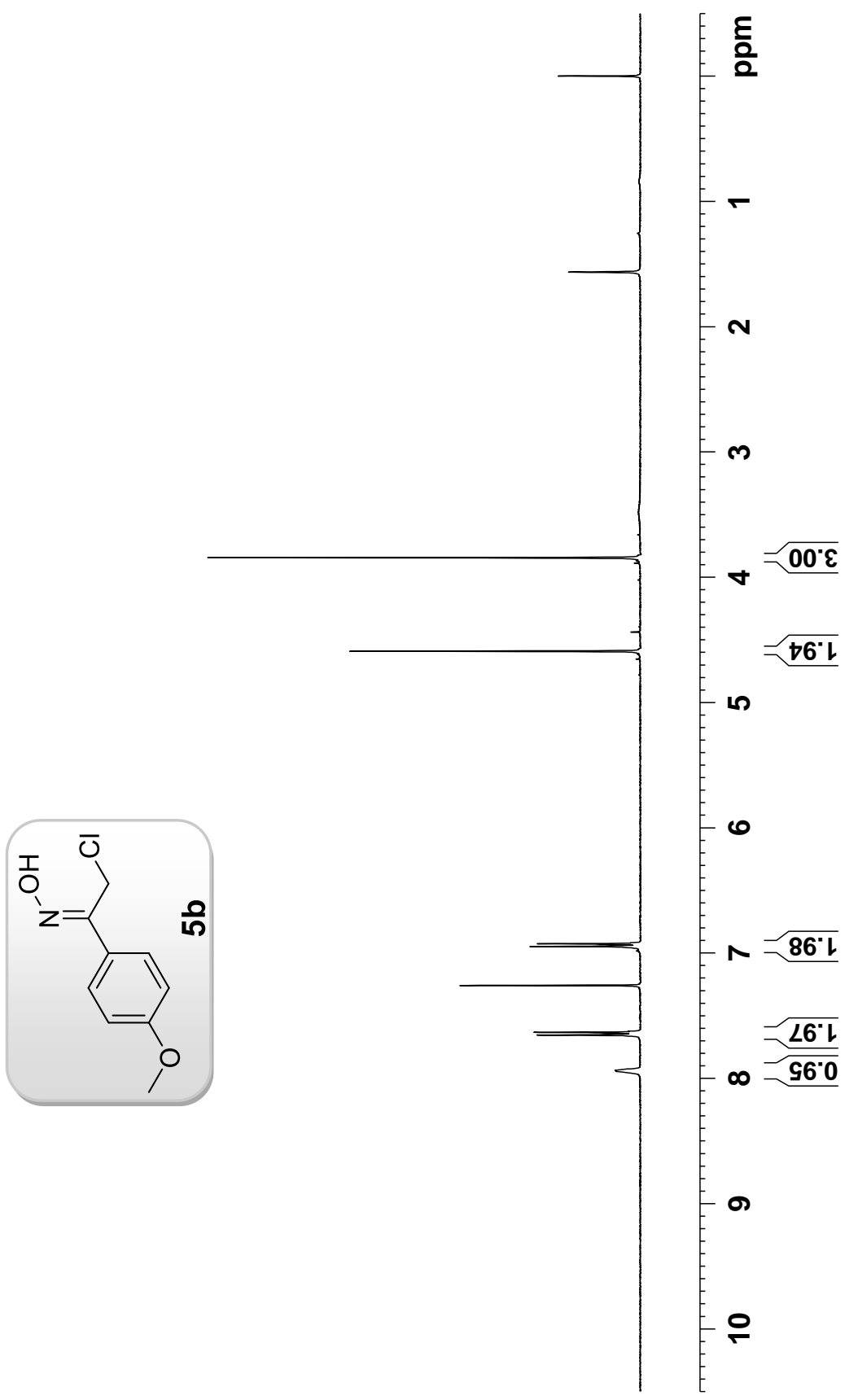

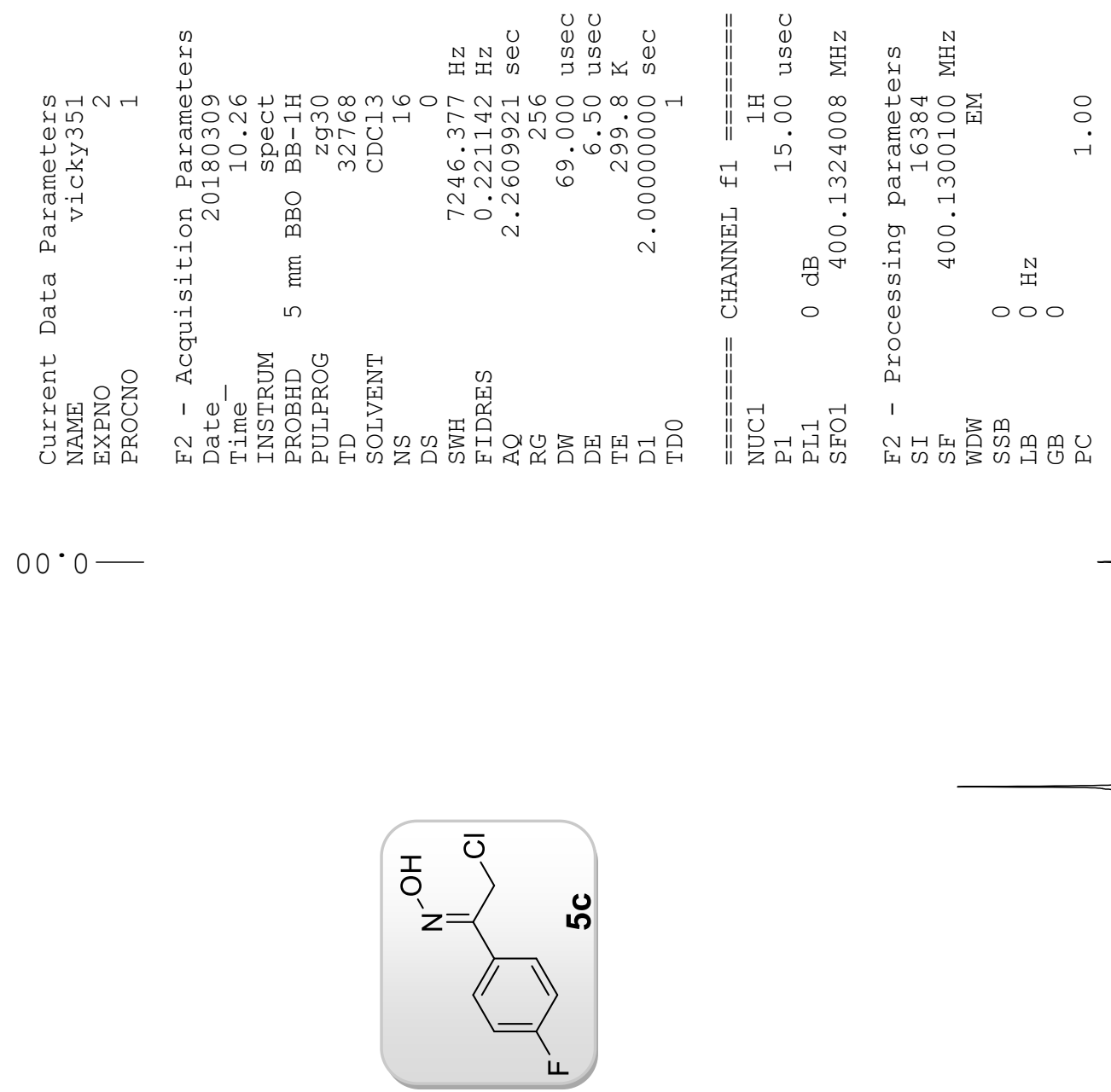

$\varepsilon \varepsilon \cdot 8$

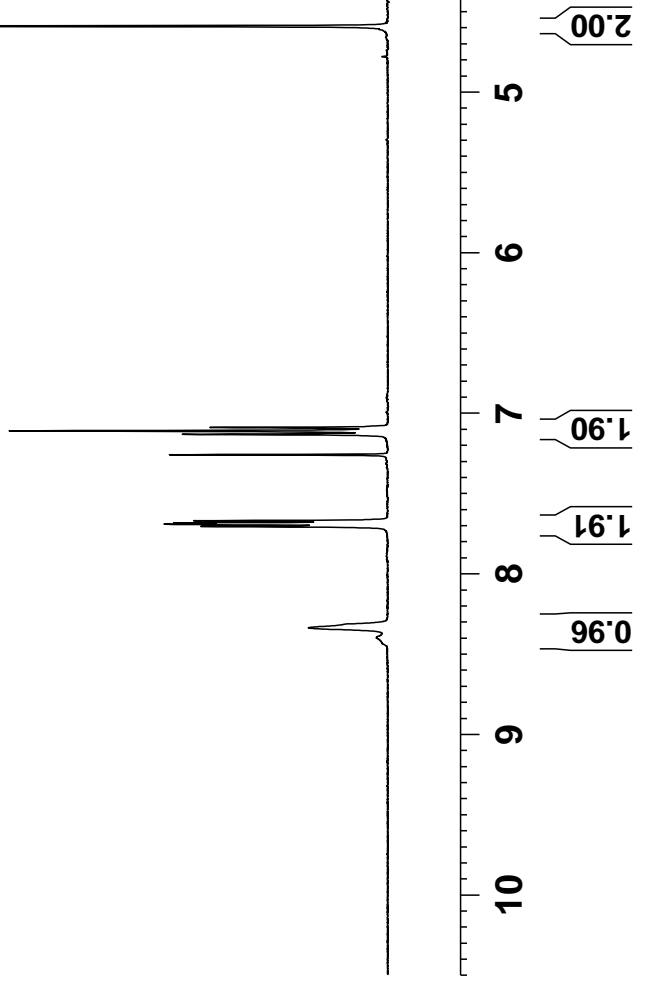




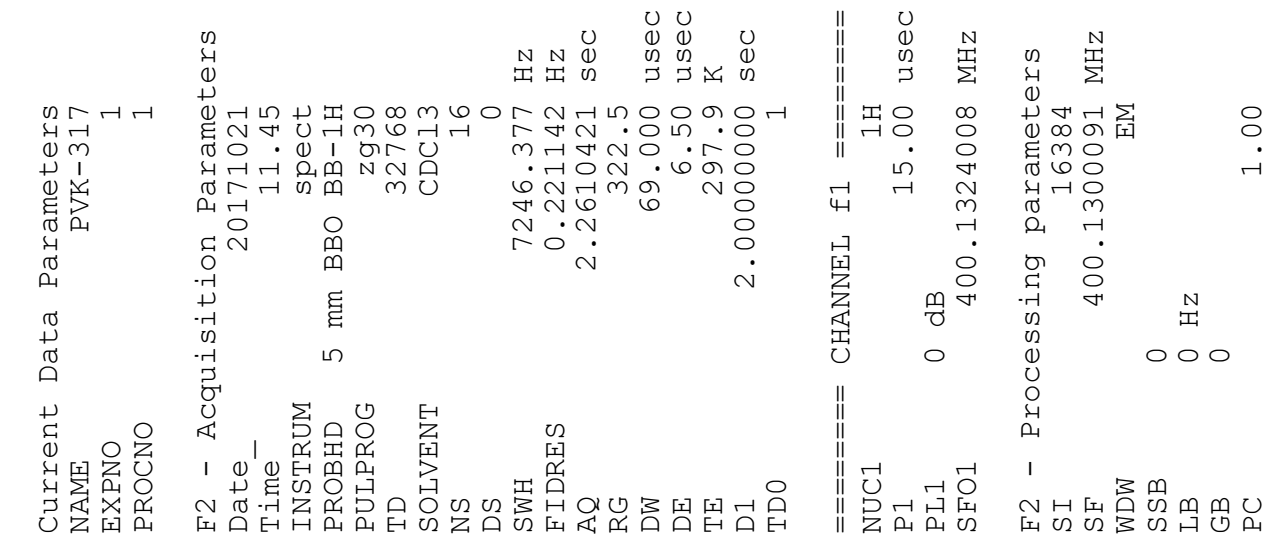

$000 \cdot 0$

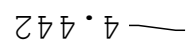

8ย9.

I9 $2^{\circ} L-$

$\varepsilon 88^{\circ}$

$906^{\circ} \mathrm{L}$

TLO.8

Б七 $1 \cdot 8=$

$992 \cdot 8-$

$48 Z^{\circ} 8$

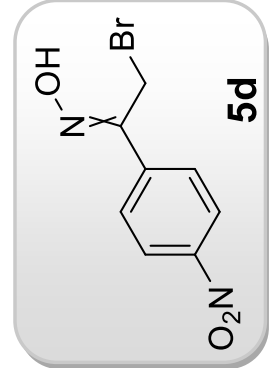

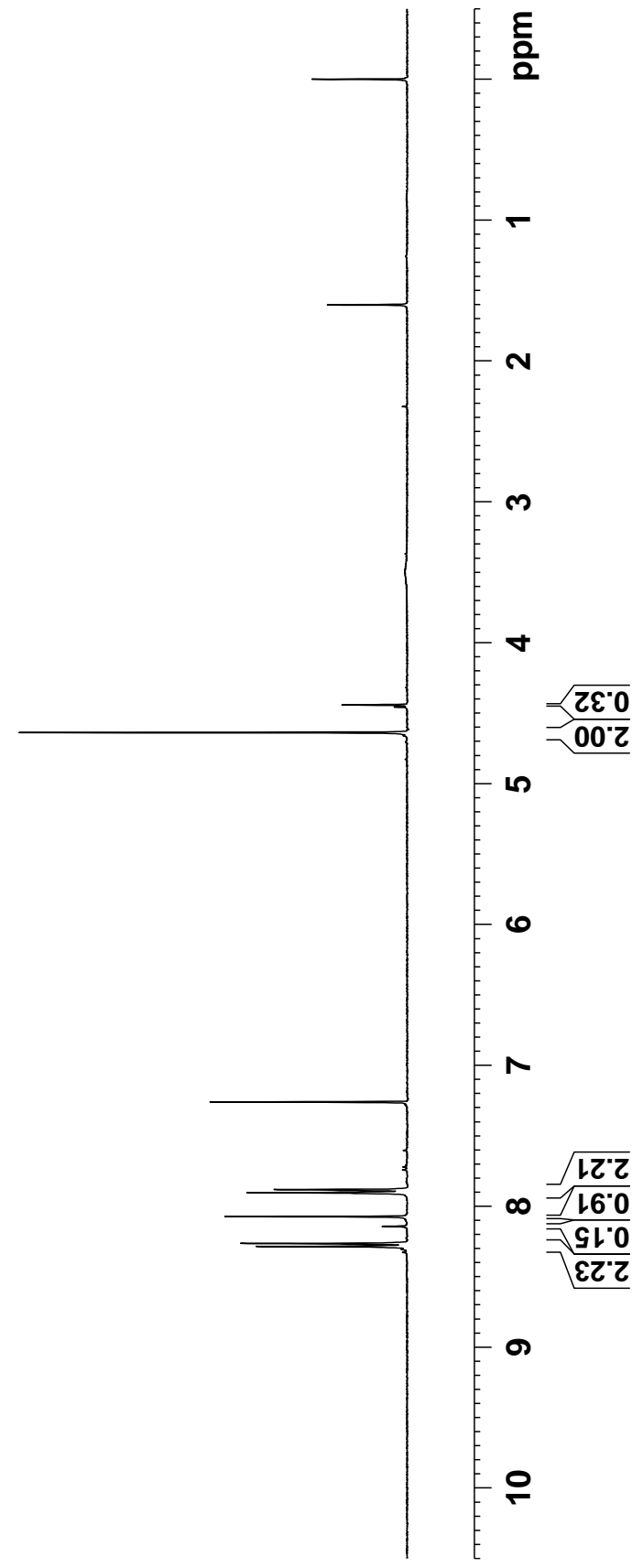



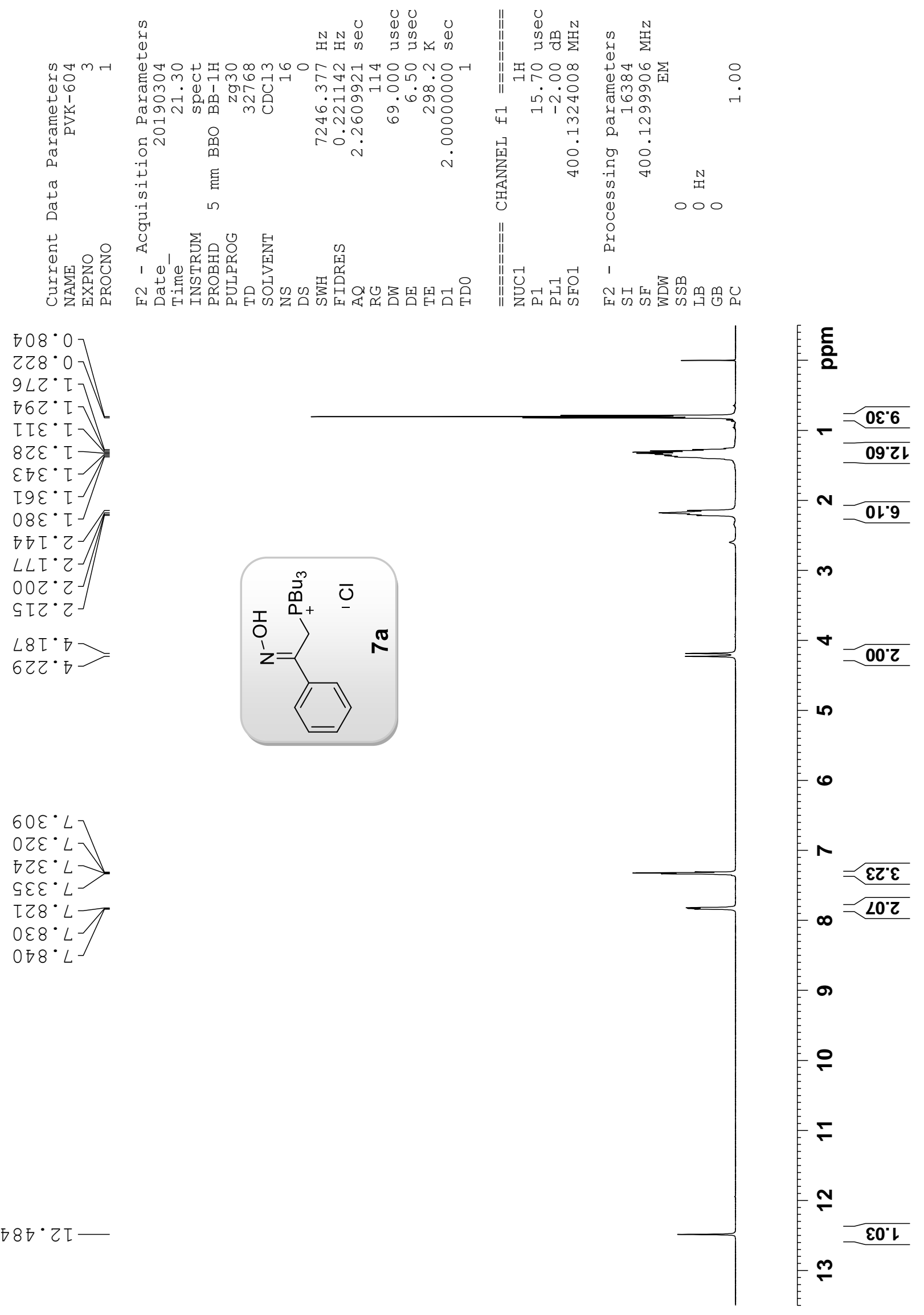

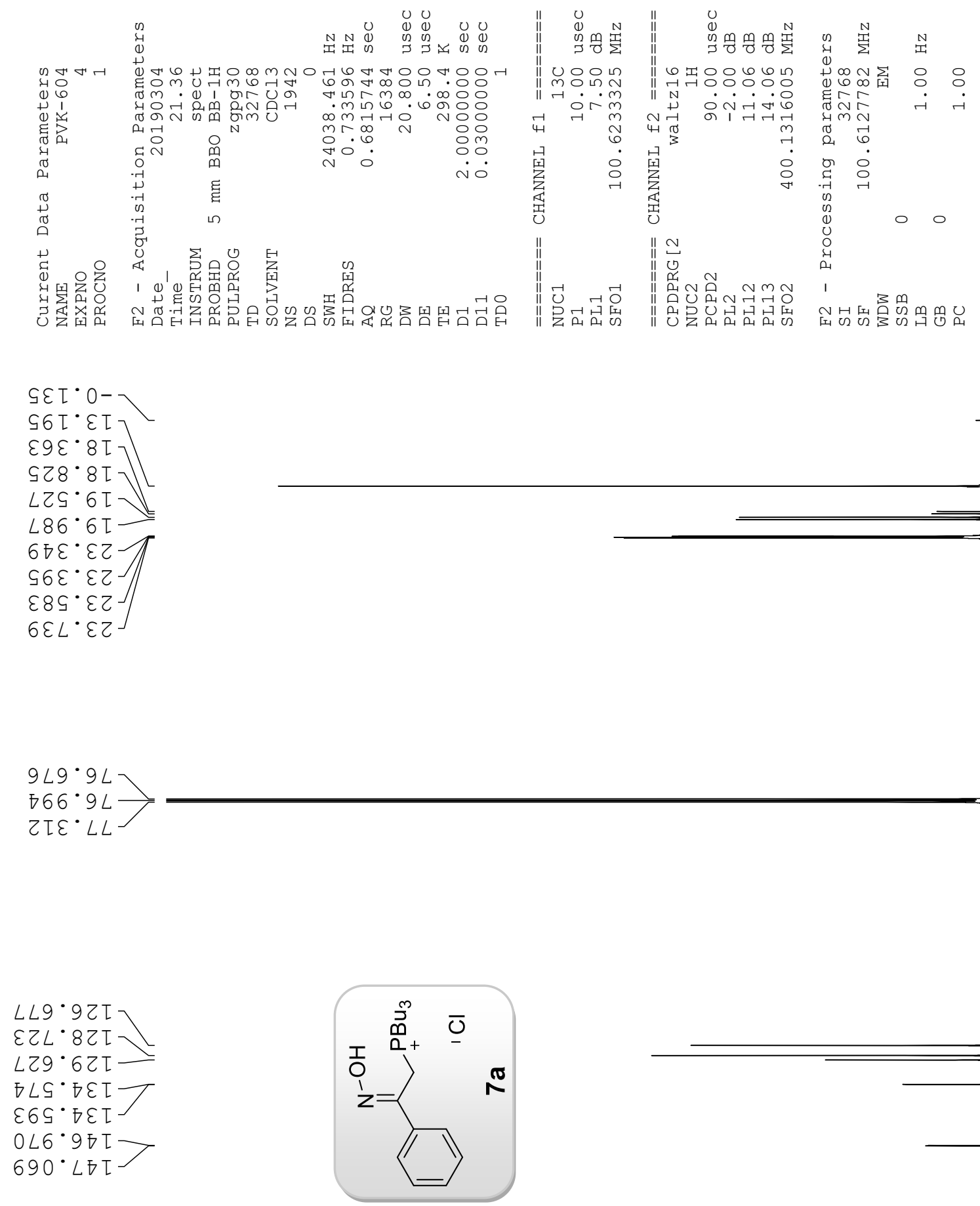

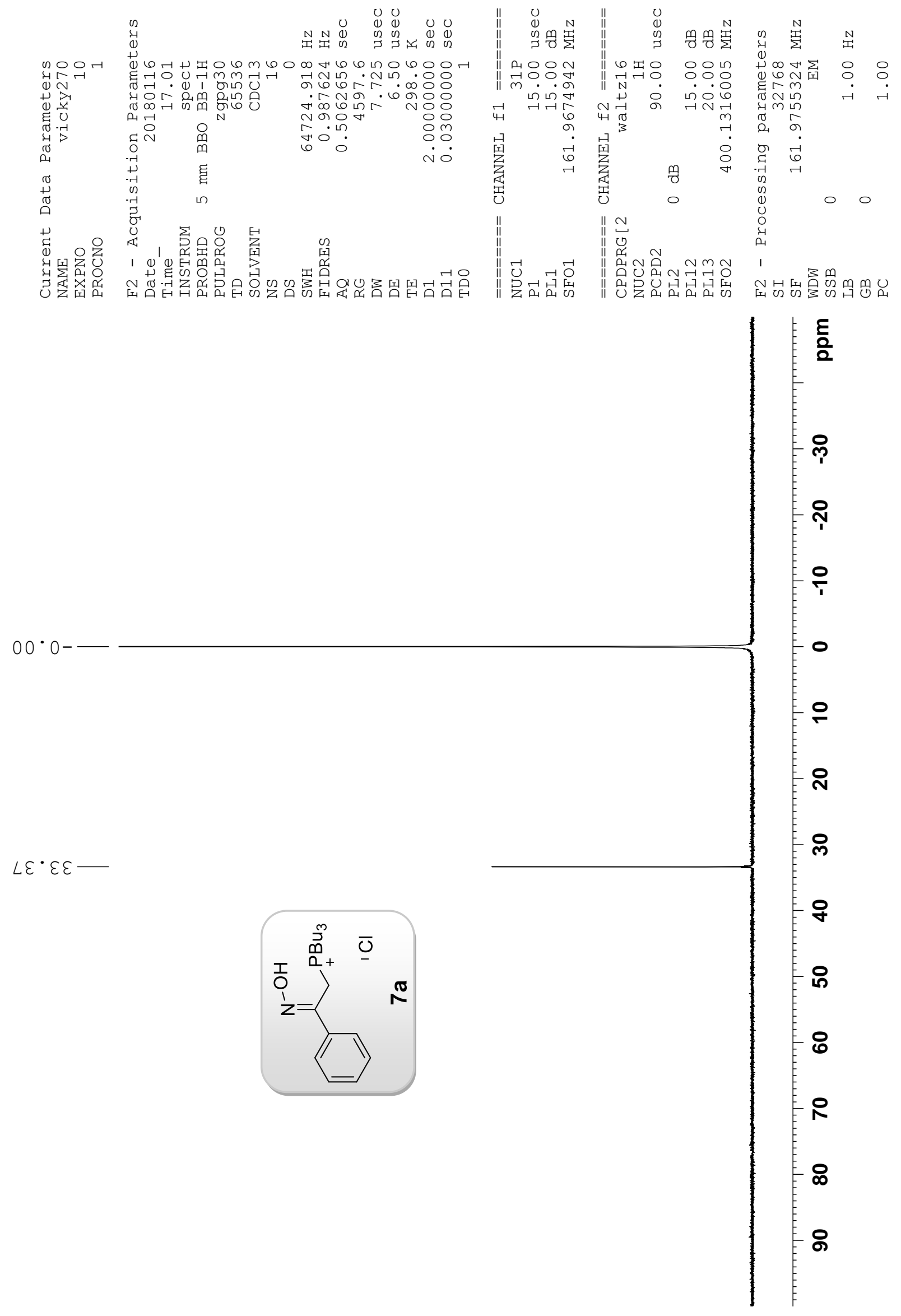

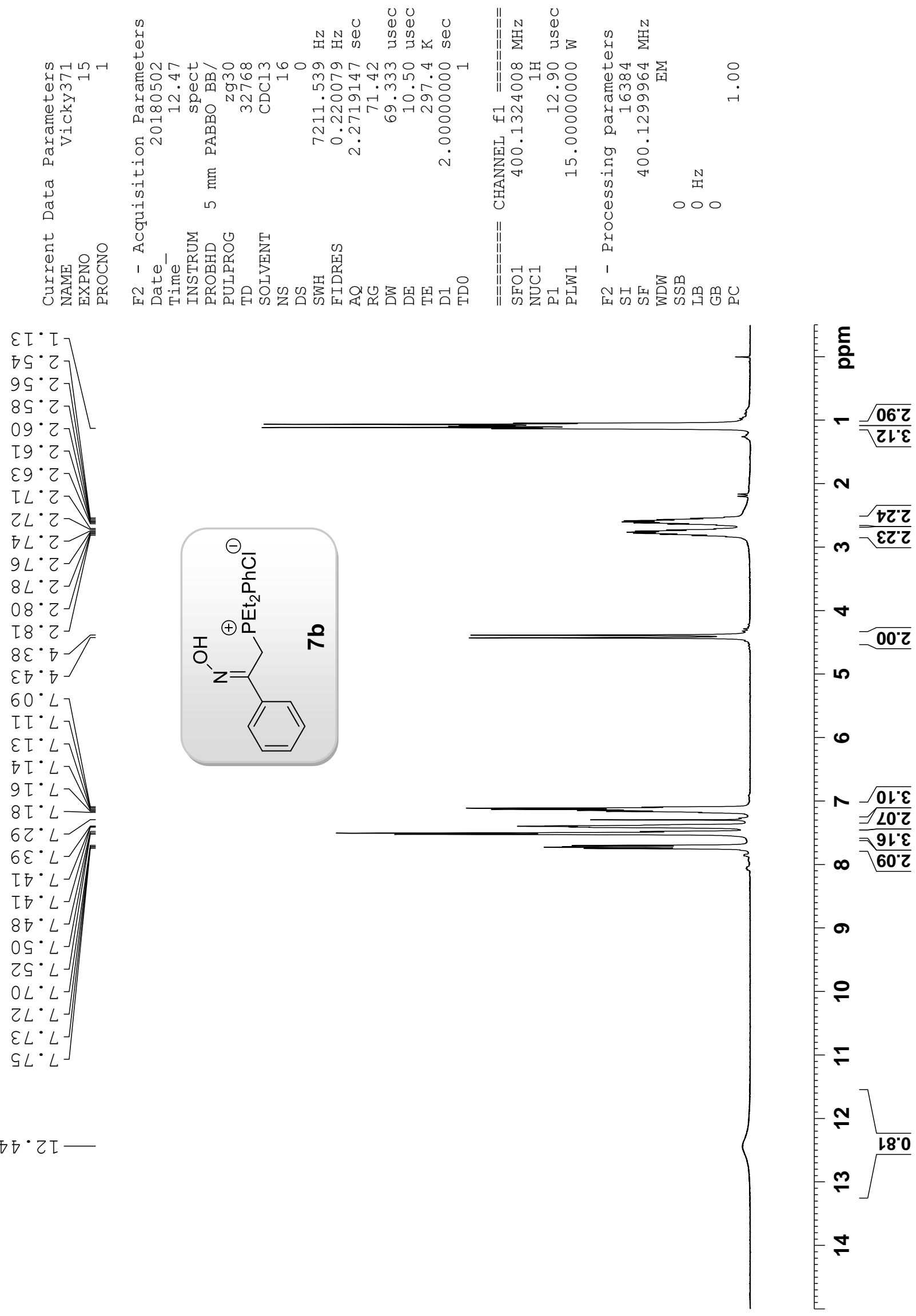

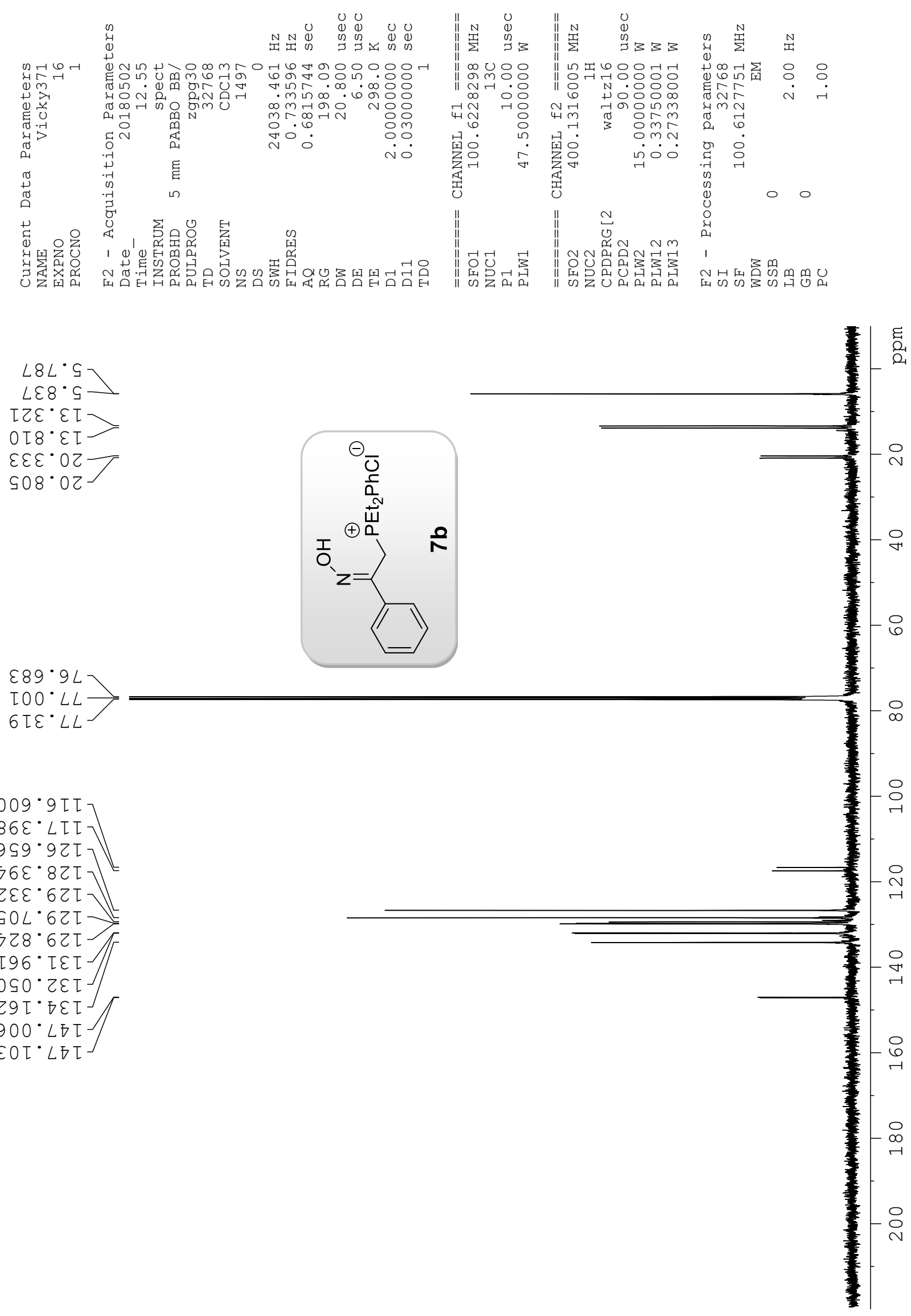


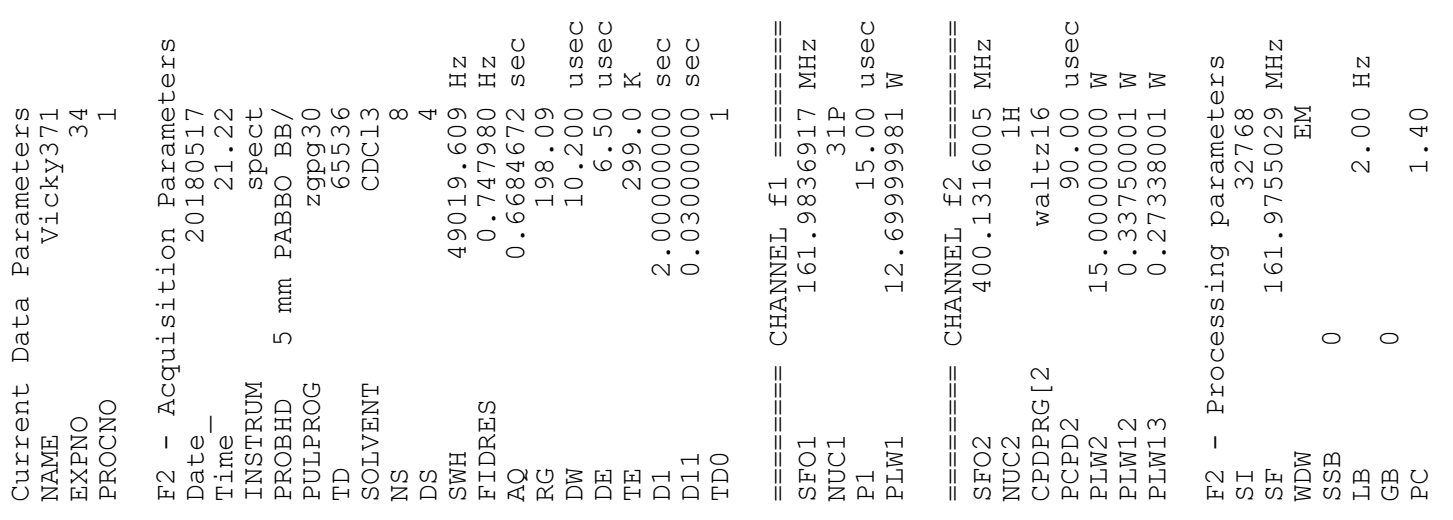

$900 \cdot 0-$
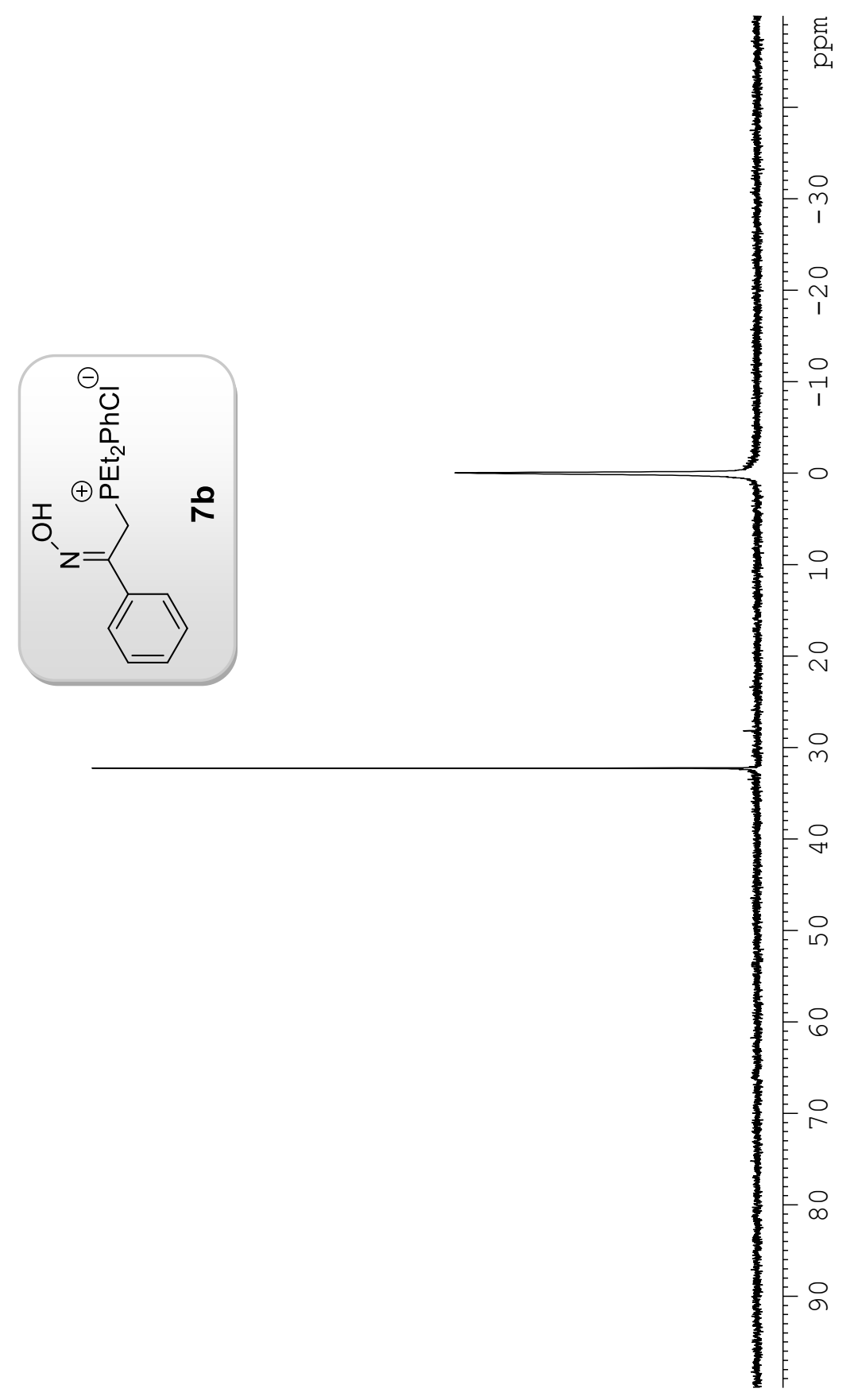

$\varepsilon\llcorner 乙 \cdot 乙 \varepsilon-$ 


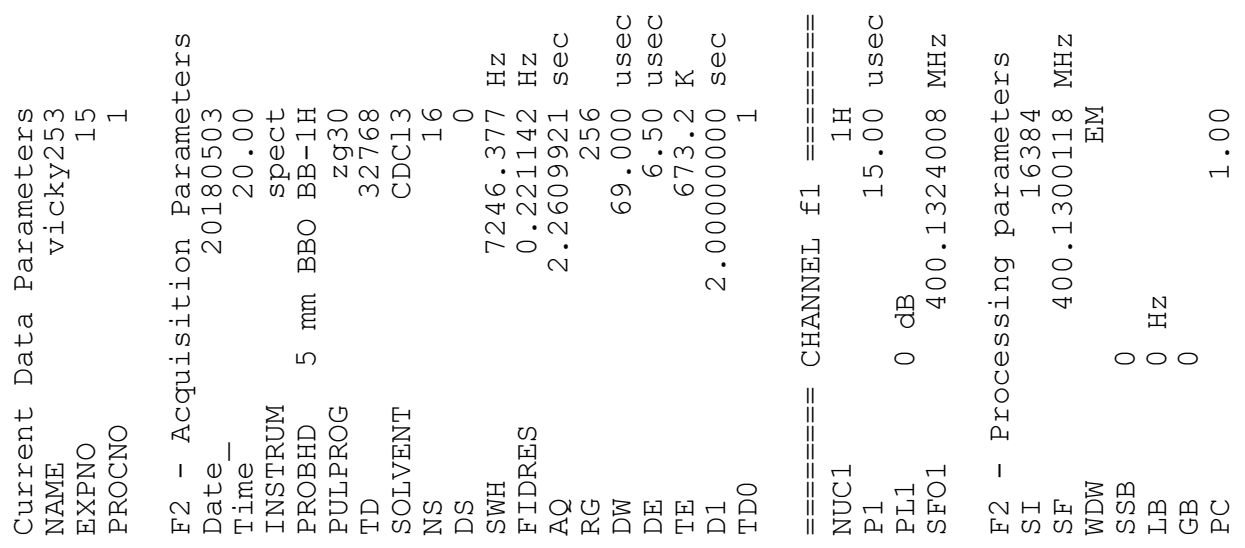

$600 \cdot 0-$

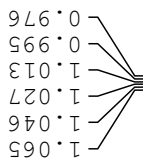

$868^{\circ} 2$

$9 โ 6^{\circ} \mathrm{r}$

โع $6^{\circ} 2$

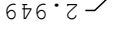

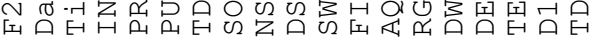

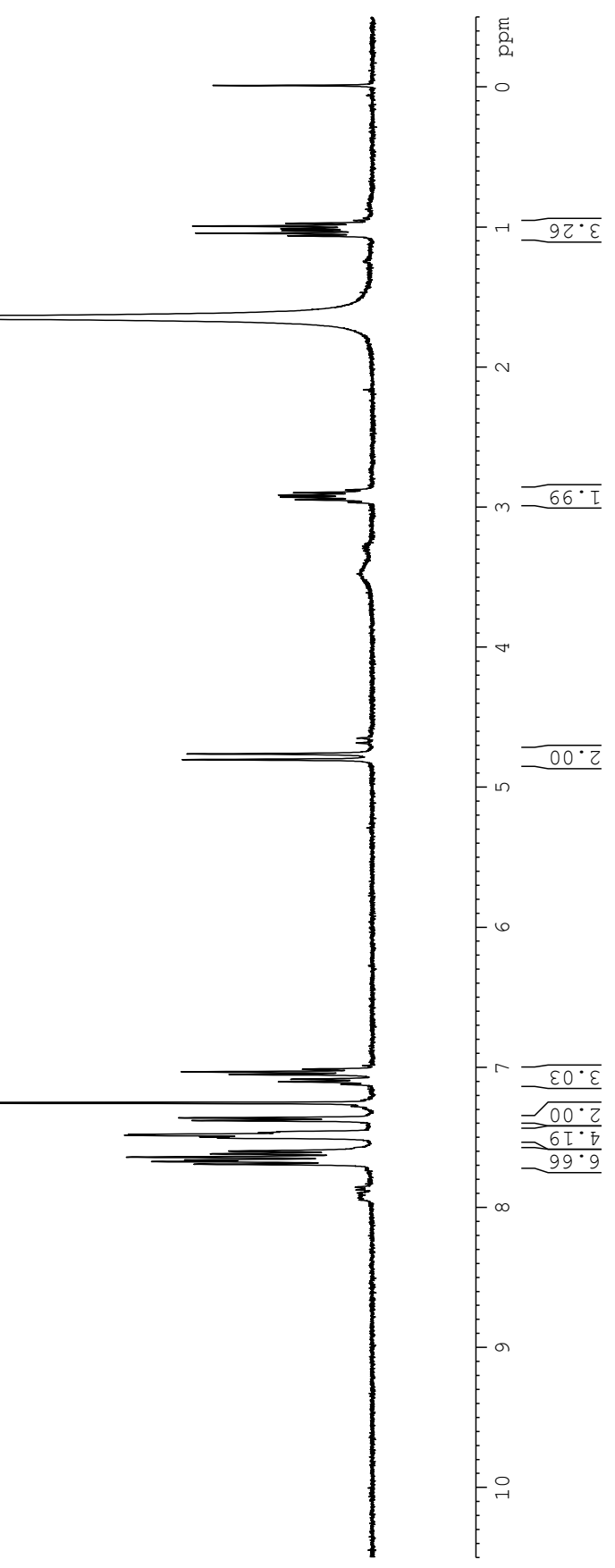

$\varepsilon 9 L^{\circ} \cdot \square$
万08. $=$
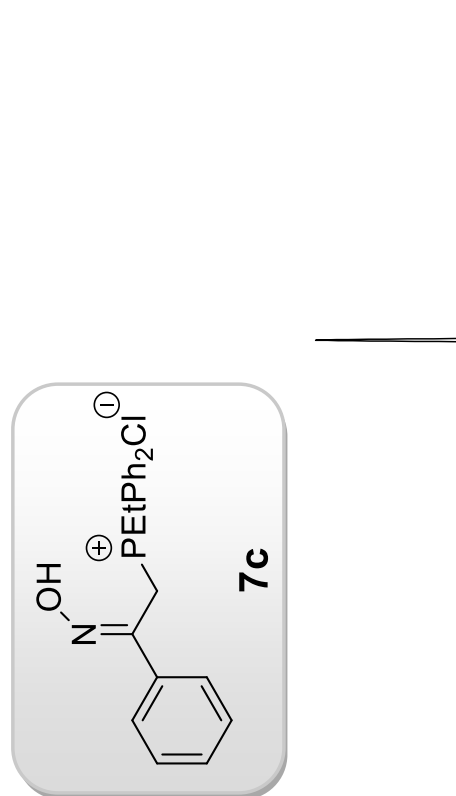

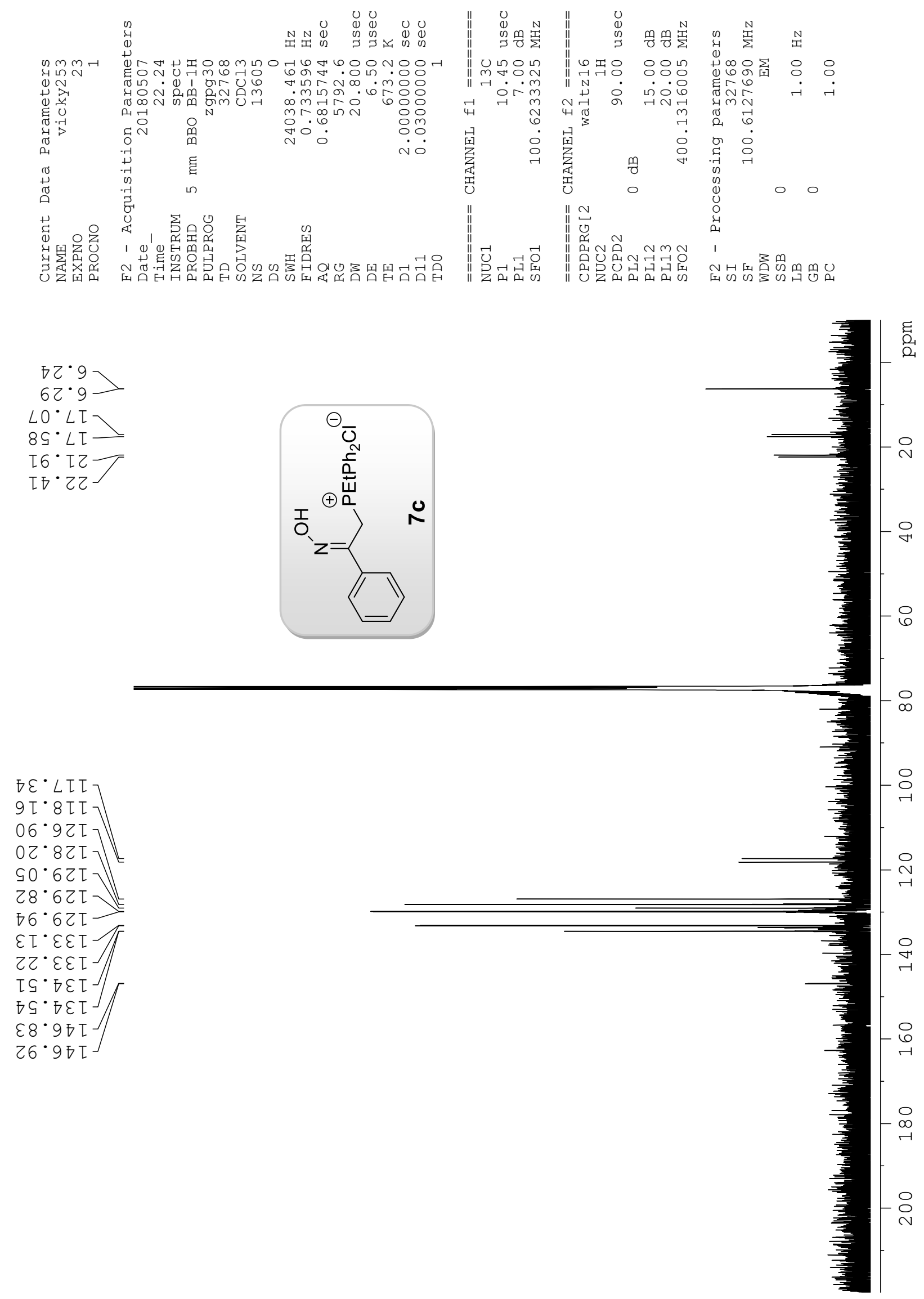


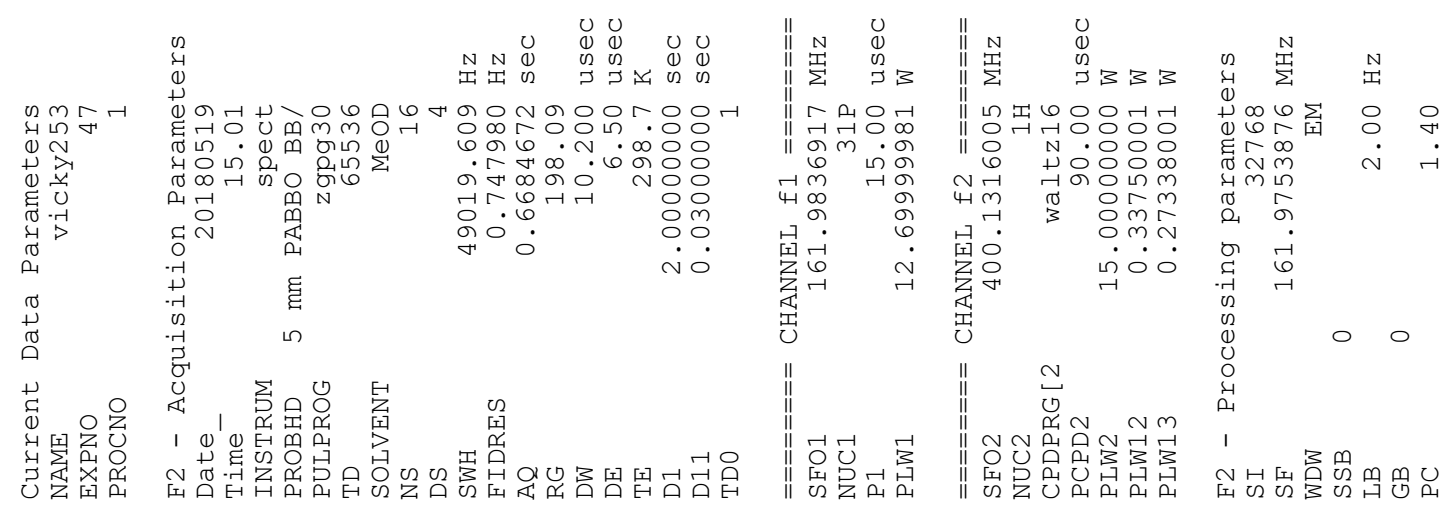

$00 \cdot 0-$

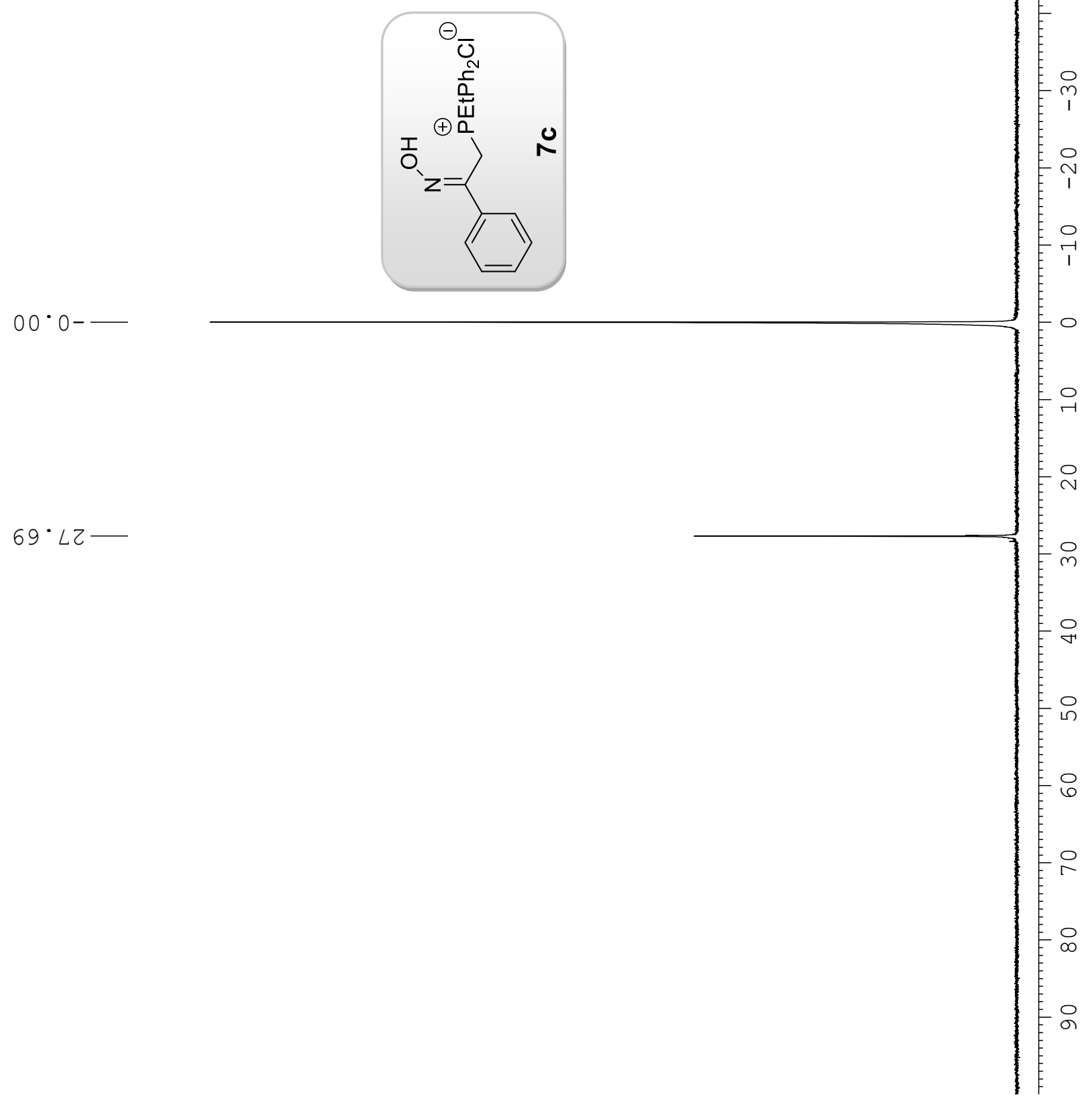

$69 \cdot \mathrm{LZ}$

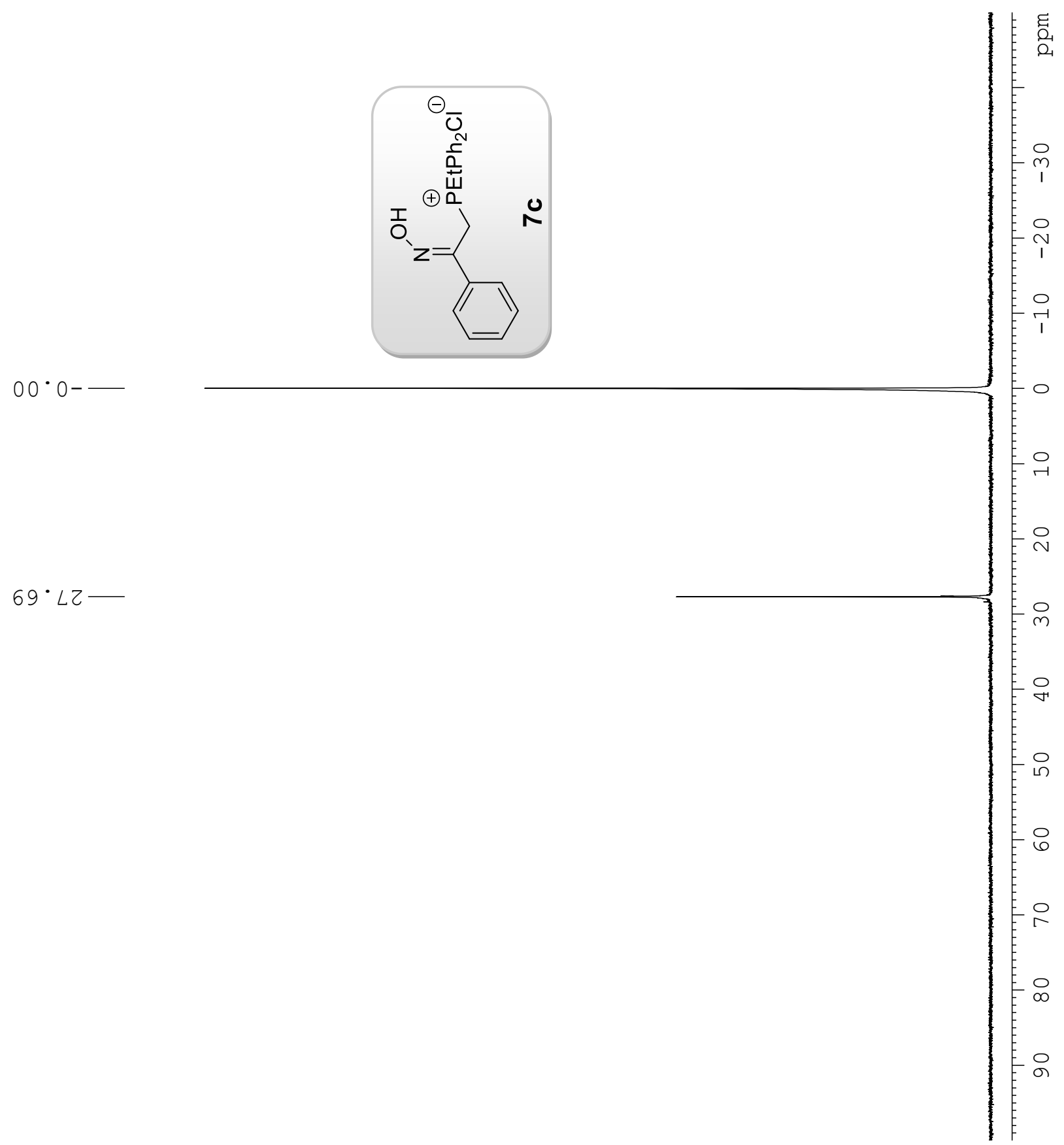



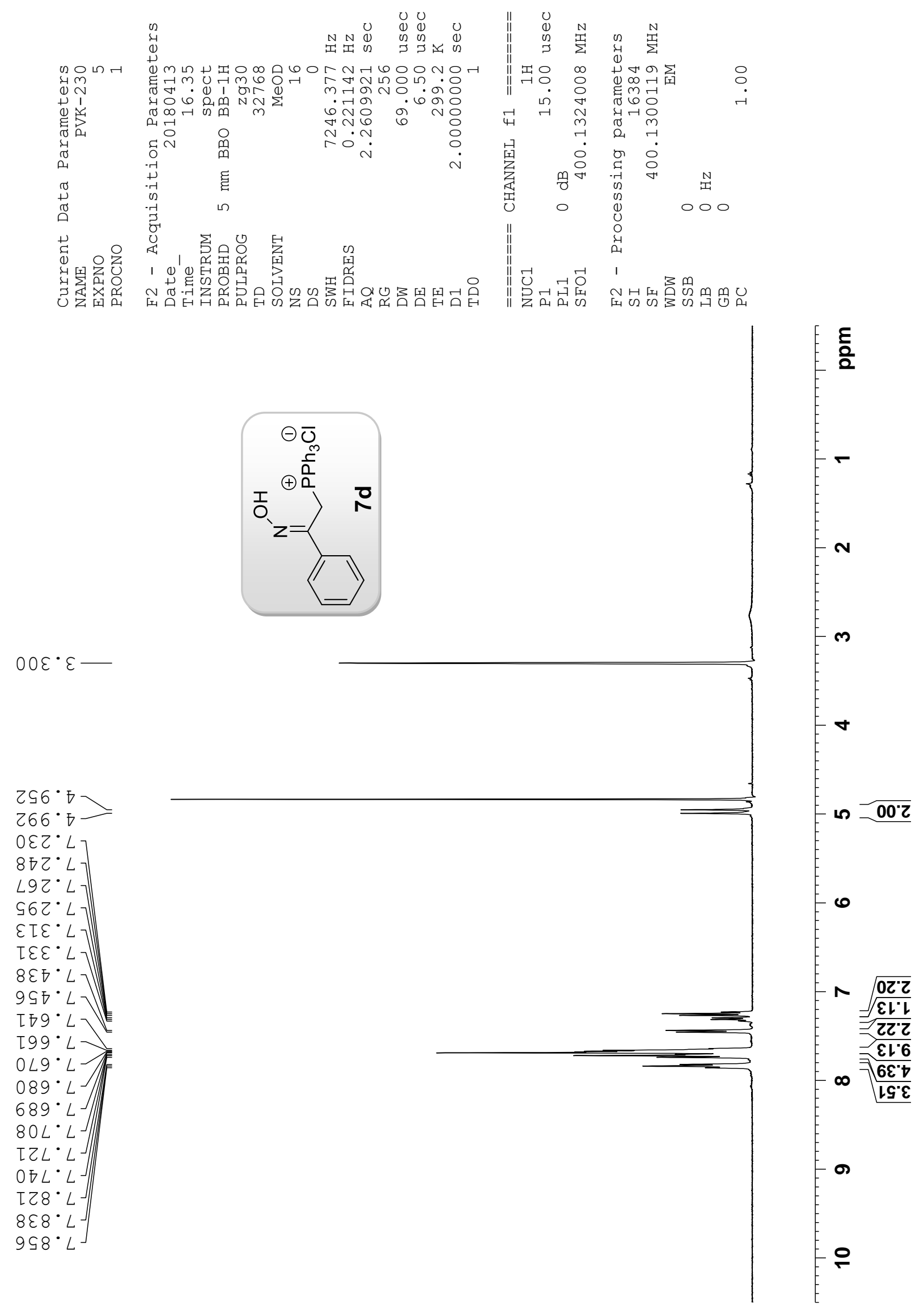

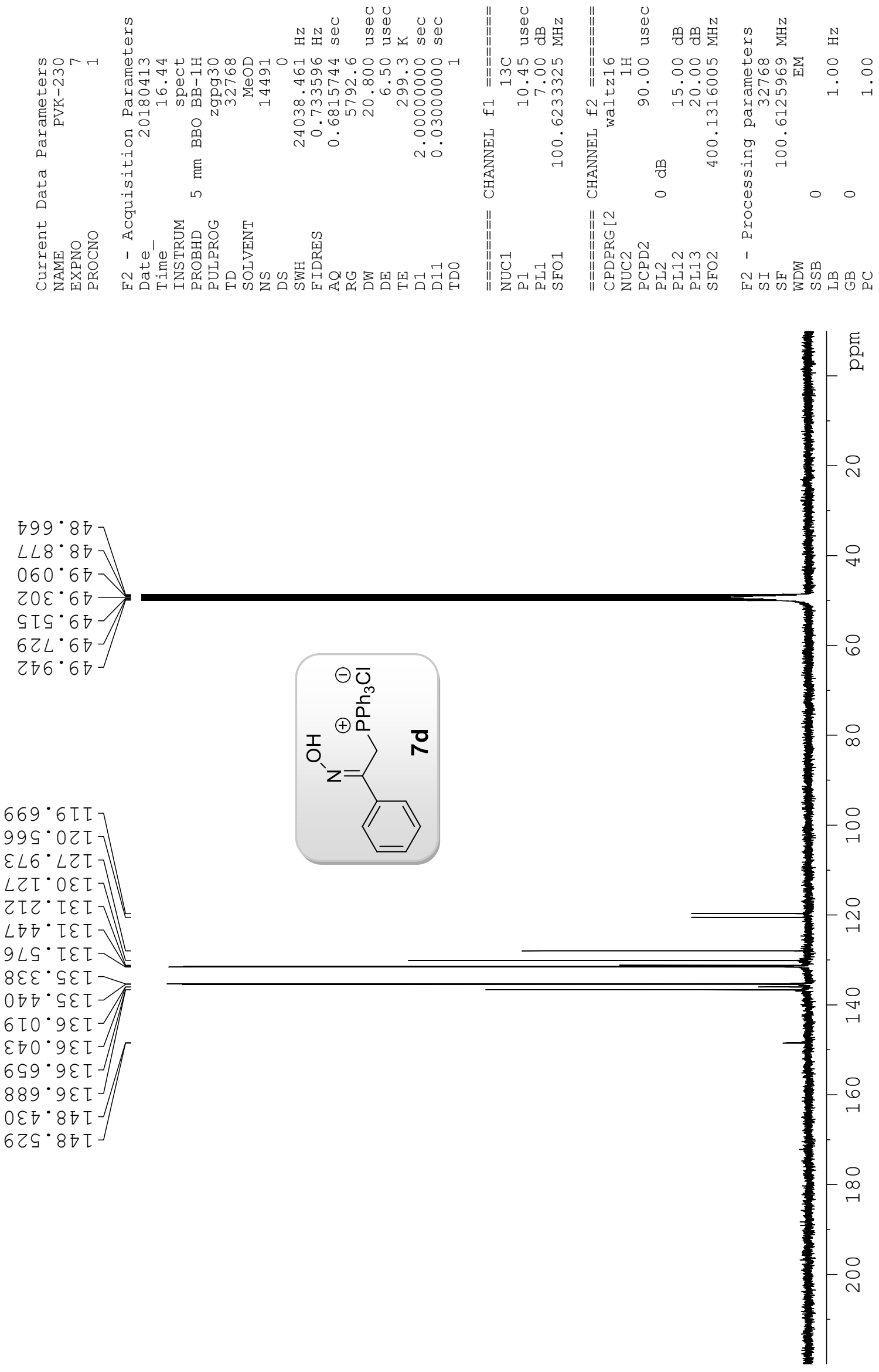

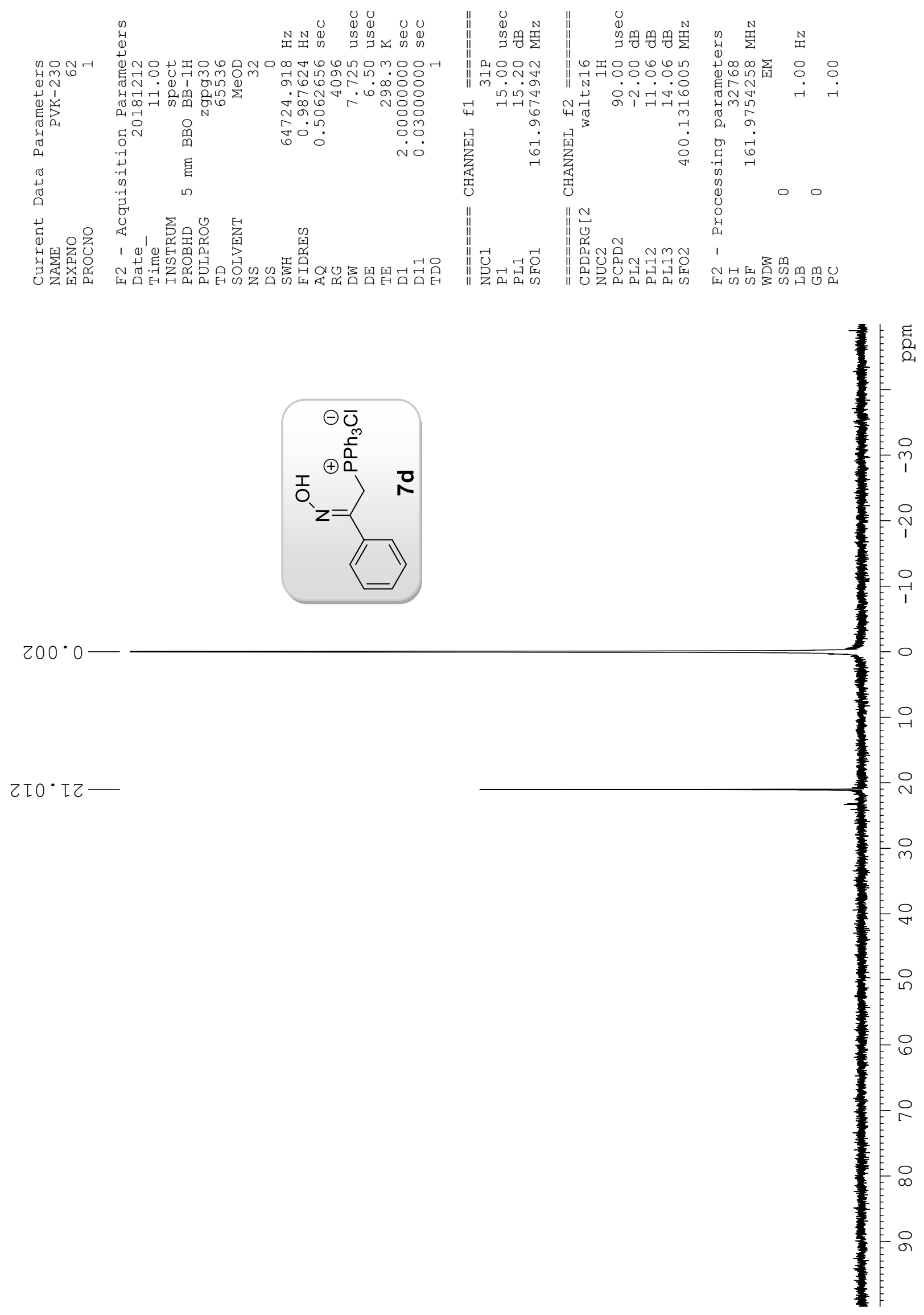


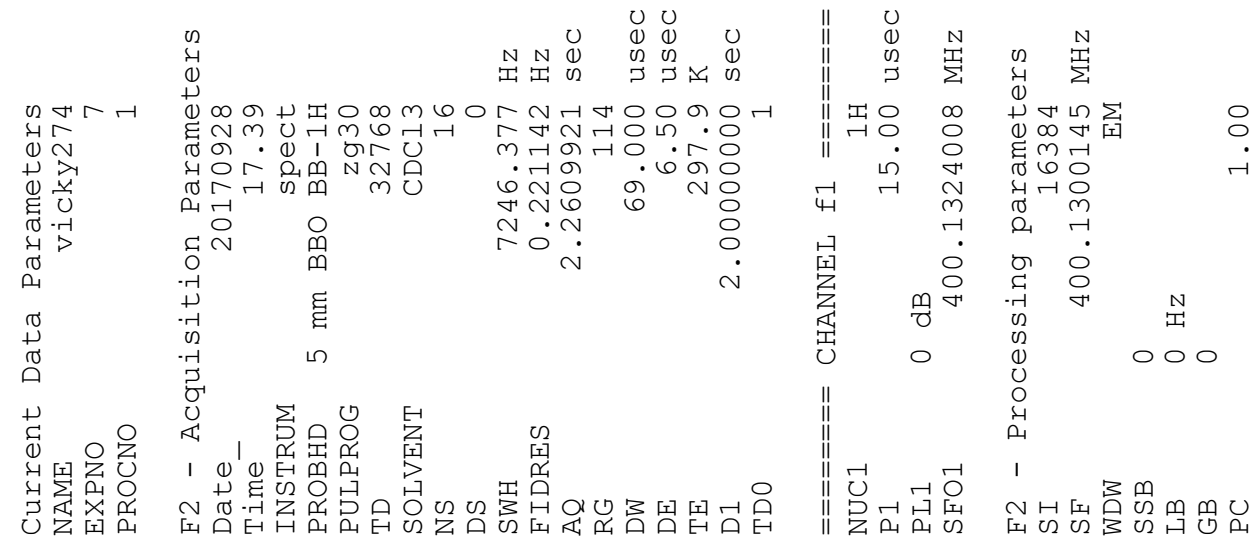

$00 \cdot 0-$

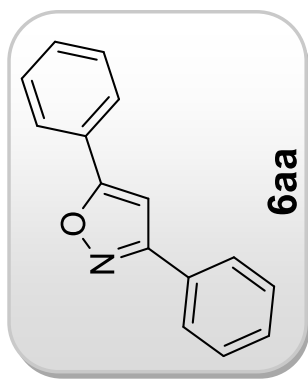

틍

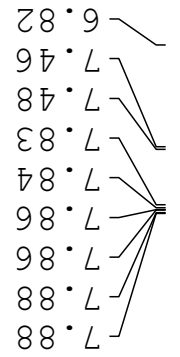



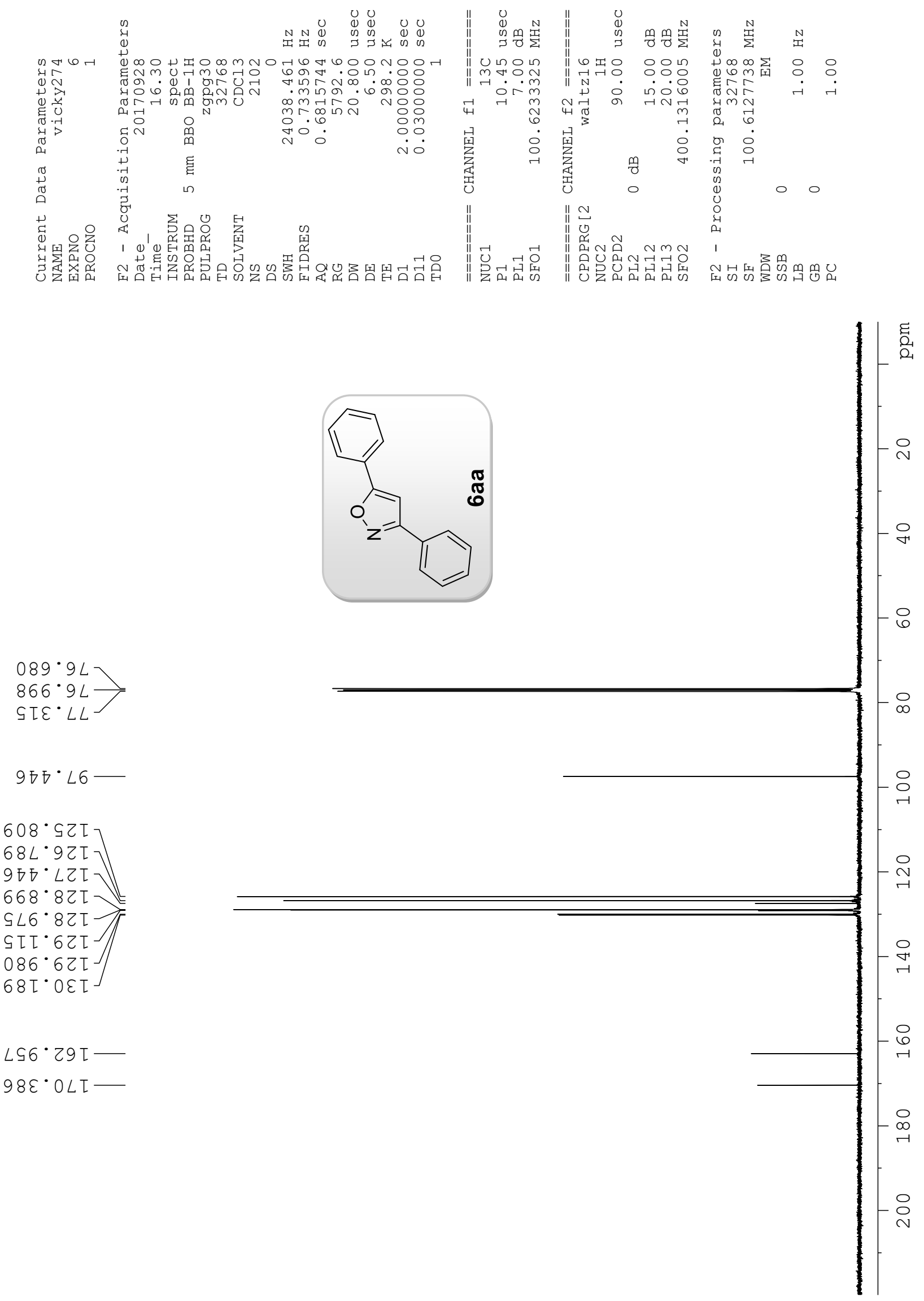

| 


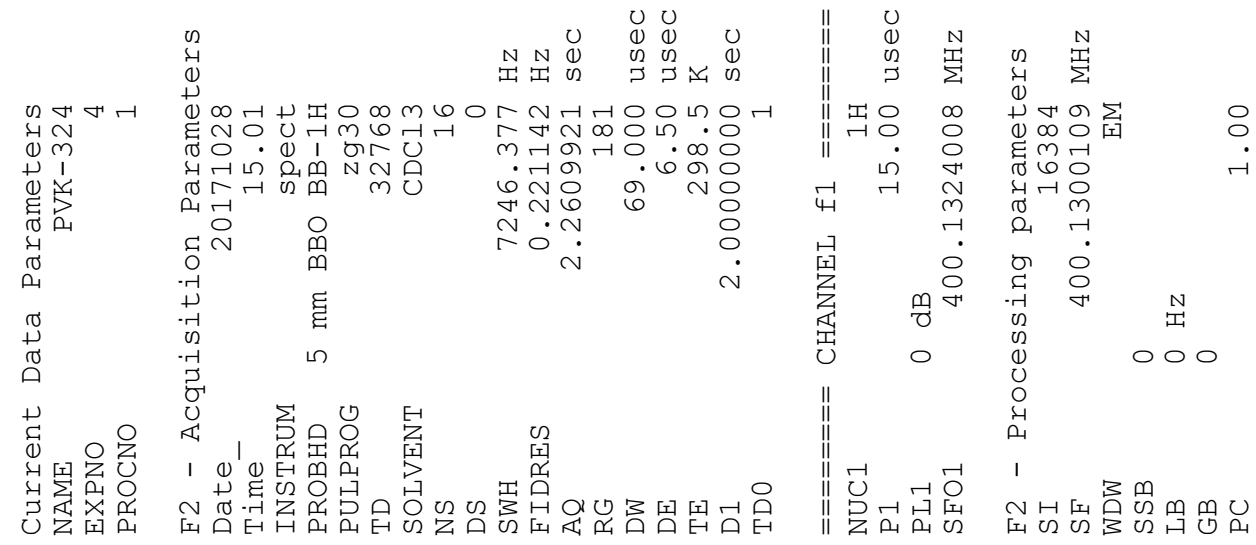

$00 \cdot 0-$

$\angle 8^{\circ} \mathcal{E}$
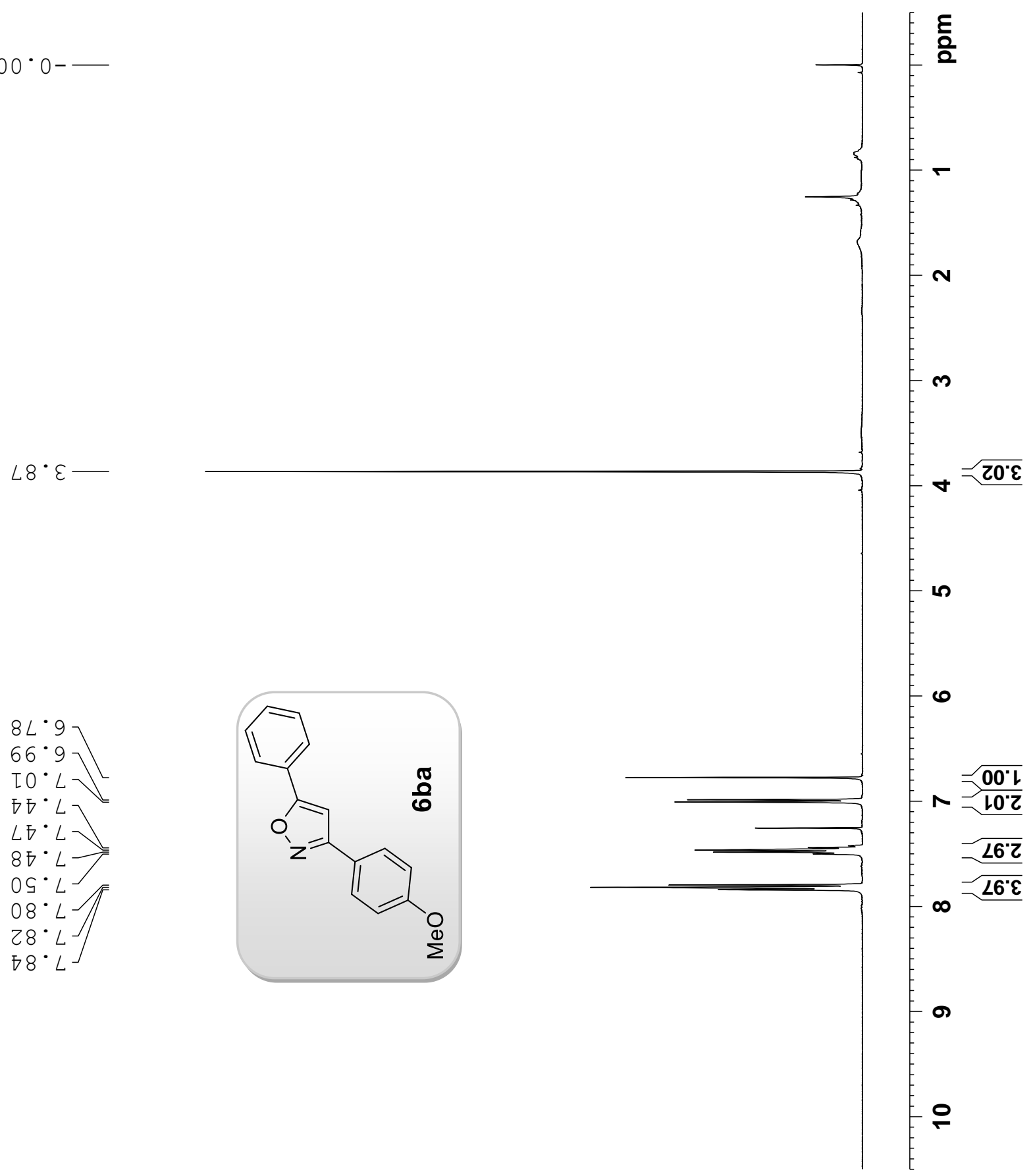

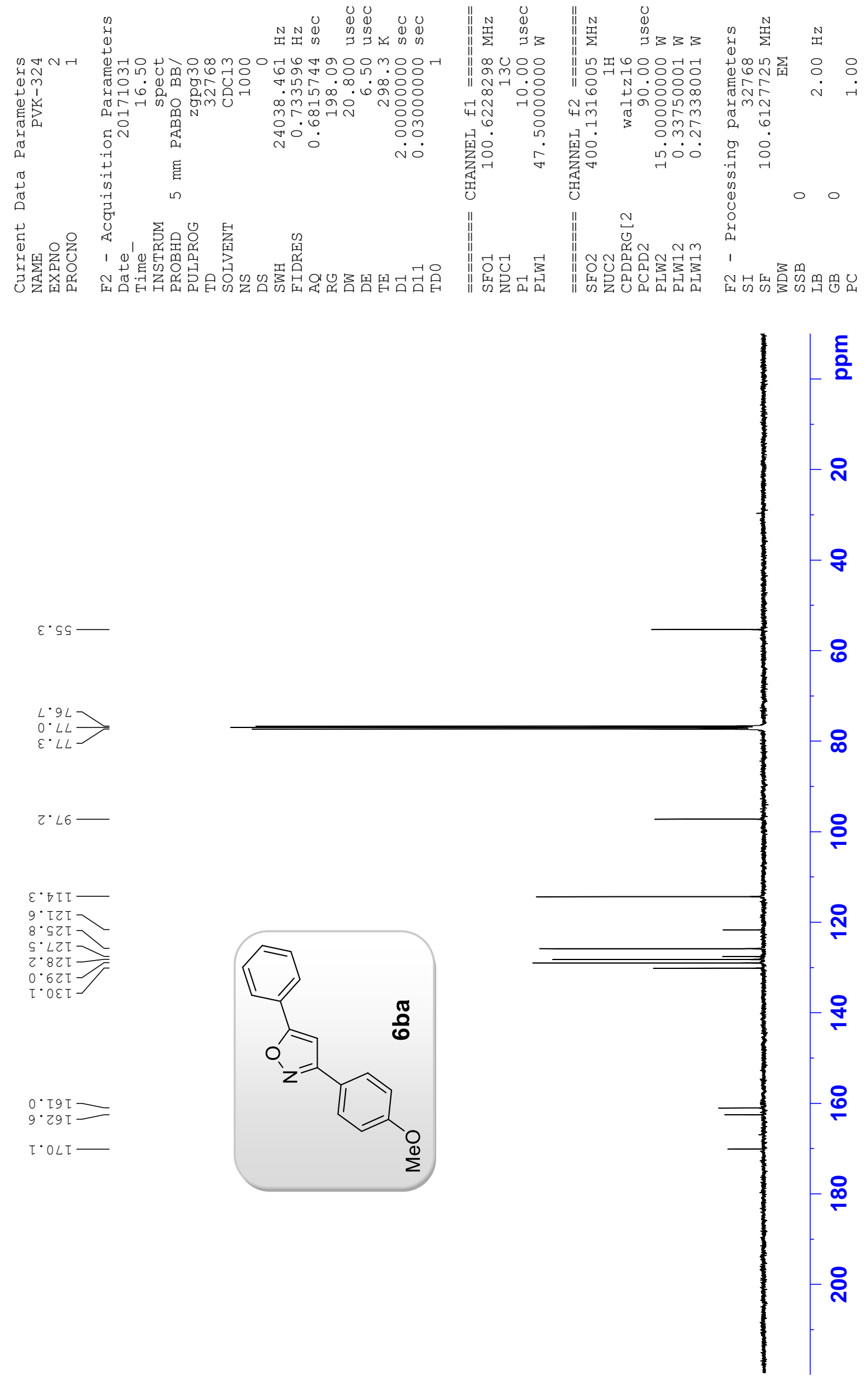


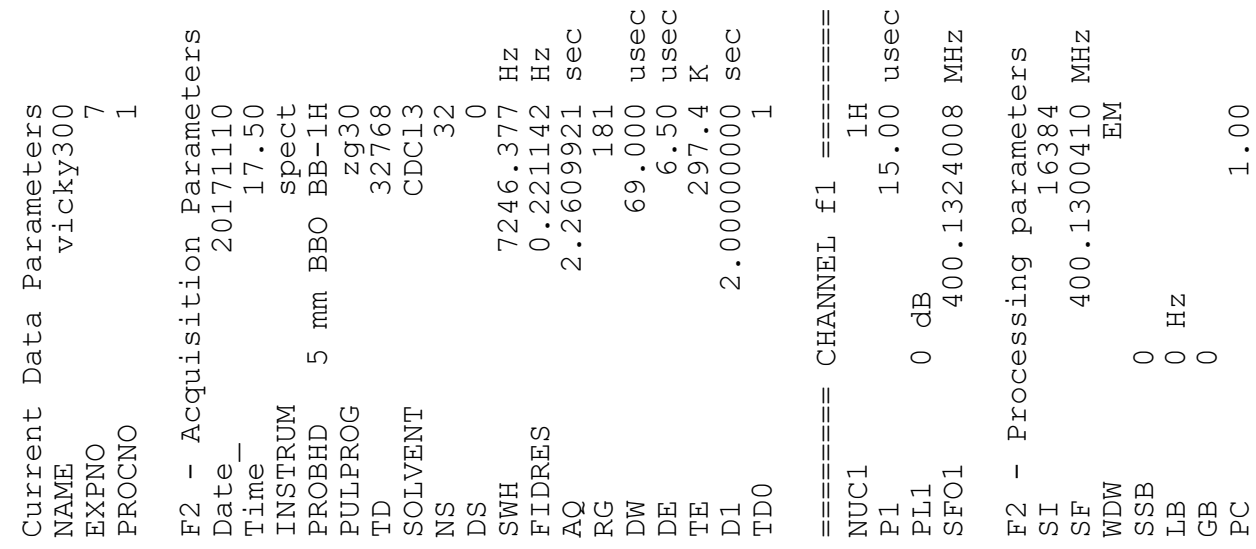

$00 \cdot 0$
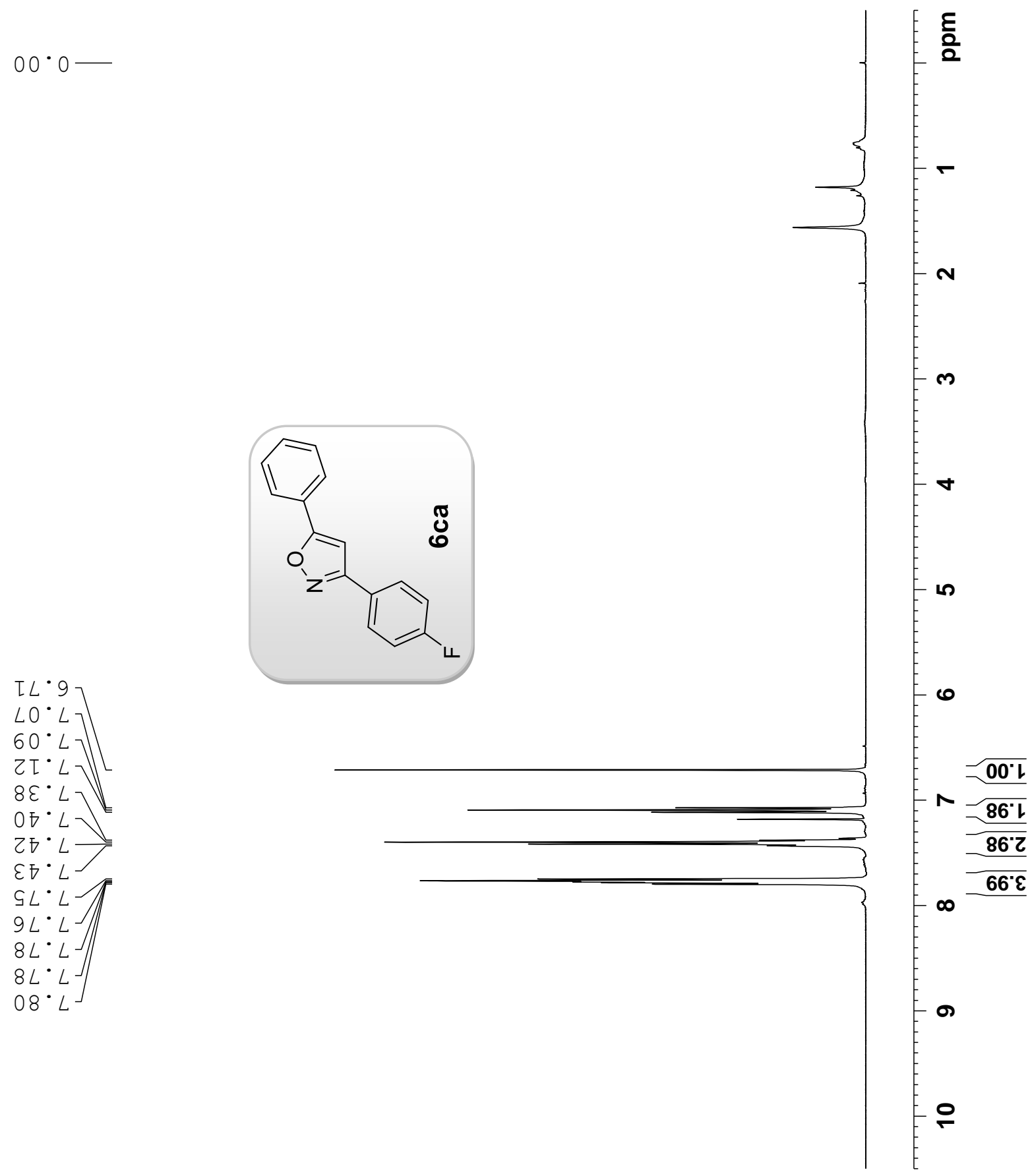

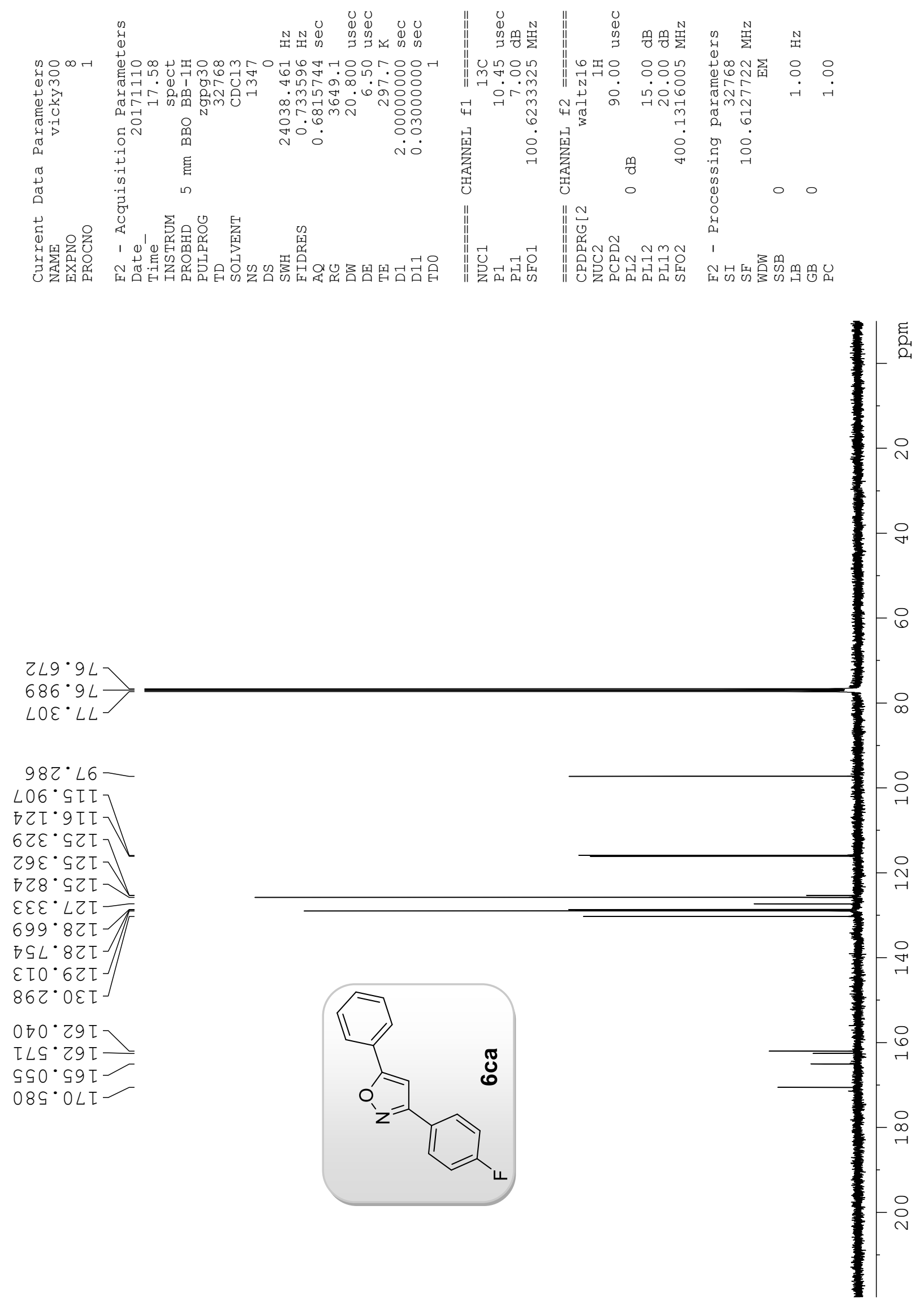

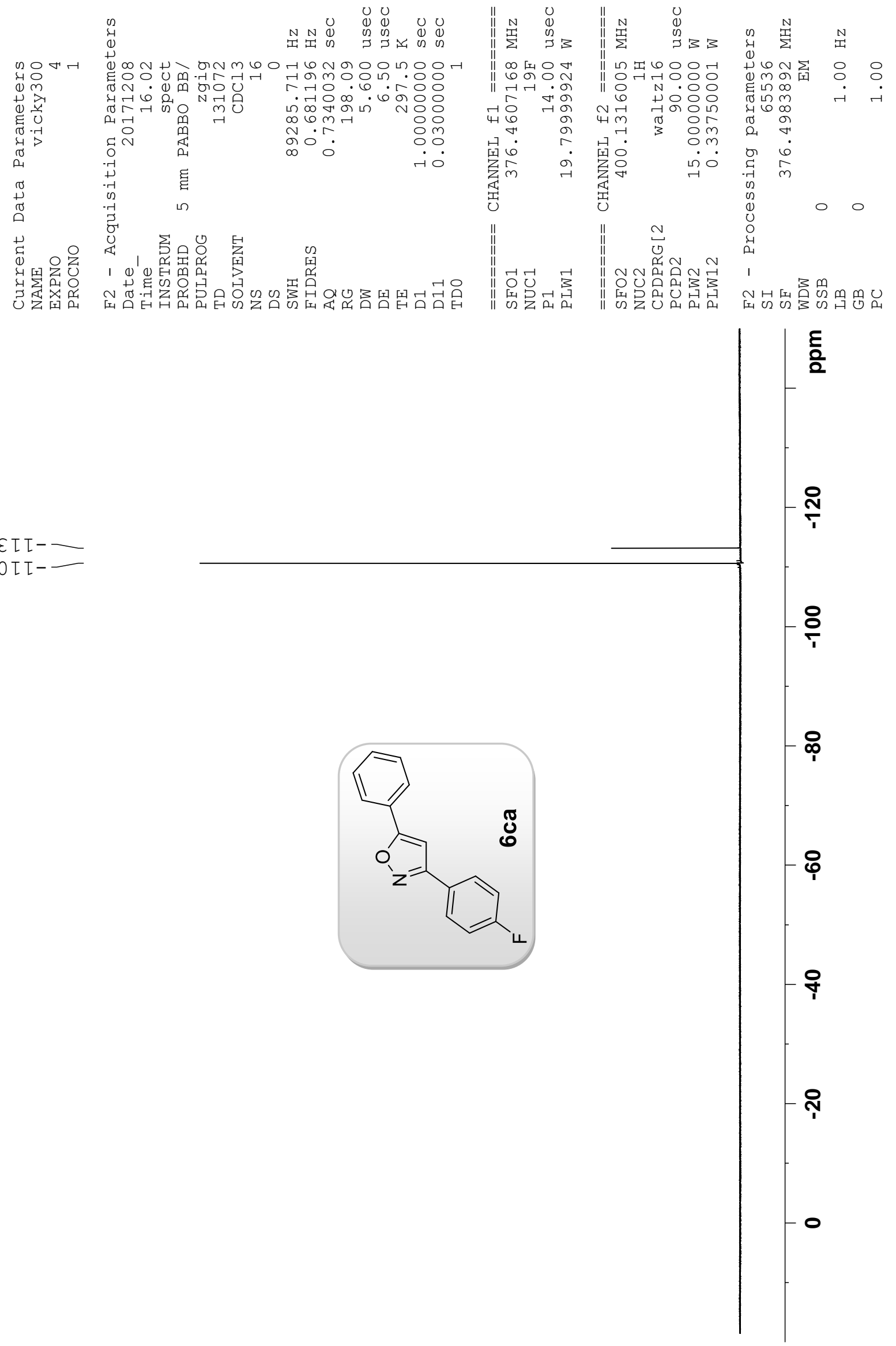


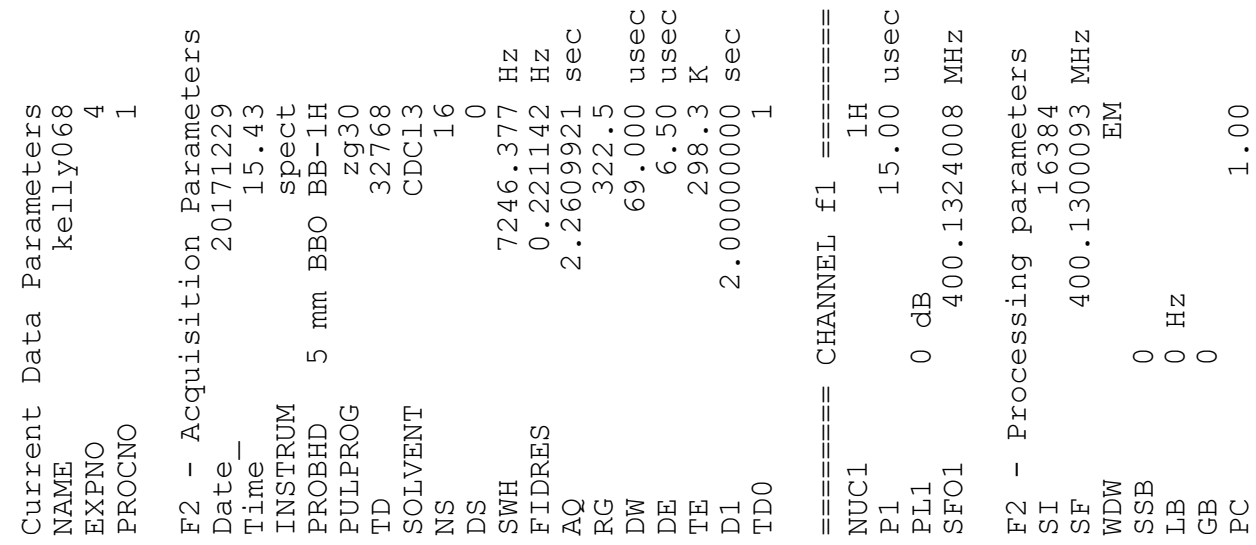

$00 \cdot 0-$
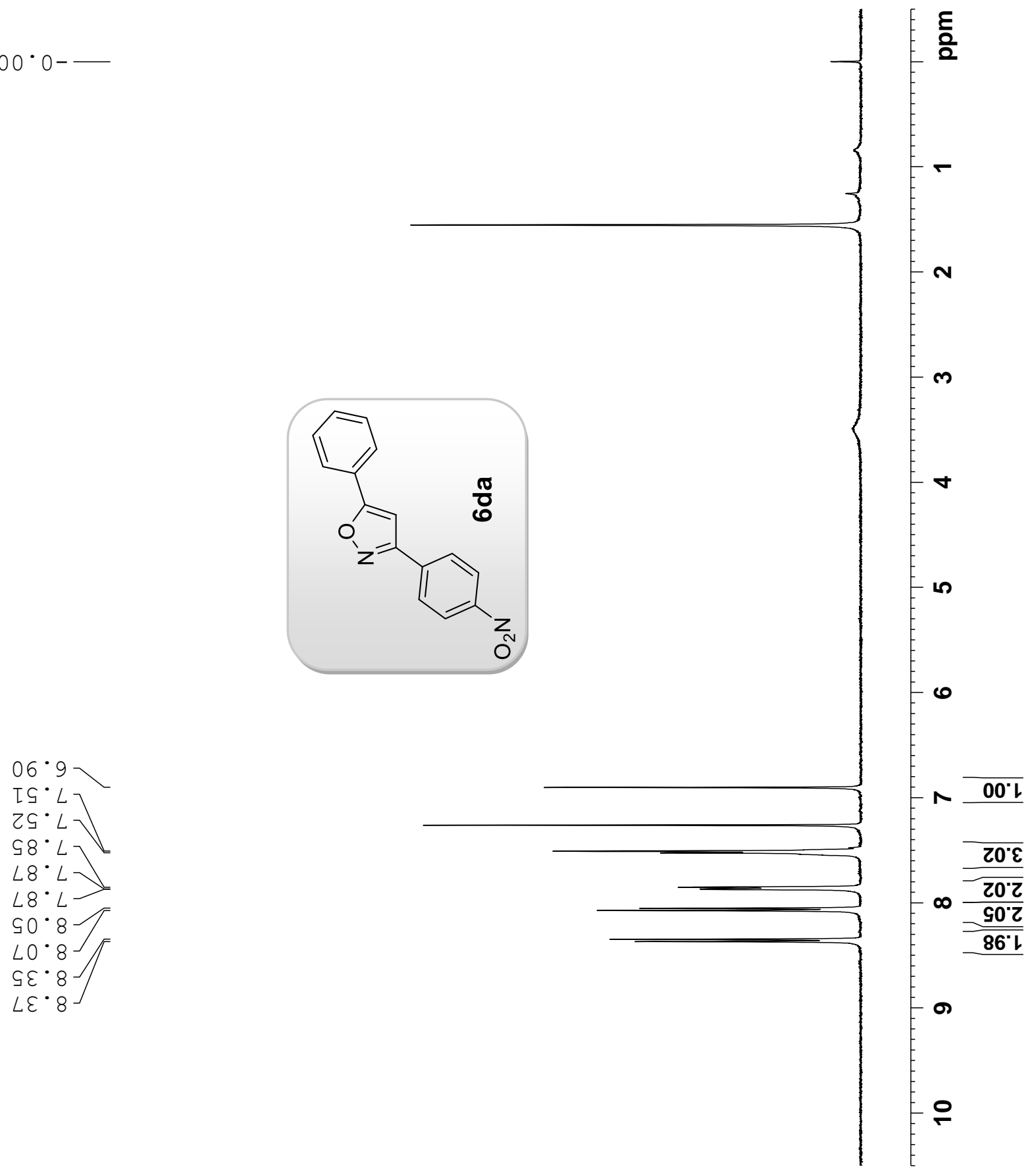

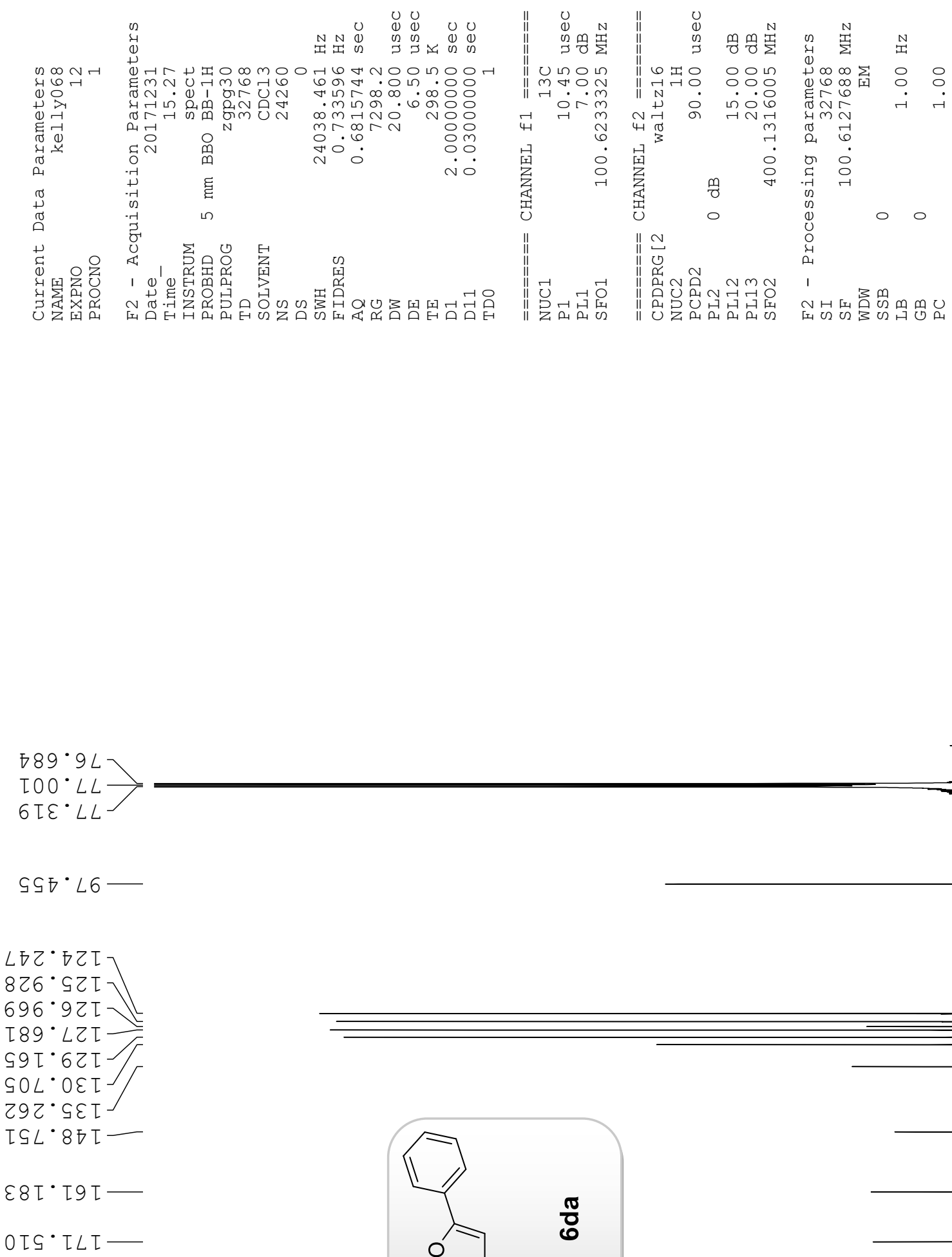

OIS TLI

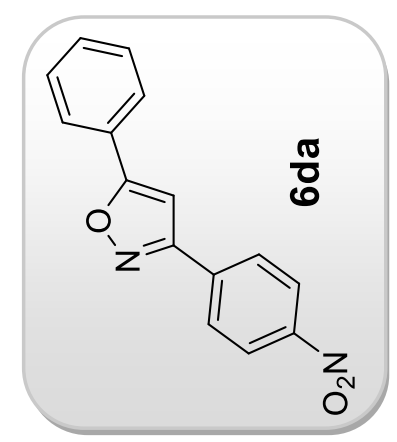




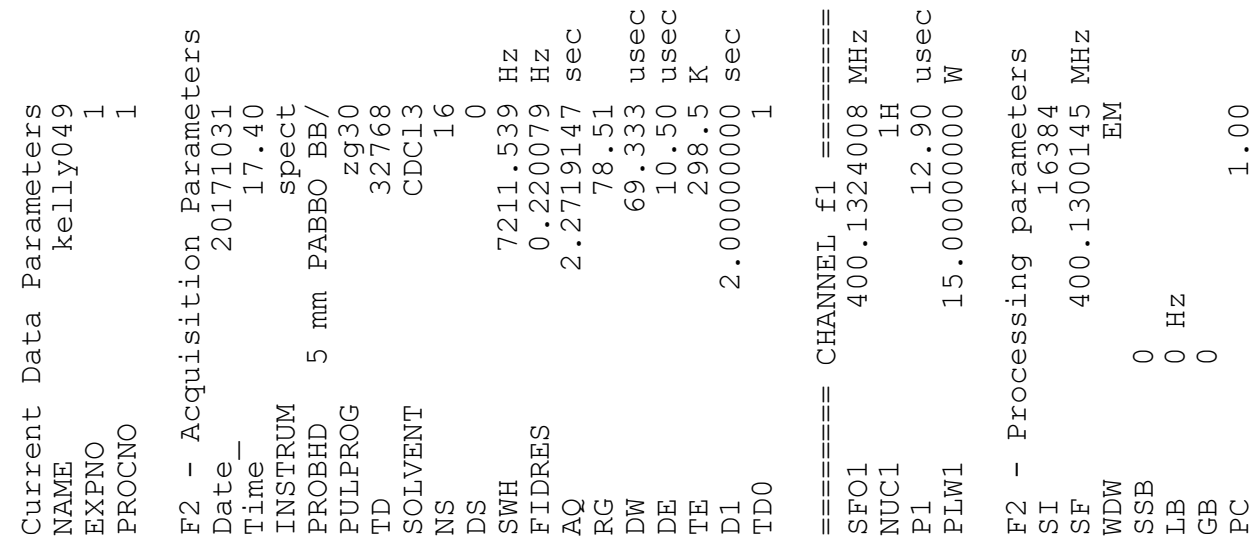

$00 \cdot 0-$

ても・て

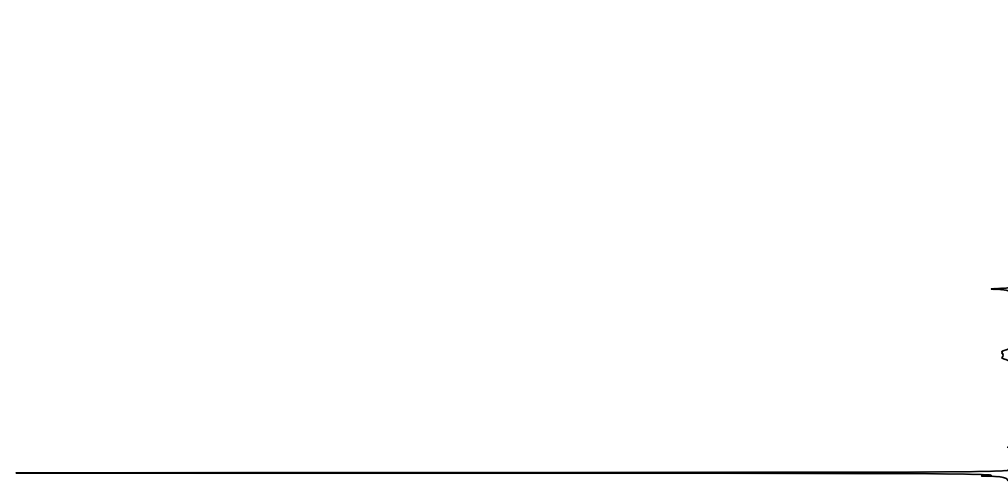

등
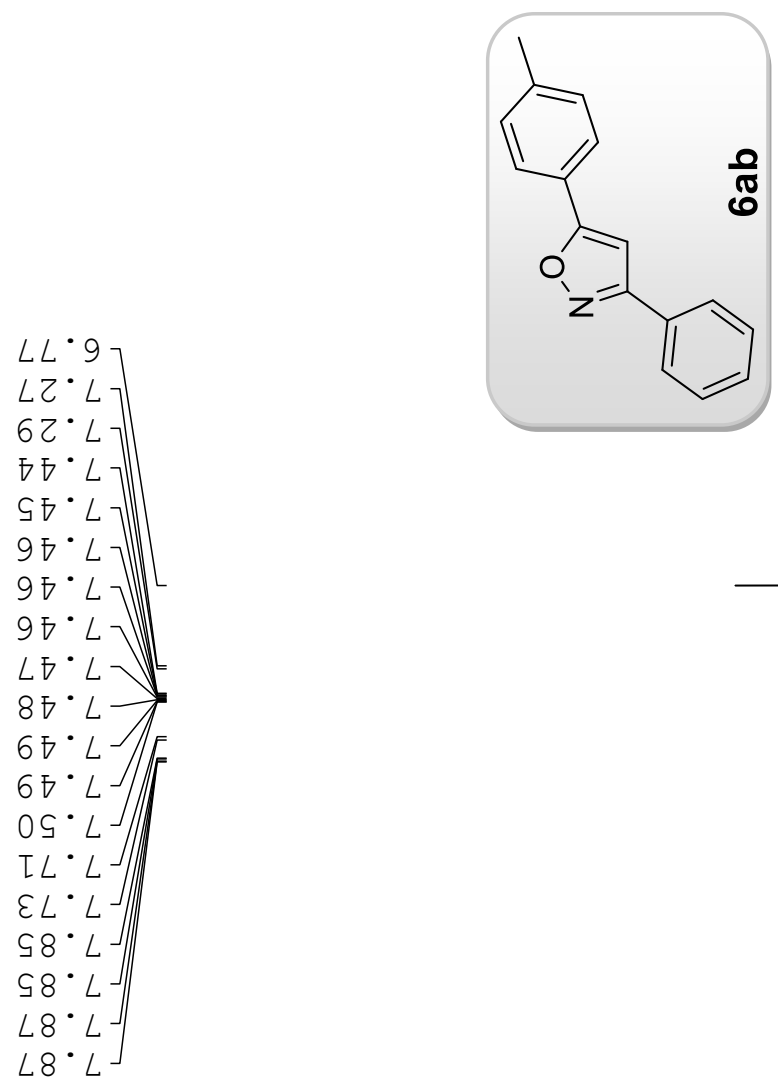

N

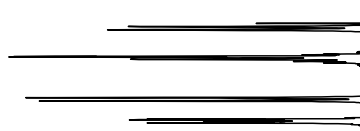

$N$ 

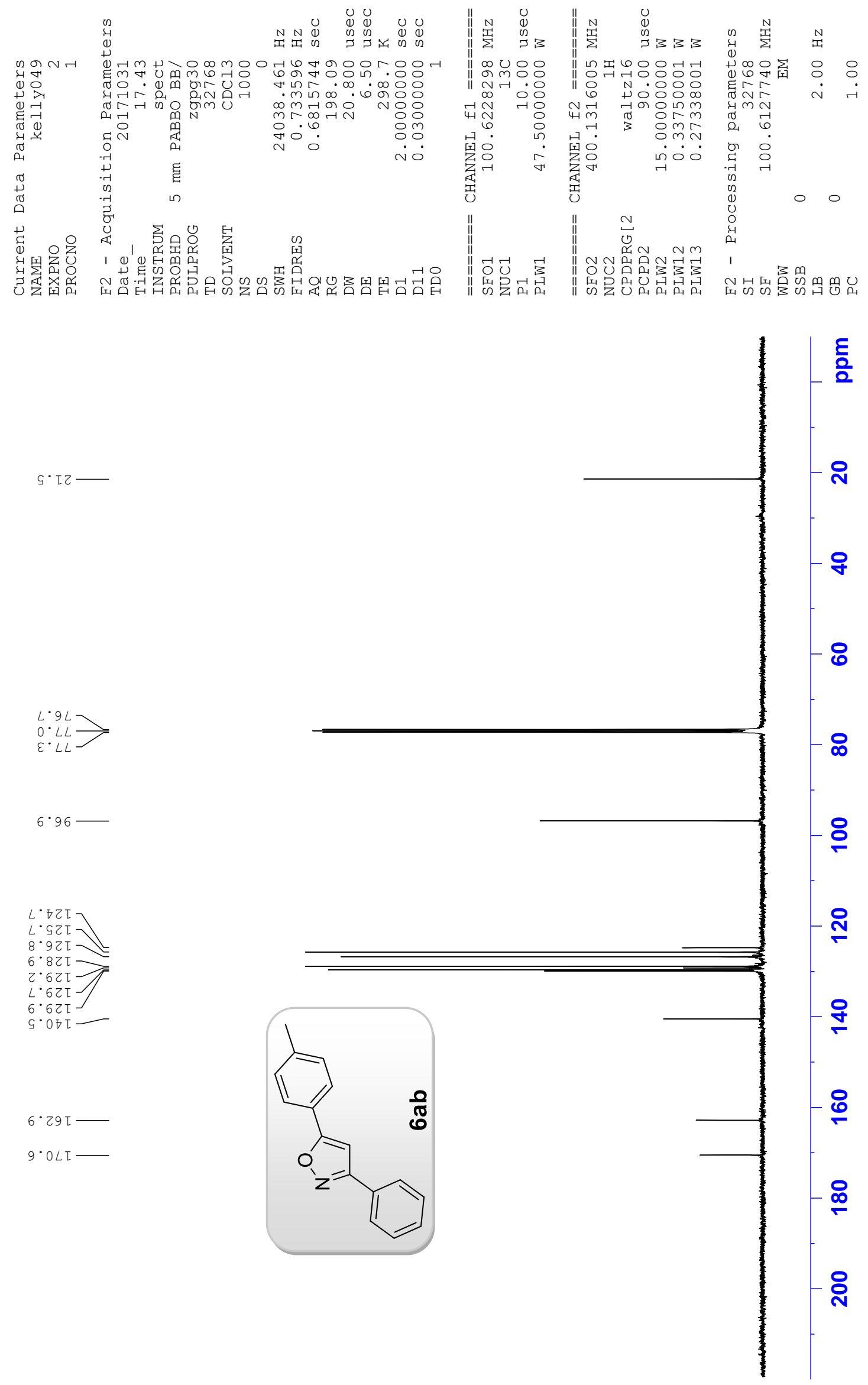

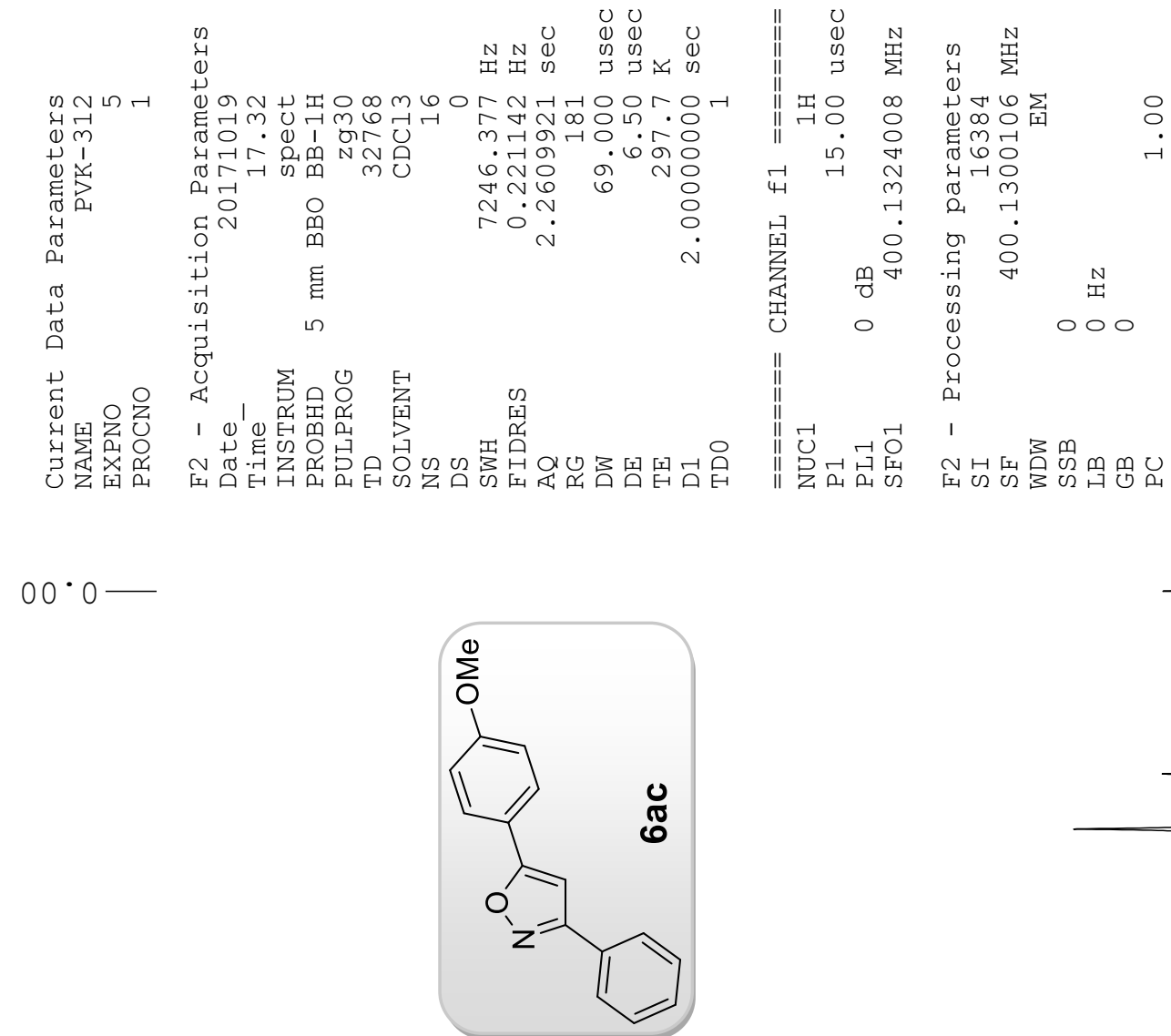

$\angle 8^{\circ} \varepsilon-$

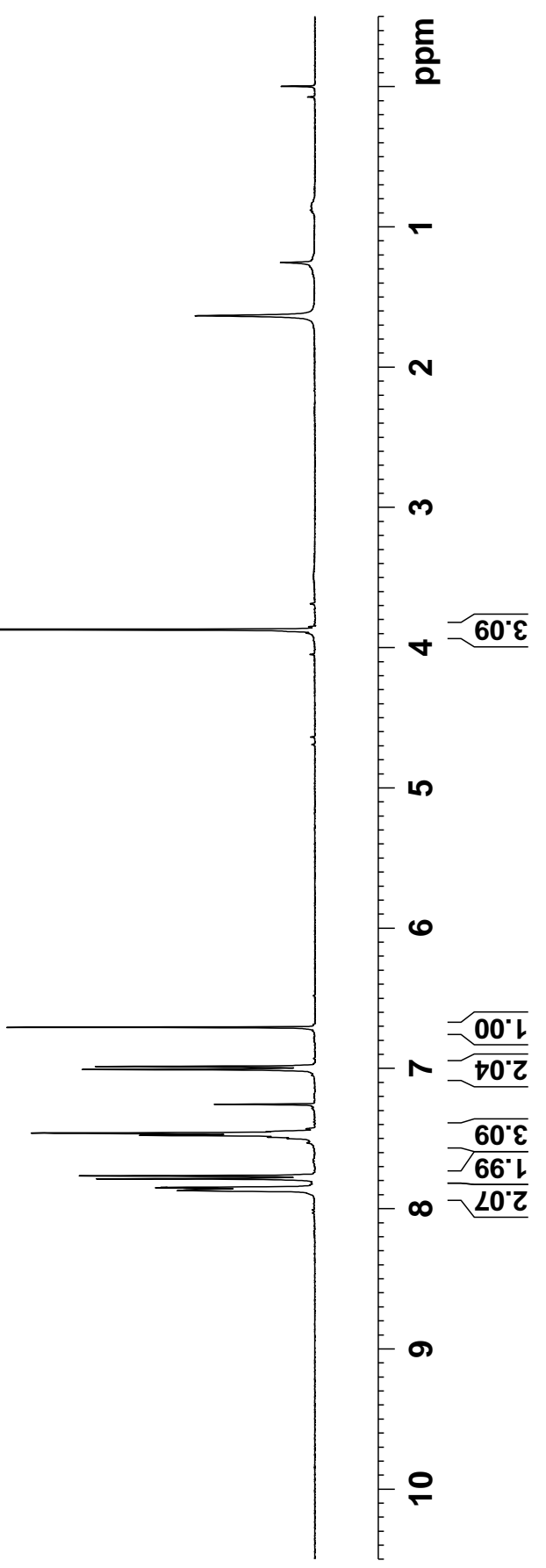

$[L \cdot 9$
$66 \cdot 9$
$20 \cdot L]$
$97 \cdot L]$
$87 \cdot L]$
$L L \cdot L$
$6 L \cdot L]$
$98 \cdot L$
$98 \cdot L$ 

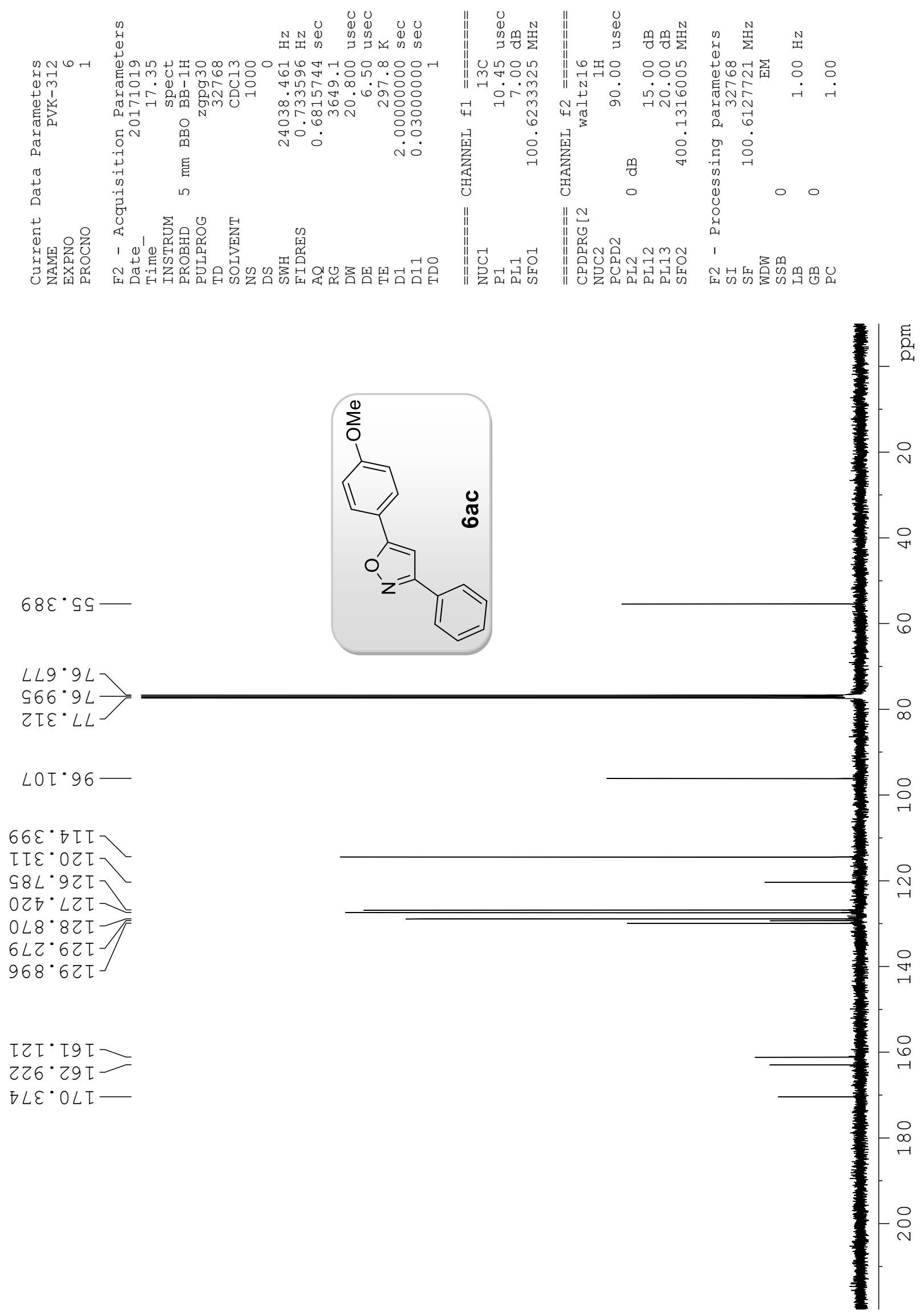


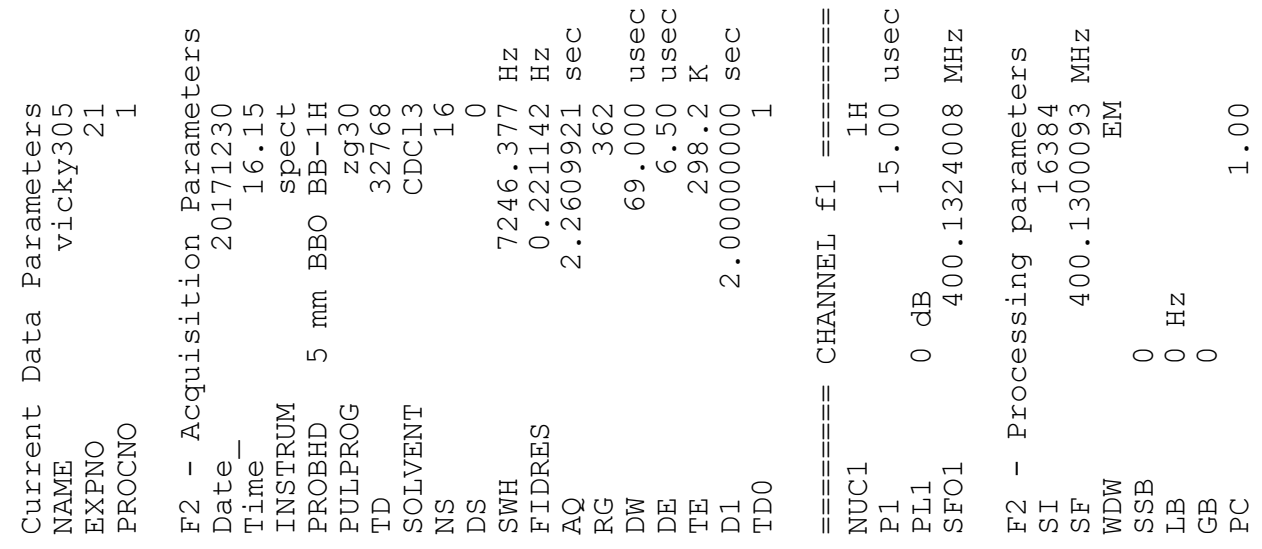

$00 \cdot 0-$

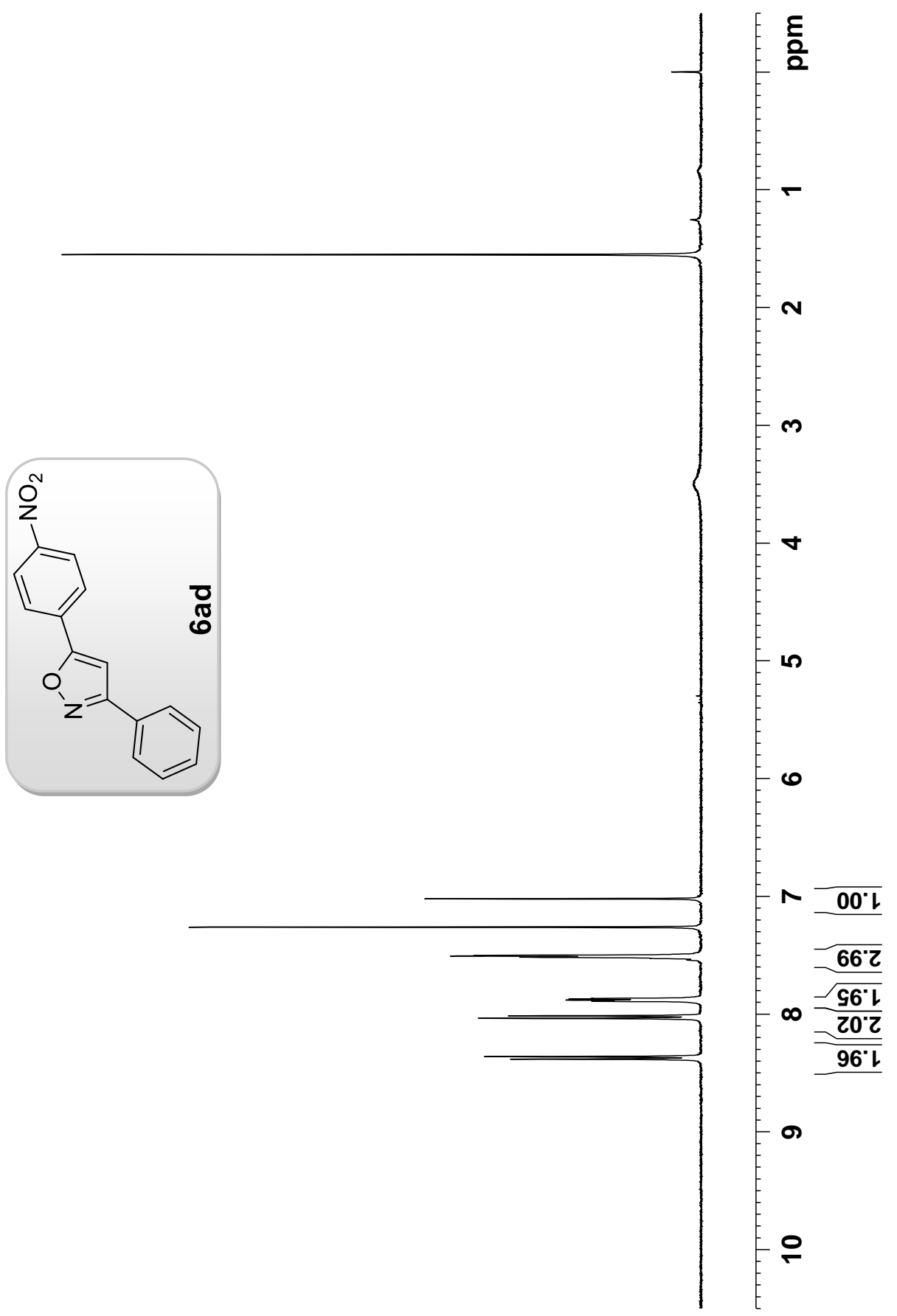



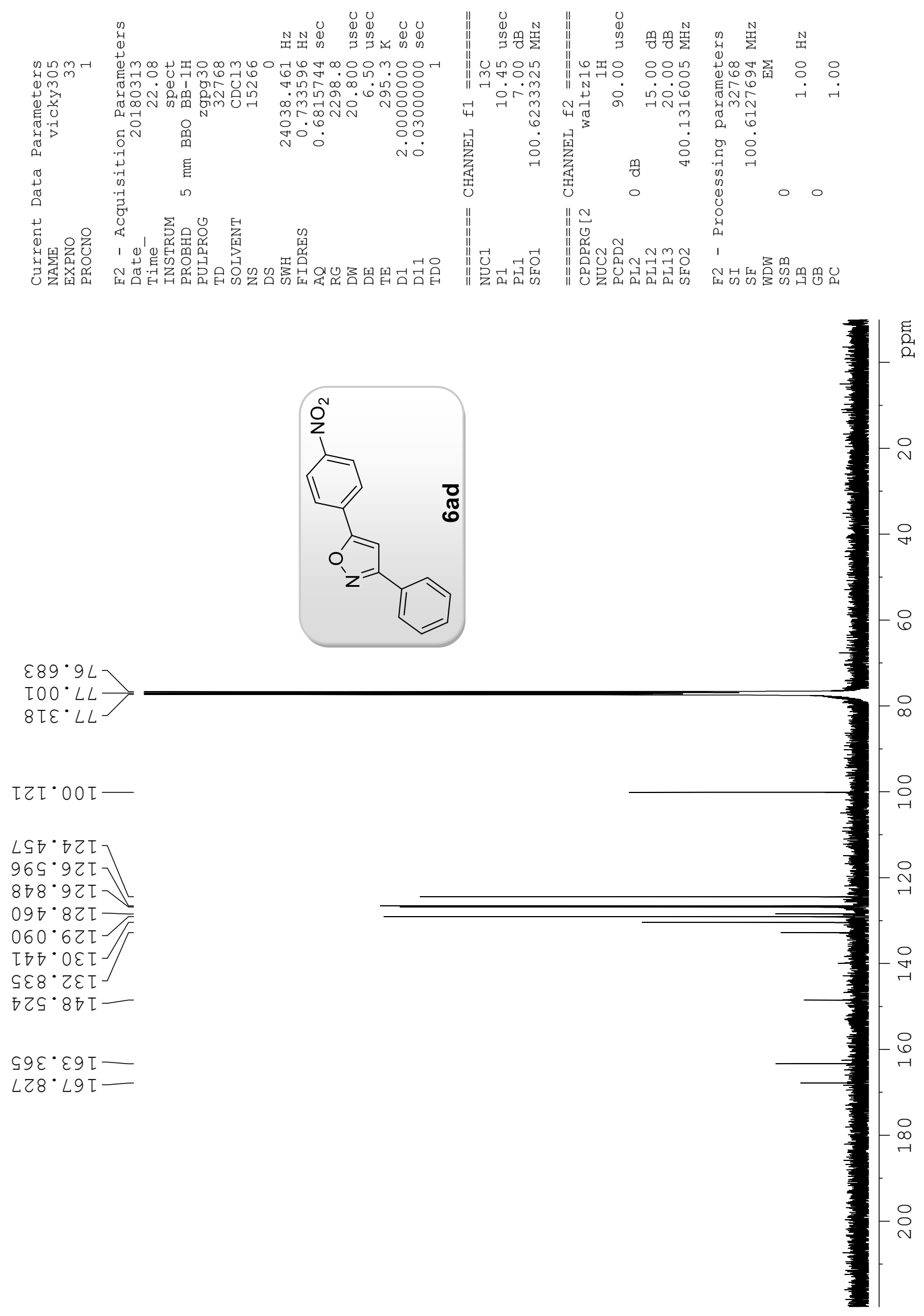


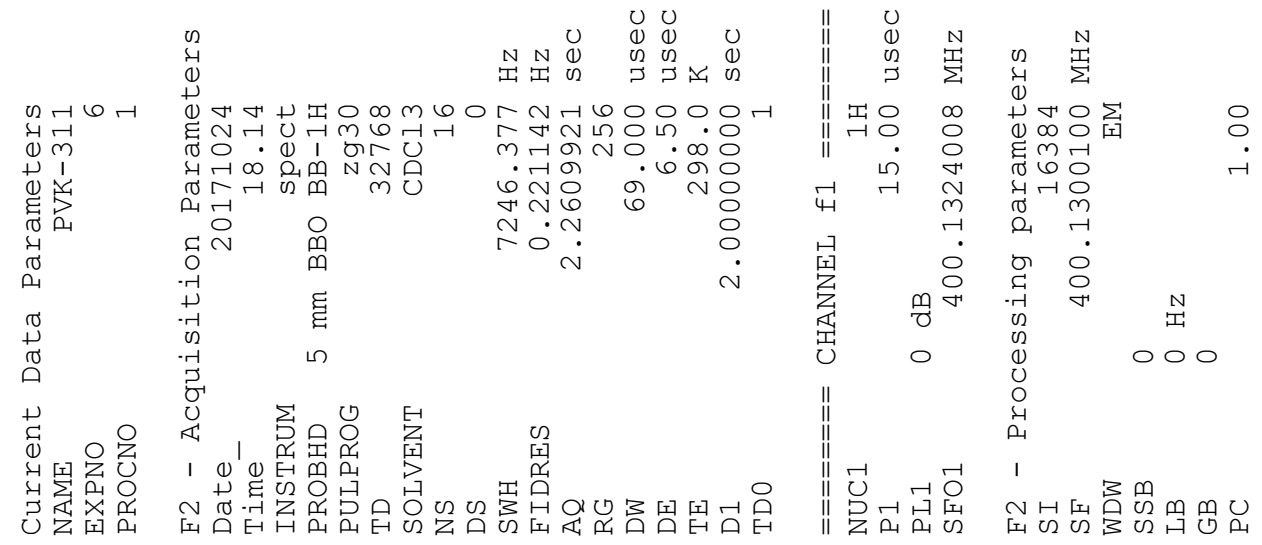

$00 \cdot 0-$
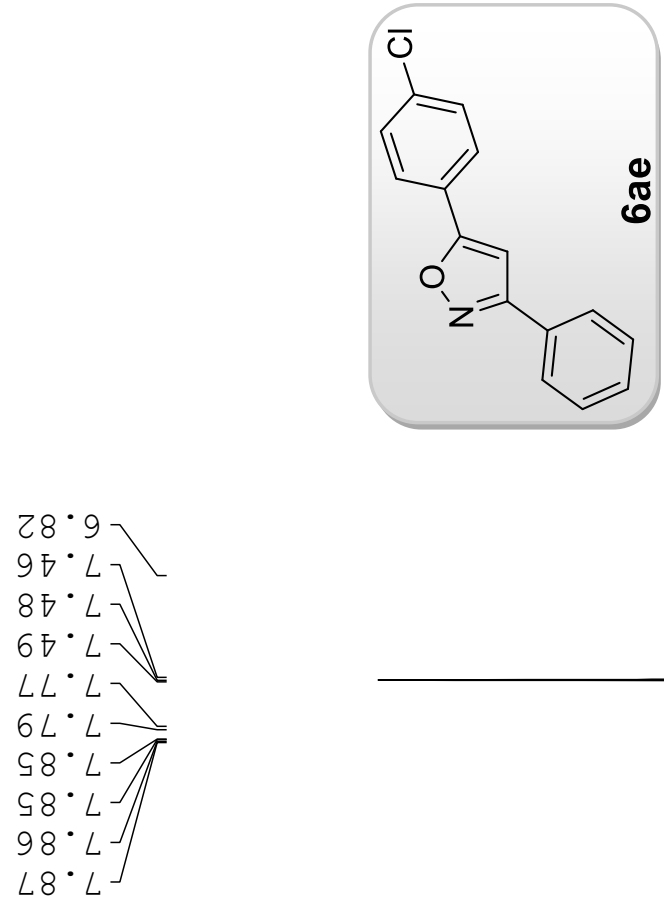

m

$\underset{\mathbb{0}}{0}$

$\{E$ 을

N

$\checkmark$

1)

0

00.1

S0.9

66.1

$\infty$

$90^{\circ} \mathrm{Z}$

$\sigma$

으 

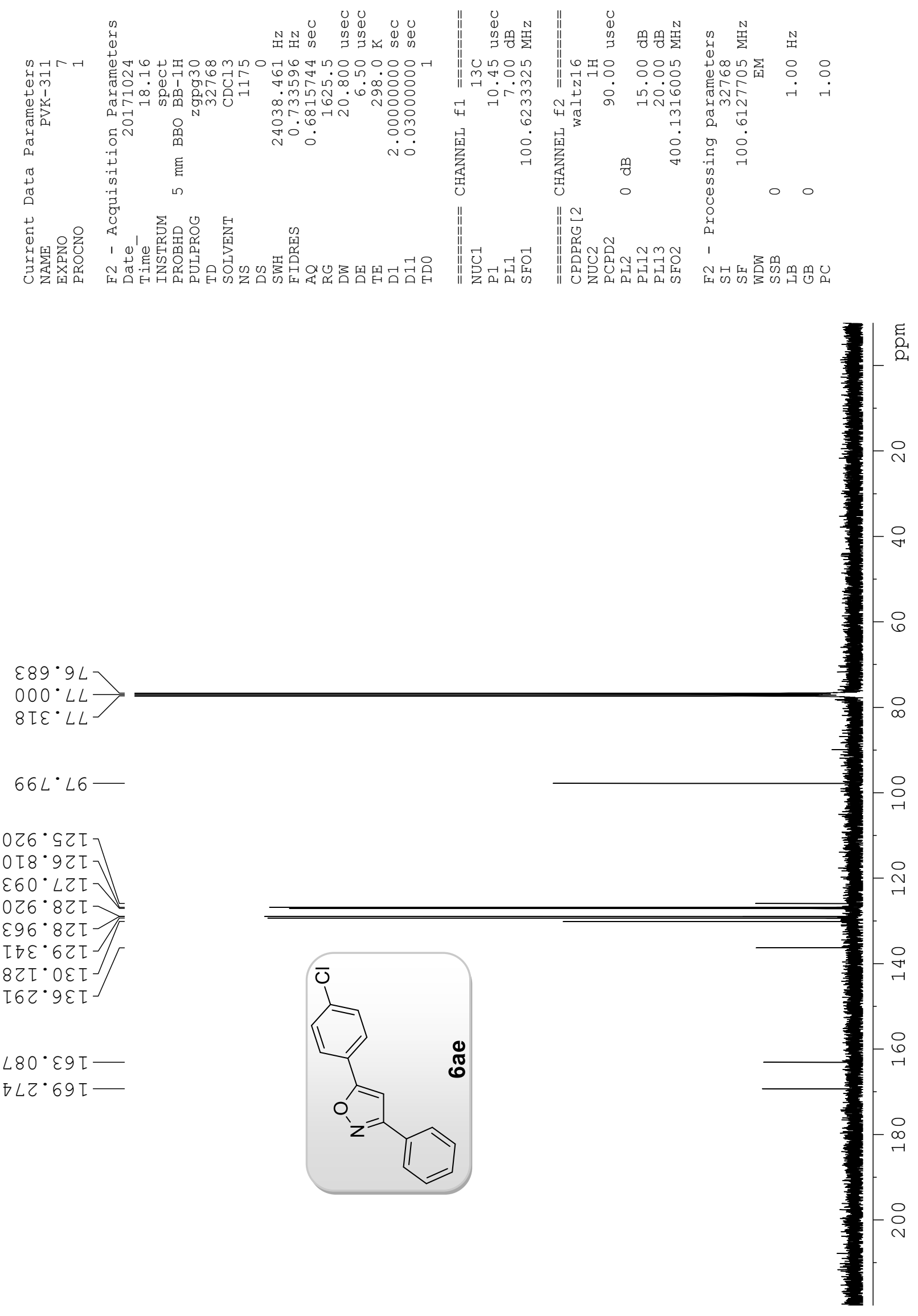


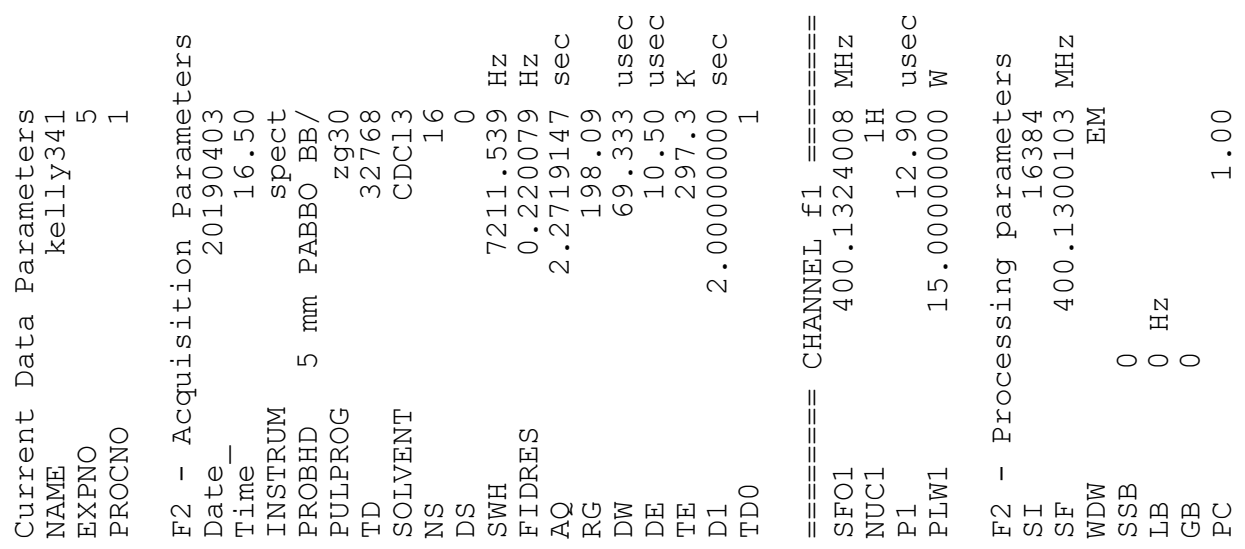

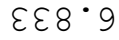

$89 \tau^{\circ}$

S9⿱艹一兀

ZLE'L

$\left.987^{\circ} L\right]$

$067^{\circ} L$

$860^{\circ} L \sqrt{ }$

$\operatorname{LOS} \cdot L$

$S[9 \cdot L / /$

$\angle 69^{\circ} L^{\prime}$

$8[L \cdot L]$

7๐ $8^{\circ} L$

$878^{\circ} L$

$\angle 98^{\circ} \mathrm{L}$

โ98 $8^{\circ} L$

$898^{\circ} L$
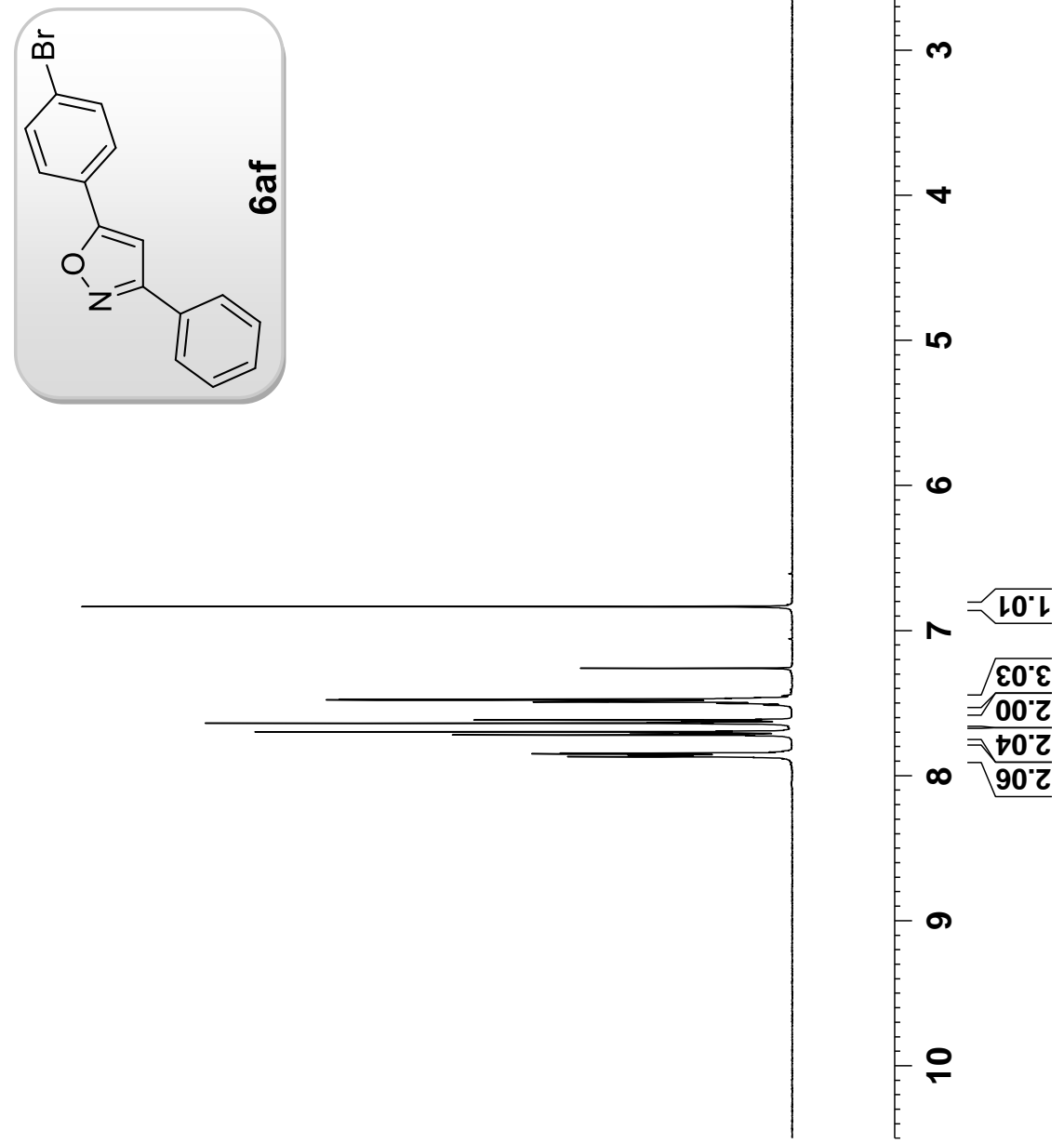

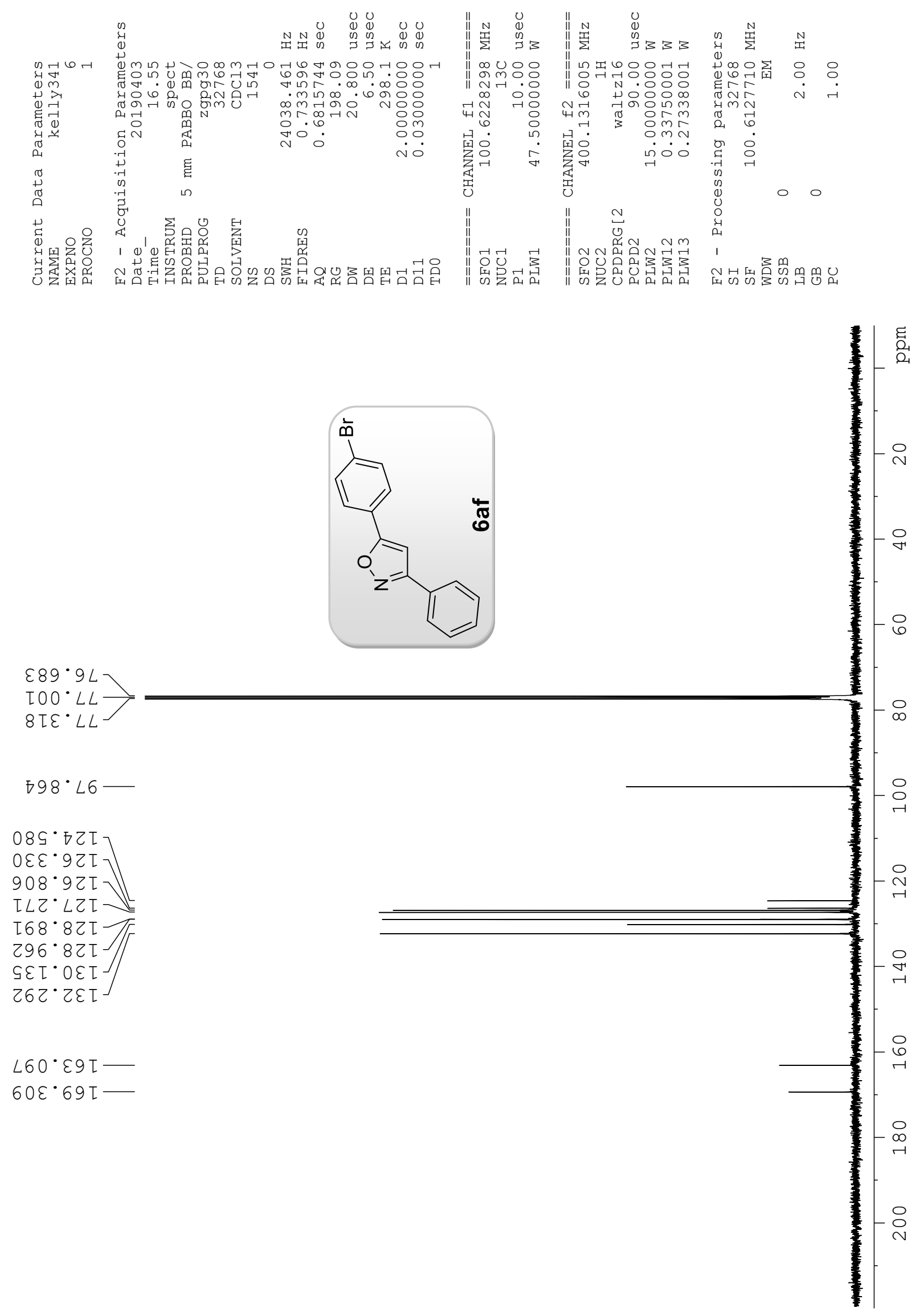


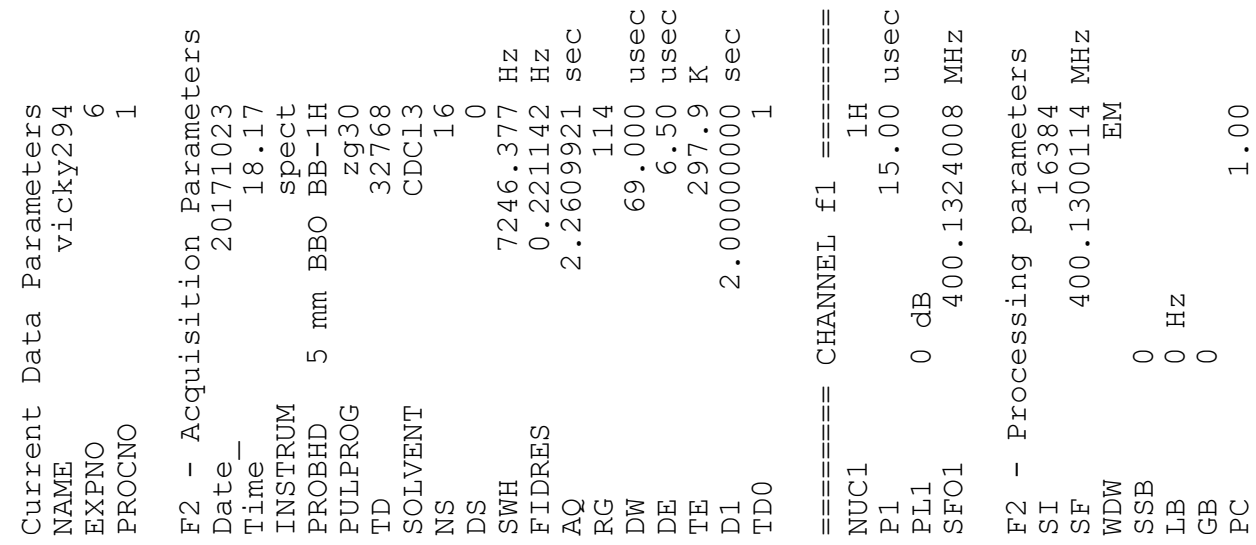

$00 \cdot 0-$
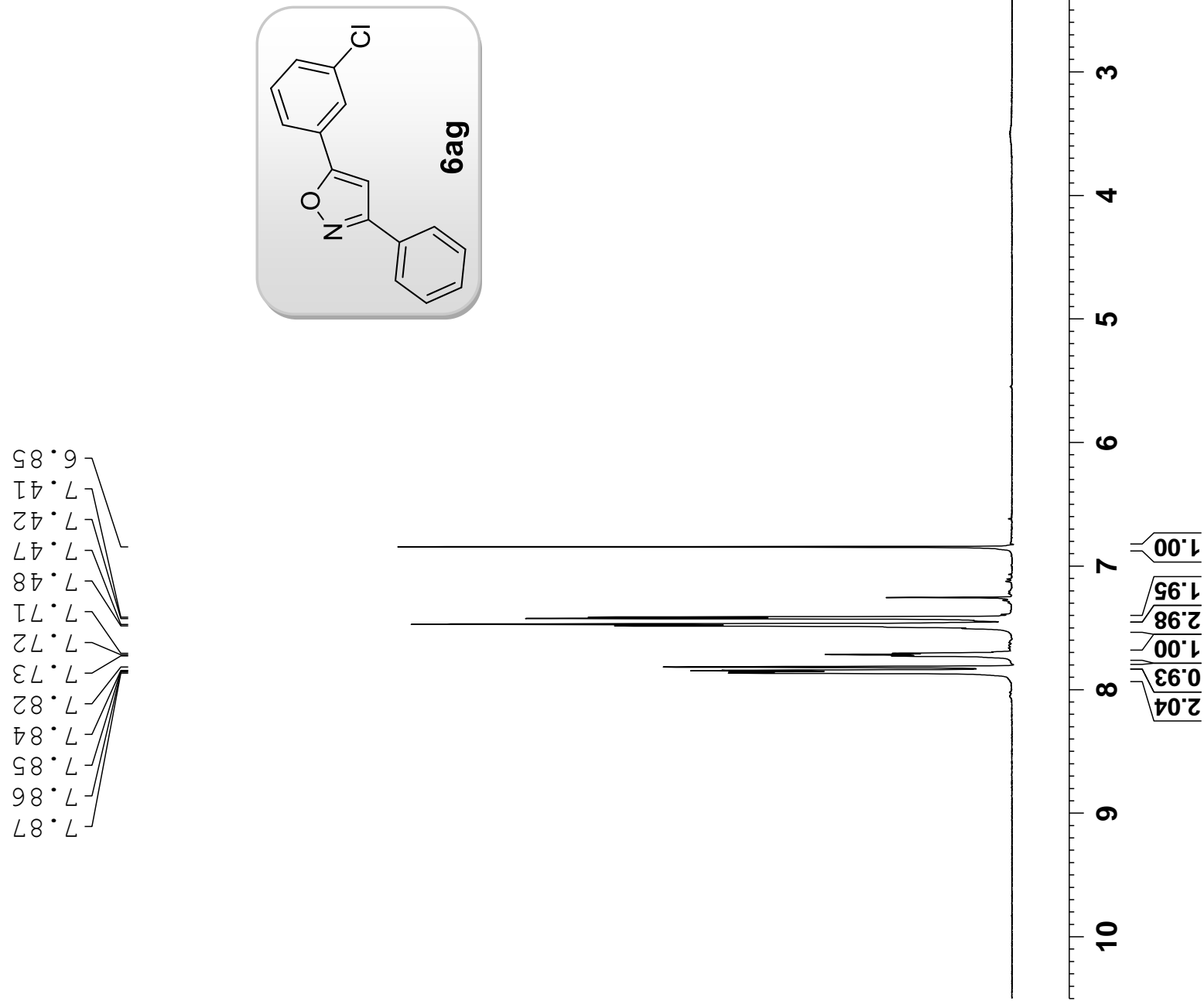

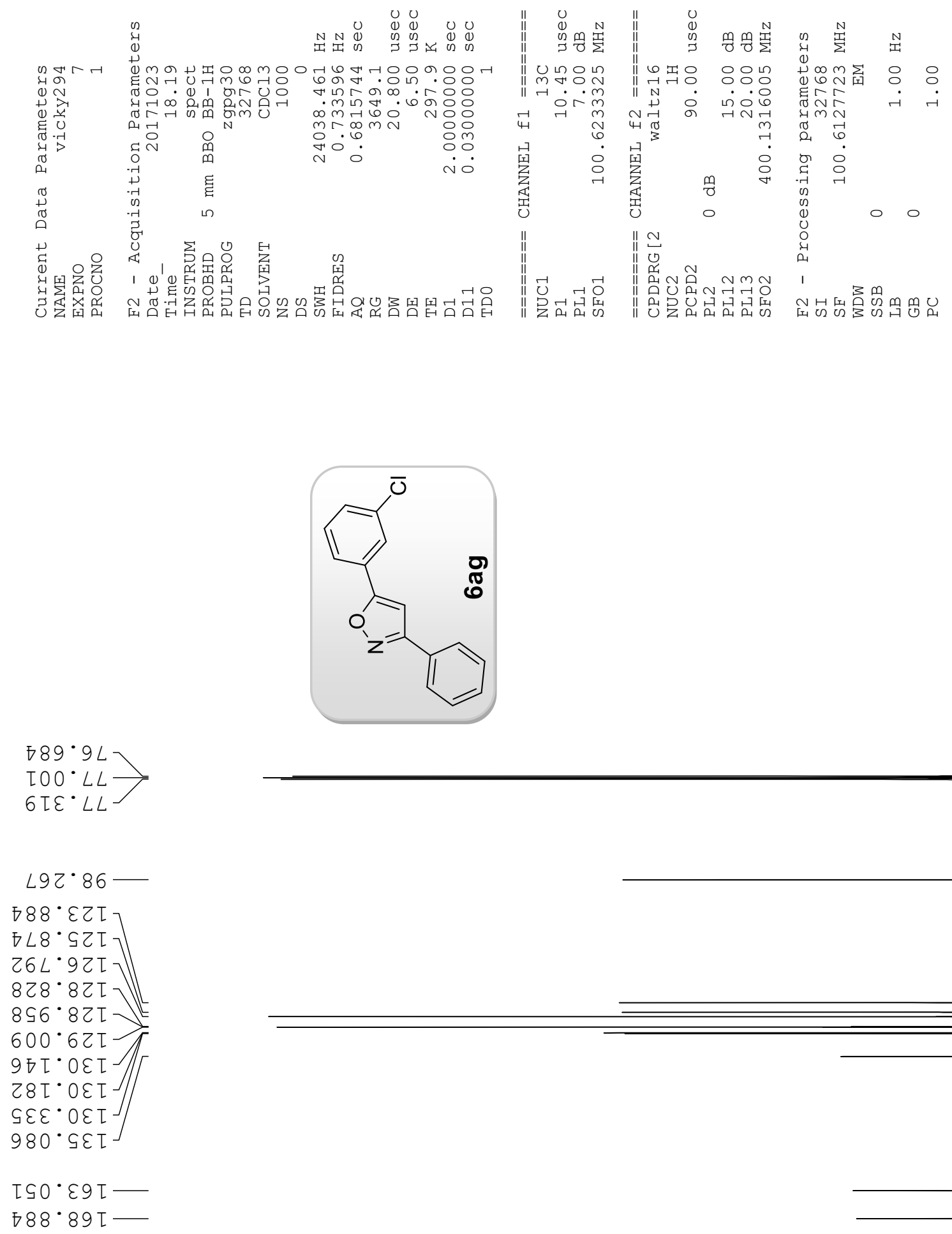

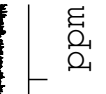



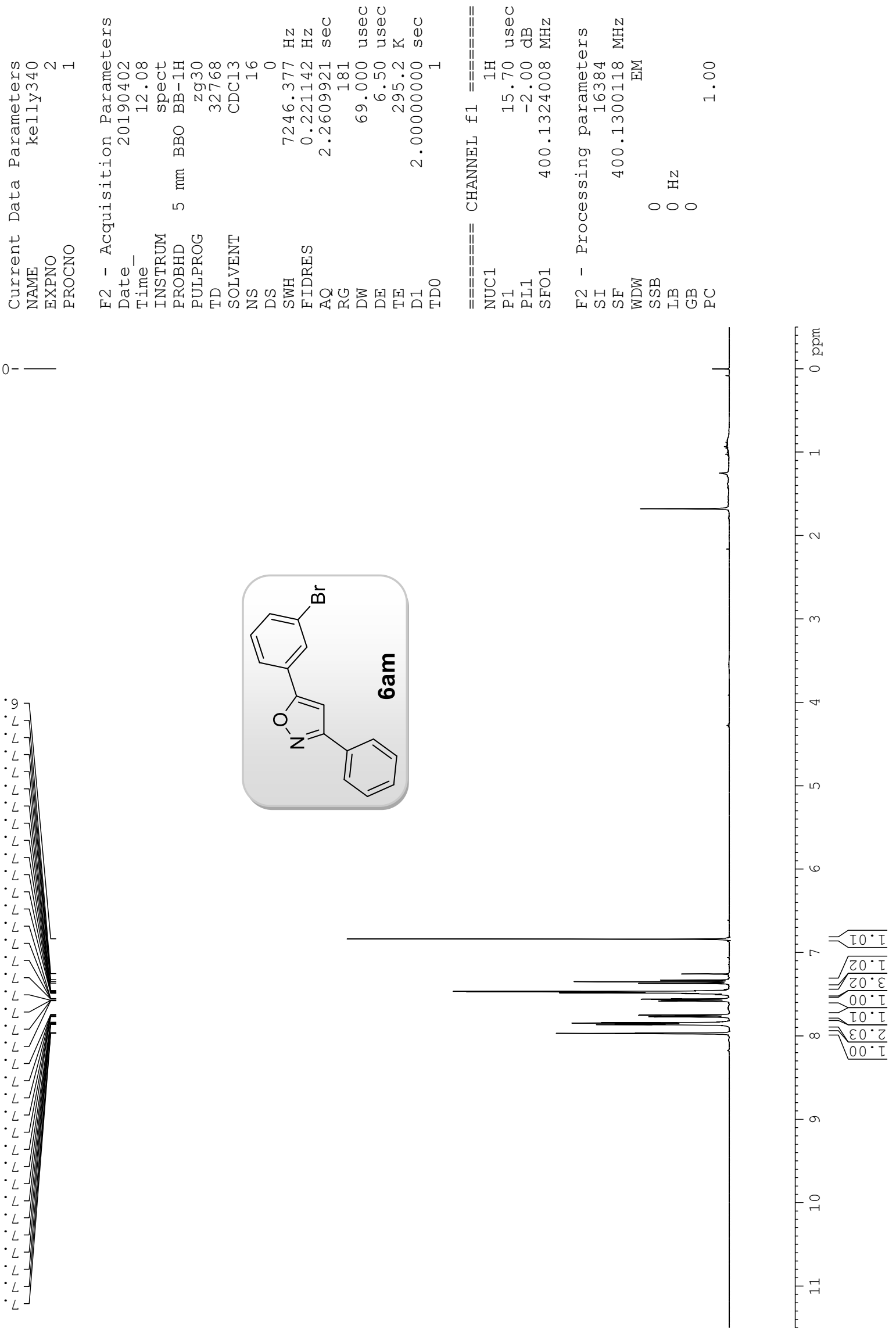

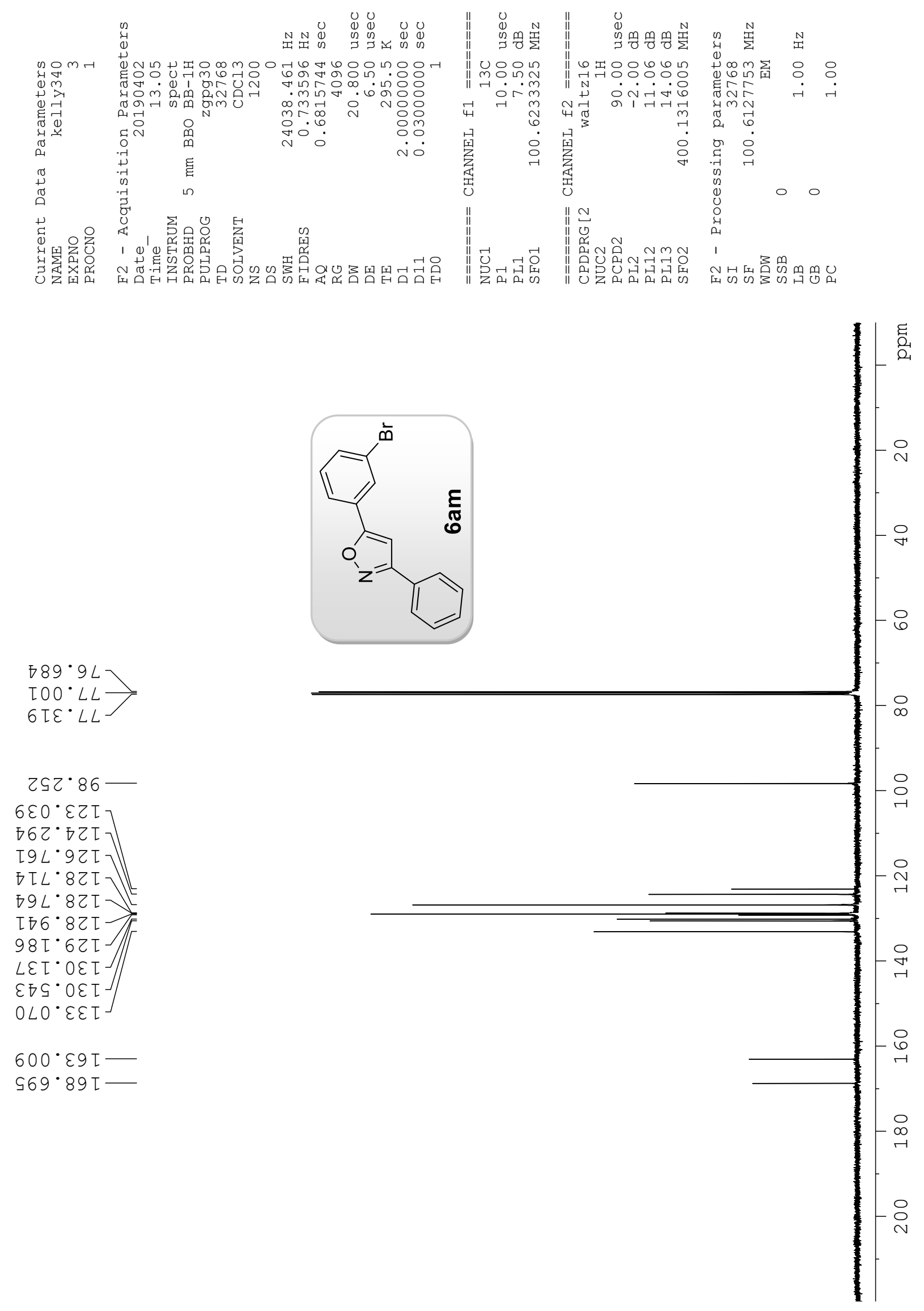


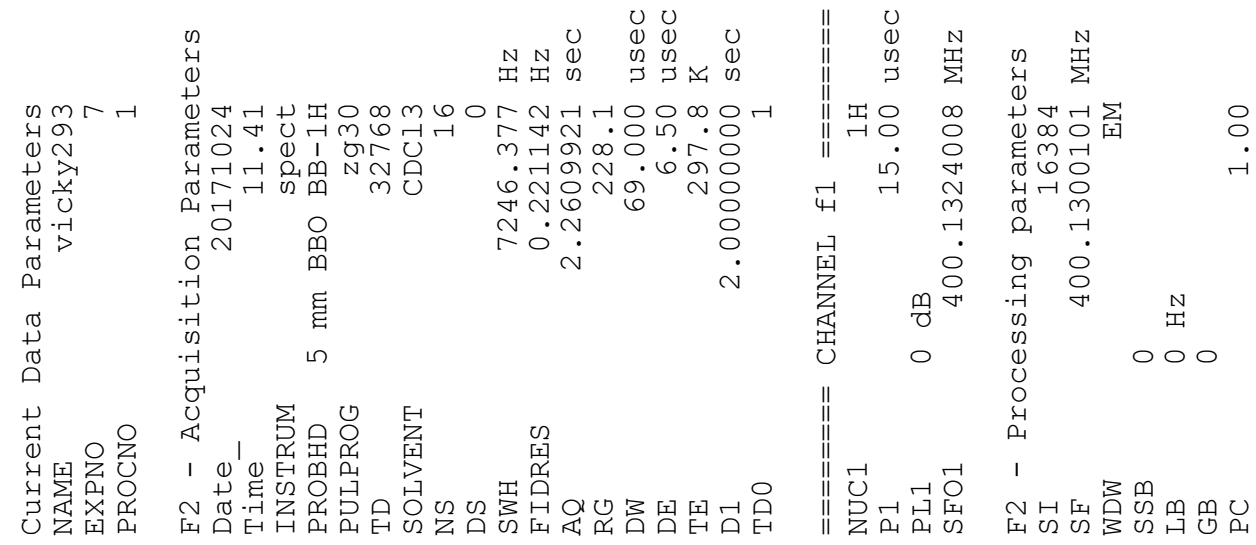

$00 \cdot 0-$
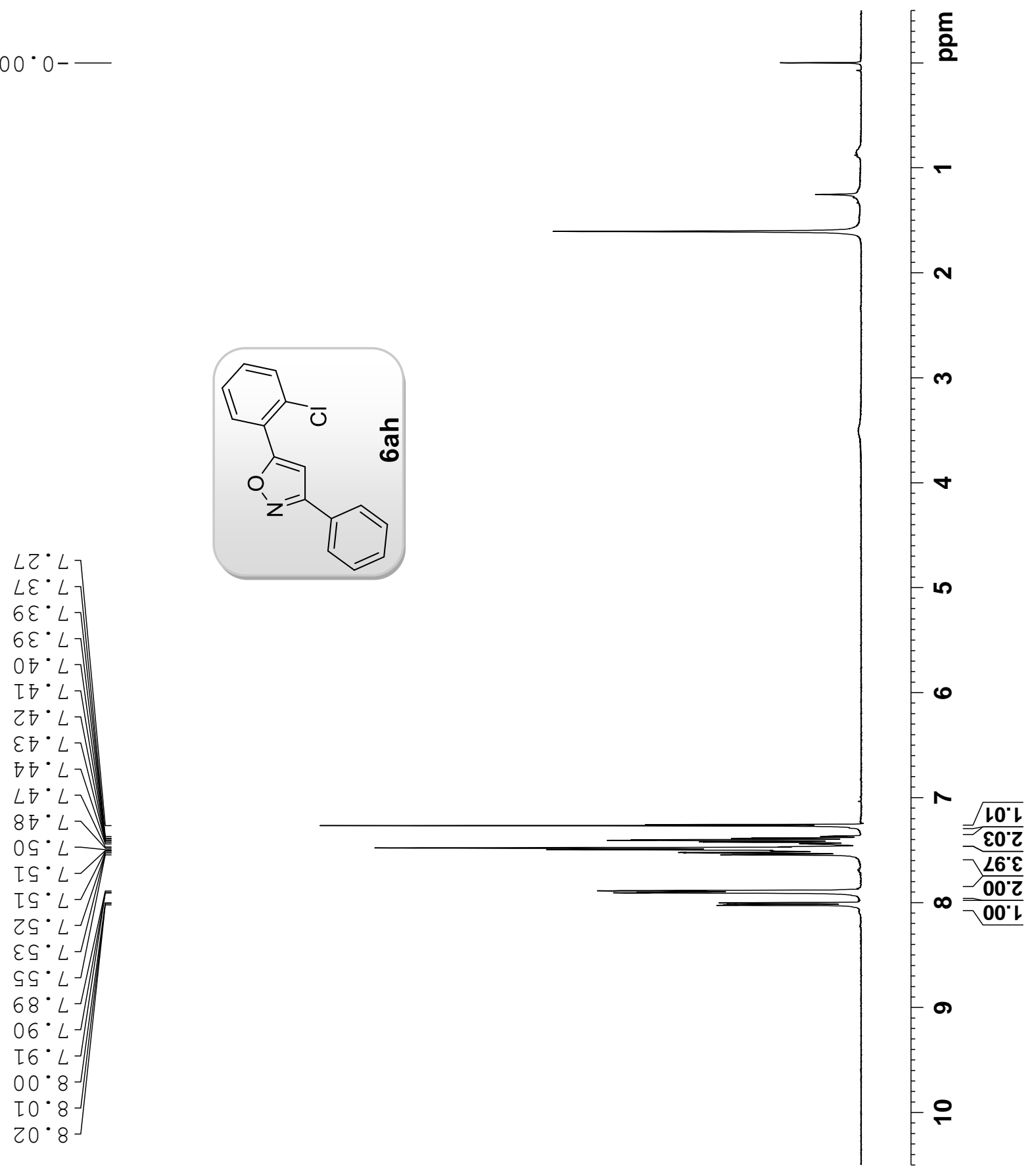


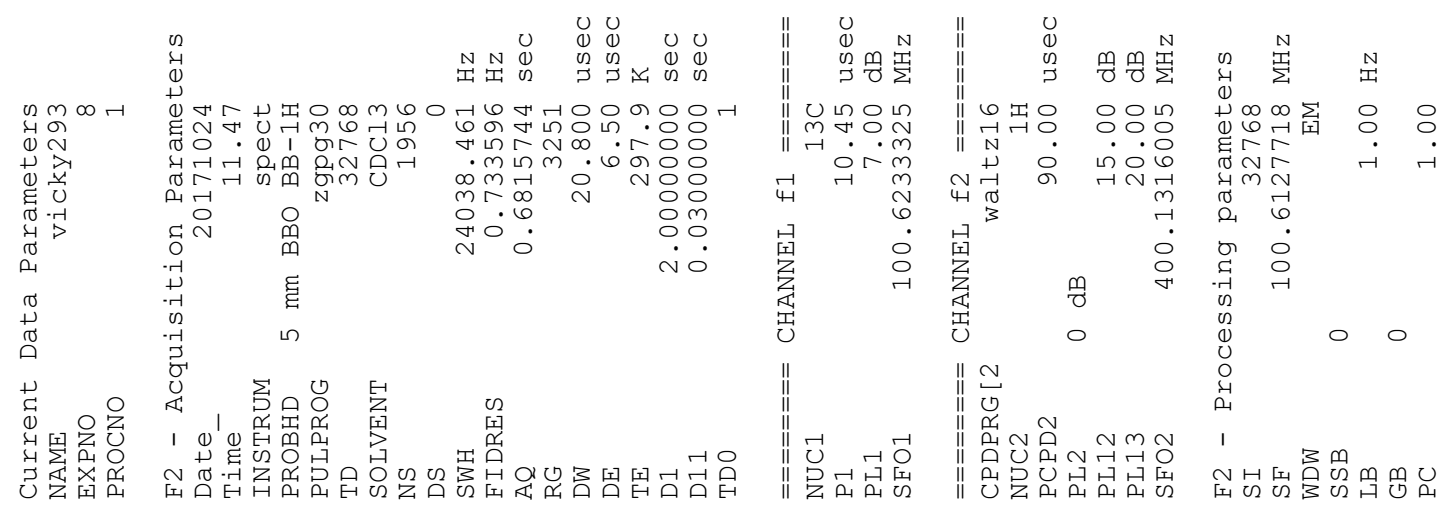

$8 L 9 \cdot 9 L$
$966 \cdot 9 L$
$\varepsilon[\varepsilon \cdot L L$

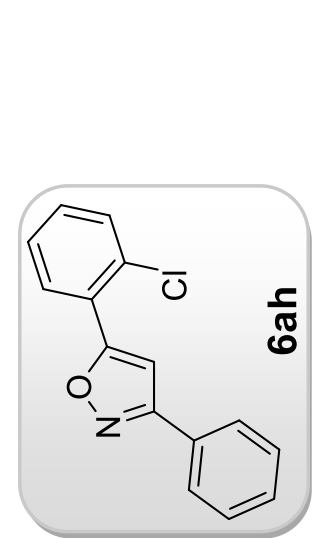

オS币・20L

$S L Z \cdot 9 Z[$
$[L 8 \cdot 9 Z[]$

$\nabla \varepsilon \tau \cdot L \tau[]$

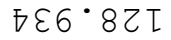

$\varepsilon \nabla 0 \cdot 6 Z \mathrm{I}$

$\angle \tau \nabla \cdot 6 \tau T$

$890^{\circ} 0 \varepsilon[$

$\left.\begin{array}{l}\left.8 \nabla 8^{\circ} 0 \varepsilon \tau\right] \\ \varepsilon\left[L^{\circ}[\varepsilon[\right.\end{array}\right]$

SE6. $29 \mathrm{I}$

$809^{\circ} 99 \mathrm{I}$

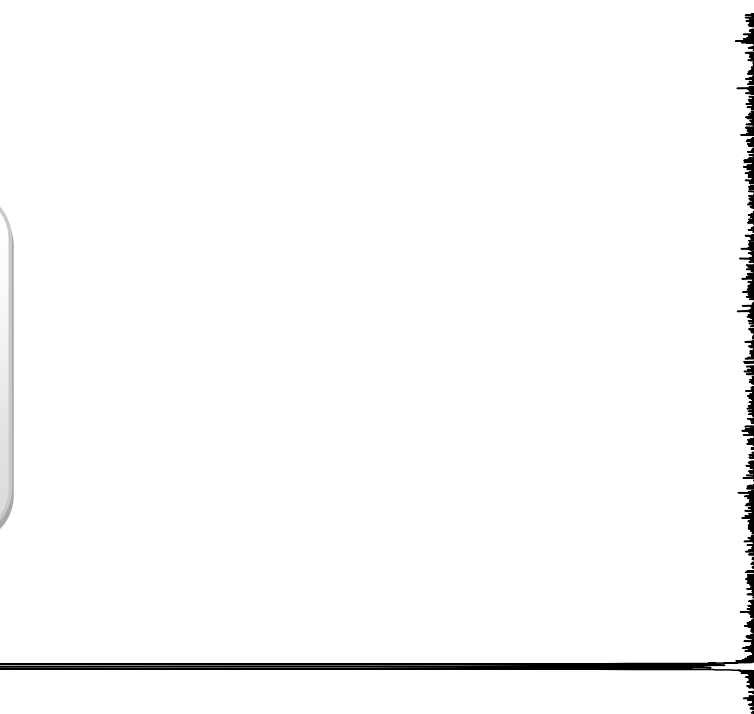




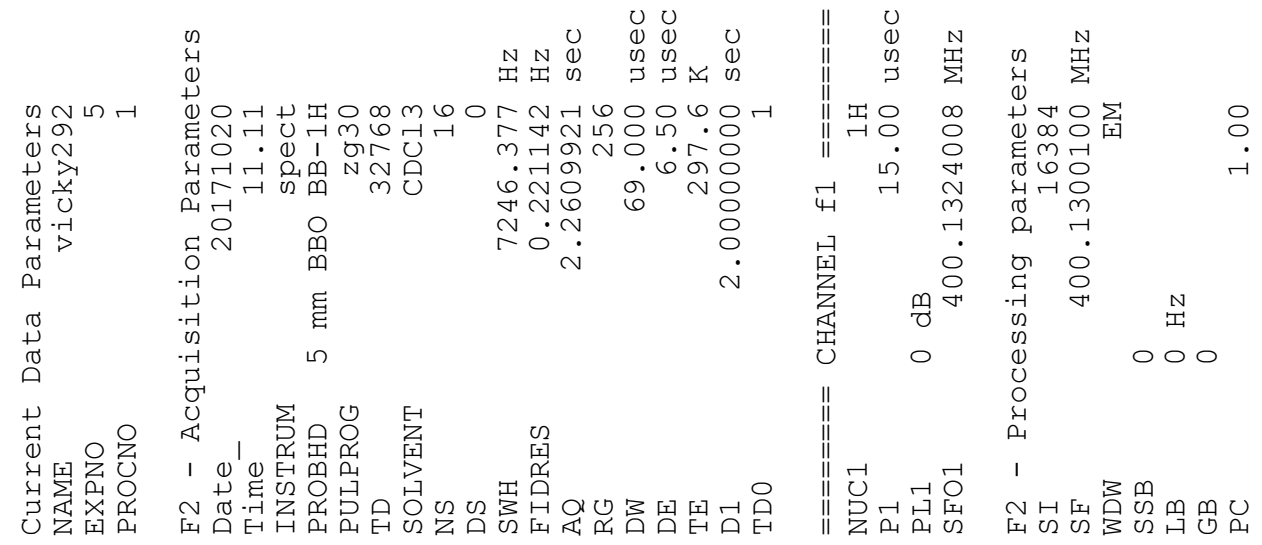

$00 \cdot 0-$
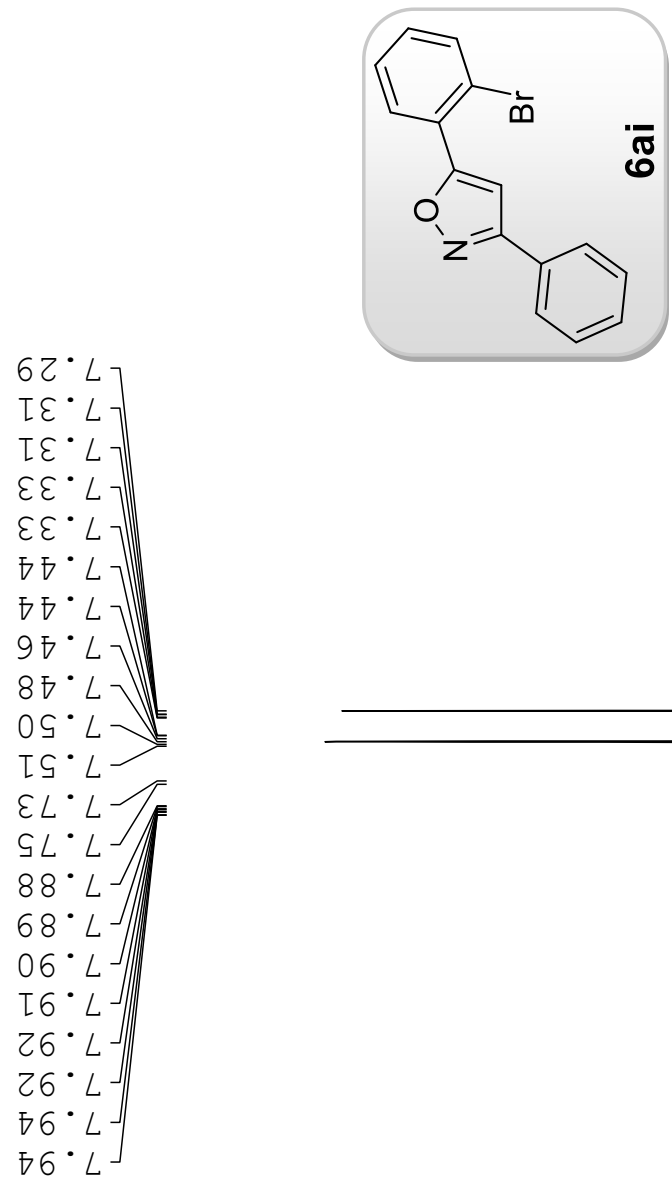

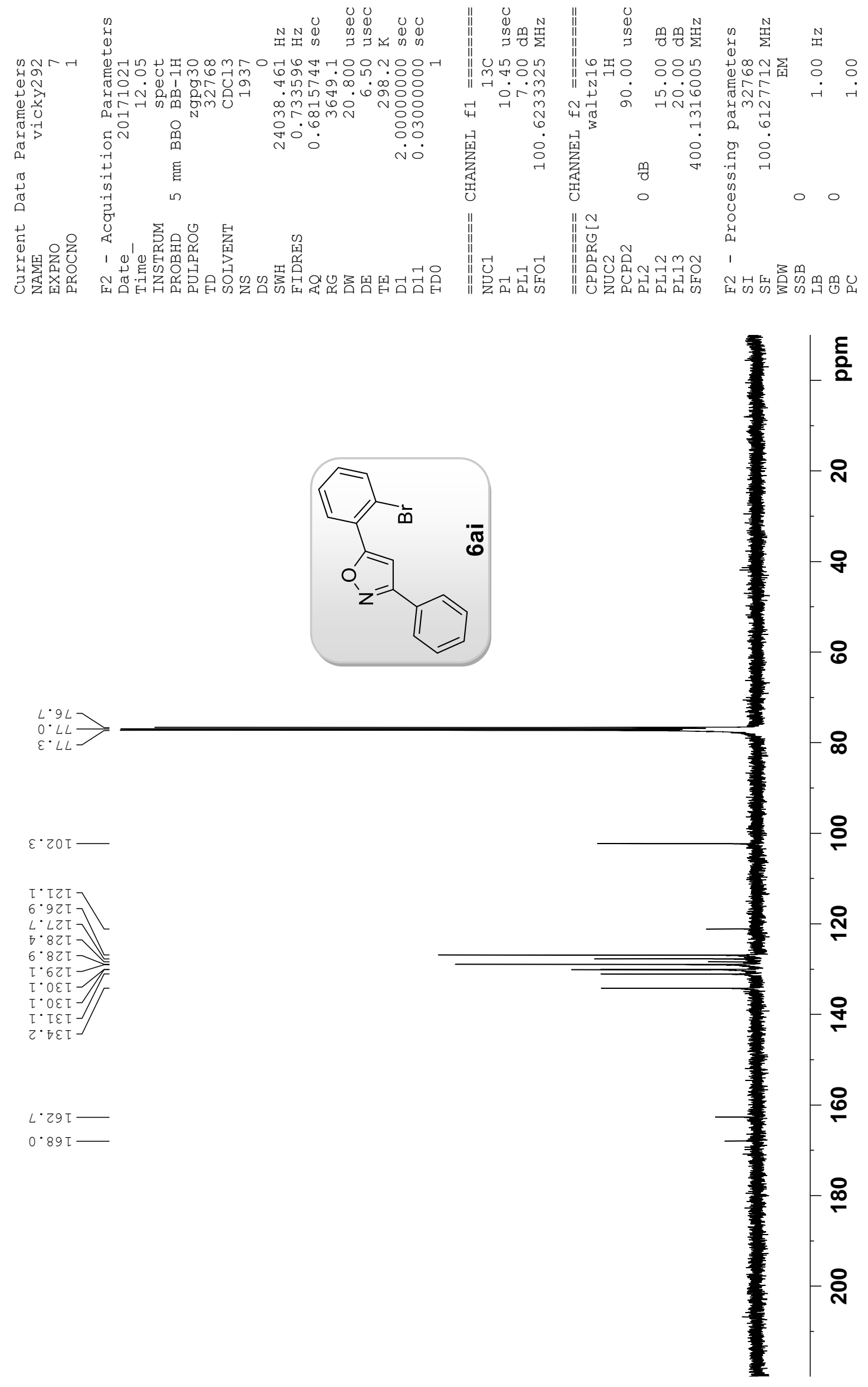

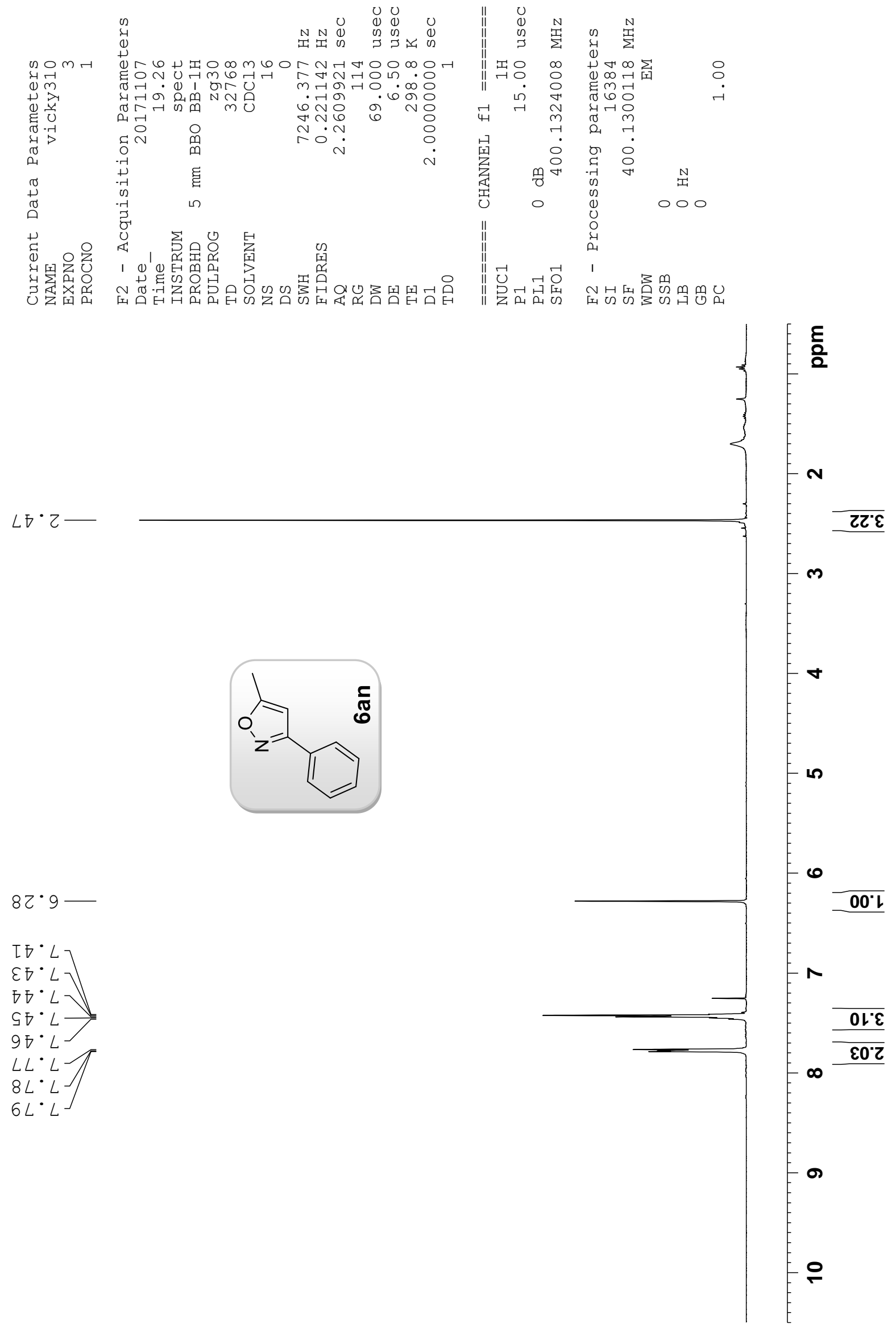

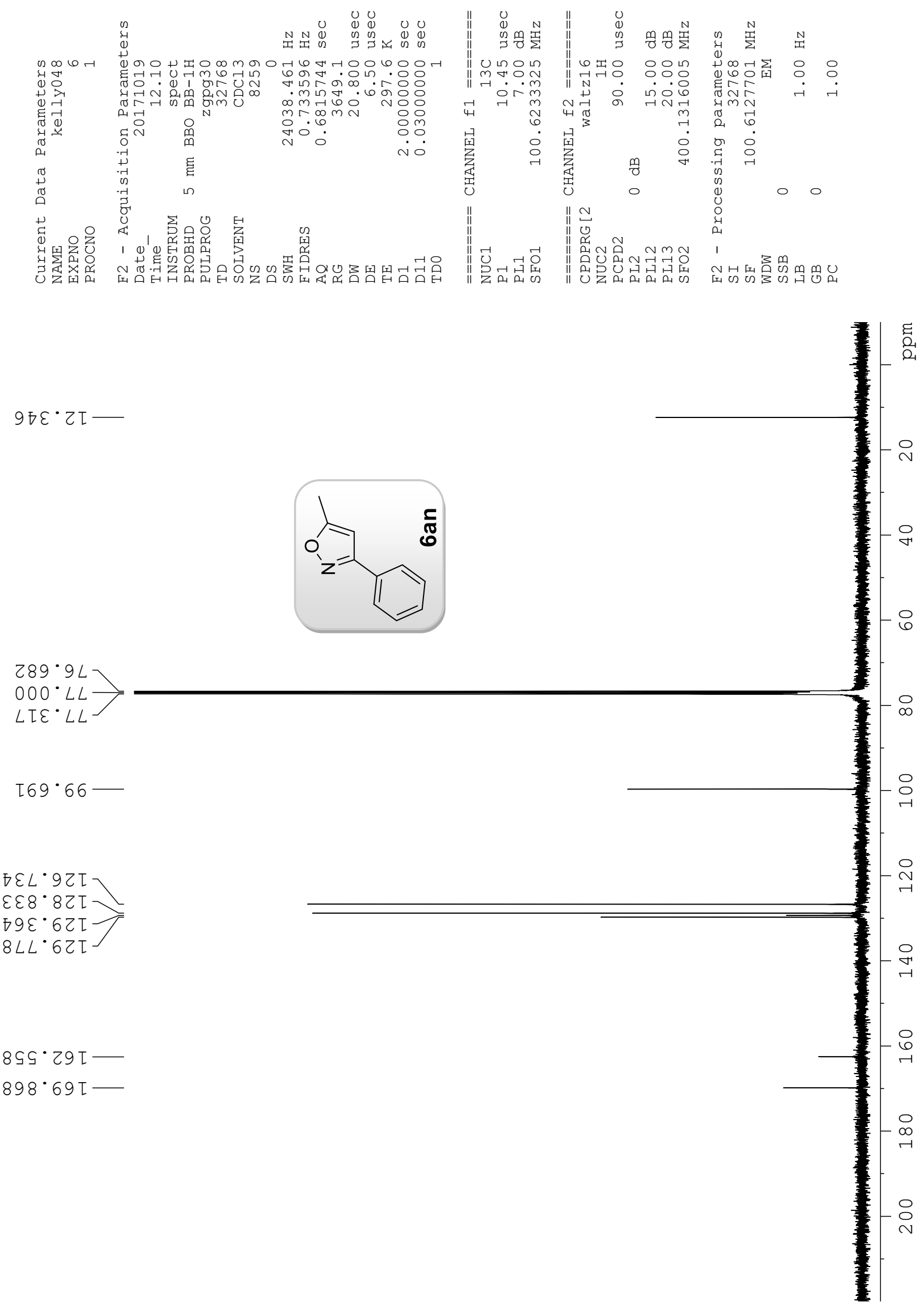


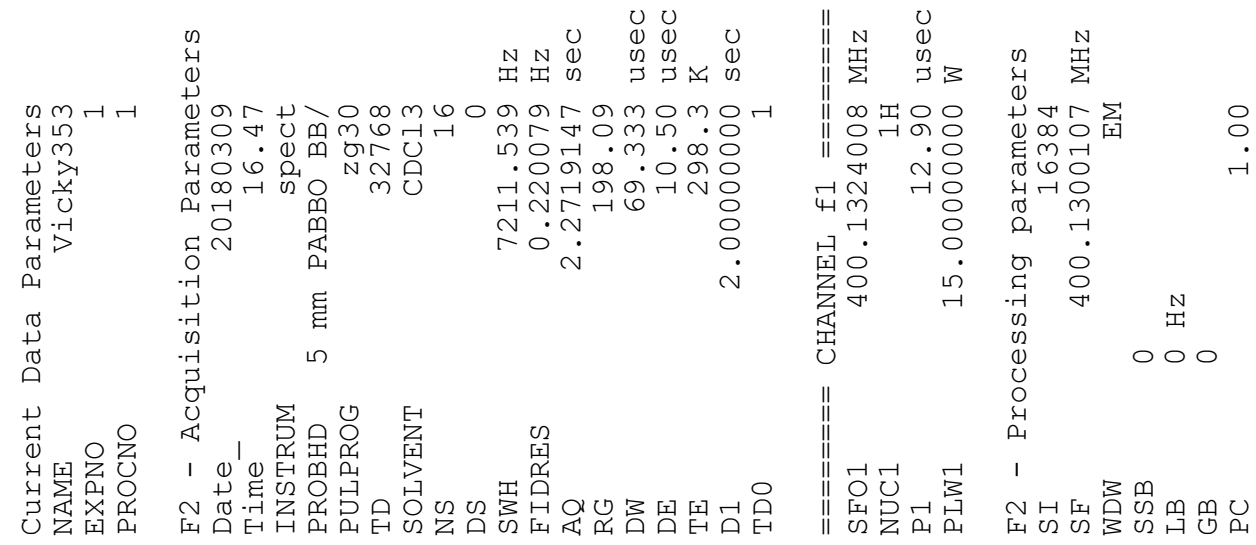

$00 \cdot 0-$
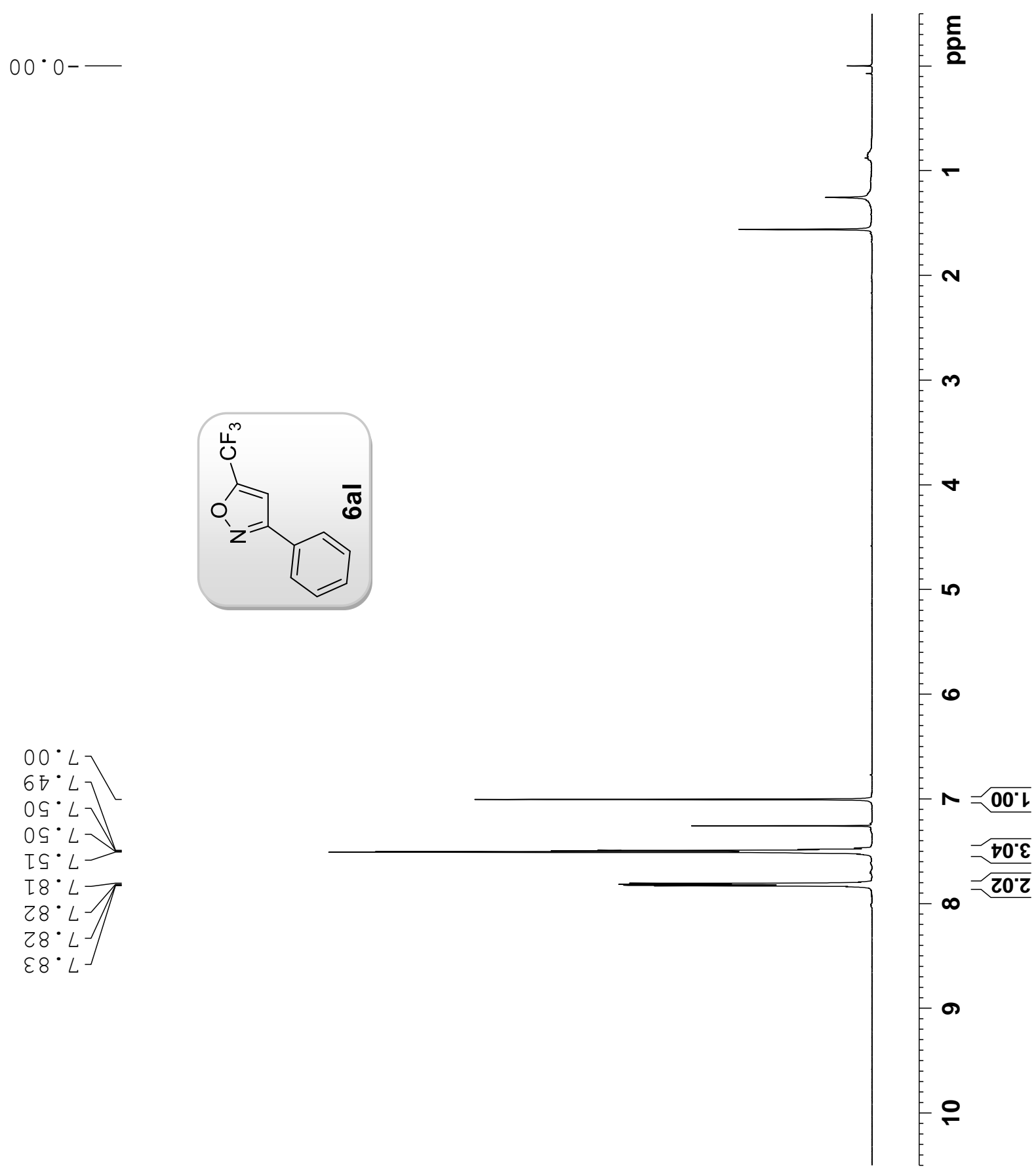

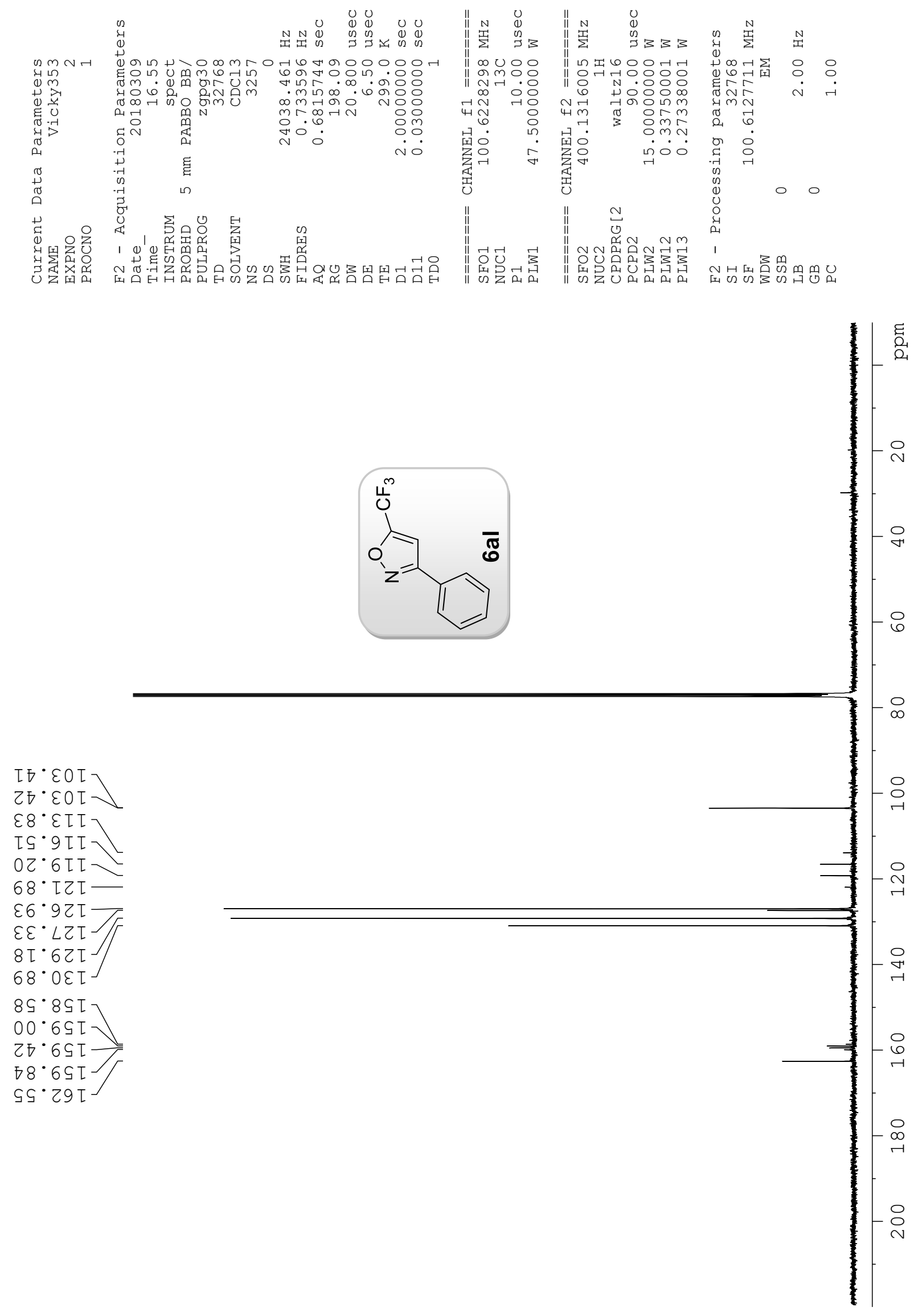


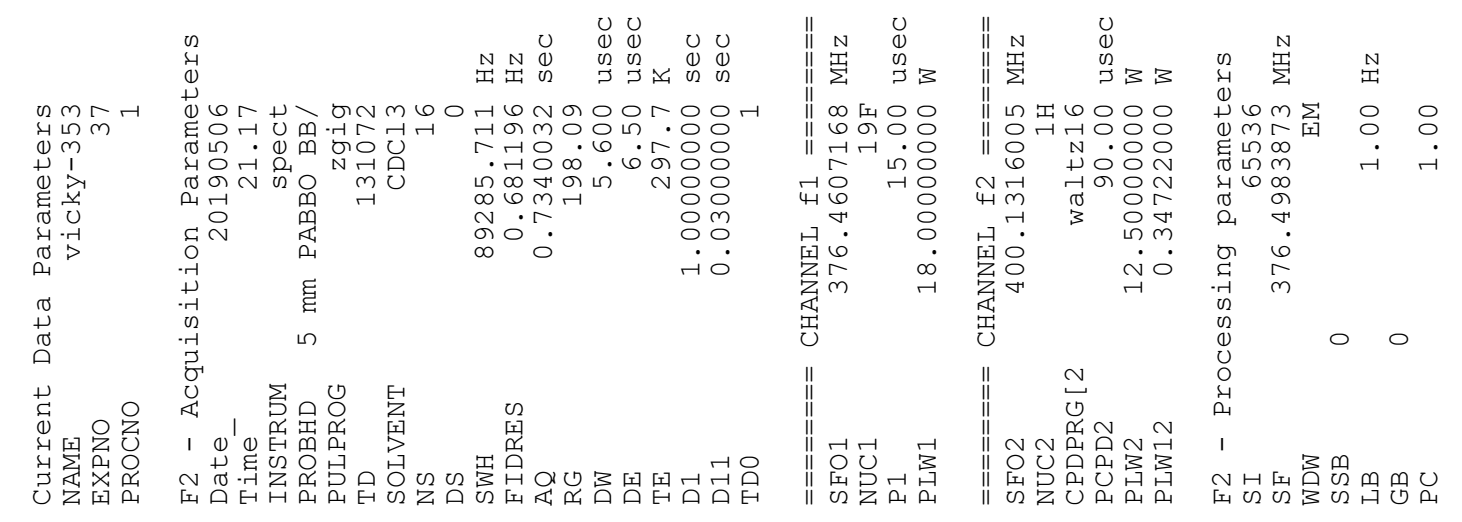

$S \tau \cdot \varepsilon \tau \tau-$

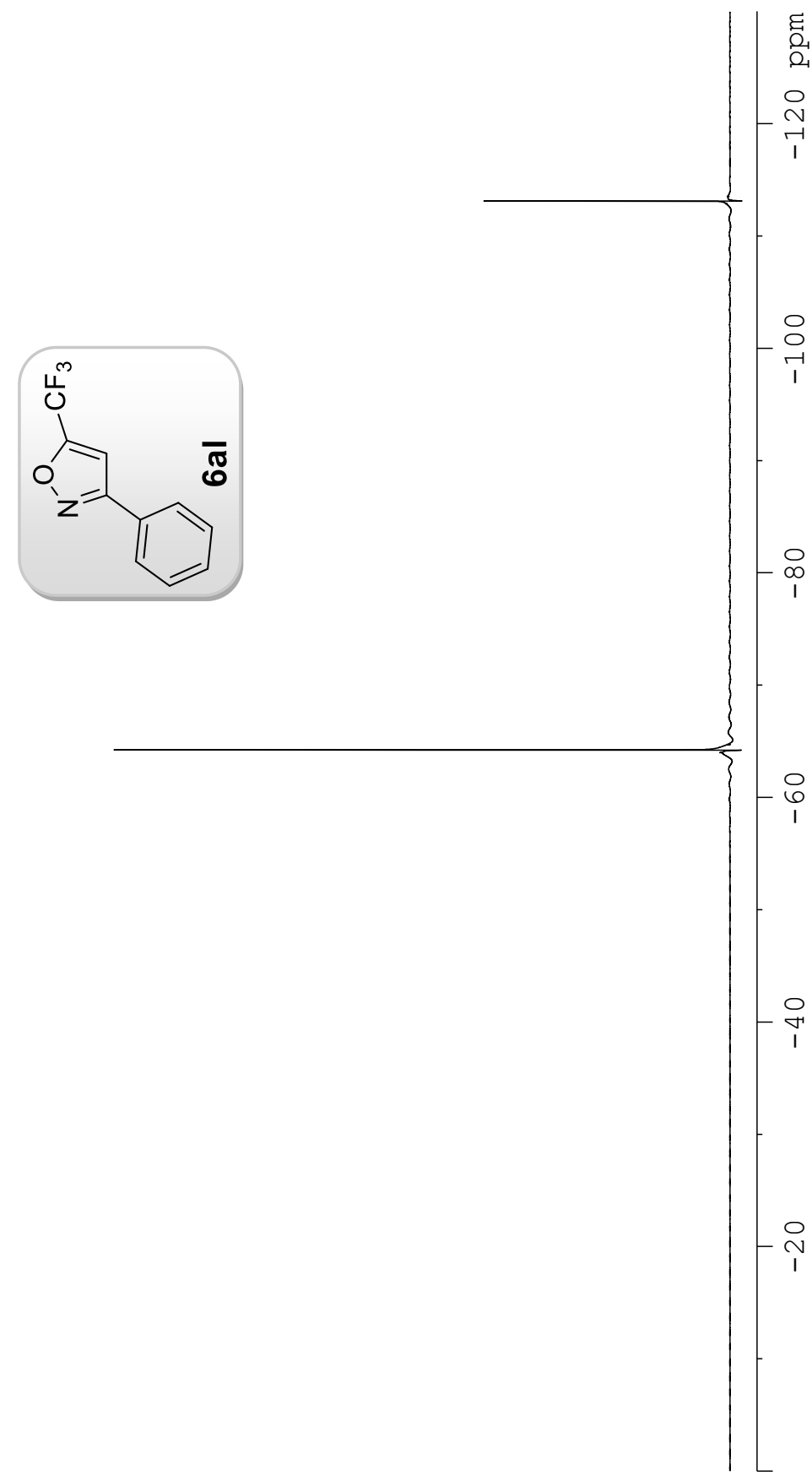

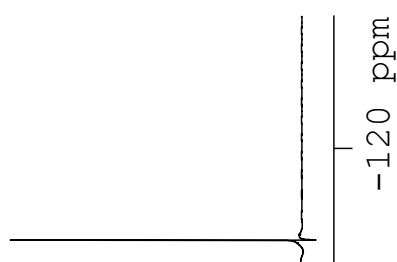

$\varsigma て \cdot \sqcap 9-$ 


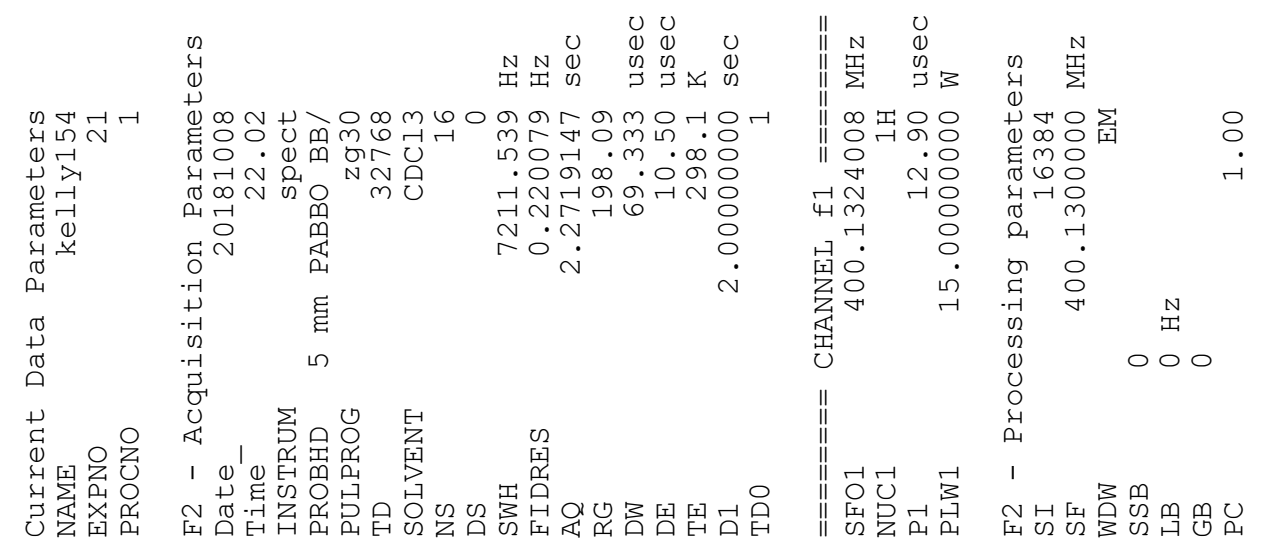

$\angle 20^{\circ} 0$

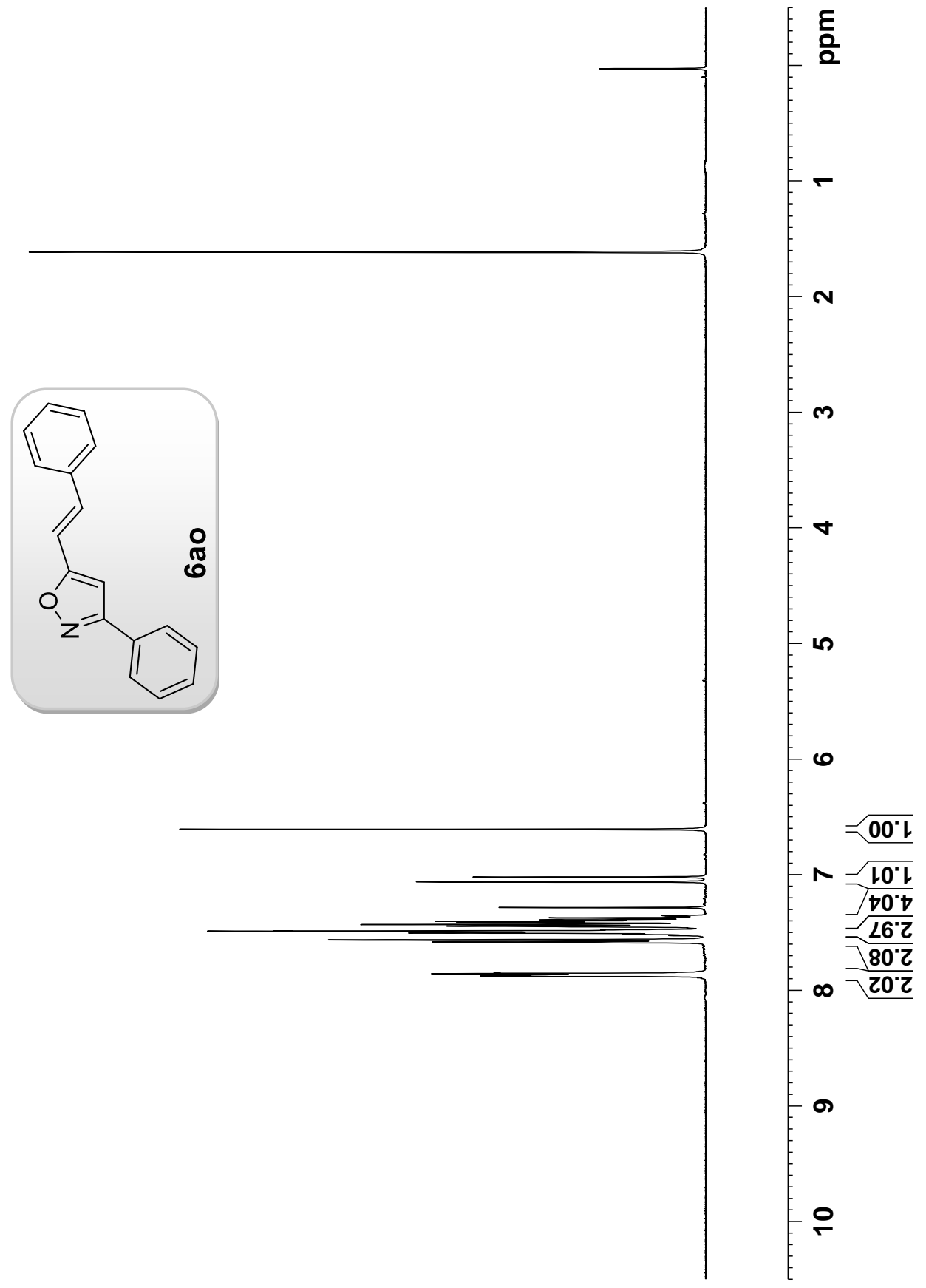



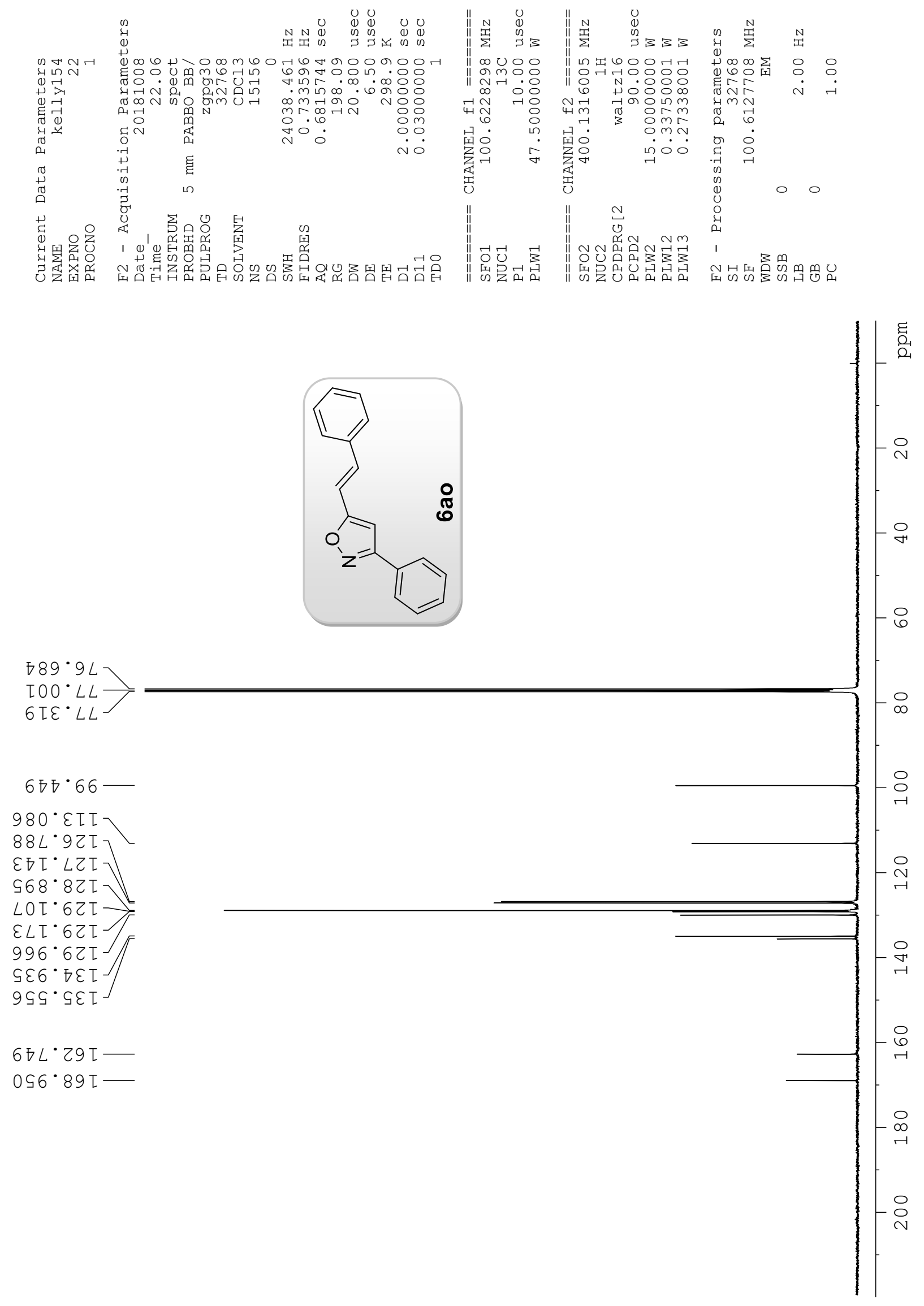


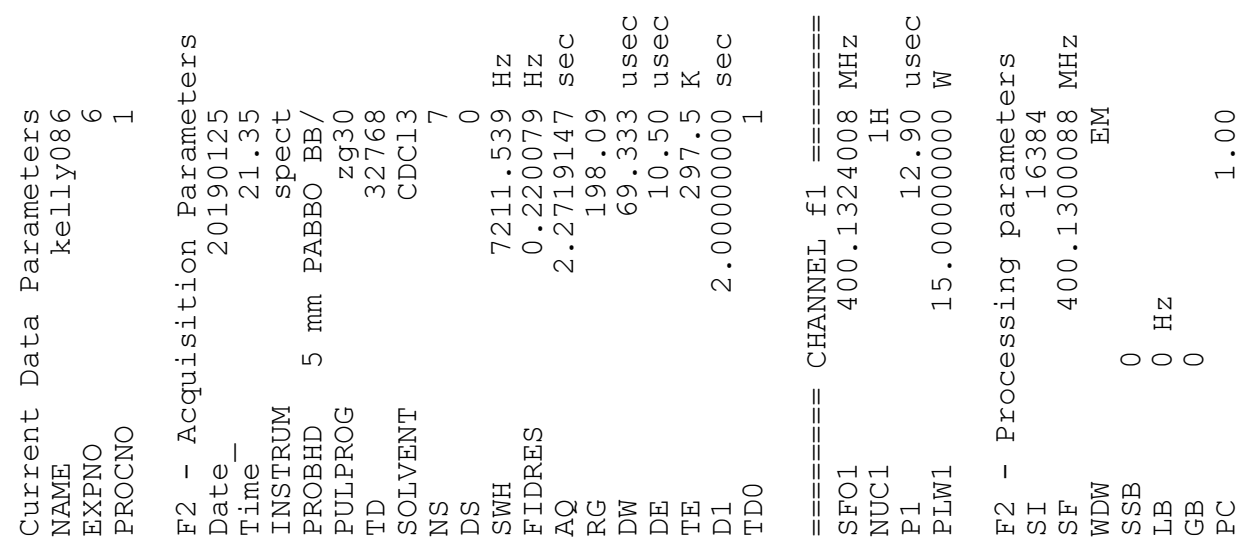

$000 \cdot 0$
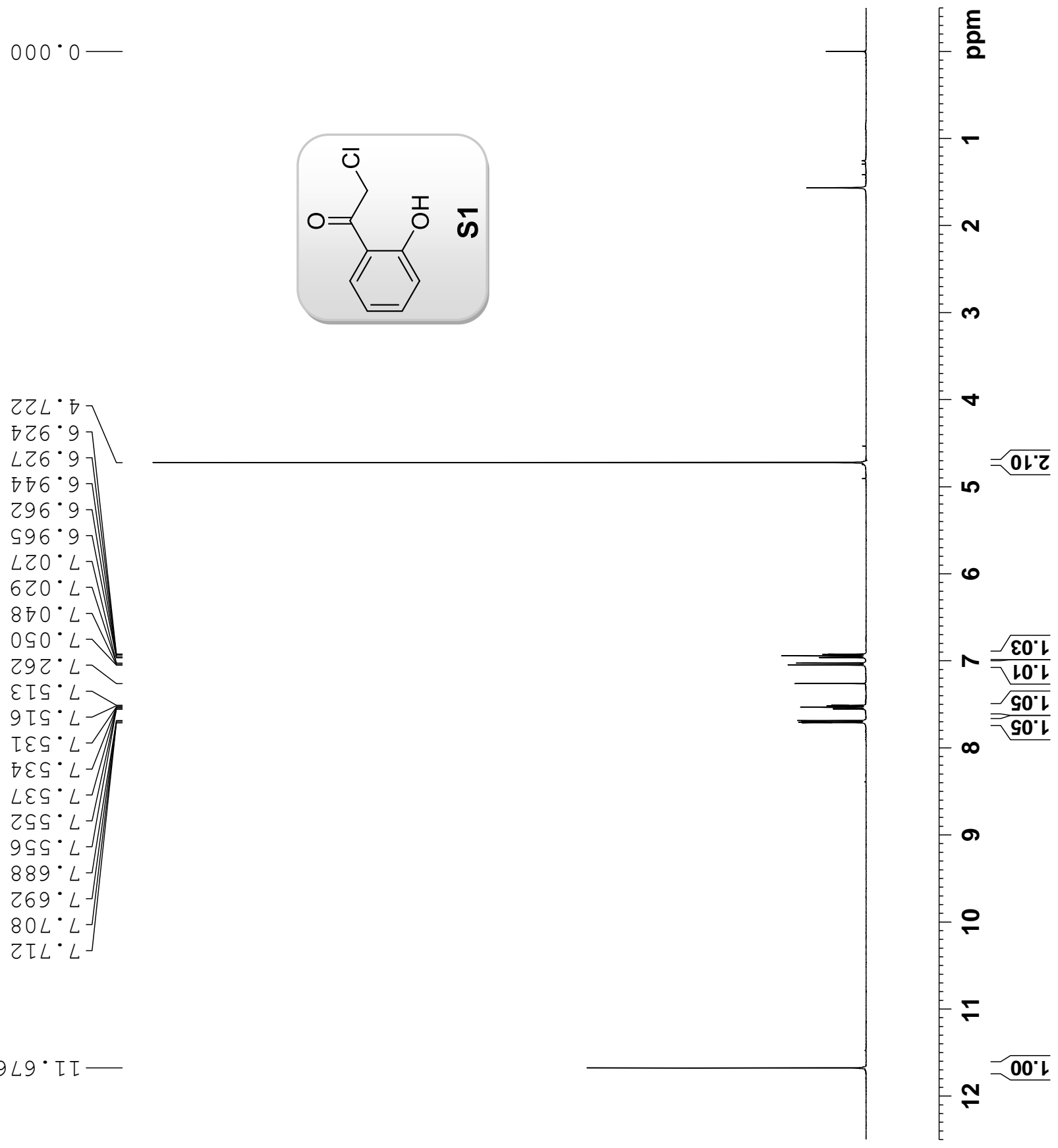

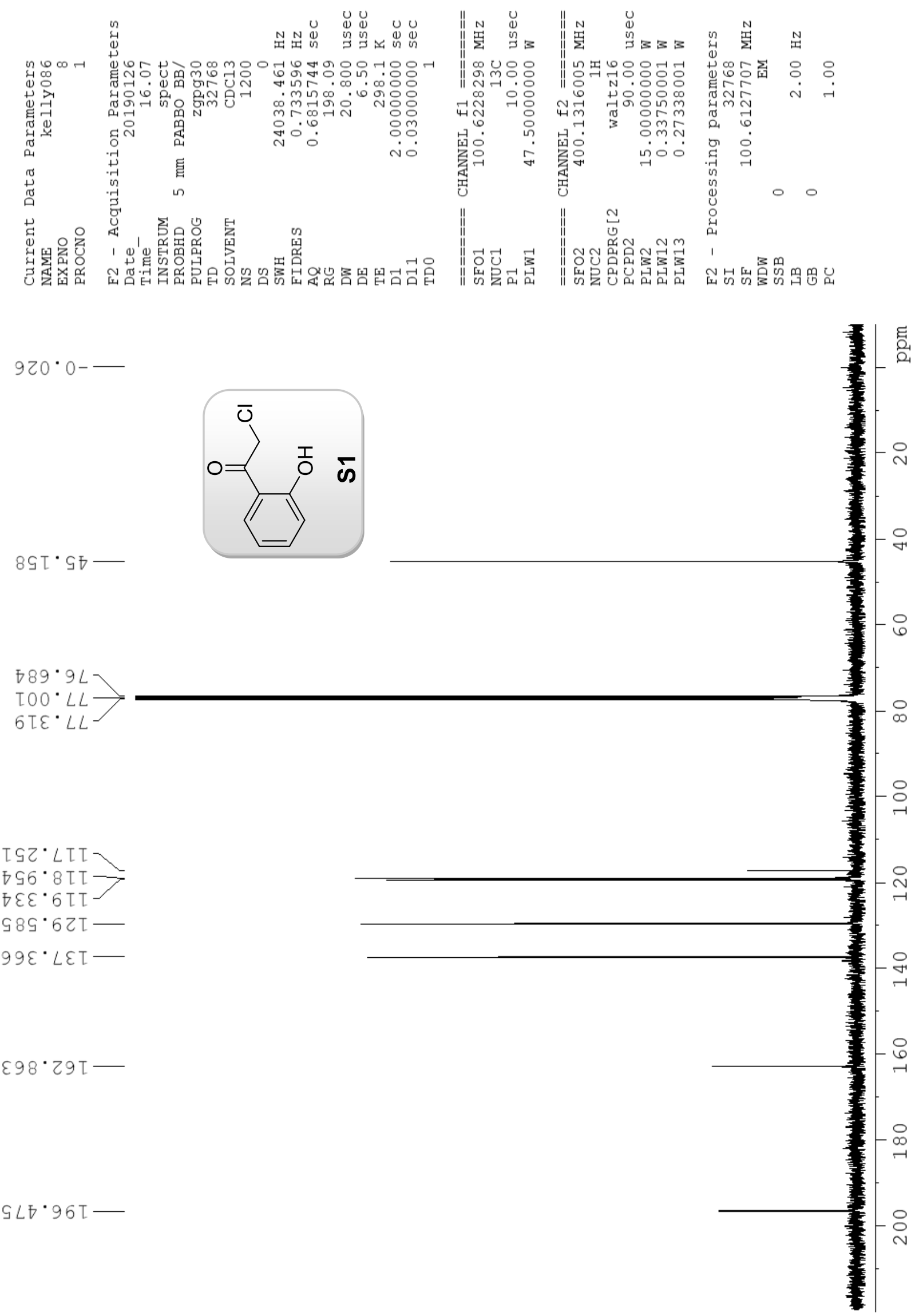


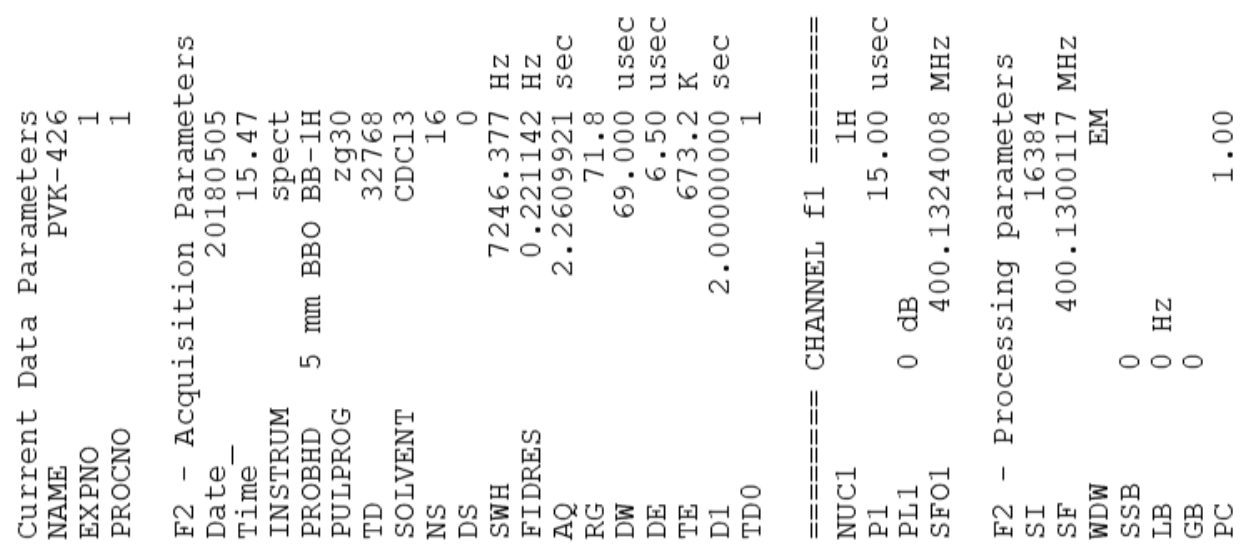

$100 \cdot 0$
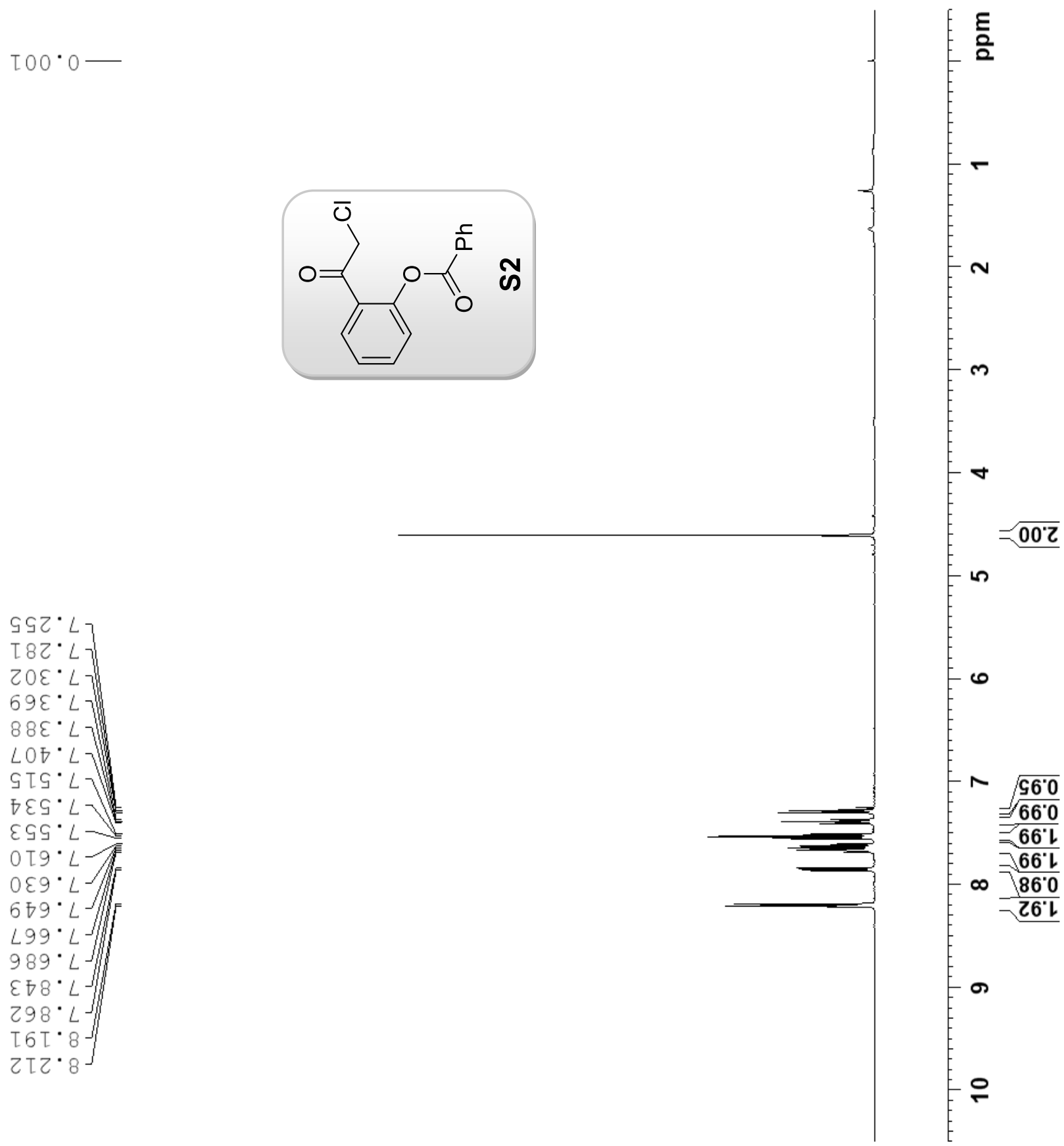

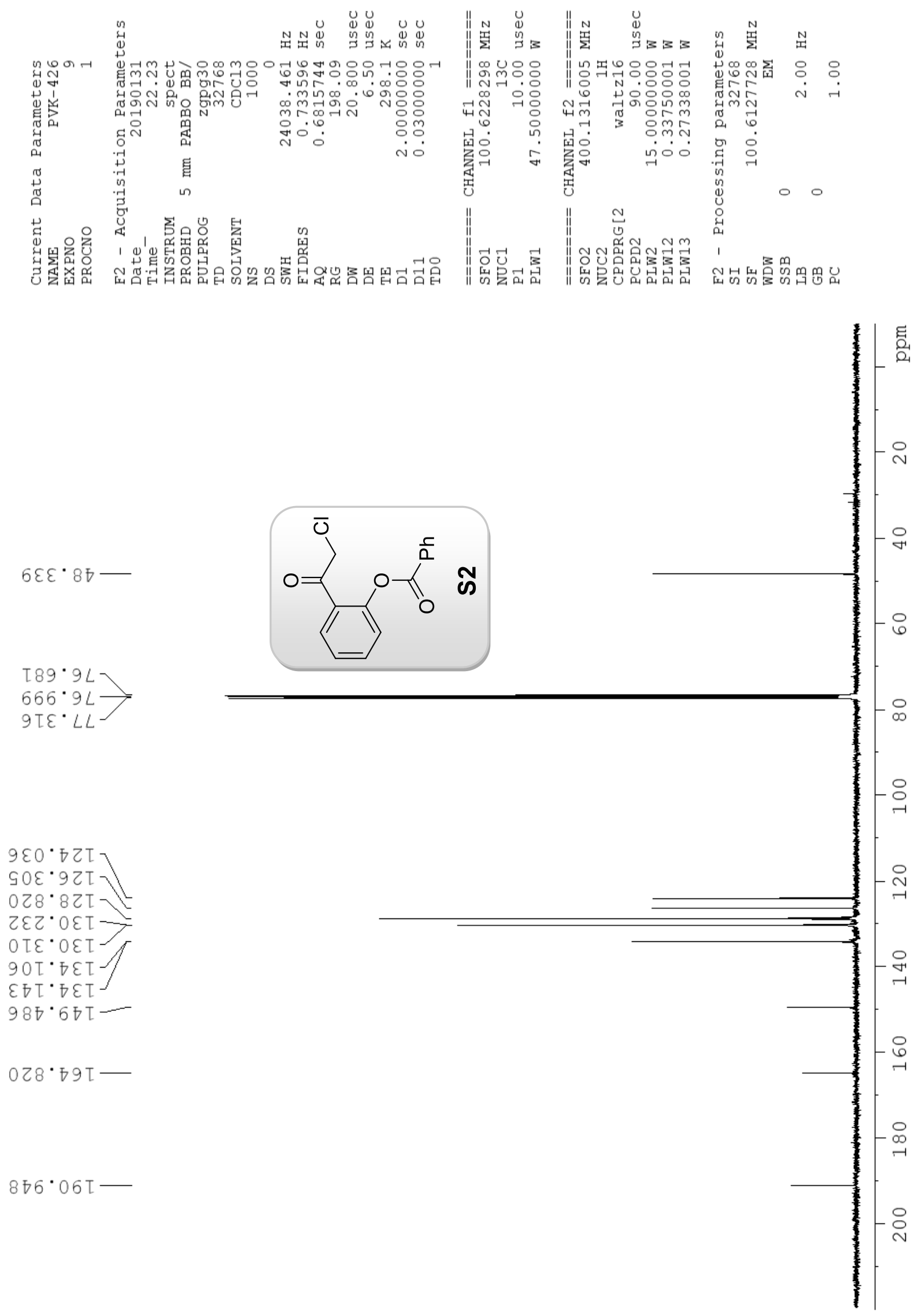

$876^{\circ} .06 \tau-$ 

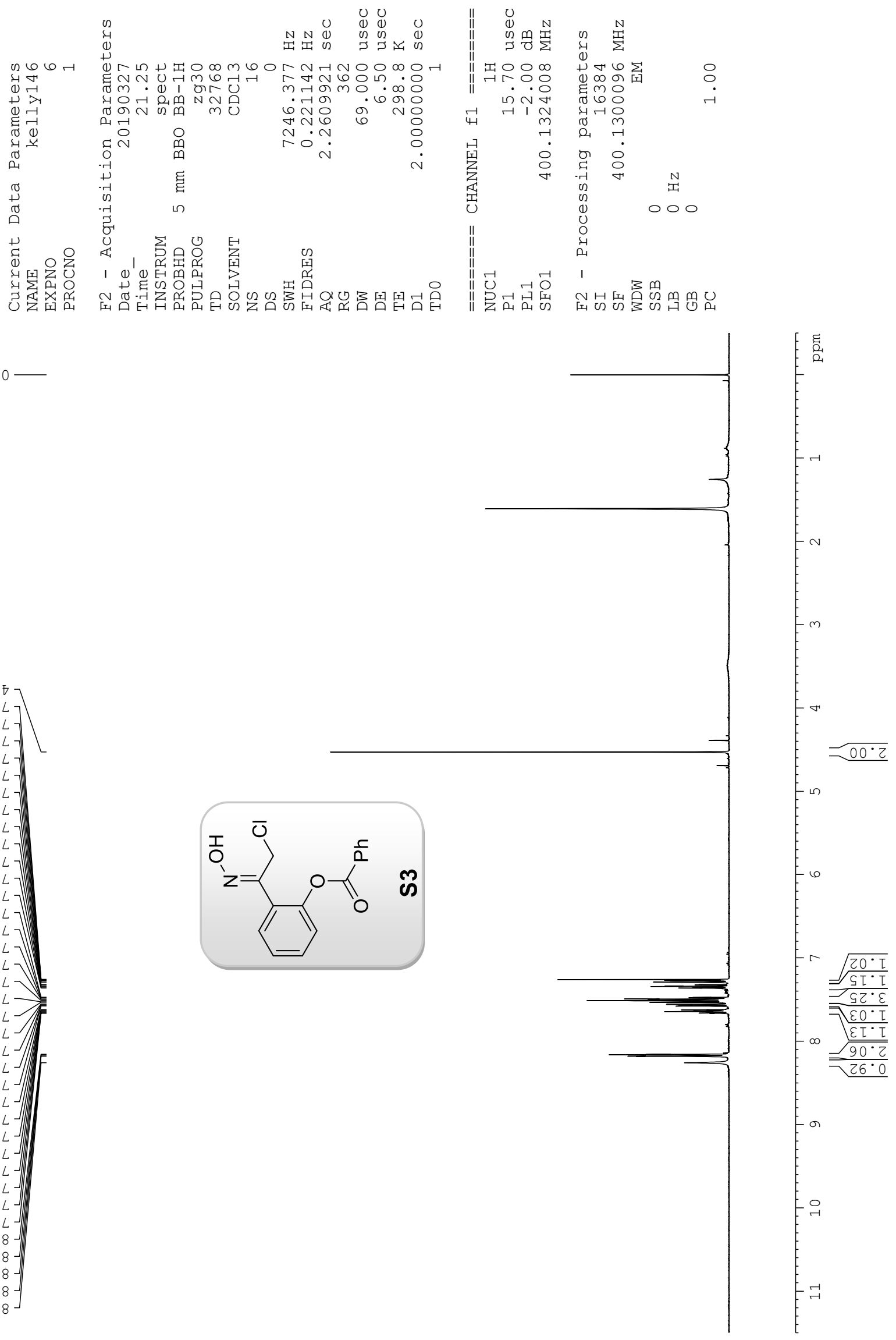


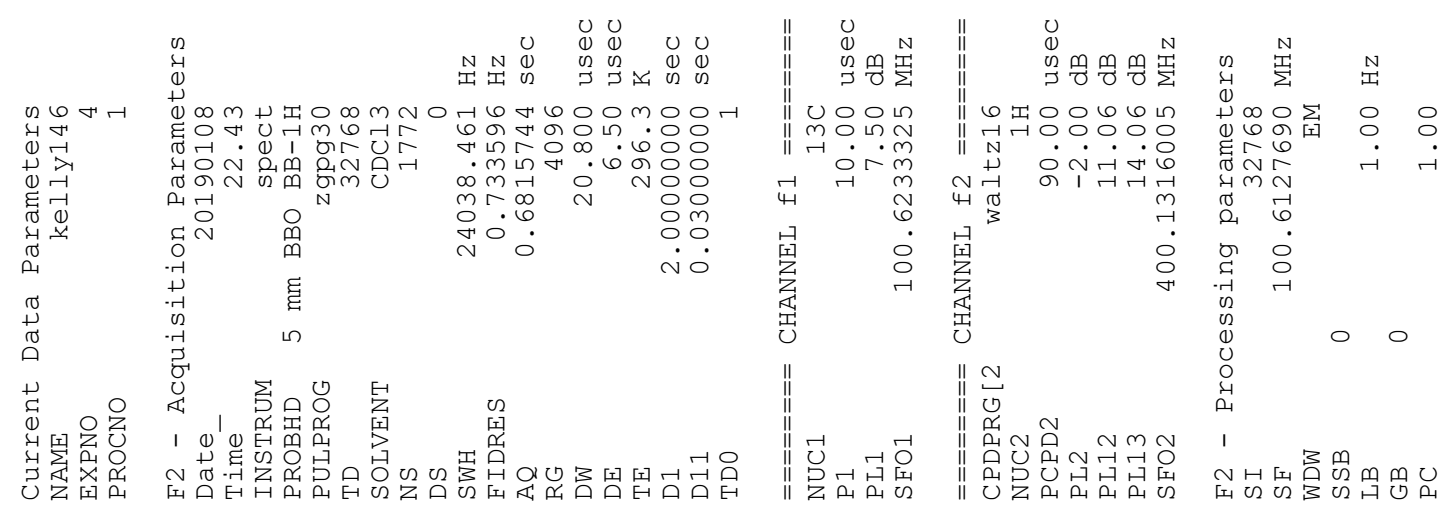

$890 \cdot 9 \varepsilon-$
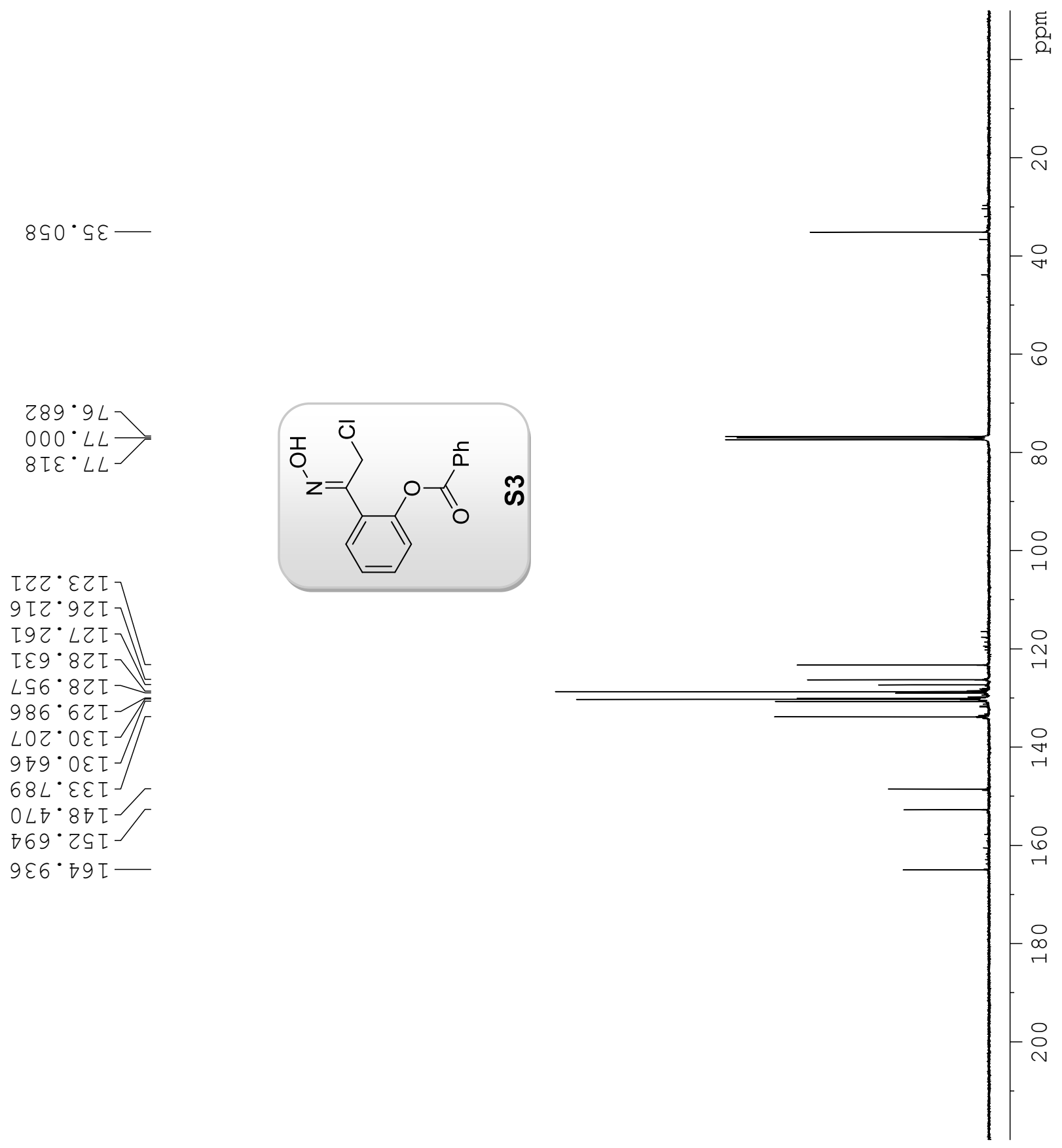


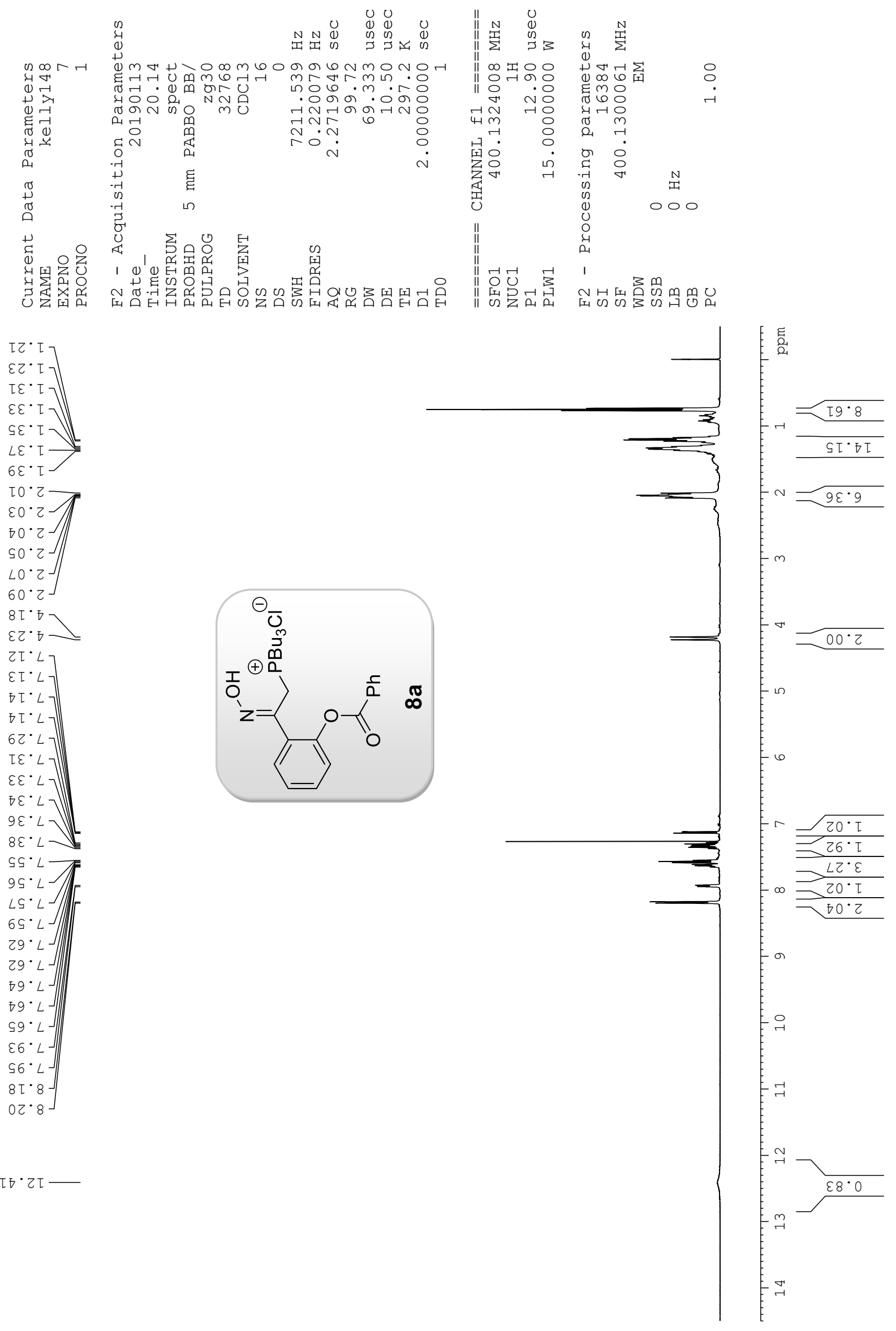



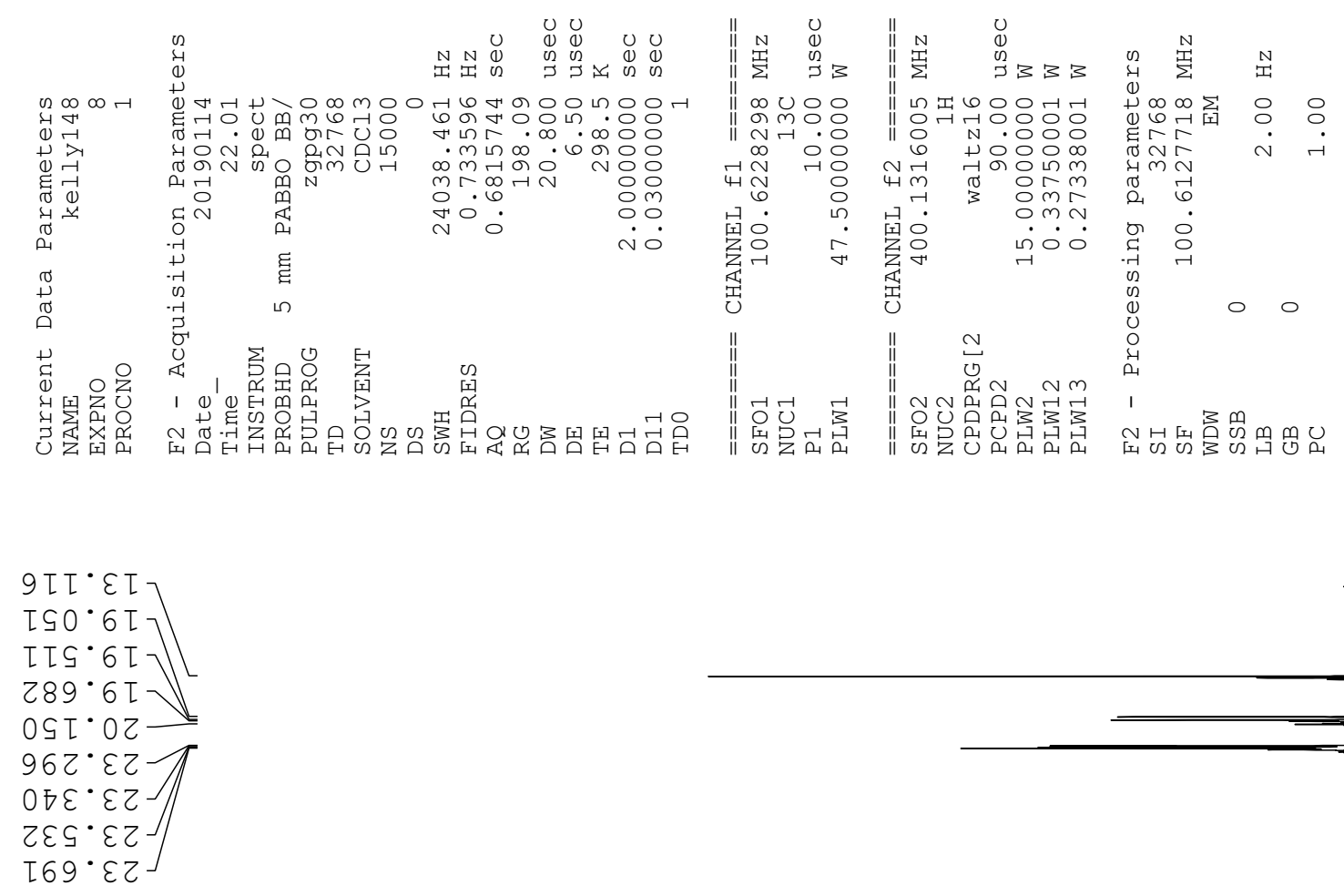

$\varepsilon 89^{\circ} 9 L$

$000^{\circ} L$

8 IE $L L$
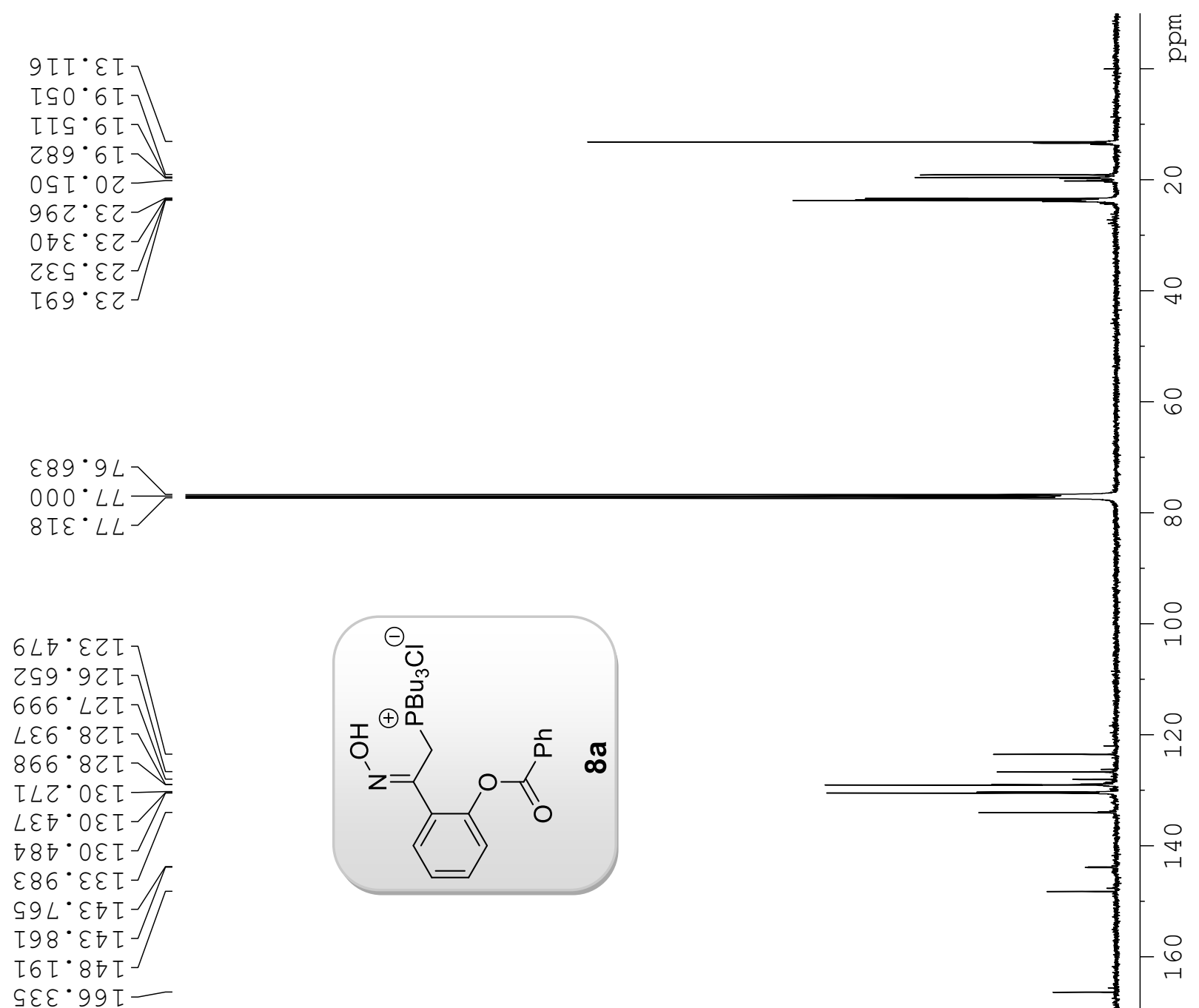


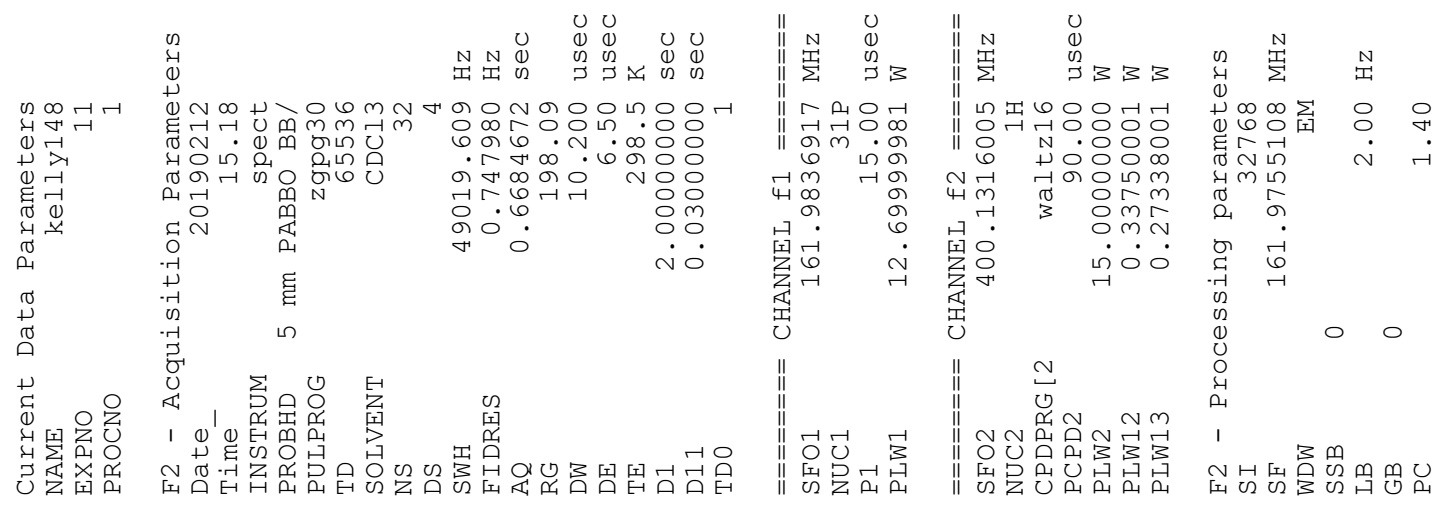

$00 \cdot 0-$

II ${ }^{\circ} \bar{\varepsilon}-$
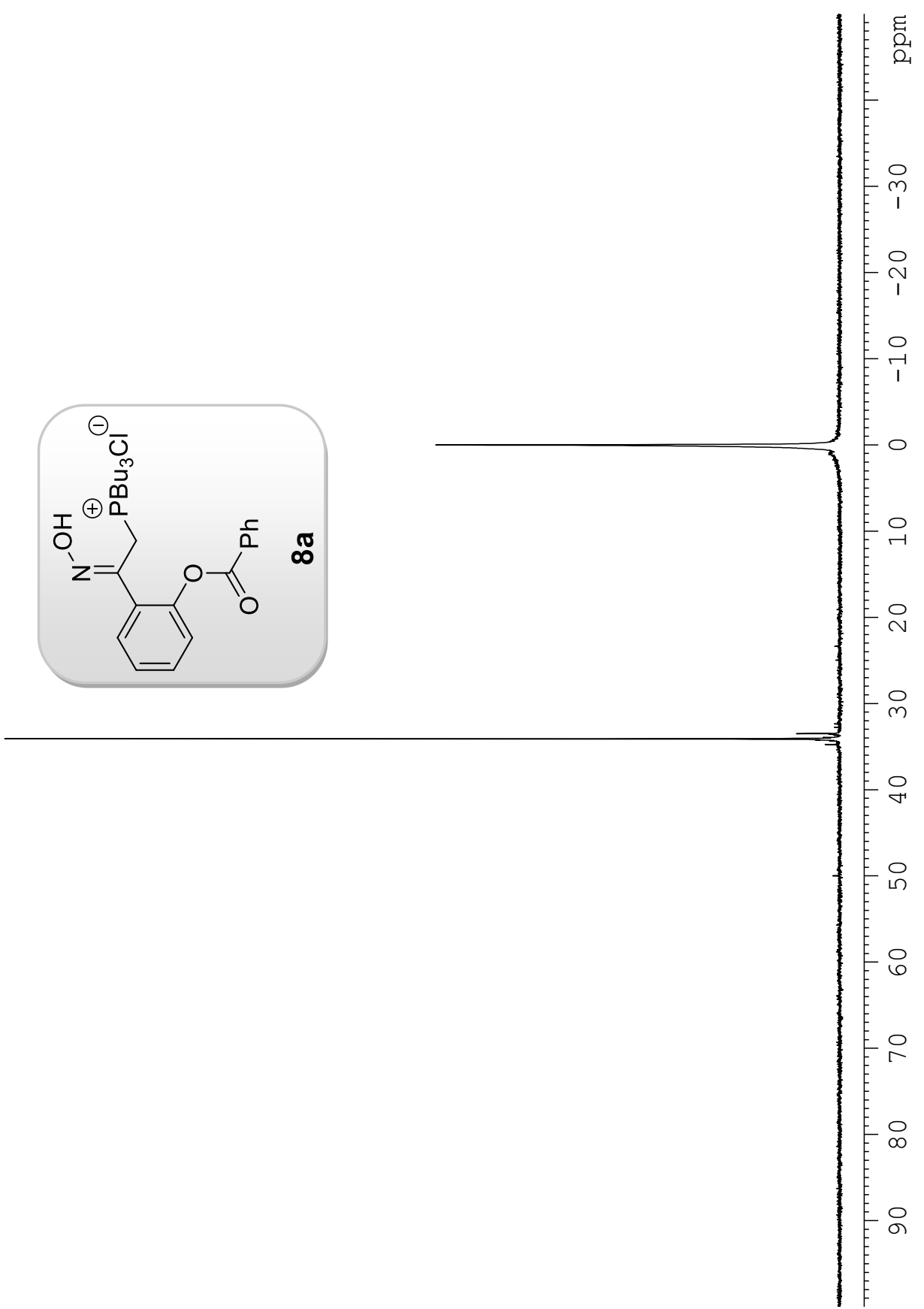

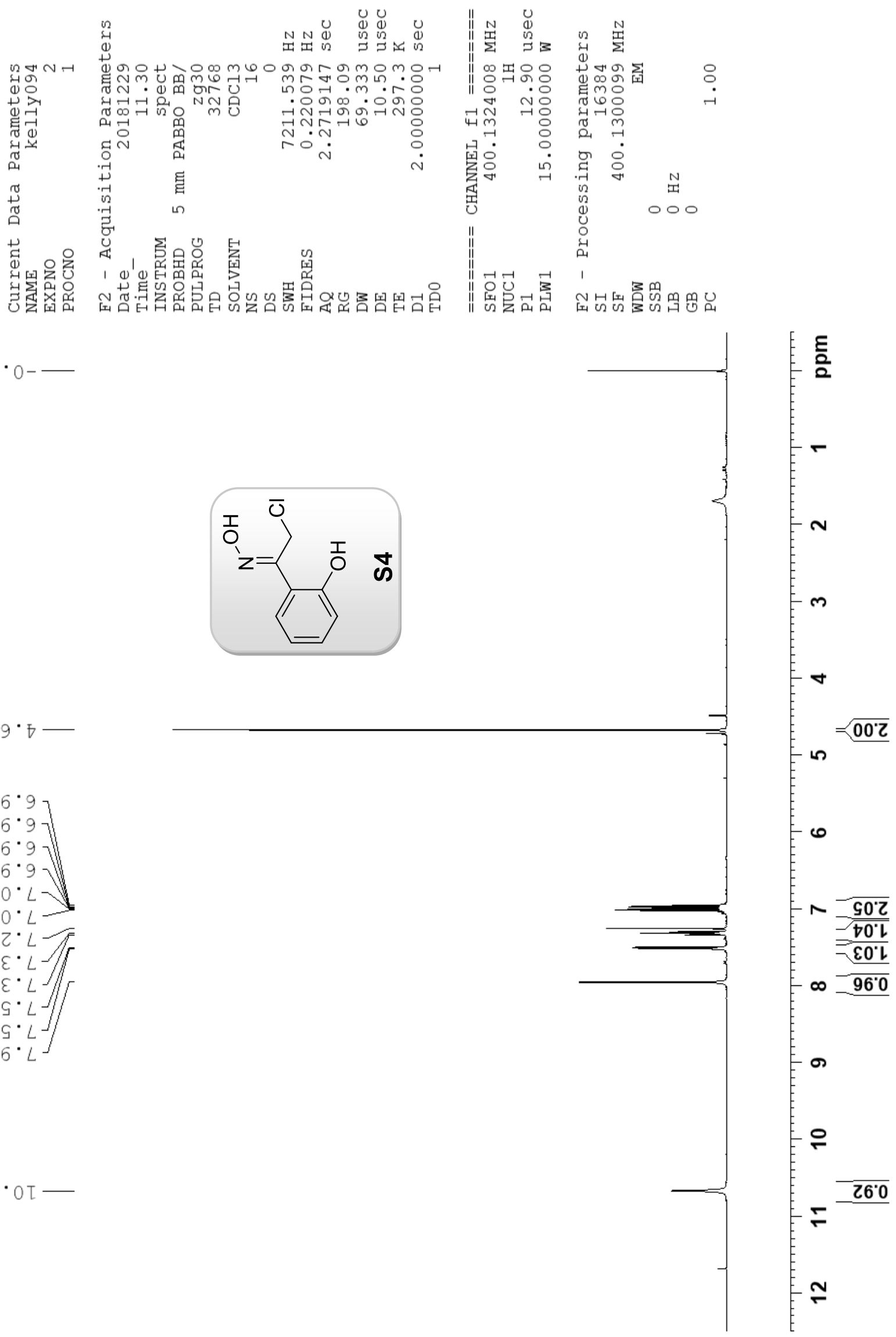

$S \angle 9^{\circ} \bar{\square}$
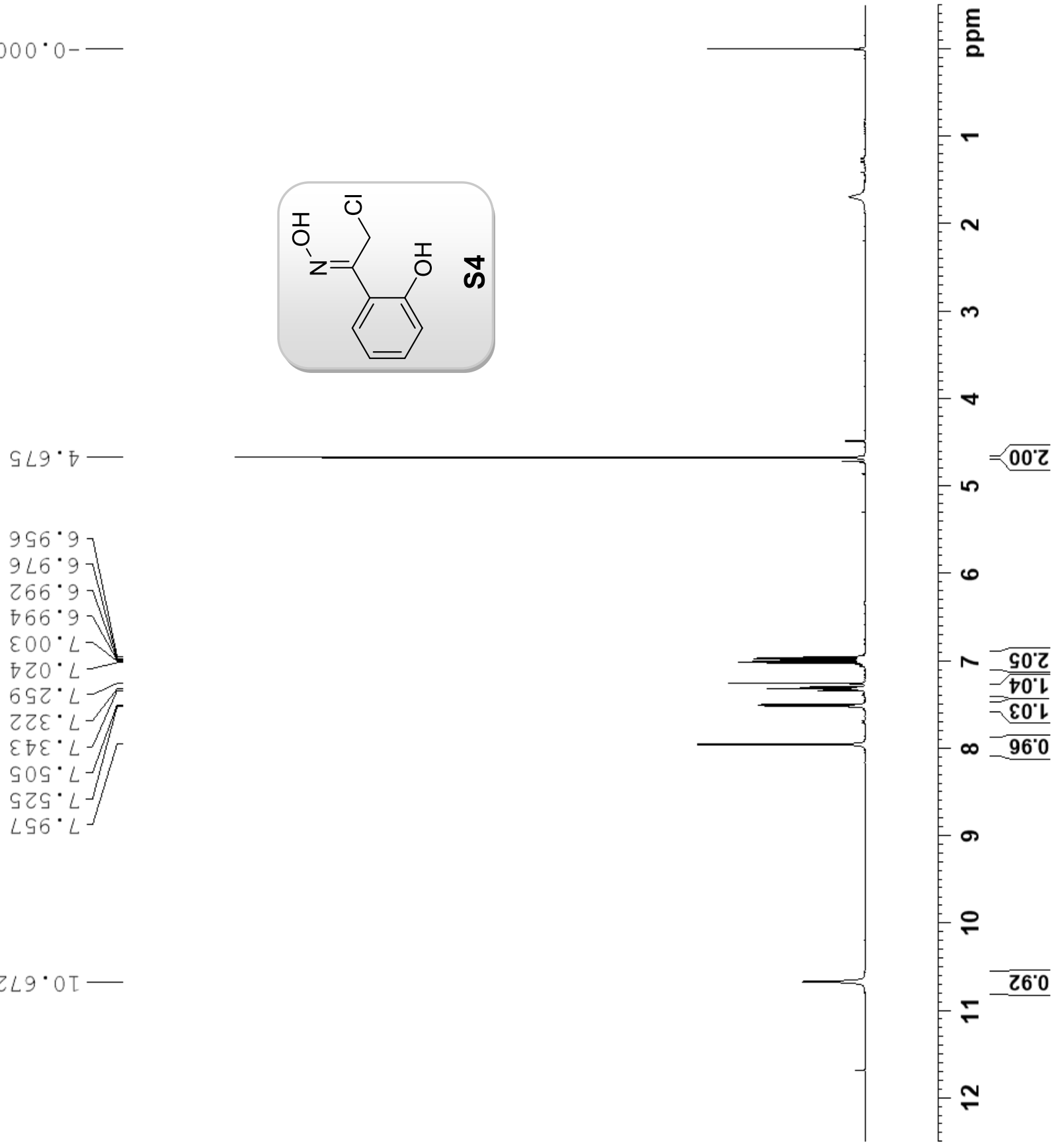


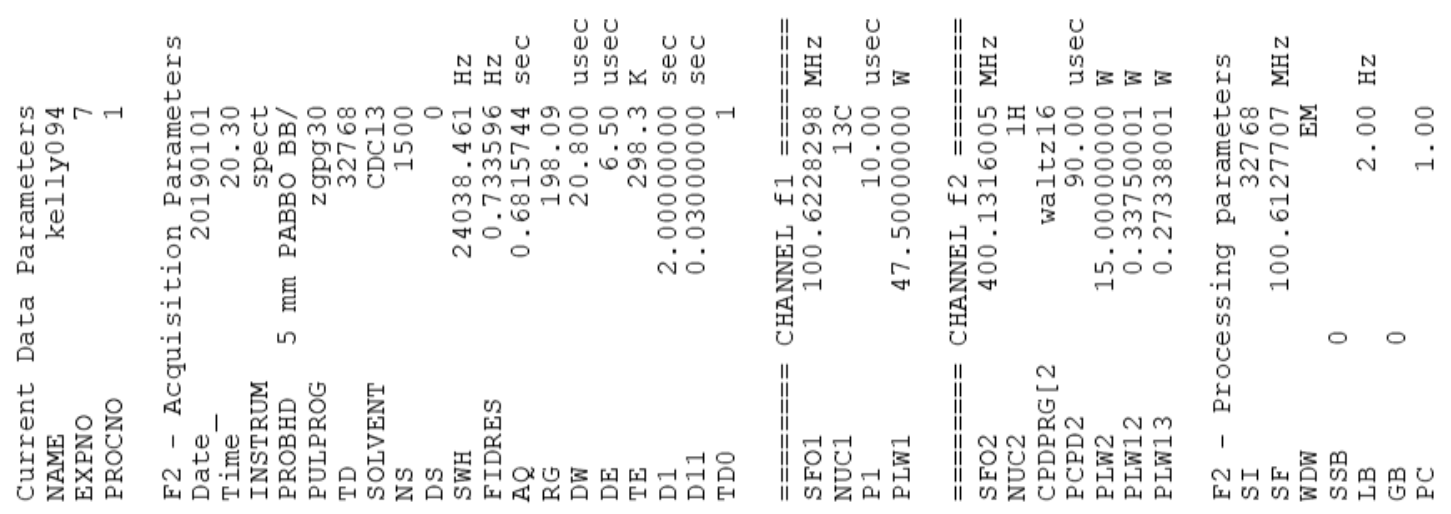

$L L Z \cdot O \varepsilon-$

$789^{\circ} 9 L$

TOO $L L$

$6 T \varepsilon^{\circ} L L$

$609^{\circ} \mathrm{SIT}$

SOL LIT

6 $\angle 9 \cdot 6$ T $~$

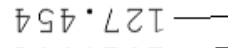

$799^{\circ}$ TET -

$0 D Z \cdot \angle G I D=$

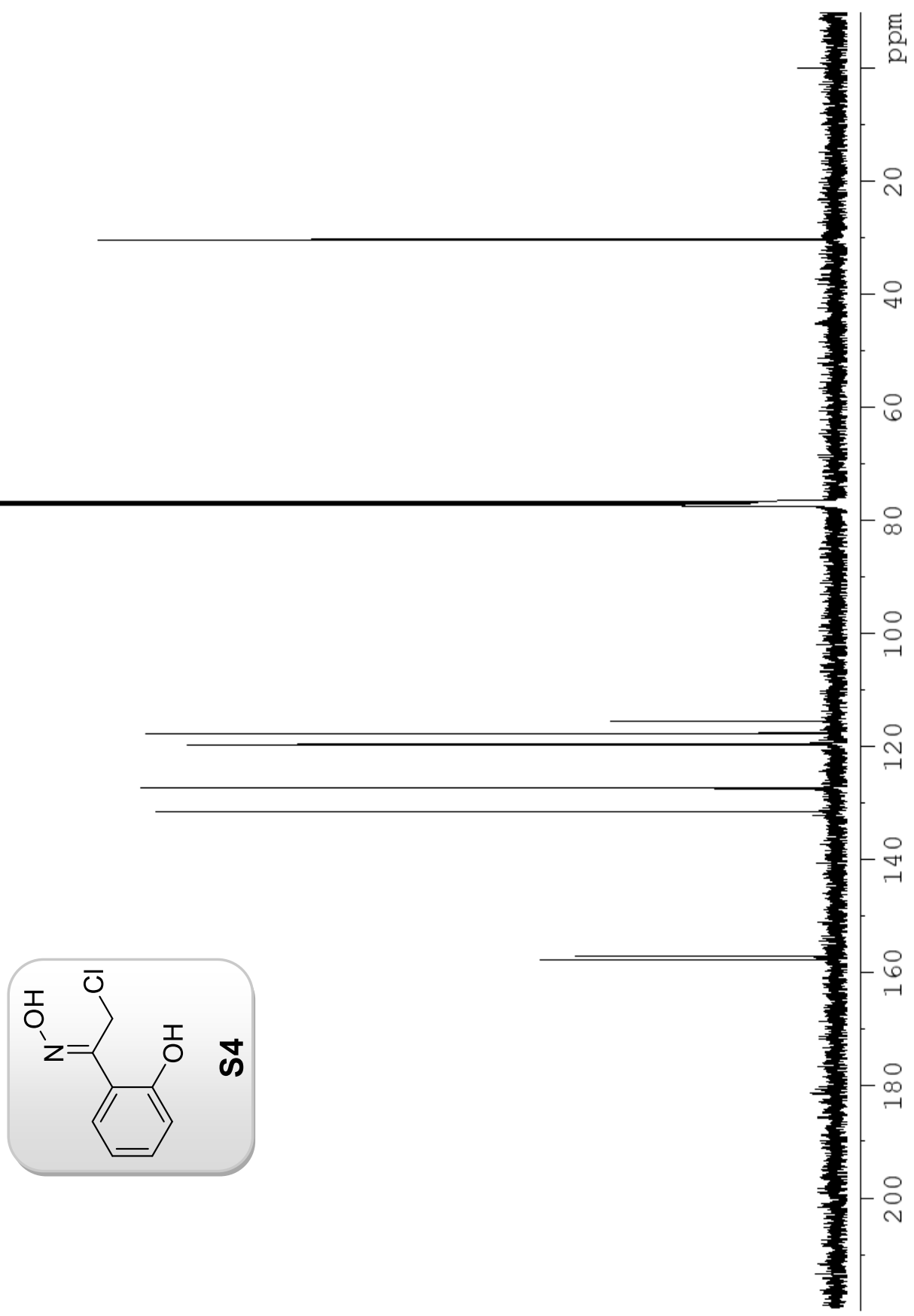




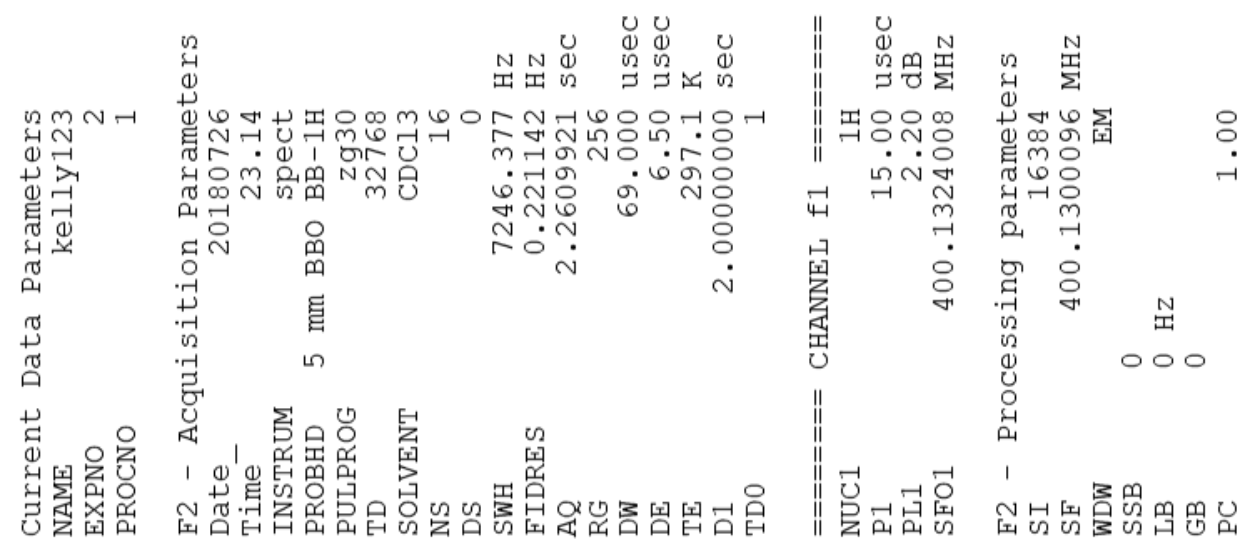

$000 \cdot 0$

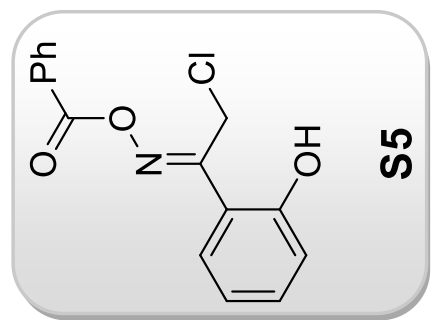

SSL. T

$786^{\circ} 97$

$\varepsilon 00^{\circ} L$

$\varepsilon 20^{\circ} \mathrm{L}$

$\left.20 T^{\circ} L\right]$

$\varepsilon 乙 \tau^{\circ} L$

$S 6 \varepsilon^{\circ} L$

$\varepsilon[\nabla \cdot L]$

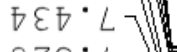

$\left.829^{\circ} L\right]$

$\angle \nabla G \cdot L$ F

$899^{\circ} L-$

$\varepsilon L S^{\circ} L / \int$

$689^{\circ} L$

$\varepsilon 69^{\circ} L$

$\angle S 9^{\circ} L$

$9 \angle 9^{\circ} \mathrm{L}$

$769^{\circ} \mathrm{L}$

$67 \tau \cdot 8$

$\angle 9 T \cdot 8 \mathrm{~J}$

$088^{\circ} 0 \mathrm{~T}$

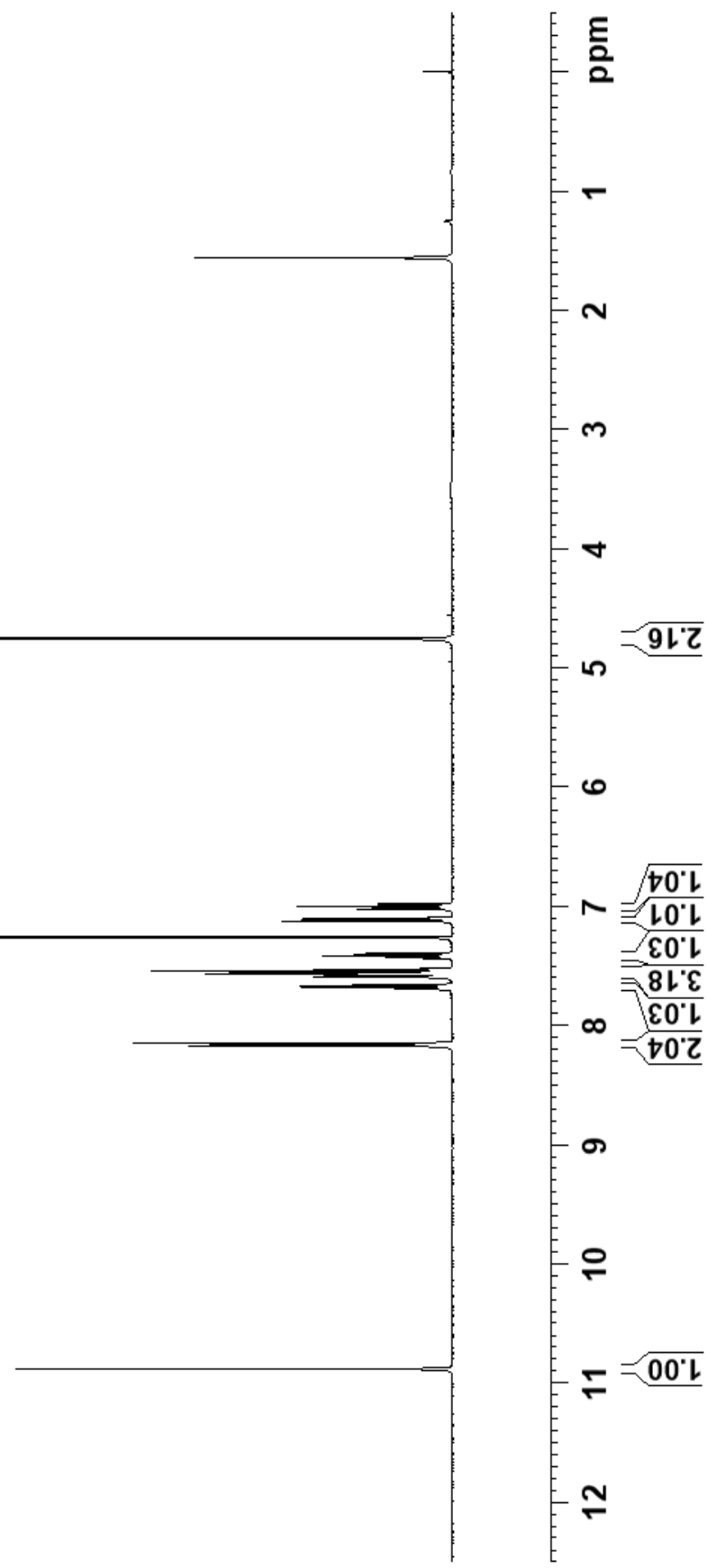



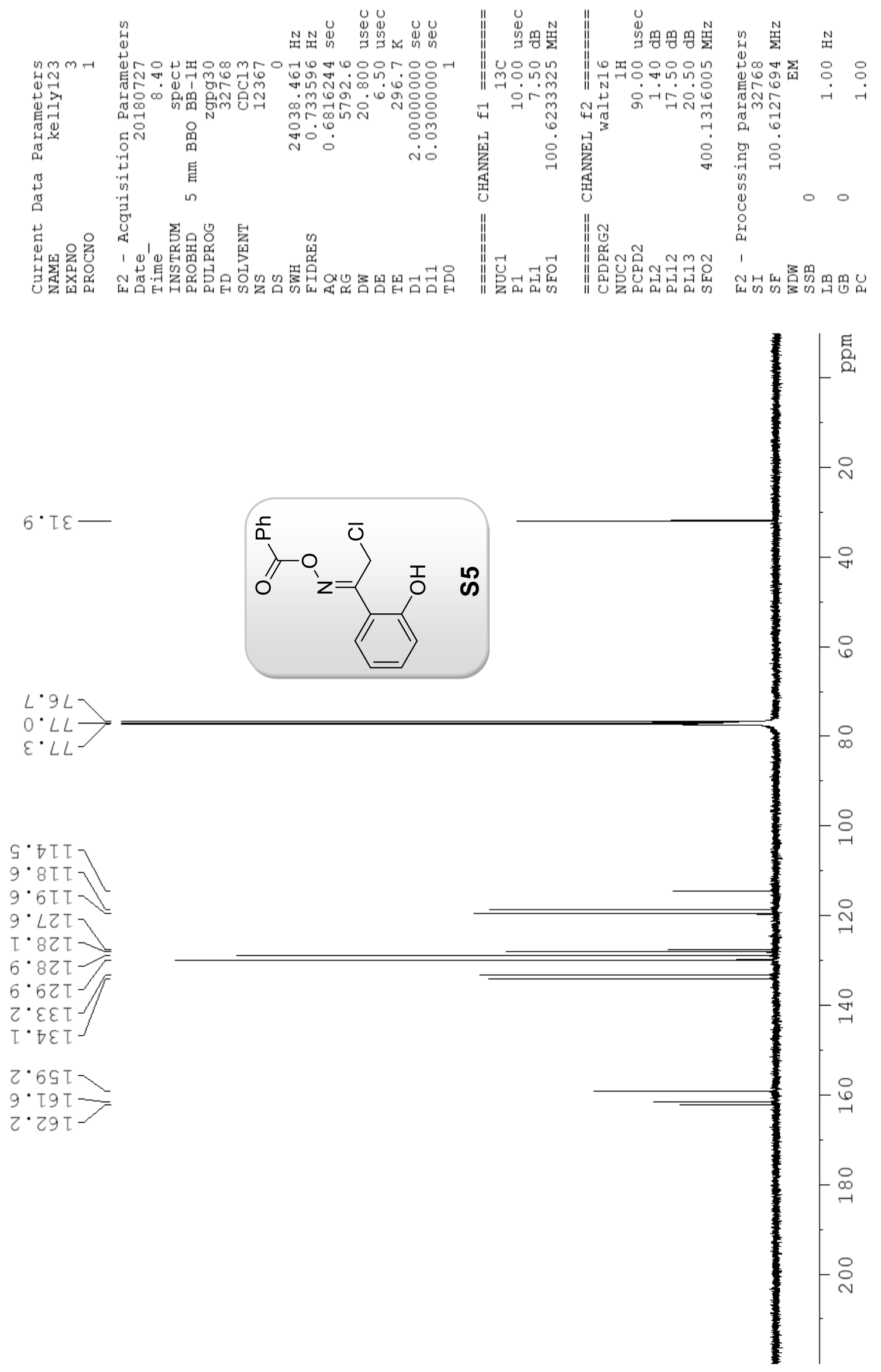

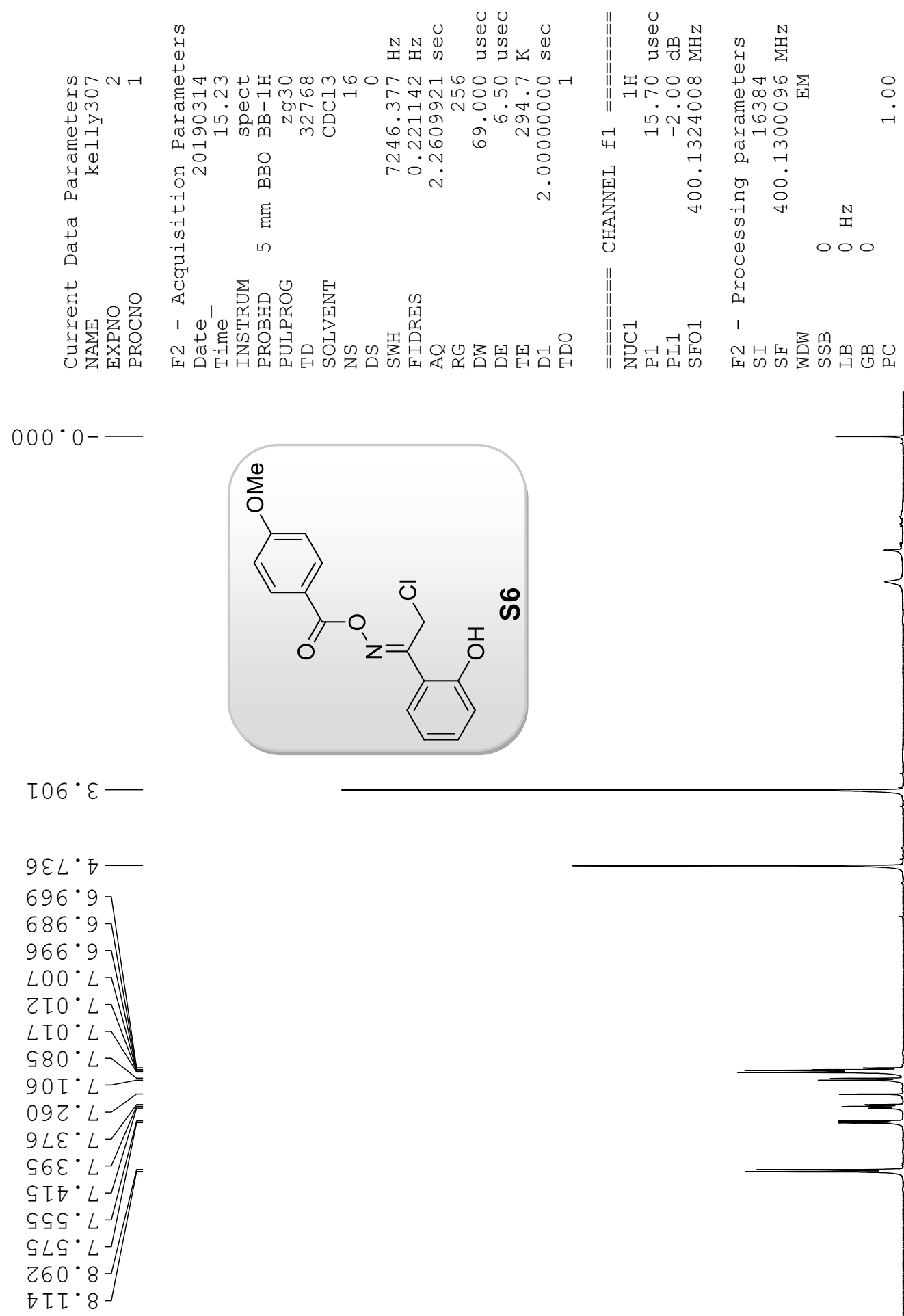

흥

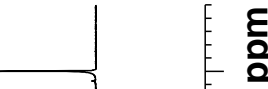

$\angle 0^{\circ} \varepsilon$

$10^{\circ} \mathrm{Z}$

N

क

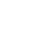

$-6$

$\wedge \frac{\sqrt{96^{\circ} \mathrm{Z}}}{00^{\circ} \mathrm{L}}$

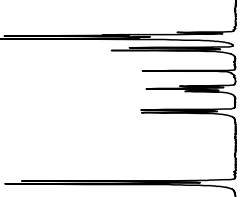

$- \infty \longdiv { 0 0 ^ { \circ } \mathrm { z } }$

$a$

으

$=\square 6^{\circ} 0$

SE $6^{\circ} 0 \tau$

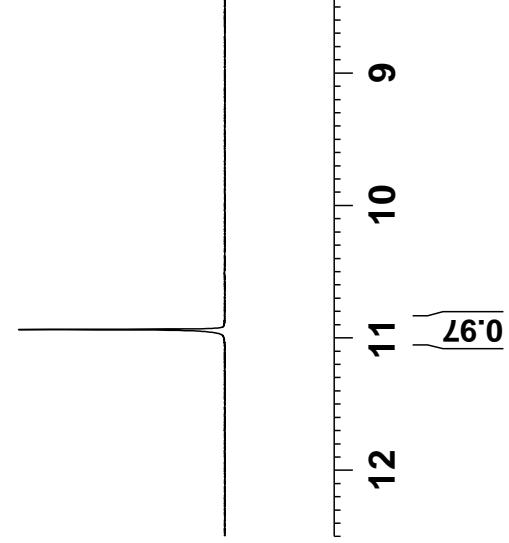



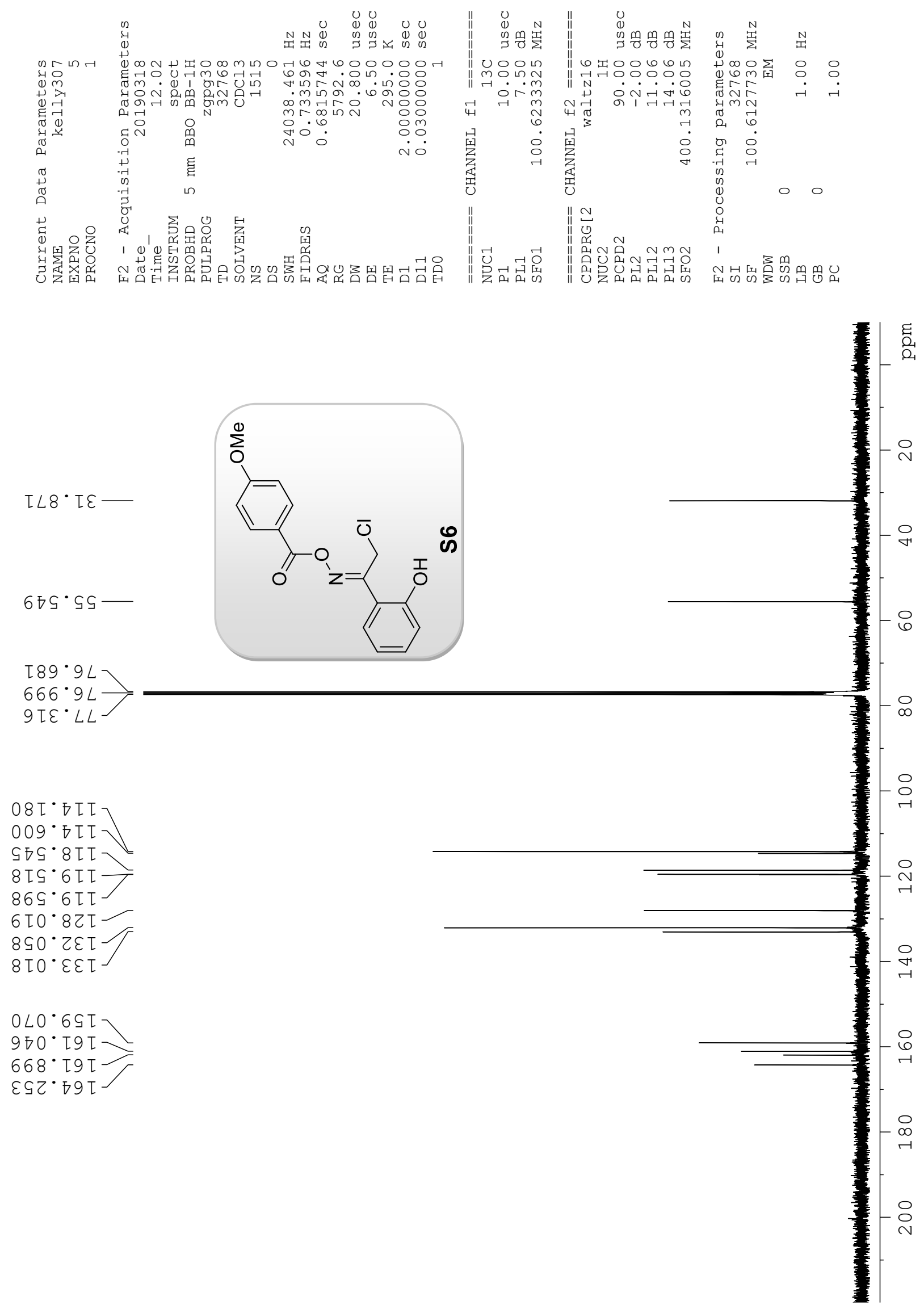

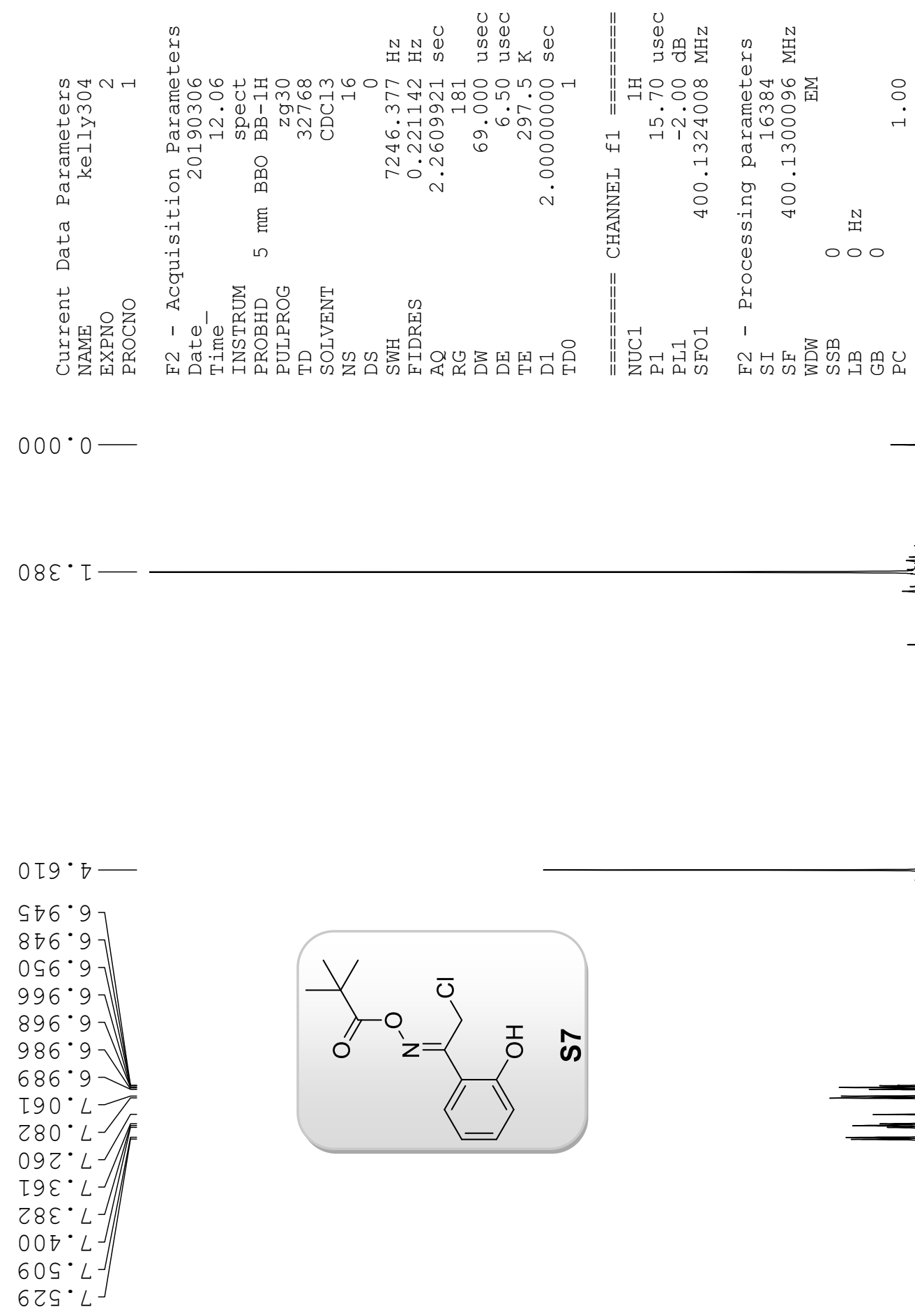

0 I $8^{\circ} 0 \tau$

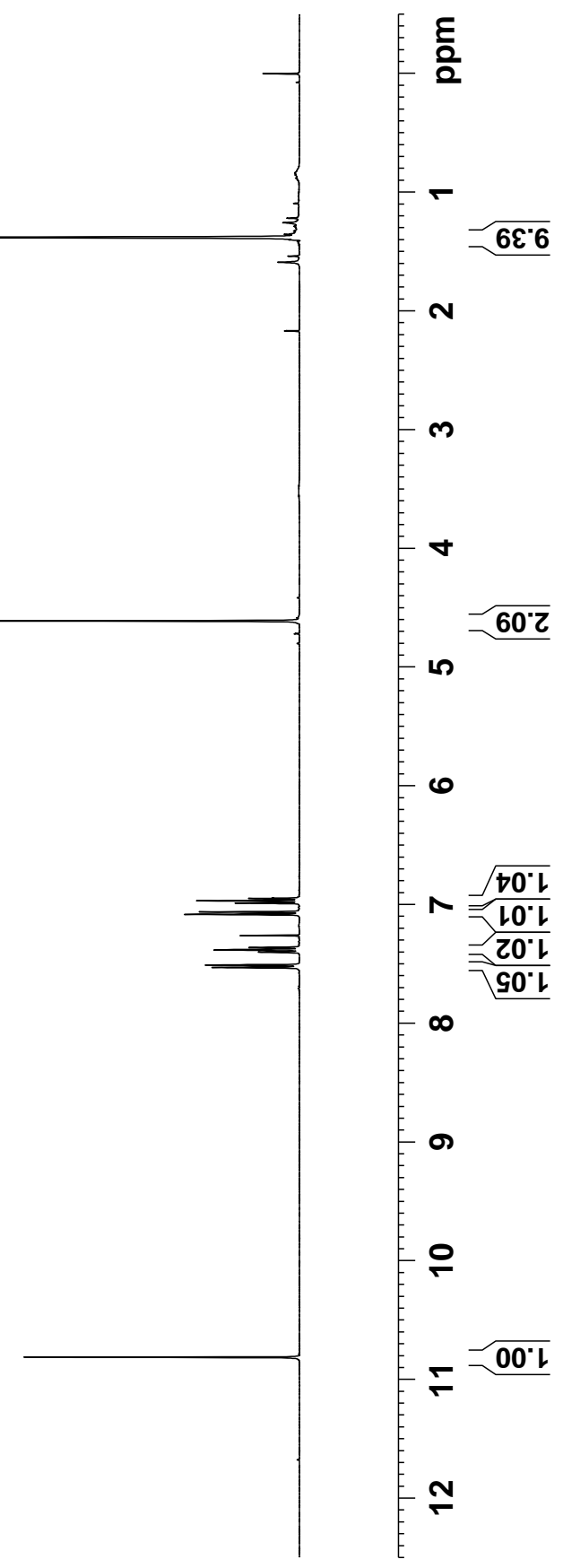



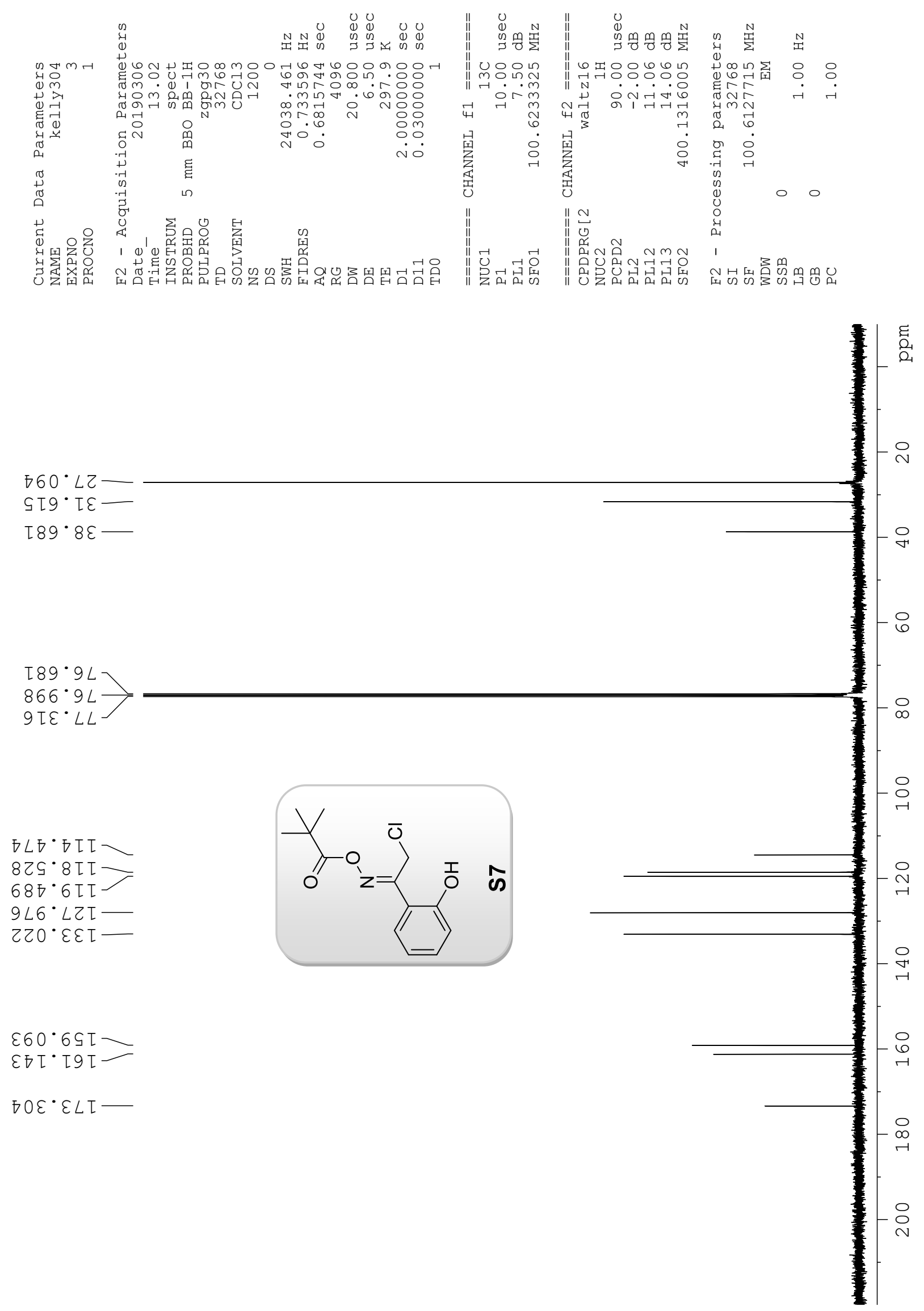


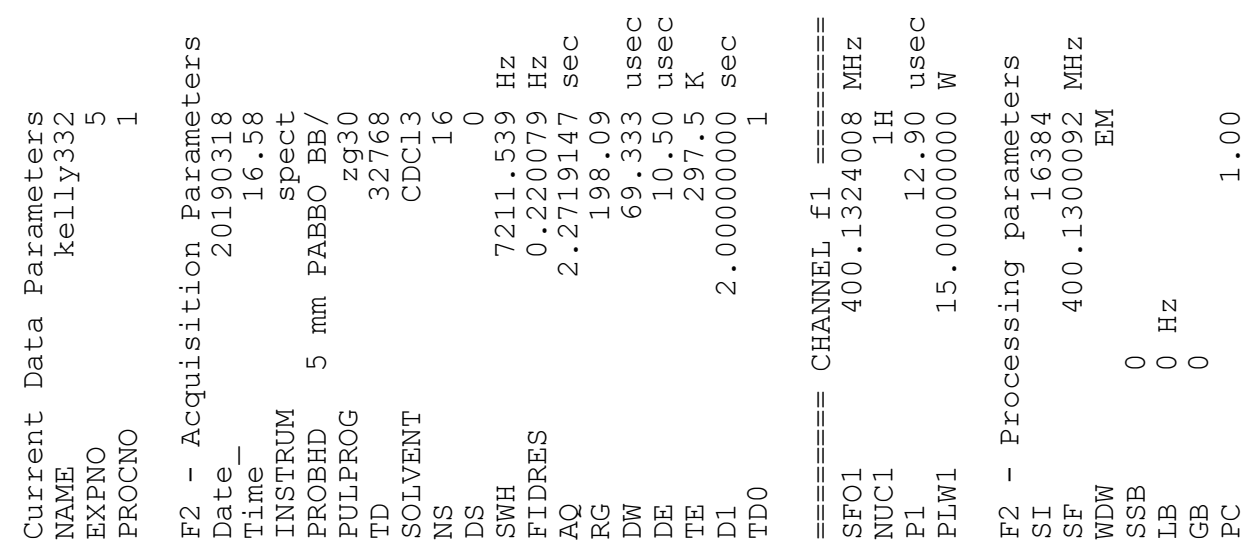

$000 \cdot 0$

$80 \varepsilon^{*} 乙$

$079^{\circ} \overline{7}$

$996 \cdot 9$

$\angle S 6^{\circ} 9$

2L6.9]

$S L 6 \cdot 9$

$\angle L 6 \cdot 9$

$\varepsilon 66^{\circ} 9$

$966^{\circ} 9$

$690^{\circ} L$

[9 $\left.0^{\circ} \mathrm{L}\right]$

$080^{\circ} \mathrm{L}$

$280^{\circ} \mathrm{L}$

I9 ${ }^{\circ} L$

$99 \varepsilon \cdot L$

$\varsigma 8 \varepsilon^{\circ} L$

$88 \varepsilon^{\circ} L^{J}$

โ6E $\varepsilon^{\circ} L$

SOF. L

$607^{\circ} L$

929. $L$

OES $L$

LDS $L$

$96 L \cdot 0 I-$
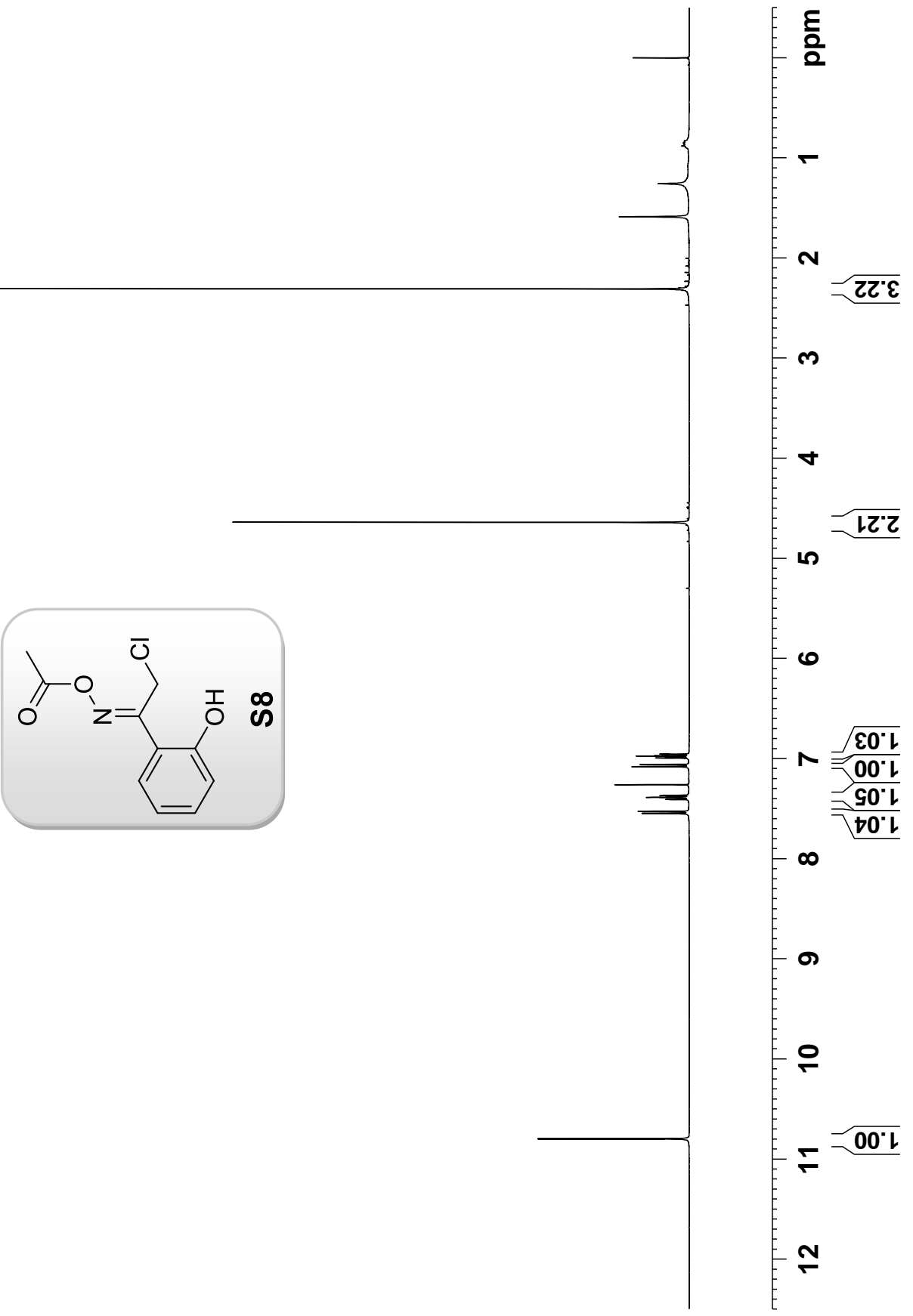

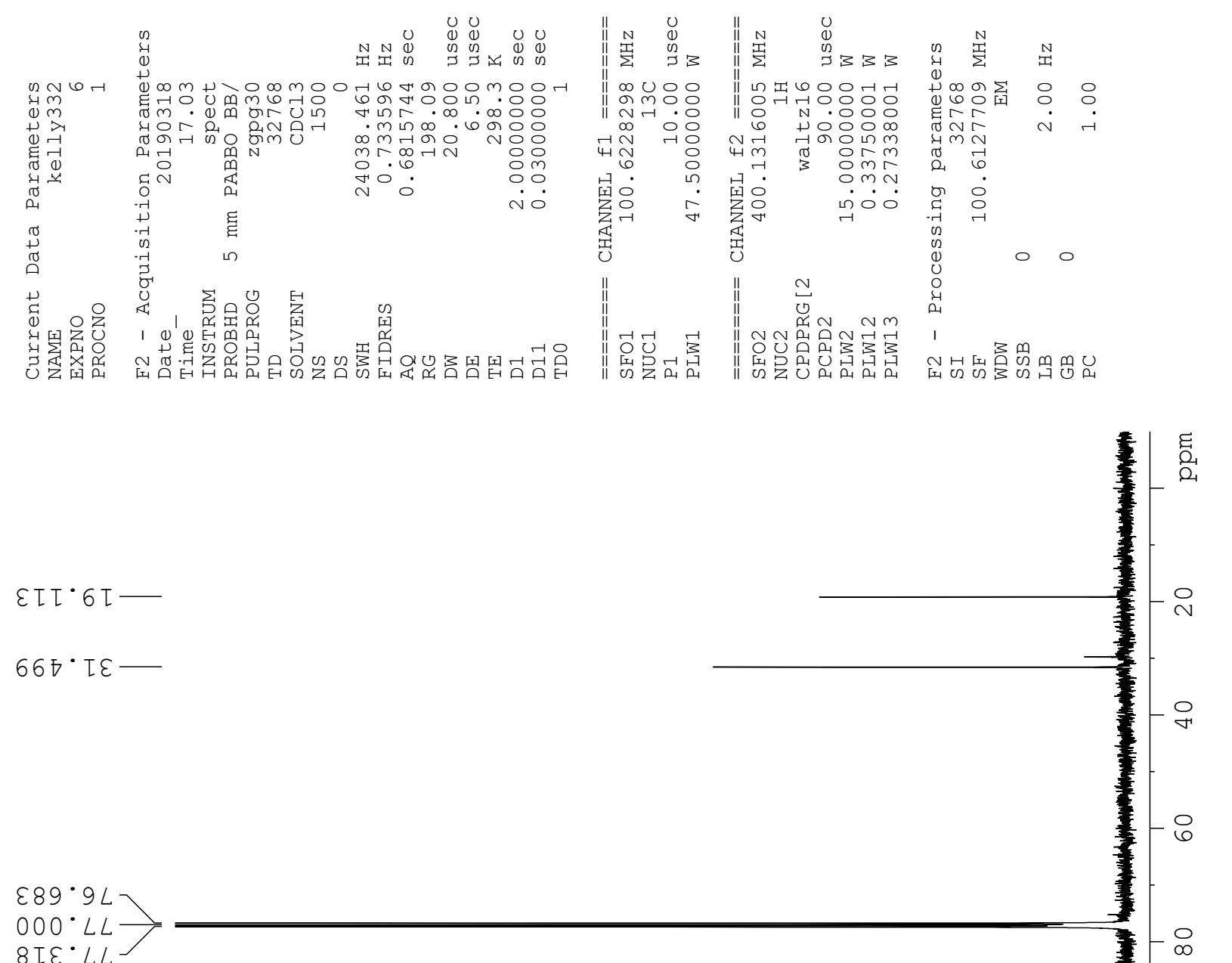

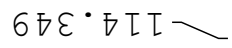

7ஏ ${ }^{\circ} 8 I I-$

S6I.8ZI

$\varepsilon 80^{\circ} \varepsilon \varepsilon \tau$

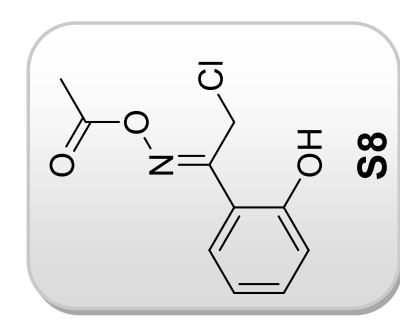

$160 \cdot 6 \mathrm{ST}$

758.09T

$986^{\circ} 99 \mathrm{I}$

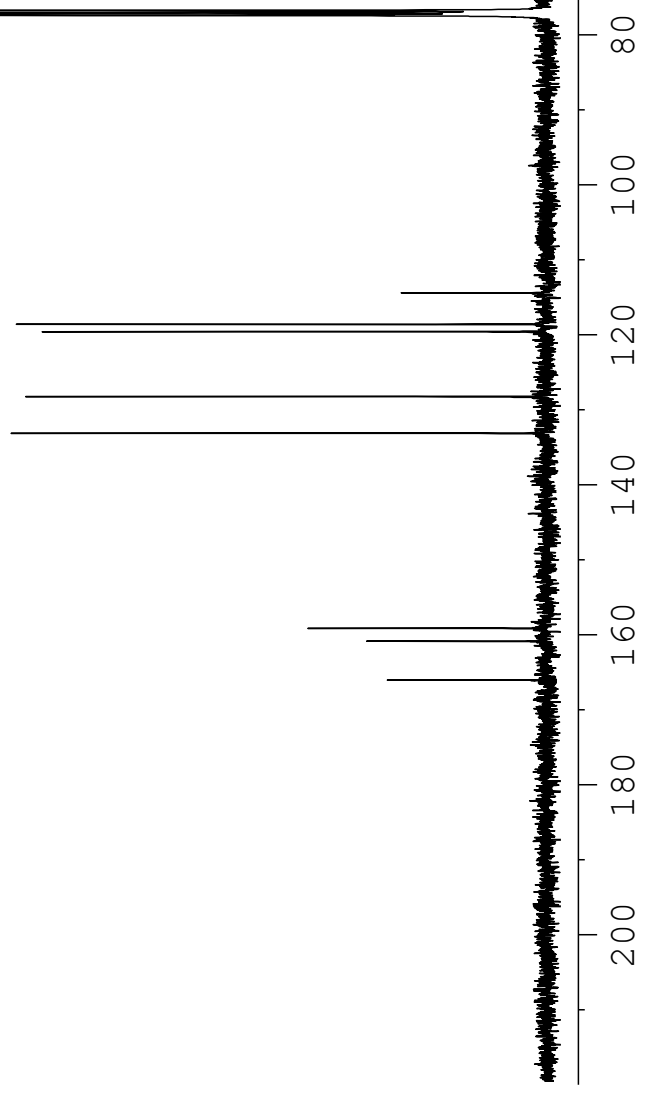




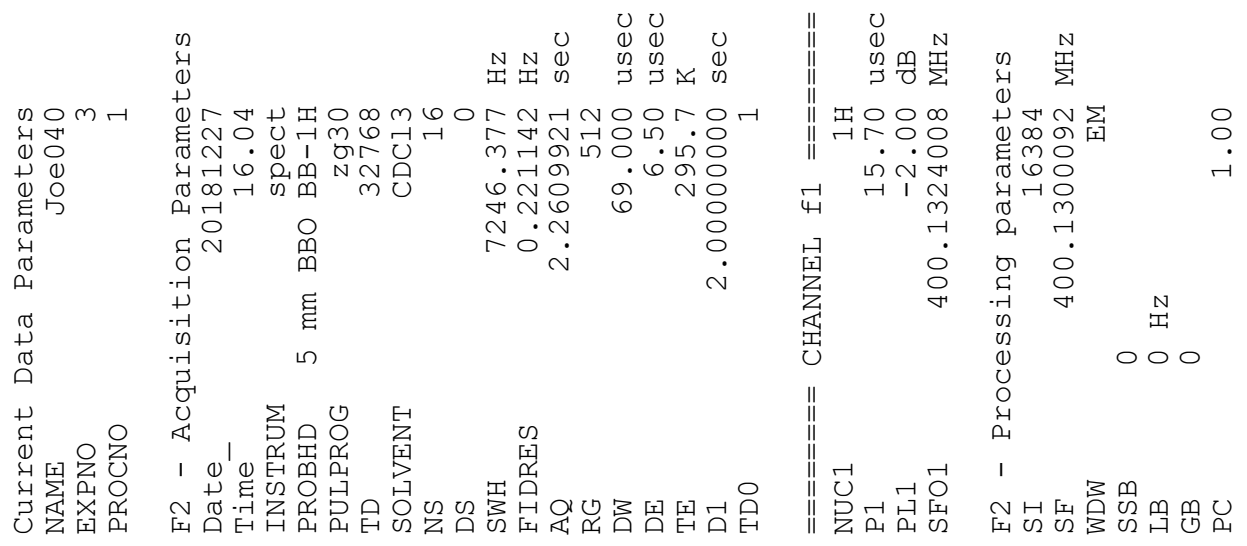

$000 \cdot 0$
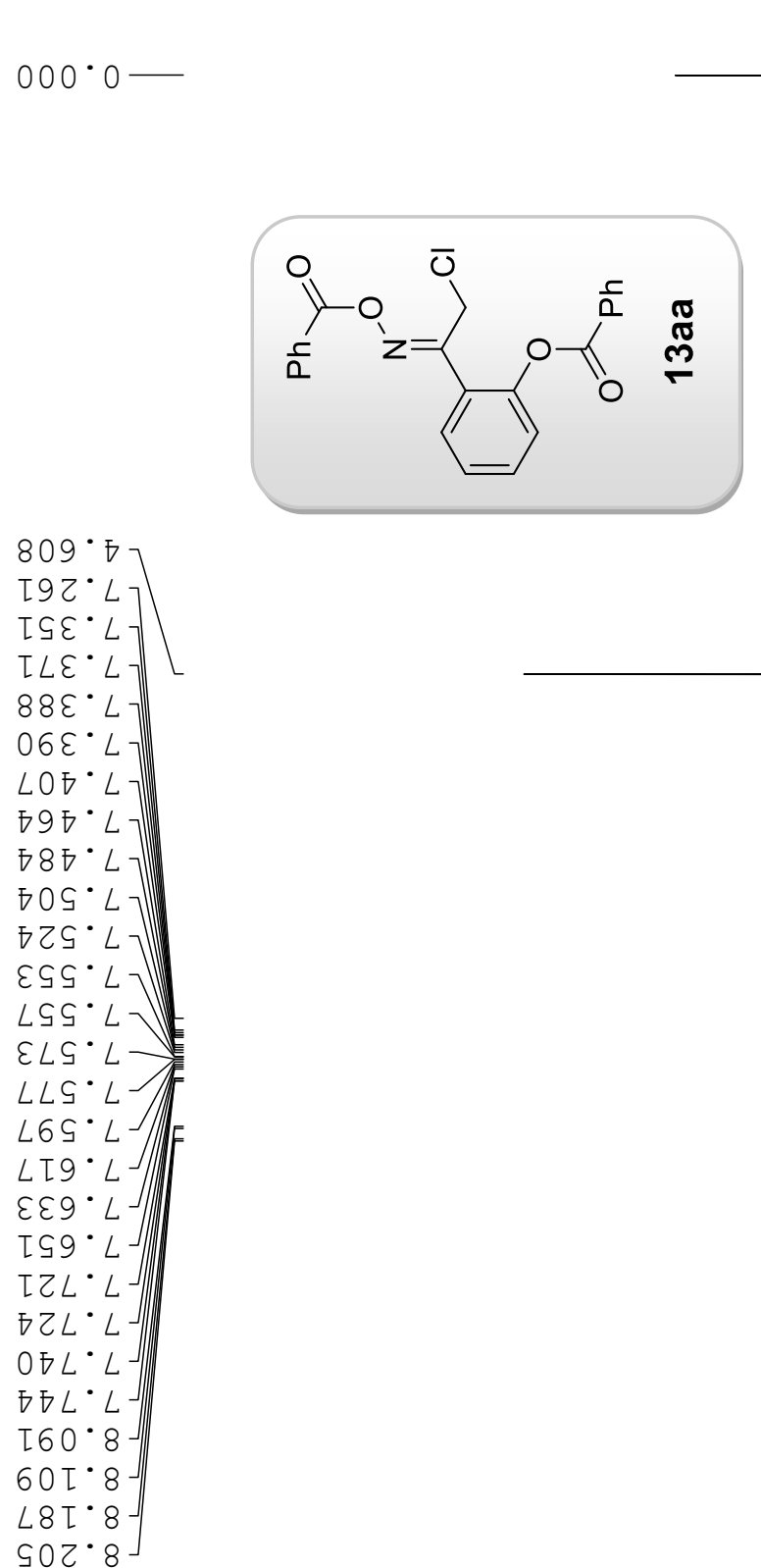

흥

00

- 10

0 

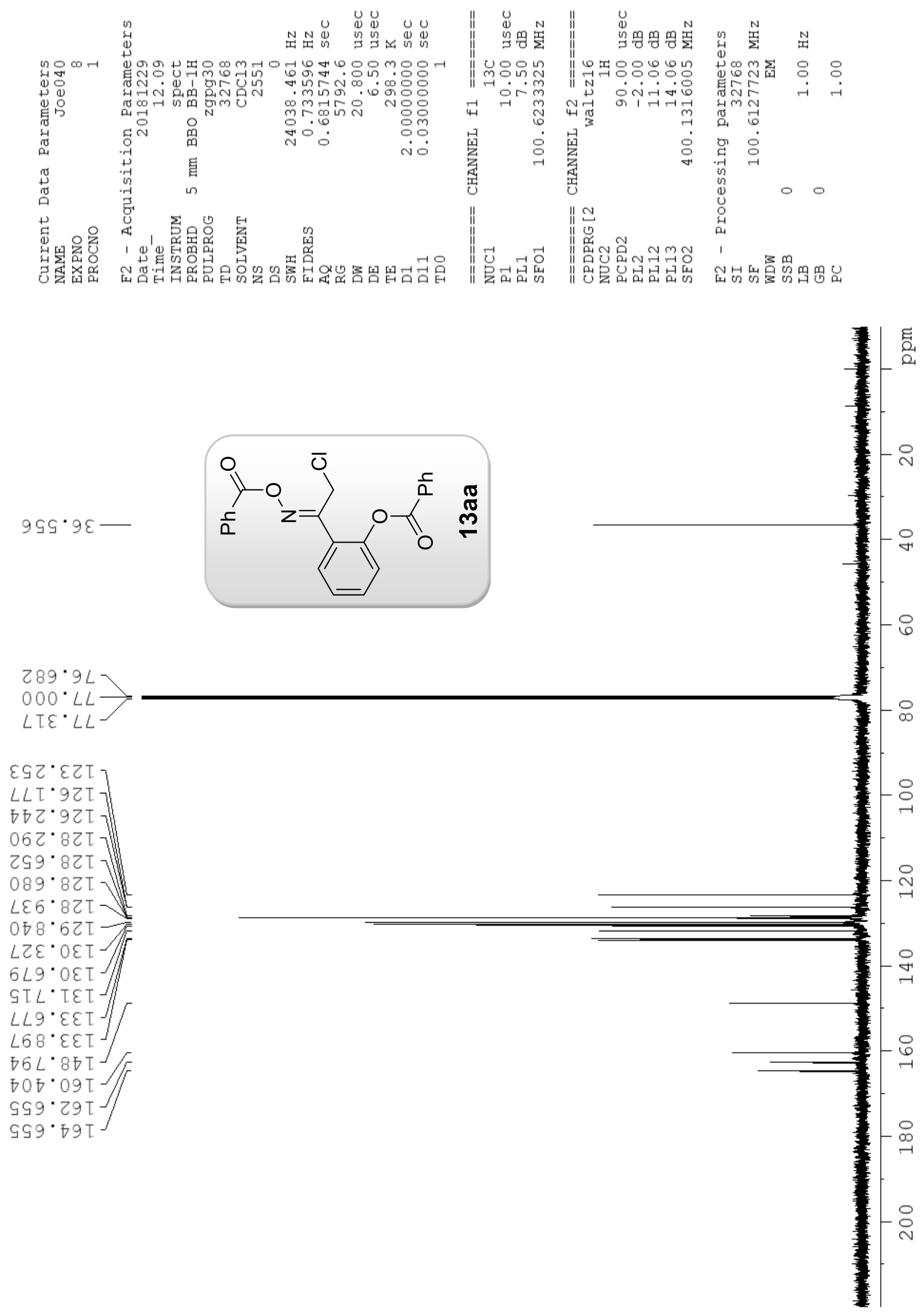

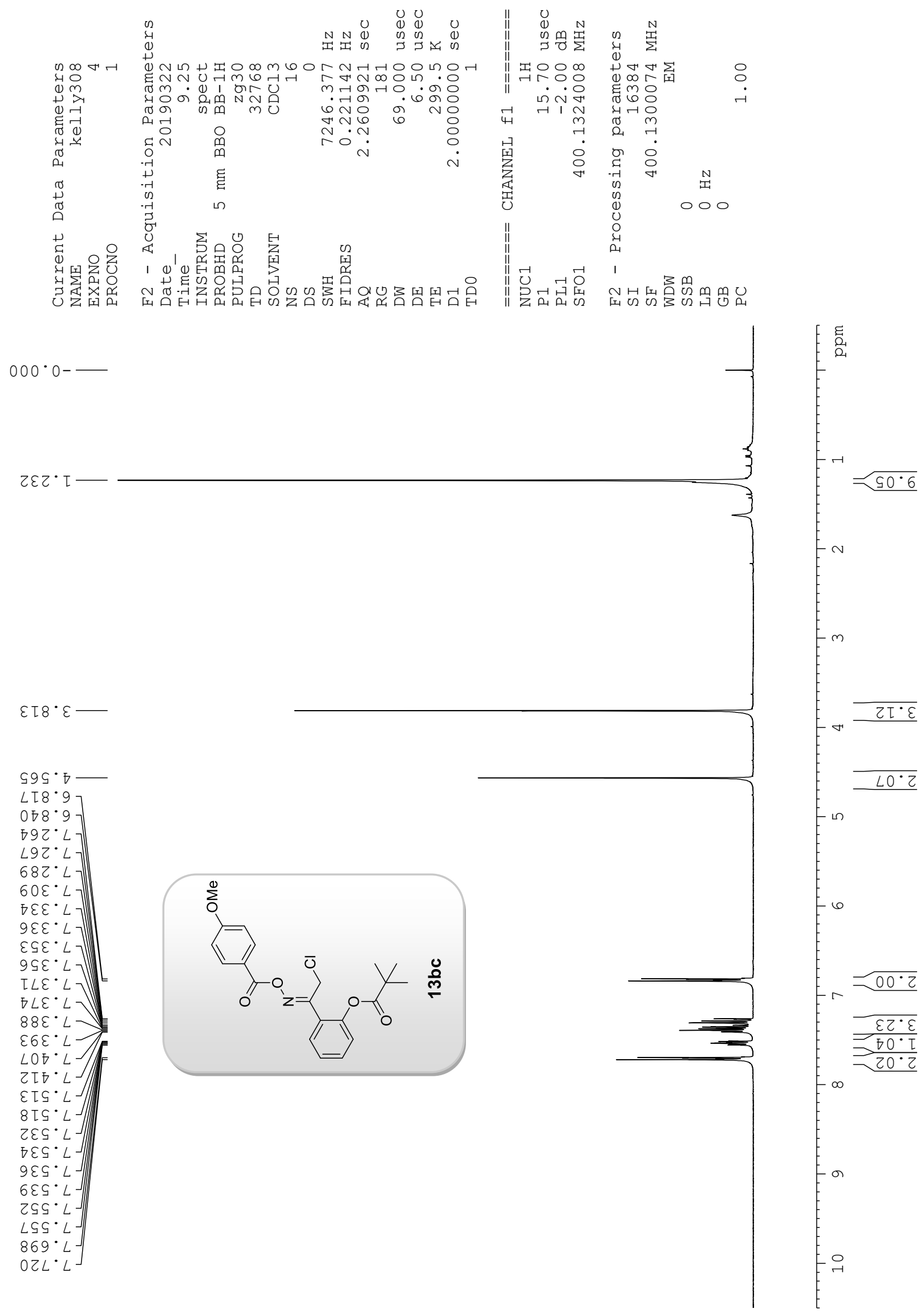

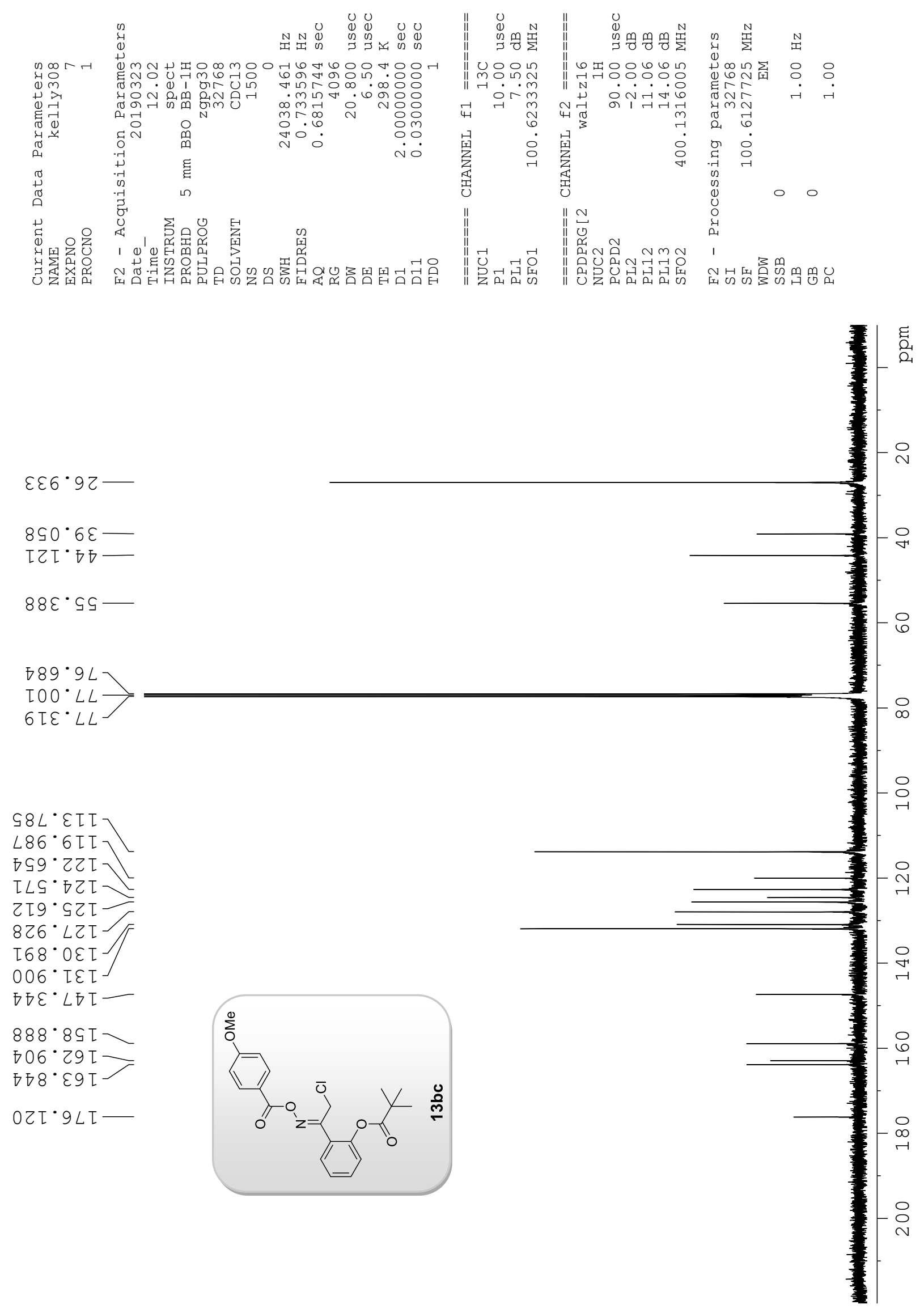

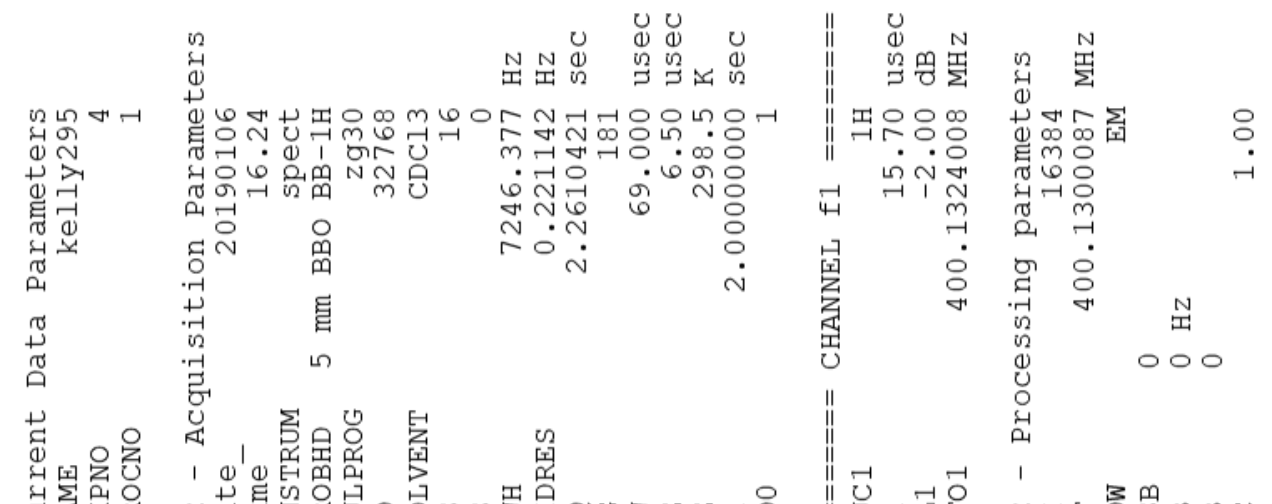

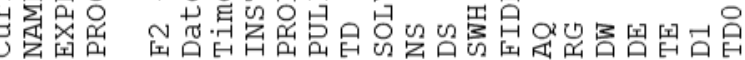

||

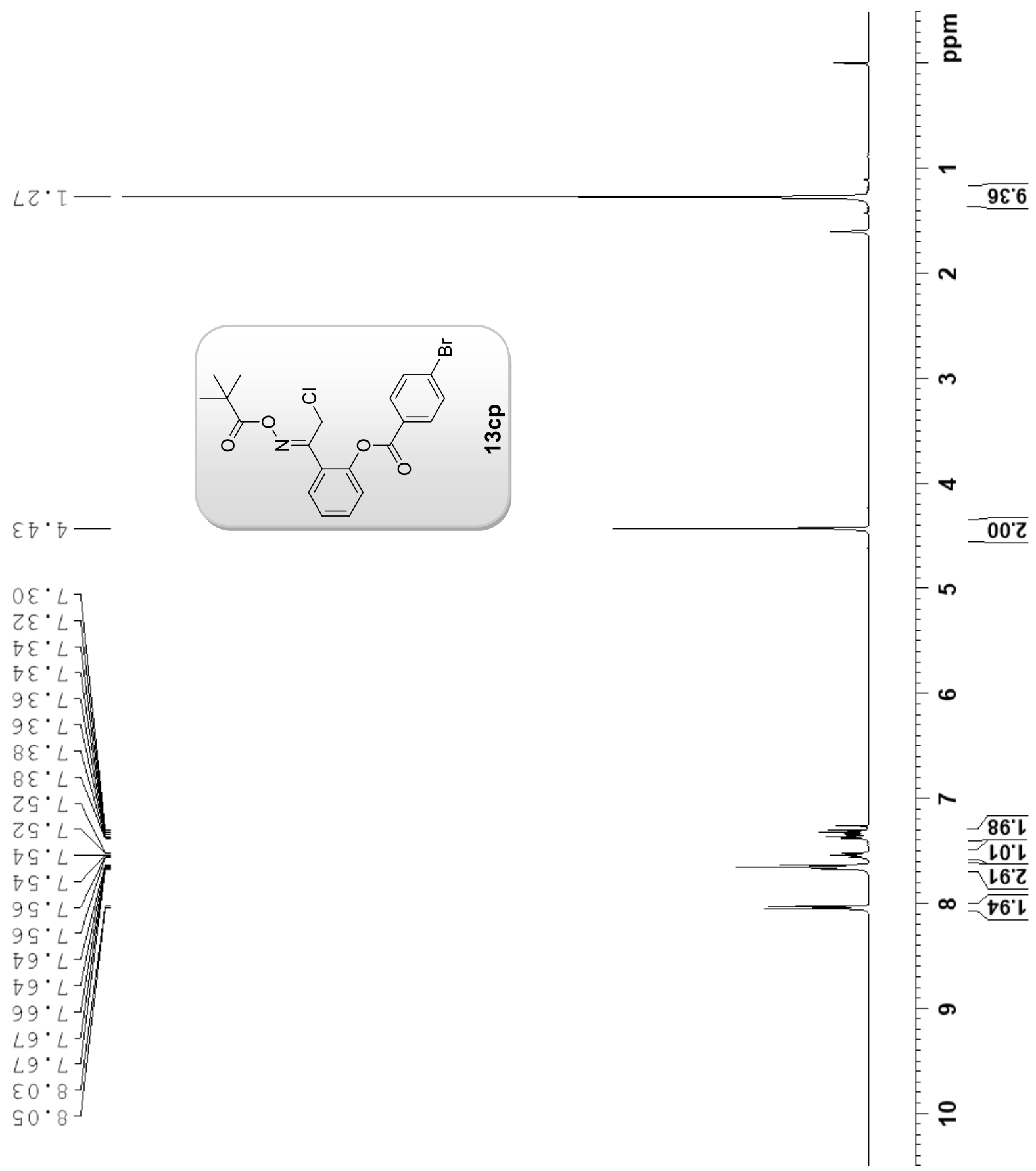



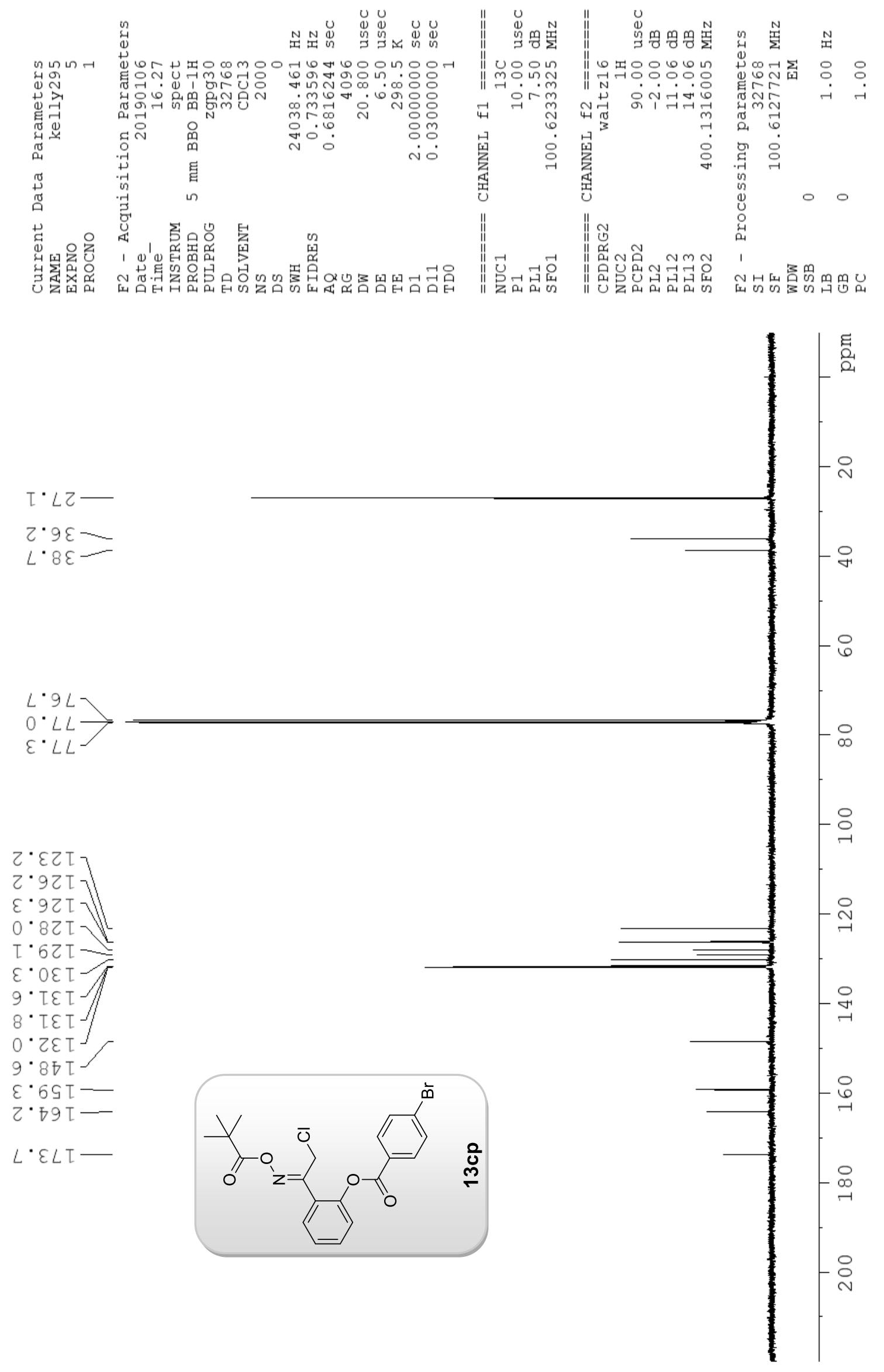

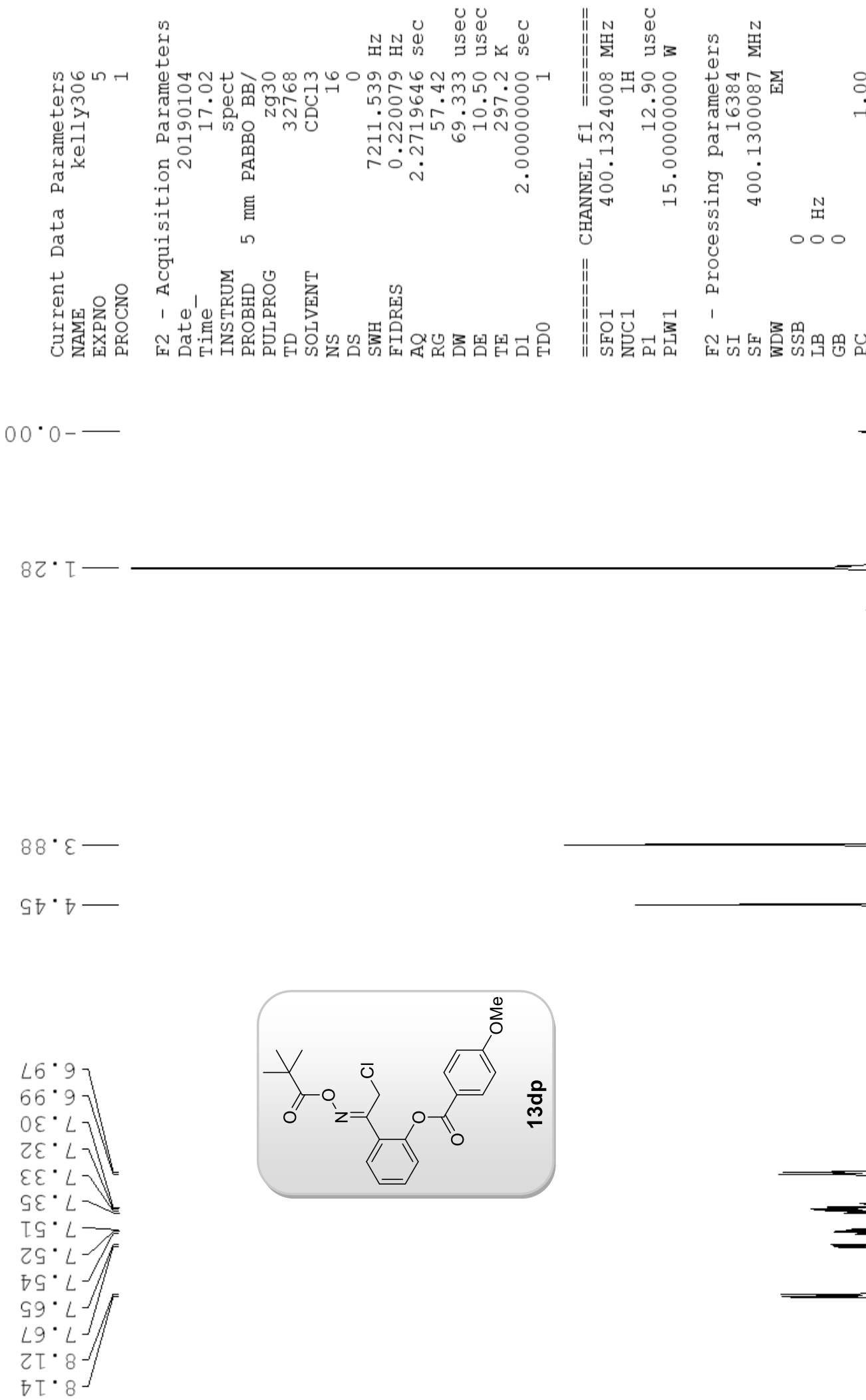

틈

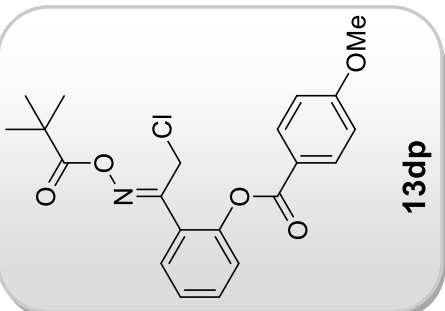



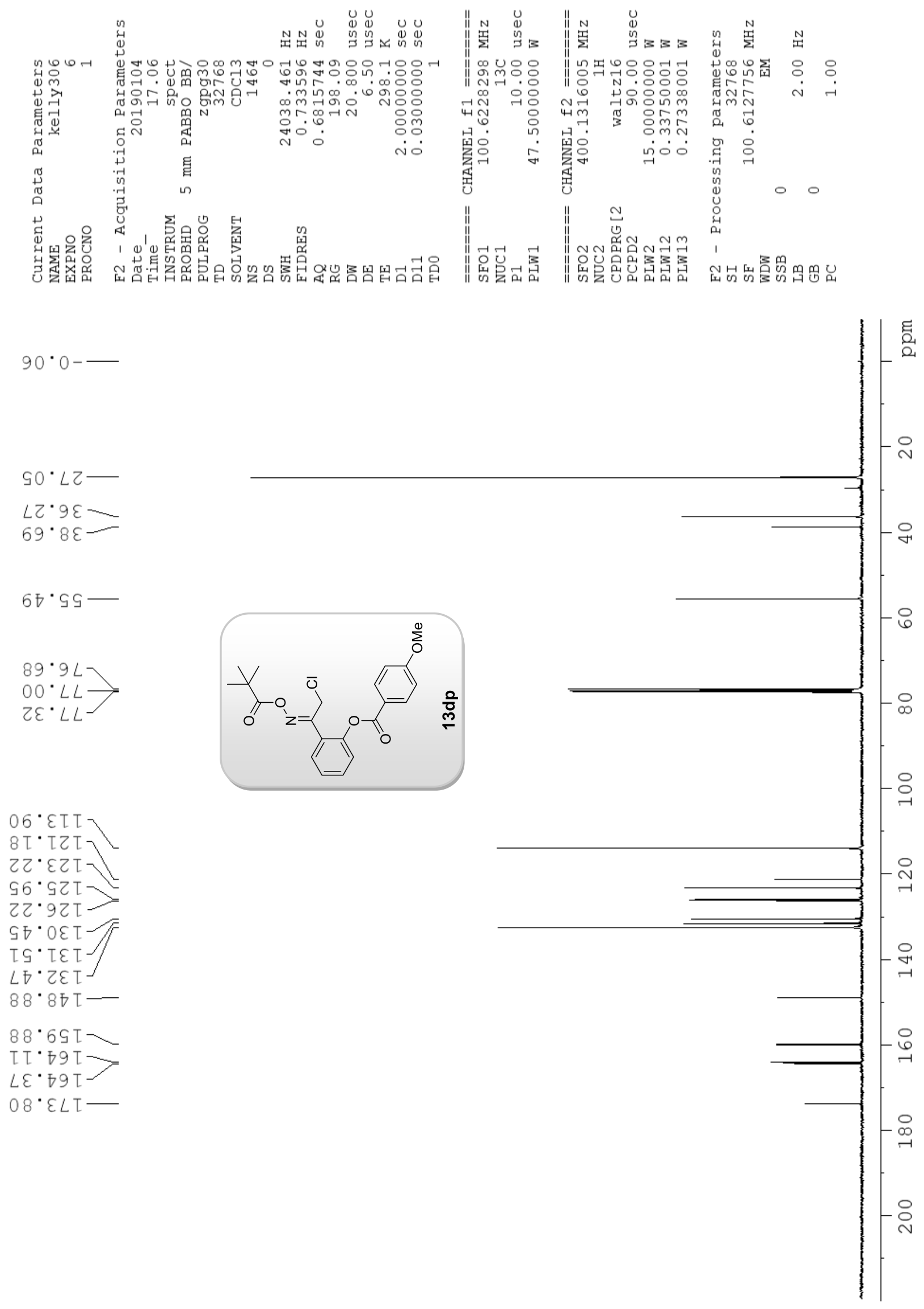


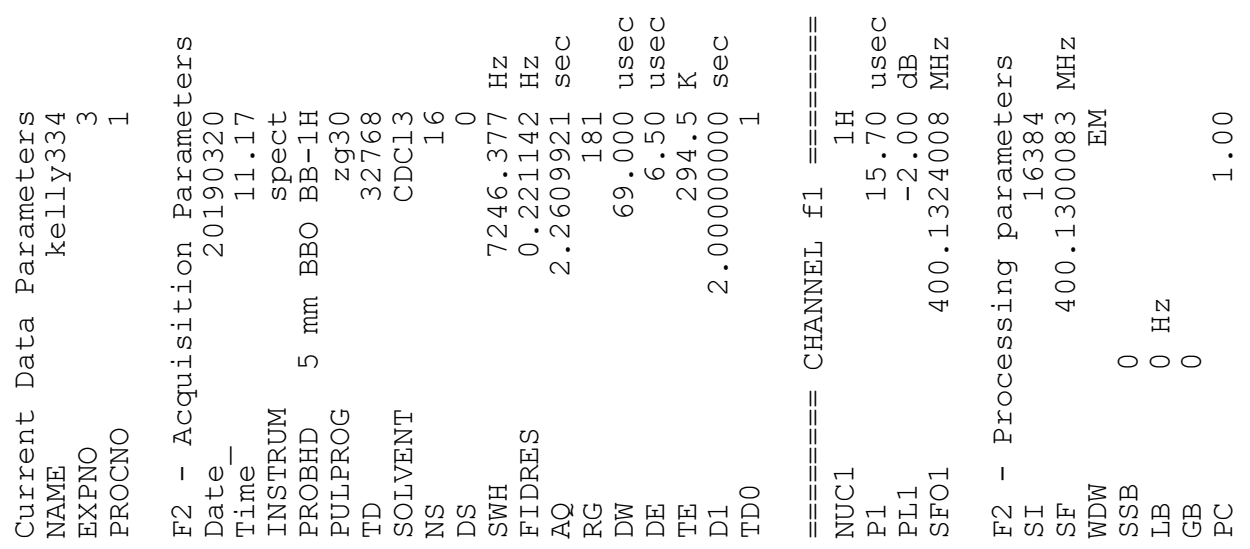

$000 \cdot 0$

$06 I \cdot 2$
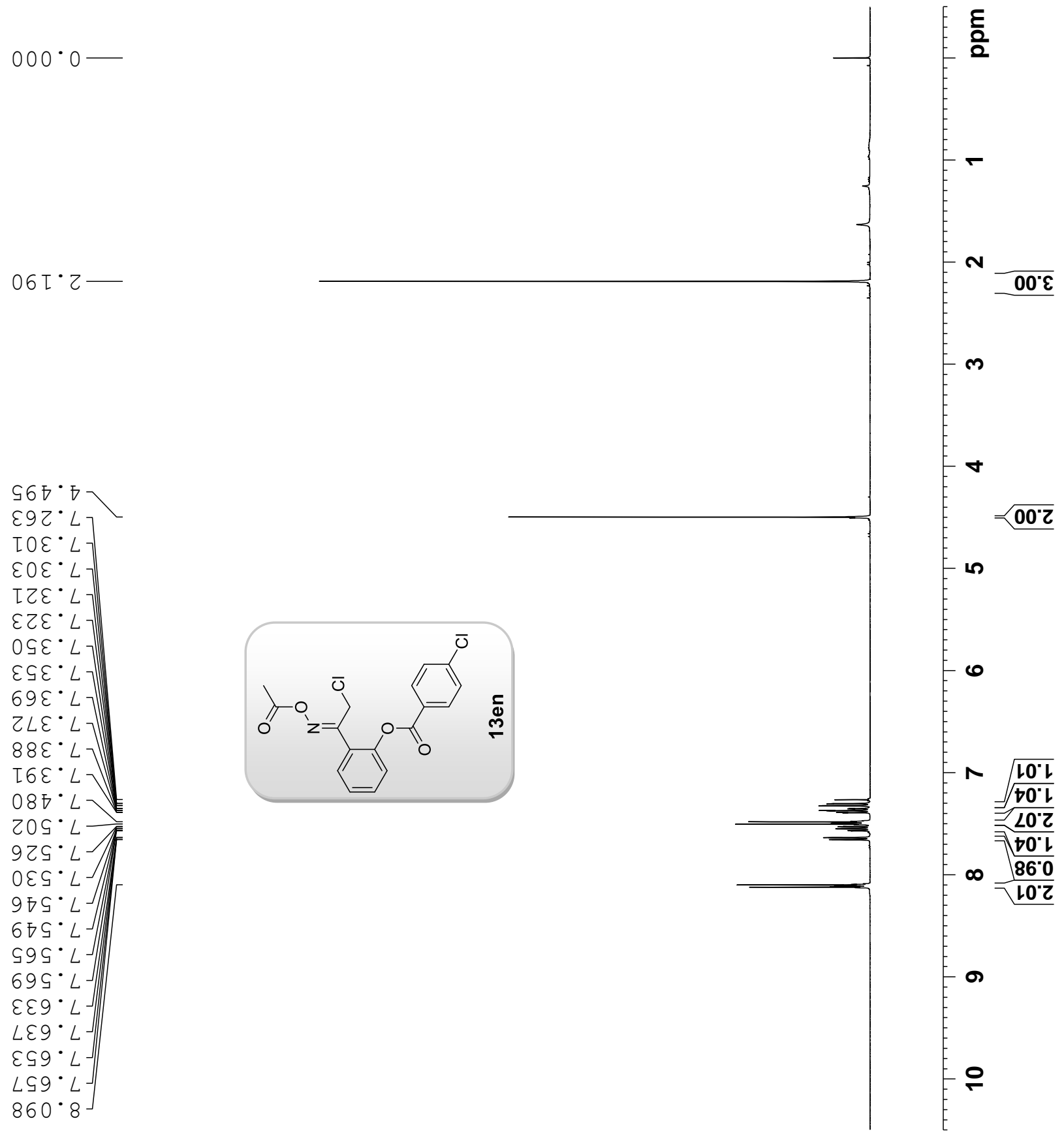

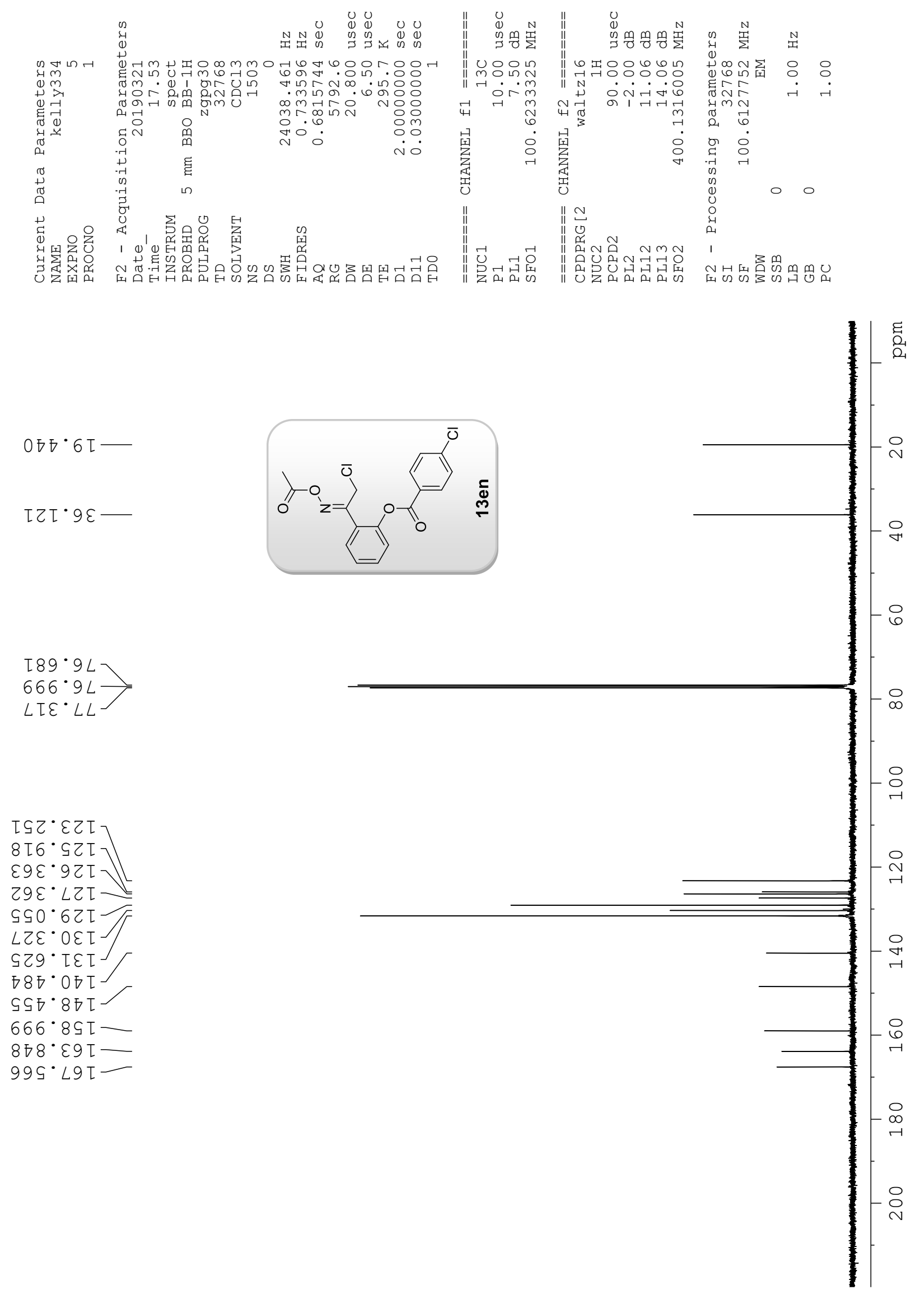

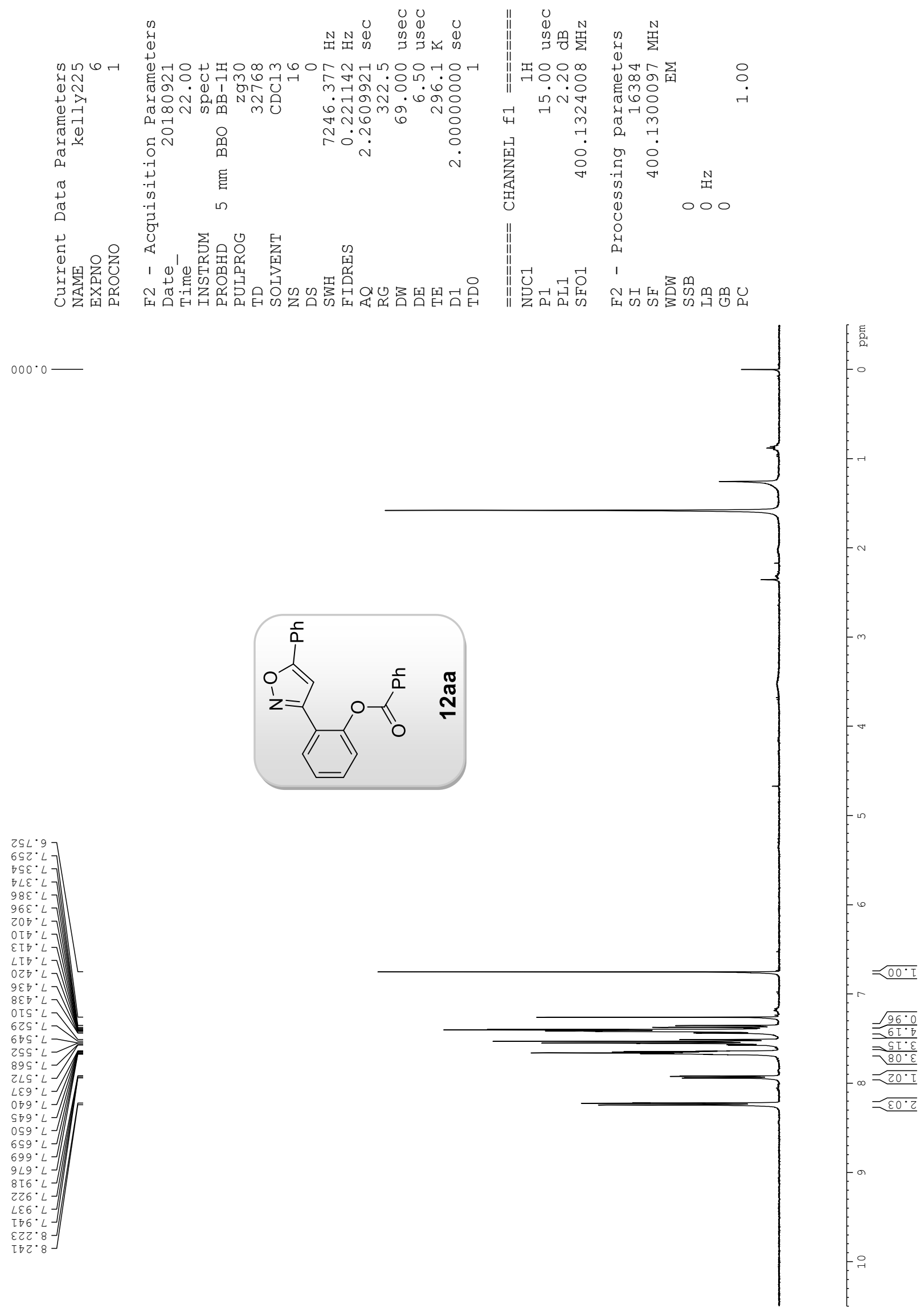

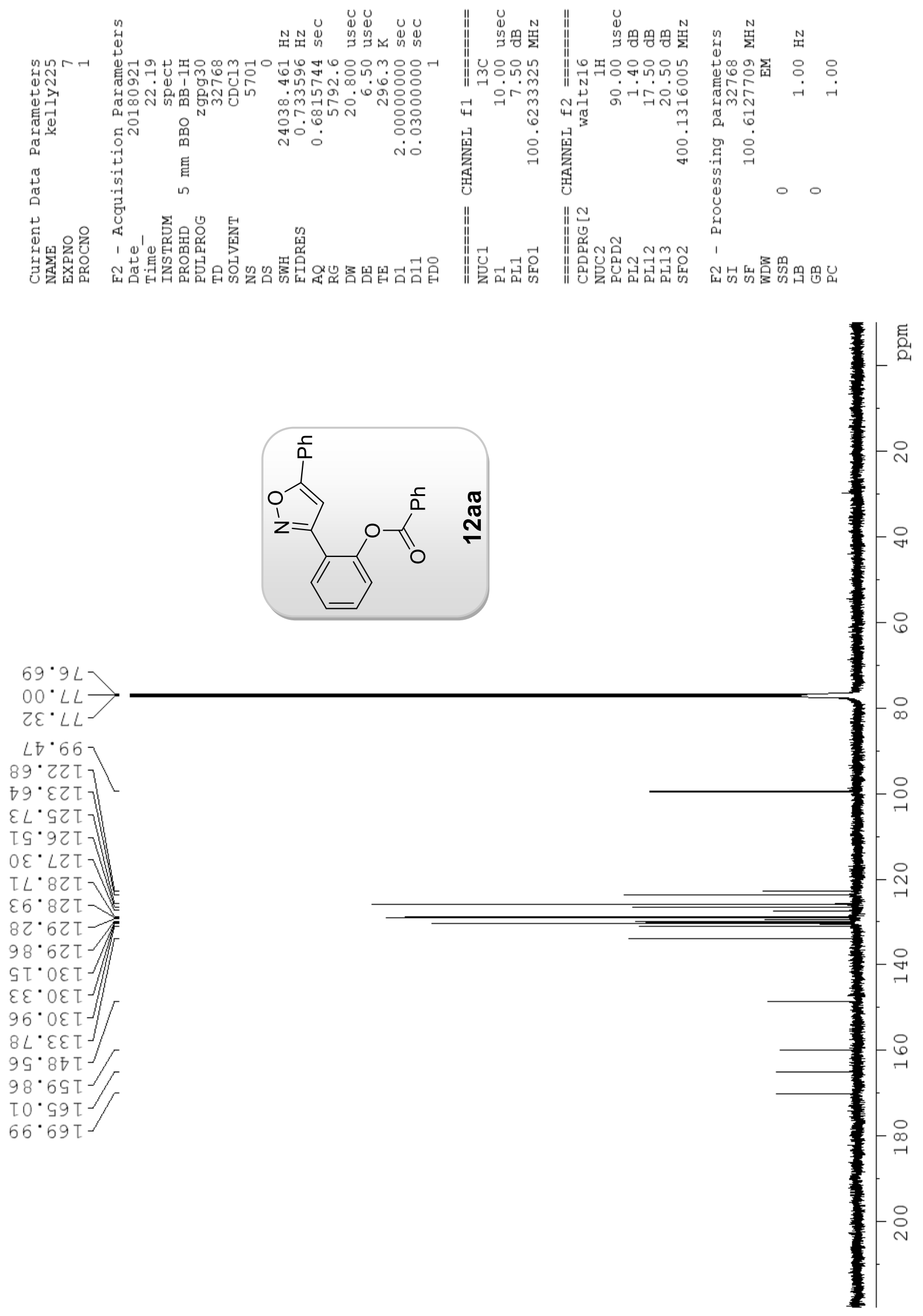


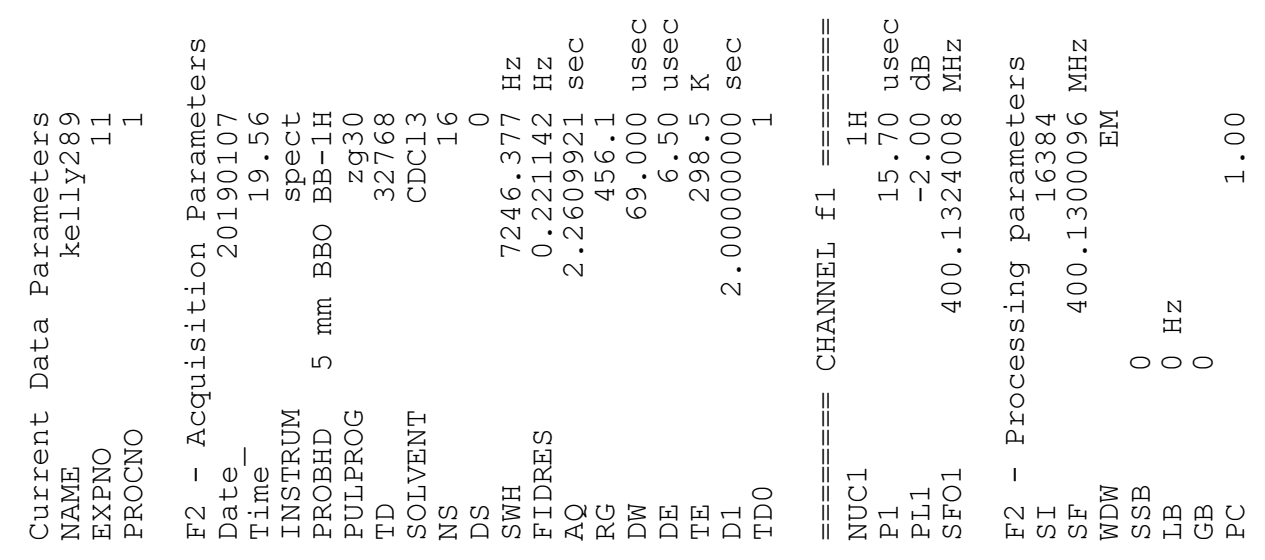

$000 \cdot 0$
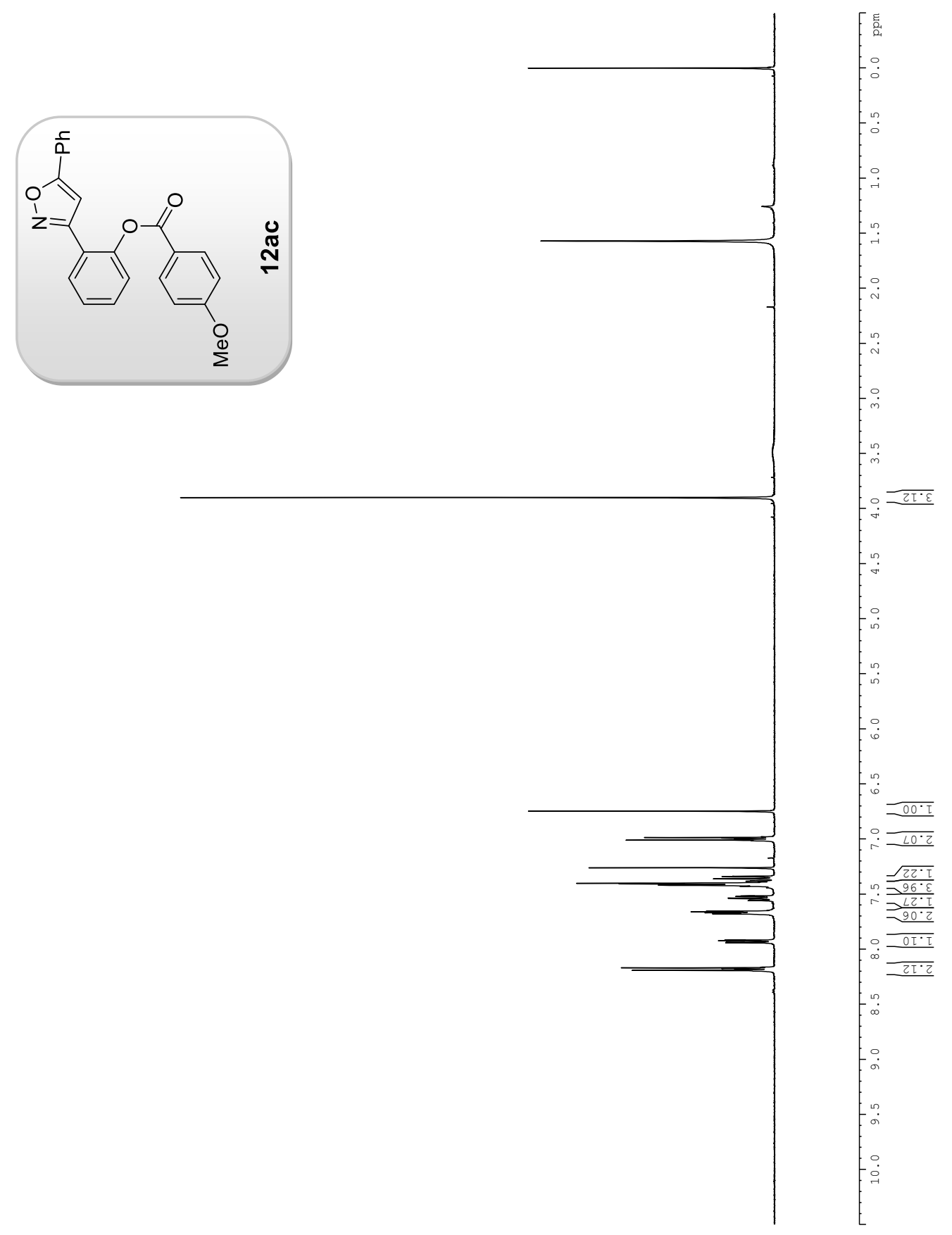


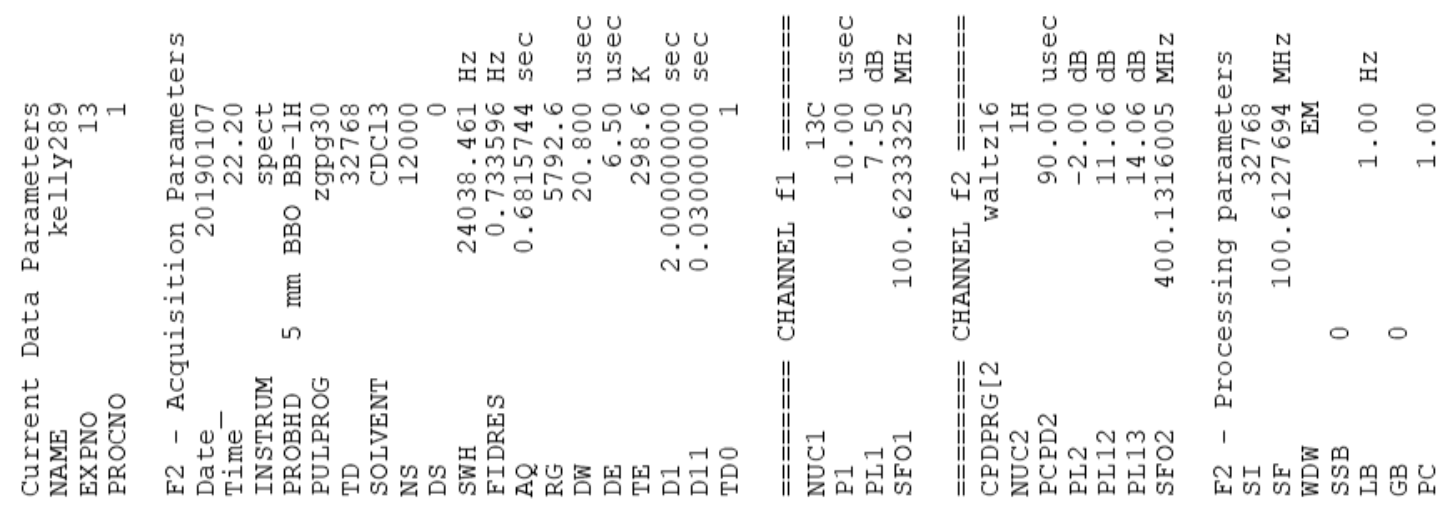

$9 \mathrm{TO}^{\circ} \mathrm{O}-$
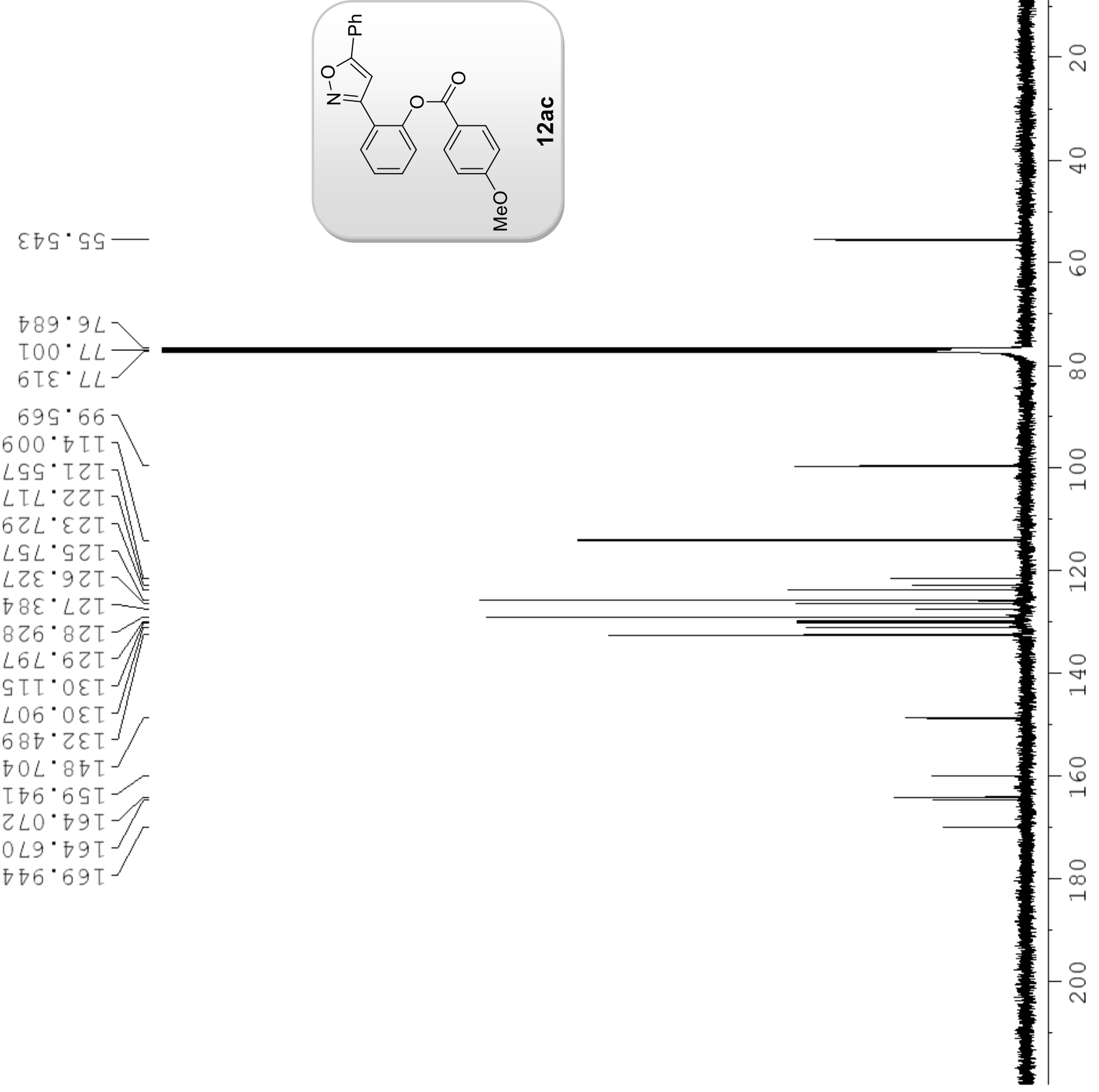

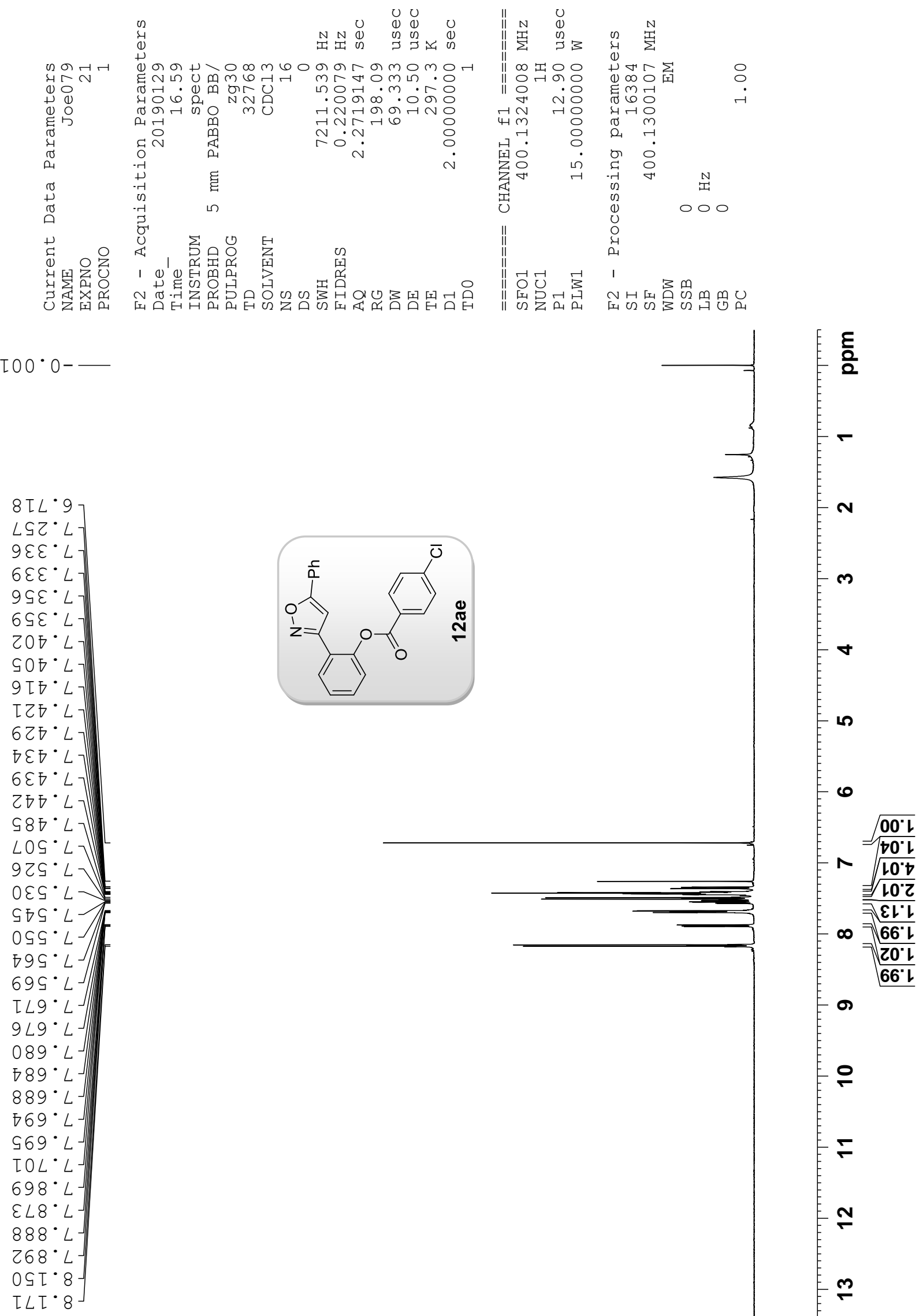

0

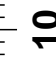

-

$\stackrel{9}{2}$ 

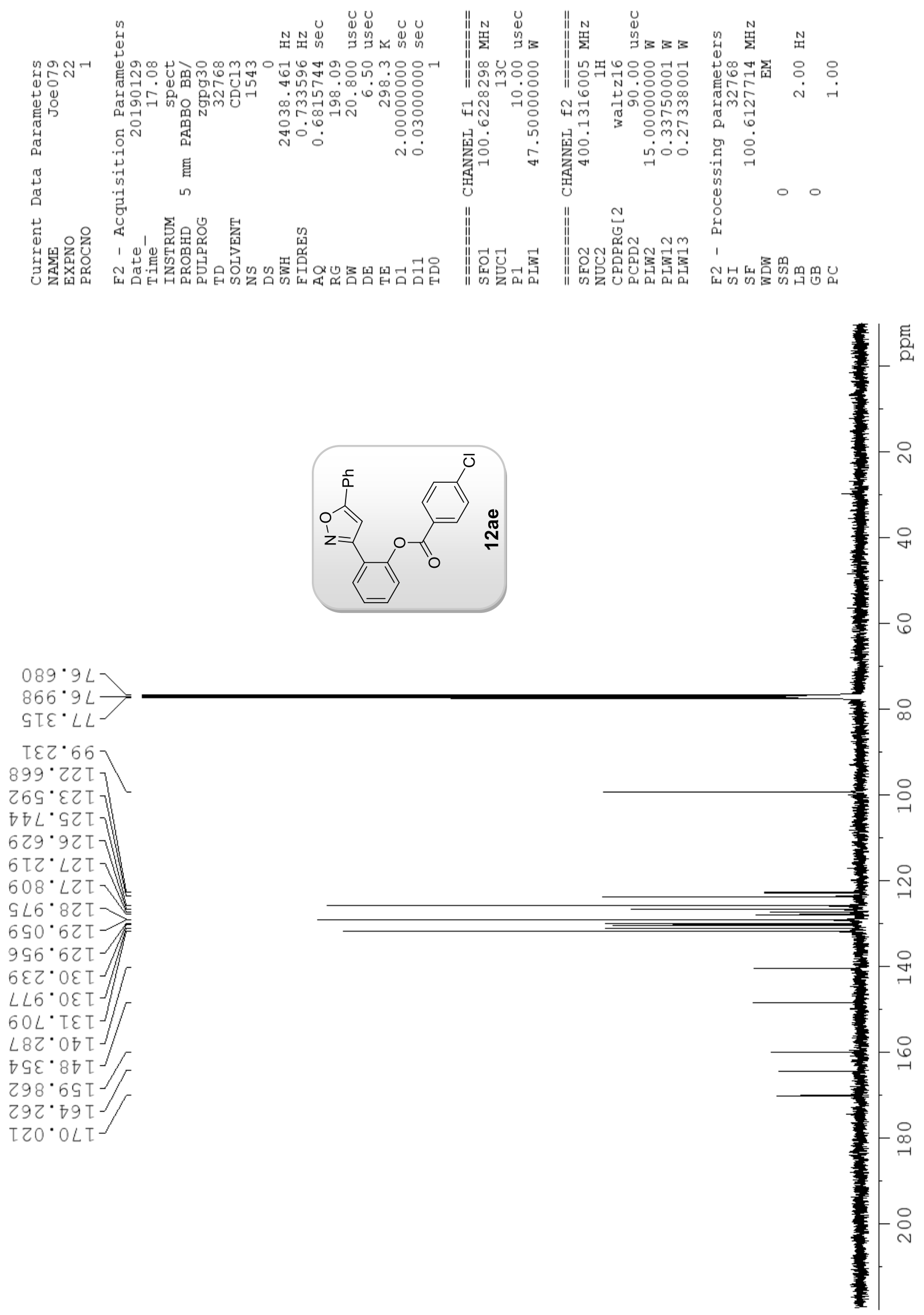

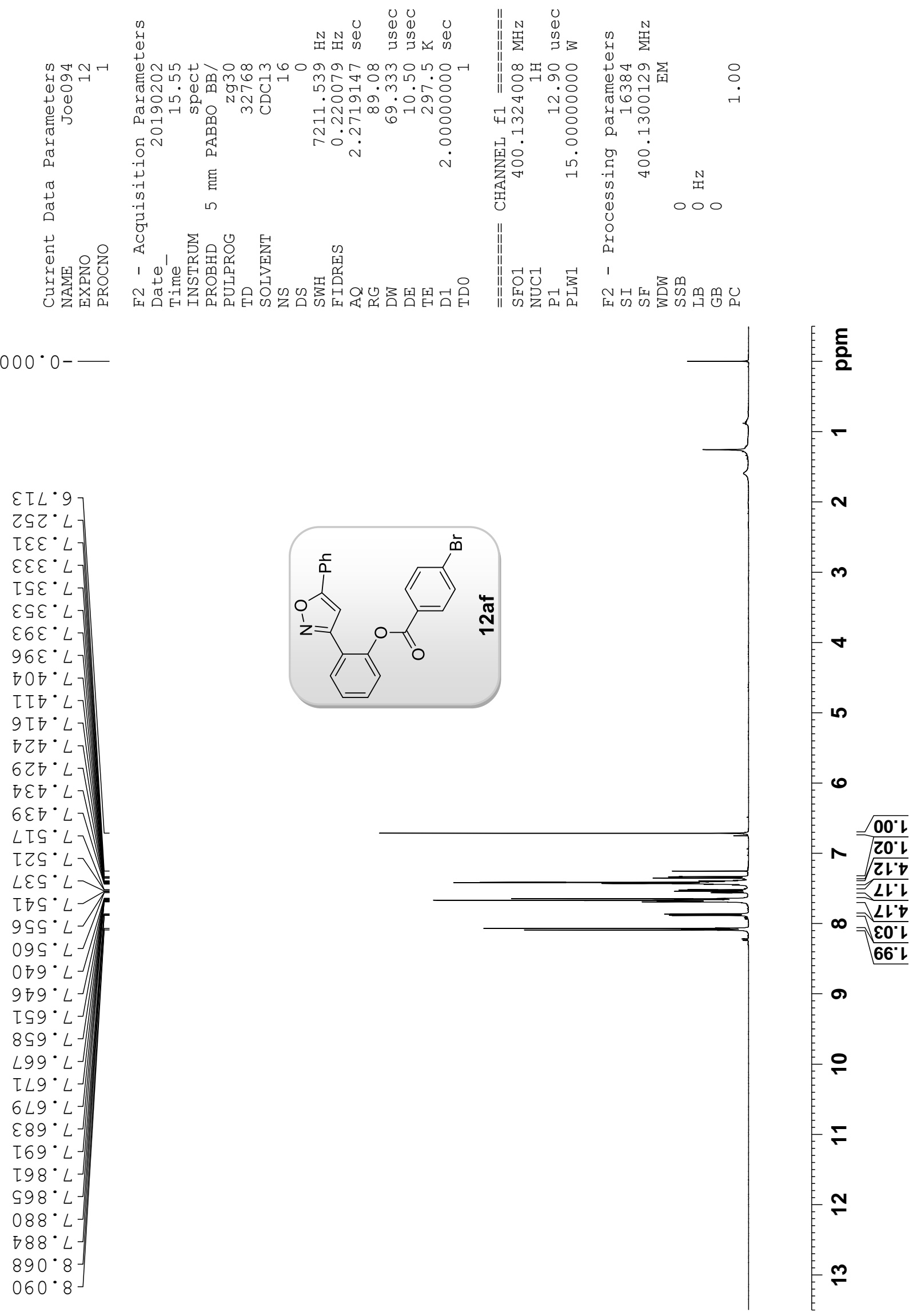

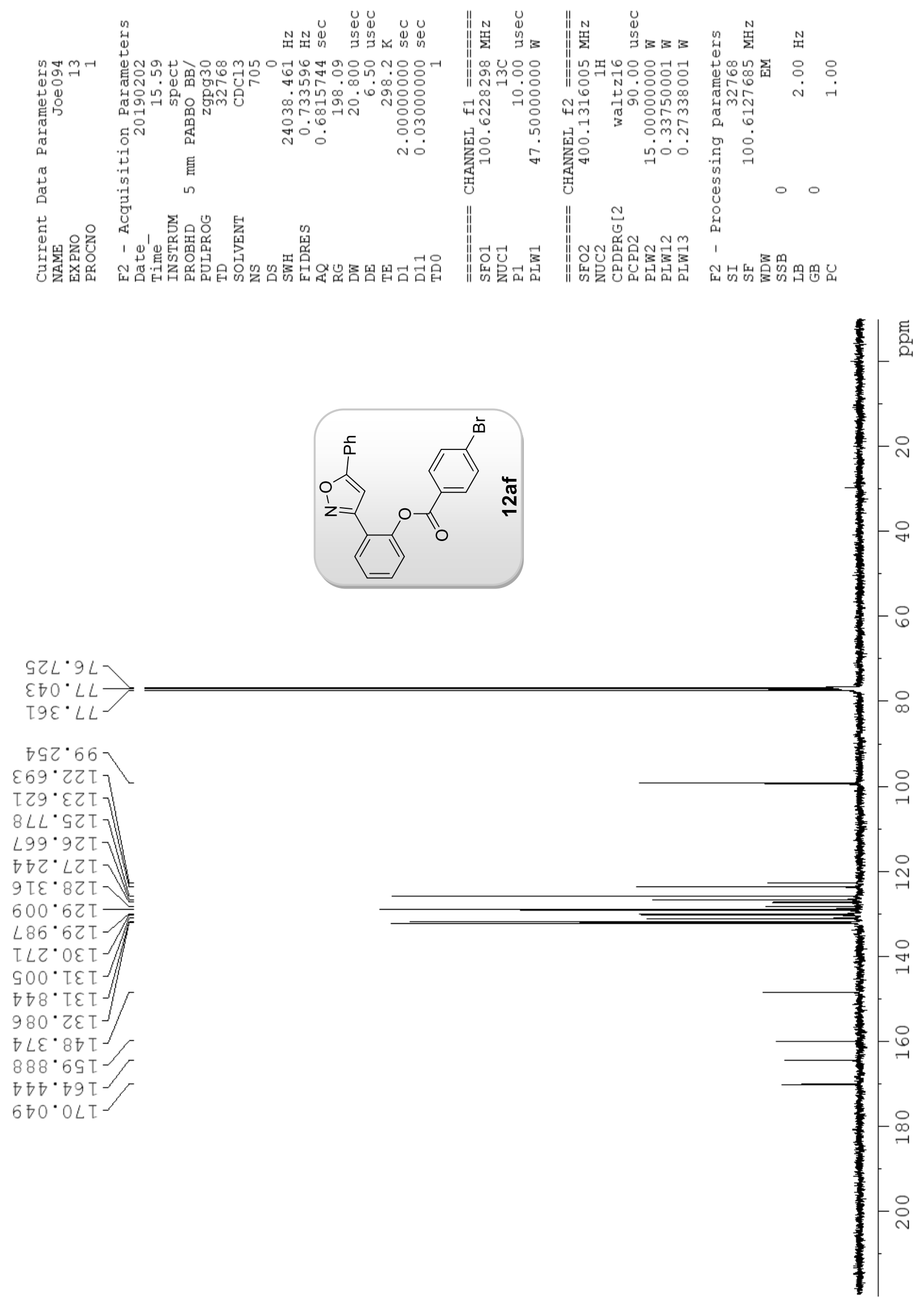


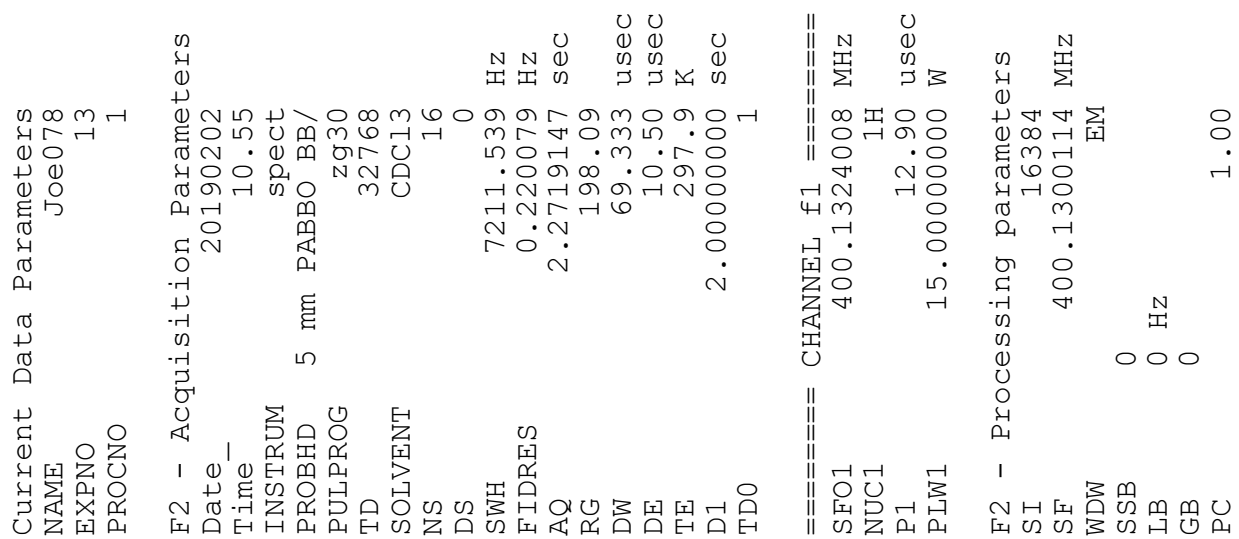
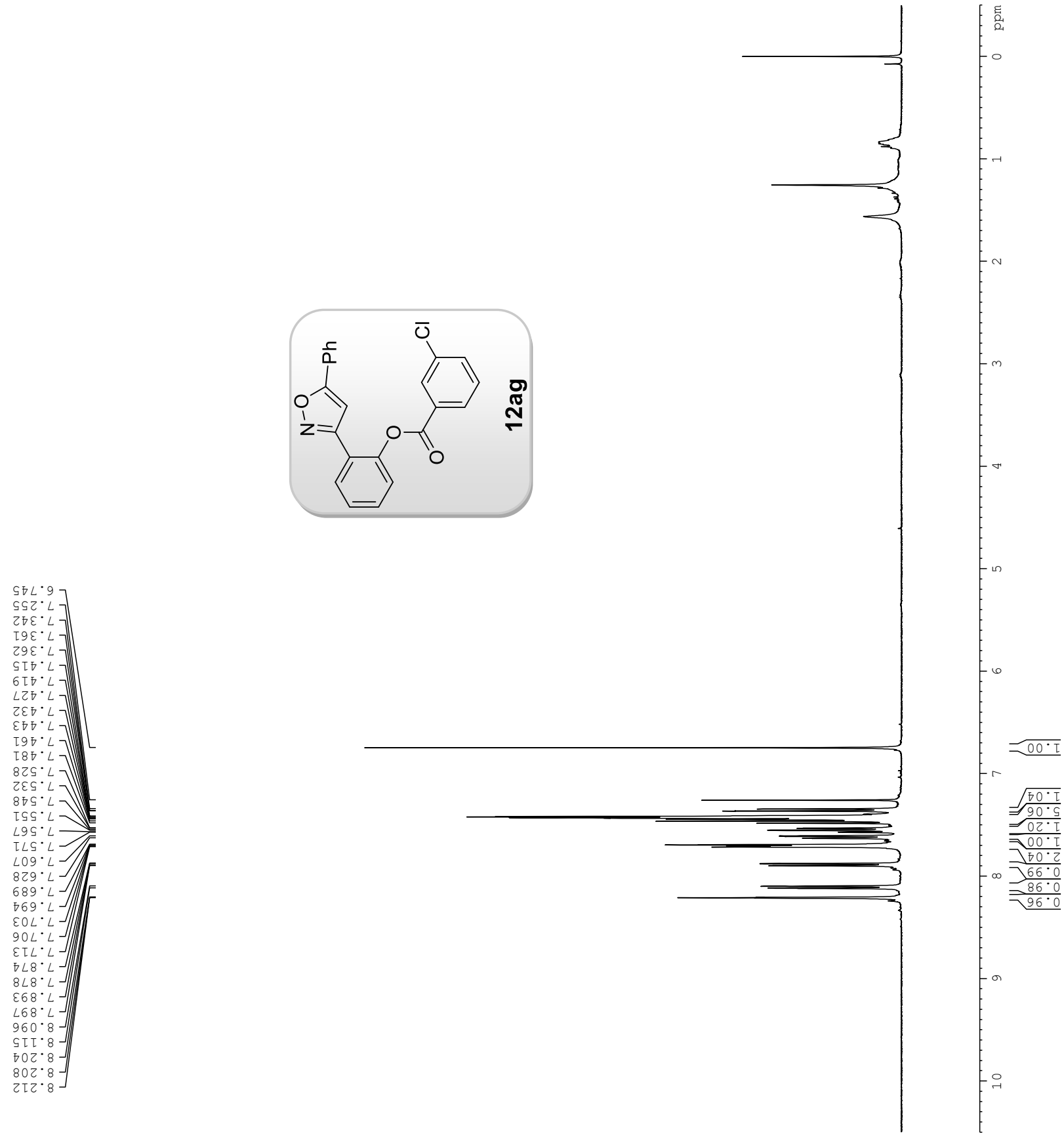

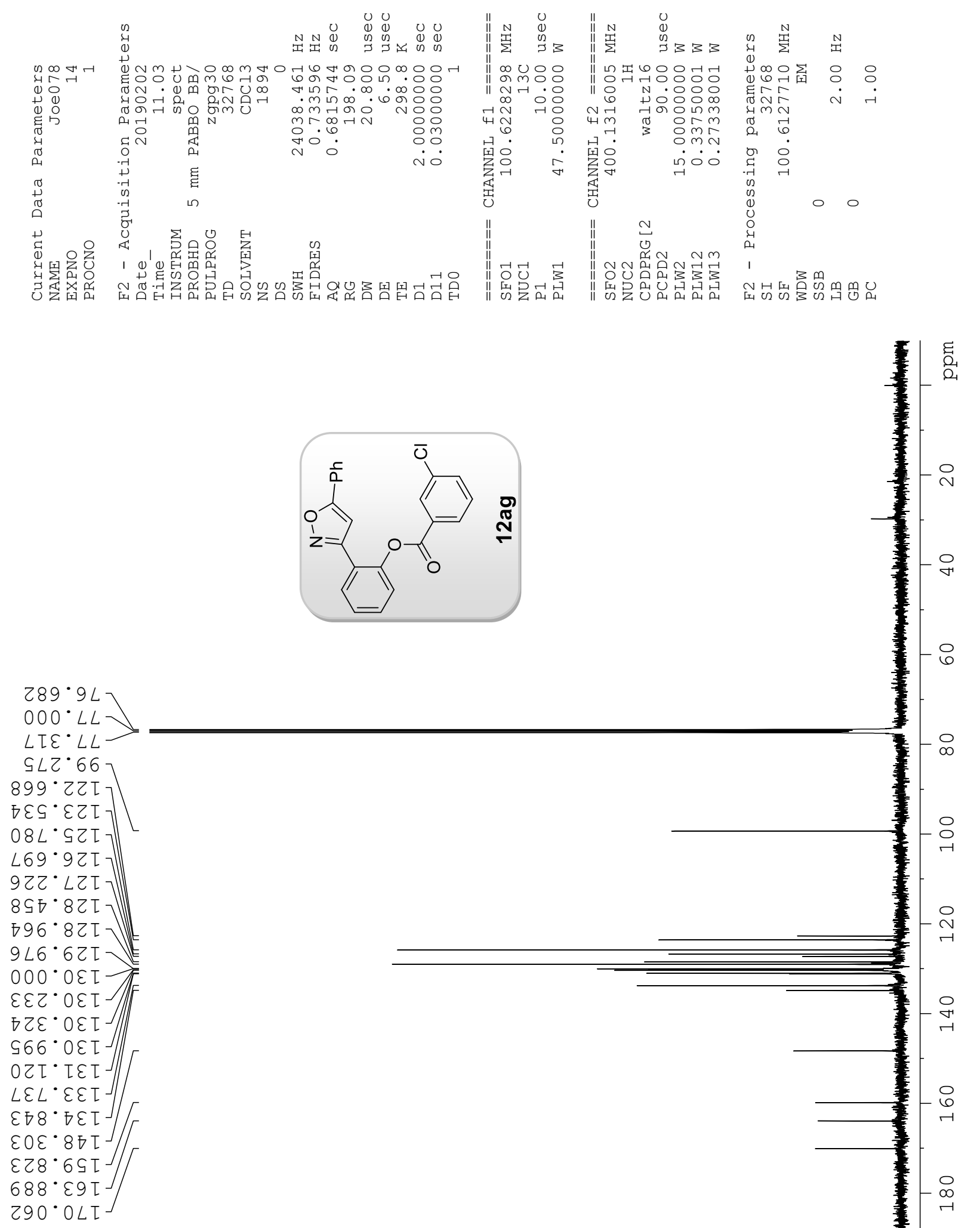


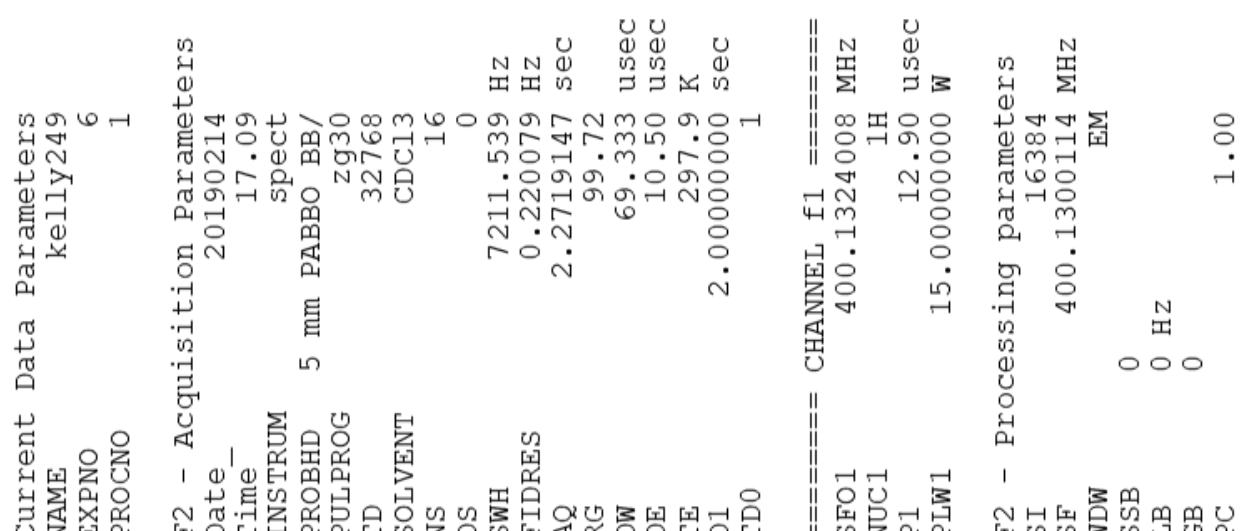

$000 \cdot 0$

$97 \varepsilon \cdot 乙 \longrightarrow$
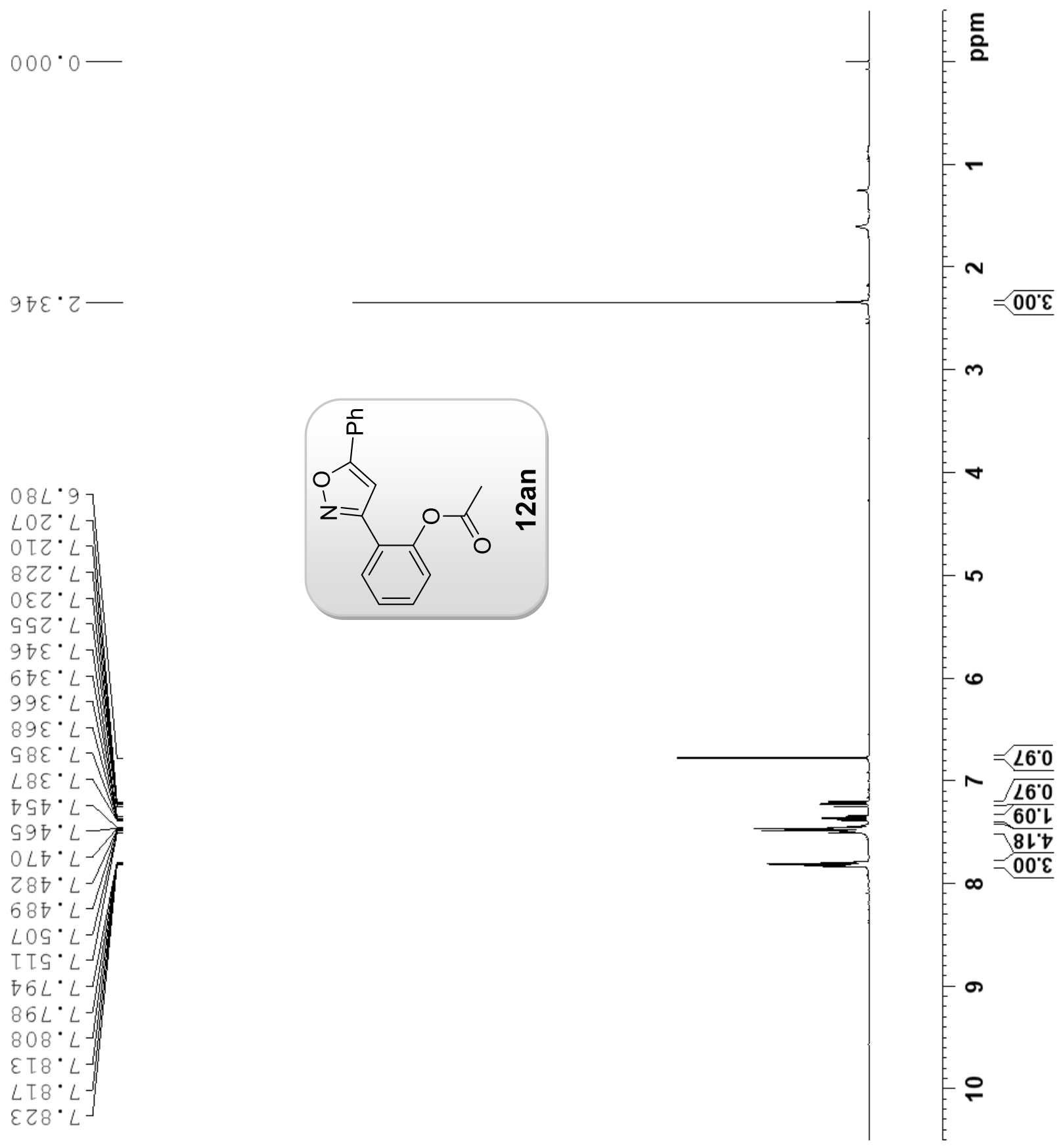

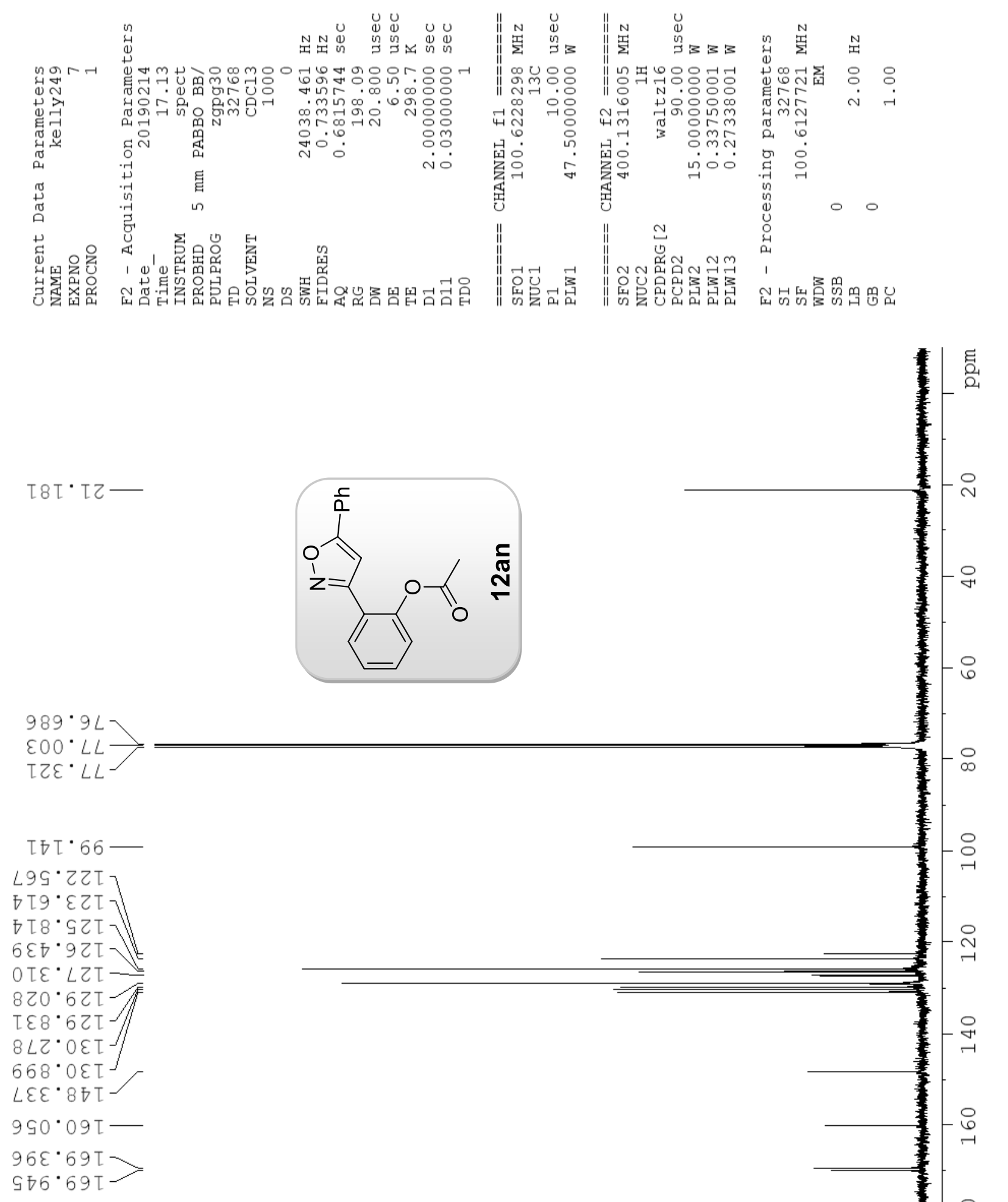


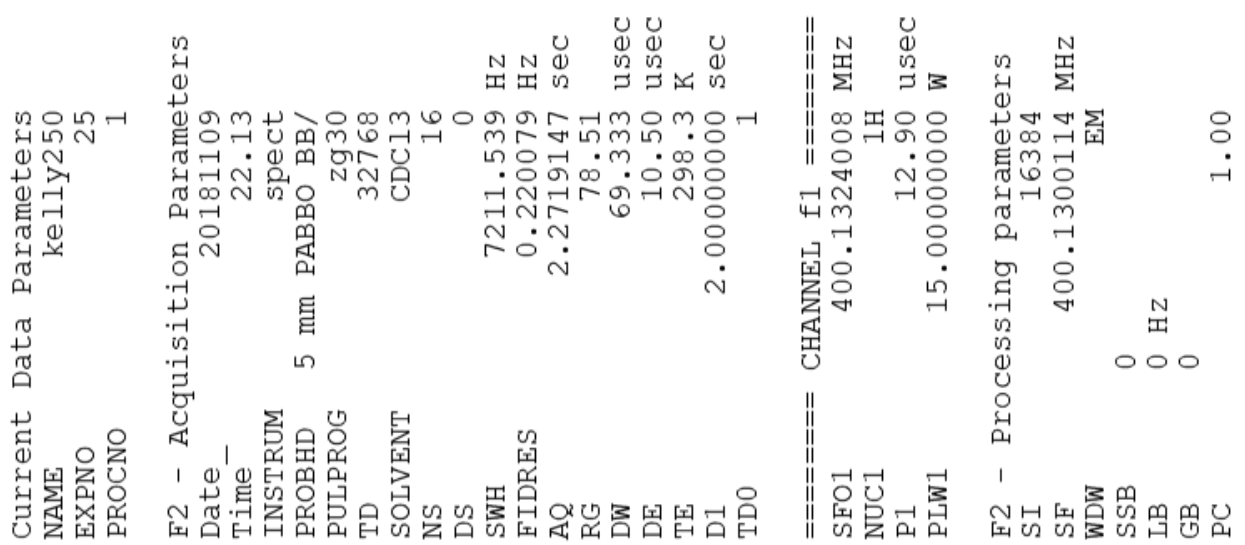

$000 \cdot 0$

$6 \nabla \varepsilon \cdot \tau$
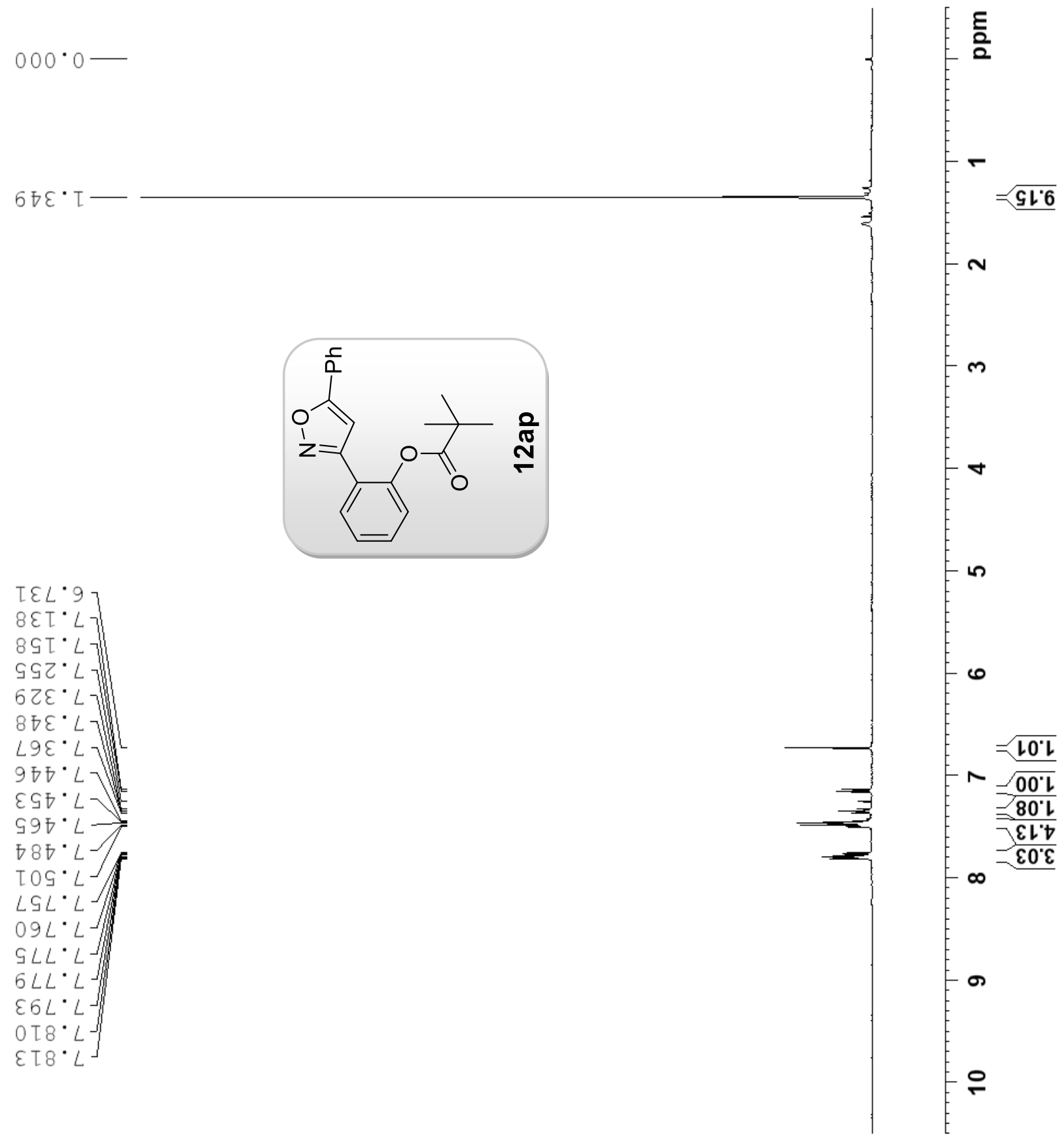

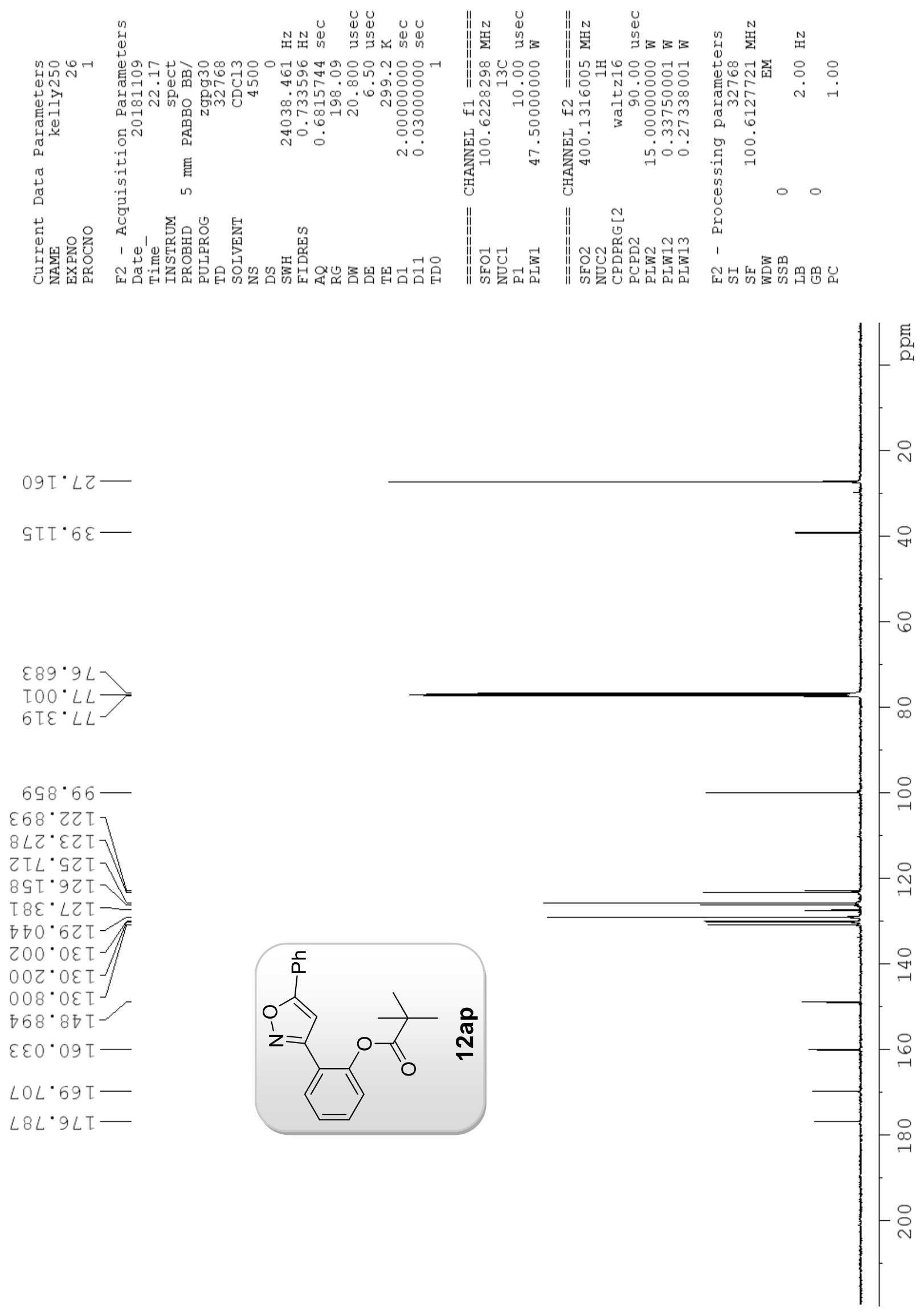

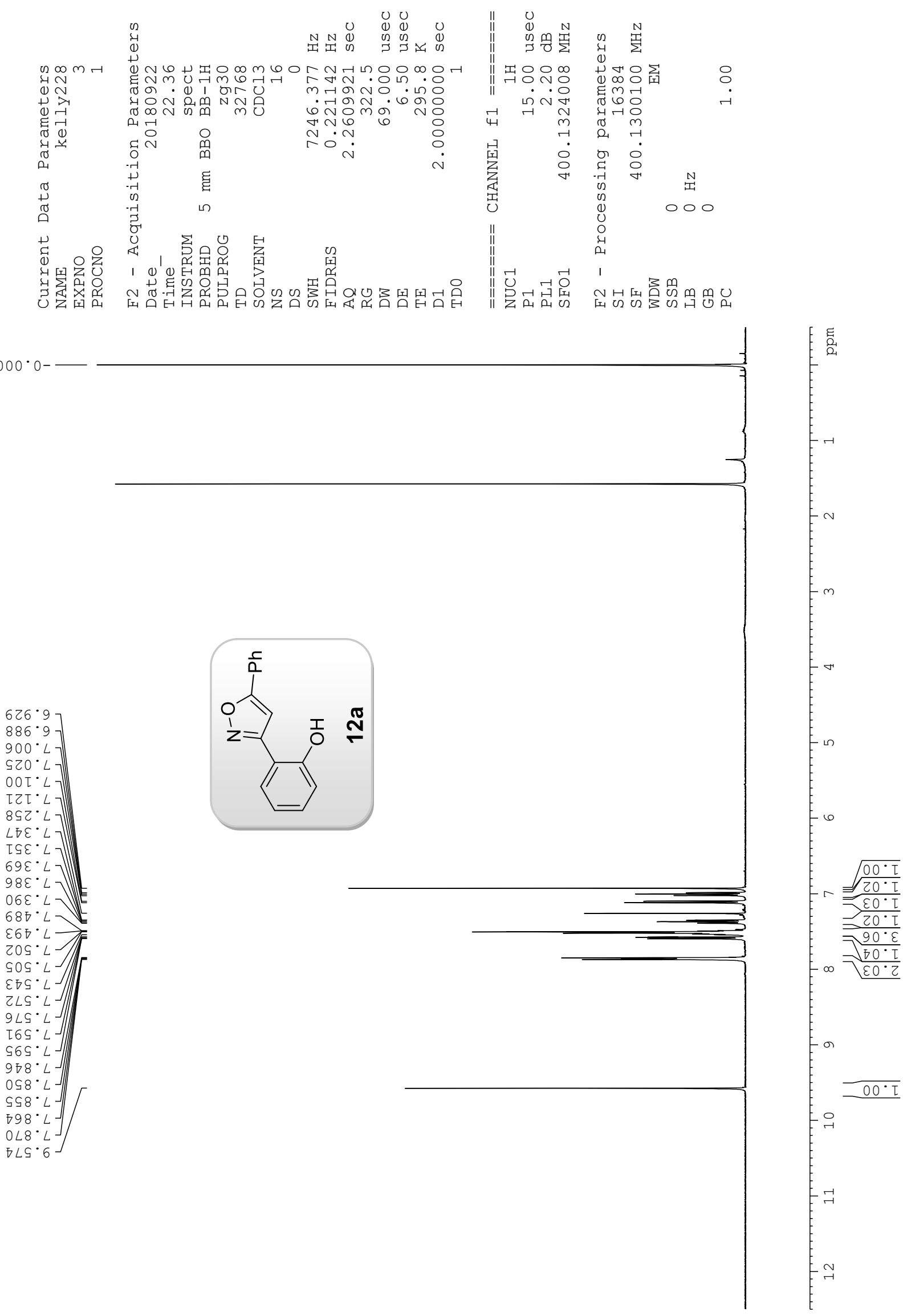

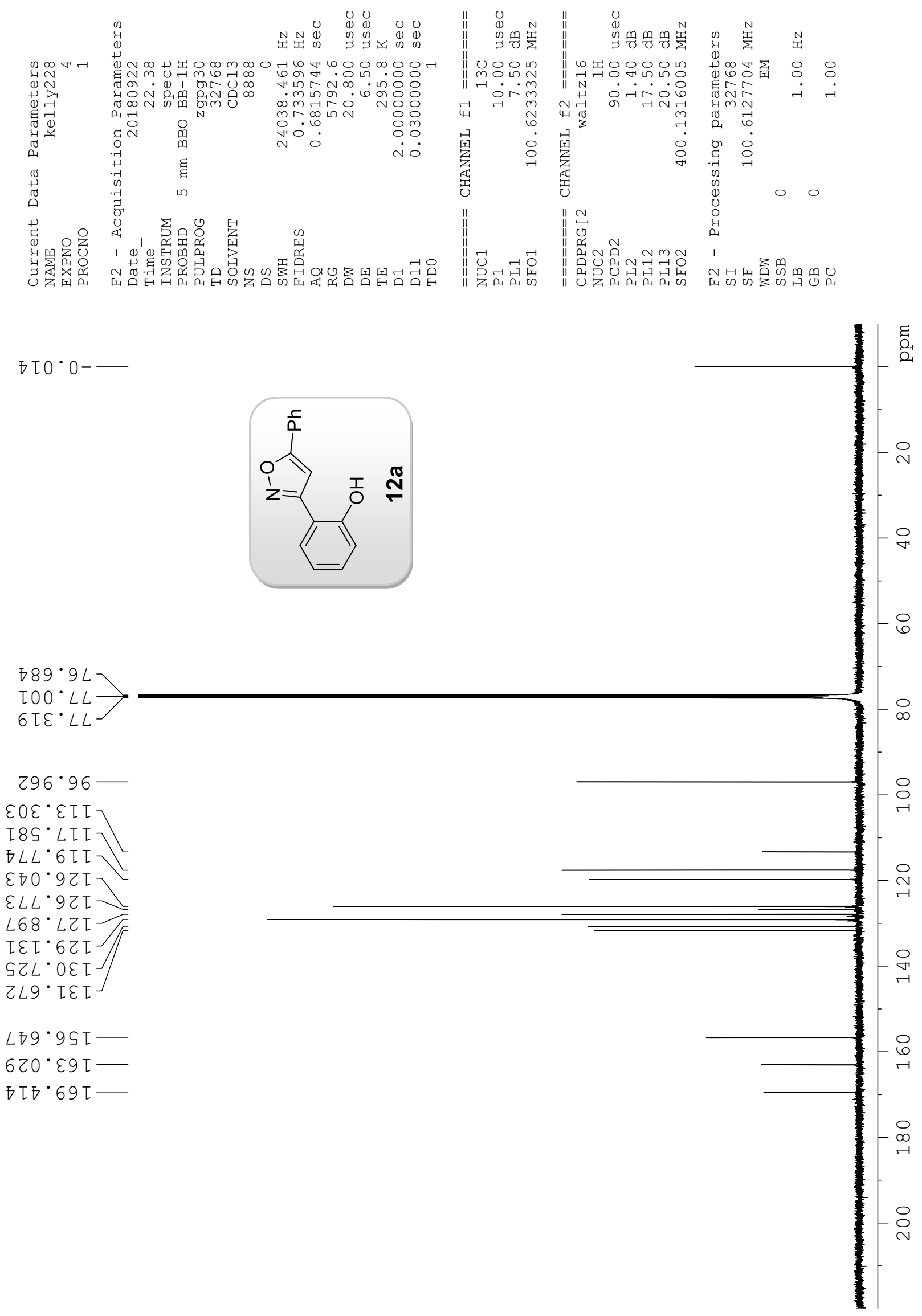


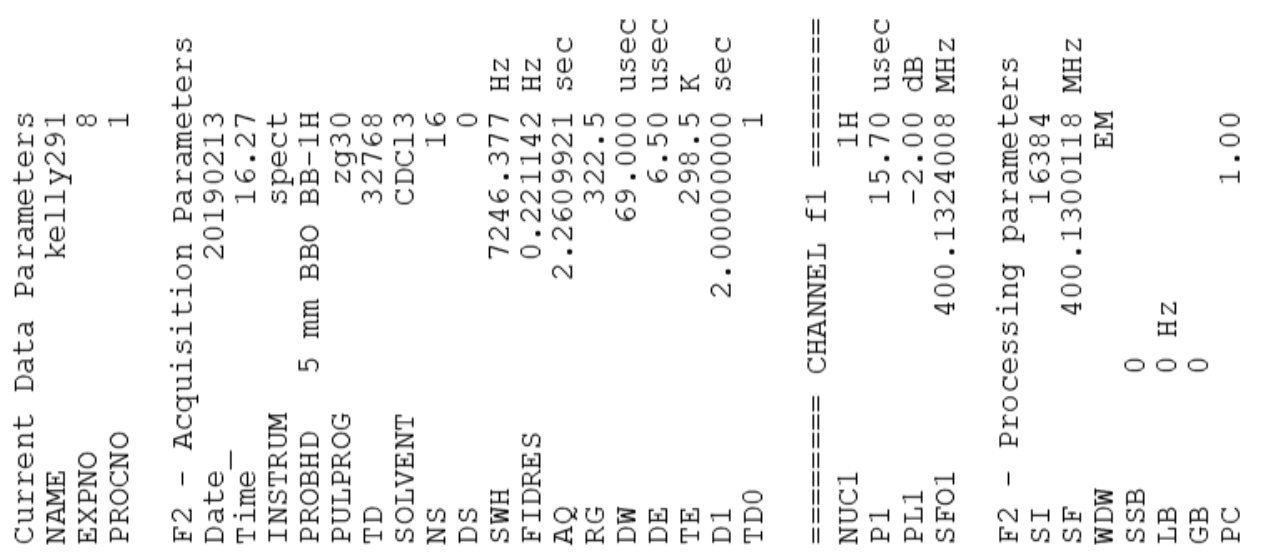

$\varepsilon 00^{\circ} 0-$
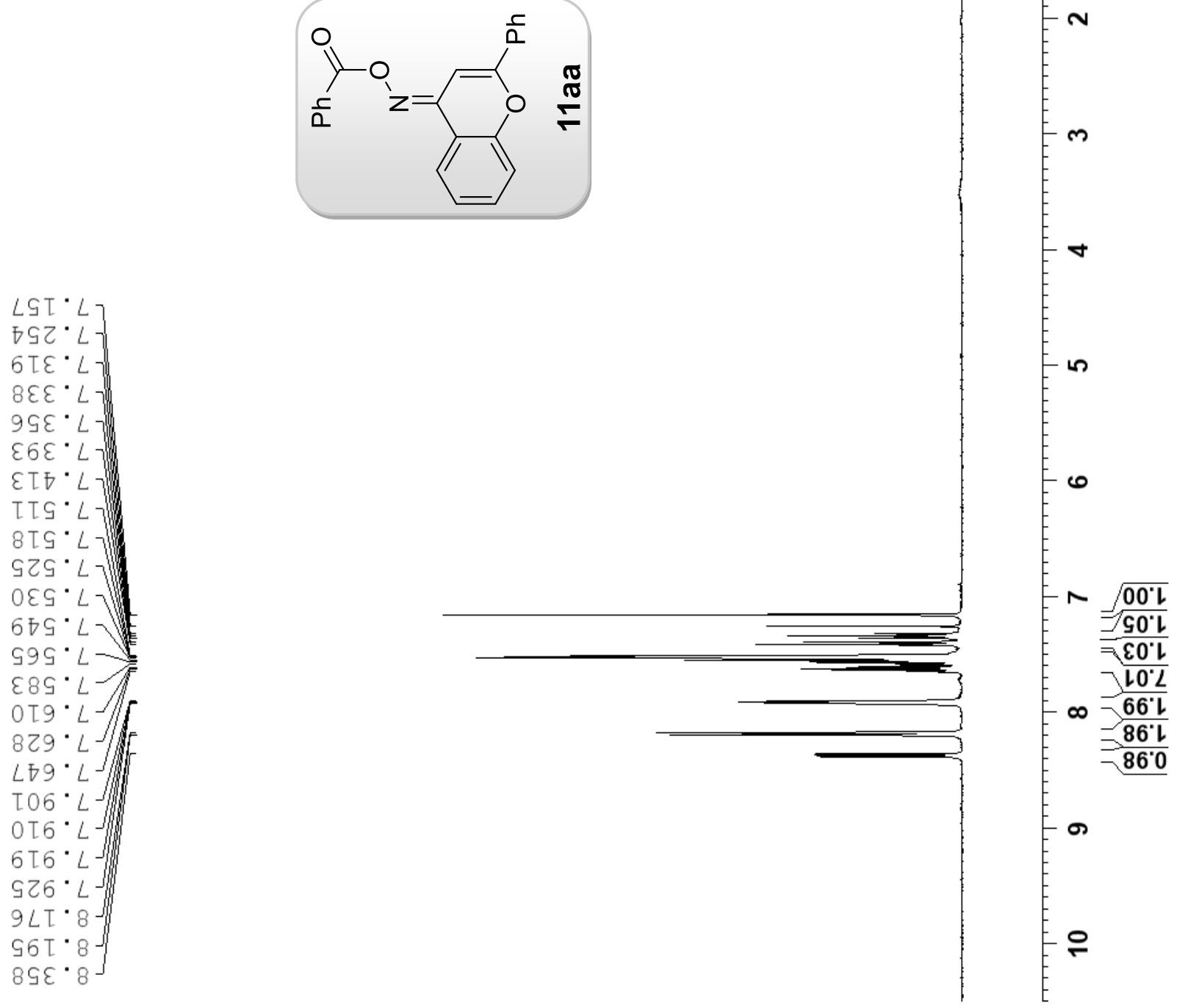

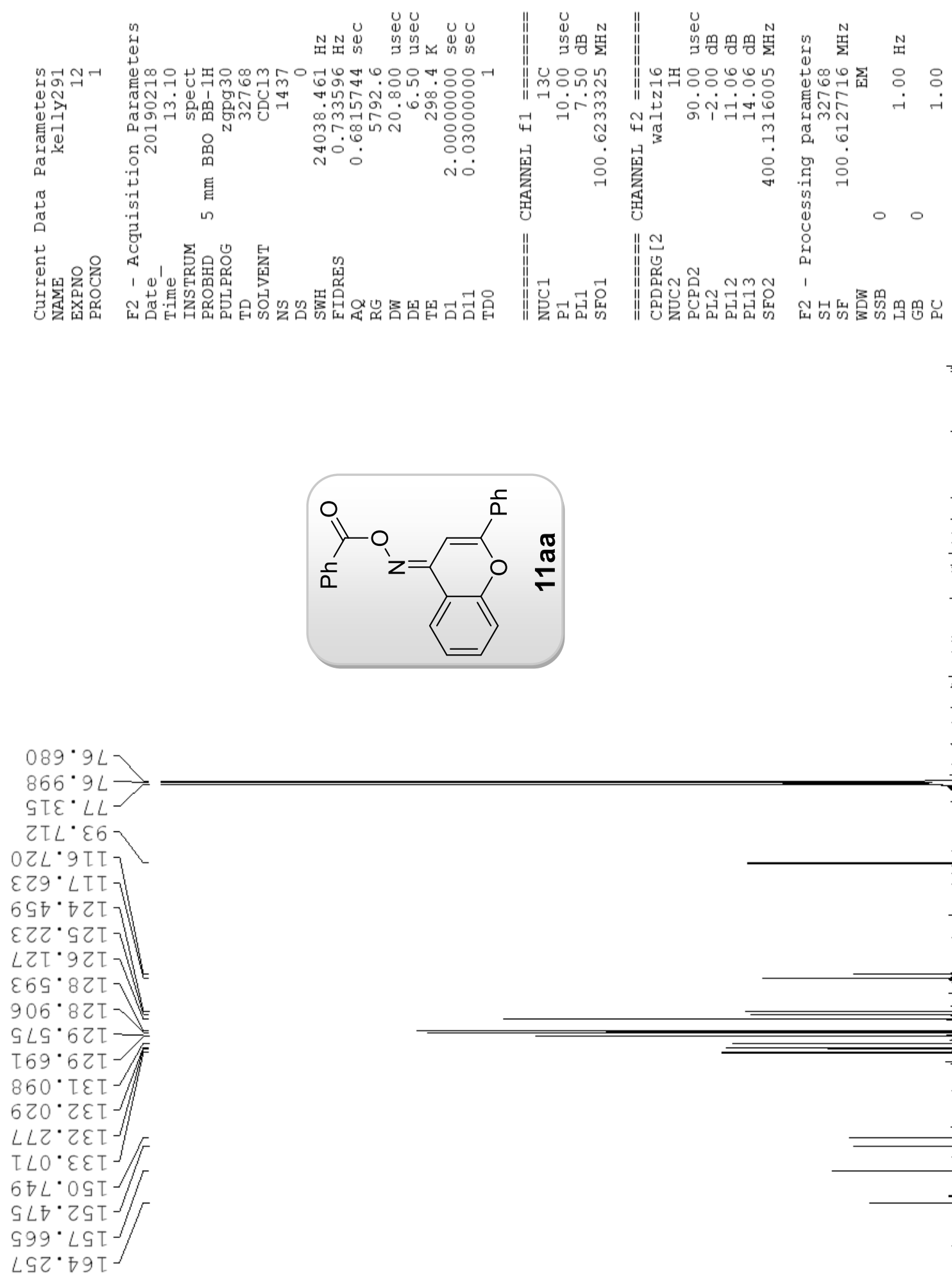


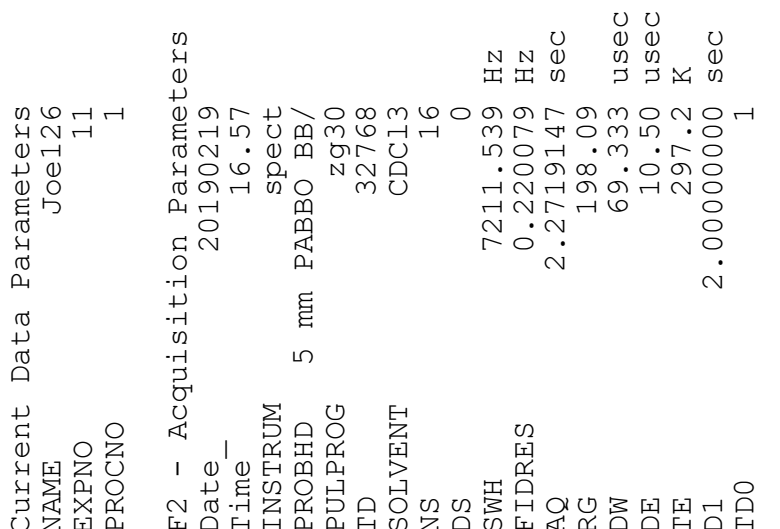

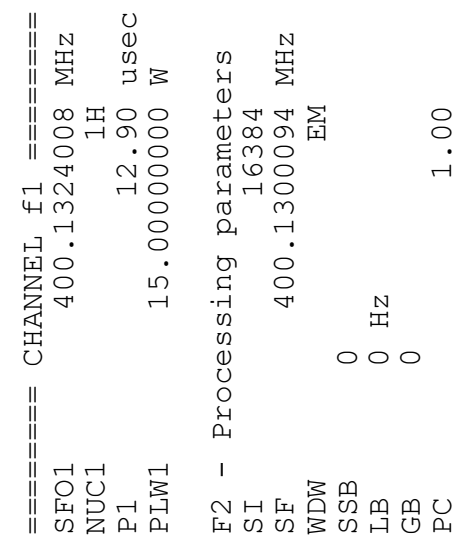

I00 $0-$
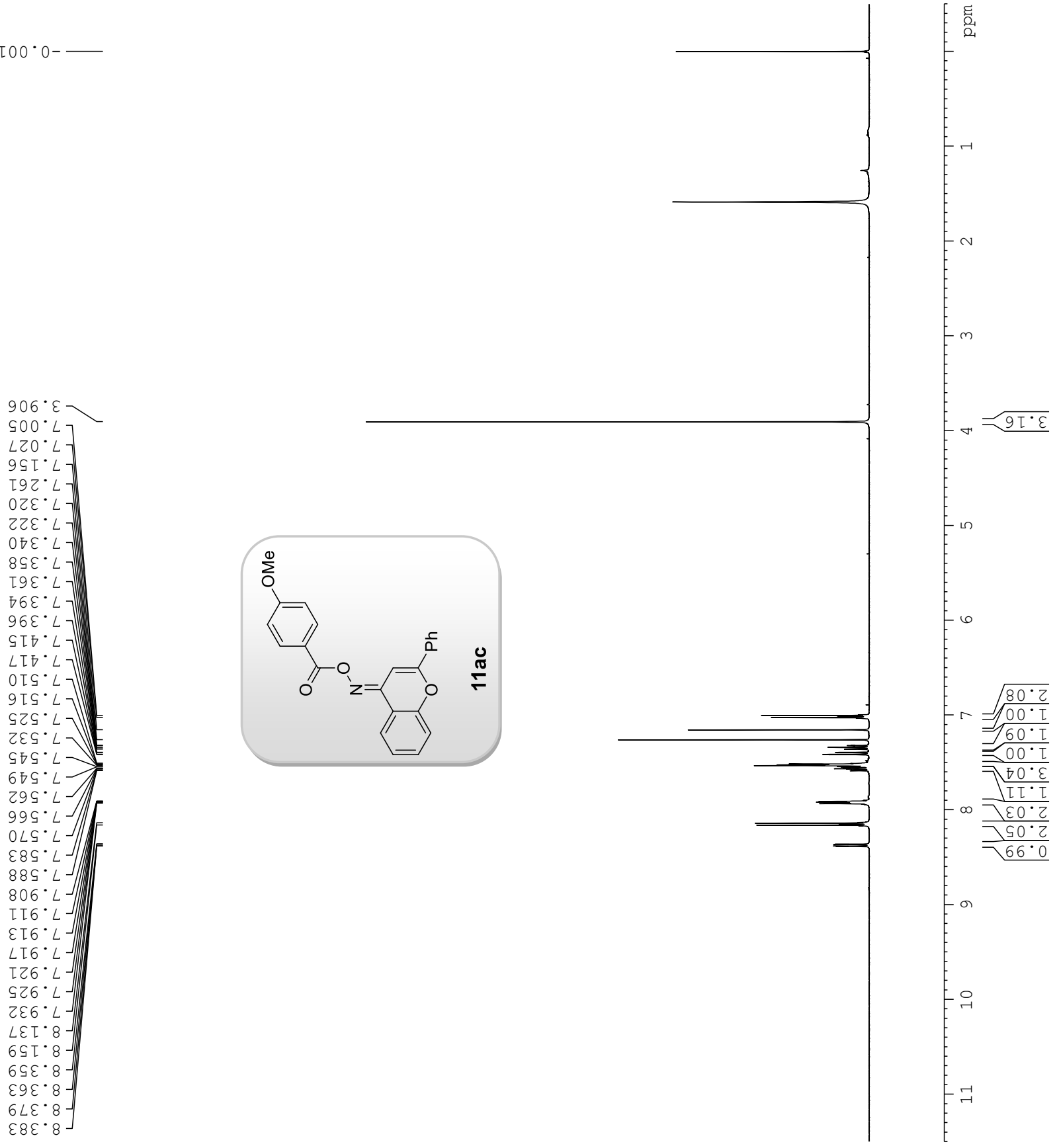


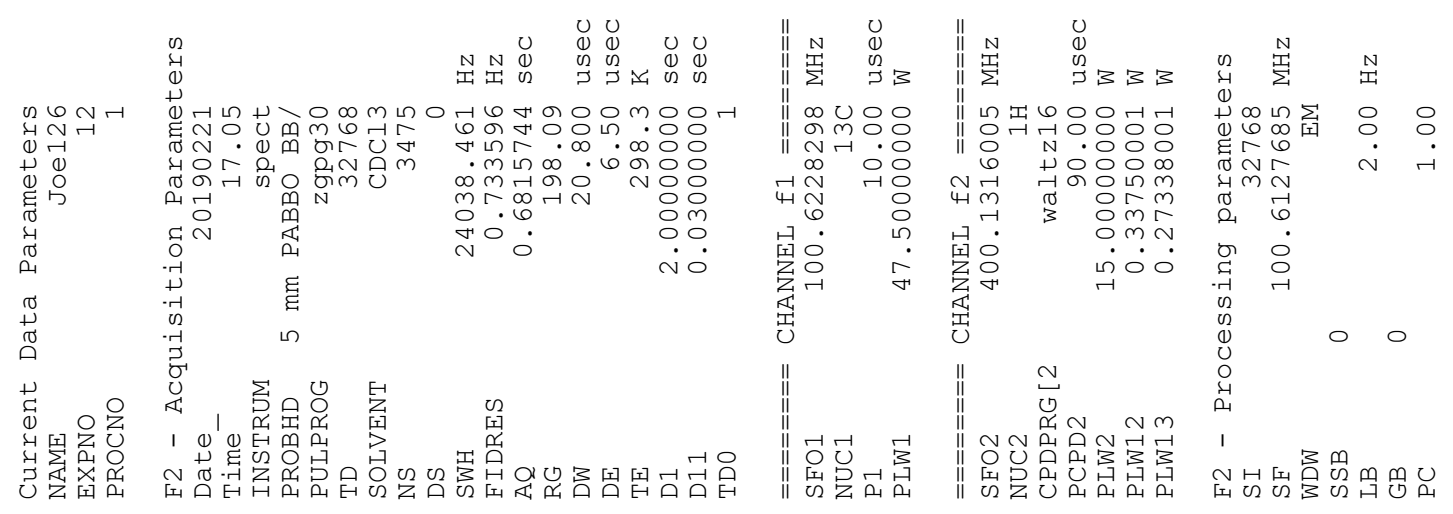

LIO $0^{\circ}-$

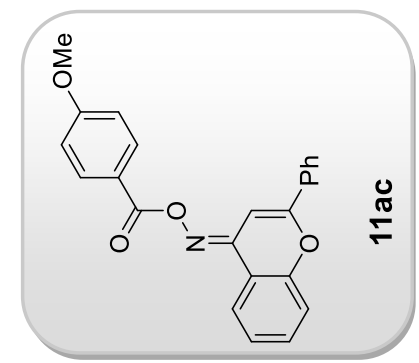

$967 \cdot 99$

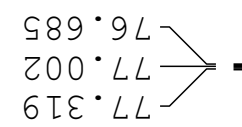

$0[8 \cdot \varepsilon 6$

I06. $\varepsilon\left[\tau_{]}\right.$

$\nabla S 8^{\circ} 9[[]$

$\varepsilon 09^{\circ} L\left[I_{]}\right]$

$6 乙 6^{\circ}$ [乙 [ ]

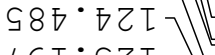

$L 6 I \cdot S Z I]$
$9 z I \cdot 9 z I$

906.8ZI

$0 \varepsilon 9^{\circ}\left[\varepsilon[]^{\circ}\right.$

$\varepsilon \subseteq 6^{\circ}[\varepsilon[]$

$\angle 9 \varepsilon^{\circ} \mathrm{ZE} \mathrm{I}$

EOS OST

ZLD'乙SI

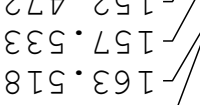

LEO $\nabla 9[$

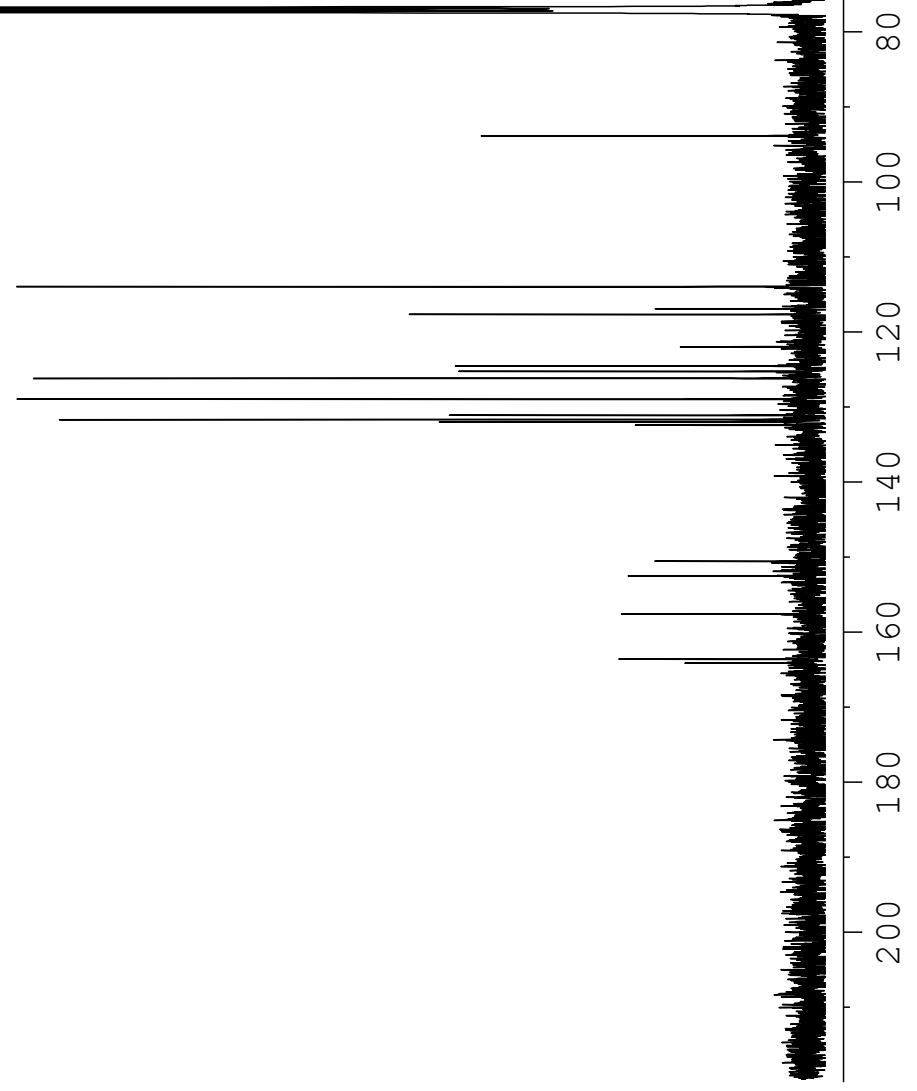



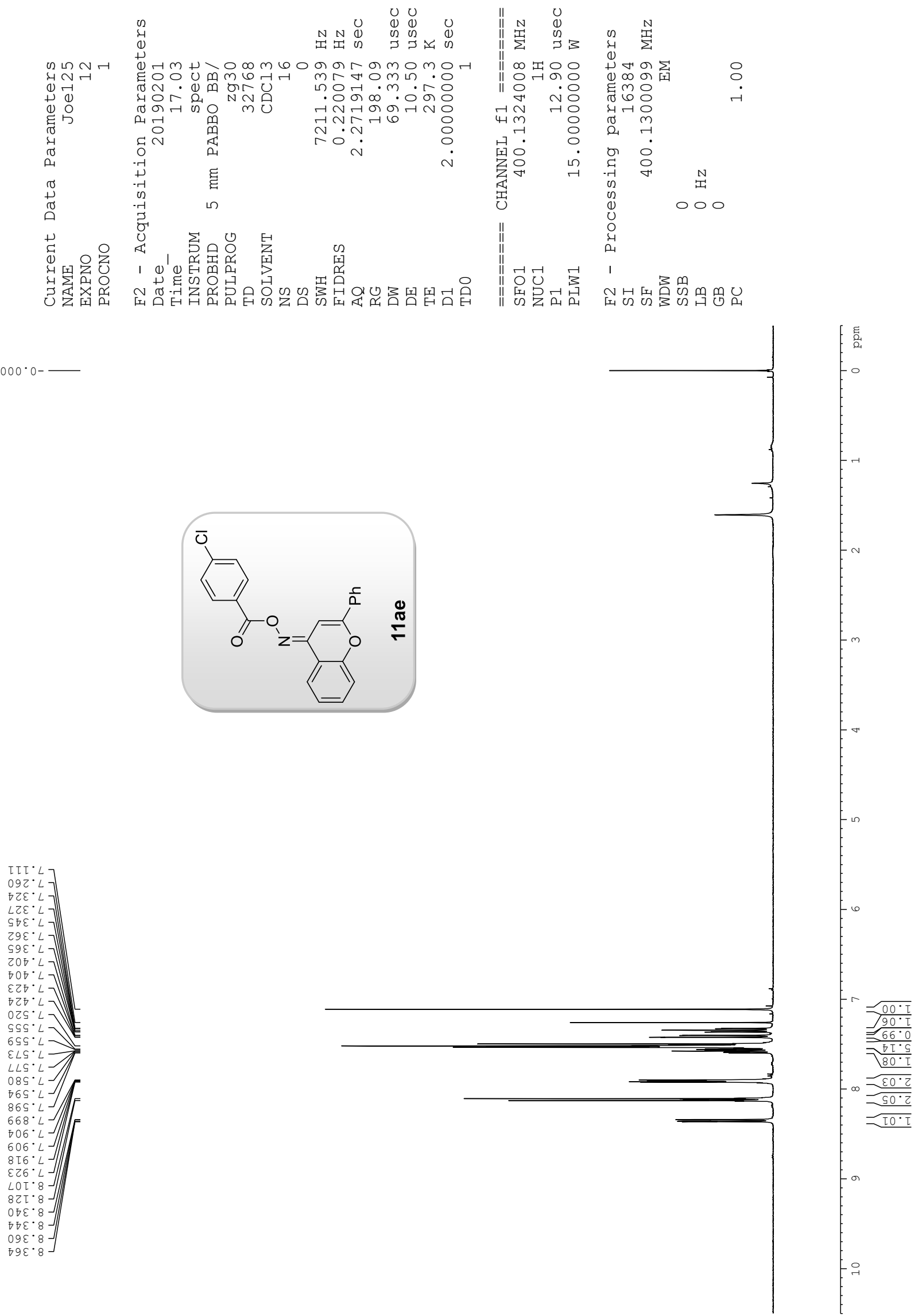

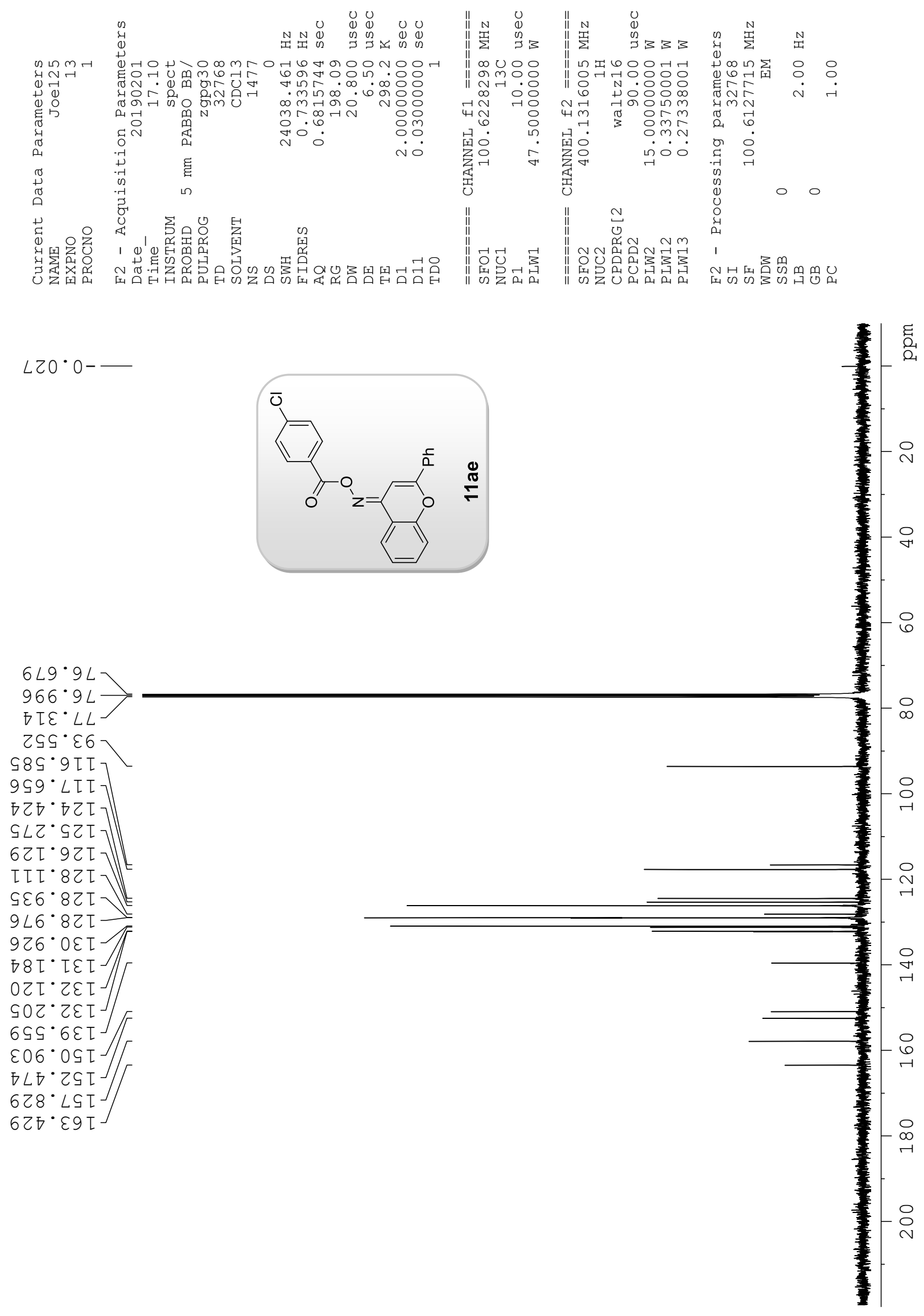


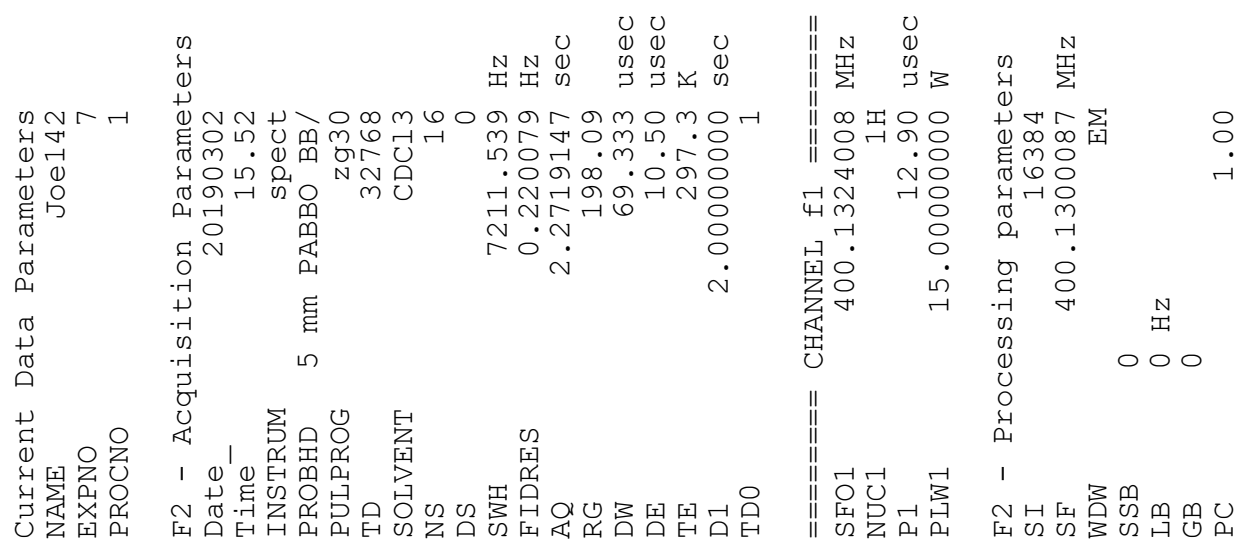

$000 \cdot 0$

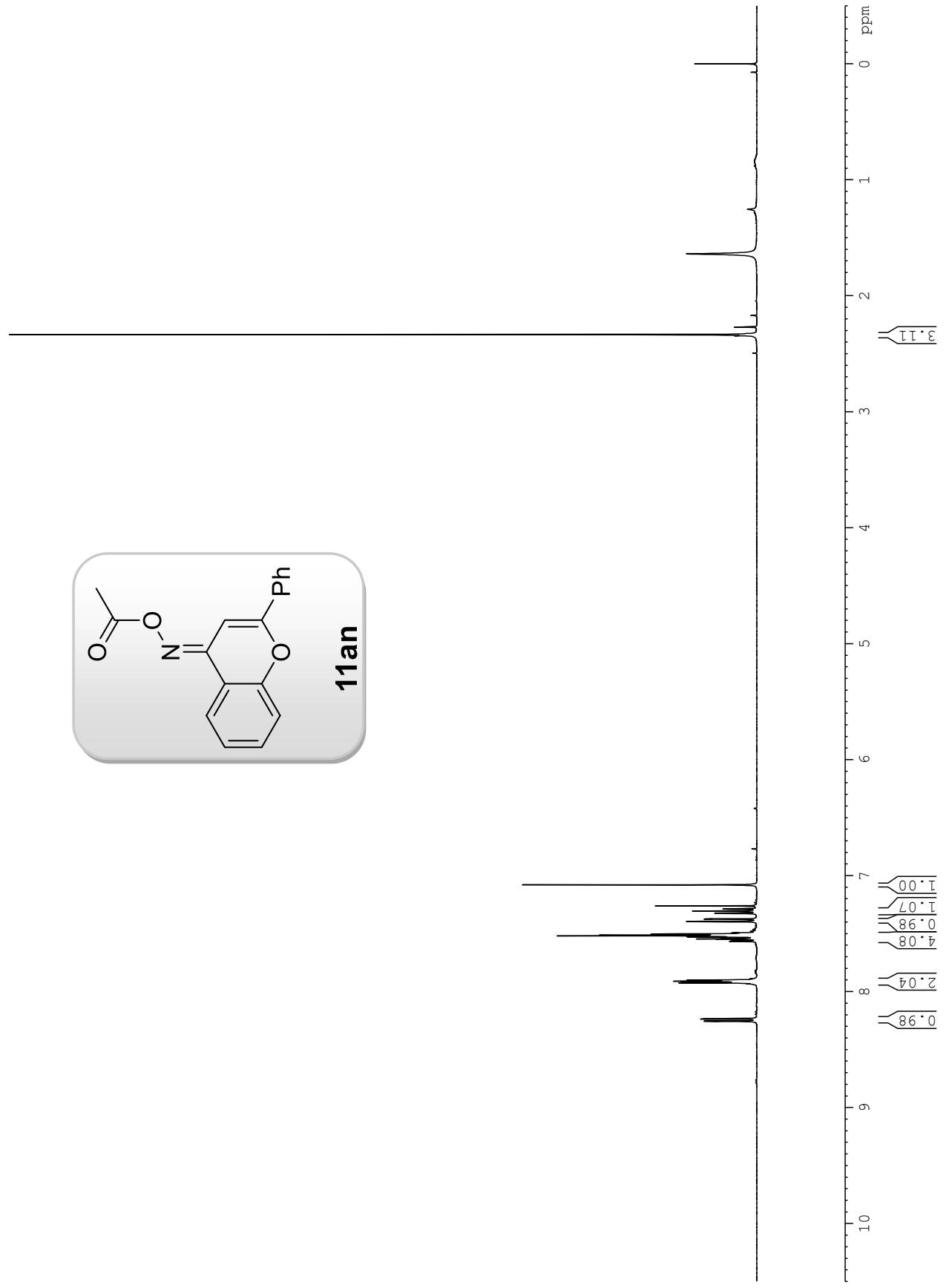




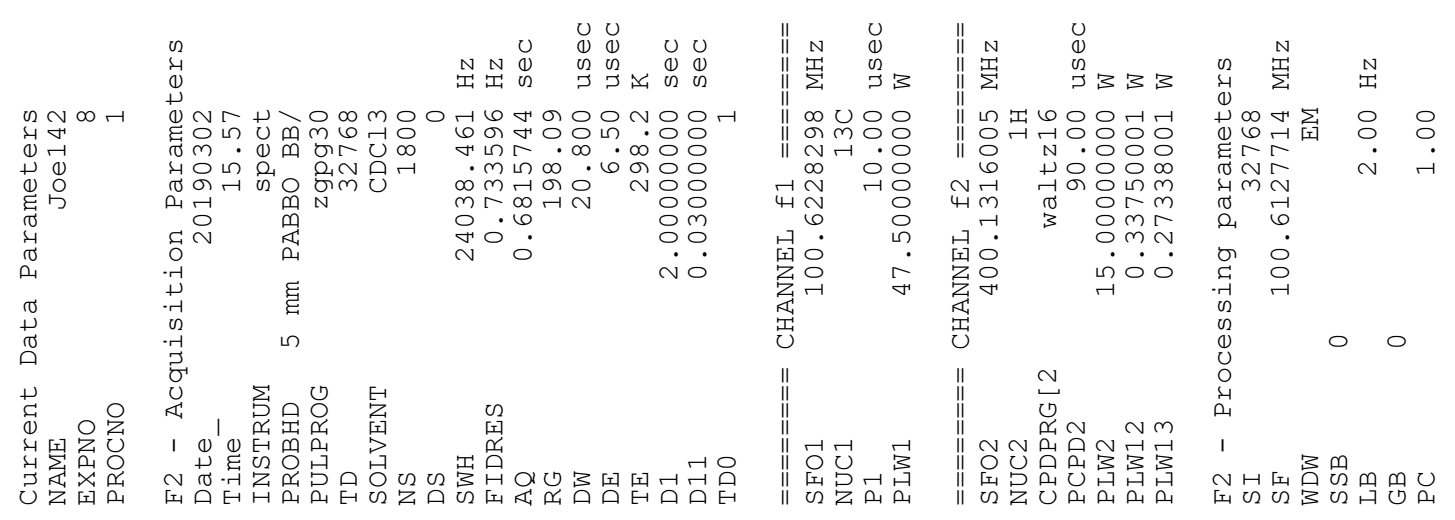

$200 \cdot 02-$

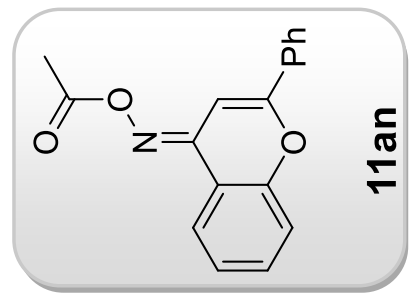

$289^{\circ} 9 L$
$000^{\circ} L L \backslash$
$L I \varepsilon^{\circ} L L$

$6 \tau 9 \cdot \varepsilon 6$

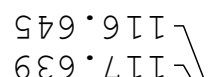

$09[\cdot \nabla 2 \tau]$

9ZI・9zI
$060 \cdot 92 I]$

$\angle \nabla 8 \cdot 8 乙 T$

Z๐0 $\mathrm{\circ \varepsilon \tau}$

$\varsigma\left[6^{\circ}[\varepsilon[]\right.$
$0 \nabla \tau \cdot \tau \varepsilon[$

ZIS.6口I

9LF'2SI

$9 \nabla \nabla \cdot L S I$

$8 I 7 \cdot 69 I$

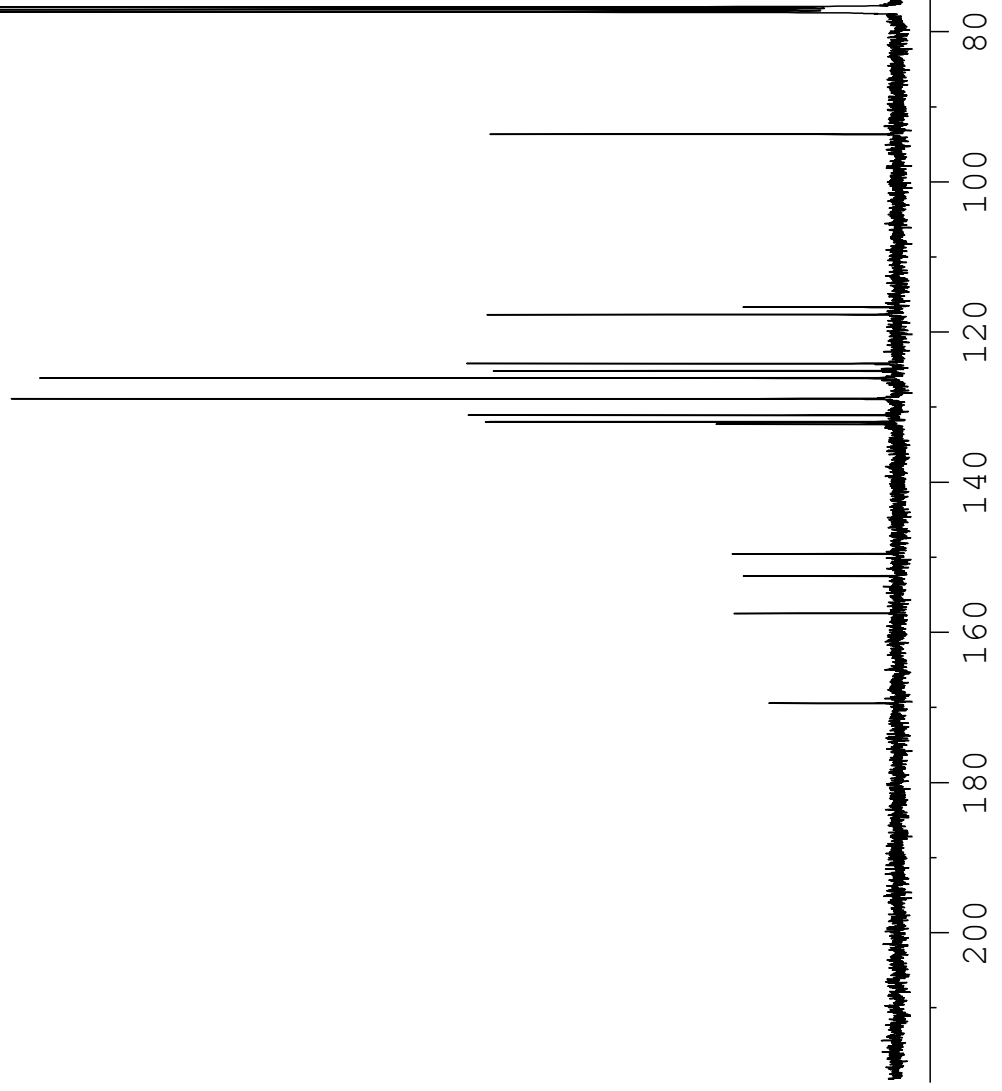




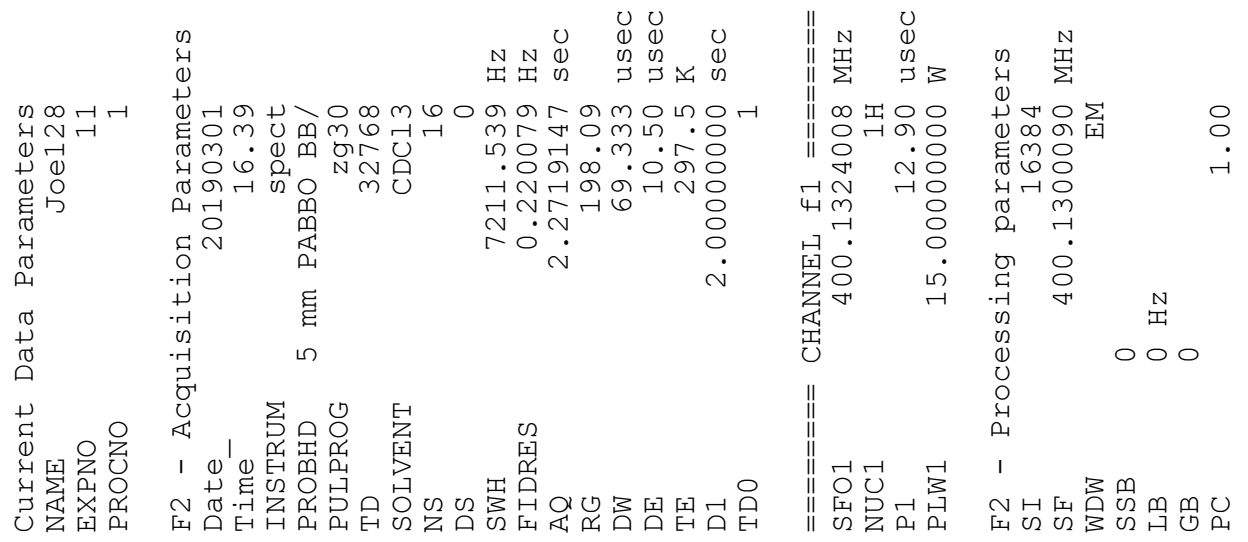

$00 \cdot 0-$

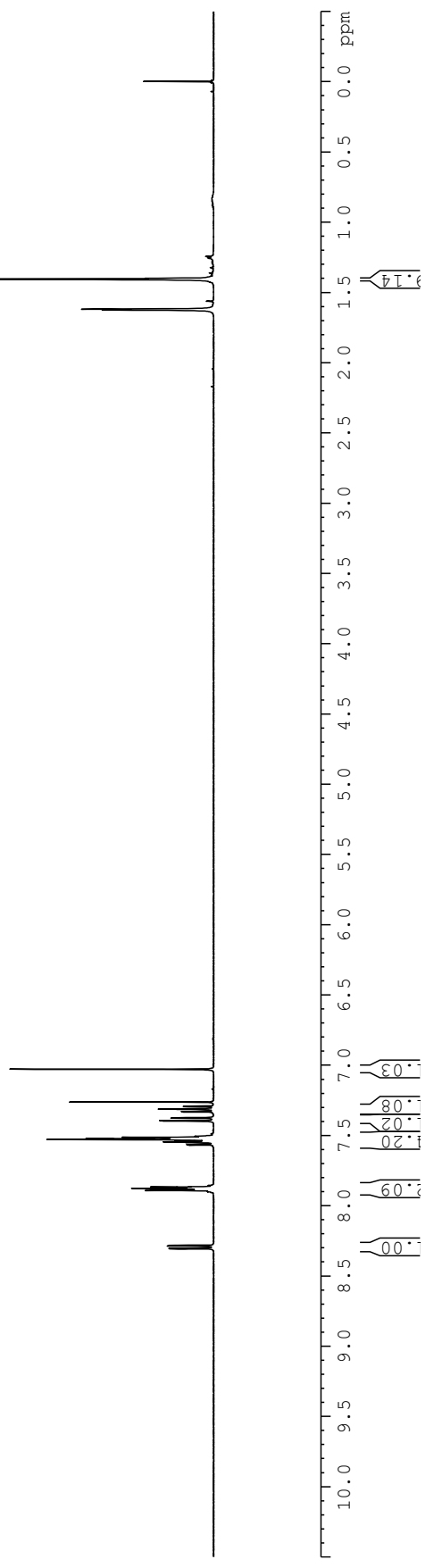



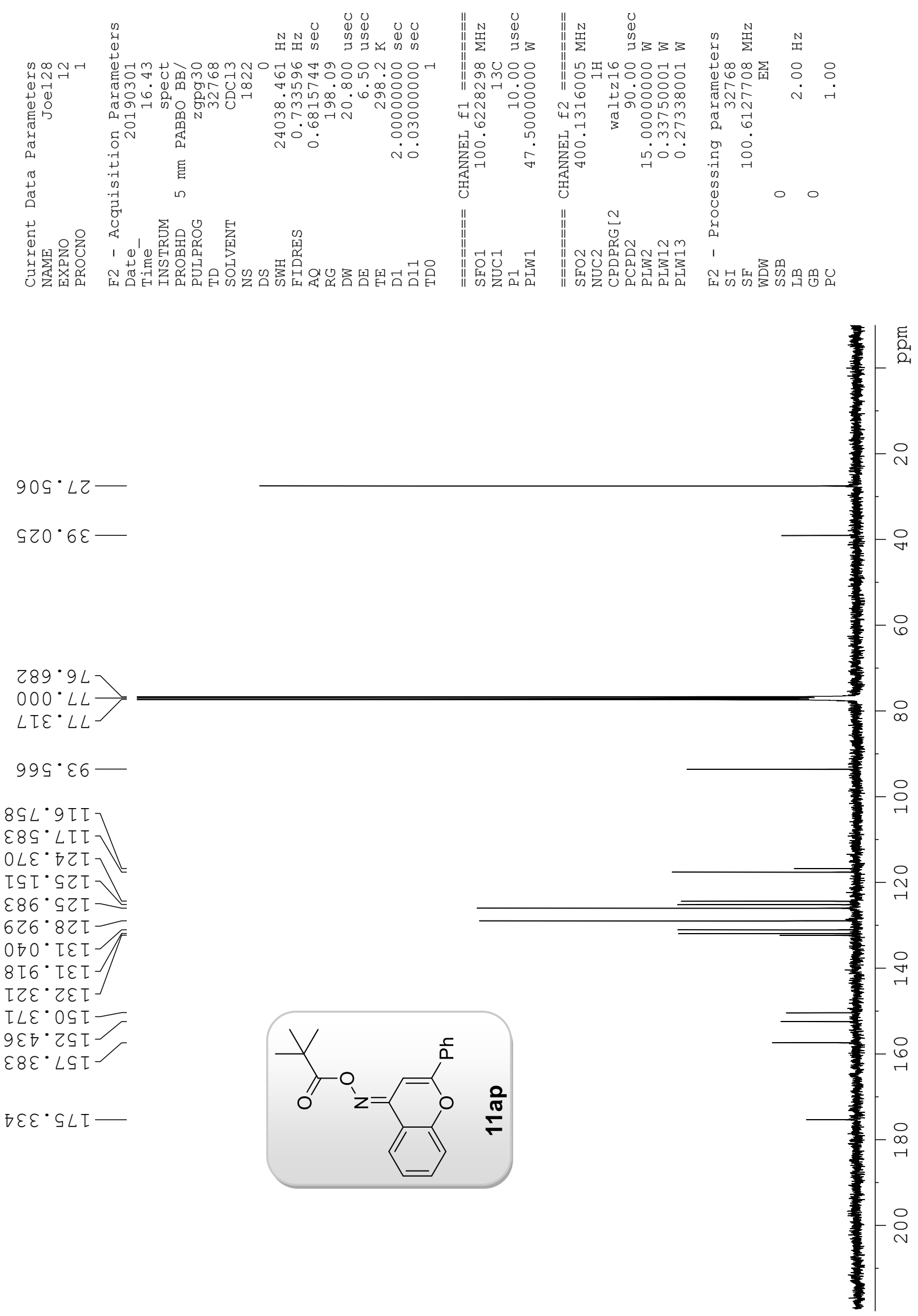


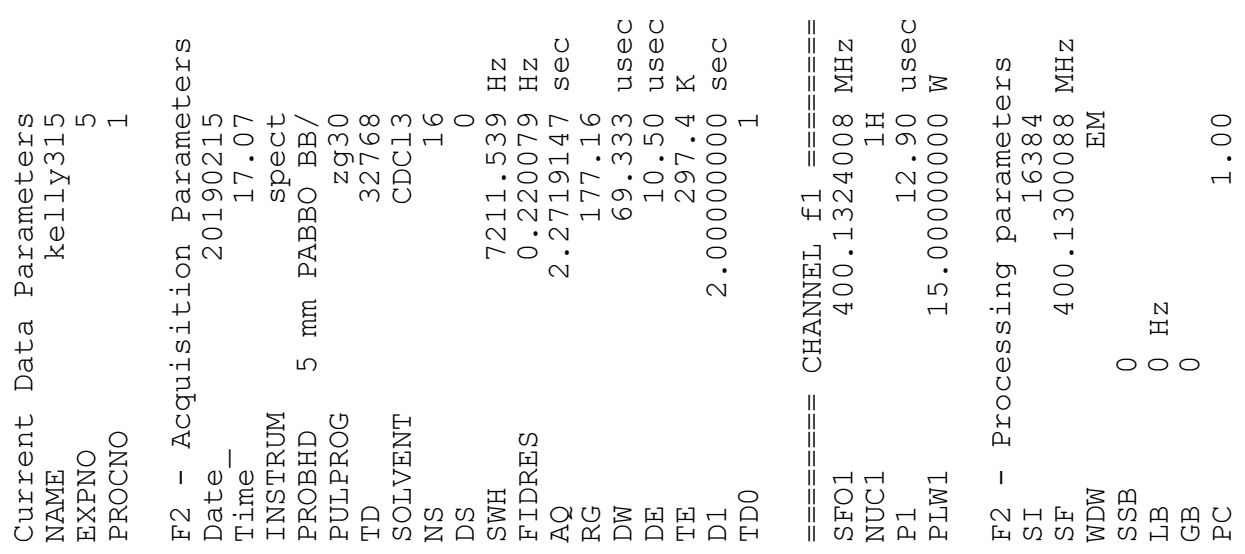

$000 \cdot 0$

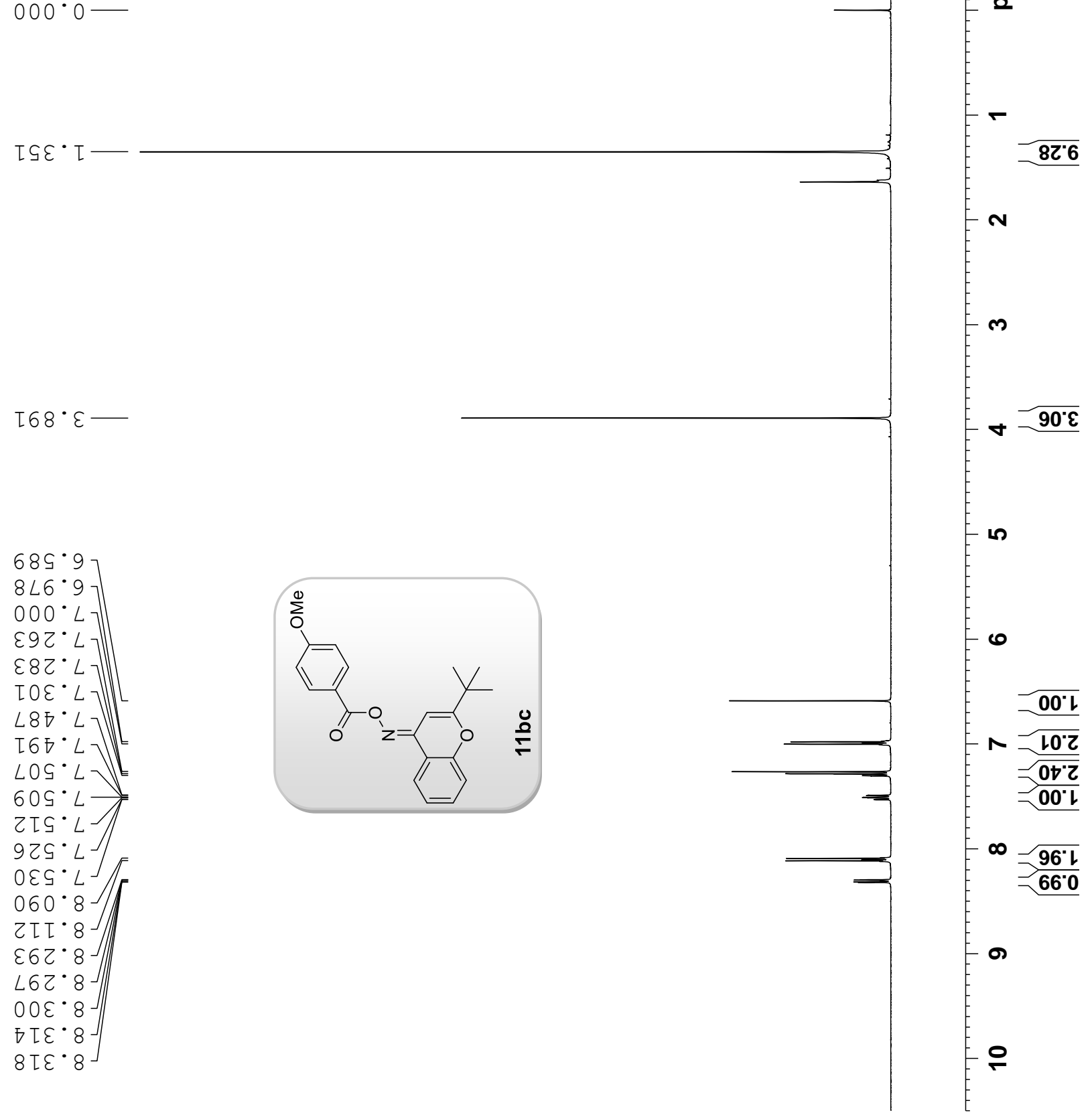

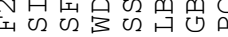




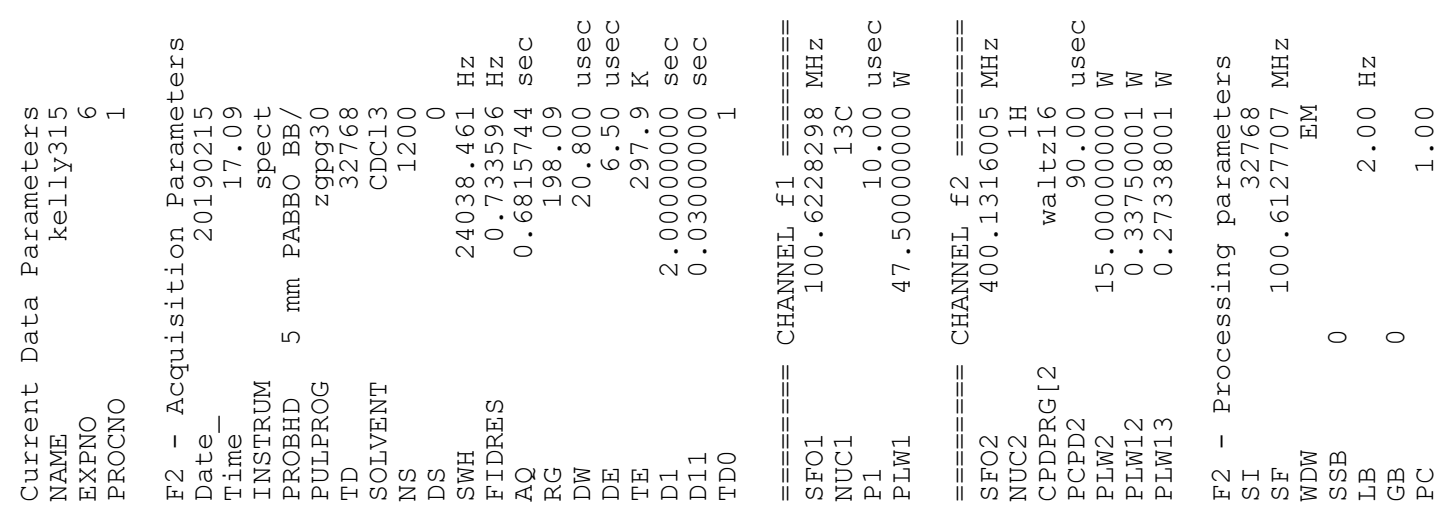

IZO $0-$

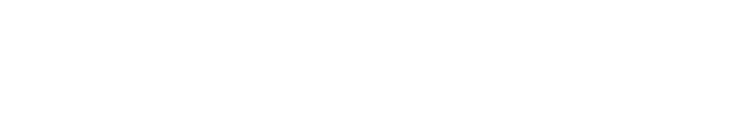

น乙8・して -

$662 \cdot 9 \varepsilon$

$097 \cdot 99$

$989^{\circ} 9 L$

$200^{\circ} L$

OZE $L L$

$488^{\circ} โ 6$

$\left.\begin{array}{l}\varepsilon \nabla 8 \cdot \varepsilon[I \\ 0 乙 S^{*} \cdot 9[\tau]\end{array}\right]$

$26 \tau^{\circ} \operatorname{LIT} L$

LEO ZZI

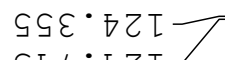

$\varepsilon \varpi し \cdot \nabla 乙 I$

EOS [ET

OZ8.0ST -

$9 \angle 9 \cdot 2 G T$

$9[\overline{1} \varepsilon 9[$

LOO. $79 I \longrightarrow$

$L I 9^{\circ} 69 T$

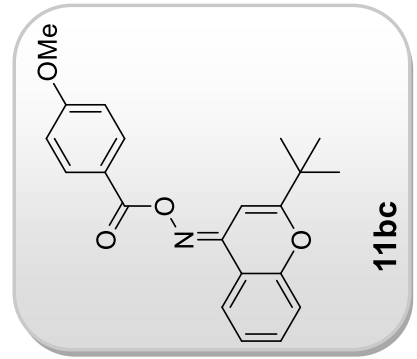




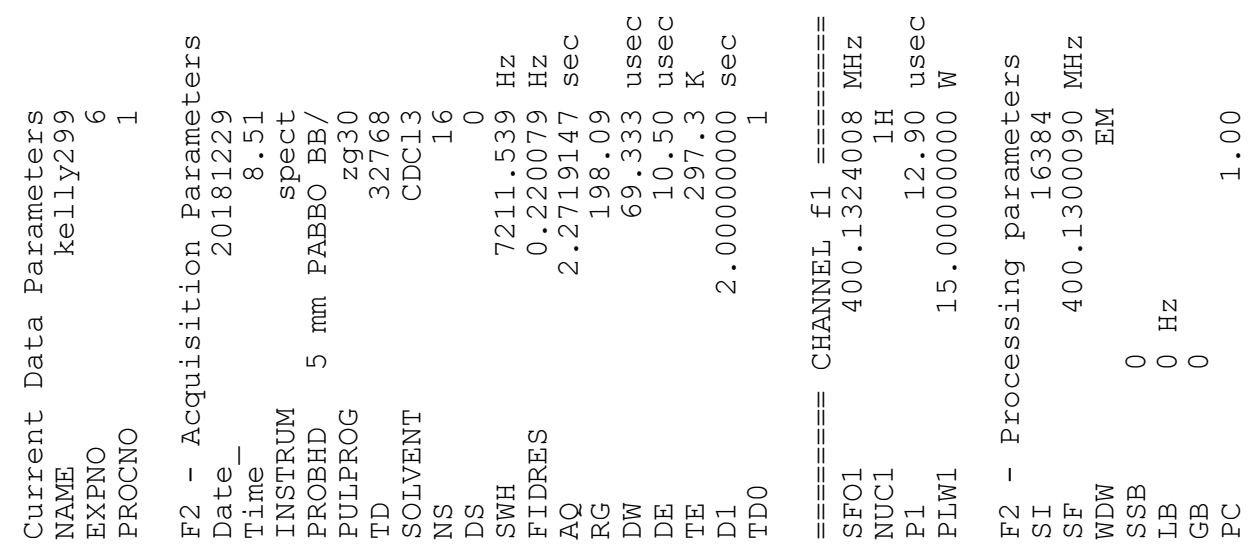

I00 $0-$

TOF
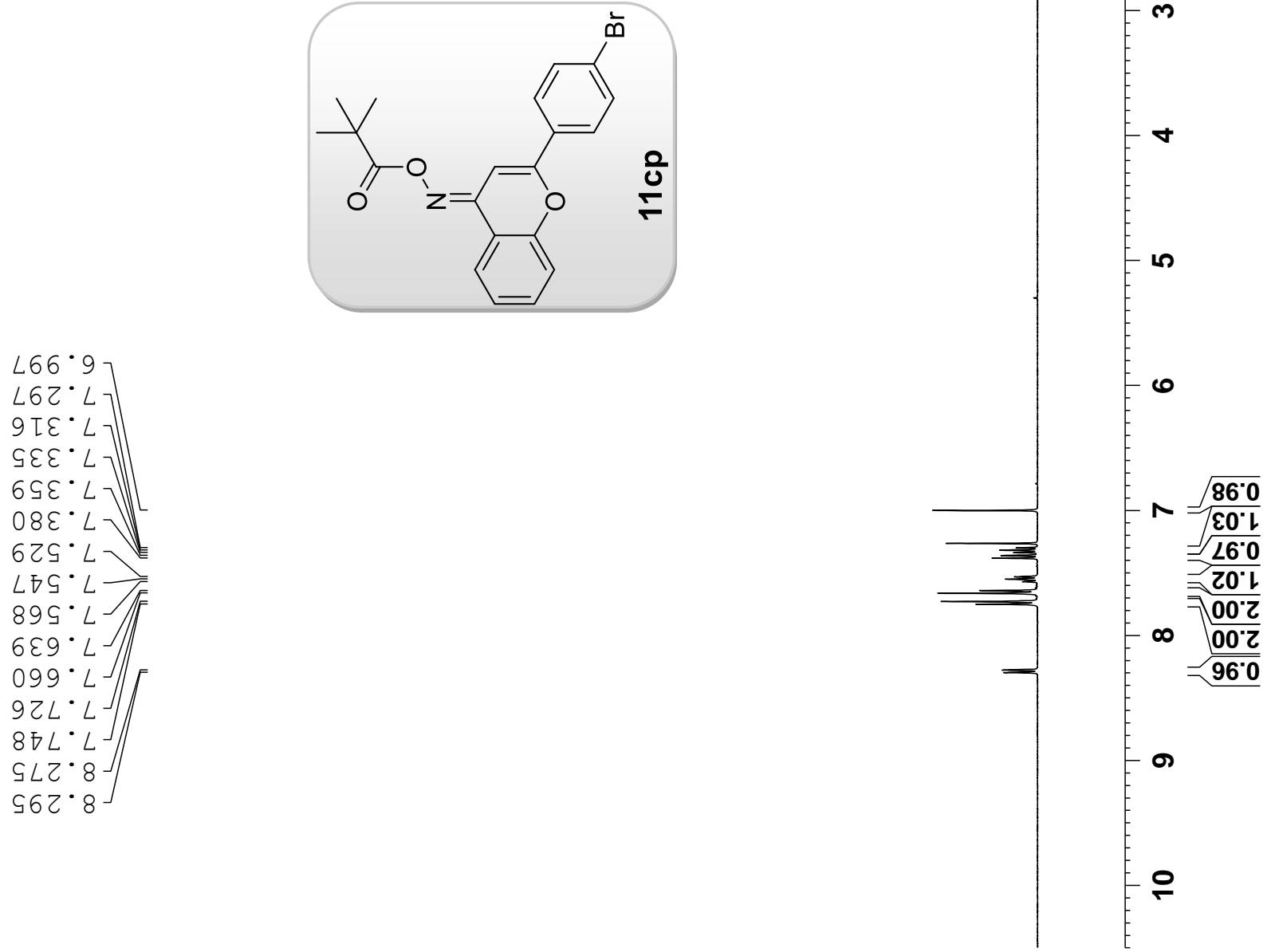

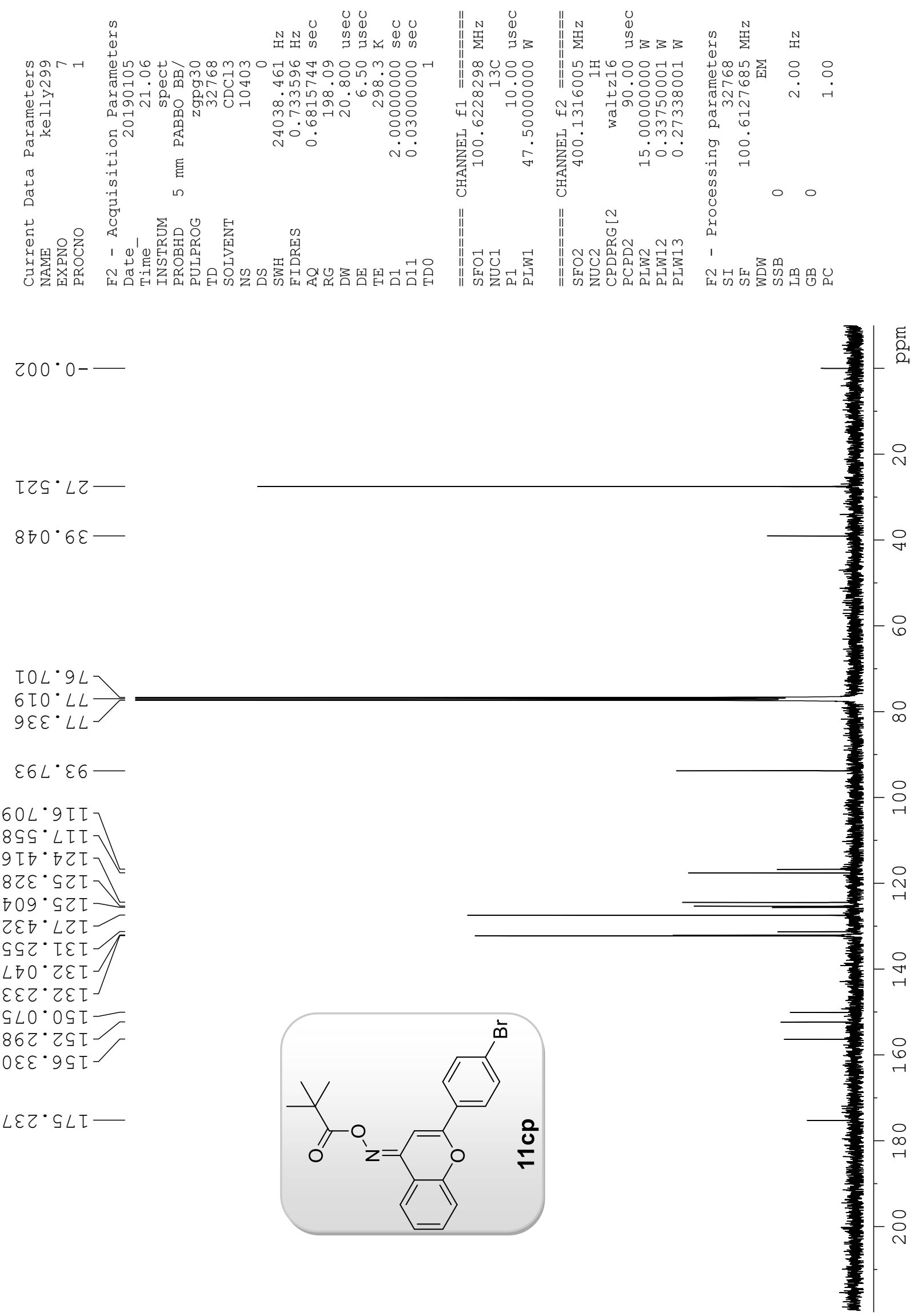


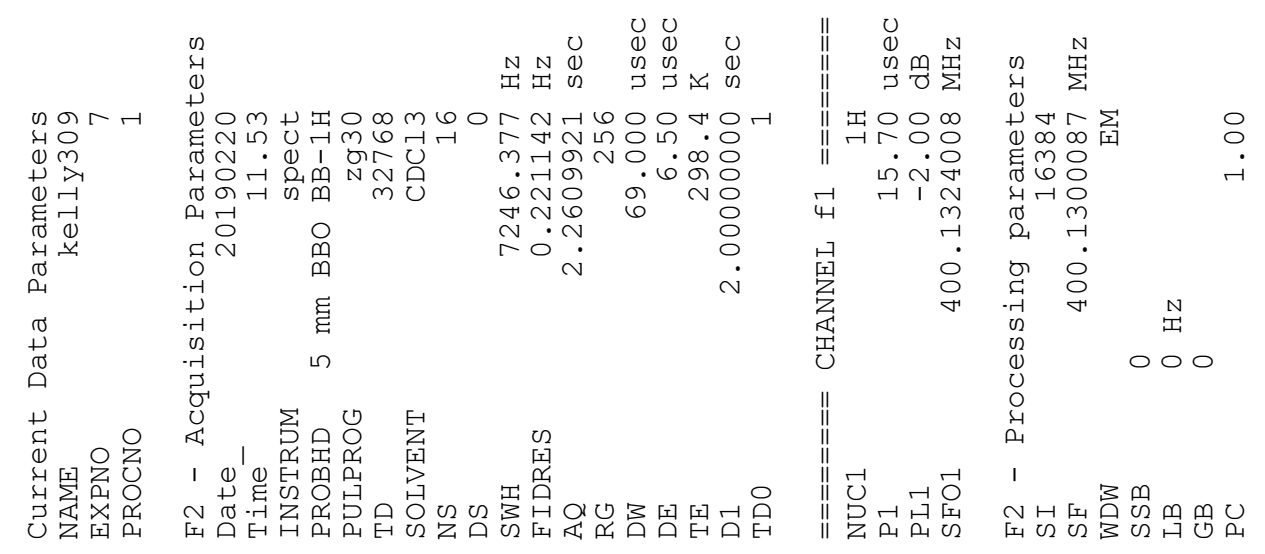

$000 \cdot 0$

$688^{\circ} \varepsilon$
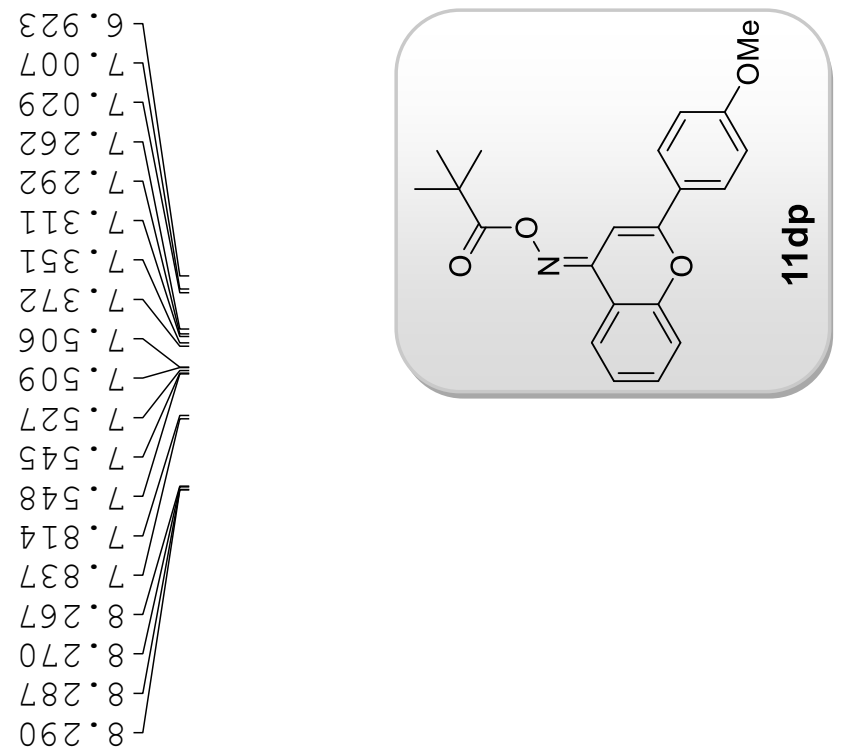

万0

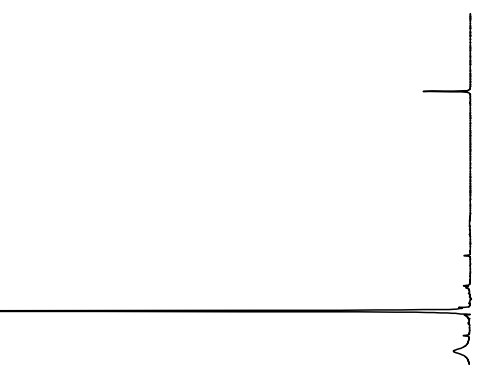

흘

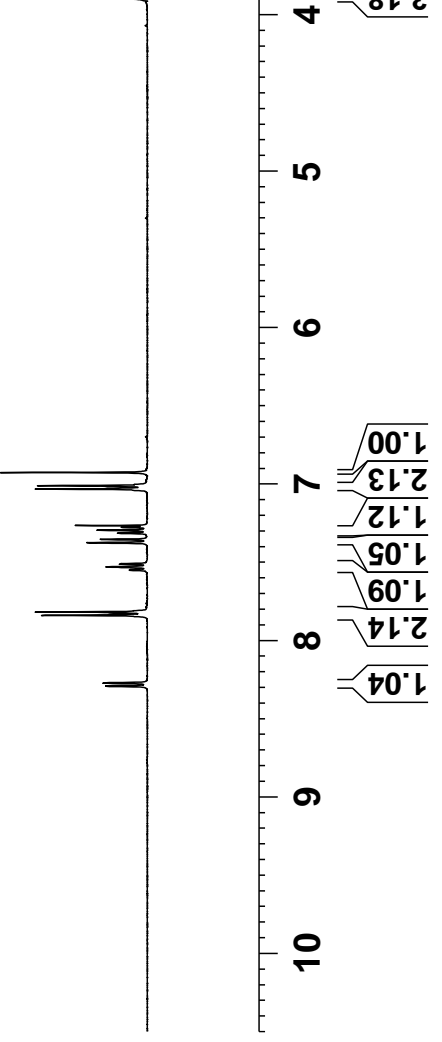



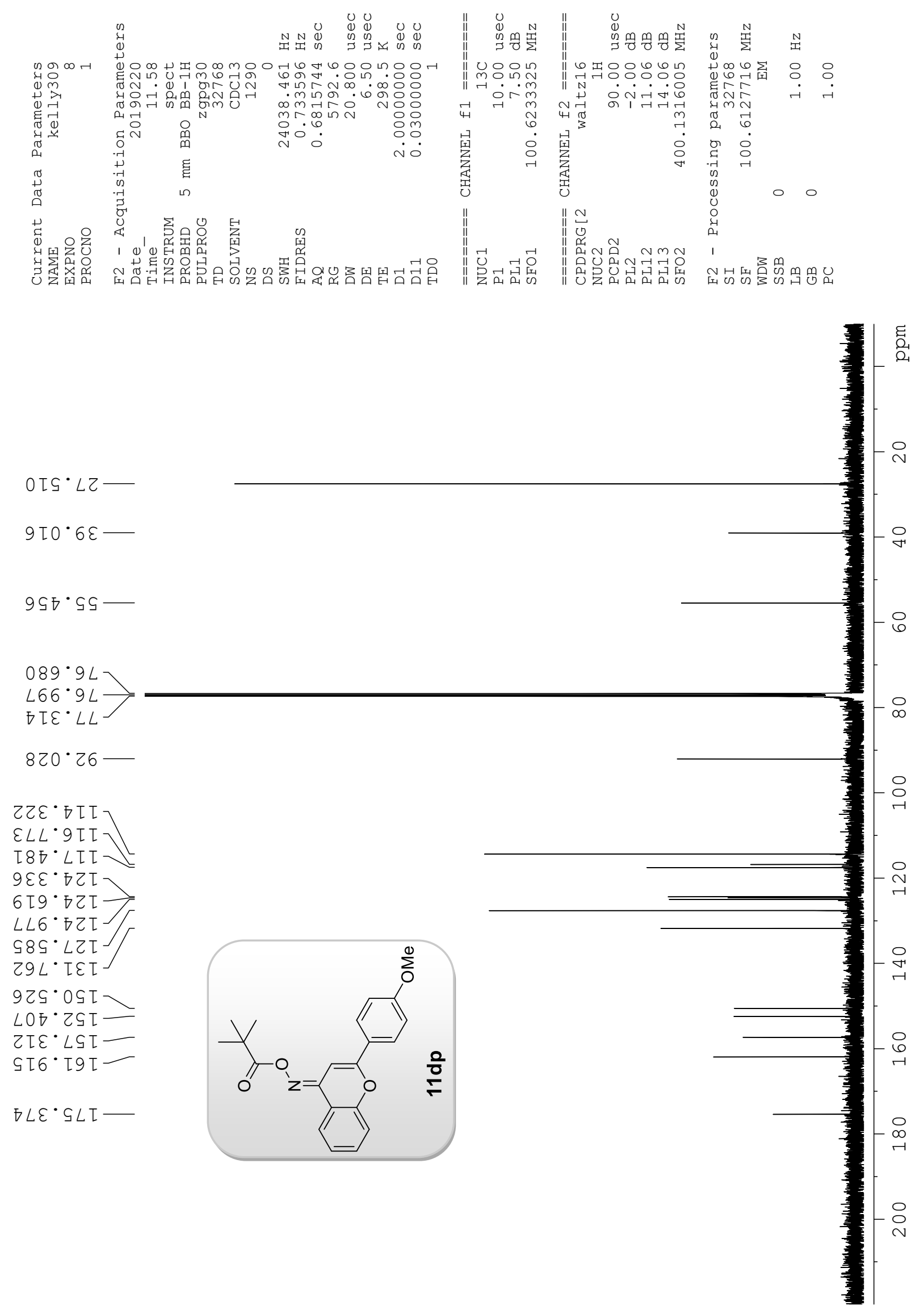


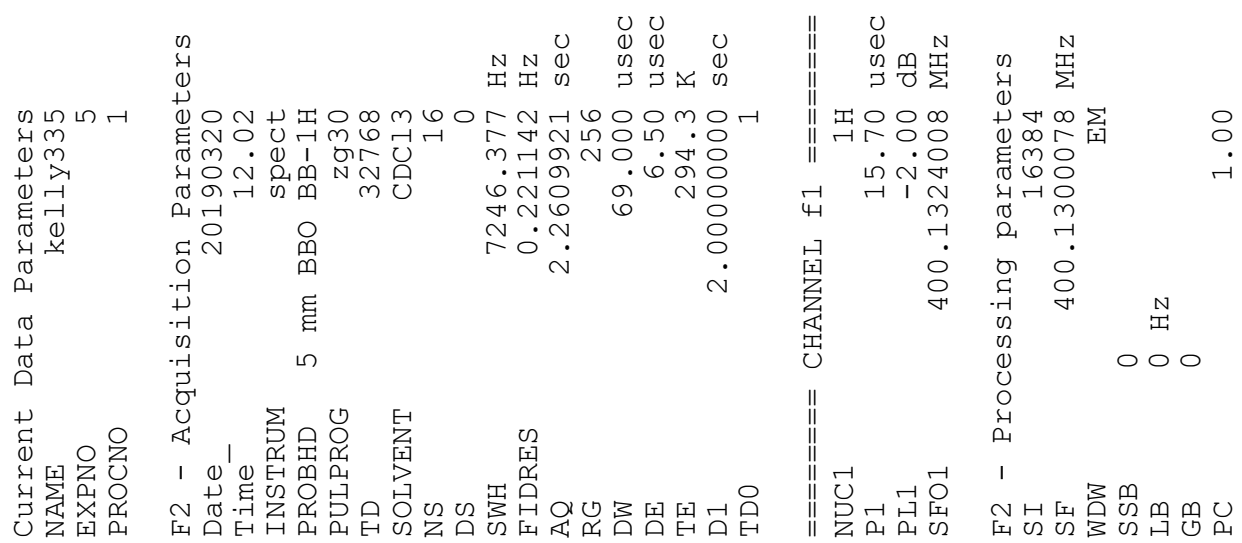

$000 \cdot 0-$

$L \mathcal{L} \mathcal{E}^{\circ} \mathrm{Z}$

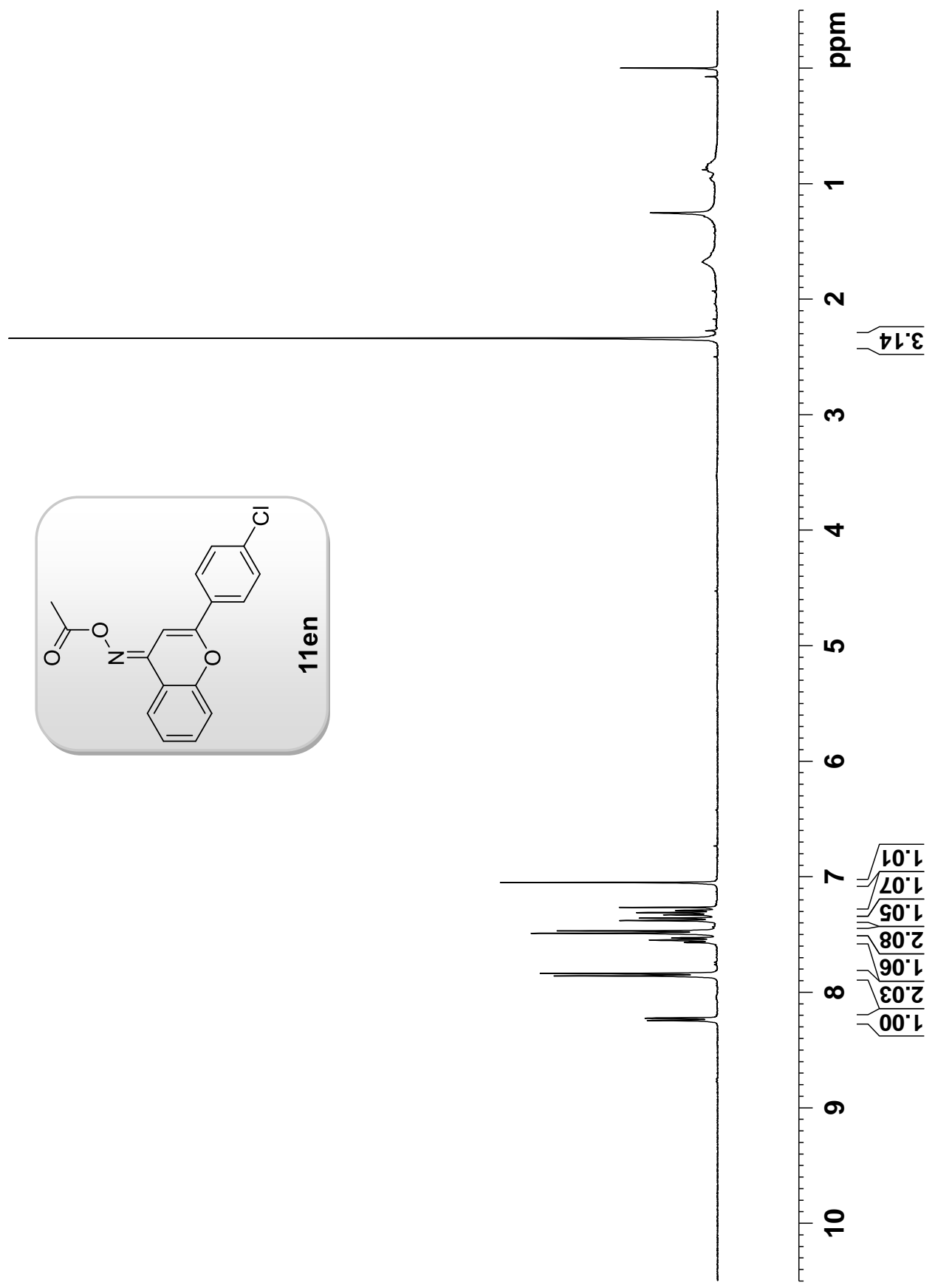




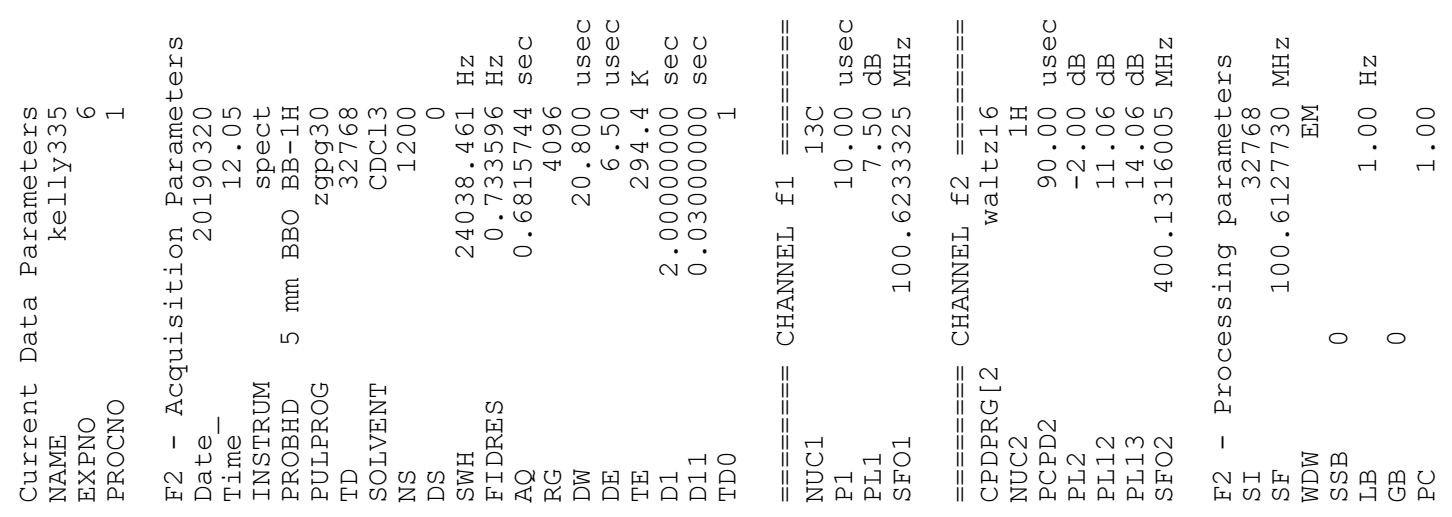

IE O $0^{-}-$

I I0 OZ -

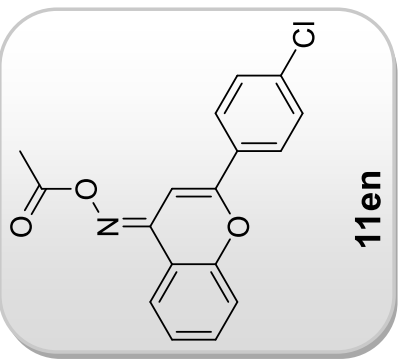

$089^{\circ} 9 L$

$866^{\circ} 9$

SIE $L L$

$\varepsilon S L \cdot \varepsilon \sigma-$

[ES 9 [I

$\left.\begin{array}{l}6 \angle S^{\circ} \angle I T \\ \nabla \nabla[\cdot \nabla Z T\end{array}\right]$

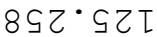

Ђ乙E $\angle 乙 I$

$9 \varepsilon \tau \cdot 6 Z \tau$

[ย9 $0 \varepsilon[/$

$\left.600^{\circ} \mathrm{C \varepsilon I}\right]$

ZLI· $6 \square \mathrm{I}$

$062 \cdot 2 \mathrm{TI}$

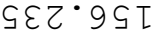

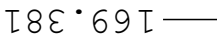

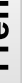

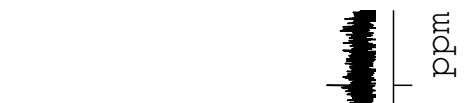




\section{Scanned copies of EI Mass Spectra for compounds 12 and 11:}
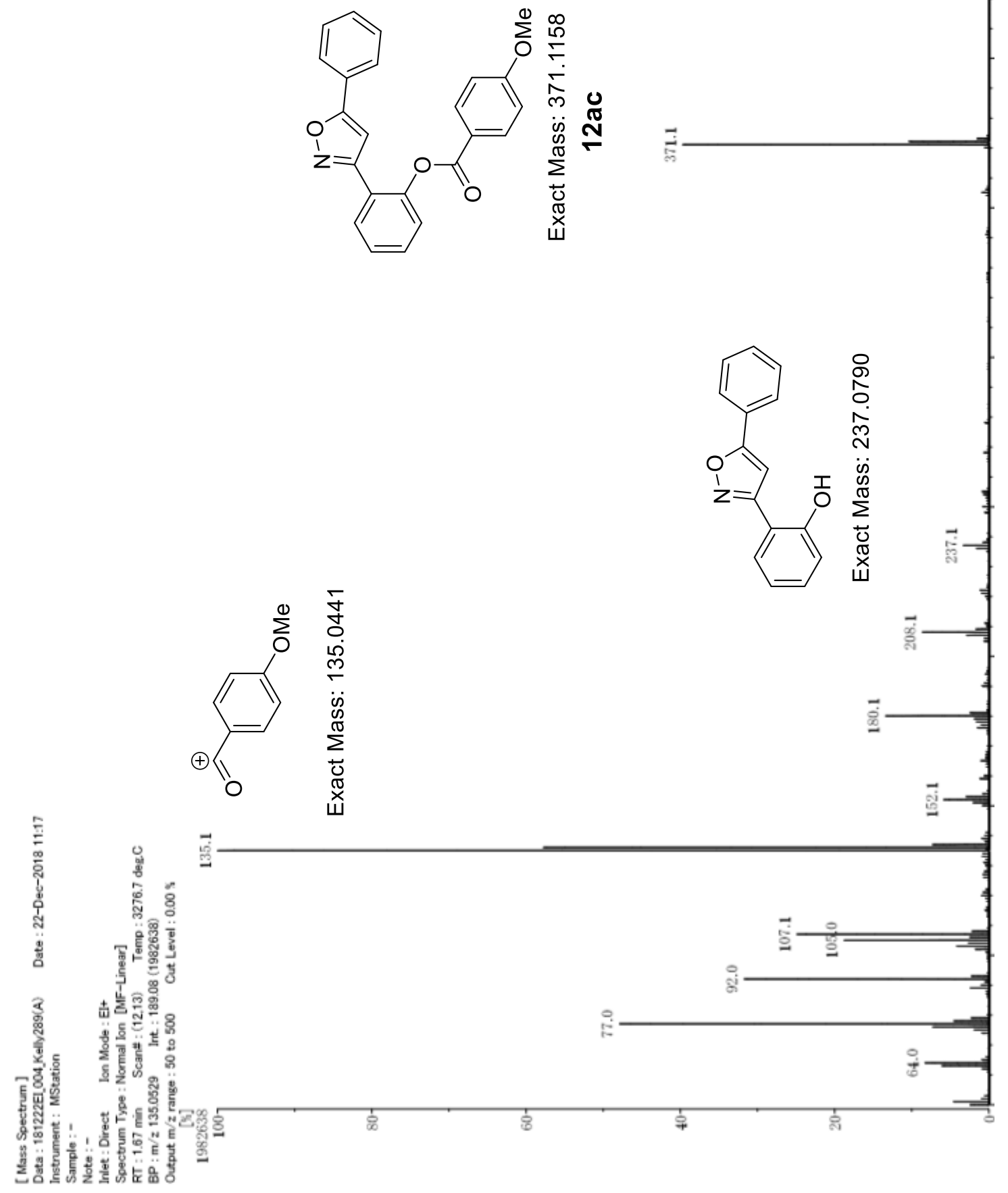

离 


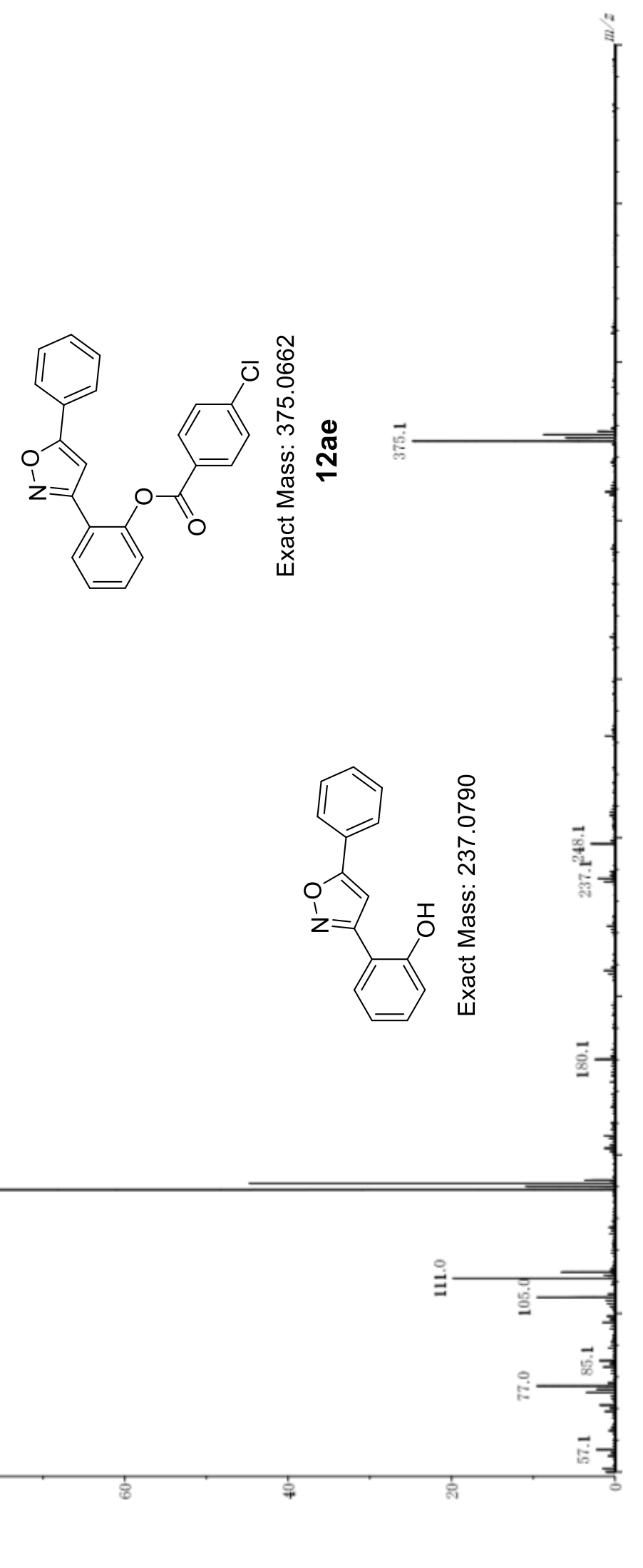




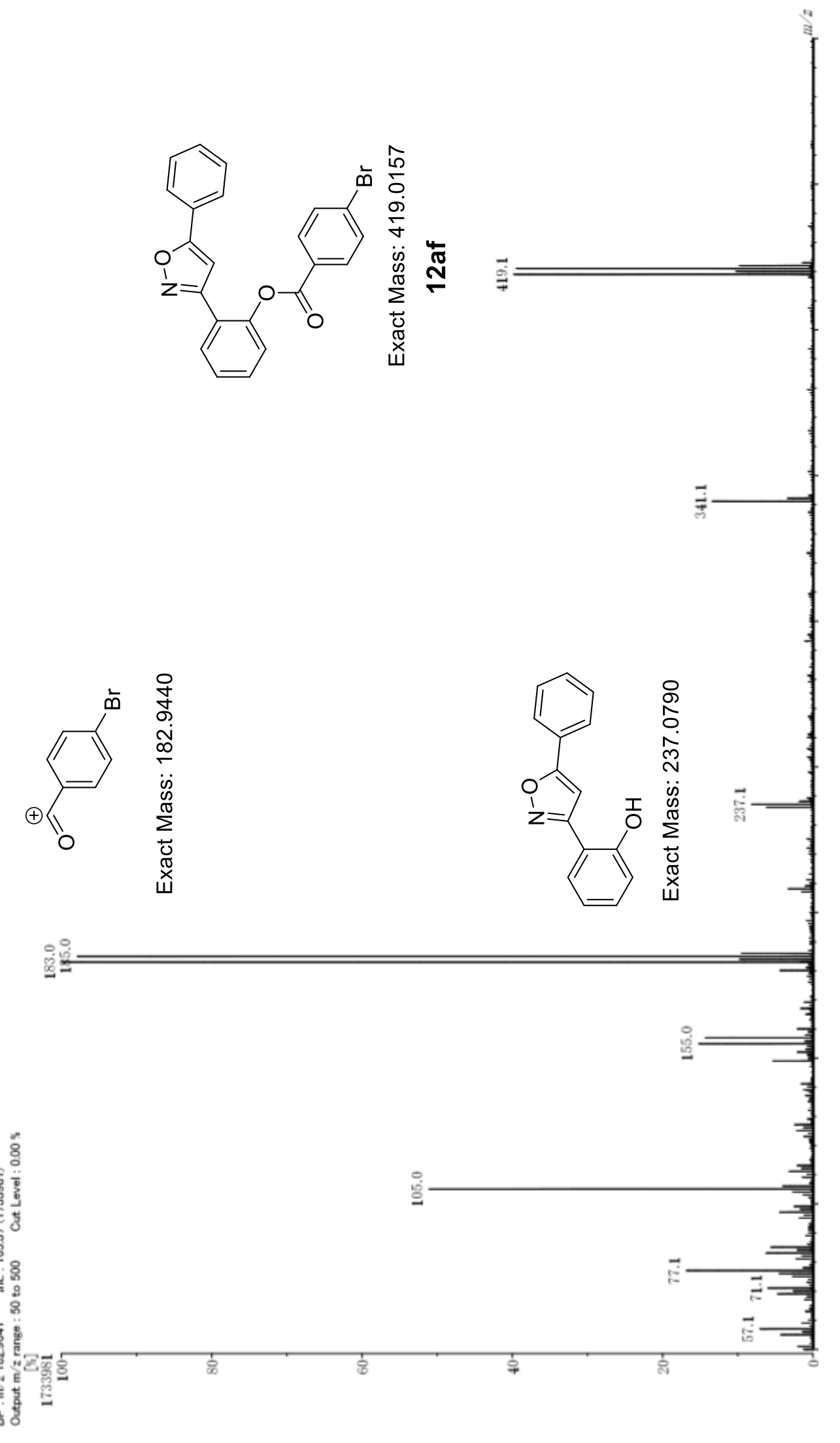



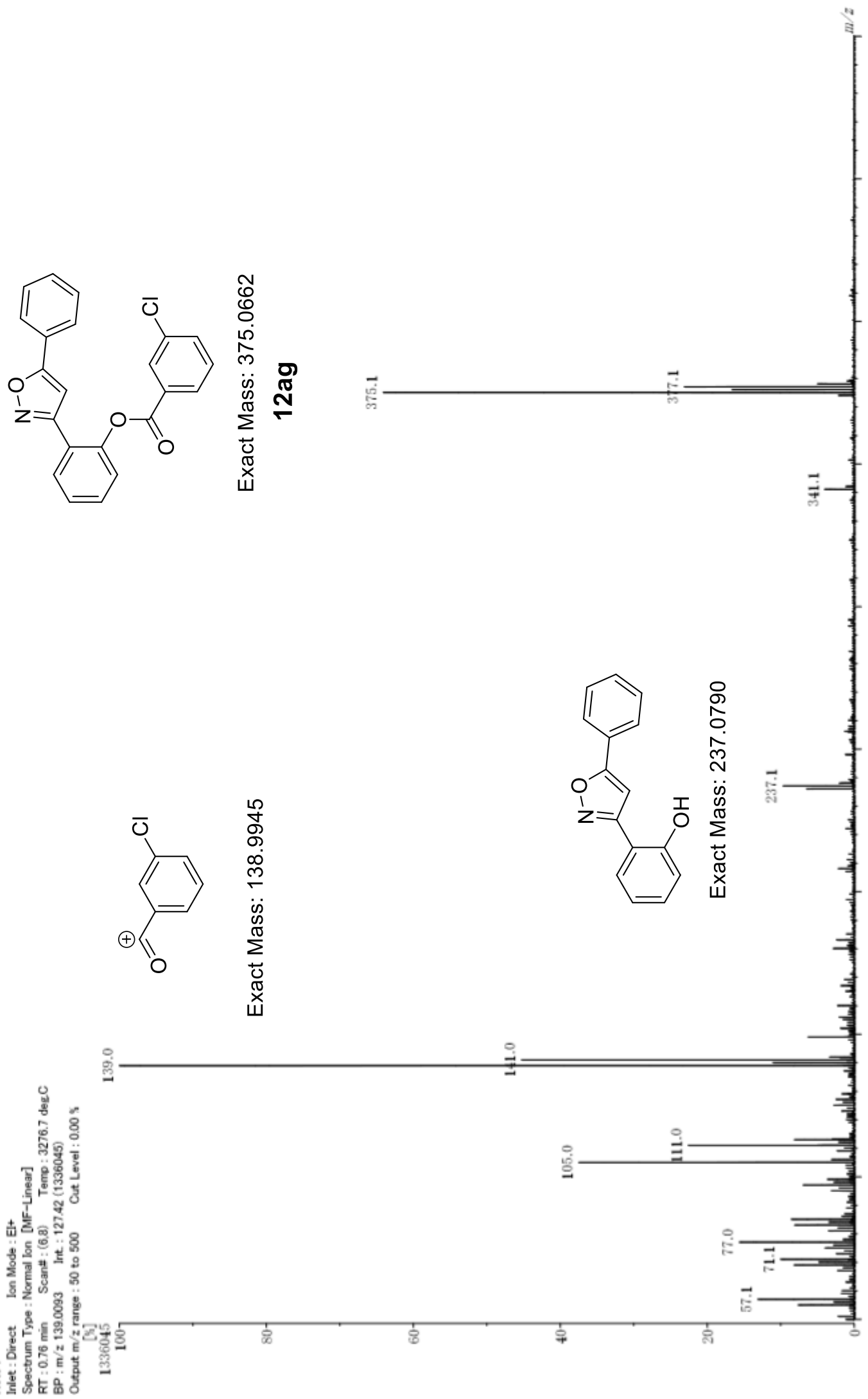


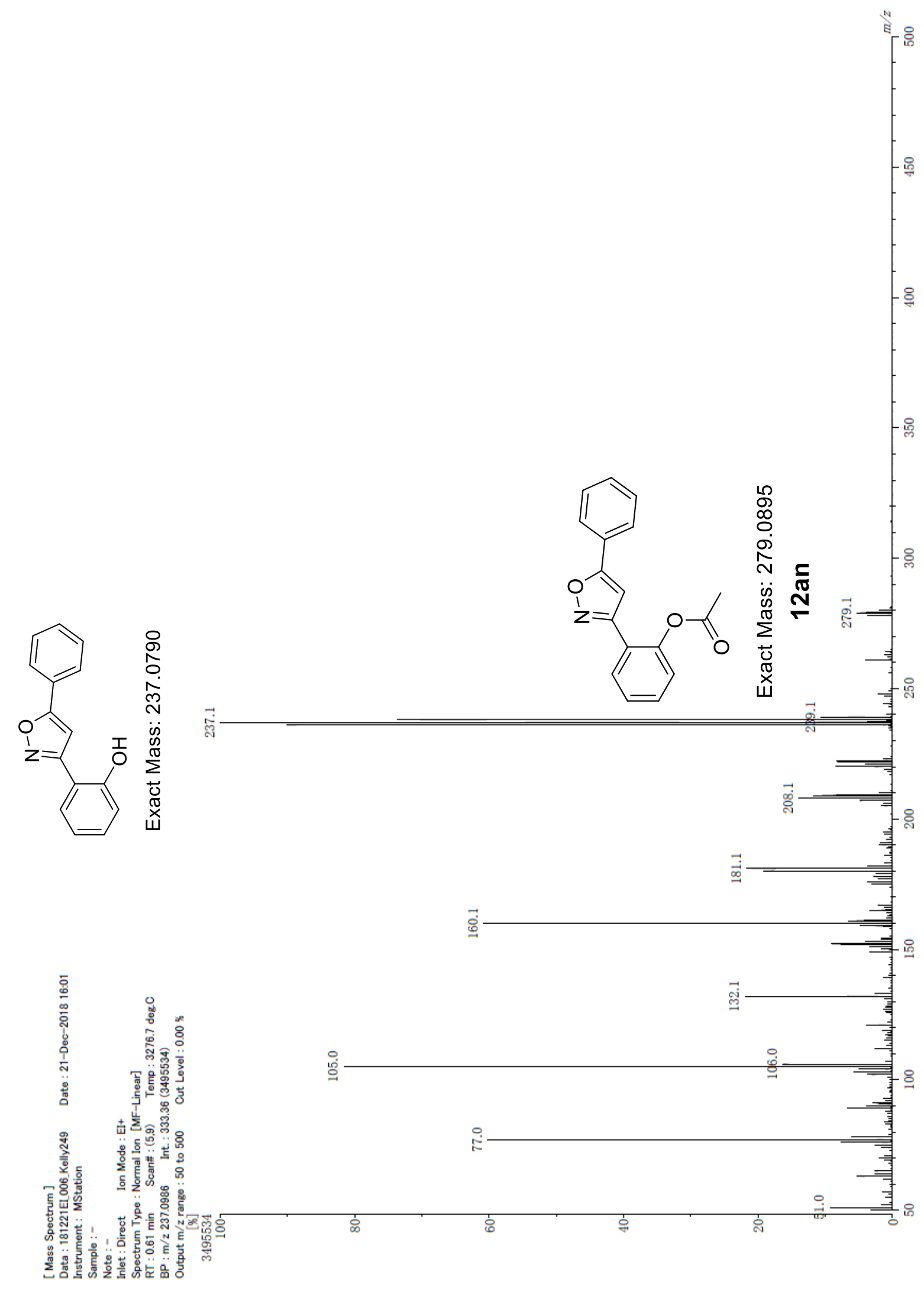



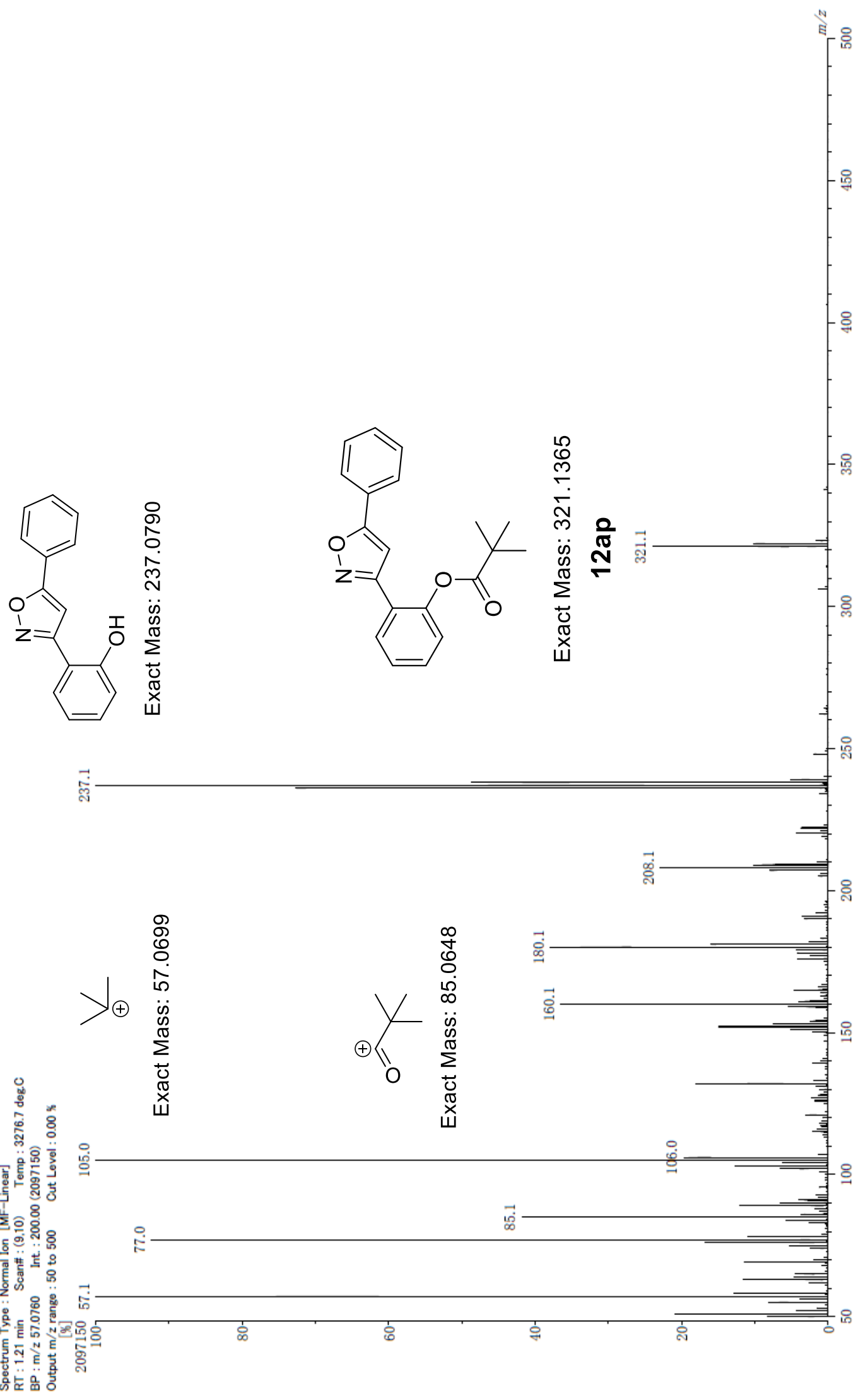


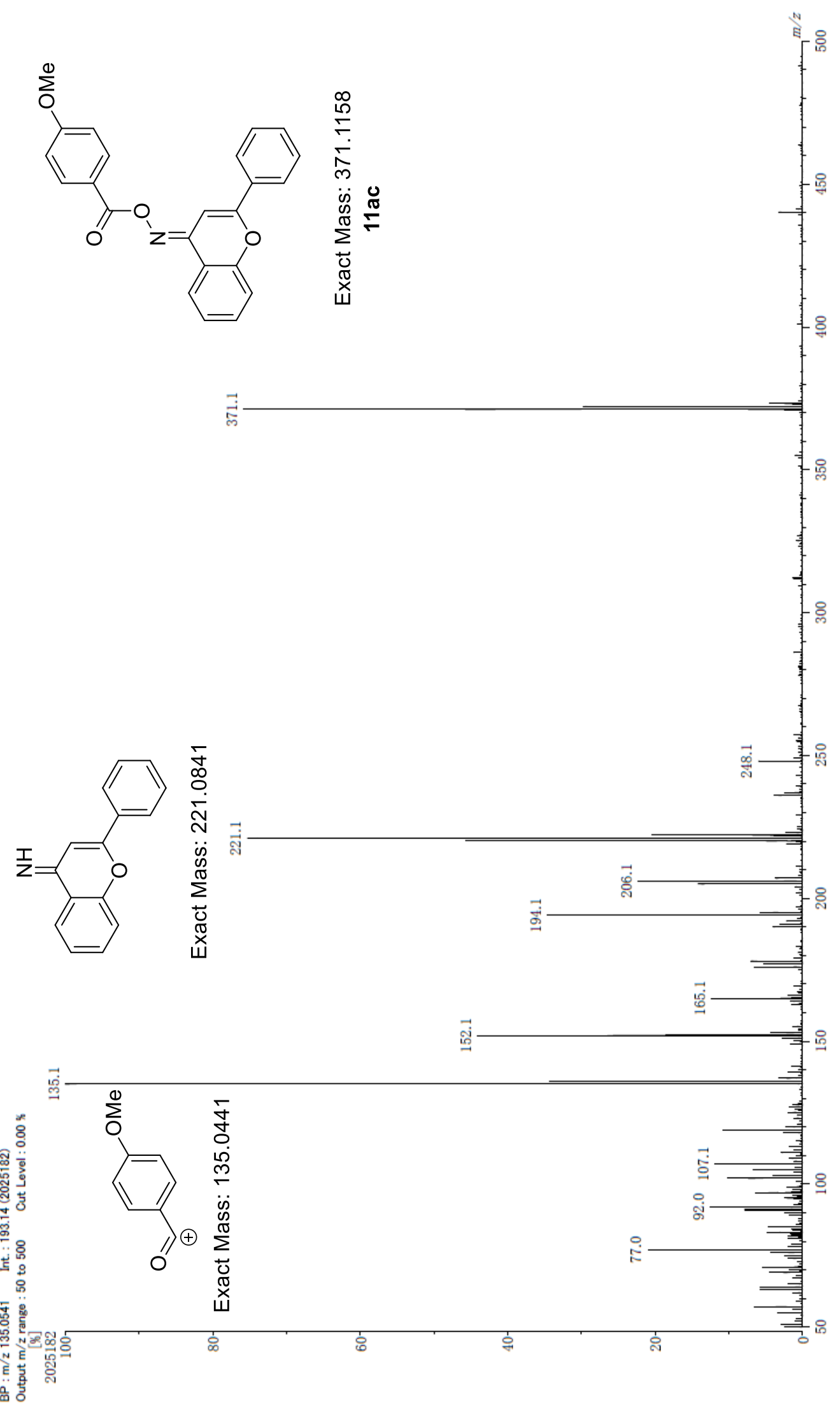



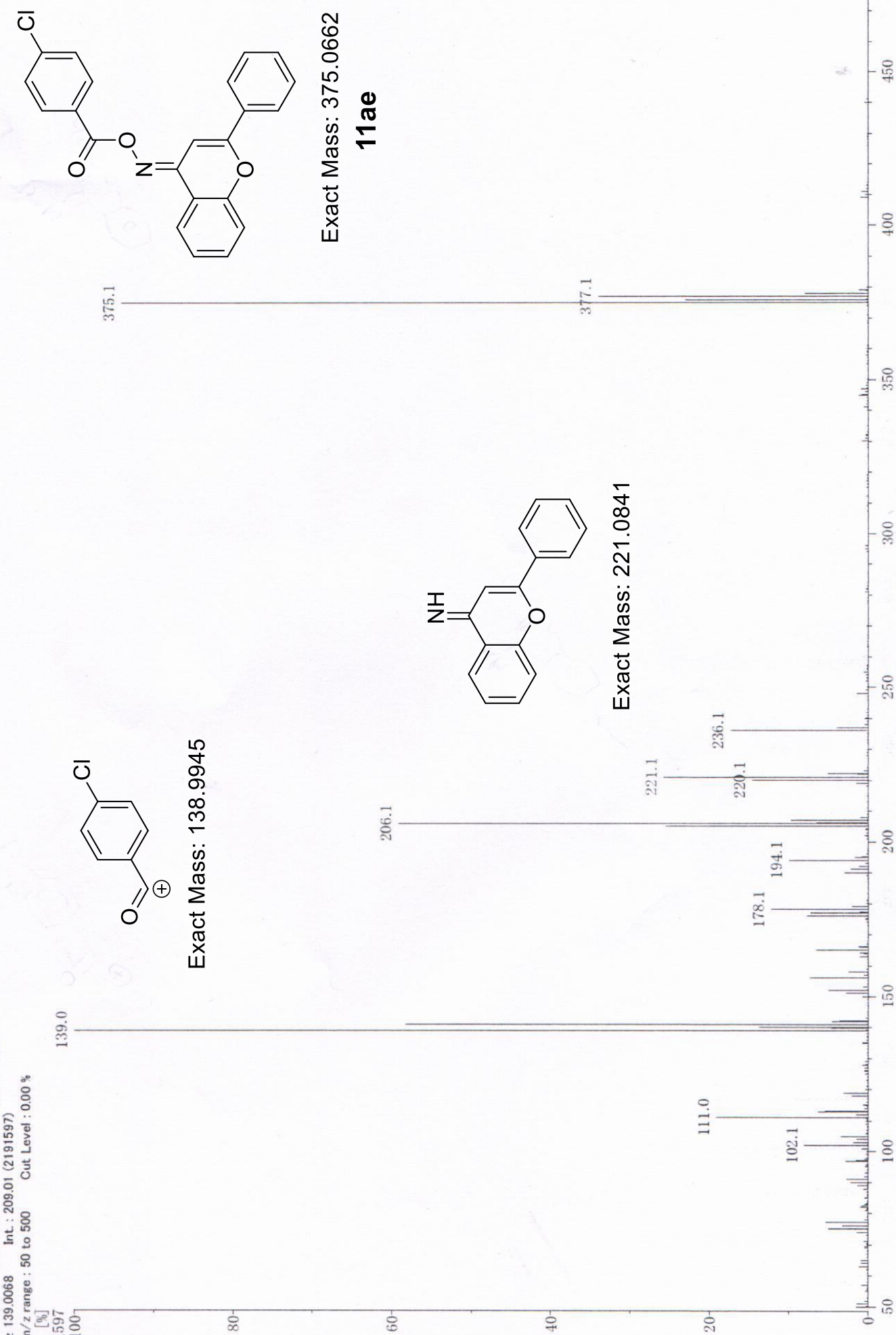

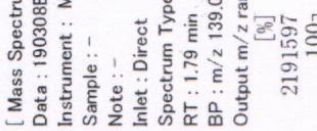




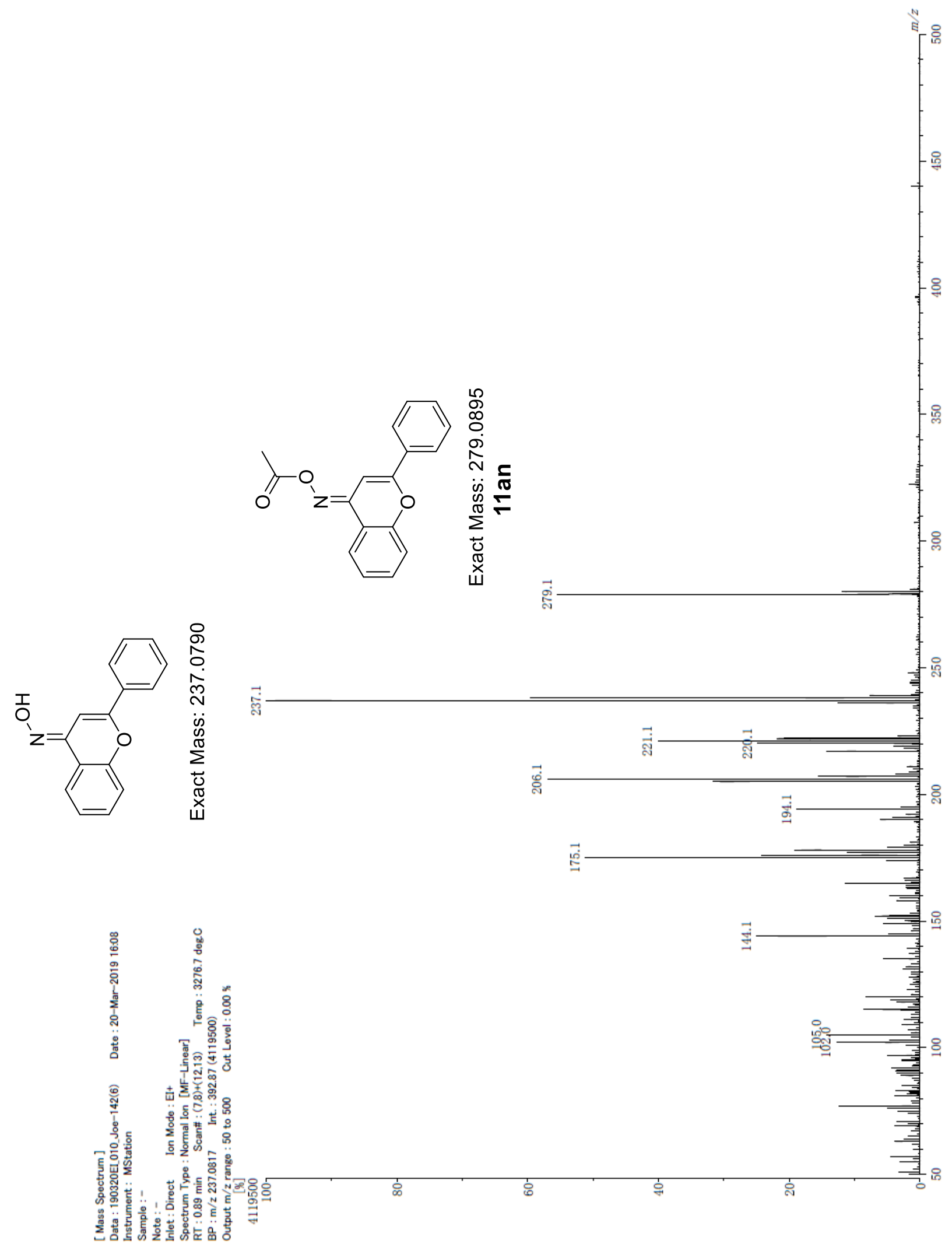



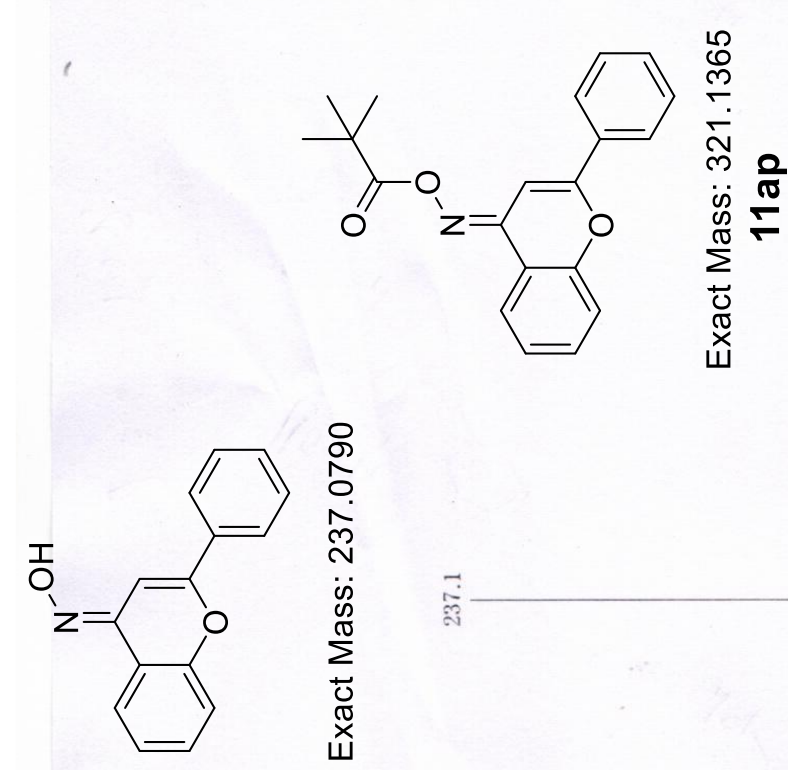

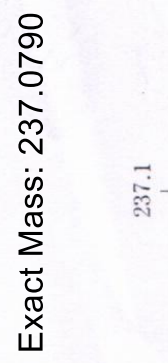

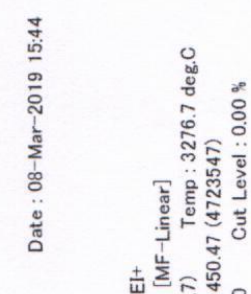

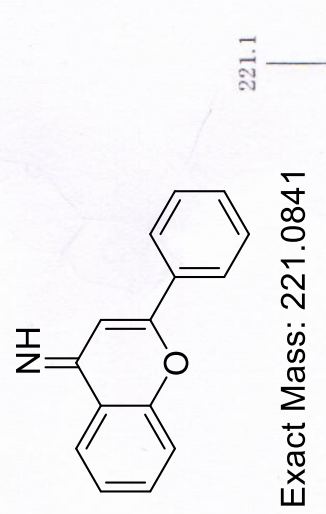

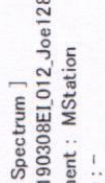

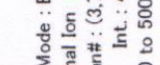

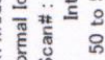

政

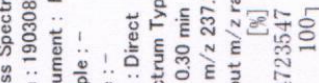

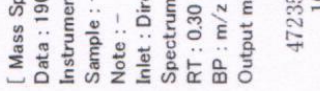

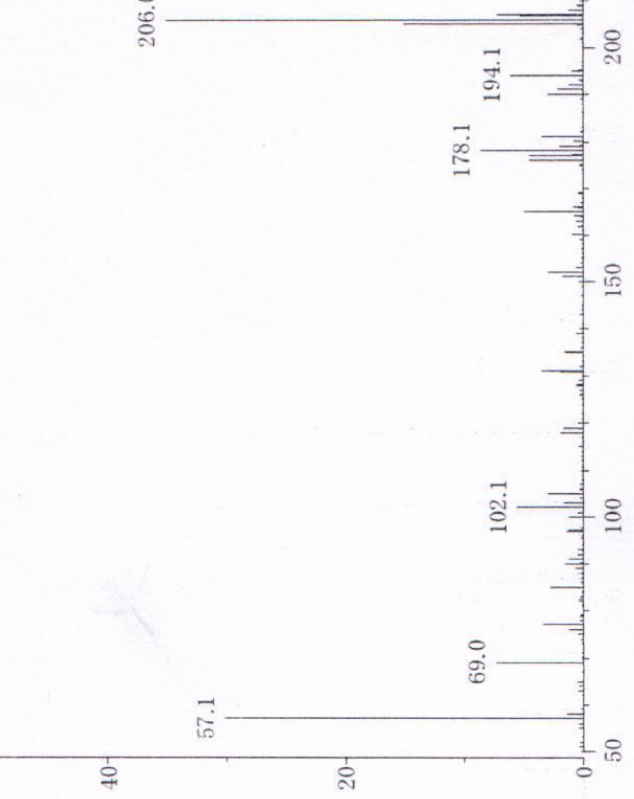

